\title{
"ONE ROTTEN APPLE SPOILS THE WHOLE BARREL" : THE PLANT HORMONE ETHYLENE, THE SMALL MOLECULE AND ITS COMPLEXITY
}

EDITED BY: Domenico De Martinis, Tomotsugu Koyama and Caren Chang PUBLISHED IN: Frontiers in Plant Science

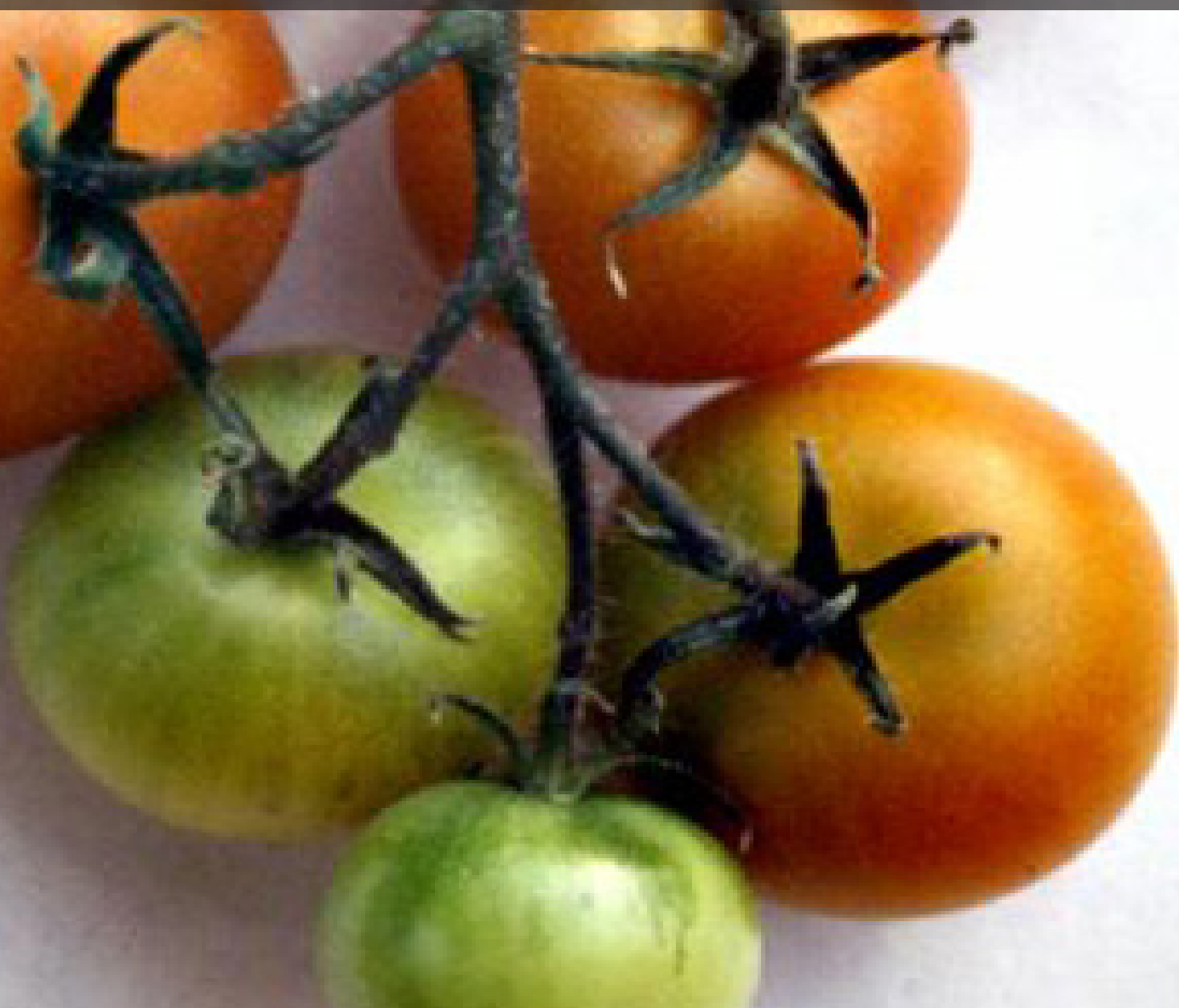

frontiers Research Topics 


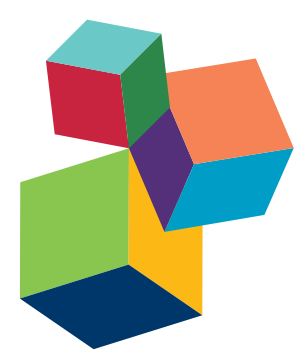

Frontiers Copyright Statement

(c) Copyright 2007-2015 Frontiers Media SA. All rights reserved.

All content included on this site, such as text, graphics, logos, button icons, images, video/audio clips, downloads, data compilations and software, is the property of or is licensed to Frontiers Media SA

("Frontiers") or its licensees and/or subcontractors. The copyright in the text of individual articles is the property of their respective authors, subject to a license granted to Frontiers.

The compilation of articles constituting this e-book, wherever published, as well as the compilation of all other content on this site, is the exclusive property of Frontiers. For the conditions for downloading and copying of e-books from Frontiers' website, please see the Terms for Website Use. If purchasing Frontiers e-books from other websites or sources, the conditions of the website concerned apply.

Images and graphics not forming part of user-contributed materials may not be downloaded or copied without permission.

Individual articles may be downloaded and reproduced in accordance with the principles of the CC-BY licence subject to any copyright or other notices. They may not be re-sold as an e-book.

As author or other contributor you grant a CC-BY licence to others to reproduce your articles, including any graphics and third-party materials supplied by you, in accordance with the Conditions for Website Use and subject to any copyright notices which you include in connection with your articles and materials.

All copyright, and all rights therein, are protected by national and international copyright laws.

The above represents a summary only. For the full conditions see the Conditions for Authors and the Conditions for Website Use.

ISSN 1664-8714 ISBN 978-2-88919-623-4

DOI 10.3389/978-2-88919-623-4

\section{About Frontiers}

Frontiers is more than just an open-access publisher of scholarly articles: it is a pioneering approach to the world of academia, radically improving the way scholarly research is managed. The grand vision of Frontiers is a world where all people have an equal opportunity to seek, share and generate knowledge. Frontiers provides immediate and permanent online open access to all its publications, but this alone is not enough to realize our grand goals.

\section{Frontiers Journal Series}

The Frontiers Journal Series is a multi-tier and interdisciplinary set of open-access, online journals, promising a paradigm shift from the current review, selection and dissemination processes in academic publishing. All Frontiers journals are driven by researchers for researchers; therefore, they constitute a service to the scholarly community. At the same time, the Frontiers Journal Series operates on a revolutionary invention, the tiered publishing system, initially addressing specific communities of scholars, and gradually climbing up to broader public understanding, thus serving the interests of the lay society, too.

\section{Dedication to Quality}

Each Frontiers article is a landmark of the highest quality, thanks to genuinely collaborative interactions between authors and review editors, who include some of the world's best academicians. Research must be certified by peers before entering a stream of knowledge that may eventually reach the public - and shape society; therefore, Frontiers only applies the most rigorous and unbiased reviews.

Frontiers revolutionizes research publishing by freely delivering the most outstanding research, evaluated with no bias from both the academic and social point of view. By applying the most advanced information technologies, Frontiers is catapulting scholarly publishing into a new generation.

\section{What are Frontiers Research Topics?}

Frontiers Research Topics are very popular trademarks of the Frontiers Journals Series: they are collections of at least ten articles, all centered on a particular subject. With their unique mix of varied contributions from Original Research to Review Articles, Frontiers Research Topics unify the most influential researchers, the latest key findings and historical advances in a hot research area! Find out more on how to host your own Frontiers Research Topic or contribute to one as an author by contacting the Frontiers Editorial Office: researchtopics@frontiersin.org 


\section{“ONE ROTTEN APPLE SPOILS THE WHOLE BARREL" : THE PLANT HORMONE ETHYLENE, THE SMALL MOLECULE AND ITS COMPLEXITY}

Topic Editors:

Domenico De Martinis, ENEA, Italian National Agency for New Technologies, Energy and Sustainable Economic Development, Rome, Italy

Tomotsugu Koyama, Suntory Foundation for Life Sciences, Kyoto, Japan

Caren Chang, University of Maryland, College Park, USA

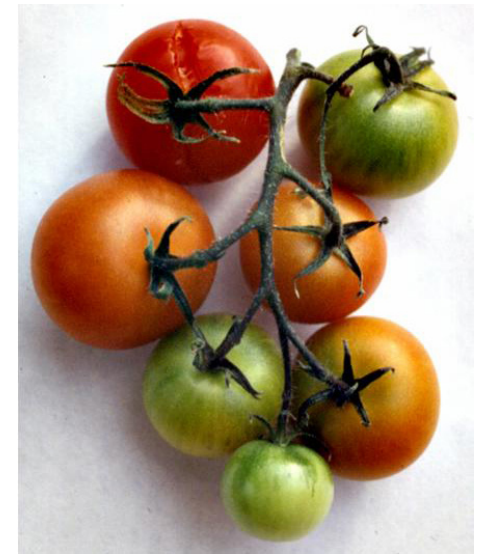

Image taken by Domenico de Martinis.
The gaseous molecule ethylene (C2H4), which is small in size and simple in structure, is a plant hormone most often associated with fruit ripening yet has a diversity of effects throughout the plant life cycle. While its agricultural effects were known even in ancient Egypt, the complexity of its mode of action and the broad spectrum of its effects and potential uses in plant physiology remain important scientific challenges today. In the last few decades, the biochemical pathway of ethylene production has been uncovered, ethylene perception and signaling have been molecularly dissected, ethylene-responsive transcription factors have been identified and numerous effects of ethylene have been described, ranging from water stress, development, senescence, reproduction plant-pathogen interactions, and of course, ripening. Thus ethylene is involved in plant development, in biotic and abiotic stress, and in reproduction. There is no stage in plant life that is not affected by ethylene, modulated by a complex and fascinating molecular machinery.

Citation: De Martinis, D., Koyama, T., Chang, C., eds. (2015). "One Rotten Apple Spoils the Whole Barrel": The Plant Hormone Ethylene, the Small Molecule and its Complexity. Lausanne: Frontiers Media. doi: 10.3389/978-2-88919-623-4 


\section{Table of Contents}

05 Ethylene is all around

Domenico De Martinis, Tomotsugu Koyama and Caren Chang

07 Shedding light on ethylene metabolism in higher plants

Maria A. Rodrigues, Ricardo E. Bianchetti and Luciano Freschi

23 Tomato ACS4 is necessary for timely start of and progression through the climacteric phase of fruit ripening

Suzanne W. Hoogstrate, Lambertus J. A. van Bussel, Simona M. Cristescu, Eric Cator, Celestina Mariani, Wim H. Vriezen and Ivo Rieu

30 Genetic introgression of ethylene-suppressed transgenic tomatoes with higherpolyamines trait overcomes many unintended effects due to reduced ethylene on the primary metabolome

Anatoly P. Sobolev, Anil Neelam, Tahira Fatima, Vijaya Shukla, Avtar K. Handa and Autar K. Mattoo

40 Heterologous over-expression of ACC SYNTHASE8 (ACS8) in Populus tremula x P. alba clone 717-1B4 results in elevated levels of ethylene and induces stem dwarfism and reduced leaf size through separate genetic pathways

Jonathan M. Plett, Martin Williams, Gaetan LeClair, Sharon Regan and Tannis Beardmore

56 The roles of ethylene and transcription factors in the regulation of onset of leaf senescence

Tomotsugu Koyama

64 To grow old: regulatory role of ethylene and jasmonic acid in senescence Joonyup Kim, Caren Chang and Mark L. Tucker

71 1-aminocyclopropane-1-carboxylic acid (ACC) in plants: more than just the precursor of ethylene!

Bram Van de Poel and Dominique Van Der Straeten

82 The role of ethylene and ROS in salinity, heavy metal, and flooding responses in rice

Bianka Steffens

87 Loss of the ETR1 ethylene receptor reduces the inhibitory effect of far-red light and darkness on seed germination of Arabidopsis thaliana

Rebecca L. Wilson, Arkadipta Bakshi and Brad M. Binder

100 An alternate route of ethylene receptor signaling

Jingyi Zhang, Jing Yu and Chi-Kuang Wen 
106 Novel connections and gaps in ethylene signaling from the ER membrane to the nucleus

Young-Hee Cho and Sang-Dong Yoo

113 Ethylene, a key factor in the regulation of seed dormancy

Françoise Corbineau, Qiong Xia, Christophe Bailly and Hayat El-Maarouf-Bouteau

126 Plant-Agrobacterium interaction mediated by ethylene and superAgrobacterium conferring efficient gene transfer

Satoko Nonaka and Hiroshi Ezura 


\title{
Ethylene is all around
}

\author{
Domenico De Martinis $^{1}{ }^{*}$, Tomotsugu Koyama ${ }^{2}$ and Caren Chang ${ }^{3}$ \\ ${ }^{1}$ ENEA Italian National Agency for New Technologies, Energy and Sustainable Economic Development, Rome, Italy \\ 2 Suntory Foundation for Life Sciences, Osaka, Japan \\ ${ }^{3}$ Department of Cell Biology and Molecular Genetics, University of Maryland, College Park, MD, USA \\ *Correspondence: domenico.demartinis@enea.it
}

Edited and reviewed by:

Andreas P. M. Weber, Heinrich-Heine-Universität, Germany

Keywords: ethylene, plant biology, plant developmental biology, fruit ripening, plant reproduction, plant senescence, plant stress biology, plant-microbe interactions

The small and simple gaseous molecule ethylene $\left(\mathrm{C}_{2} \mathrm{H}_{4}\right)$ is usually associated with ripening and senescence events in plants. Its effects have been known for ages (literally), and in the last century there have been some major breakthroughs in ethylene biology. These breakthroughs include the discovery that the molecule that hastened the ripening of fruits and caused growth distortions in plants was indeed ethylene (early 1900's), the demonstration that while generally associated with leaking gas mains, ethylene is produced by plants themselves (1930) and finally, the description of the ethylene biosynthesis pathway by Shang Fa Yang (the "Yang cycle" 1979) (Bradford, 2008).

Yet, it was at the end of the Twentieth century that research on ethylene received massive attention with the understanding of how the ethylene chemical signal is perceived and processed by plants via complex mechanisms and that almost all physiological events in plants are influenced by ethylene.

This is probably why ethylene research in plant science is so broad and active; biosynthesis, perception, signal transduction and mode of action indeed keep scientists from different disciplines very busy.

This activity and breadth in our Frontiers research topic are clearly in evidence with the involvement of scientists describing many of these aspects of ethylene biology.

Metabolism is reviewed by Rodrigues and colleagues (Shedding light on ethylene metabolism in higher plants) describing both ethylene biosynthesis and the mechanisms controlling it, as well as the influence of light on ethylene evolution.

Ethylene biosynthesis and ripening is approached by Hoogstrate and colleagues, analyzing a single ACC synthase gene, ACS4, and demonstrating how ACS4 is necessary for the normal progression of tomato fruit ripening and how mutation of this gene (out 12 ACS genes in the tomato genome) may provide a useful means for altering ripening traits.

The effects of ethylene on the fruit metabolome and the resulting fruit quality are not fully understood. In their study, Sobolev et al. approach the issue, showing that ripening-associated metabolic changes are both ethylene dependent and independent, and that the fruit metabolome is under the control of multiple regulators.

The role of ACS is also described in the model plant, poplar. In their study, Plett et al. describe how ethylene affects the important agronomic trait of plant height. The study also describes the effect of ethylene on leaf senescence, an important biological event that is more broadly described in the reviews by Tomotsugu Koyama and Kim et al. in terms of physiological events and molecular regulation.

The ethylene precursor, ACC itself, also serves as a substrate for several other derivatives. Van de Poel and Van Der Straeten describe the potential role of ACC as more than just the precursor of ethylene but as a means for plants to quickly communicate between organs, whenever ethylene diffusion is too slow.

Besides ripening, numerous other events are regulated by ethylene. Bianka Steffens describes flooding, one of the main abiotic stresses resulting in crop yield loss, a worldwide problem in different landscapes. Some crop species endure soil waterlogging for some hours, others for days or months. Flooding can be associated with water quality changes (salinity, $\mathrm{pH}$, and heavy metal concentration). One metabolic response is the induction of ethylene production and reactive oxygen species (ROS) that together play an important role in mediating numerous specific growth or cell death responses.

Ethylene perception is described in the original research paper by Wilson et al. In their paper, the authors also describe a physiological effect of loss of ethylene perception that reduces the effect of far-red light and darkness on seed germination.

A possible mode of action of signal translation is addressed in the perspective by Zhang et al. and the review by Cho and Yoo, while ethylene effects on germination are reviewed by Corbineau et al.

Last but not least, the important role of ethylene in plantmicrobe interaction with the biotech relevant Agrobacterium is reviewed by Nonaka and Ezura.

The breadth of participation has been as broad as the topic itself. This research topic arrives after approximately 10 months from the initiative of Frontiers in Plant Science to invite us to serve as guest associate editors. During those months we contacted about one hundred scientists worldwide including established experts in the fields as well as "young guns." We eventually published 13 articles involving authors from Europe, North and South America, and Asia. The result is an issue with a broad view that matches and complements the others topics in the "signaling" area.

In this topic, the fascinating biology of ethylene has led us to approach, in modern terms, questions posed about 100 years ago. How many more questions will arise? It must be noted that 
ethylene is also massively produced and widely used in chemical industry and can even be released by humans (Cristescu et al., 2014).

Chemistry and Medicine other than Biology are therefore the domain of research and development for ethylene; and the more we study, the more we discover that ethylene, indeed, is all around.

\section{REFERENCES}

Bradford, K. J. (2008). Shang Fa Yang: pioneer in plant ethylene biochemistry. Plant Sci. 175, 2-7. doi: 10.1016/j.plantsci.2008.01.005

Cristescu, S. M., Kiss, R., te Lintel Hekkert, S., Dalby, M., Harren, F. J. M., Risby, T. H., et al. (2014). Real-time monitoring of endogenous lipid peroxidation by exhaled ethylene in patients undergoing cardiac surgery. Am. J. Physiol. Lung Cell. Mol. Physiol. 307, L509-L515. doi: 10.1152/ajplung.00168
Conflict of Interest Statement: The authors declare that the research was conducted in the absence of any commercial or financial relationships that could be construed as a potential conflict of interest.

Received: 29 January 2015; accepted: 29 January 2015; published online: 12 February 2015.

Citation: De Martinis D, Koyama T and Chang C (2015) Ethylene is all around. Front. Plant Sci. 6:76. doi: 10.3389/fpls.2015.00076

This article was submitted to Plant Physiology, a section of the journal Frontiers in Plant Science.

Copyright (C) 2015 De Martinis, Koyama and Chang. This is an open-access article distributed under the terms of the Creative Commons Attribution License (CC BY). The use, distribution or reproduction in other forums is permitted, provided the original author(s) or licensor are credited and that the original publication in this journal is cited, in accordance with accepted academic practice. No use, distribution or reproduction is permitted which does not comply with these terms. 


\title{
Shedding light on ethylene metabolism in higher plants
}

\author{
Maria A. Rodrigues, Ricardo E. Bianchetti and Luciano Freschi * \\ Laboratory of Plant Physiology, Institute of Biosciences, Department of Botany, University of São Paulo, São Paulo, Brazil
}

\section{Edited by:}

Domenico De Martinis, Italian

National Agency for New

Technologies, Energy and

Sustainable Economic

Development, Italy

Reviewed by:

Vasileios Fotopoulos, Cyprus

University of Technology, Cyprus

Filip Vandenbussche, Ghent

University, Belgium

\section{${ }^{*}$ Correspondence:}

Luciano Freschi, Laboratory of Plant

Physiology, Institute of Biosciences, Department of Botany, University of

São Paulo, Matao Street 277, São

Paulo CEP 05508-090, Brazil

email:freschi@usp.br

\begin{abstract}
Ethylene metabolism in higher plants is regulated by a wide array of endogenous and environmental factors. During most physiological processes, ethylene levels are mainly determined by a strict control of the rate-limiting biosynthetic steps responsible for the production of 1-aminocyclopropane-1-carboxylic acid (ACC) and its subsequent conversion to ethylene. Responsible for these reactions, the key enzymes ACC synthase and ACC oxidase are encoded by multigene families formed by members that can be differentially regulated at the transcription and post-translational levels by specific developmental and environmental signals. Among the wide variety of environmental cues controlling plant ethylene production, light quality, duration, and intensity have consistently been demonstrated to influence the metabolism of this plant hormone in diverse plant tissues, organs, and species. Although still not completely elucidated, the mechanisms underlying the interaction between light signal transduction and ethylene evolution appears to involve a complex network that includes central transcription factors connecting multiple signaling pathways, which can be reciprocally modulated by ethylene itself, other phytohormones, and specific light wavelengths. Accumulating evidence has indicated particular photoreceptors as essential mediators in light-induced signaling cascades affecting ethylene levels. Therefore, this review specifically focuses on discussing the current knowledge of the potential molecular mechanisms implicated in the light-induced responses affecting ethylene metabolism during the regulation of developmental and metabolic plant responses. Besides presenting the state of the art in this research field, some overlooked mechanisms and future directions to elucidate the exact nature of the light-ethylene interplay in higher plants will also be compiled and discussed.
\end{abstract}

Keywords: ACC, ACO, ACS, ethylene, light signaling, photoreceptors, phytochrome

\section{INTRODUCTION}

Light is one of the most influential and versatile environmental stimuli controlling plant life. It varies not only in quantity (fluence) but also in quality (wavelength), periodicity (photoperiod), and direction (unidirectional or diffuse). As photoautotroph and sessile organisms, most higher plants rely on sophisticated and plastic mechanisms to use light as both an energy source and abiotic signal to control decisive developmental adjustments. Not unexpectedly, during evolution plants have evolved a variety of photosensory systems that perceive the light environment and integrate this information into intrinsic developmental programs. Acting at the interface between the external and the internal plant environments, four main families of photoreceptors are responsible for the perception of light signals and their transduction through an array of gene expression modifications that will ultimately lead to adjustments in the plant growth and morphogenic patterns-a sequence of light-triggered processes collectively known as photomorphogenesis (Neff et al., 2000; Ma et al., 2001; Gyula et al., 2003; Franklin et al., 2005; Franklin and Quail, 2010).

During photomorphogenic responses the initial light cue is often translated into changes in the hormonal homeostasis in particular tissues or even throughout the entire plant. Hence, by modulating their hormonal status plants can rapidly adjust growth fitness to constantly changing environments (Li et al., 2004; Achard et al., 2006; Wolters and Jürgens, 2009). The early development of seedlings, which in nature is often skotomorphogenic, and the transition to photomorphogenic growth upon light exposure is one of the best-studied light-controlled processes in eudicotyledons, and it is tightly regulated by an intricate interplay between light signals and plant hormones. In fact, most of the current knowledge about light signaling pathways and ethylene biosynthesis, signal transduction, and response pathways has arisen from experimental approaches based on the etiolated seedling system. The simplicity of the light-induced responses in dark-grown seedlings made it ideal for quickly identifying photomorphogenic mutants in the model plant Arabidopsis thaliana (Liscum and Hangarter, 1993; Stepanova and Ecker, 2000; Li et al., 2004; Stepanova and Alonso, 2005; Chen and Chory, 2011; Boron and Vissenberg, 2014). In addition, the triple response phenotype of etiolated seedlings triggered by ethylene has also been proven as a simple and useful trait to screen for ethylene mutants in the same model plant (Bleecker et al., 1988; Guzman and Ecker, 1990; Lin et al., 2009). 
Ethylene is an important growth regulator of numerous developmental aspects during plant life cycle (e.g., both vegetative and reproductive development, and responses to biotic and abiotic stresses), playing a key role in signaling pathways responsible for adaptive adjustments of plant's fitness in a continuously fluctuating array of environmental signals. Moreover, ethylene biosynthesis is highly regulated by both developmental and external inputs, and stress-induced ethylene production can mediate multiple physiological and morphological responses involved in redirecting all required resources from standard growth to promote plant defense, resistance, resource forage, and/or "escape" mechanisms, including senescence, abscission, and plastic alterations in tissue/organ elongation and shoot-root ratios. However, the variable degree of plasticity observed in such ethylene-mediated responses seems to be dependent on the environmental challenge and species-specific features (Pierik et al., 2006, 2007; Lin et al., 2009; Wolters and Jürgens, 2009).

The development of photomorphogenic and hormonal mutant collections together with a considerable improvement in the experimental methods employed in molecular and genetics research over the last years have paved the way for elegant studies that have begun to shed light on the "black box" of how light signaling controls ethylene production to coordinate plant development. For example, it has been shown that light can modulate ethylene biosynthesis through particular photoreceptor-mediated pathways. Moreover, some transcription factors have been identified as potential integrators for light signaling and ethylene biosynthesis modulation. Furthermore, many regulators of ethylene biosynthesis at both transcriptional (Zhang et al., 2009; Wan et al., 2011; Xiao et al., 2013) and post-transcriptional levels (Wang et al., 2002, 2004; Chae et al., 2003; Liu and Zhang, 2004; Argueso et al., 2007; Joo et al., 2008; Prasad et al., 2010; Wan et al., 2011) have also been documented in the last decades. Besides controlling ethylene biosynthesis, light also influences ethylene signaling and, although not covered in this review, this is also an important aspect that has received increasing attention in the last decades.

Here we have compiled the major controlling mechanisms of both light signaling and ethylene biosynthesis and discussed the latest information on the potential signaling networks connecting both these pathways, with an emphasis on the emerging evidence of molecular mechanisms regulating the transcription and posttranslational modifications of key participants in the ethylene biosynthetic pathway.

\section{LIGHT PERCEPTION AND SIGNALING MECHANISMS}

Light perception and signal transduction are responsible for modulating many processes throughout the plant life cycle and in diverse environmental contexts. Among the different wavelengths detected by the plant photosensory system, red light (RL), blue light (BL), UV-A and UV-B radiation are particularly informative and their perception involves four main families of informationtransducing photoreceptors: the RL-absorbing phytochromes, the UV-A/BL-absorbing cryptochromes and phototropins, and the recently identified UVR8 receptors, which essentially perceive UV-B radiation. These signaling molecules provide the plants with information concerning various aspects of the light environment, thereby playing a vital role in plant survival and optimal growth (Franklin et al., 2005; Franklin and Quail, 2010; Rizzini et al., 2011). Detailed signaling mechanisms triggered by photoreceptors have been the focus of excellent reviews (Quail, 2002; Gyula et al., 2003; Chory, 2010; Chen and Chory, 2011); therefore, only general information about this theme will be provided here.

\section{IDENTITY AND GENERAL FEATURES OF PLANT PHOTORECEPTORS}

Phytochromes are the RL and far-red light (FRL) photoreceptors that play essential roles during plant photomorphogenesis (Chen and Chory, 2011). They are part of a chromoprotein multigene family, which in Arabidopsis is divided into photodegradable (phytochrome A-PHYA) and photostable (phytochrome B, C, D, and E-PHYB, PHYC, PHYD, and PHYE) types. These photoreceptors are formed by the association of one globular apoprotein and one chromophore which confers the properties of activation in response to the RL spectrum and photoreversibility upon FRL exposure (Wagner et al., 2005). The perception of RL is mediated by the chromophore (Wagner et al., 2005), which is synthesized in plastids and combined with the phytochrome apoproteins in the cytosol. Under dark conditions, the chromophore is maintained in a stable-inactive conformation $\left(\mathrm{PHY}_{\mathrm{Pr}}\right)$ whereas the $\mathrm{RL}$ triggers the photoconversion of the inactive state $\mathrm{PHY} P$ into the active form $\left(\mathrm{PHY}_{\mathrm{Pfr}}\right)$, resulting in the phytochrome translocation from the cytoplasm into the nucleus (Ulijasz et al., 2010; Song et al., 2011; Figure 1). Despite the relatively wellconserved structure, PHYA and PHYB have different response patterns in terms of light stimulation and action mechanisms. PHYA-mediated responses are mainly triggered by FRL and this phytochrome does not show photoreversibility (Quail, 2002). Once in the active form, PHYA $\mathrm{Pfr}$ associates with the proteins FAR RED ELONGATED HYPOCOTYL (FHR) and FHR-LIKE (FHL) and transiently accumulates in the nucleus (Kircher et al., 2002; Pfeiffer et al., 2012). On the other hand, PHYB is primarily activated by RL, presents R/FR photoreversibility, and accumulates in the nucleus for longer periods than PHYA (Gil et al., 2000). Furthermore, distinct members of the PHY family can display a certain degree of organ specificity in response to RL (Tepperman et al., 2004).

The cryptochromes (CRY) are nuclear proteins that primarily respond to BL and UV-A radiation (Ahmad and Cashmore, 1997) and present an intricate interaction with phytochromes during the control of numerous photomorphogenic responses (Guo et al., 2001; Facella et al., 2008). In Arabidopsis, cryptochrome 1 (CRY1) is photostable and acts mainly at high light fluence (Yang et al., 2000; Herbel et al., 2013) whereas cryptochrome 2 (CRY2) is photodegradable and plays an important role at relatively lower light fluence (Lin et al., 1998; Guo et al., 2001; Gyula et al., 2003). CRY1/2 phosphorylation and subsequent molecular modifications are triggered soon after BL/UV-A exposure, facilitating the CRY interaction with other proteins (Kondoh et al., 2011). Such BL/UV-A-driven modifications in the CRY molecules can be reversed in complete darkness or upon exposure to green radiation (Sellaro et al., 2010).

In contrast, phototropins 1 and 2 are membrane-localized photoreceptors that undergo auto-phosphorylation in response 


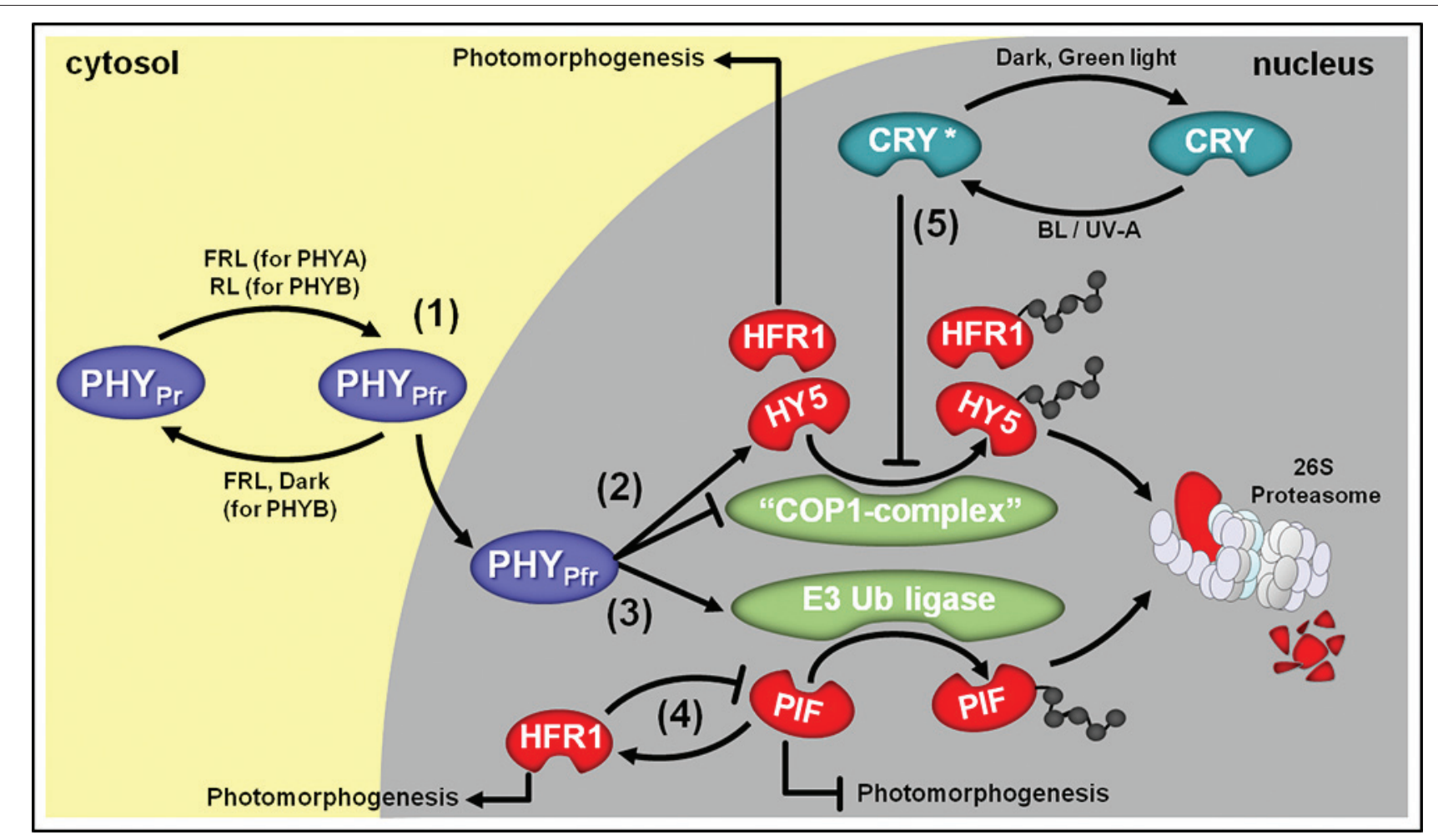

FIGURE 1 | Simplified overview of light signal transduction via phytochromes (PHY) and cryptochromes (CRY) and their main interacting proteins. (1) Upon far-red light (FRL) or red light $(R L)$ irradiation, cytosolic PHYAPr and $\mathrm{PHYB}_{\mathrm{Pr}}$, respectively, are converted into their active Pfr forms, which migrate to the nucleus. (2) In the cell

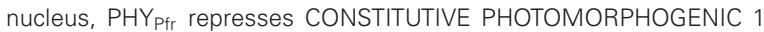
(COP1) E3-complex ("COP1 complex"), which is responsible for targeting positive components of the light signal transduction pathway such as LONG HYPOCOTYL 5 (HY5) and LONG HYPOCOTYL IN FAR-RED 1 (HFR1) to proteasomal degradation. The expression of genes coding for HY5 and HFR1 is also promoted by $\mathrm{PHY}$ Pfr and these transcription factors are responsible for stimulating the expression of numerous photomorphogenesis-associated genes. (3) In parallel, $\mathrm{PHY} \mathrm{Pfr}_{\mathrm{fr}}$ activates an unidentified E3 Ubiquitin (Ub) ligase responsible for targeting light signal transduction repressor proteins such as PHYTOCHROME-INTERACTING FACTORS (PIFs) to degradation via 26S proteasome. (4) PIFs are also known to stimulate HFR1 and HFR1 blocks PIFs transcriptional activity. (5) Upon blue light (BL) or UV-A exposure, nuclear-localized CRY is converted into active $\mathrm{CRY}^{*}$, which also repress "COP1 complex" action. to BL/UV-A radiation (Christie, 2007) and play an important role in many phototropic responses in plants (Kinoshita et al., 2001; Sakai et al., 2001). In the case of the UVR8 receptors, the perception of UV-B radiation occurs via tryptophan residues present at the protein homodimeric interface which leads to the monomerization and subsequent association of the UVR8 monomers with CONSTITUTIVE PHOTOMORPHOGENESIS 1 (COP1), which functions as an E3 ubiquitin ligase in multiple protein complexes and represents a master repressor during light signaling cascades. Such protein interaction will ultimately result in differential gene expression (Rizzini et al., 2011; Wu et al., 2012), and its potential outputs will be discussed later in the context of light signaling mechanisms controlling the transcription of genes involved in ethylene biosynthesis.

\section{HUB TRANSCRIPTION FACTORS UNDER PHYTOCHROME CONTROL}

Downstream of the RL perception, an increasing number of transcription factors has been identified as central integrators of light and multiple internal signals to optimize plant development. A key mechanism by which PHYs regulate gene expression is by modulating the protein stability of target transcription factors in the nucleus. One of the most important $\mathrm{PHY}_{\mathrm{Pfr}^{-}}$ mediated signaling pathways relies on the regulation of a multigene family of basic helix-loop-helix transcription factors known as PHYTOCHROME INTERACTION FACTOR (PIF). Accordingly, under inductive light conditions $\mathrm{PHYA}_{\mathrm{Pfr}}$ and/or $\mathrm{PHYB}_{\mathrm{Pfr}}$ migrates to the nucleus, where they can physically interact with PIF proteins and promote their ubiquitination and subsequent degradation via proteasome 26S (Figure 1). Under continuous darkness, PIF proteins inhibit the transcription of many genes associated with photomorphogenic responses; therefore, PHY activation and migration to the nucleus followed by its positive influence on PIF degradation represents a major point of transcriptional control of such genes under PIF-dependent inhibition (Ni et al., 1999; Khanna et al., 2004; Shen et al., 2007; Leivar et al., 2008; Lorrain et al., 2008; Chen and Chory, 2011). Besides playing a central role during the scoto-to-photomorphogenesis transition, PIFs are also involved in many developmental processes triggered by low R/FR conditions, such as shade avoidance responses, during which they control the transcription of genes coding for 
important proteins such as PIF3-LIKE 1 (PIL-1) and ARABIDOPSIS THALIANA HOMEOBOX PROTEIN 2 (ATHB2), and some components of the biosynthetic routes of plant hormones such as auxins, gibberellin, and ethylene (Martínez-Garcia et al., 2000; Leivar et al., 2009).

Active $\mathrm{PHYA}_{\mathrm{Pfr}}$ and $\mathrm{PHYB}_{\mathrm{Pfr}}$ are also known to increase the levels of LONG HYPOCOTYL 5 (HY5), which is another crucial transcription factor associated with light signaling and several photomorphogenic responses in plants. Hence, under continuous darkness HY5 undergoes very rapid destabilization mediated by COP1 (Gyula et al., 2003; Chory, 2010; Boron and Vissenberg, 2014). In fact, COP1 usually acts in association with other regulatory proteins ("COP1 complex") promoting the ubiquitindependent degradation of both HY5 and PHYs, thereby representing an important element in the post-translational regulation of the PHY-dependent signaling cascades. In an intricate crosstalk with PHY, CRY also plays a fundamental role in the stabilization of HY5 since both CRY1 and CRY2 have been shown to increase the half-life of this protein by directly interacting with COP1 and promoting its destabilization or removal from the cell nucleus (Figure 1), which ultimately leads to an extended window of time for the stimulatory action of HY5 on the transcription of genes associated with photomorphogenic responses. Moreover, CRY also increases the half-life of PHYA by destabilizing the COP1 complex (Schwechheimer and Deng, 2000; Gyula et al., 2003; Chory, 2010; Fankhauser and Ulm, 2011; Boron and Vissenberg, 2014).

\section{INFLUENCE OF DIVERSE LIGHT REGIMES ON ETHYLENE EVOLUTION}

Light influence on ethylene evolution rate has been consistently demonstrated in several plants and can either stimulate or inhibit ethylene production depending on the tissue, organ, species, plant developmental phase, and the nature of light signal (Abeles et al., 1992; Corbineau et al., 1995; Halliday and Fankhauser, 2003; Kurepin et al., 2010). For example, European production of lilies can be hampered by long periods of low light, which induce higher production of ethylene, causing the abscission of developing flower buds (Abeles et al., 1992). On the other hand, potato plants treated with continuous light for faster tuber development displayed up to 15-fold increase in ethylene production, while ethylene levels rapidly dropped when these plants were transferred back to the $12 \mathrm{~h}$ light/dark photoperiod (Wheeler and Tibbitts, 1986). Despite the physiological response, most lighttriggered adjustments in ethylene production seem to be mainly under PHY and CRY control (Vandenbussche et al., 2003; Kurepin et al., 2010).

$\mathrm{PHY}_{\mathrm{Pfr}}$-mediated signaling usually represses ethylene emission in several monocotyledons and eudicotyledons, as reported for etiolated seedlings of pea (Pisum sativum; Goeschl et al., 1967; Kang and Burg, 1972; Steed et al., 2004), light-grown seedlings of sorghum (Sorghum bicolor; Finlayson et al., 1998, 1999), coleoptiles and apical segments of etiolated rice (Oryza sativa) seedlings (Imaseki et al., 1971), light-grown leaves of both oat (Avena sativa; Corbineau et al., 1995) and wheat (Triticum aestivum; Jiao et al., 1987), and adult plants of both tobacco (Nicotiana tabacum; Pierik et al., 2004a) and Arabidopsis thaliana (Vandenbussche et al., 2003; Bours et al., 2014). Given its important role in
RL perception and signaling, PHYB is the photoreceptor more closely associated with the negative regulation of ethylene levels in several plant models (Finlayson et al., 1998; Vandenbussche et al., 2003). In addition, the inhibitory effect of RL on ethylene emission depends on plant light exposure duration, radiation fluence (Imaseki et al., 1971; Pierik et al., 2004b; Kurepin et al., 2010), and some light-evoked adjustments on the plant circadian clock (Finlayson et al., 1998). However, in some plant systems PHYA also seems to play a prominent role in repressing ethylene production by direct action and/or via CRY1 repression, as reported for de-etiolating phyAphyB mutant pea seedlings (Foo et al., 2006).

\section{CENTRAL METABOLIC INTERMEDIATES AND ENZYMES IN ETHYLENE BIOSYNTHESIS}

Ethylene is a relatively simple unsaturated two-carbon gas which can be produced by numerous non-biological chemical reactions catalyzed by heat, oxidation, light, or ionizing radiation. In fact, non-biological model systems were systematically employed during the initial search for metabolic components and steps involved in ethylene production (Yang and Hoffman, 1984; Abeles et al., 1992). Such approaches led to the unexpected discovery that ethylene could be chemically generated from the amino acid methionine and its derivatives (Yang et al., 1966). Following this breakthrough, significant advances were achieved in ethylene research with biological systems, providing the basis of knowledge regarding the ethylene biosynthetic pathway (Yang and Hoffman, 1984; Abeles et al., 1992; Kende, 1993).

Ethylene biosynthesis in higher plants is now well characterized by a relatively simple metabolic pathway, which is, however, coordinated with some other equally important synthetic pathways involved in the plant metabolism regulation (e.g., polyamines). The identification of the intermediate components in ethylene biosynthesis allowed further elucidation of the two committed reactions in this pathway, which comprise the rate-limiting enzymes 1-aminocyclopropane-1-carboxylic acid (ACC) synthase (ACS, EC 4.4.1.14) and ACC oxidase (ACO, EC 1.4.3; Yang and Hoffman, 1984; Kende, 1993; Zarembinski and Theologis, 1994; Argueso et al., 2007; McClellan and Chang, 2008; Harpaz-Saad et al., 2012). Both ACS and ACO are encoded by multigene families whose members have been well characterized in some plant species and they are recognized as major players in ethylene biosynthetic regulation. However, as we will discuss in more detail below, the regulatory mechanisms of ethylene biosynthesis usually converge on the modulation of ACS proteins (Fluhr and Mattoo, 1996; Wang et al., 2002).

\section{YANG CYCLE AND SYNTHETIC PATHWAYS ASSOCIATED WITH ETHYLENE PRODUCTION}

Ethylene biosynthesis in planta starts with the conversion of L-methionine to $S$-adenosyl methionine (AdoMet or SAM) in a reaction catalyzed by the enzyme L-methionine-Sadenosyltransferase (SAM synthetase, EC 2.5.1.6; Figure 2). The subsequent conversion of SAM to ACC is catalyzed by ACS and is generally considered the first committed and ratelimiting step in ethylene biosynthesis. The following crucial step in this pathway is catalyzed by ACO in a reaction that 


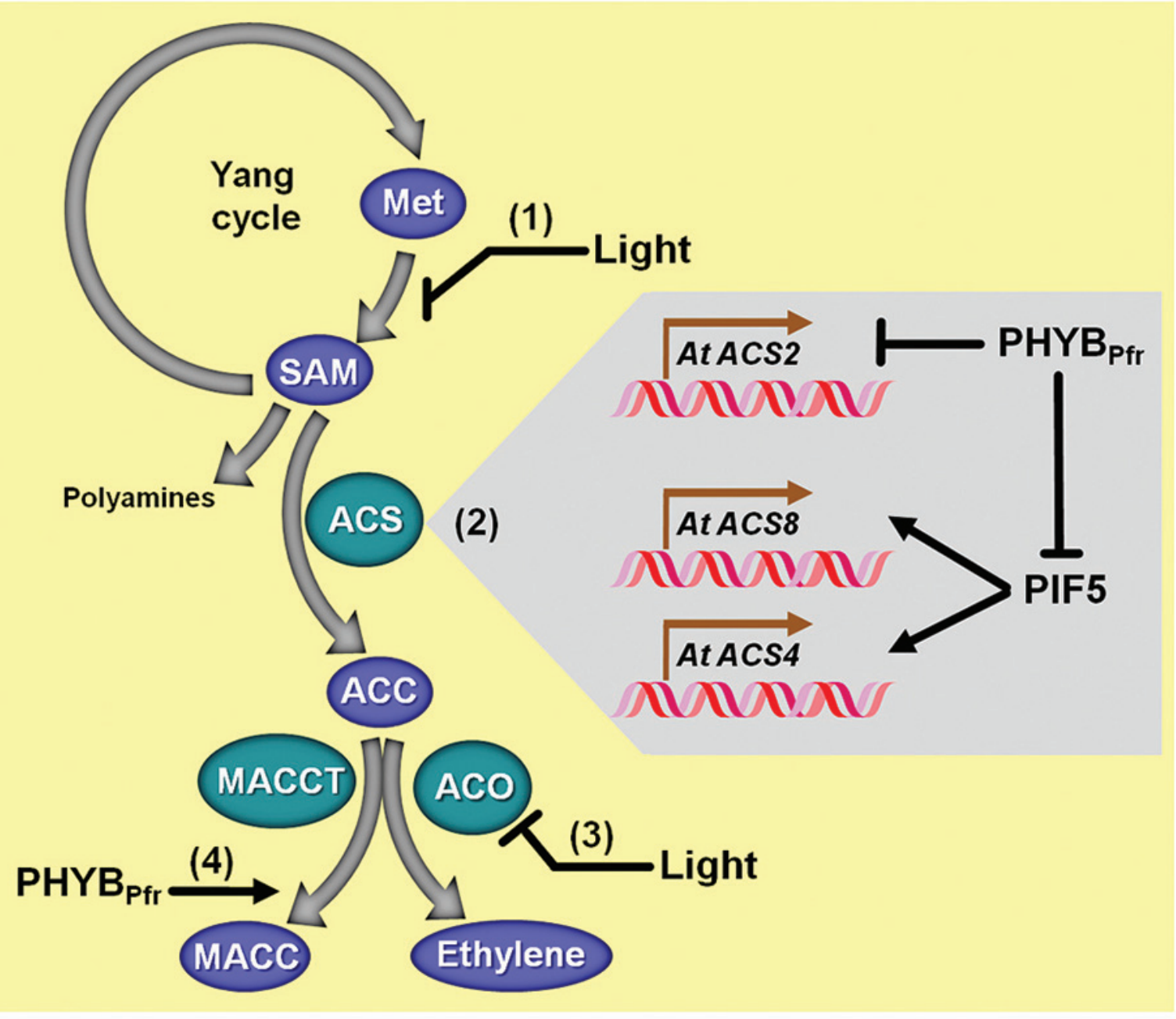

FIGURE 2 | Schematic representation of light-evoked impacts on ethylene biosynthetic pathway. (1) Light irradiation negatively impacts the conversion of methionine (Met) to S-adenosyl methionine (SAM). (2) Light also affects the transcription of particular 1-aminocyclopropane 1-carboxylic acid (ACC) synthases (ACS), responsible for converting SAM into ACC. In Arabidopsis, whereas ACS2 gene transcription is negatively controlled by $\mathrm{PHYB}_{\mathrm{Pfr}}, A C S 8 / 4$ gene transcription is stimulated by PIF5, and, accordingly, PIF5 protein stability is negatively influenced by active phytochrome $B$
$\left(\mathrm{PHYB}_{\mathrm{Pfr}}\right)$. (3) Light is also known to inhibit the transcription and activity of ACC oxidase (ACO), whose activity converts $A C C$ into ethylene. (4) $\mathrm{PHYB} P f$ also stimulates the conversion of ACC into the non-volatile ACC metabolite 1-malonyl aminocyclopropane-1-carboxylic acid (MACC) via MACC transferase (MACCT). Biosynthetic enzymes and metabolic substrates or products are represented with green and blue ovals, respectively. Light-dependent transcriptional control of particular ACS genes is highlighted by the gray area of the figure. converts ACC to ethylene, $\mathrm{CO}_{2}, \mathrm{HCN}$, and $\mathrm{H}_{2} \mathrm{O}$ (Adams and Yang, 1977, 1979; Lürssen et al., 1979; Yang and Hoffman, 1984; McKeon and Yang, 1987; Argueso et al., 2007; Lin et al., 2009). Behind this relatively linear sequence of few reactions hides a fundamental metabolic mechanism that prevents potential depletion of the methionine pools when a high rate of ethylene production is required. Such metabolic detail is significant because methionine, the biological precursor of ethylene, is a very scarce sulfur-containing amino acid and the amounts of sulfur available are usually limited in plants. In this sense, the methylthio-group from methionine needs to be recycled after SAM production. This is possible because the conversion of SAM to ACC by ACS also produces $5^{\prime}$-methylthioadenosine
(MTA) as a by-product that retains the reduced methylthiogroup and is readily recycled back to methionine through the Yang cycle. Therefore, the Yang cycle facilitates the occurrence of high rates of ethylene biosynthesis without influencing the steady-state levels of methionine pool (Yang and Hoffman, 1984; Miyazaki and Yang, 1987; Argueso et al., 2007; Zheng et al., 2013).

Besides being the direct precursor of ethylene, ACC is also suggested as a cell-signaling molecule per se. ACC is a soluble molecule that seems to be translocated throughout different plant organs, and its translocation within flowers has been suggested to play an important role in floral senescence (Abeles et al., 1992; Harpaz-Saad et al., 2012; Yoon and Kieber, 2013a). Hence, 
it appears logical that most tissues can actively regulate the endogenous ACC levels during plant development. This function is mainly controlled by ACS and ACO; however, the cellular pool of ACC can be also modulated by other metabolic reactions leading to its conjugation (Yang and Hoffman, 1984; McKeon and Yang, 1987; Martin and Saftner, 1995; Peiser and Yang, 1998; Staswick and Tiryaki, 2004; Kombrink, 2012). Accordingly, ACC can be converted into ACC derivates, such as jasmonic acid-ACC (JA-ACC), $\gamma$-glutamyl-ACC (GACC), and 1-(malonylamino) cyclopropane-1-carboxylate (MACC). Both GACC and JA-ACC comprise minor moieties in the pool of ACC derivates, whose potential biological functions still remain poorly characterized. In this sense, the physiological significance of such derivates might be underestimated (Staswick and Tiryaki, 2004; Kombrink, 2012). Conversely, MACC formation seems to be a metabolic sink that allows depletion of ACC levels under certain conditions. This reaction is catalyzed by ACC- $N$-malonyltransferase (MACCT; Figure 2), an enzyme that displays increased activity during late stages of tomato fruit ripening (Yang and Hoffman, 1984). Some hypotheses suggest MACC as an end metabolite derived from ACC that can be easily accumulated (Yang and Hoffman, 1984; McKeon and Yang, 1987), while others consider MACC as a means for temporary storage of ACC in a non-reactive form which could be hydrolyzed back to ACC when needed for ethylene production (Hoffman et al., 1982; Jiao et al., 1986; Hanley et al., 1989).

\section{LIGHT SIGNALING ON METABOLIC REGULATION OF THE ETHYLENE PRECURSOR LEVELS}

One of the mechanisms involved in the $\mathrm{PHY}_{\mathrm{Pfr}}$-dependent regulation of ethylene production in plants precisely relies on regulating the abundance of the immediate ethylene precursor ACC. Although still not fully characterized at the molecular level, the active form $\mathrm{PHYB}_{\mathrm{Pfr}}$ has been shown to promote the rapid conjugation of ACC into MACC during the light-induced seedling de-etiolation of wheat, thereby decreasing the internal ACC pool available for ethylene formation (Jiao et al., 1987; Figure 2). Interestingly, these events occurred without any modification in the extractable MACCT activity, indicating that light might have exerted its inductive effects by a mechanism other than increasing the MACCT levels (Jiao et al., 1987). Such mechanism could represent some potential post-translational control of MACCT yet unknown.

\section{POST-TRANSLATIONAL MECHANISMS CONTROLLING ETHYLENE BIOSYNTHESIS}

Both ACS and ACO family members are suggested to be at certain degree under post-translational regulation by proteasomemediated degradation (Chen et al., 2005). However, particular attention has been devoted to unraveling the molecular structure and regulatory mechanisms of ACS proteins in Arabidopsis. For this reason, the following topics will mainly focus on discussing the central mechanisms involved in both transcriptional and post-translational control of ACS members in this plant model. Likewise, some evidence on ACS and ACO regulation in other species will be also comparatively discussed when opportune.
Briefly, ACS proteins belong to the $\alpha$-superfamily of pyridoxal5 -phosphate-dependent enzymes, which are represented in Arabidopsis by eight active members differentially regulated by several developmental and environmental signals. In addition to these active ACS enzymes, the Arabidopsis genome encodes an additional ACS (ACS1) that is catalytically inactive (Yamagami et al., 2003; Tsuchisaka and Theologis, 2004). Based primarily on the C-terminal sequence domains, ACS proteins are usually classified into three main groups which depict distinct regulatory features affecting the stability of the respective ACS proteins (Chae and Kieber, 2005; Yoshida et al., 2005). In Arabidopsis, type I ACS proteins (ACS2 and ACS6) have an extended C-terminus that contains phosphorylation sites (four conserved serine residues) targeted by mitogen-activated protein kinases (MPK) and calcium-dependent protein kinases (CDPK; Figure 3). Type II group includes isoforms (ACS4, ACS5, ACS8, and ACS9) with a shorter C-terminus that only contains a putative CDPK target site. Type III class is represented by one single member, ACS7, which has a very short C-terminal domain with no recognized phosphorylation sites (Tatsuki and Mori, 2001; Sebastià et al., 2004; Liu and Zhang, 2004; Chae and Kieber, 2005). Interestingly, this criterion of ACS classification has also been used for tomato ACS enzymes (Yoshida et al., 2005, 2006), indicating a general degree of structural conservation within ACS family. However, tomato encodes 10 LeACS isoenzymes with particular functional patterns (Yoshida et al., 2005, 2006).

\section{ACS PHOSPHORYLATION}

Several experimental approaches have consistently suggested an important role for ACS phosphorylation and dephosphorylation during ethylene biosynthesis (Spanu et al., 1994; Tuomainen et al., 1997; Zhong and Burns, 2003), which regulates protein stability in an ACS isoform-dependent manner (Joo et al., 2008; Hahn and Harter, 2009; Figure 3). Genetic and biochemical studies have indicated CDPK and/or MPK as important molecules that cooperatively regulate stability of most ACS proteins and thus ethylene production, in response to developmental and environmental stimuli (Tatsuki and Mori, 2001; Liu and Zhang, 2004; Huang et al., 2013). As discussed previously, particular residues at the Cterminal domain of different isoforms of ACS represent putative target sites for phosphorylation, with MPK usually targeting three Ser residues that are distinct from the CDPK target site in the Cterminal extended region of a subset of ACS isoenzymes (Liu and Zhang, 2004; Sebastià et al., 2004; Joo et al., 2008; Huang et al., 2013).

Although very little information is available on CDPK signaling mechanisms during ACS regulation, it is widely accepted that the MPK3/6 module of MPK kinase (MKK) cascades plays relevant roles in the regulation of ethylene biosynthesis (Liu and Zhang, 2004; Joo et al., 2008; Xu et al., 2008; Hahn and Harter, 2009; Ju and Chang, 2012). Accordingly, the MPK3/6 module is mostly under control of MKK4, MKK5, and MKK9, constituting an important step in various signaling pathways involved in stressinduced responses in plants (Xu et al., 2008; Yoo et al., 2008; Beckers et al., 2009). For example, the stress-activated MKK4/5 signaling cascade can positively regulate ethylene biosynthesis by 


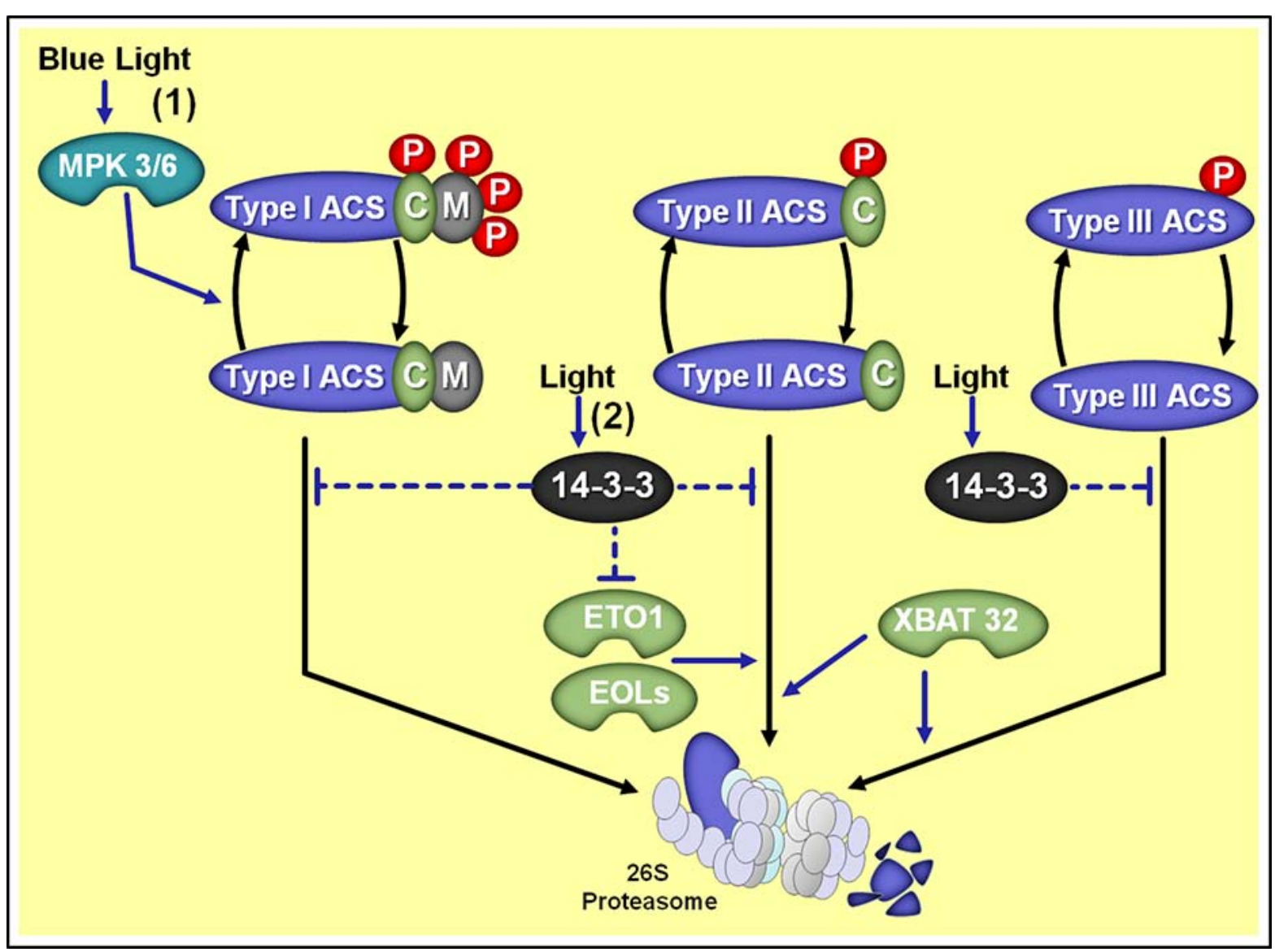

FIGURE 3 | Overview of the distinct regulatory mechanisms controlling stability of type I, II, and III 1-aminocyclopropane 1-carboxylic acid synthases (ACS) and their regulation by light. (1) Blue light stimulates mitogen-activated protein kinases 3 and 6 (MPK 3/6) which in turn phosphorylate type I ACSs, thereby preserving ACSs from $26 \mathrm{~S}$

proteasome-mediated degradation. (2) Light also stimulates 14-3-3 proteins which influence ACSs protein stabilization by both direct protein interaction and destabilization of ETHYLENE-OVERPRODUCER1 (ETO1) and ETO1/2-LIKE (EOL1/2) responsible for targeting type II ACS proteins for proteasomal degradation. Moreover, the RING-type E3 ligase XBAT32 affects both type II and III ACSs degradation. Protein phosphorylation is represented with red ovals and " $P$ " letters. Phosphorylation sites targeted by calcium-dependent protein kinases (CDPK) are represented with green ovals and "C" letters. Phosphorylation sites targeted by MPK are represented with gray ovals and " $M$ " letters. Note that type I ACS have both CDPK ("C") and MPK ("M") phosphorylation sites, type II ACS lacks only the MPK ("M") phosphorylation site and type III ACS lacks both phosphorylation sites activating MPK6 (Liu and Zhang, 2004). In addition, ACS6 was initially identified as a potential substrate for MPK3/6 phosphorylation (Feilner et al., 2005) and further studies have confirmed that the MPK3/6 module is associated with enhanced stability of both type I ACS2 and ACS6 (Liu and Zhang, 2004; Joo et al., 2008; Figure 3). Interestingly, the autocatalytic ethylene production is often stress related and at least in Arabidopsis relies on post-translational regulation of type I ACSs by MPK3/6 cascade (Vandenbussche et al., 2012). Furthermore, the MKK9-MPK3/6 module is also suggested as a potential regulatory mechanism of ACS2/6 stabilization in some particular biological contexts (Popescu et al., 2009), supporting previous suggestions of the possible role of MKK9 and MPKs in ethylene biosynthesis control (Joo et al., 2008; Xu et al., 2008).

Therefore, ACS phosphorylation appears to be a major mechanism controlling the enzyme activity by increasing protein stability (Tsuchisaka and Theologis, 2004; Chae and Kieber, 2005;
Argueso et al., 2007). Besides, tobacco plants under stressful conditions display a rapid increase in ethylene production mediated by an active NtMEK2 (MPKK)/SIPK (MPK) cascade that triggers a dramatic increase in ACS activity, which is followed by the transcriptional activation of a subgroup of ACS and ACO genes (Kim et al., 2003). As discussed in the following sections, recent data also suggest that phosphorylation might increase ACS affinity to directly interact with other proteins that influence ACS stability (Yoon and Kieber, 2013b).

\section{ACS DEGRADATION}

As previously discussed, the ubiquitin-26S proteasome has been linked to diverse functions in plants, including hormone signaling, photomorphogenesis, and stress-triggered responses (Yi and Deng, 2005; Stone and Callis, 2007; Vierstra, 2009; Santner and Estelle, 2010). In fact, many aspects of ethylene biosynthesis are also highly regulated at the post-transcriptional 
level by degradation of proteins controlled by the ubiquitin-26S proteasome system. Genetic studies in Arabidopsis have begun to reveal a number of ethylene biosynthetic enzymes which are targeted for proteasomal degradation, including the type II ACS proteins and the type III ACS7 (Wang et al., 2004; Yoshida et al., 2005; Christians et al., 2009; Lyzenga et al., 2012). Although not fully understood, ample evidence indicates that protein phosphorylation preserves ACS proteins from $26 \mathrm{~S}$ proteasome-mediated degradation (Figure 3), providing a direct and rapid mechanism to change ethylene production (Spanu et al., 1994; Kelley and Estelle, 2012).

Details on post-translational regulation of ACS proteins have been derived from analyses of the ethylene-overproducing (eto13) mutants of Arabidopsis. Accordingly, dark-grown eto seedlings display the triple-response phenotype even in the absence of exogenous ethylene application and produces 10- to 40-fold more ethylene in the dark than the wild-type seedlings (Guzman and Ecker, 1990; Woeste et al., 1999; Chae et al., 2003; Wang et al., 2004). The increased ethylene biosynthesis in eto 1 and eto 2 mutations results from two different modifications at the C-terminal region of ACS5 isoform, while eto3 mutation is the result of a single amino acid change at the C-terminal region of ACS9 (Chae et al., 2003; Chae and Kieber, 2005). Such mutations were identified to cause disruptions within the TOE (for target of ETO1) domain of these ACS, which is recognized as a target site for ubiquitin-26S proteasome degradation. In fact, ETHYLENEOVERPRODUCER1 (ETO1)/ETO1/2-LIKE (EOL1/2) genes code for CULLIN-3 E3 ubiquitin ligases which recognize and directly interact with the TOE domain of type II ACS proteins, targeting them for rapid degradation via 26S proteasome (Vogel et al., 1998; Chae et al., 2003; Wang et al., 2004; Yoshida et al., 2005, 2006; Christians et al., 2009; Figure 3). Additionally, the stability of both ACS7 (type III) and ACS4 (type II) was recently found to be turned over in a $26 \mathrm{~S}$ proteasome-dependent manner through the participation of a RING-type E3 ligase named XBAT32 (Lyzenga et al., 2012), which also negatively modulates the abundance of ACS proteins and ethylene biosynthesis (Prasad and Stone, 2010; Prasad et al., 2010; Lyzenga et al., 2012). As the type III ACS7 lacks a C-terminal extension with the TOE sequence (Lyzenga et al., 2012), XBAT32 seems to represent a still unknown TOE-independent mechanism controlling ACS stability.

Adding an extra level of complexity in the ubiquitin-26S proteasome-dependent ACS stability control, recent findings revealed that ACS stability is also affected by ACS interaction with 14-3-3 proteins (Yoon and Kieber, 2013c; Figure 3). In this sense, 14-3-3 seems to interact with multiple isoforms from all three classes of ACS proteins, and this interaction increases the stability of the ACS proteins (Yoon and Kieber, 2013b). The molecular mechanism behind such regulation appears to involve the direct interaction of 14-3-3 with ACS proteins by decreasing their degradation by a still unknown ETO1/EOL-independent mechanism. These findings support the previous hypothesis that there is at least one further system acting to degrade type II ACS proteins in addition to the ETO1/EOLs, and the 14-3-3 proteins seem to antagonize this second degradation pathway (Lyzenga et al., 2012; Yoon and Kieber, 2013c). However, 14-3-3 proteins also seem to facilitate increased ACS stability by their interaction with the ETO1/EOLs E3 ligases which down-regulates their stability, thus increasing their degradation in an ubiquitin/proteasomedependent manner and, consequently, decreasing the abundance of the ubiquitin ligases that target a subset of ACS proteins for degradation (Yoon and Kieber, 2013b).

In rice, the type II OsACS1 can interact with 14-3-3 proteins and the C-terminal domain of this enzyme was presumably phosphorylated by OsCDPK on a 14-3-3 recognition motif, suggesting that the phosphorylated OsACS1 may interact with rice 14-3-3 proteins, thus preventing ETO1 from binding to OsACS1 and induce this enzyme degradation (Yao et al., 2007). Therefore, such a regulatory mechanism might explain why after CDPKdependent phosphorylation ACS proteins display increased protein stability (Yao et al., 2007; Yoon and Kieber, 2013c). Moreover, some evidence has indicated that 14-3-3 proteins interacted with $\mathrm{ACO} 2$ and $\mathrm{ACO} 4$ proteins in a yeast two-hybrid system, thus indicating that 14-3-3 proteins may regulate the ethylene biosynthesis pathway by modulating both ACS and ACO proteins (Huang et al., 2013).

\section{LIGHT SIGNALING AND THE POST-TRANSLATIONAL CONTROL OF ACS AND ACO PROTEINS}

Recently, Yoon and Kieber (2013c) reported that etiolated seedlings of Arabidopsis submitted to light treatment present a rapid (within $2 \mathrm{~h}$ ) increase in the levels ACS5 proteins without corresponding changes in ACS5 transcripts, suggesting that light stimuli act by increasing ACS5 stability in this plant system. Accordingly, light treatment triggers the opposite effect on EOL2 protein levels, which exhibit a significant and rapid reduction as soon as $2 \mathrm{~h}$ after light exposure. These authors have suggested that the mechanism by which 14-3-3 proteins control ACS stability could also be regulated by light stimuli; however, such hypothetical connection remains uncertain. Furthermore, some evidence indicates that the particular isoform $\mathrm{SbACO} 2$ from sorghum might show a light-regulated post-transcriptional regulation throughout the diurnal cycle. This suggestion was based on the observation that diurnal fluctuations in SbACO2 transcript abundance were translated into diel changes in enzymatic activity under unshaded environment, but not under simulated highshade conditions (Finlayson et al., 1999). Additionally, recent findings have shown significant connections between MPK and light signaling pathways in plants which indicate the MKK3MPK6 cascade being actively regulated by BL signaling at several levels (Sethi et al., 2014). Therefore, it is tempting to speculate that MKK3-MPK6-dependent cascades might possibly be implicated in the interplay between light signaling and ACS posttranslational control (Figure 3).

\section{TRANSCRIPTIONAL MECHANISMS CONTROLLING ETHYLENE BIOSYNTHESIS}

Transcriptional regulation of ACS and ACO genes is one of the pivotal mechanisms controlling ethylene biosynthesis (Wan et al., 2011). As previously discussed, although regulatory mechanisms affecting ACS activity are generally considered the crucial regulatory point for ethylene production, increasing evidence has indicated that modulation of $A C O$ expression can also represent 
a significant point of control for ethylene production under particular circumstances (Rudus et al., 2013; Xiao et al., 2013). Accordingly, a number of studies have reported that both ACS and ACO family members can be differentially expressed in diverse plant organ/tissues, distinct developmental phases, and in response to different environmental stimuli (Liang et al., 1992; Kende, 1993; Zarembinski and Theologis, 1994; Vahala et al., 1998; Wang et al., 2002; Argueso et al., 2007). Differences in the expression levels of each ACS and ACO genes might be an important means for adjusting differential ethylene production within a particular organ or plant tissue. For instance, the ACS multigene family of Arabidopsis has a prominent member-specific spatial regulation, suggesting a tissue-specific diversity of ethylene production (Tsuchisaka and Theologis, 2004). Likewise, members of the ACO gene family of tomato (Solanum lycopersicum) are also differentially regulated at the transcriptional level during climacteric fruit ripening, indicating distinct roles played by each ACO homolog during different tomato fruit developmental stages (Rudus et al., 2013; Xiao et al., 2013).

Additionally, both ACS and ACO expression can be directly and/or indirectly controlled by several signaling connections with other plant hormones such as auxin, brassinosteroid, and gibberellin (Guzman and Ecker, 1990; Joo et al., 2006). In this sense, auxin has been widely recognized as one of the most significant hormones controlling ethylene biosynthesis through transcriptional regulation of several ACS genes (Abel et al., 1995; Tsuchisaka and Theologis, 2004; Stepanova et al., 2007). In Arabidopsis, with the exception of $A C S 1 / 7 / 9$ genes, the transcription of all other ACS members coding for functional enzymes are induced by auxin (Yamagami et al., 2003). In fact, the auxinethylene crosstalk often occurs via reciprocal regulation at biosynthetic level (Stepanova et al., 2007) with auxin apparently acting mostly via up-regulation of ACS4 transcription in Arabidopsis (Liang et al., 1992; Abel et al., 1995; Zhu and Guo, 2008). On the other hand, some data on the crosstalk between auxin and ethylene during transcriptional regulation of two ACO genes in rice $(O s A C O 2 / 3)$ have indicated that auxin effects on ethylene biosynthesis are highly dependent of the ethylene status itself. For example, $\mathrm{OsACO} 3$ is induced by ethylene, but not in the presence of auxin, whereas OsACO2 is induced by auxin, but in a reduced extent when in the presence of ethylene (Chae et al., 2000). Hence, auxin induction of ACO transcripts is generally considered an indirect effect of auxin-stimulated ethylene production resulting from an increase in ACS activity (Peck and Kende, 1995). Hence, direct regulation of ACO transcription seems to be mainly induced by ethylene itself by an autocatalytic process that will be discussed in the next sections of this review.

\section{ETHYLENE CONTROLLING ITS OWN BIOSYNTHESIS VIA ACS AND ACO TRANSCRIPTION}

As mentioned previously, ethylene can modulate its own metabolism (Tsuchisaka et al., 2009) and this process usually occurs in an extremely flexible, developmentally and environmentally sensitive manner. Several studies have been reported on either positive or negative signaling feedback loops wherein ethylene regulates its own production (Sisler et al., 1985; Nakajima et al., 1990; Rottmann et al., 1991; Van Der Straeten et al., 1992; Woodson et al., 1992; Lincoln et al., 1993; RodriguesPousada et al., 1999; Wang et al., 2002; Zhong and Burns, 2003). Therefore, depending on the plant tissue and the developmental/environmental context, ethylene can either restrain (autoinhibition or system 1) or promote (autocatalysis or system 2) its own biosynthesis. Moreover, it has also been suggested that particular members of ACS gene family can play specific roles in these two systems of ethylene production in plants (Yang and Hoffman, 1984; Liu et al., 1985; Nakatsuka et al., 1998; Vandenbussche et al., 2012).

Negative feedback is particularly active during vegetative growth under non-stressful conditions and non-climacteric fruit development while autocatalytic ethylene production is usually related to stressful conditions, senescence and climacteric fruit ripening (McMurchie et al., 1972; Nakatsuka et al., 1998; Vandenbussche et al., 2012). During tomato fruit ripening different $A C S$ and $A C O$ genes are induced at particular stages of ripening. For example, LeACS2 and LeACS4 are mainly associated with autocatalytic ethylene production while LeACS6 is mostly related to the auto-inhibitory system responsible for the maintenance of basal ethylene production (Nakatsuka et al., 1998; Barry et al., 2000; Cara and Giovannoni, 2008).

In Arabidopsis, the auto-inhibitory system also operates through the tightly controlled activities of several ACS proteins, which generate the basal ethylene levels in a coordinated manner (Yoshii and Imaseki, 1982; Tsuchisaka et al., 2009). The transcription of ACS6 gene in this same species is generally associated with stress-induced conditions, in which ethylene autocatalysis plays an important role (Tsuchisaka et al., 2009; Vandenbussche et al., 2012). Furthermore, at least two ACO genes are ethylene inducible in Arabidopsis, suggesting that a feedback mechanism is in place to ensure that there will be no limitations to ethylene production once the precursor ACC is produced (Alonso et al., 2003; Zhong and Burns, 2003; Chen et al., 2005). Interestingly, the autocatalytic ethylene production in Arabidopsis is usually mediated by MPK cascade modules associated with stress-triggered signaling pathways. For example, besides posttranslationally controlling ACS protein stability, the MPK3/6 module plays an important role in the ethylene autocatalytic pathway by inducing the transcription of the WRKY33 transcription factor which, in turn, directly interacts with ACS2/6 promoters (Smékalová et al., 2014). In addition, besides triggering ACS2/6 transcription up-regulation, the MKK9-MPK3/6 module also positively regulates the transcript abundance of several ERF (ETHYLENE RESPONSE FACTORS) genes (Xu et al., 2008; Yoo et al., 2008).

ERF Or EREBP (ETHYLENE RESPONSE ELEMENTBINDING PROTEIN) genes represent one of the largest families of transcription factors in the plant kingdom which regulates a diverse array of processes (including ethylene production) in response to multiple developmental and environmental cues (Smékalová et al., 2014). These plant-specific transcription factors function as trans-acting regulators at the last step of ethylene signaling pathway, and usually exhibit highly conserved motifs that includes AP2 (APETALA2)/ERF DNAbinding domain, RAV (related to ABI3/VP1), and DREB 
(dehydration-responsive element binding protein). These ERF domains can specifically interact with cis-elements in promoters of the ethylene-responsive genes, such as GCC box and/or dehydration-responsive elements/C-repeat (DRE/CRT) motifs, thus regulating the expression of different downstream genes (Ohme-Takagi and Shinshi, 1995; Park et al., 2001; Huang et al., 2004; Lin et al., 2009; Zhang et al., 2009; Kohli et al., 2013). In addition, some ERFs can induce the transcription of additional transcription factors, inducing a transcription-factor cascade that might facilitate the signal amplification and fine-tuning of signal output (Chen et al., 2005).

Several studies have already shown that ERFs participate in a feedback regulation of ethylene production by modulating the expression of ethylene biosynthesis genes during fruit ripening (Zhang et al., 2009; Sharma et al., 2010; Lee et al., 2012; Pirrello et al., 2012). For example, ectopic expression of the ERF.B3$S R D X$ dominant repressor in tomato caused reduction in ethylene biosynthesis associated with the down-regulation of ACS and ACO genes, which presented the GCC box and DRE/CRT motifs at their promoters (Liu et al., 2013; Pirrello et al., 2012). In addition, LeERF2/TERF2 activates ethylene biosynthesis by promoting the expression of ACS genes in tobacco and of both ACS and ACO genes in tomato (Zhang et al., 2009). On the other hand, the ERF repressor SIAP2a opposes tomato ripening by suppressing the expression of ACS2/4 and ACO1 genes, then causing down-regulation of ethylene biosynthesis (Chung et al., 2010). Interestingly, distinct MaERFs of banana (Musa acuminata) can be differentially expressed in a tissue-dependent manner during fruit ripening while different MaERFs appeared to regulate the transcription of particular ACS and/or ACO in either positive (MaERF9-induced activation of MaACS1) or negative (MaERF11-induced repression of MaACS1 and MaACO1) way (Xiao et al., 2013). Therefore, although the important role of multiple ERFs in the regulation of fruit ripening has been consistently described by several studies, little information is available about the signaling pathways involved in ERF regulation and the additional mechanisms modulating their target genes.

Furthermore, ERF proteins also play important roles in plant response to a variety of stress-related stimuli that involves modulation of ethylene synthesis (Xu et al., 2006; Wang et al., 2008; Hattori et al., 2009; Yaish et al., 2010). For instance, the ERF genes SUB1A and OSDERF1 of rice are, respectively, positive and negative regulators in drought response. Upon drought stress, the expression of both these ERFs is modulated, affecting in turn the ethylene production via alterations in the expression of ethylene biosynthesis genes (Xu et al., 2006; Fukao et al., 2011; Wan et al., 2011). Consequently, it has become clear that ERF transcription factors are the primary targets mediating stressinduced responses involving changes in ethylene signaling and/or biosynthesis. However, very little is known about the signaling mechanisms controlling ERF proteins in ethylene biosynthesis at the transcriptional level (Zhang et al., 2009), which highlights the great demand to identify transcription factors and other signaling elements involved in ethylene biosynthesis adjustments. The potential regulatory role of these transcription factors during the light-induced pathway leading to the regulation of $A C S$ and/or ACO transcription will be discussed below.

\section{LIGHT SIGNALING AND THE TRANSCRIPTIONAL CONTROL OF ACS AND ACO ENZYMES}

PHYB is well known to module ethylene emission by repressing the transcription of genes encoding particular ACS isoenzymes (Figure 2). Illustrating this regulatory mechanism, mutations in $p h y A$ and $p h y B$ provide, respectively, increases on transcription of ACS1 in pea (Foo et al., 2006) and ACS2 in Arabidopsis (Bours et al., 2014). In fact, the genome-expression profiling of Arabidopsis revealed the light-regulated suppression of $A C S, A C O$, and SAM synthase transcripts (Ma et al., 2001). Accordingly, in this plant species the RL-evoked inhibition of ethylene emission not only depends on the repression of ACS2 gene transcription, but is also strongly associated with the PHYB-dependent degradation of PIF proteins, such as PIF4 and particularly PIF5. Interestingly, PIF1 and PIF3 biochemically interact with both PHYA and PHYB, whereas PIF4 and PIF5 are only degraded when associated with active PHYB and this PHYB-mediated degradation of PIF4 and PIF5 is considered to be an important mechanism responsible for controlling ethylene production in plant responses triggered by either darkness or low R/FR conditions, such as skotomorphogenic growth and shade avoidance responses (Lorrain et al., 2008; Shin et al., 2009).

As previously described, PIF5 protein is constitutively localized in the nucleus, where it exerts a repressive influence on the transcription of genes associated with photomorphogenic responses and presents a positive impact on genes associated with low R/FR responses, such as ACS4/8 (Figure 2). In agreement, Arabidopsis plants over-expressing PIF5 exhibited up to 300 -fold increases in ACS4 transcripts even in the presence of light (Khanna et al., 2007). Although less prominent than observed for the ACS4 expression, PIF5 also positively influences ACS 8 expression, which is the most abundantly expressed ACS gene in Arabidopsis and is the ACS gene most strongly controlled by endogenous ethylene and circadian clock (Yamagami et al., 2003; Thain et al., 2004). Due to such prominent impact on ethylene production, the PIF5 over-expression in Arabidopsis resulted in the triple response phenomenon characteristically triggered by excessive ethylene production. Moreover, PIF5 is also known to destabilize PHYB by promoting its degradation via proteasome 26S, and this PIF5-triggered degradation of PHYB might accentuate even more the ethylene production in conditions of high PIF5 protein abundance (Khanna et al., 2007).

Furthermore, it has become clear that an integrated, multihormonal network is a common signaling mechanism involved in ethylene biosynthesis control (Chae et al., 2000). For example, besides acting as repressors of gibberellins (GA) responses, DELLA proteins can also physically interact with PIF proteins, thereby inhibiting PIF responses via a PHY-independent mechanism. Such a DELLA-dependent PIF suppression mainly takes place under complete darkness, when both these proteins are at abundant levels. Thus, the influence of GA on the transcriptional regulation of some ethylene-related genes, such as ACS8, seems to be associated with the DELLA destabilization and consequent repression of PIF degradation. Another overlap between ethylene and GA consists of the fact that active $\mathrm{PHYB}_{\mathrm{Pfr}}$ represses active GA biosynthesis, which in turn decreases ethylene emission via DELLA-dependent and independent pathways (Keller et al., 
2011). Accordingly, low R/FR conditions promote DELLA degradation, leading to increased PIF-related responses (de Lucas et al., 2008) which include the stimulation of ethylene biosynthesis. Moreover, light responses triggered by low fluence radiation, such as shade avoidance, are well known to involve both auxins and ethylene, during which an intensive crosstalk between these phytohormones and PHYB is suggested (Vandenbussche et al., 2003; Millenaar et al., 2009; Pierik et al., 2009). For example, Arabidopsis mutants exhibiting low auxin sensitivity, such as auxin resistant 2 (axr2) and (axr1-3), usually present phenotypical similarities to wild-type plants maintained under complete darkness or low R/FR radiation. Treatment of these mutants with ethylene has been shown to revert this phenotype, suggesting that low R/FR leads to increased auxin levels, and these elevated auxin levels are responsible for stimulating ethylene production under these conditions. Additionally, auxin and low R/FR apparently share the same signaling pathways to increase ACS6/8 transcript abundance (Vandenbussche et al., 2003). However, some contrasting results obtained with low R/FR-treated Arabidopsis plants (Millenaar et al., 2009; Pierik et al., 2009) have indicated that signaling interactions among PHYs, auxins, and ethylene during low-light induced responses can be highly plastic and dependent on specific experimental conditions (i.e., plant material obtained by distinct growth methods, different periods of exposure to the light treatment).

Adding even more complexity to the crosstalk between light signaling and regulation of ethylene biosynthesis in plants, it is currently known that LONG HYPOCOTYL IN FAR-RED1 (HFR1) is particularly relevant during low F/FR responses. Accordingly, HFR1 is a nuclear protein structurally similar to PIF3 (Fairchild et al., 2000), which is phosphorylated by COP1 and usually marked for degradation under complete darkness (Figure 1). However, in the presence of light, especially under BL- or FRL-rich radiation, this protein remains much more stable due to the presence of active PHYA and CRYs. Some FRL-induced responses that are associated with modifications in ethylene emission (e.g., repression of ACS8 transcription) are apparently influenced by the biochemical interaction between PHYA-HFR1 or via the heterodimerization of HFR1 with PIF3 (Fairchild et al., 2000). However, it is also possible that such FRL-induced responses associated with modifications in ethylene evolution occurs through a more indirect pathway involving the HFR1-induced repression of genes and/or enzymes of other plant hormone biosynthetic pathways, such as GA and auxin (Sessa et al., 2005). Recent work has also suggested that PIFs and COP1 complexes synergistically repress photomorphogenesis in the dark, indicating that PIF proteins might inhibit HY5 by direct and indirect mechanisms (Xu et al., 2014). Furthermore, COP1 was reported as capable of physically interact with PIF3-LIKE1 (PIL1) and promote PIL1 degradation via the 26S proteasome, whereas PHYB physically interacts with PIL1 and enhances PIL1 protein accumulation upon RL irradiation, possibly via suppressing the COP1-PIL1 association (Jang et al., 2010; Luo et al., 2014).

Since PIF and COP1/HY5-mediated pathways are generally considered the two main light signaling branches downstream of the photoreceptors (Figure 1), it is likely that these components participate as central integrators between signaling pathways mediated by light and hormones cues (Yamamoto et al., 1998; Lau and Deng, 2010). In fact, some studies have consistently indicated PIF and COP/HY5 as hub steps of convergence and integration of signaling information mediated by both light and hormones. For example, such a PIF/COP/HY5-mediated network appears to control the opposite effects induced by ethylene on hypocotyl elongation of etiolated Arabidopsis seedlings when grown under darkness (elongation promotion) or light exposure (elongation repression; Feng et al., 2008; Liang et al., 2012). Interestingly, both these signaling steps are located downstream of ETHYLENE INSENSITIVE 3 (EIN3) during ethylene-regulated hypocotyl development in dark-grown seedlings (Ang and Deng, 1994; An et al., 2010). The transcription factors EIN3 and EIN3-LIKE 1 (EIL1) are intermediates in the ethylene signaling transduction responsible for the subsequent activation of $E R F$ genes during multiple physiological processes (An et al., 2010; Liang et al., 2012). Moreover, COP1 appears to positively regulate the accumulation of EIN3 protein through a yet non-identified regulatory mechanism (Zhong et al., 2009) while COP1 affects the transcription of EIN3 downstream genes such as ERF1 (Solano et al., 1998; Liang et al., 2012).

\section{CONCLUSION}

Light has been acknowledged for some time as an important environmental cue capable of modulating ethylene biosynthesis in higher plants. While substantial progress has been made in identifying the metabolic components and enzymatic steps involved in ethylene biosynthetic route, our current understanding of how ethylene production is controlled by light signaling pathways is still very limited. Over the past decades, the identification of several mutants affected in particular aspects of ethylene production and/or photomorphogenic responses has facilitated significant advances in both research fields. As a result, some important breakthroughs on mechanistically explaining how light perception and signal transduction can modulate ethylene biosynthesis have been recently achieved, particularly in terms of the lightevoked changes in transcriptional and post-translational regulation of ACS and ACO enzymes.

Besides the direct effects of light-associated proteins on particular ethylene biosynthetic elements, we might also remain openminded to conceive relatively more complex interconnection nodes, in which other plant hormones act in between the light perception and the actual modification of ethylene biosynthetic steps. Exciting new models of light-ethylene interaction networks might probably emerge when the combinatory influence of distinct photoreceptors (e.g., phytochromes and cryptochromes) or different environmental stimuli (e.g., light and biotic or abiotic stresses) started to be considered. Moreover, our knowledge about the mechanistic interplays between light and ethylene production might become even more complex as the research currently performed mainly in few plant models (e.g., Arabidopsis, tomato, pea) is extended to a wider range of species. Given the profuse influence of both light and ethylene on plant development, growth, and metabolism, studying the interplay between these stimuli is unarguably a promising research field for years to come. 


\section{ACKNOWLEDGMENTS}

Work in the authors' laboratory has been supported by Fundação de Amparo a Pesquisa do Estado de São Paulo (grant no. 2013/18056-2, São Paulo Research Foundation - FAPESP), Conselho Nacional de Desenvolvimento Científico e Tecnológico (CNPq), and Coordenação de Aperfeiçoamento de Pessoal de Nível Superior (CAPES).

\section{REFERENCES}

Abel, S., Nguyen, M. D., Chow, W., and Theologis, A. (1995). ACS4, a primary indoleacetic acid-responsive gene encoding 1-aminocyclopropane-1carboxylate synthase in Arabidopsisthaliana. Structural characterization, expression in Escherichia coli, and expression characteristics in response to auxin. J. Biol. Chem. 270, 19093-19099. doi: 10.1074/jbc.270.32.19093

Abeles, F. B., Morgan, P. W., and Salveit, M. E. (1992). Ethylene in Plant Biology. San Diego: Academic Press.

Achard, P., Cheng, H., De Grauwe, L., Decat, J., Schoutteten, H., Moritz, T., et al. (2006). Integration of plant responses to environmentally activated phytohormonal signals. Science 311, 91-94. doi: 10.1126/science.1118642

Adams, D. O., and Yang, S. F. (1977). Methionine metabolism in apple tissue: implication of $S$-adenosylmethionine as an intermediate in the conversion of methionine to ethylene. Plant Physiol. 60, 892-896. doi: 10.1104/pp.60. 6.892

Adams, D. O., and Yang, S. F. (1979). Ethylene biosynthesis: identification of aminocyclopropane-1-carboxylic acid as an intermediate in the conversion of methionine to ethylene. Proc. Natl. Acad. Sci. U.S.A. 76, 170-174. doi: 10.1073/pnas.76.1.170

Ahmad, M., and Cashmore, A. R. (1997). The blue-light receptor cryptochrome 1 shows functional dependence on phytochrome A or phytochrome B in Arabidopsis thaliana. Plant J. 11, 421-427. doi: 10.1046/j.1365-313X.1997. 11030421.x

Alonso, J. M., Stepanova, A. N., Solano, R., Wisman, E., Ferrari, S., Ausubel, F. M., et al. (2003). Five components of the ethylene-response pathway identified in a screen for weak ethylene-insensitive mutants in Arabidopsis. Proc. Natl. Acad. Sci. U.S.A. 100, 2992-2997. doi: 10.1073/pnas.0438070100

An, F., Zhao, Q., Ji, Y., Li, W., Jiang, Z., Yu, X., et al. (2010). Ethyleneinduced stabilization of ETHYLENE INSENSITIVE3 and EIN3-LIKE1 is mediated by proteasomal degradation of EIN3 binding F-box 1 and 2 that requires EIN2 in Arabidopsis. Plant Cell 22, 2384-2401. doi: 10.1105/tpc.110. 076588

Ang, L. H., and Deng, X. W. (1994). Regulatory hierarchy of photomorphogenic loci: allele-specific and light-dependent interaction between the HY5 and COP1 loci. Plant Cell 6, 613-628. doi: 10.1105/tpc.6.5.613

Argueso, C. T., Hansen, M., and Kieber, J. J. (2007). Regulation of ethylene biosynthesis. J. Plant Growth Regul. 26, 92-105. doi: 10.1007/s00344-007-0013-5

Barry, C. S., Llop-Tous, M. I., and Grierson, D. (2000). The regulation of 1aminocyclopropane-1-carboxylic acid synthase gene expression during the transition from system-1 to system-2 ethylene synthesis in tomato. Plant Physiol. 123, 979-986. doi: 10.1104/pp.123.3.979

Beckers, G. J. M., Jaskiewicz, M., Liu, Y., Underwood, W. R., He, S. Y., Zhang, S., et al. (2009). Mitogen-activated protein kinases 3 and 6 are required for full priming of stress responses in Arabidopsis thaliana. Plant Cell 21, 944-953. doi: 10.1105/tpc. 108.062158

Bleecker, A. B., Estelle, M. A., Somerville, C., and Kende, H. (1988). Insensitivity to ethylene conferred by a dominant mutation in Arabidopsis thaliana. Science 241, 1086-1089. doi: 10.1126/science.241.4869.1086

Boron, A. K., and Vissenberg, K. (2014). The Arabidopsis thaliana hypocotyl, a model to identify and study control mechanisms of cellular expansion. Plant Cell Rep. 33, 697-706. doi: 10.1007/s00299-014-1591-x

Bours, R., van Zanten, M., Pierik, R., Bouwmeester, H., and van der Krol, A. (2014). Antiphase light and temperature cycles affect PHYTOCHROME B-controlled ethylene sensitivity and biosynthesis, limiting leaf movement and growth of Arabidopsis. Plant Physiol. 163, 882-895. doi: 10.1104/pp.113. 221648

Cara, B., and Giovannoni, J. J. (2008). Molecular biology of ethylene during tomato fruit development and maturation. Plant Sci. 175, 106-113. doi: 10.1016/j.plantsci.2008.03.021
Chae, H. S., Cho, Y. G., Park, M. Y., Lee, M. C., Eun, M. Y., Kang, B. G., et al. (2000). Hormonal cross-talk between auxin and ethylene differentially regulates the expression of two members of the 1-aminocyclopropane-1-carboxylate oxidase gene family in rice (Oryza sativa L.). Plant Cell Physiol. 41, 354-362. doi: $10.1093 / \mathrm{pcp} / 41.3 .354$

Chae, H. S., Faure, F., and Kieber, J. J. (2003). The eto1, eto2, and eto3 mutations and cytokinin treatment increase ethylene biosynthesis in Arabidopsis by increasing the stability of ACS protein. Plant Cell 15, 545-559. doi: 10.1105/tpc.006882

Chae, H. S., and Kieber, J. J. (2005). Eto Brute? Role of ACS turnover in regulating ethylene biosynthesis. Trends Plant Sci. 10, 291-296. doi: 10.1016/j.tplants. 2005.04.006

Chen, M., and Chory, J. (2011). Phytochrome signaling mechanisms and the control of plant development. Trends Cell Biol. 21, 664-671. doi: 10.1016/j.tcb. 2011.07.002

Chen, Y. F., Etheridge, N., and Schaller, G. E. (2005). Ethylene signal transduction. Ann. Bot. 95, 901-915. doi: 10.1093/aob/mci100

Chory, J. (2010). Light signal transduction: an infinite spectrum of possibilities. Plant J. 61, 982-991. doi: 10.1111/j.1365-313X.2009.04105.x

Christians, M. J., Gingerich, D. J., Hansen, M., Binder, B. M., Kieber, J. J., and Vierstra, R. D. (2009). The BTB ubiquitin ligases ETO1, EOL1 and EOL2 act collectively to regulate ethylene biosynthesis in Arabidopsis by controlling type-2 ACC synthase levels. Plant J. 57, 332-345. doi: 10.1111/j.1365313X.2008.03693.x

Christie, J. M. (2007). Phototropin blue-light receptors. Annu. Rev. Plant Biol. 58, 21-45. doi: 10.1146/annurev.arplant.58.032806.103951

Chung, M. Y., Vrebalov, J., Alba, R., Lee, J., McQuinn, R., Chung, J. D., et al. (2010). A tomato (Solanum lycopersicum) APETALA2/ERF gene, SIAP2a, is a negative regulator of fruit ripening. Plant J. 64, 936-947. doi: 10.1111/j.1365313X.2010.04384.x

Corbineau, F., Rudnicki, R. M., Goszczynska, D. M., and Come, D. (1995). The effect of light quality on ethylene production in leaves of oat seedlings (Avena sativa L.). Environ. Exp. Bot. 35, 227-233. doi: 10.1016/0098-8472(94) 00052-7

de Lucas, M., Daviere, J. M., Rodriguez-Falcon, M., Pontin, M., Iglesias-Pedraz, J. M., Lorrain, S., et al. (2008). A molecular framework for light and gibberellin control of cell elongation. Nature 451, 480-484. doi: 10.1038/nature06520

Facella, P., Lopez, L., Carbone, F., Galbraith, D. W., Giuliano, G., and Perrotta, G. (2008). Diurnal and circadian rhythms in the tomato transcriptome and their modulation by cryptochrome photoreceptors. PLOS ONE 3:e2798. doi: 10.1371/journal.pone.0002798

Fairchild, C. D., Schumaker, M. A., and Quail, P. H. (2000). HFR1 encodes an atypical bHLH protein that acts in phytochrome A signal transduction. Genes Dev. 14, 2377-2391. doi: 10.1101/gad.828000

Fankhauser, C., and Ulm, R. (2011). Light-regulated interactions with SPA proteins underlie cryptochrome-mediated gene expression. Genes Dev. 25, 1004-1009. doi: 10.1101/gad.2053911

Feilner, T., Hultschig, C., Lee, J., Meyer, S., Immink, R. G., Koenig, A., et al. (2005). High throughput identification of potential Arabidopsis mitogenactivated protein kinases substrates. Mol. Cell. Proteomics 4, 1558-1568. doi: 10.1074/mcp.M500007-MCP200

Feng, S., Martinez, C., Gusmaroli, G., Wang, Y., Zhou, J., Wang, F., et al. (2008). Coordinated regulation of Arabidopsis thaliana development by light and gibberellins. Nature 451, 475-479. doi: 10.1038/nature06448

Finlayson, S. A., Jung, I. J., Mullet, J. E., and Morgan, P. W. (1999). The mechanism of rhythmic ethylene production in sorghum. The role of phytochrome B and simulated shading. Plant Physiol. 119, 1083-1089. doi: 10.1104/pp.119.3. 1083

Finlayson, S. A., Lee, I. J., and Morgan, P. W. (1998). Phytochrome B and the regulation of circadian ethylene production in sorghum. Plant Physiol. 116, 1725. doi: 10.1104/pp.116.1.17

Fluhr, R., and Mattoo, A. K. (1996). Ethylene-biosynthesis and perception. Crit. Rev. Plant Sci. 15, 479-523.

Foo, E., Ross, J. J., Davies, N. W., Reid, J. B., and Weller, J. L. (2006). A role for ethylene in the phytochrome-mediated control of vegetative development. Plant J. 46, 911-921. doi: 10.1111/j.1365-313X.2006.02754.x

Franklin, K. A., Larner, V. S., and Whitelam, G. C. (2005). The signal transducing photoreceptors of plants. Int. J. Dev. Biol. 49, 653-664. doi: 10.1387/ijdb.051989kf 
Franklin, K. A., and Quail, P. H. (2010). Phytochrome functions in Arabidopsis development. J. Exp. Bot. 61, 11-24. doi: 10.1093/jxb/erp304

Fukao, T., Yeung, E., and Bailey-Serres, J. (2011). The submergence tolerance regulator SUB1A mediates crosstalk between submergence and drought tolerance in rice. Plant Cell 23, 412-427. doi: 10.1105/tpc.110.080325

Gil, P., Kircher, S., Adam, E., Bury, E., Kozma-Bognar, L., Schäfer, E., et al. (2000). Photocontrol of subcellular partitioning of phytochrome-B:GFP fusion protein in tobacco seedlings. Plant J. 22, 135-145. doi: 10.1046/j.1365313x.2000.00730.x

Goeschl, J. D., Pratt, H. P., and Bonner, B. A. (1967). An effect of light on the production of ethylene and the growth of the plumular portion of etiolated pea seedlings. Plant Physiol. 42, 1077-1080. doi: 10.1104/pp.42.8.1077

Guo, H., Mockler, T., Duong, H., and Lin, C. (2001). SUB1, an Arabidopsis $\mathrm{Ca}^{2+}$. binding protein involved in cryptochrome and phytochrome co-action. Science 291, 487-490. doi: 10.1126/science.291.5503.487

Guzman, P., and Ecker, J. (1990). Exploiting the triple response of Arabidopsis to identify ethylene-related mutants. Plant Cell 2, 513-523. doi: $10.1105 /$ tpc.2.6.513

Gyula, P., Schäfer, E., and Nagy, F. (2003). Light perception and signalling in higher plants. Curr. Opin. Plant Biol. 6, 446-452. doi: 10.1016/S1369-5266(03) 00082-7

Hahn, A., and Harter, K. (2009). Mitogen-activated protein kinase cascades and ethylene: signaling, biosynthesis, or both? Plant Physiol. 149, 1207-1210. doi: 10.1104/pp.108.132241

Halliday, K. J., and Fankhauser, C. (2003). Phytochrome-hormonal signalling networks. New Phytol. 157, 449-463. doi: 10.1046/j.1469-8137.2003.00689.x

Hanley, K. M., Meir, S., and Bramlage, W. J. (1989). Activity of ageing carnation flower parts and the effects of 1-(malonylamino)cyclopropane-1-carboxylic acid-induced ethylene. Plant Physiol. 91, 1126-1130. doi: 10.1104/pp.91.3. 1126

Harpaz-Saad, S., Yoon, G. M., Mattoo, A. K., and Kieber, J. J. (2012). The formation of ACC and competition between polyamines and ethylene for SAM. Annu. Plant Rev. 44, 53-81. doi: 10.1002/9781118223086.ch3

Herbel, V., Orth, C., Wenzel, R., Ahmad, M., Bittl, R., and Batschauer, A. (2013). Lifetimes of Arabidopsis cryptochrome signaling states in vivo. Plant J. 74, 583592. doi: $10.1111 /$ tpj. 12144

Hattori, Y., Nagai, K., Furukawa, S., Song, X.-J., Kawano, R., Sakakibara, H., et al. (2009). The ethylene response factors SNORKEL1 and SNORKEL2 allow rice to adapt to deep water. Nature 460, 1026-1030. doi: 10.1038/nature08258

Hoffman, N. E., Yang, S. F., and McKeon, T. (1982). Identification and metabolism of 1-(malonylamino)cyclopropane-1-carboxylic acid as a major conjugate of 1-aminocyclopropane-1-carboxylic acid, an ethylene precursor in higher plants. Biochem. Biophys. Res. Commun. 104, 765-770. doi: 10.1016/0006291X(82)90703-3

Huang, S.-J., Chang, C.-L., Wang, P.-H., Tsai, M.-C., Hsu, P.-H., and Chang, I.F. (2013). A type III ACC synthase, ACS7, is involved in root gravitropism in Arabidopsis thaliana. J. Exp. Bot. 64, 4343-4360. doi: 10.1093/jxb/ert241

Huang, Z., Zhang, Z., Zhang, X., Zhang, H., Huang, D., and Huang, R. (2004). Tomato TERF1 modulates ethylene response and enhances osmotic stress tolerance by activating expression of downstream genes. FEBS Lett. 573, 110-116. doi: 10.1016/j.febslet.2004.07.064

Imaseki, H., Pjon, C.-J., and Furuya, M. (1971). Phytochrome action in Oryza sativa L. - red and far red reversible effect on the production of ethylene in excised coleoptiles. Plant Physiol. 48, 241-244. doi: 10.1104/pp.48.3.241

Jang, I.-C., Henriques, R., Seo, H. S., Nagatani, A., and Chua, N.-H. (2010). Arabidopsis PHYTOCHROME INTERACTING FACTOR proteins promote phytochrome B polyubiquitination by COP1 E3 ligase in the nucleus. Plant Cell 22, 2370-2383. doi: 10.1105/tpc.109.072520

Jiao, X. Z., Philosoph-Hadas, S., Su, L. Y., and Yang, S. F. (1986). The conversion of 1-(malonylamino)cyclopropane-1-carboxylic acid to 1aminocyclopropane-1-carboxylic acid in plant tissues. Plant Physiol. 81, 637-641. doi: 10.1104/pp.81.2.637

Jiao, X. Z., Yip, W. K., and Yang, S. F. (1987). The effect of light and phytochrome on 1-aminocyclopropane-1-carboxylic acid metabolism in etiolated wheat seedling leaves. Plant Physiol. 85, 643-647. doi: 10.1104/pp.85.3.643

Joo, S., Liu, Y., Lueth, A., and Zhang, S. (2008). MPK phosphorylation-induced stabilization of ACS6 protein is mediated by the non-catalytic C-terminal domain, which also contains the cis-determinant for rapid degradation by the $26 \mathrm{~S}$ proteasome pathway. Plant J. 54, 129-140. doi: 10.1111/j.1365-313X.2008.03404.x
Joo, S., Seo, Y. S., Kim, S. M., Hong, D. K., Park, K. Y., and Kim, W. T. (2006). Brassinosteroid induction of AtACS4 encoding an auxin responsive 1aminocyclopropane-1-carboxylate synthase 4 in Arabidopsis seedlings. Physiol. Plant. 126, 592-604. doi: 10.1111/j.1399-3054.2005.00602.x

Ju, C., and Chang, C. (2012). Advances in ethylene signalling: protein complexes at the endoplasmic reticulum membrane. AoB Plants 2012:pls031. doi: 10.1093/aobpla/pls031

Kang, B. G., and Burg, S. P. (1972). Involvement of ethylene in phytochromemediated carotenoid synthesis. Plant Physiol. 49, 631-633. doi: 10.1104/ pp.49.4.631

Keller, M. M., Jaillais, Y., Pedmale, U. V., Moreno, J. E., Chory, J., and Ballar, C. L. (2011). Cryptochrome 1 and phytochrome $B$ control shade-avoidance responses in Arabidopsis via partially independent hormonal cascades. Plant J. 67, 195207. doi: 10.1111/j.1365-313X.2011.04598.x

Kelley, D. R., and Estelle, M. (2012). Ubiquitin-mediated control of plant hormone signaling. Plant Physiol. 160, 47-55. doi: 10.1104/pp.112.200527

Kende, H. (1993). Ethylene biosynthesis. Annu. Rev. Plant Physiol. Plant Mol. Biol. 44, 283-307. doi: 10.1146/annurev.pp.44.060193.001435

Khanna, R., Huq, E., Kikis, E. A., Al-Sady, B., Lanzatella, C., and Quail, P. H. (2004). A novel molecular recognition motif necessary for targeting photoactivated phytochrome signaling to specific basic helix-loop-helix transcription factors. Plant Cell 16, 3033-3044. doi: 10.1105/tpc.104.025643

Khanna, R., Shen, Y., Marion, C. M., Tsuchisaka, A., Theologis, A., Schäfer, E., et al. (2007). The basic helix-loop-helix transcription factor PIF5 acts on ethylene biosynthesis and phytochrome signaling by distinct mechanisms. Plant Cell 19, 3915-3929. doi: 10.1105/tpc.107.051508

Kim, J., Yi, H., Choi, G., Shin, B., Song, P.-S., and Choi, G. (2003). Functional characterization of phytochrome interacting factor 3 in phytochrome-mediated light signal transduction. Plant Cell 15, 2399-2407. doi: 10.1105/tpc.014498

Kinoshita, T., Doi, M., Suetsugu, N., Kagawa, T., Wada, M., and Shimazaki, K. (2001). Phot1 and phot2 mediate blue light regulation of stomatal opening. Nature 414, 656-660. doi: 10.1038/414656a

Kircher, S., Gil, P., Kozma-Bognár, L., Fejes, E., Speth, V., Husselstein-Muller, T., et al. (2002). Nucleocytoplasmic partitioning of the plant photoreceptors phytochrome A, B, C, D, and E is regulated differentially by light and exhibits a diurnal rhythm. Plant Cell 14, 1541-1555. doi: 10.1105/tpc.001156

Kohli, A., Sreenivasulu, N., Lakshmanan, P., and Kumar, P. P. (2013). The phytohormone crosstalk paradigm takes center stage in understanding how plants respond to abiotic stresses. Plant Cell Rep. 32, 945-957. doi: 10.1007/s00299013-1461-y

Kombrink, E. (2012). Chemical and genetic exploration of jasmonate biosynthesis and signaling paths. Planta 236, 1351-1366. doi: 10.1007/s00425-012-1705-Z

Kondoh, M., Shiraishi, C., Müller, P., Ahmad, M., Hitomi, K., Getzoff, E. D., et al. (2011). Light-induced conformational changes in full-length Arabidopsis thaliana cryptochrome. J. Mol. Biol. 413, 128-137. doi: 10.1016/j.jmb.2011.08.031

Kurepin, L. V., Walton, L. J., Yeung, E. C., Chinnappa, C. C., and Reid, D. M. (2010). The interaction of light irradiance with ethylene in regulating growth of Helianthus annuus shoot tissues. Plant Growth Regul. 62, 43-50. doi: 10.1007/s10725-010-9483-8

Lau, O. S., and Deng, X. W. (2010). Plant hormone signaling lightens up: integrators of light and hormones. Curr. Opin. Plant Biol. 13, 571-577. doi: 10.1016/j.pbi.2010.07.001

Lee, J. M., Joung, J.-G., McQuinn, R., Chung, M.-Y., Fei, Z., Tieman, D., et al. (2012). Combined transcriptome, genetic diversity and metabolite profiling in tomato fruit reveals that the ethylene response factor SIERF6 plays an important role in ripening and carotenoid accumulation. Plant J. 70, 191-204. doi: 10.1111/j.1365-313X.2011.04863.x

Leivar, P., Monte, E., Al-Sady, B., Carle, C., Storer, A., Alonso, J. M., et al. (2008). The Arabidopsis phytochrome-interacting factor PIF7, together with PIF3 and PIF4, regulates responses to prolonged red light by modulating phyB levels. Plant Cell 20, 337-352. doi: 10.1105/tpc.107.052142

Leivar, P., Tepperman, J. M., Monte, E., Calderon, R. H., Liu, T. L., and Quail, P. H. (2009). Definition of early transcriptional circuitry involved in lightinduced reversal of PIF-imposed repression of photomorphogenesis in young Arabidopsis seedlings. Plant Cell 21, 3535-3553. doi: 10.1105/tpc.109.070672

Li, H., Johnson, P., Stepanova, A., Alonso, J. M., and Ecker, J. R. (2004). Convergence of signaling pathways in the control of differential cell growth in Arabidopsis. Dev. Cell 7, 193-204. doi: 10.1016/j.devcel.2004.07.002 
Liang, X., Abel, S., Keller, J. A., Shen, N. F., and Theologis, A. (1992). The 1aminocyclopropane-1-carboxylate synthase gene family of Arabidopsis thaliana. Proc. Natl. Acad. Sci. U.S.A. 89, 11046-11050. doi: 10.1073/pnas.89.22.11046

Liang, X., Wang, H., Mao, L., Hu, Y., Dong, T., Zhang, Y., et al. (2012). Involvement of COP1 in ethylene- and light-regulated hypocotyl elongation. Planta 236, 1791-1802. doi: 10.1007/s00425-012-1730-y

Lin, C., Yang, H., Guo, H., Mockler, T., Chen, J., and Cashmore, A. R. (1998). Enhancement of blue-light sensitivity of Arabidopsis seedlings by a blue light receptor cryptochrome 2. Proc. Natl. Acad. Sci. U.S.A. 95, 2686-2690. doi: 10.1073/pnas.95.5.2686

Lin, Z. F., Zhong, S. L., and Grierson, D. (2009). Recent advances in ethylene research. J. Exp. Bot. 60, 3311-3336. doi: 10.1093/jxb/erp204

Lincoln, J. E., Campbell, A. D., Oetiker, J., Rottmann, W. H., Oeller, P. W., Shen, N. F., et al. (1993). LE-ACS4, a fruit ripening and wound-induced 1-aminocyclopropane-1-carboxylate synthase gene of tomato (Lycopersicum esculentum). Expression in Escherichia coli, structural characterization, expression characteristics, and phylogenetic analysis. J. Biol. Chem. 268, 19422-19430.

Liscum, E., and Hangarter, R. P. (1993). Light-stimulated apical hook opening in wild-type Arabidopsis thaliana seedlings. Plant Physiol. 101, 567-572.

Liu, M., Pirrello, J., Kesari, R., Mila, I., Roustan, J.-P., Li, Z., et al. (2013). A dominant repressor version of the tomato Sl-ERF.B3 gene confers ethylene hypersensitivity via feedback regulation of ethylene signaling and response components. Plant J. 76, 406-419. doi: 10.1111/tpj.12305

Liu, Y., Hoffman, N. E., and Yang, S. F. (1985). Promotion by ethylene of the capability to convert 1-aminocyclopropane-1-carboxylic acid to ethylene in preclimacteric tomato and cantaloupe fruit. Plant Physiol. 77, 407-411. doi: 10.1104/pp.77.2.407

Liu, Y., and Zhang, S. (2004). Phosphorylation of 1-aminocyclopropane-1carboxylic acid synthase by MPK6, a stress-responsive mitogen activated protein kinase, induces ethylene biosynthesis in Arabidopsis. Plant Cell 16, 3386-3399. doi: 10.1105/tpc.104.026609

Lorrain, S., Allen, T., Duek, P. D., Whitelam, G. C., and Fankhauser, C. (2008). Phytochrome-mediated inhibition of shade avoidance involves degradation of growth-promoting bHLH transcription factors. Plant J. 53, 312-323. doi: 10.1111/j.1365-313X.2007.03341.X

Luo, Q., Lian, H.-L., He, S.-B., Li, L., Jia, K.-P., and Yang, H.-Q. (2014). COP1 and phyB physically interact with PILl to regulate its stability and photomorphogenic development in Arabidopsis. Plant Cell 26, 2441-2456. doi: 10.1105/tpc.113.121657

Lürssen, K., Naumann, K., and Schroder, R. (1979). 1-aminocyclopropane-1carboxylic acid. An intermediate in ethylene biosynthesis in higher plants. $Z$. Pflanzenphysiol. 92, 285-294. doi: 10.1016/S0044-328X(79)80011-2

Lyzenga, W. J., Booth, J. K., and Stone, S. L. (2012). The Arabidopsis RINGtype E3 ligase XBAT32 mediates the proteasomal degradation of the ethylene biosynthetic enzyme, 1-aminocyclopropane-1-carboxylate synthase 7. Plant J. 71, 23-34. doi: 10.1111/j.1365-313X.2012.04965.x

Ma, L., Li, J., Qu, L., Hager, J., Chen, Z., Zhao, H., et al. (2001). Light control of Arabidopsis development entails coordinated regulation of genome expression and cellular pathways. Plant Cell 13, 2589-2607. doi: 10.1105/tpc.13.12.2589

Martin, M. N., and Saftner, R. A. (1995). Purification and characterization of 1-aminocyclopropanecarboxylic acid N-malonyltransferase from tomato fruit. Plant Physiol. 108, 1241-1249.

Martínez-Garcia, J. F., Huq, E., and Quail, P. H. (2000). Direct targeting of light signals to a promoter element-bound transcription factor. Science 288, 859-863. doi: 10.1126/science.288.5467.859

McClellan, C. A., and Chang, C. (2008). The role of protein turnover in ethylene biosynthesis and response. Plant Sci. 175, 24-31. doi: 10.1016/j.plantsci.2008. 01.004

McKeon, T. A., and Yang, S.-F. (1987). "Biosynthesis and metabolism of ethylene," in Plant Hormones and Their Role in Plant Growth and Development, ed. P. J. Davies (Dordrecht: Kluwer Academic Publishers), 94-112. doi: 10.1007/978-94009-3585-3_6

McMurchie, E. J., McGlasson, W. B., and Eaks, I. L. (1972). Treatment of fruit with propylene gives information about the biogenesis of ethylene. Nature 237, 235236. doi: $10.1038 / 237235 \mathrm{a} 0$

Millenaar, F. F., van Zanten, M., Cox, M. C. H., Pierik, R., Voesenek, L. A. C. J., and Peeters, A. J. M. (2009). Differential petiole growth in Arabidopsis thaliana: photocontrol and hormonal regulation. New Phytol. 184, 141-152. doi: 10.1111/j.1469-8137.2009.02921.x
Miyazaki, J. H., and Yang, S. F. (1987). The methionine salvage pathway in relation to ethylene and polyamine biosynthesis. Physiol. Plant. 69, 366-370. doi: 10.1111/j.1399-3054.1987.tb04302.x

Nakajima, N., Mori, H., Yamazaki, K., and Imaseki, H. (1990). Molecular cloning and sequence of a complementary DNA encoding 1-aminocyclopropane-1carboxylate synthase induced by tissue wounding. Plant Cell Physiol. 31, 10211029.

Nakatsuka, A., Murachi, S., Okunishi, H., Shiomi, S., Nakano, R., Kubo, Y., et al. (1998). Differential expression and internal feedback regulation of 1-aminocyclopropane-1-carboxylate synthase, 1-aminocyclopropane1-carboxylate oxidase, and ethylene receptor genes in tomato fruit during development and ripening. Plant Physiol. 118, 1295-1305. doi: 10.1104/pp.118. 4.1295

Neff, M. M., Fankhauser, C., and Chory, J. (2000). Light: an indicator of time and place. Genes Dev. 14, 257-271. doi: 10.1101/gad.14.3.257

Ni, M., Tepperman, J. M., and Quail, P. H. (1999). Binding of phytochrome B to its nuclear signalling partner PIF3 is reversibly induced by light. Nature 400, 781-784. doi: 10.1038/23500

Ohme-Takagi, M., and Shinshi, H. (1995). Ethylene-inducible DNA binding proteins that interact with an ethylene-responsive element. Plant Cell 7, 173-182. doi: 10.1105/tpc.7.2.173

Park, J. M., Park, C. J., Lee, S. B., Ham, B. K., Shin, R., and Paek, K. H. (2001). Overexpression of the tobacco Tsil gene encoding an EREBP/AP2-type transcription factor enhances resistance against pathogen attack and osmotic stress in tobacco. Plant Cell 13, 1035-1046. doi: 10.1105/tpc.13.5.1035

Peck, S. C., and Kende, H. (1995). Sequential induction of the ethylene biosynthetic enzymes by indole-3-acetic acid in etiolated peas. Plant Mol. Biol. 28, 293-301. doi: $10.1007 / \mathrm{BF} 00020248$

Peiser, G., and Yang, S. F. (1998). Evidence for 1-(malonylamino)-cyclopropane-1carboxylic acid being the major conjugate of aminocyclopropane-1-carboxylic acid in tomato fruit. Plant Physiol. 116, 1527-1532. doi: 10.1104/pp.116.4.1527

Pfeiffer, A., Nagel, M.-K., Popp, C., Wüst, F., Bindics, J., Viczián, A., et al. (2012). Interaction with plant transcription factors can mediate nuclear import of phytochrome B. Proc. Natl. Acad. Sci. U.S.A. 109, 5892-5897. doi: 10.1073/pnas.1120764109

Pierik, R., Whitelam, G. C., Voesenek, L. A. C. J., de Kroon, H., and Visser, E. J. W. (2004a). Canopy studies on ethylene-insensitive tobacco identify ethylene as a novel element in blue light and plant-plant signalling. Plant J. 38, 310-319. doi: 10.1111/j.1365-313X.2004.02044.x

Pierik, R., Cuppens, M. L. C., Voesenek, L. A. C. J., and Visser, E. J. W. (2004b). Interactions between ethylene and gibberellins in phytochromemediated shade avoidance responses in tobacco. Plant Physiol. 136, 2928-2936. doi: 10.1104/pp.104.045120

Pierik, R., Djakovic-Petrovic, T., Keuskamp, D. H., de Wit, M., and Voesenek, L. A. C. J. (2009). Auxin and ethylene regulate elongation responses to neighbor proximity signals independent of gibberellin and DELLA proteins in Arabidopsis. Plant Physiol. 149, 1701-1712. doi: 10.1104/pp.108.133496

Pierik, R., Sasidharan, R., and Voesenek, L. A. C. J. (2007). Growth control by ethylene: adjusting phenotypes to the environment. J. Plant Growth Regul. 26, 188-200. doi: 10.1007/s00344-006-0124-4

Pierik, R., Tholen, D., Poorter, H., Visser, E. J. W., and Voesenek, L. A. C. J. (2006). The Janus face of ethylene: growth inhibition and stimulation. Trends Plant Sci. 11, 176-183. doi: 10.1016/j.tplants.2006.02.006

Pirrello, J., Prasad, B. C. N., Zhang, W., Chen, K., Mila, I., Zouine, M., et al. (2012). Functional analysis and binding affinity of tomato Ethylene Response Factors provide insight on the molecular bases of plant differential responses to ethylene. BMC Plant Biol. 12:190. doi: 10.1186/1471-2229-12-190

Popescu, S., Popescu, G. V., Bachan, S., Zhang, Z., Gerstein, M., Snyder, M., et al. (2009). MPK target networks in Arabidopsis thaliana revealed using functional protein microarrays. Genes Dev. 23, 80-92. doi: 10.1101/gad.1740009

Prasad, M. E., Schofield, A., Lyzenga, W., Liu, H., and Stone, S. L. (2010). Arabidopsis RING E3 ligase XBAT32 regulates lateral root production through its role in ethylene biosynthesis. Plant Physiol. 153, 1587-1596. doi: 10.1104/pp.110.156976

Prasad, M. E., and Stone, S. L. (2010). Further analysis of XBAT32, an Arabidopsis RING E3 ligase, involved in ethylene biosynthesis. Plant Signal. Behav. 5, 14251429. doi: 10.4161/psb.5.11.13294

Quail, P. H. (2002). Phytochrome photosensory signalling networks. Nat. Rev. Mol. Cell. Biol. 3, 85-93. doi: 10.1038/nrm728 
Rizzini, L., Favory, J.-J., Cloix, C., Faggionato, D., O’Hara, A., Kaiserli, E., et al. (2011). Perception of UV-B by the Arabidopsis UVR8 protein. Science 332, $103-$ 106. doi: 10.1126/science. 1200660

Rodrigues-Pousada, R., Van Caeneghem, W., Chauvaux, N., Van Onckelen, H., Van Montagu, M., and Van Der Straeten, D. (1999). Hormonal cross-talk regulates the Arabidopsis thaliana 1-aminocyclopropane-1-carboxylate synthase gene 1 in a developmental and tissue-dependent manner. Physiol. Plant. 105, 312-320. doi: 10.1034/j.1399-3054.1999.105217.x

Rottmann, W. H., Peter, G. F., Oeller, P. W., Keller, J. A., Shen, N. F., Nagy, B. P., et al. (1991). 1-aminocyclopropane-1-carboxylate synthase in tomato is encoded by a multigene family whose transcription is induced during fruit and floral senescence. J. Mol. Biol. 222, 937-961. doi: 10.1016/0022-2836(91) 90587-V

Rudus, I., Sasiak, M., and Kepczynski, J. (2013). Regulation of ethylene biosynthesis at the level of 1-aminocyclopropane-1-carboxylate oxidase (ACO) gene. Acta Physiol. Plant. 35, 295-307. doi: 10.1007/s11738-012-1096-6

Sakai, T., Kagawa, T., Kasahara, M., Swartz, T. E., Christie, J. M., Briggs, W. R., et al. (2001). Arabidopsis nphl and npl1: blue light receptors that mediate both phototropism and chloroplast relocation. Proc. Natl. Acad. Sci. U.S.A. 98, 69696974. doi: 10.1073/pnas.101137598

Santner, A., and Estelle, M. (2010). The ubiquitin-proteasome system regulates plant hormone signaling. Plant J. 61, 1029-1040. doi: 10.1111/j.1365313X.2010.04112.x

Schwechheimer, C., and Deng, X. W. (2000). The COP/DET/FUS proteinsregulators of eukaryotic growth and development. Semin. Cell Dev. Biol. 11, 495-503. doi: 10.1006/scdb.2000.0203

Sebastià, C. H., Hardin, S. C., Clouse, S. D., Kieber, J. J., and Huber, S. C. (2004). Identification of a new motif for CDPK phosphorylation in vitro that suggests ACC synthase may be a CDPK substrate. Arch. Biochem. Biophys. 428, 81-91. doi: 10.1016/j.abb.2004.04.025

Sellaro, R., Crepy, M., Trupkin, S. A., Karayekov, E., Buchovsky, A. S., Rossi, C., et al. (2010). Cryptochrome as a sensor of the blue/green ratio of natural radiation in Arabidopsis. Plant Physiol. 154, 401-409. doi: 10.1104/pp.110.160820

Sessa, G., Carabelli, M., Sassi, M., Ciolfi, A., Possenti, M., Mittempergher, F., et al. (2005). A dynamic balance between gene activation and repression regulates the shade avoidance response in Arabidopsis. Genes Dev. 19, 2811-2815. doi: 10.1101/gad.364005

Sethi, V., Raghuram, B., Sinha, A. K., and Chattopadhyay, S. (2014). A mitogenactivated protein kinase cascade module, MKK3-MPK6 and MYC2, is involved in blue light-mediated seedling development in Arabidopsis. Plant Cell 26, 33433357. doi: 10.1105/tpc.114.128702

Sharma, M. K., Kumar, R., Solanke, A. U., Sharma, R., Tyagi, A. K., and Sharma, A. K. (2010). Identification, phylogeny, and transcript profiling of ERF family genes during development and abiotic stress treatments in tomato. Mol. Genet. Genomics 284, 455-475. doi: 10.1007/s00438-010-0580-1

Shen, Y., Khanna, R., Carle, C. M., and Quail, P. H. (2007). Phytochrome induces rapid PIF5 phosphorylation and degradation in response to red-light activation. Plant Physiol. 145, 1043-1051. doi: 10.1104/pp.107.105601

Shin, J., Kim, K., Kang, H., Zulfugarov, I. S., Bae, G., Lee, C. H., et al. (2009). Phytochromes promote seedling light responses by inhibiting four negativelyacting phytochrome-interacting factors. Proc. Natl Acad. Sci. U.S.A. 106, 76607665. doi: 10.1073/pnas.0812219106

Sisler, E. C., Goren, R., and Huberman, M. (1985). Effect of 2,5-norbornadiene on abscission and ethylene production in citrus leaf explants. Physiol. Plant. 63, 114-120. doi: 10.1111/j.1399-3054.1985.tb02828.x

Smékalová, V., Doskocilová, A., Komis, G., and Šamaj, J. (2014). Crosstalk between secondary messengers, hormones and MPK modules during abiotic stress signalling in plants. Biotechnol. Adv. 32, 2-11. doi: 10.1016/j.biotechadv.2013. 07.009

Solano, R., Stepanova, A., Chao, Q., and Ecker, J. R. (1998). Nuclear events in ethylene signaling: a transcriptional cascade mediated by ETHYLENEINSENSITIVE3 and ETHYLENE-RESPONSE FACTOR1. Genes Dev. 12, 37033714. doi: 10.1101/gad.12.23.3703

Song, C., Psakis, G., Lang, C., Mailliet, J., Gärtner, W., Hughes, J., et al. (2011). Two ground state isoforms and a chromophore D-ring photoflip triggering extensive intramolecular changes in a canonical phytochrome. Proc. Natl. Acad. Sci. U.S.A. 108, 3842-3847. doi: 10.1073/pnas. 1013377108

Spanu, P., Grosskopf, D. G., Felix, G., and Boller, T. (1994). The apparent turnover of 1-aminocyclopropane-1-carboxylate synthase in tomato cells is regulated by protein phosphorylation and dephosphorylation. Plant Physiol. 106, 529-535.

Staswick, P. S., and Tiryaki, I. (2004). The oxylipin signal jasmonic acid is activated by an enzyme that conjugates it to isoleucine in Arabidopsis. Plant Cell 16, 2117 2127. doi: $10.1105 /$ tpc.104.023549

Steed, C. L., Taylor, L. K., and Harrison, M. A. (2004). Red light regulation of ethylene biosynthesis and gravitropism in etiolated pea stems. Plant Growth Regul. 43, 117-125. doi: 10.1023/B:GROW.0000040116.10016.c3

Stepanova, A. N., and Alonso, J. M. (2005). Ethylene signalling and response pathway: a unique signalling cascade with a multitude of inputs and outputs. Physiol. Plant. 123, 195-206. doi: 10.1111/j.1399-3054.2005.00447.x

Stepanova, A. N., and Ecker, J. R. (2000). Ethylene signaling: from mutants to molecules. Curr. Opin. Plant Biol. 3, 353-360. doi: 10.1016/S1369-5266(00) 00096-0

Stepanova, A. N., Yun, J., Likhacheva, A. V., and Alonso, J. M. (2007). Multilevel interactions between ethylene and auxin in Arabidopsis roots. Plant Cell 19, 2169-2185. doi: 10.1105/tpc.107.052068

Stone, S. L., and Callis, J. (2007). Ubiquitin ligases mediate growth and development by promoting protein death. Curr. Opin. Plant Biol. 10, 624-632. doi: 10.1016/j.pbi.2007.07.010

Tatsuki, M., and Mori, H. (2001). Phosphorylation of tomato 1aminocyclopropane-1-carboxylic acid synthase, LE-ACS2, at the C-terminal region. J. Biol. Chem. 276, 28051-28057. doi: 10.1074/jbc.M101543200

Tepperman, J. M., Hudson, M. E., Khanna, R., Zhu, T., Chang, S. H., Wang, X., et al. (2004). Expression profiling of phyB mutant demonstrates substantial contribution of other phytochromes to red light-regulated gene expression during seedling de-etiolation. Plant J. 38, 725-739. doi: 10.1111/j.1365313X.2004.02084.x

Thain, S. C., Vandenbussche, F., Laarhoven, L. J. J., Dowson-Day, M. J., Wang, Z. Y., Tobin, E. M., et al. (2004). Circadian rhythms of ethylene emission in Arabidopsis. Plant Physiol. 136, 3751-3761. doi: 10.1104/pp.104. 042523

Tsuchisaka, A., and Theologis, A. (2004). Unique and overlapping expression patterns among the Arabidopsis 1-amino-cyclopropane-1-carboxylate synthase gene family members. Plant Physiol. 136, 2982-3000. doi: 10.1104/pp.104. 049999

Tsuchisaka, A., Yu, G. X., Jin, H. L., Alonso, J. M., Ecker, J. R., Zhang, X. M., et al. (2009). A combinatorial interplay among the 1-aminocyclopropane-1carboxylate isoforms regulates ethylene biosynthesis in Arabidopsis thaliana. Genetics 183, 979-1003. doi: 10.1534/genetics.109.107102

Tuomainen, J., Betz, C., Kangasjärvi, J., Ernst, D., Yin, Z.-H., Langebartels, C., et al. (1997). Ozone induction of ethylene emission in tomato plants: regulation by differential accumulation of transcripts for biosynthesis enzymes. Plant J. 12, 1151-1162. doi: 10.1046/j.1365-313X.1997.12051151.x

Ulijasz, A. T., Cornilescu, G., Cornilescu, C. C., Zhang, J., Rivera, M., Markley, J. L., et al. (2010). Structural basis for the photoconversion of a phytochrome to the activated Pfr form. Nature 463, 250-254. doi: 10.1038/nature 08671

Vahala, J., Schalgnhaufer, C. D., and Pell, E. J. (1998). Induction of ACC synthase cDNA by ozone in light-grown Arabidopsis thaliana leaves. Physiol. Plant. 103, 45-50. doi: 10.1034/j.1399-3054.1998.1030106.x

Vandenbussche, F., Vaseva, I., Vissenberg, K., and Van Der Straeten, D. (2012). Ethylene in vegetative development: a tale with a riddle. New Phytol. 194, 895909. doi: 10.1111/j.1469-8137.2012.04100.x

Vandenbussche, F., Vriezen, W. H., Smalle, J., Laarhoven, L. J., Harren, F. J., and Van Der Straeten, D. (2003). Ethylene and auxin control the Arabidopsis response to decreased light intensity. Plant Physiol. 133, 517-527. doi: 10.1104/pp.103.022665

Van Der Straeten, D., Rodrigues-Pousada, R. A., Villarroel, R., Hanley, S., Goodman, H., and Van Montagu, M. (1992). Cloning, genetic mapping, and expression analysis of an Arabidopsis thaliana gene that encodes 1aminocyclopropane-1-carboxylate. Proc. Natl. Acad. Sci. U.S.A. 89, 9969-9973. doi: 10.1073/pnas.89.20.9969

Vierstra, R. D. (2009). The ubiquitin-26S proteasome system at the nexus of plant biology. Nat. Rev. Mol. Cell Biol. 10, 385-397. doi: 10.1038/nrm2688

Vogel, J. P., Woeste, K. W., Theologis, A., and Kieber, J. J. (1998). Recessive and dominant mutations in the ethylene biosynthetic gene ACS5 of Arabidopsis confer cytokinin insensitivity and ethylene overproduction, respectively. Proc. Natl. Acad. Sci. U.S.A. 95, 4766-4771. doi: 10.1073/pnas.95.8.4766 
Wagner, J. R., Brunzelle, J. S., Forest, K. T., and Vierstra, R. D. (2005). A light-sensing knot revealed by the structure of the chromophorebinding domain of phytochrome. Nature 438, 325-331. doi: 10.1038/nature 04118

Wan, L., Zhang, J., Zhang, H., Zhang, Z., Quan, R., Zhou, S., et al. (2011). Transcriptional activation of OsDERF1 in OsERF3 and OsAP2-39 negatively modulates ethylene synthesis and drought tolerance in rice. PLoS ONE 6:e25216. doi: 10.1371/journal.pone.0025216

Wang, K. L., Li, H., and Ecker, J. R. (2002). Ethylene biosynthesis and signaling networks. Plant Cell 14, S131-S151. doi: 10.1105/tpc.001768

Wang, K. L.-C., Yoshida, H., Lurin, C., and Ecker, J. R. (2004). Regulation of ethylene gas biosynthesis by the Arabidopsis ETO1 protein. Nature 428, 945-950. doi: 10.1038/nature02516

Wang, Q., Guan, Y., Wu, Y., Chen, H., Chen, F., and Chu, C. (2008). Overexpression of a rice OsDREB1F gene increases salt, drought, and low temperature tolerance in both Arabidopsis and rice. Plant Mol. Biol. 67, 589-602. doi: 10.1007/s11103008-9340-6

Wheeler, R. M., and Tibbitts, T. W. (1986). Growth and tuberization of potato (Solanum tuberosum L.) under continuous light. Plant Physiol. 80, 801-804. doi: 10.1104/pp.80.3.801

Woeste, K. E., Ye, C., and Kieber, J. J. (1999). Two Arabidopsis mutants that overproduce ethylene are affected in the post-transcriptional regulation of 1aminocyclopropane-1-carboxylic acid synthase. Plant Physiol. 119, 521-530. doi: 10.1104/pp.119.2.521

Wolters, H., and Jürgens, G. (2009). Survival of the flexible: hormonal growth control and adaptation in plant development. Nat. Rev. Genet. 10, 305-317. doi: 10.1038/nrg2558

Woodson, W. R., Park, K. Y., Drory, A., Larsen, P. B., and Wang, H. (1992). Expression of ethylene biosynthetic pathway transcripts in senescing carnation flowers. Plant Physiol. 99, 526-532. doi: 10.1104/pp.99.2.526

Wu, D., Hu, Q., Yan, Z., Chen, W., Yan, C., Huang, X., et al. (2012). Structural basis of ultraviolet-B perception by UVR8. Nature 484, 214-219. doi: 10.1038/nature10931

Xiao, Y.-Y., Chen, J.-Y., Kuang, J.-F., Shan, W., Xie, H., Jiang, Y.-M., et al. (2013). Banana ethylene response factors are involved in fruit ripening through their interactions with ethylene biosynthesis genes. J. Exp. Bot. 64, 2499-2510. doi: 10.1093/jxb/ert108

Xu, K., Xu, X., Fukao, T., Canlas, P., Maghirang-Rodriguez, R., Heuer, S., et al. (2006). SublA is an ethylene-response-factor-like gene that confers submergence tolerance to rice. Nature 442, 705-708. doi: 10.1038/nature 04920

Xu, J., Li, Y., Wang, Y., Liu, H., Lei, L., Yang, H., et al. (2008). Activation of MPK kinase 9 induces ethylene and camalexin biosynthesis and enhances sensitivity to salt stress in Arabidopsis. J. Biol. Chem. 283, 26996-27006. doi: 10.1074/jbc.M801392200

Xu, X., Paik, I., Zhu, L., Bu, Q., Huang, X., Deng, X. W., et al. (2014). PHYTOCHROME INTERACTION FACTOR1 enhance the E3 ligase activity of CONSTITUTIVE PHOTOMORPHOGENIC1 to synergistically repress photomorphogenesis in Arabidopsis. Plant Cell 26, 1992-2006. doi: 10.1105/tpc.114.125591

Yaish, M. W., El-Kereamy, A., Zhu, T., Beatty, P. H., Good, A. G., Bi, Y. M., et al. (2010). The APETALA-2-like transcription factor OsAP2-39 controls key interactions between abscisic acid and gibberellin in rice. PLoS Genet. 6:e1001098. doi: 10.1371/journal.pgen.1001098

Yamagami, T., Tsuchisaka, A., Yamada, K., Haddon, W. F., Harden, L. A., and Theologis, A. (2003). Biochemical diversity among the 1-aminocyclopropane1-carboxylate synthase isoenzymes encoded by the Arabidopsis gene family. J. Biol. Chem. 278, 49102-49112. doi: 10.1074/jbc.M308297200

Yamamoto, Y. Y., Matsui, M., Ang, L. H., and Deng, X. W. (1998). Role of a COP1 interactive protein in mediating light-regulated gene expression in Arabidopsis. Plant Cell 10, 1083-1094. doi: 10.1105/tpc.10.7.1083

Yang, H. Q., Wu, Y. J., Tang, R. H., Liu, D., Liu, Y., and Cashmore, A. R. (2000). The $\mathrm{C}$ termini of Arabidopsis cryptochromes mediate a constitutive light response. Cell 103, 815-827. doi: 10.1016/S0092-8674(00)00184-7

Yang, S. F., and Hoffman, N. E. (1984). Ethylene biosynthesis and its regulation in higher plants. Annu. Rev. Plant Physiol. 35, 155-189. doi: 10.1146/ annurev.pp.35.060184.001103
Yang, S. F., Ku, H. S., and Pratt, H. K. (1966). Ethylene production from methionine as mediated by flavin mononucleotide and light. Biochem. Biophys. Res. Commun. 24, 729-743. doi: 10.1016/0006-291X(66)90387-1

Yao, Y., Du, Y., Jiang, L., and Liu, J. Y. (2007). Interaction between ACC synthase 1 and 14-3-3 proteins in rice: a new insight. Biochemistry 72, 1003-1007. doi: $10.1134 /$ S000629790709012X

Yi, C., and Deng, X. W. (2005). COP1-from plant photomorphogenesis to mammalian tumorigenesis. Trends Cell Biol. 15, 618-625. doi: 10.1016/j.tcb.2005.09.007

Yoo, S.-D., Cho, Y. H., Tena, G., Xiong, Y., and Sheen, J. (2008). Dual control of nuclear EIN3 by bifurcate MPK cascades in $\mathrm{C}_{2} \mathrm{H}_{4}$ signalling. Nature 451, 789795. doi: 10.1038/nature06543

Yoon, G. M., and Kieber, J. J. (2013a). 1-Aminocyclopropane-1-carboxylic acid as a signalling molecule in plants. AoB Plants 5:plt017. doi: 10.1093/aobpla/plt017

Yoon, G. M., and Kieber, J. J. (2013b). 14-3-3 regulates 1-aminocyclopropane-1carboxylate synthase protein turnover in Arabidopsis. Plant Cell 25, 1016-1028. doi: $10.1105 /$ tpc.113.110106

Yoon, G. M., and Kieber, J. J. (2013c). ACC synthase and its cognate E3 ligase are inversely regulated by light. Plant Signal. Behav. 8:e26478. doi: $10.4161 /$ psb. 26478

Yoshida, H., Nagata, M., Saito, K., Wang, K. L., and Ecker, J. R. (2005). Arabidopsis ETO1 specifically interacts with and negatively regulates type 2 1-aminocyclopropane-1-carboxylate synthases. BMC Plant Biol. 5:14. doi: 10.1186/1471-2229-5-14

Yoshida, H., Wang, K. L., Chang, C. M., Mori, K., Uchida, E., and Ecker, J. R. (2006). The ACC synthase TOE sequence is required for interaction with ETO1 family proteins and destabilization of target proteins. Plant Mol. Biol. 62, 427-437. doi: 10.1007/s11103-006-9029-7

Yoshii, H., and Imaseki, H. (1982). Regulation of auxin-induced ethylene biosynthesis. Repression of inductive formation of 1-aminocyclopropane-1carboxylate synthase by ethylene. Plant Cell Physiol. 23, 639-649.

Zarembinski, T. I., and Theologis, A. (1994). Ethylene biosynthesis and action: a case of conservation. Plant Mol. Biol. 26, 1579-1597. doi: 10.1007/BF00016491

Zhang, Z. J., Zhang, H. W., Quan, R. D., Wang, X. C., and Huang, R. F. (2009). Transcriptional regulation of ethylene response factor LeERF2 in the expression of ethylene biosynthesis genes controls ethylene production in tomato and tobacco. Plant Physiol. 150, 365-377. doi: 10.1104/pp.109.135830

Zheng, Z., Guo, Y., Novák, O., Dai, X., Zhao, Y., Ljung, K., et al. (2013). Coordination of auxin and ethylene biosynthesis by the aminotransferase VAS 1. Nat. Chem. Biol. 9, 244-248. doi: 10.1038/nchembio.1178

Zhong, G. V., and Burns, J. K. (2003). Profiling ethylene-regulated gene expression in Arabidopsis thaliana by microarray analysis. Plant Mol. Biol. 53, 117-131. doi: 10.1023/B:PLAN.0000009270.81977.ef

Zhong, S., Zhao, M., Shi, T., Shi, H., An, F., Zhao, Q., et al. (2009). EIN3/EIL1 cooperate with PIF1 to prevent photo-oxidation and to promote greening of Arabidopsis seedlings. Proc. Natl. Acad. Sci. U.S.A. 106, 21431-21436. doi: 10.1073/pnas.0907670106

Zhu, Z., and Guo, H. (2008). Genetic basis of ethylene perception and signal transduction in Arabidopsis. J. Integr. Plant Biol. 50, 808-815. doi: 10.1111/j.17447909.2008.00710.x

Conflict of Interest Statement: The authors declare that the research was conducted in the absence of any commercial or financial relationships that could be construed as a potential conflict of interest.

Received: 16 September 2014; accepted: 07 November 2014; published online: 01 December 2014.

Citation: Rodrigues MA, Bianchetti RE and Freschi L (2014) Shedding light on ethylene metabolism in higher plants. Front. Plant Sci. 5:665. doi: 10.3389/fpls.2014.00665 This article was submitted to Plant Physiology, a section of the journal Frontiers in Plant Science.

Copyright (C) 2014 Rodrigues, Bianchetti and Freschi. This is an open-access article distributed under the terms of the Creative Commons Attribution License (CC BY). The use, distribution or reproduction in other forums is permitted, provided the original author(s) or licensor are credited and that the original publication in this journal is cited, in accordance with accepted academic practice. No use, distribution or reproduction is permitted which does not comply with these terms. 


\title{
Tomato ACS4 is necessary for timely start of and progression through the climacteric phase of fruit ripening
}

\author{
Suzanne W. Hoogstrate ${ }^{1}$, Lambertus J. A. van Bussel ${ }^{1}$, Simona M. Cristescu ${ }^{2}$, Eric Cator ${ }^{3}$, \\ Celestina Mariani ${ }^{1}$, Wim H. Vriezen ${ }^{4}$ and Ivo Rieu ${ }^{1 *}$ \\ ${ }^{1}$ Department of Molecular Plant Physiology, Institute for Water and Wetland Research, Radboud University, Nijmegen, Netherlands \\ ${ }_{2}^{2}$ Department of Molecular and Laser Physics, Institute for Molecules and Materials, Radboud University, Nijmegen, Netherlands \\ ${ }^{3}$ Department of Applied Stochastics, Institute for Mathematics, Astrophysics and Particle Physics, Radboud University, Nijmegen, Netherlands \\ ${ }^{4}$ Molecular Breeding, Bayer Crop Science Vegetable Seeds, Nunhem, Netherlands
}

\section{Edited by: \\ Domenico De Martinis, ENEA Italian \\ National Agency for New \\ Technologies, Energy and \\ Sustainable Economic \\ Development, Italy \\ Reviewed by: \\ Ning Li, The Hong Kong University of Science and Technology, China Alisdair Fernie, Max Planck Institut for Plant Physiology, Germany}

\section{*Correspondence:}

Ivo Rieu, Department of Molecular Plant Physiology, Institute for Water and Wetland Research, Radboud University, Nijmegen,

Heyendaalseweg 135, 6525 AJ

Nijmegen, Netherlands

e-mail: i.rieu@science.ru.nl
Climacteric fruit ripening, as it occurs in many fruit crops, depends on a rapid, autocatalytic increase in ethylene production. This agriculturally important process has been studied extensively, with tomato simultaneously acting both as a model species and target crop for modification. In tomato, the ethylene biosynthetic genes ACC SYNTHASE2 (ACS2) and ACS4 are highly expressed during fruit ripening, with a combined loss of both ACS2 and ACS4 activity preventing generation of the ethylene burst necessary for fruit ripening. However, the individual roles and importance of ACS2 and ACS4 have not been determined. In this study, we examined specifically the role of ACS4 by comparing the phenotype of an acs 4 mutant firstly with that of the wild-type, and secondly with two novel ripening-inhibitor (rin) mutants. Ethylene production during ripening was significantly reduced in both acs4-1, and rin lines, with rin genotypes showing the weaker ethylene burst. Also i) the time between anthesis and the start of fruit ripening and ii) the time required to progress through ripening were significantly longer in acs4-1 than in the wild type, but shorter than in the strongest rin mutant. The delay in ripening was reflected in the lower expression of ripening-related transcripts during the mature green and light red ripening stages. Furthermore, expression of ACS2 and ACS4 was strongly dependent on a functional RIN gene, while ACS2 expression was largely independent of ACS4. Altogether, we show that ACS4 is necessary for normal progression of tomato fruit ripening and that mutation of this gene may provide a useful means for altering ripening traits.

Keywords: $A C S 2, A C S 4$, climacteric fruit ripening, ethylene, RIN, tomato

\section{INTRODUCTION}

Climacteric fruit ripening is a mechanism by which fully grown fruits go through a final phase of changes in texture, color, smell, and taste. It is accompanied by a burst in respiration and a rapid, autocatalytic increase in the production of the gaseous plant hormone ethylene (Alexander and Grierson, 2002). Because of the importance of climacteric fruits for the human diet (e.g., tomato, apple, banana, mango, avocado, and passion fruit) this type of ripening has been studied extensively, with tomato (Solanum lycopersicum) serving as both a model species and target crop for modification.

Research on non-ripening tomato mutants has provided detailed insights into the regulation of climacteric ripening. One of the best studied of these tomato mutants is ripening-inhibitor (rin), in which virtually all measured ripening phenomena, like the ethylene burst, change in color, fruit softening and the production of flavor compounds are inhibited (Tigchelaar et al., 1978). The dramatic phenotypic effect of the rin mutant suggests that RIN is a master regulator for many ripening related processes. RIN/rin hybrids are widely used in commercial tomato production because of their extended shelf-life (Vrebalov et al.,
2002). Exogenous ethylene can still activate ethylene response genes in a rin background, but less effectively than in the wild type (Lincoln and Fischer, 1988), suggesting that RIN also has ethylene-independent functions and thus acts upstream of both ethylene- and non-ethylene-mediated ripening processes. In 2002, Vrebalov et al. showed that disruption of the gene LeMADS-RIN is responsible for the ripening phenotype of the rin mutant (Vrebalov et al., 2002). RIN is part of the MADS-box transcription factor family, known to function as DNA-binding protein dimers consisting of two interacting MADS monomers (Ng and Yanofsky, 2001; Ito et al., 2008; Smaczniak et al., 2012). Other well studied genes involved in the regulation of tomato fruit ripening are COLORLESS NONRIPENING (CNR), an SBP-type transcription factor (Manning et al., 2006) and NONRIPENING (NOR), a NAC transcription factor (Seymour et al., 2013). Complex interactions exist between RIN, CNR, and NOR, with NOR acting upstream and downstream RIN (Martel et al., 2011; Osorio et al., 2011) and CNR being both a target of RIN as well as a necessary factor for RIN DNA binding activity (Martel et al., 2011). Furthermore, RIN probably acts as part of a protein complex containing another ripening-associated 
MADS-box protein, TAGL1 (Giovannoni, 2007; Vrebalov et al., 2009).

RIN has been shown to directly interact with promoters of genes involved in the major pathways associated with ripening, like ethylene biosynthesis, ethylene perception, downstream ethylene response, cell wall metabolism, and carotenoid biosynthesis (Fujisawa et al., 2011, 2012; Martel et al., 2011). The induction of ethylene biosynthesis is an essential process in the ripening of climatic fruit (Alexander and Grierson, 2002). Ethylene is synthesized from methionine, which is converted to Sadenosyl-L-methionine in the Yang-cycle (Adams and Yang, 1979). S-adenosyl-L-methionine is processed into 1-aminocyclopropane1-carboxylic acid (ACC) by ACC synthase (ACS) and subsequently the ACC is oxidized by ACC oxidases (ACO) to form ethylene (Kende, 1993 and references therein). Because ethylene is involved in a wide range of developmental processes throughout the plant, ethylene synthesis, perception and signaling must be tightly controlled (Lin et al., 2009). There are at least 12 ACS and 7 ACO genes in the tomato genome with specific temporal and spatial expression patterns (Seymour et al., 2013). It is suggested that the production of ethylene is regulated by two different systems (McMurchie et al., 1972). System 1 is pre-climacterically active and is responsible for providing the basal levels of ethylene which are found in developing fruits and vegetative tissues. ACS1 $a$ and ACS6 are thought to fulfill the function of ACC synthase in the auto-inhibitory ethylene synthesis of system 1 (Zarembinski and Theologis, 1994; Oetiker et al., 1997; Barry et al., 2000). When the fruit has reached a stage at which it is ripening-competent it goes through a transitional phase in which the expression of ACS1a is temporarily enhanced and the expression of ACS4 is induced. The RIN protein is thought to play a role during this transitional phase via the direct transcriptional activation of ACS2 and ACS4 (Barry et al., 2000; Fujisawa et al., 2011, 2012; Martel et al., 2011). Higher concentrations of ethylene trigger system 2, the system active during climacteric ripening of fruit and characterized by an increase in the expression of ACS2 and ACS4. Upon activation of system 2, the expression of ACS1a and ACS6 decreases and the ethylene production becomes auto-stimulatory (Barry et al., 2000). Consequently, the hyper-activation of ACS2 and ACS4 expression causes a burst of ethylene. This big increase in ethylene production promotes the ripening process and is essential for normal completion of ripening in climacteric-ripening plants.

Tomato plants expressing an antisense gene targeting ACS2 show strong down regulation of both ACS2 and ACS4. The antisense fruit slowly develop an orange color but never turn red and soft nor develop an aroma (Oeller et al., 1991). They have reduced ripening-related ethylene synthesis, down to $0.1 \%$ of control fruit, and show no respiratory burst. Treatment of the antisense plants with exogenous ethylene rescues the phenotype, induces the respiratory burst and initiates the ripening process (Oeller et al., 1991). These plants show that down-regulating ACS2 and ACS4 has a strong effect on ethylene production and ripening. However, the individual roles and importance of these genes have not been determined. In this study, we examined a newly generated acs4 mutant with a homozygous mutation introducing a stop-codon in the first half of the ACS4 coding sequence. We studied the effect of the mutation on ethylene production of the fruit, ripening timing and the expression of ripening-related genes.

\section{MATERIALS AND METHODS PLANT MATERIAL}

Solanum lycopersicum var. TPAADASU was used for all experiments. For the ethylene measurements, plants were grown under standard greenhouse conditions, with $16 \mathrm{~h} /$ day and $8 \mathrm{~h} / \mathrm{night}$ (assimilation lights used during the day at less than $250 \mathrm{~W} / \mathrm{m}^{2}$ ) and temperature kept above $20 / 18^{\circ} \mathrm{C}$ (day/night). For the experiment regarding ripening time and gene expression analysis, plants were grown under standard greenhouse conditions, with $16 \mathrm{~h} /$ day and $8 \mathrm{~h} /$ night (assimilation lights used during the day at less than $300-350 \mathrm{~W} / \mathrm{m}^{2}$ ) and temperature kept above $20.5 / 15.5^{\circ} \mathrm{C}$ (day/night). Genotypes were randomly distributed in the greenhouse. Self-pollination was stimulated by artificial vibration of flower clusters three times per week to get homogeneous fertilization and fruit size. Fruit stages were based on color, as determined by a single person throughout the experiments. Used stages were: mature green $(M G)$, turning $(T)$, orange $(O)$, light red $(L R)$ and red (R) (Gillaspy et al., 1993; Steinhauser et al., 2010). Because the coloring of the different lines was not uniform, the turning stage represented the fruits that were halfway in between mature green and orange. This included fruits that had a green skin with marks of orange and fruits that were more uniformly yellow. Green fruit of the wild type were harvested on average 36 days after pollination.

\section{MUTANT GENERATION AND IDENTIFICATION}

Mutations were identified by screening a TPAADASU EMS M2 mutant population for two PCR fragments with the TILLING protocol adapted for a HRM LightScanner platform as described previously (Gady et al., 2009). The acs4-1 mutant presented here was identified in a gene fragment amplified using the following primers: $5^{\prime}$-GCTATCGAAGAGGCCTATGAAAAAGG-3' (forward) and 5'-CACAAATTCATCGTCAGACAACATG-3' (reverse). Both mutations in the RIN gene were identified in a PCR fragment generated with primers $5^{\prime}$-TTGATGAAATTG ATTTTCTTGTTG-3' (forward) and 5'-AGCAAGTTGATCAAGA ATGTGTT- $3^{\prime}$ (reverse). PCRs were performed as follows on fourfold genomic DNA pools and, after positive pool selection, on single family genomic DNA: $94^{\circ} \mathrm{C}$ for 2 min; 40 cycles of $94^{\circ} \mathrm{C}$ for $5 \mathrm{~s}, 68^{\circ} \mathrm{C}$ for $10 \mathrm{~s}$, and $72^{\circ} \mathrm{C}$ for $10 \mathrm{~s}$; a final denaturation step of $30 \mathrm{~s}$ at $94^{\circ} \mathrm{C}$; and renaturation by cooling to $30^{\circ} \mathrm{C}$. RT-PCR to analyze rin-3 transcript length was done with a standard PCR program, using primers $5^{\prime}$-CCAAGACATTGGAGAGATACCA-3' (forward) and 5'-TTTGCCTCAATGATGAATCC-3' (reverse).

\section{ETHYLENE MEASUREMENTS}

Three individuals per genotype were used. After harvesting, fruits were left to recover for $1 \mathrm{~h}$ (for the stages $\mathrm{T}, \mathrm{O}$ and $\mathrm{LR}$ ) or $3 \mathrm{~h}$ (for stage MG) before being weighted and placed into glass cuvettes connected on-line with a laser-based ethylene detector (ETD-300, Sensor Sense B.V. Nijmegen, the Netherlands) (Cristescu et al., 2008). For mature green fruit the air flow was set at $1 \mathrm{~L} / \mathrm{h}$, for all other stages at $4 \mathrm{~L} / \mathrm{h}$. Ethylene measurement was performed as described before (Nitsch et al., 2012). The samples were measured at least twice for 10-12 min and a representative measurement was selected. Four to six biological replicas were used per stage and line. The ethylene emission was corrected for flow, weight, and background ethylene in the air. 


\section{RIPENING TIME MEASUREMENTS}

For the mutant rin-3, two individuals were used; for the wild type and the rin-2 and acs4-1 mutant lines five. On specific time points during a period of 4 weeks the stage of each individual fruit (MG, T, O, LR and R) was determined. Based on these observations a model was developed to estimate the effect of the genotype on the number of days between anthesis and the start of ripening as well as on the ripening time (i.e., number of days between the first signs of ripening and the red stage) for the different plant lines in this study. As covariates the plant number, which truss the fruit was on and the fruit number were considered. The data was censored, since it was not known which day the fruit entered in a particular phase, only the phase at a fixed number of time points was known. Since this limited the amount of information in the data, not all possible interactions were considered. Furthermore, some plants carried very few fruits at the end of the experiment; the model therefore included a random effect for the plant number. $Y=\left(Y^{(1)}, Y^{(2)}\right)^{t}$ was defined as the two time points at which the plant entered the two stages of interest (i.e., first sign of ripening and actual ripening), $p$ as an index indicating the plant number, $b$ as an index indicating the truss and finally $f$ as an index indicating the fruit number, forming the following model:

$$
\left(\begin{array}{c}
Y_{p b f}^{(1)} \\
Y_{p b f}^{(2)}
\end{array}\right)=\left(\begin{array}{c}
\mu^{(1)}+\alpha_{m(p)}^{(1)}+\beta_{b}^{(1)}+\gamma_{f}^{(1)} \\
\mu^{(2)}+\alpha_{m(p)}^{(2)}+\beta_{b}^{(2)}+\gamma_{f}^{(2)}
\end{array}\right)+Z_{p}+U_{p b f} .
$$

Here, $m(p)$ is the genetic modification of plant $p$, while $\mu, \alpha_{m(p)}$, $\beta_{b}$, and $\gamma_{f}$ are parameters of the linear model, and $Z_{p}$ is a random effect corresponding to each plant, such that

$$
Z_{p} \sim N_{2}\left(\left(\begin{array}{l}
0 \\
0
\end{array}\right), \sum_{0}\right)
$$

where $\Sigma_{0}$ is an unknown 2-by-2 covariance matrix. Finally, $U_{m p b f}$ is an independent random fluctuation

$$
U_{p b f} \sim N_{2}\left(\left(\begin{array}{l}
0 \\
0
\end{array}\right), \sum\right)
$$

with $\Sigma$ unknown. Due to the censoring of the data $L^{(i)}=L_{m p b f}^{(i)}$ and $R^{(i)}=R_{m p b f}^{(i)}$ are defined as the last time the fruit was observed before, respectively after the relevant phase started (i C; $L \leq Y \leq R$ ). The log-likelihood of all observations as a function of the parameters is given by:

$$
\begin{array}{r}
\sum_{p=1}^{26} \log \left(\mathbb { E } _ { \sum _ { 0 } } \left(\prod _ { b , f } \mathbb { P } _ { \sum } \left(U+\mu+\alpha_{m(p)}+\beta_{b}+\gamma_{f}\right.\right.\right. \\
\left.\left.\left.+Z \in\left[L_{p b f}^{(1)}, R_{p b f}^{(1)}\right] \times\left[L_{p b f}^{(2)}, R_{p b f}^{(2)}\right]\right)\right)\right),
\end{array}
$$

Where $U$ under $\mathrm{P}_{\sigma}$ has a $\mathrm{N}(0, \Sigma)$-distribution and $Z$ under $E_{\sigma 0}$ has a $\mathrm{N}\left(0, \Sigma_{0}\right)$-distribution. This model was used on the data of 26 plants (including 9 plants from non-presented genotypes) and a full factorial design with respect to the truss, the fruit and the plant. Maximum likelihood was applied to estimate the parameters of this model.

\section{REAL-TIME QUANTITATIVE RT-PCR ANALYSIS}

Fruits were obtained from at least two individuals per genotype. Directly after harvesting the fruit, the pericarp tissue was collected, cut into small pieces, frozen in liquid nitrogen and stored at $-80^{\circ} \mathrm{C}$. The pieces were ground into powder with piston and mortar in liquid nitrogen to keep the tissue frozen. $200 \mathrm{mg}$ of powder was used to isolate total RNA (RNeasy Plant Mini kit, Qiagen). The RNA was treated with DNase (TURBO DNA-free, Ambion) and $0.9 \mu \mathrm{g}$ was used for reverse transcription (iScript cDNA Synthesis kit, BioRad). The cDNA equivalent of $20 \mathrm{ng}$ of total RNA was used in a $25 \mu \mathrm{l}$ PCR reaction on a thermocycler (CFX iCycler, BioRad) with cybergreen (iQ SYBR Green Supermix, BioRad). Three to four biological replicas of each sample type were analyzed with two technical replicates. Absence of genomic DNA and primer dimers was confirmed by analysis of RT-minus and water control samples and by examination of dissociation curves. PCR primers were designed using a computer program (Beacon Designer 7.51) and are listed in Supplemental Table 1. Quantitative gene expression data was analyzed according to Rieu and Powers (2009). To normalize the qPCR data, five reference genes were used (EF-1 $\alpha$, Actin2-7, RPL8, UBQ11, and $G A P D H 3)$ and the stability of these reference genes across samples was confirmed using geNORM software $\left(\mathrm{V}_{5 / 6}=0.15\right.$; Vandesompele et al., 2002). Primer pair efficiencies were estimated by analysis of the amplification curves with LinReg v12.1 software (Ramakers et al., 2003) and the average efficiency of all reactions on a plate was used in calculations (Cook et al., 2004).

\section{STATISTICAL ANALYSIS}

Ethylene evolution data of the different genotypes was compared using one-way ANOVA with LSD on log-transformed data. To test hypothesis on differences in ripening time parameters, likelihood ratio testing (with asymptotic $\chi^{2}$-distributions) was applied to the ripening model described above. For analysis of the qPCR results, log-transformed normalized relative quantities were compared using a one-way ANOVA with LSD. In case of the LR stage, two datasets were combined following mean centering.

\section{RESULTS}

\section{GENERATION AND IDENTIFICATION OF NEW ALLELES OF ACS4 AND}

\section{RIN}

Using the TILLING strategy (Gady et al., 2009), a new allele of ACS4, named acs4-1, was identified in tomato. The allele had an A-to-T mutation at bp 610 of the open-reading frame resulting in a premature stop codon $\left[\mathrm{K}^{204}\right.$ (AAA) to stop (TAA)] and a truncated protein (Figure 1A). The truncated protein is likely to represent a null-allele, as it ends in the middle of the canonical aminotransferase class I/classII domain (IPR004839, aa 25-439), before the cofactor binding site (IPR004838, aa 279-292). In the same way, two novel RIN alleles were identified and named rin-2 and rin-3. The rin-2 mutation results in an amino-acid substitution $\mathrm{Leu}^{112}$ (CTA) to Pro ${ }^{112}$ (CCA) in the conserved K-box 

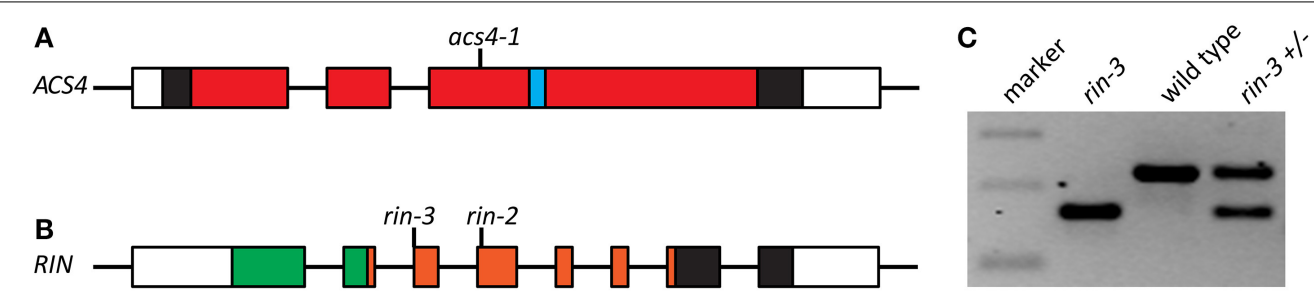

FIGURE 1 | Gene models and mutant description. Gene models of ACS4 (A) and RIN (B), indicating the sites of mutations in the alleles presented in this study. Exons are indicated as boxes and depicted in relative sizes, introns not. Coding sequence is indicated as filled boxes; red: the canonical aminotransferase class I/classII domain (IPR004839); blue: the cofactor binding site (IPR004838); green: MADS-box domain (IPR002100); orange: K-box (IPR002487). (C) RT-PCR showing reduced length of the rin-3 transcript. The 100-, 200- and 300-bp bands of the DNA marker are shown.
(IPR002487), which promotes protein dimerization. The mutation in rin-3 is a substitution of the last nucleotide ( $G$ to $A$ ) of intron 2. This nucleotide is part of the splicing acceptor site and cDNA sequencing showed that the mutation leads to an mRNA that misses the 62-bp long exon 3 (Figures 1B,C). This, in turn, introduces a stop codon in the ORF of the mutated gene, five codons downstream of $\mathrm{Q}^{88}$. The truncated protein still contains the complete MADS-box domain (IPR002100) for DNA binding, but has lost most of the K-box, making it a likely null-allele. These two new rin mutant lines are unique in that they are solely mutated in the LeMADS-RIN locus and therefore differ from the classical rin mutant, in which the mutation consists of a deletion of both LeMADS-RIN and the neighboring LeMADS-MACROCALYX (LeMADS-MC), the latter being is associated with sepal development (Vrebalov et al., 2002).

\section{ETHYLENE EMISSION}

As a characteristic of the climacteric fruit ripening process, ethylene production was strongly enhanced during the ripening phase in the wild-type tomato fruits (Figure 2). To determine the effect of the new mutations in ACS4 and RIN on the production of ethylene in the fruit, ethylene emission was measured at two ripening stages, i.e., turning and light red (Figure 2). The rin-2 and -3 mutant fruits showed a 90-95\% reduction in ethylene emission at the analyzed stages, as compared to the similar stage in the wild-type $(P<0.001)$, confirming that both alleles have severely reduced RIN function, but produced more ethylene than maturegreen wild-type fruit $(P<0.001)$. acs $4-1$ produced about a third to a quarter of the ethylene of the wild type at the turning and light red stages, respectively $(P<0.05)$.

\section{RIPENING TIMES}

To estimate the effect of the genotype on both the period before the ripening process is initiated and the time required to progress through ripening, we developed a model, taking as covariates (i) the plant individual, (ii) the position of the truss on the plant and (iii) the position of the fruit in the truss (see Materials and Methods). Likelihood ratio tests showed that the position of the truss was not a significant covariate, and therefore this factor was removed from the equation. There was an effect of the position of the fruit, where the fruits formed earlier on a truss (closer to the stem) ripened somewhat faster. Using the model we found that the rin-2 mutant line showed a significant increase in both,

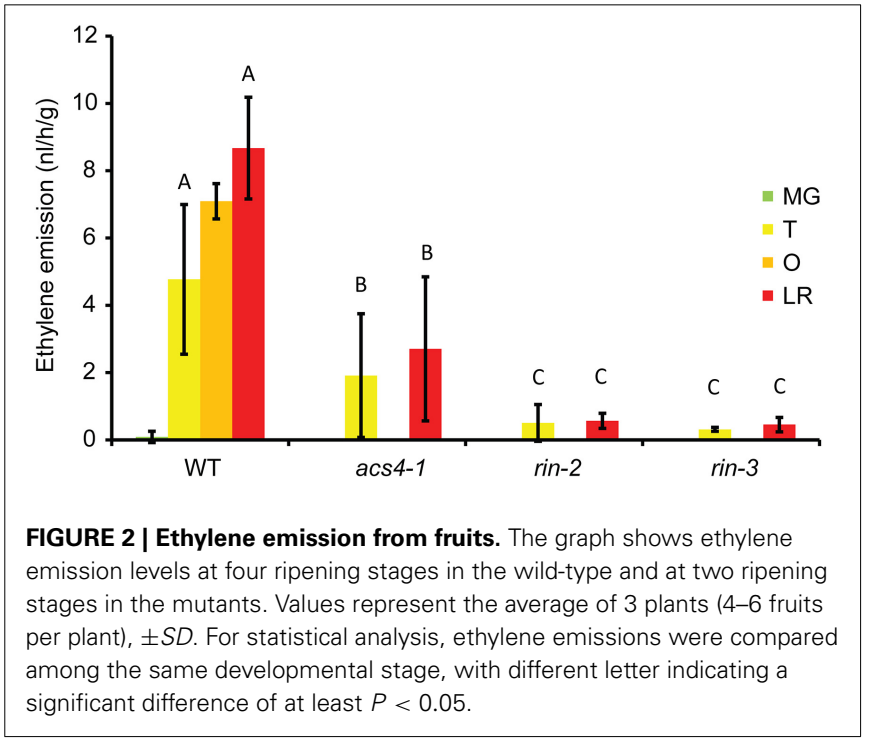

the average time until fruit ripening started and the length of the climacteric ripening phase, as compared to the wild type (Figures 3A,B, also see Supplemental Table 2). The rin-3 mutant also had an increased ripening time but showed no significant delay in the appearance of the first sign of ripening. The acs4-1 mutant was delayed in both processes, but less so than the rin-2 mutant.

\section{EXPRESSION OF RIPENING RELATED GENES}

To determine the effect of the mutations on the expression of ripening-related genes, quantitative RT-PCR was used to measure the transcript levels of ACS2, ACS4, expansin1 (EXP1), phytoene synthase 1 (PSY1) and polygalacturinase $(P G)$ in fruits before and during the ripening phase (Figure 4). Each of these genes is known to function during fruit ripening. PSY1 is needed for the production of carotenoids (Bird et al., 1991) and PG and EXP1 are involved in the softening of the fruit (cell wall metabolism) (DellaPenna et al., 1989; Rose et al., 1997). As expected, the expression of all five genes was strongly up-regulated from the green to the light red fruit stage in the wild-type, from around 18 times (ACS4) to almost 1000 times (PG). The rin mutants showed a significant reduction in expression of all these transcripts during fruit ripening, and in rin-2 the expression of four out of the 


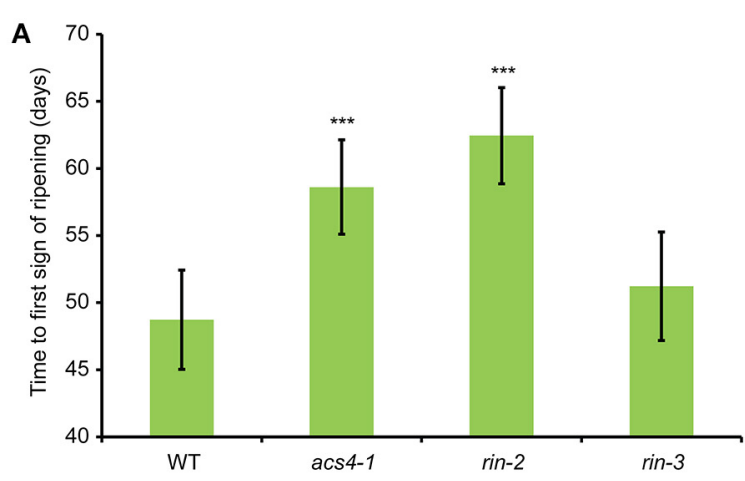

FIGURE 3 | Fruit ripening times. (A) Estimation of the number of days from open flower until the first sign of ripening, $\pm S D(n=24-60$ fruits, i.e., $10-12$ fruits from 2-5 plants per genotype). (B) Estimation of the length of the

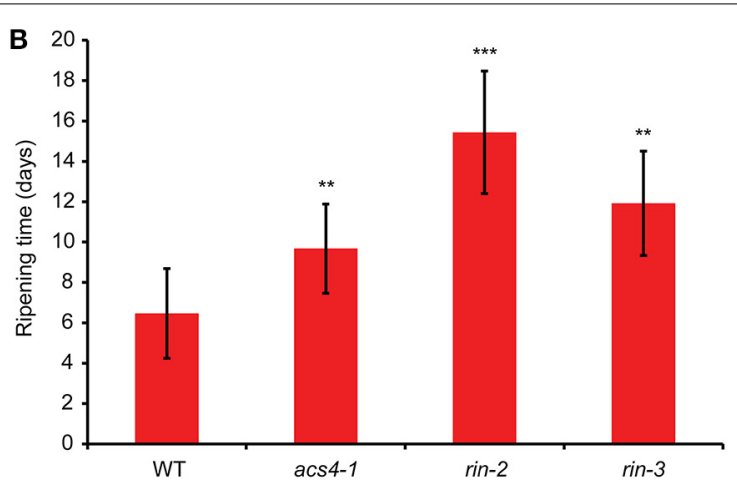

climacteric ripening phase (ripening time), $\pm S D$ ( $n=28-30$ fruits, i.e., 5-6 fruits from 5 plants per genotype each). ${ }^{* *}$, significantly different from the same stage in the wild type, $P<0.01$; ${ }^{* * *}, P<0.001$. five genes was already down-regulated in mature green fruit. In the acs4-1 mutant EXP1 and PSY1 expression was significantly lower than in the wild type in the light red fruit stage, but no differences were detected before the start of ripening. ACS2 and $P G$ showed the same trend, although not statistically significant. We also examined the system-1 ACS gene ACS6. In the wild-type, the expression of this gene was down-regulated during ripening $(P<0.01)$. Contrary to the genes mentioned above, the regulation of ACS6 was not affected by the rin mutations, and also not by the acs $4-1$ mutation.

\section{DISCUSSION}

By measuring the ethylene production, ripening timing and ripening-related gene expression in an acs4-1 mutant and comparing it to control genotypes, we gained insight into the role of the tomato system-2 ACC synthase gene ACS4 in climacteric fruit ripening.

As control genotypes, we used two novel rin alleles, both in the TPAADASU background. As described here, rin-2 and -3 are severely affected in all measured ripening characteristics, indicating that they have reduced RIN function. The phenotypes, however, are weaker than described for the original rin mutant. In the latter, ACS2, ACS4, EXP1, PG, and PSY1 expression is not or hardly up-regulated during ripening (DellaPenna et al., 1989; Rose et al., 1997; Barry et al., 2000; Fujisawa et al., 2011, 2012; Martel et al., 2011), ethylene production does not rise above the pre-climacteric levels (i.e., about $1-2 \%$ of the orange to red stages in the wild type), even after 120 days of development, and fruits do not color beyond yellow (Robinson and Tomes, 1968; Herner and Sink, 1973; Tigchelaar et al., 1978). By contrast, significant up-regulation of the tested ripening-related transcripts was seen in rin-2 and -3 and fruit of the mutants still produced $5-10 \%$ of wild-type ethylene during ripening, well above preclimacteric levels (Figure 2). Most tellingly, rin-2 and -3 fruit was able to complete fully the ripening process (Figure 3). As rin mutants are still ethylene responsive, the ripening-related gene expression and ripening progression could in theory be caused by exogenous ethylene from ethylene-producing neighboring plants during cultivation. However, because exogenous ethylene does not enhance ethylene production in rin (Herner and Sink, 1973), a more likely explanation might be that system-2 ethylene production is less RIN dependent in TPAADASU than in VF36, the original rin background or that the LeMADS-MC protein, which is not affected in rin-2 and -3, might play a redundant role in system-2 ethylene production in the absence of functional RIN protein. Public transcriptome sequencing data (http://www.ncbi. nlm.nih.gov/Traces/sra/?study=SRP010775) show that this gene is relatively active in the fruit at breaker stage (Tomato Genome Consortium, 2012). Also, we found that expression of the system1 gene ACS6 was down-regulated upon ripening in rin-2 and -3 similar to wild type fruits, whereas Barry et al. (2000) did not see such down-regulation in rin. However, our results are similar to those on rin published in a public database (http://www. ncbi.nlm.nih.gov/Traces/sra/?study=SRP004923). These differences are likely to be due to differences in the stage analyzed. Further study is needed to clarify these aspects.

The acs4-1 mutant, too, had a significantly lower rate of ethylene production, delayed ripening and reduced ethylenedependent gene expression. These effects, however, were less severe than described for an ACS2/ACS4 silenced line, which produced virtually no ethylene and did not ripen beyond the orange stage in up to 120 days (Oeller et al., 1991). Together, this shows that ACS4 has a unique function in the production of system-2 ethylene and climacteric ripening in tomato, but that there is an additional, important role for ACS2. Although ACS2 expression was suggested to depend on ACS4 expression during the transition from the system-1 to system-2 ethylene synthesis (Barry et al., 2000), no significant reduction in its expression was found in the acs4-1 mutant (Figure 4). Together with the finding that both ACS4 and ACS2 are direct targets of RIN (Martel et al., 2011), this does not support the idea that ACS4 has an essential role in the system-1-to-2 transition. However, it should be noted that the fact that fruits were always compared at similar ripening stages, instead of at similar time points during development, dampens observed differences between genotypes.

In summary, we conclude that ACS4 has an important role in the climacteric ripening of tomato fruit. A $\mathrm{K}^{204}$ truncation of the acs 4 protein leads to a significant delay in the ripening process 


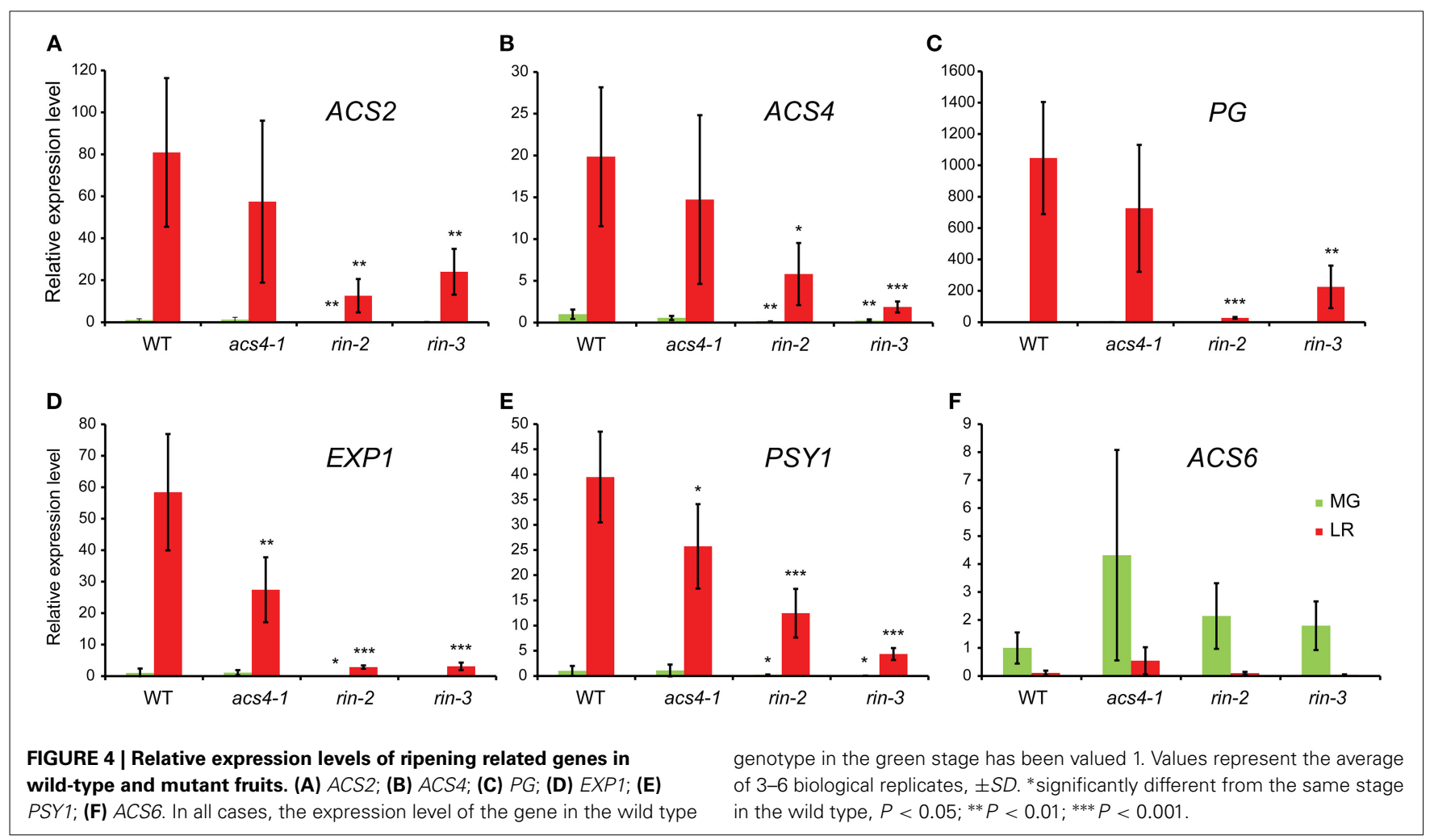

on multiple levels. This allele, as well as the new rin alleles, may be useful to increase tomato shelf life and reduce post-harvest losses.

\section{ACKNOWLEDGMENTS}

The authors thank Gerard van der Weerden (Experimental garden and gene bank, Radboud University Nijmegen, The Netherlands) and his staff for taking excellent care of the plants used in this study, Jurre Floors for technical assistance and William Teale for comments on the manuscript.

\section{SUPPLEMENTARY MATERIAL}

The Supplementary Material for this article can be found online at: http://www.frontiersin.org/journal/10.3389/fpls. 2014.00466/abstract

\section{REFERENCES}

Adams, D. O., and Yang, S. F. (1979). Ethylene biosynthesis: identification of 1-aminocyclopropane-1-carboxylic acid as an intermediate in the conversion of methionine to ethylene. Proc. Natl. Acad. Sci. U.S.A. 76, 170-174. doi: 10.1073/pnas.76.1.170

Alexander, L., and Grierson, D. (2002). Ethylene biosynthesis and action in tomato: a model for climacteric fruit ripening. J. Exp. Bot. 53, 2039-2055. doi: 10.1093/ jxb/erf072

Barry, C. S., Llop-Tous, M. I., and Grierson, D. (2000). The Regulation of 1aminocyclopropane-1-carboxylic acid synthase gene expression during the transition from system-1 to system-2 ethylene synthesis in tomato. Plant Physiol. 123, 979-986. doi: 10.1104/pp.123.3.979

Bird, C. R., Ray, J. A., Fletcher, J. D., Boniwell, J. M., Bird, A. S., Teulieres, C., et al. (1991). Using antisense RNA to study gene function: inhibition of carotenoid biosynthesis in transgenic tomatoes. Nat. Biotechnol. 9, 635-639. doi: $10.1038 /$ nbt0791-635
Cook, P., Fu, C., Hickey, M., Han, E. S., and Miller, K. S. (2004). SAS programs for real-time RT-PCR having multiple independent samples. Biotechniques 37, 990-995. Available online at: http://www.biotechniques. com/BiotechniquesJournal/2004/December/SAS-programs-for-real-time-RTPCR-having-multiple-independent-samples/biotechniques-117478.html

Cristescu, S. M., Persijn, S. T., te Lintel Hekkert, S., and Harren, F. J. M (2008). Laser-based systems for trace gas detection in life sciences. Appl. Phys. B 92, 343-349. doi: 10.1007/s00340-008-3127-y

DellaPenna, D., Lincoln, J. E., Fischer, R. L., and Bennett, A. B. (1989). Transcriptional analysis of polygalacturonase and other ripening associated genes in rutgers, rin, nor, and Nr tomato fruit. Plant Physiol. 90, 1372-1377. doi: $10.1104 /$ pp.90.4.1372

Fujisawa, M., Nakano, T., and Ito, Y. (2011). Identification of potential target genes for the tomato fruit-ripening regulator RIN by chromatin immunoprecipitation. BMC Plant Biol. 11:26. doi: 10.1186/1471-2229$11-26$

Fujisawa, M., Shima, Y., Higuchi, N., Nakano, T., Koyama, Y., Kasumi, T., et al. (2012). Direct targets of the tomato-ripening regulator RIN identified by transcriptome and chromatin immunoprecipitation analyses. Planta 235, 1107-1122. doi: 10.1007/s00425-011-1561-2

Gady, A. L., Hermans, F. W., van de Wal, M. H., van Loo, E. N., Visser, R. G., and Bachem, C. W. (2009). Implementation of two high through-put techniques in a novel application: detecting point mutations in large EMS mutated plant populations. Plant Methods 5, 13. doi: 10.1186/1746-4811-5-13

Gillaspy, G., Ben-David, H., and Gruissem, W. (1993). Fruits - a developmental perspective. Plant Cell 5, 1439-1451. doi: 10.1105/tpc.5.10.1439

Giovannoni, J. J. (2007). Fruit ripening mutants yield insights into ripening control. Curr. Opin. Plant Biol. 10, 283-289. doi: 10.1016/j.pbi.2007. 04.008

Herner, R. C., and Sink, K. C. Jr. (1973). Ethylene production and respiratory behavior of the rin tomato mutant. Plant Physiol. 52, 38-42. doi: 10.1104/pp. 52.1 .38

Ito, Y., Kitagawa, M., Ihashi, N., Yabe, K., Kimbara, J., Yasuda, J., et al. (2008). DNAbinding specificity, transcriptional activation potential, and the rin mutation effect for the tomato fruit-ripening regulator RIN. Plant J. 55, 212-223. doi: 10.1111/j.1365-313XX.2008.03491.x 
Kende, H. (1993). Ethylene biosynthesis. Annu. Rev. Plant Physiol. Plant Mol. Biol. 44, 283-307. doi: 10.1146/annurev.pp.44.060193.001435

Lin, Z., Zhong, S., and Grierson, D. (2009). Recent advances in ethylene research. J. Exp. Bot. 60, 3311-3336. doi: 10.1093/jxb/erp204

Lincoln, J. E., and Fischer, R. L. (1988). Regulation of gene expression by ethylene in wild-type and rin tomato (Lycopersicon esculentum) fruit. Plant Physiol. 88, 370-374. doi: 10.1104/pp.88.2.370

Manning, K., Tör, M., Poole, M., Hong, Y., Thompson, A. J., King, G. J., et al. (2006). A naturally occurring epigenetic mutation in a gene encoding an SBP-box transcription factor inhibits tomato fruit ripening. Nat. Genet. 38, 948-952. doi: $10.1038 / \mathrm{ng} 1841$

Martel, C., Vrebalov, J., Tafelmeyer, P., and Giovannoni, J. J. (2011). The tomato MADS-box transcription factor RIPENING INHIBITOR interacts with promoters involved in numerous ripening processes in a COLORLESS NONRIPENING-dependent manner. Plant Physiol. 157, 1568-1579. doi: 10.1104/pp.111.181107

McMurchie, E. J., McGlasson, W. B., and Eaks, I. L. (1972). Treatment of fruit with propylene gives information about the biogenesis of ethylene. Nature 237, 235-236. doi: $10.1038 / 237235 \mathrm{a} 0$

Ng, M., and Yanofsky, M. F. (2001). Function and evolution of the plant MADS-box gene family. Nat. Rev. Genet. 2, 186-195. doi: 10.1038/35056041

Nitsch, L., Kohlen, W., Oplaat, C., Charnikhova, T., Cristescu, S., Michieli, P., et al. (2012). ABA-deficiency results in reduced plant and fruit size in tomato. J. Plant Physiol. 169, 878-883. doi: 10.1016/j.jplph.2012.02.004

Oeller, P. W., Lu, M. W., Taylor, L. P., Pike, D. A., and Theologis, A. (1991). Reversible inhibition of tomato fruit senescence by antisense RNA. Science 254, 437-439. doi: 10.1126/science. 1925603

Oetiker, J. H., Olson, D. C., Shiu, O. Y., and Yang, S. F. (1997). Differential induction of seven 1-aminocyclopropane-1-carboxylate synthase genes by elicitor in suspension cultures of tomato (Lycopersicon esculentum). Plant Mol. Biol. 34, 275-286. doi: 10.1023/A:1005800511372

Osorio, S., Alba, R., Damasceno, C. M. B., Lopez-Casado, G., Lohse, M., Inés Zanor, M., et al. (2011). Systems biology of tomato fruit development: combined transcript, protein, and metabolite analysis of tomato transcription factor (nor, rin) and ethylene receptor $(\mathrm{Nr})$ mutants reveals novel regulatory interactions. Plant Physiol. 157, 405-425. doi: 10.1104/pp.111.175463

Ramakers, C., Ruijter, J. M., Lekanne Deprez, R. H., and Moorman, A. F. M. (2003). Assumption-free analysis of quantitative real-time polymerase chain reaction (PCR) data. Neurosci. Lett. 339, 62-66. doi: 10.1016/S0304-3940(02)01423-4

Rieu, I., and Powers, S. J. (2009). Real-time quantitative RT-PCR: design, calculations, and statistics. Plant Cell 21, 1031-1033. doi: 10.1105/tpc.109.066001

Robinson, R. W., and Tomes, M. L. (1968). Ripening inhibitor: a gene with multiple effect on ripening. Tomato Genet. Coop. 18, 36-37.

Rose, J. K. C., Lee, H. H., and Bennett, A. B. (1997). Expression of a divergent expansin gene is fruit-specific and ripening-regulated. Proc. Natl. Acad. Sci. U.S.A. 94, 5955-5960. doi: 10.1073/pnas.94.11.5955

Seymour, G. B., Chapman, N. H., Chew, B. L., and Rose, J. K. C. (2013). Regulation of ripening and opportunities for control in tomato andother fruits. Plant Biotechnol. J. 11, 269-278. doi: 10.1111/j.1467-7652.2012.00738.x
Smaczniak, C., Immink, R. G., Angenent, G. C., and Kaufmann, K. (2012). Developmental and evolutionary diversity of plant MADS-domain factors: insights from recent studies. Development 139, 3081-3098. doi: 10.1242/dev. 074674

Steinhauser, M. C., Steinhauser, D., Koehl, K., Carrari, F., Gibon, Y., Fernie, A. R., et al. (2010). Enzyme activity profiles during fruit development in tomato cultivars and Solanum pennellii. Plant Physiol. 153, 80-98. doi: 10.1104/pp.110. 154336

Tigchelaar, E. C., McGlasson, W. B., and Buescher, R. W. (1978). Genetic regulation of tomato fruit ripening. HortScience 13, 508-513.

Tomato Genome Consortium. (2012). The tomato genome sequence provides insights into fleshy fruit evolution. Nature 485, 635-641. doi: 10.1038/nature 11119

Vandesompele, J., de Preter, K., Pattyn, F., Poppe, B., van Roy, N., de Paepe, A., et al. (2002). Accurate normalization of real-time quantitative RT-PCR data by geometric averaging of multiple internal control genes. Genome Biol. 3:research0034. doi: 10.1186/gb-2002-3-7-research0034

Vrebalov, J., Pan, I. L., Arroyo, A. J. M., McQuinn, R., Chung, M. Y., Poole, M., et al. (2009). Fleshy fruit expansion and ripening are regulated by the tomato SHATTERPROOF gene TAGL1. Plant Cell 21, 3041-3062. doi: 10.1105/tpc.109. 066936

Vrebalov, J., Ruezinsky, D., Padmanabhan, V., White, R., Medrano, D., Drake, R., et al. (2002). A MADS-box gene necessary for fruit ripening at the tomato Ripening-Inhibitor (Rin) locus. Science 296, 343-346. doi: 10.1126/science. 1068181

Zarembinski, T., and Theologis, A. (1994). Ethylene biosynthesis and action: a case of conservation. Plant Mol. Biol. 26, 1579-1597. doi: 10.1007/BF00016491

Conflict of Interest Statement: Patents concerning mutations in the tomato ACS2, ACS4 and RIN genes were published under publication numbers WO2014079896 A1, WO2014049002 A1 and WO2013156204 Al, respectively (Nunhems B. V., Wim $\mathrm{H}$. Vriezen et al). The authors declare that the research was conducted in the absence of any commercial or financial relationships that could be construed as a potential conflict of interest.

Received: 17 July 2014; accepted: 27 August 2014; published online: 16 September 2014. Citation: Hoogstrate SW, van Bussel LJA, Cristescu SM, Cator E, Mariani C, Vriezen WH and Rieu I (2014) Tomato ACS4 is necessary for timely start of and progression through the climacteric phase of fruit ripening. Front. Plant Sci. 5:466. doi: 10.3389/ fpls.2014.00466

This article was submitted to Plant Physiology, a section of the journal Frontiers in Plant Science.

Copyright (c) 2014 Hoogstrate, van Bussel, Cristescu, Cator, Mariani, Vriezen and Rieu. This is an open-access article distributed under the terms of the Creative Commons Attribution License (CC BY). The use, distribution or reproduction in other forums is permitted, provided the original author(s) or licensor are credited and that the original publication in this journal is cited, in accordance with accepted academic practice. No use, distribution or reproduction is permitted which does not comply with these terms. 


\title{
Genetic introgression of ethylene-suppressed transgenic tomatoes with higher-polyamines trait overcomes many unintended effects due to reduced ethylene on the primary metabolome
}

\author{
Anatoly P. Sobolev' ${ }^{1}$ Anil Neelam ${ }^{2}$, Tahira Fatima ${ }^{2}$, Vijaya Shukla ${ }^{2}$, Avtar K. Handa ${ }^{3}$ and \\ Autar K. Mattoo ${ }^{*}$ * \\ ' Laboratory of Magnetic Resonance "Annalaura Segre," Institute of Chemical Methodologies, National Research Council, Rome, Italy \\ 2 Sustainable Agricultural Systems Laboratory, The Henry A. Wallace Agricultural Research Center, Agricultural Research Service, United States Department of \\ Agriculture, Beltsville, MD, USA \\ ${ }^{3}$ Department of Horticulture, Purdue University, West Lafayette, IN, USA
}

\section{Edited by:}

Domenico De Martinis, ENEA Italian National Agency for New

Technologies, Energy and Sustainable Economic Development, Italy

\section{Reviewed by:}

Athanassios Molassiotis, Aristotle University of Thessaloniki, Greece Angelos K. Kanellis, Aristotle University of Thessaloniki, Greece

\section{${ }^{*}$ Correspondence:}

Autar K. Mattoo, Sustainable Agricultural Systems Laboratory, The Henry A. Wallace Agricultural Research Center, Agricultural Research Service, United States Department of Agriculture, Beltsville, MD 20705, USA

e-mail: autar.mattoo@ars.usda.gov
Ethylene regulates a myriad physiological and biochemical processes in ripening fruits and is accepted as the ripening hormone for the climacteric fruits. However, its effects on metabolome and resulting fruit quality are not yet fully understood, particularly when some of the ripening-associated biochemical changes are independent of ethylene action. We have generated a homozygous transgenic tomato genotype (2AS-AS) that exhibits reduced ethylene production as a result of impaired expression of 1 aminocyclopropane-1-carboxylate synthase 2 gene by its antisense RNA and had a longer shelf life. Double transgenic hybrid $(2 \mathrm{AS}-\mathrm{AS} \times 579 \mathrm{HO})$ developed through a genetic cross between 2AS-AS and $579 \mathrm{HO}$ (Mehta etal., 2002) lines resulted in significantly higher ethylene production than either the WT or 2AS-AS fruit. To determine the effects of reduced ethylene and introgression of higher polyamines' trait, the metabolic profiles of ripening fruits from WT (556AZ), 2AS-AS, and 2AS-AS $\times 579 \mathrm{HO}$ lines were determined using ${ }^{1} \mathrm{H}-\mathrm{NMR}$ spectroscopy. The levels of Glu, Asp, AMP, Adenosine, Nucl1, and Nucl2 increased during ripening of the WT fruit. The increases in Glu, Asp, and AMP levels were attenuated in 2AS-AS fruit but recovered in the double hybrid with higher ethylene and polyamine levels. The ripening-associated decreases in Ala, Tyr, Val, Ile, Phe, malate, and myo-inositol levels in the 2AS-AS line were not reversed in the double hybrid line suggesting a developmental/ripening regulated accumulation of these metabolites independent of ethylene. Significant increases in the levels of fumarate, formate, choline, Nucl1, and Nucl2 at most stages of ripening fruit were found in the double transgenic line due to introgression with higher-polyamines trait. Taken together these results show that the ripening-associated metabolic changes are both ethylene dependent and independent, and that the fruit metabolome is under the control of multiple regulators, including ethylene and polyamines.

Keywords: ACC synthase, fruit ripening, LeACS2, metabolome, polyamines, spermidine, spermine, transgenics

\section{INTRODUCTION}

Ethylene is a gaseous plant hormone involved in regulating various aspects of plant growth, development, and senescence (Mattoo and Suttle, 1991; Abeles et al., 1992). Among myriad processes that ethylene regulates its role is plant aging, fruit ripening and cell death is pivotal. Plants have evolved to tightly regulate the production of ethylene, which seems to be achieved via the presence of a family of genes encoding key enzymes in ethylene biosynthesis, and by differential regulation of their transcripts (see Fluhr and Mattoo, 1996; Harpaz-Saad et al., 2012; Gapper et al., 2014). Ethylene biosynthesis in higher plants mostly involves the conversion of methionine to $S$-adenosylmethionine (SAM) catalyzed by SAM synthase, SAM is then converted to 1-aminocyclopropane-1-carboxylate (ACC) catalyzed by ACC synthase, and finally, ACC is oxidized to ethylene by ACC oxidase (Harpaz-Saad et al., 2012; Grierson, 2014).

The reverse genetics approach demonstrated that ethylene is indeed a ripening hormone and ACC synthase is a ratelimiting enzyme in the pathway (Oeller et al., 1991). Treatments with chemicals such as inhibitors of ethylene biosynthesis (e.g., aminoethoxyvinylglycine) or perception [e.g., silver salts or 1-methylcyclopropene (1-MCP)] were shown to delay fruit ripening (Mattoo and Suttle, 1991; Abeles et al., 1992). Genetic approach established that silencing ACC synthase or ACC oxidase using the antisense RNA technology leads to delayed ripening and increased shelf life of fruits (Hamilton et al., 1990; Oeller et al., 1991), or by overexpression of a bacterial ACC dreaminess, which hydrolyzes ACC (Klee, 1993). In summary, other genes such as those that 
encode SAM hydrolase and N-ACC malonyltransferase, which catalyze either the synthesis or breakdown of precursors of ethylene biosynthesis and whose expression was manipulated all decreased ethylene (see Fluhr and Mattoo, 1996). Genetic control of ethylene action was also achieved by silencing ethylene receptors and thereby the ethylene signaling transduction pathway (Fluhr and Mattoo, 1996; Wilkinson et al., 1997). However, it is recognized that ethylene-dependent (Gapper et al., 2014), as well as ethyleneindependent processes (Hiwasa-Tanase and Ezura, 2014), regulate ripening. In addition to ethylene, other hormones also play a role in the ripening process (Davies and Böttcher, 2014; Hiwasa-Tanase and Ezura, 2014).

Post-harvest losses of fruit and vegetables are significant and therefore stemming such losses has been an on-going activity among horticulturalists and geneticists alike. Early on, increasing the post-harvest life of fruits involved chemical treatment, use of plastic films and treatment with waxes and inhibitors of ethylene biosynthesis or action (Paliyath et al., 2008; Nath et al., 2014). Enhancing the quality attributes particularly nutrients that potentially benefit human and animal health, and flavor/aroma components that add to the produce value has generated considerable research interest among nutraceutical and horticultural industries (Paliyath et al., 2008; Nath et al., 2014).

The association of ethylene with metabolism of sugars and organic acids (Defilippi et al., 2004) as well as aroma volatiles (Bauchot et al., 1998; Flores et al., 2002; Defilippi et al., 2005) brought out an important aspect of fruit physiology. Particularly, such studies highlighted the importance of analyzing nutrients (metabolites) and other quality attributes of fruit and vegetable crops whose shelf-life is extended by treatment with chemical inhibitors of ethylene biosynthesis or by silencing genes that prevent ethylene production. Metabolomics approach has been variously applied to discern the dynamics of metabolic processes in fruits (Carrari et al., 2006; Klie et al., 2013), methyl jasmonate-associated (Kausch et al., 2012) and polyamineassociated metabolic processes (Mattoo et al., 2006) and substrate fluxes into ethylene and polyamine biosynthesis pathways (Lasanajak etal., 2013), as well as to analyze alleles responsible for metabolic traits (Perez-Fons et al., 2014).

Ethylene deficient fruit has been shown to have a prolonged shelf life (Oeller et al., 1991). The question we asked was whether this benefit occurs at the expense of a changed metabolome since ethylene association with metabolic pathways is better appreciated now than previously thought. Therefore, we engineered an ethylene-deficient tomato line by introducing an antisense gene of ACC synthase gene as originally described by Oeller et al. (1991), and also crossed it with a previously described high polyamine tomato line (Mehta et al., 2002; Mattoo et al., 2006) to produce a double transgenic tomato line. A metabolomics approach was then applied to delineate primary metabolites whose levels are affected in the single-event transgenic tomato fruit in comparison to the double transgenic tomato and the azygous control line.

\section{MATERIALS AND METHODS PLANT MATERIAL}

The following tomato lines (Solanum lycopersicum cv. Ohio 8245) were developed in Ohio 8245 background and analyzed: Azygous control line (556AZ); transgenic 2AS-AS line developed (Methods described below) to anti-sense ACC synthase 2 gene (ACS2) under the constitutive CaMV 35S promoter; and 579X2AS-HO line which is a genetic cross between 2AS-AS and $579 \mathrm{HO}$ (see below). Transgenic tomato line $579 \mathrm{HO}$ is an independent genotype that expresses yeast SAM decarboxylase (SAMdc) gene under ripening-specific E8 promoter and accumulates polyamines spermidine (SPD) and spermine (SPM), at the cost of putrescine levels, in a fruit ripening-specific manner (Mehta et al., 2002). All the lines were grown in a temperature-controlled greenhouse at the USDA-ARS, Beltsville Agricultural Research Center, Beltsville, MD, USA, under natural lighting. Fruits at the following stages were collected for analysis: Mature green $(\mathrm{G})$, breaker $(B)$, turning $(\mathrm{T})$, pink $(\mathrm{P})$, and red-ripe $(\mathrm{R})$ stages as described previously (Mehta et al., 2002). USDA color chart was used to classify ripening stages

https://ucanr.org/repository/view.cfm?article $=83755 \% 20 \&$ gro upid=9. The pericarp tissue was peeled, weighed, and frozen in liquid nitrogen before lyophilizing (Mattoo et al., 2006).

\section{DEVELOPING TOMATO PLANTS SUPPRESSED FOR ACC SYNTHASE GENE (2AS-AS LINE) AND ITS SIBLING (2AS-HO x 579HO LINE) - A GENETIC CROSS BETWEEN 2AS-AS AND 579HO TRANSGENIC LINES}

Anti-sense ACS2 transgenic tomato line, 2AS-AS, was developed by Agrobacterium mediated transformation. Primers (ACC-SN: 5'AGTGTCGACCCATGGTTAACGAACTAATGGTGAGGG3' and ACC-XP: 5'GCGTCTAGACACGTGATGGGATTTGAGATTGCAA AGACC3') with restriction sites (Nco I and Pml I, respectively) for cloning were designed to span the complete coding region of LeACS2 gene (Accession \#: X59145). LeACS2 coding region was amplified by PCR with Pfu DNA polymerase (Stratagene) from isolated tomato ACS2 cDNA clone. PCR amplified LeACS2 was digested with Pml I and Nco I and cloned into pCambia 1303 binary vector, down stream of a full-length CAMV 35S promoter (CAMBIA, Canberra, ACT, Australia). This resulted in cloning of LeACS2 coding region in anti-sense orientation with respect to $35 \mathrm{~S}$ promoter (Figure 1A). Binary construct with anti-sense ACS2 gene was transferred into Agrobacterium strain, LBA4404, by electroporation and subsequently used to transform (Pfitzner, 1998) 10-day old cotyledons of tomato (Ohio 8245). Initially, transgenic calli were regenerated and selected on $10 \mathrm{mg} / \mathrm{L}$ hygromycin but shoots were regenerated from callus using a lower hygromycin concentration $(5 \mathrm{mg} / \mathrm{L})$. Subsequently, the regenerated shoots were rooted on $1 \mathrm{mg} / \mathrm{L}$ hygromycin and the plantlets were transferred to soil. The transgenic plants (Figure 1B) were screened by PCR using primers (35SST5PF: 5' AGGACCTAACAGAACTCGCC3' and ACC-XP: 5' GCGTCTAGACACGTGATGGGATTTGAGATTGCAAAGACC3') spanning the $35 \mathrm{~S}$ promoter and anti-sense ACS gene to confirm the presence of promoter and anti-sense ACS transgene. Segregating progeny of T1 and T2 plants containing anti-sense ACS transgene, 2AS-AS, were further selected by PCR.

The selection of $2 \mathrm{AS}-\mathrm{AS} \times 579 \mathrm{HO}$ heterozygote plants (Figure 4A) was made based on the PCR detection of both transgenes (Mehta et al., 2002). Then, F1 plants were selfed and segregating F2 seeds were collected. Evaluation of independent F2 seedlings for the presences of both $y S A M d c$ and anti-SlACS2 


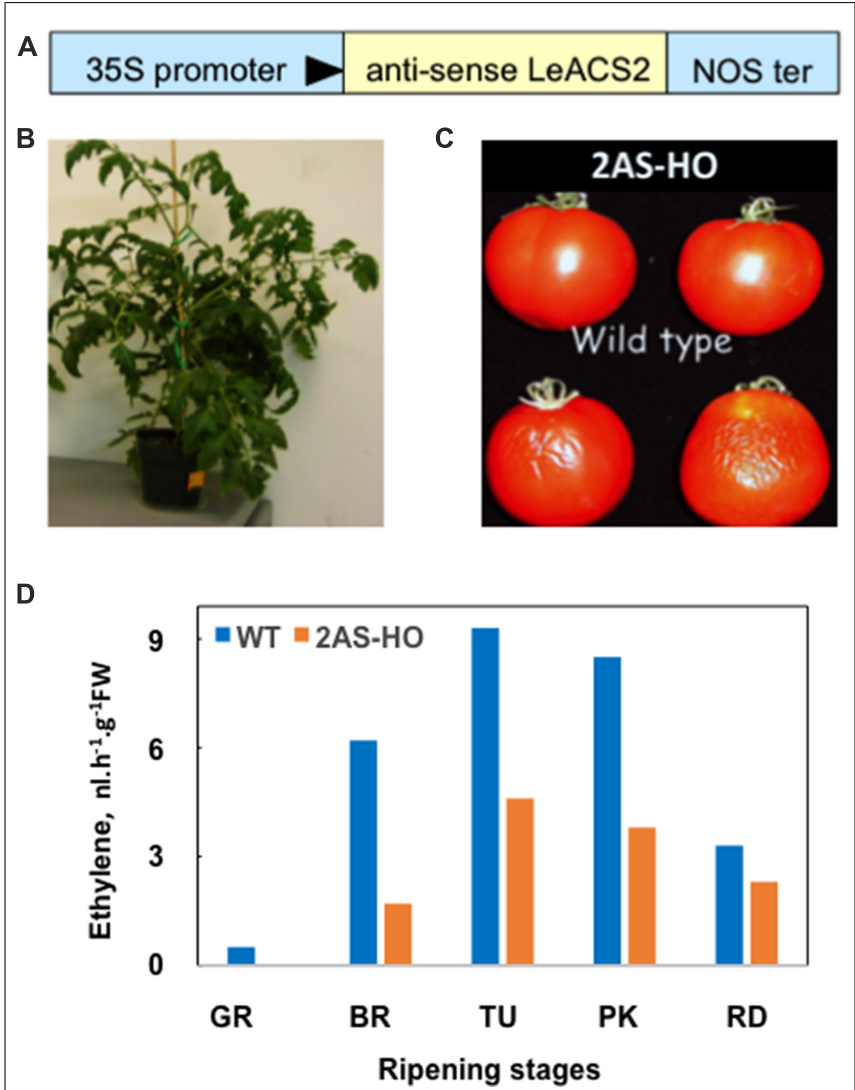

FIGURE 1 | Generation and characterization of transgenic SIACS2 antisense tomato. (A) Anti-sense SIACS2 construct in pCambia/1303 was made as described in Section "Materials and Methods." Ohio 8245 tomato cultivar was transformed with SIACS2-antisense construct using an Agrobacterium based transformation and transgenic plants were selected using hygromycin as selectable marker. (B) Phenotype of one of SIACS2-antisense tomato plant. (C) Phenotype of SIACS2-antisense (Upper panel) and WT (Lower panel) fruits taken 45 days of storage at room temperature following green stage. (D) Rate of ethylene evolution from fruits of WT and transgenic ACS2-antisense fruits. Each time point represents the average from two fruits. Fruits from SIACS2 antisense transgenic tomato line produced $\sim 50 \%$ lesser ethylene than the WT line.

transgenes was re-tested by PCR. The selected F2 plants harboring both $y S A M d c$ and SlACS2 transgenes were grown to fruit maturity, and F3 seeds were collected individually from each line. Seeds from each of the independent F3 were germinated and resulting seedling populations evaluated for the transgenes presence as mentioned above. Two lines were obtained in which further segregation of the two transgenes did not occur. One robust one, $2 \mathrm{AS}-\mathrm{AS} \times 579 \mathrm{HO}$, homozygous for these transgenes, was selected and grown to maturity. Seed collected from this line were used to grow plants for analysis presented here.

\section{ANALYSIS OF ETHYLENE}

All ethylene measurements were carried out on whole fruits as they attainted indicated ripening stage based on color change (https://ucanr.org/repository/view.cfm?article $=83755 \% 20 \&$ grou pid=9). Ethylene production in the tomato fruits was measured by gas chromatography with a FID (Mehta et al., 2002).

\section{NMR ANALYSIS OF FRUIT METABOLITES}

Samples for NMR measurement were prepared as previously described (Sobolev et al., 2003; Mattoo et al., 2006). Briefly, dry powder $(25 \mathrm{mg})$ from each sample was rapidly dissolved in $1 \mathrm{~mL}$ of $0.4 \mathrm{M}$ sodium phosphate buffer prepared in $\mathrm{D}_{2} \mathrm{O}$ containing known amounts of an authentic standard, 3-(trimethylsilyl)1-propanesulfonic acid sodium salt (TSP), $\mathrm{pH}$ 6.5, and EDTA $(0.01 \mathrm{mM})$. The solution was centrifuged at $10,000 \mathrm{rpm}$ for $7 \mathrm{~min}$ and the supernatant filtered to remove any insoluble material. NMR spectra of extracts were recorded at $300 \mathrm{~K}$ on a Bruker AVANCE 600 spectrometer operating at the proton frequency of $600.13 \mathrm{MHz}$. Proton spectra were referenced to the TSP signal $(d=0.00 \mathrm{ppm})$. Proton signals were acquired by co-adding 512 transients with a recycle delay of $3 \mathrm{~s}$. The strong water signal was suppressed by using a NOESY-presat scheme with solvent presaturation during relaxation delay and mixing time (Braun et al., 1998). The one-dimensional spectra were run using $45^{\circ}$ flip angle pulses of $6 \mathrm{~ms}, 32 \mathrm{~K}$ data points. After Fourier-transformation and manual phase correction the baseline correction was performed using automatic cubic-spline correction with 25 points distributed over the spectrum. The assignment of ${ }^{1} \mathrm{H}$ NMR spectra was performed as previously described (Sobolev et al., 2003; Mattoo et al., 2006; Table 1). Additionally, the signals of adenosine, adenosine monophosphate (AMP), tyrosine, histidine, and tryptophan were assigned using literature data (Fan, 1996) and by addition of the corresponding standard compounds (Table 1). The singlet at $8.531 \mathrm{ppm}$ was attributed to both adenosine triphosphate (ATP) and adenosine diphosphate (ADP). The intensities of three unassigned signals denoted as $\mathrm{B}, \mathrm{Nucl} 1$, and $\mathrm{Nucl} 2$ were also used in the statistical analysis. The intensity of 30 selected resonances attributed to 30 metabolites (Sobolev et al., 2003; Mattoo et al., 2006) was referenced to the intensity of the internal standard, TSP at $0.00 \mathrm{ppm}$. The spectra of two to five fruits were analyzed for each ripening stage.

\section{STATISTICAL ANALYSIS}

Data of ${ }^{1} \mathrm{H}-\mathrm{NMR}$ in solution were subjected to statistical analysis by using Statistical software package for Windows (1997; edition by Statsoft) to determine if and to what extent the selected variables were able to distinguish between the different tomatoes and their ripening stages. ANOVA was used to validate the differences. ANOVA and PCA were used to treat the data (Martens and Martens, 2001).

\section{RESULTS \\ ANTI-SENSE LEACS2 TOMATO LINE IS ATTENUATED IN ETHYLENE PRODUCTION AND HAS LONGER SHELF LIFE}

Fruits from the wild type (WT) and anti-sense LeACS2 line, 2ASAS (line 2.5AS), were found to produce maximum ethylene at the turning stage (Mehta et al., 2002); however, 2AS-AS line produced only $50 \%$ of ethylene that produced by the WT (Figure 1D). Also, the fruits from ethylene-deficient line had a significantly longer shelf-life as compared to the WT fruits (Figure 1C). These data are consistent with established findings in the literature showing that ethylene suppression enhances the shelf life of tomato fruits (Oeller et al., 1991; Paliyath et al., 2008; Nath et al., 2014). 
Table 1 | List of resonances and variables used for the evaluation of the various metabolites.

\begin{tabular}{|c|c|c|}
\hline Variable No. & Chemical shift, ppm & Compound \\
\hline 1 & 1.020 & Ile \\
\hline 2 & 1.053 & Val \\
\hline 3 & 1.345 & Thr \\
\hline 4 & 1.494 & Ala \\
\hline 5 & 2.085 & Glu \\
\hline 6 & 2.482 & Gln \\
\hline 7 & 2.806 & Asp \\
\hline 8 & 2.908 & Asn \\
\hline 9 & 6.921 & Tyr \\
\hline 10 & 7.249 & His \\
\hline 11 & 7.436 & Phe \\
\hline 12 & 7.748 & Trp \\
\hline 13 & 2.304 & GABA \\
\hline 14 & 2.530 & Citrate \\
\hline 15 & 2.415 & Succinate \\
\hline 16 & 4.316 & Malate \\
\hline 17 & 6.527 & Fumarate \\
\hline 18 & 8.462 & Formate \\
\hline 19 & 3.244 & $\beta$-Glucose \\
\hline 20 & 4.023 & Fructose \\
\hline 21 & 5.422 & Sucrose \\
\hline 22 & 3.294 & Myo-inositol \\
\hline 23 & 2.976 & ${ }^{\mathrm{a}} \mathrm{B}$ \\
\hline 24 & 3.208 & Choline \\
\hline 25 & 8.363 & Adenosine \\
\hline 26 & 8.576 & AMP \\
\hline 27 & 8.531 & ATP/ADP \\
\hline 28 & 7.843 & ${ }^{\mathrm{a}} \mathrm{Nucl} 1$ \\
\hline 29 & 7.874 & ${ }^{\mathrm{a} N u c l} 2$ \\
\hline 30 & 9.130 & Trigonelline \\
\hline
\end{tabular}

${ }^{a}$ not assigned.

\section{ALTERED METABOLIC PROFILES IN 2AS-AS LINE VERSUS 556AZ (AZYGOUS CONTROL LINE)}

Fruits from 2AS-AS line and 556AZ were harvested from greenhouse-grown plants and prepared as described in the section "Materials and Methods." Metabolic profiles at four different stages of ripening are shown in Figures 2A-C.

\section{PROFILE OF AMINO ACIDS (LINE 2AS-AS VERSUS 556AZ)}

During ripening of fruits from 556AZ control line, the levels of Glu, Asp, His, and Trp gradually increased while Thr level changed slightly, and that of Ile, Val, Gln, Asn, and Phe showed a significant decrease only in R stage (Figure 2A). In comparison, the levels of Ile, Val, Thr, Ala, Tyr, Phe, Gln, Asn, and $\gamma$-aminobutyrate (GABA) gradually decreased during ripening of fruit from the ethylenedeficient line, 2AS-AS, with the levels of His and Trp remaining constant except for Asp which increased slightly (Figure 2A). It is noted that the levels of Gln, Asn, Asp, Glu, and GABA in 2AS-AS line were lower at almost all the ripening stages as compared to the fruits from 556AZ control line, with the exception of Trp that had consistently a higher level in 2AS-AS fruit than the control 556AZ fruit throughout ripening.

\section{PROFILE OF ORGANIC ACIDS AND SUGARS (LINE 2AS-AS VERSUS 556AZ)}

Citrate and malate, the most abundant organic acids in tomato fruit, showed different pattern during ripening: the level of malate gradually decreased in fruit from both 2AS-AS and 556AZ lines, while citrate level was constant in controls and slightly decreased in 2AS-AS line (Figure 2B). In contrast to fruit from the 556AZ control line, 2AS-AS ethylene-deficient fruit had lower levels of succinate during ripening and slightly higher level of fumarate except at the green $(\mathrm{G})$ stage (Figure 2).

The levels of glucose and fructose were quite stable during ripening without any significant differences between fruit from 2AS-AS and 556AZ lines (Figure 2B). Sucrose level was maximal at green $(G)$ stage in 2AS-AS fruit 1 (about five times higher in comparison with 556AZ G fruits) but, by the breaker (B) stage, the control line (556AZ) fruit had higher levels of sucrose than the 2AS-AS fruit, declining thereafter. Thereafter, the levels decreased dramatically in both genotypes (Figure 2B). Myo-inositol level remained constant throughout the ripening of fruit from the control 556AZ line but in the ethylene-deficient 2AS-AS line myo-inositol levels were highest at the green $(\mathrm{G})$ stage and progressively decreased thereafter to being lowest at the red (R) stage (Figure 2B).

\section{PROFILE OF HETEROAROMATIC COMPOUNDS (LINE 2AS-AS VERSUS 556AZ)}

The levels of nucleosides and nucleotides also showed a few significant differences between the fruit of ethylene-deficient 2AS-AS line and that of 556AZ control line. Higher levels of adenosine and ATP + ADP at the pink (P) stage and Nucll at green $(G)$ stage were observed in the 2AS-AS line in comparison to the fruit from control line (Figure 2C).

The multivariate statistical analysis of the data was performed to obtain an overall picture of metabolic differences between ethylene-deficient transgenic fruit and that from 556AZ control line at different ripening stages. First, the Principal Component Analysis (PCA) analysis was applied. The main features of metabolite variability in the fruit from the two lines are visualized in two-dimensional scores plot (Figure 3). The obvious distinction in metabolite variability between transgenic (empty symbols) versus control fruits (filled symbols) is observed along PC3 axis. The distribution of samples along $\mathrm{PC} 1$ axis reflects the ripeness grade: the lowest and the highest PC1 scores correspond to the green $(\mathrm{G})$ and red $(\mathrm{R})$ fruits, respectively. The separation of samples according to ripeness stage is easily observable for the transgenic line, while the control samples of different ripeness grade are partially mixed. Considering the fraction of variability associated with the principal components (35\% for PC1 and $10.9 \%$ of PC 3 ), it is clear that the ripening process is the principal source of metabolite variability, whereas the transgene expression is a secondary 
A

$\square$ AZ $\square$ 2AS-AS $\square 579 \times 2$ AS-HO

Ile

Val

Thr

Ala
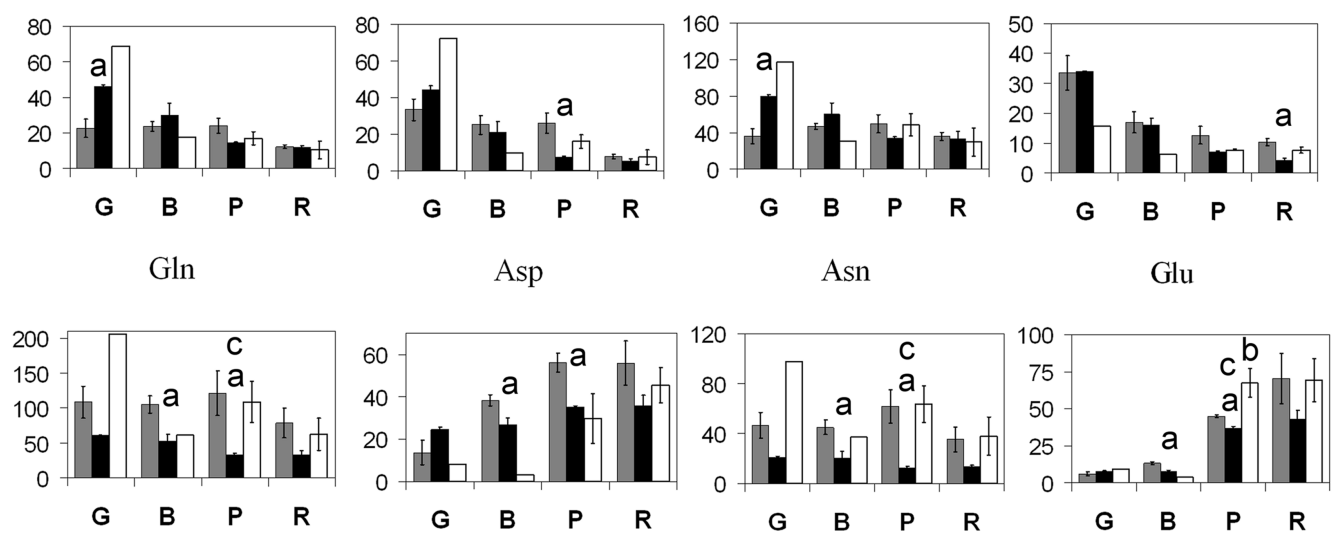

Phe

$\operatorname{Trp}$

Tyr

His
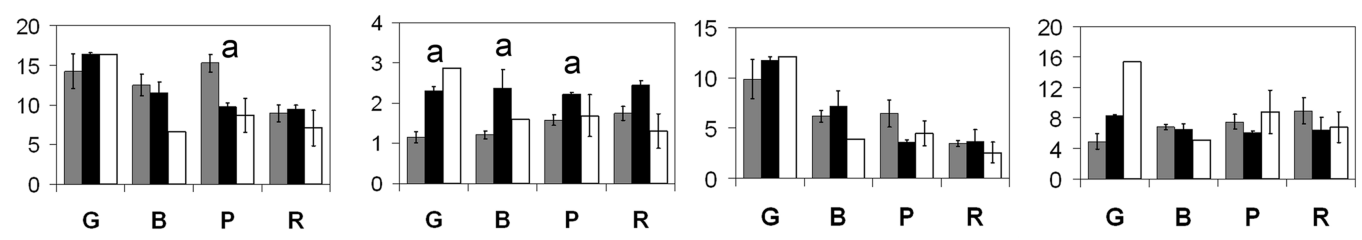

B
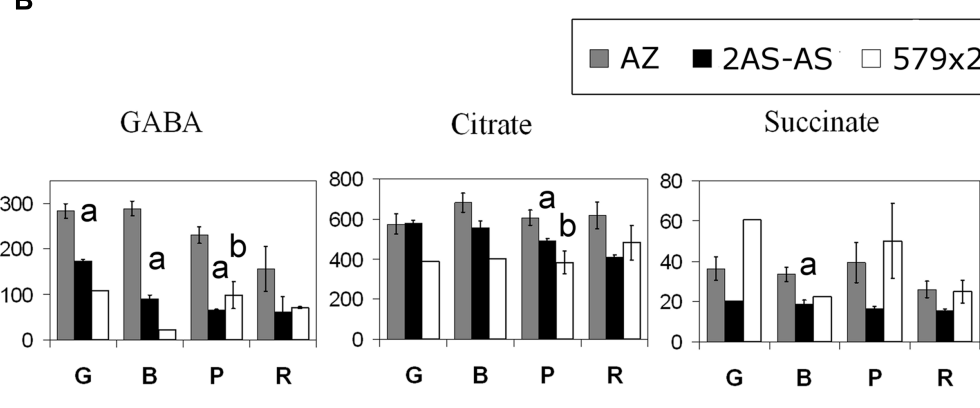

Fumarate

Formate

$\beta$-Glucose

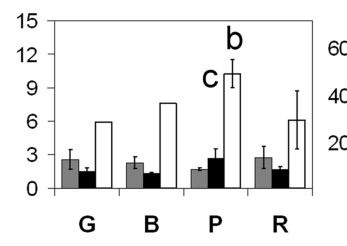

Myo-Inositol

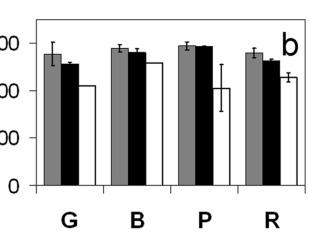

B
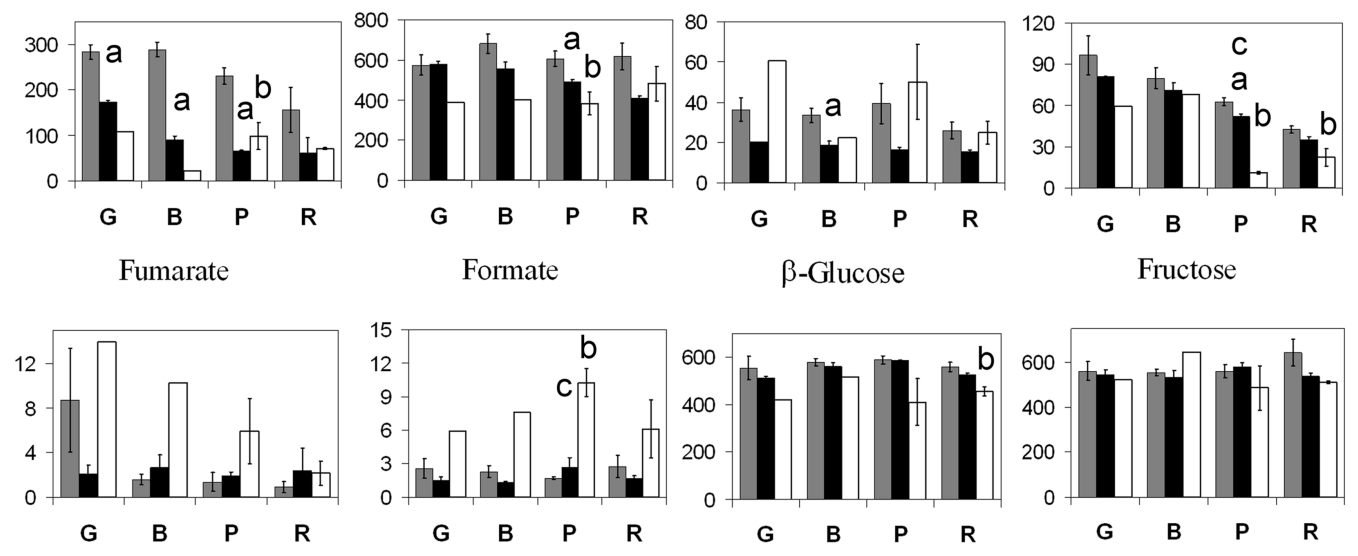

Sucrose
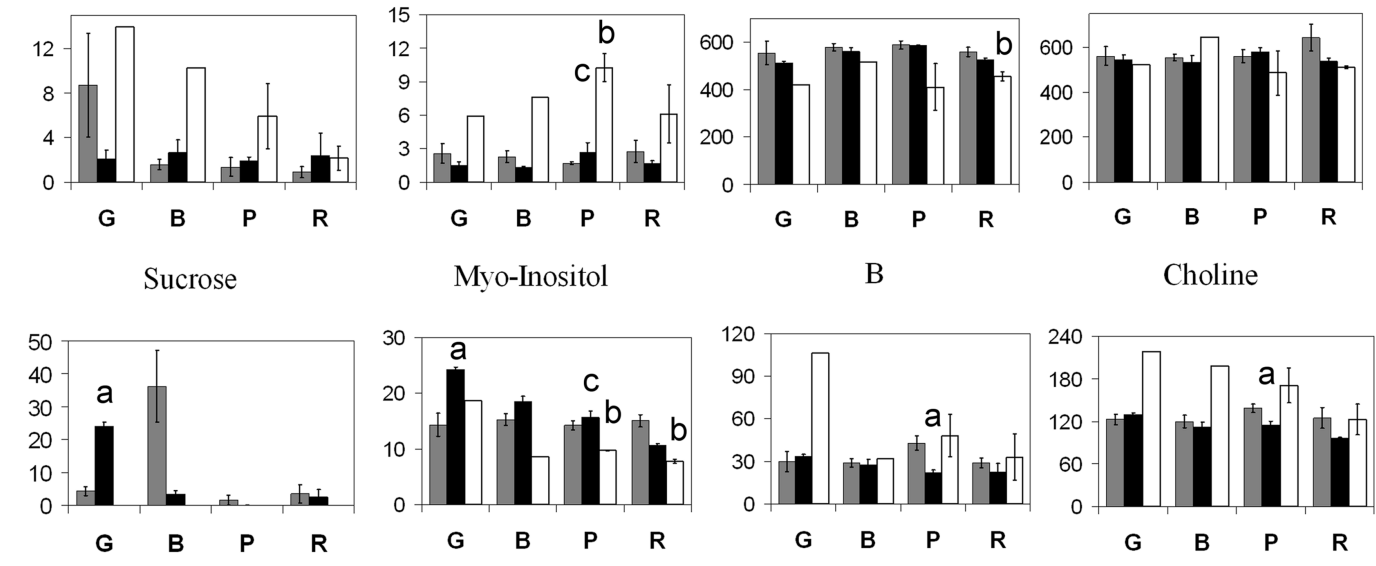

Choline

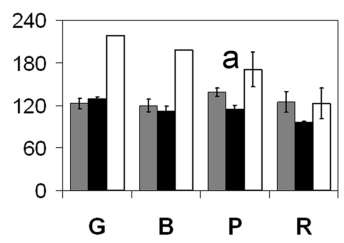

FIGURE 2 | Continued 
C

$\square$ AZ $\square 2$ AS-AS $\square 579 \times 2$ AS-HO

AMP

ATP/ADP

Adenosine
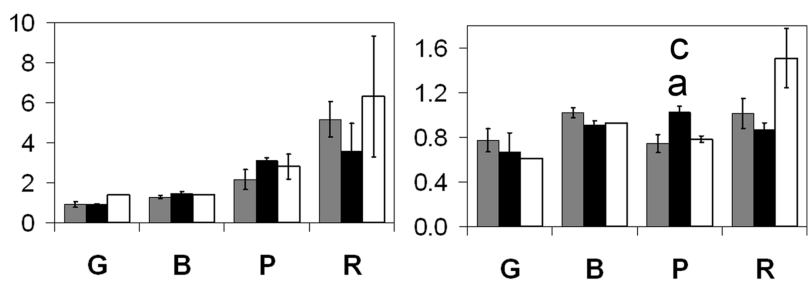

Nucl 1

Nucl2

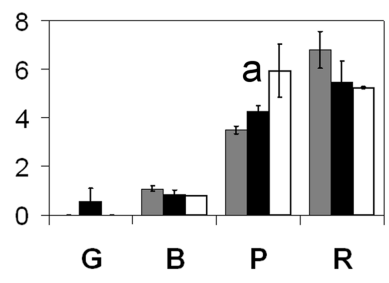

Trigonelline
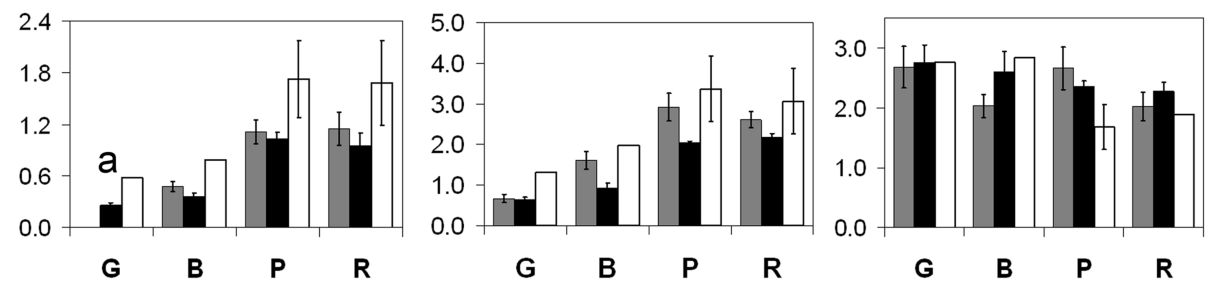

FIGURE 2 | Molecular abundance of $\mathbf{3 0}$ metabolites analyzed in fruit of 556AZ (AZ: gray boxes), 2AS-AS (black boxes), and 579X2AS-HO (white boxes) tomato lines at four ripening stages. (A) Amino acids. (B) GABA, organic acid, and sugars. (C) Miscellaneous including heteroaromatic compounds including nucleotides and ATP + ADP. Mean values and SEs are presented. The significant differences ( $p$-level $<0.05$ ) between $2 A S-A S$ and $556 \mathrm{AZ}, 2 \mathrm{AS}-\mathrm{AS} \times 579 \mathrm{HO}$ (labeled $579 \times 2 \mathrm{AS}-\mathrm{HO}$ ) and $556 \mathrm{AZ}, 2 \mathrm{AS}-\mathrm{AS}$ and $2 \mathrm{AS}-\mathrm{AS} \times 579 \mathrm{HO}$ (labeled $579 \times 2 \mathrm{AS}-\mathrm{HO}$ ) are denoted by letters 'a,' 'b,' and 'C,' respectively. G (green), B (breaker), P (pink), and R (red) refer to the stage of the fruit.

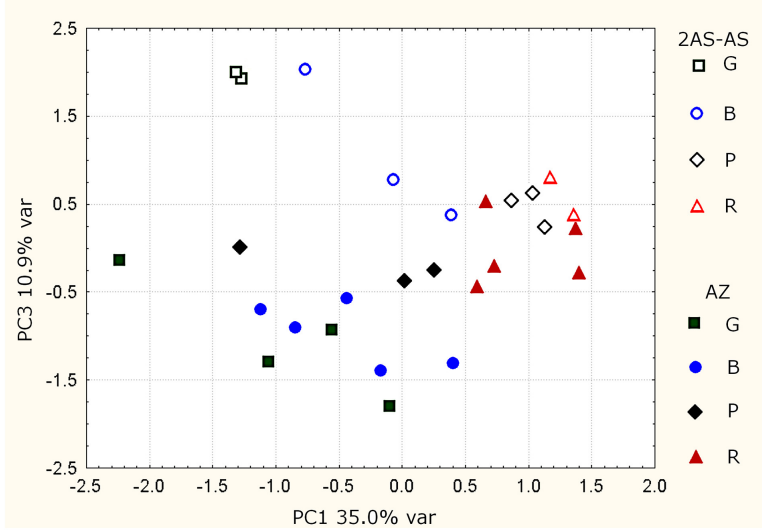

FIGURE 3 | PCA scores plot of samples from 2AS-AS (open symbols) and 556AZ (AZ, filled symbols) lines. G (green), B (breaker), P (pink), and $R$ (red) refer to the stage of the fruit.

factor influencing the metabolic profile. Nonetheless, a good and modest separation according to ripening stage of transgenic and control samples along PC1 suggests a significant influence of transgene expression on ripening. Moreover, the PCA results indicate that the distinction between transgenic and control line is less evident for red tomatoes. The predominant role of ripeness in metabolic changes can mask the influence of transgene expression; therefore, the comparison of metabolite levels in transgenic and control samples separately at different stage of ripeness is desirable.

The statistically significant differences in metabolite levels between 556AZ control and 2AS-AS transgenic fruit were calculated separately for each stage of ripening using ANOVA. Metabolites that were significantly different $(p$-level $<0.05$ ) between fruit of 556AZ and 2AS-AS lines are marked by letter "a" (Figures 2A-C). ANOVA shows the difference between two lines to be maximal for pink $(\mathrm{P})$ stage of ripeness (14 metabolites out of 30), followed by breaker (B) and green (G) stages with seven metabolites showing different level in both groups. Only Ala was at a different level in the red tomatoes of the two lines, more in the 556AZ control fruit than that in the 2AS-AS line. In total, the content of 21 metabolites out of 30 is influenced by the transgene expression and this influence is ripening-specific: only Trp and GABA show the different level in two lines throughout all the intermediate stages of ripening, whereas the difference in the content of the other 19 metabolites is observable at one or two stages of ripening only.

The ripening-induced metabolic changes are clearly seen for Ile, Val, Thr, Ala, Tyr, Phe, GABA, myo-inositol, and malate (gradual decrease from green to red tomatoes) and for Glu, Adenosine, AMP, Nucl1, and Nucl2 (gradual increment during ripening).

The influence of transgene expression on ripening process is clearly observable for Thr, Asp, Phe, and myo-inositol; Thr, Phe, and myo-inositol levels being constant in 556AZ control 
fruit (with a decrease in Phe level at red stage), whereas their levels gradually decrease during ripening of fruit from the transgenic line. Asp level slightly increases with ripening of transgenic fruit but in the 556AZ control fruit the increase is dramatic (Figures 2A,B).

\section{INTROGRESSION OF ETHYLENE-DEFICIENT 2AS-AS TOMATO LINE WITH HIGHER-POLYAMINE ACCUMULATING (579H0) TRANSGENIC LINE HIGHLIGHTS DIFFERENTIAL EFFECTS ON METABOLITE PROFILES}

In order to test which metabolite patterns in the ethylene-deficient tomato fruit are changed by adding to that line the trait of higher accumulation of polyamines, SPD and SPM (Mattoo et al., 2006), we developed a double transgenic tomato line, $2 \mathrm{AS}-\mathrm{AS} \times 579 \mathrm{HO}$, by crossing previously developed SPD (and SPM)-accumulating 579HO line (Mehta et al., 2002) and the ethylene-deficient 2ASAS line as summarized in Figure 4 (see also Materials and Methods). Surprisingly, this intervention not only reversed the ethylene deficiency in the sibling (the double transgenic line, 2ASAS $\times 579 \mathrm{HO}$, in fact, the ethylene levels were significantly higher (Figure 4C).

Previously, metabolite profiling of the higher polyaminesaccumulating line, $579 \mathrm{HO}$, produced a unique metabolite signature vis a vis the WT or azygous 556 control line, highlighting effects on the aspartate family of amino acid pathway (Mattoo et al., 2006). A similar signature was found in the fruit of the double transgenic line, $2 \mathrm{AS}-\mathrm{AS} \times 579 \mathrm{HO}$ (Figure 2A). Thus, fruit from 2AS-AS $\times 579 \mathrm{HO}$ line had higher levels of Asn (all ripening stages), Gln ( $\mathrm{G}$ and $\mathrm{P}$ stages), and Glu ( $\mathrm{P}$ and $\mathrm{R}$ stages) and lower levels of Asp, GABA ( $G$ and B stages), and Trp (from B to R stages) in comparison to fruit from ethylene-deficient 2AS-AS line (Figure 2A). The profiles of amino acids Ile, Val, Thr, Ala, Tyr, His, and Phe during ripening remained similar in $2 \mathrm{AS}-\mathrm{AS} \times 579 \mathrm{HO}$ line with respect to 2AS-AS line fruit. Significant differences $(p$ level $<0.05)$ between 2 AS-AS $\times 579 \mathrm{HO}$ and 2 AS-AS lines are marked in Figures 2A-C by letter "c."

Similarly, the profiles of organic acids and sugars were compared between the fruit from the $2 \mathrm{AS}-\mathrm{AS} \times 579 \mathrm{HO}$ line and the ethylene-deficient 2AS-AS line (Figure 2B). The level of citrate was lower and that of fumarate higher in $2 \mathrm{AS}-\mathrm{AS} \times 579 \mathrm{HO}$ line relative to 2AS-AS line at all the intermediate stages of ripening. Malate level was similar in both lines except for pink $(\mathrm{P})$ stage where its level was about five times lower in $2 \mathrm{AS}-\mathrm{AS} \times 579 \mathrm{HO}$ fruits. Succinate level was higher in 2 AS-AS $\times 579 \mathrm{HO}$ line in comparison to 2AS-AS line at green $(\mathrm{G})$ and pink $(\mathrm{P})$ stages of ripening (Figure 2B). At all the ripening stages, myo-inositol level was lower and choline level higher in the 2AS-AS $\times 579 \mathrm{HO}$ line relative to $2 \mathrm{AS}$-AS line.

Other trends noticed in heteroaromatic compounds, adenosine, AMP, ATP + ADP, Nucl1, Nucl2, and trigonelline are presented in Figure 2C. Noticeable trends that were not all found to be statistically significant, because of the noise in the data, included higher adenosine (pink, $\mathrm{P}$, stage), AMP (ripe, $\mathrm{R}$, stage), ATP + ADP (lower at pink, P, and significant drop; elevated at ripe, R), Nucl1 and Nucl2 (higher throughout ripening) and trigonelline (decreasing trend at pink, $\mathrm{P}$, and red, $\mathrm{R}$, stages) in the double transgenic line, $2 \mathrm{AS}-\mathrm{AS} \times 579 \mathrm{HO}$, relative to the ethylene-deficient 2AS-AS line (Figure 2C).

\section{DISCUSSION}

Shelf life of ripening climacteric fruits has been achieved by silencing key gene(s) in ethylene biosynthesis, perception, and signal transduction (Fluhr and Mattoo, 1996). However, whether the altered ethylene production and/or perception affects fruit metabolome or how the resulting fruit quality is achieved is not yet clearly understood. In particular, the levels of a large number of amino acids are significantly reduced in the ACC synthase-impaired tomato fruit, a reduction being more drastic than previously recognized through studies on apple fruit silenced for the expression of ACC oxidase gene (Defilippi et al., 2005). A number of these involve aromatic amino acids (Tyr, Phe, Ile, Val), aspartate family of amino acids (Asp, Asn, Thr, Gln, GABA) and energy/salvage pathway metabolites (adenosine, ATP, ADP), many among which are synthesized from substrates generated from phosphoenolpyruvate and pyruvate, tricarboxylic acid (TCA) cycle intermediates, and amination (Heldt, 2005). Other metabolites affected in ethylene-deficient tomato involve alanine, myo-inositol, and TCA cycle intermediates - citrate, succinate, and fumarate. Gln and Ile were among the metabolites similarly affected in the apple fruit silenced for the expression of ACC oxidase (Defilippi et al., 2005). The depletion of the TCA cycle intermediates in ethylene-deficient tomatoes is likely a reflection on the diminished respiration (Saltveit, 1999). Thus, multiple biochemical pathways are targeted by ethylene in tomato fruit. The synthesis (and dynamics) of these important small molecules occurs in different subcellular compartments in a plant cell, indicating a wide range of ethylene influence on the fruit metabolome (Mattoo etal., 2006; Osorio et al., 2013). Interestingly, the observation that Ile, a major precursor of aroma volatiles, is found commonly affected in relation to ethylene deficiency (Bauchot et al., 1998; Flores et al., 2002; Defilippi et al., 2005) suggests that most ethylene-deficient fruit would be impacted in flavor. However, Ile levels were found to recover to a significant level when 21-day post-harvest apple fruit were exposed to a dose of exogenous ethylene (Defilippi et al., 2005), identifying a critical link between Ile, aroma, and ethylene.

We have previously determined polyamine-responsive metabolome of tomato by analyzing metabolite profiles of the fruit engineered for the expression of heterologous yeast SAMdc gene and fruit-specific accumulation of higher polyamines, SPD, and SPM and comparing these with azygous control fruit (Mattoo et al., 2006). A comparison of metabolites impacted by polyamine addition to ethylene-deficient fruit versus ethylene-deficiency provides a window into the metabolites that these two antagonistic growth regulators (Fluhr and Mattoo, 1996) commonly target but not necessarily in the same direction. These are: Asp, Asn, Gln, Val, citrate, and fumarate. Those metabolites that were suppressed in ethylene-deficient tomato fruit but were neutral to the polyamine response include Thr, Ile, GABA, Ala, and Phe. In this regard, it was interesting to find out which changes in the metabolome of ethylene-deficient tomato fruit would occur when fruit also harbor the high polyamine trait as in the double transgenic tomato developed and presented here. In other words, which polyaminespecific metabolites would dominate and which would behave differently in the double transgenic tomato. Thus, the double 

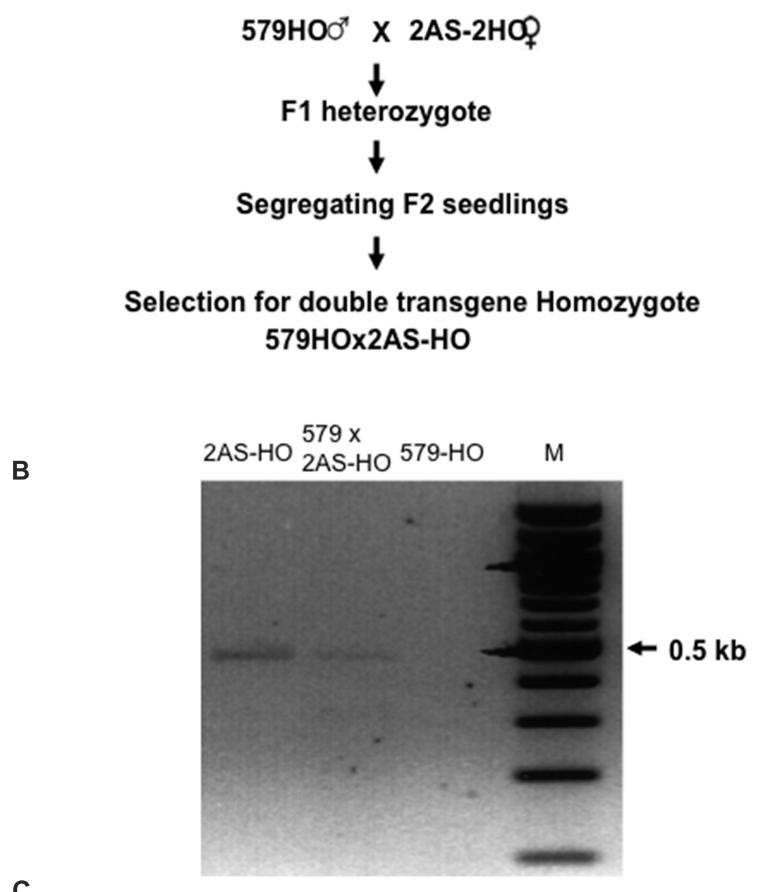

C

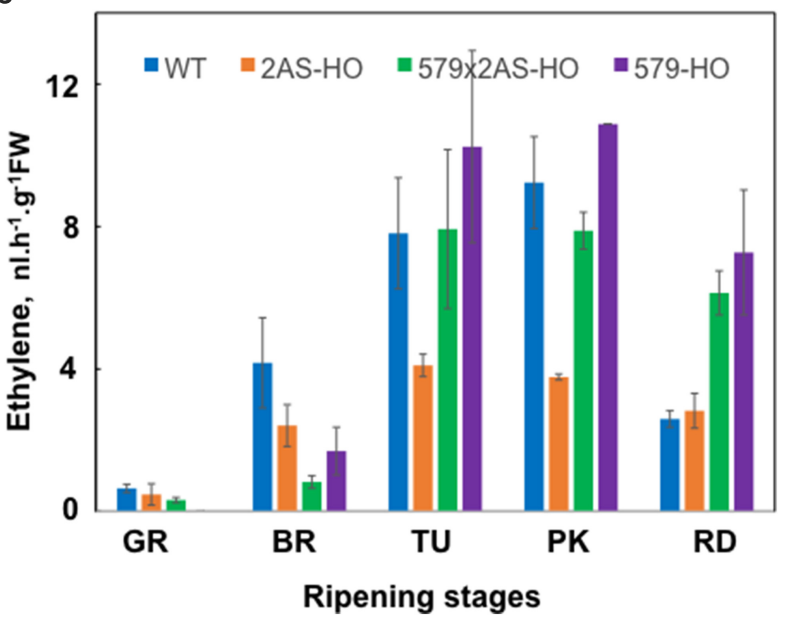

FIGURE 4 | Generation and characterization of transgenic ACS2-AS and development of double transgenic tomato line expressing both CaMV35S-ACS2-AS and E8:ySAMDC transgenes. (A) F1 line was

developed by pollinating 2AS-AS (2AS-HO) flower with pollen from $579 \mathrm{HO}$ line. Plants from the resulting heterozygous seed were characterized.

Those that showed presence of both transgenes by RT-PCR confirmed the heterozygote and then their seeds were collected. Fifty segregating seeds from F1 line were grown to maturity and from each segregating line seeds were collected individually. About 20 seedlings from each segregated seed were evaluated for the presence of both transgenes to identify homozygous lines. (B) RT-PCR of RNA from 2AS-AS (labeled 2AS-OH) and double transgene homozygous 2AS-AS $\times 579 \mathrm{HO}$ (labeled $579 \times 2 \mathrm{AS}-\mathrm{HO}$ ) showing the expression of ACS2-AS transcript. Shown is the $0.5 \mathrm{~kb}$ PCR fragment from ACS2 gene. The line $579 \mathrm{HO}$ does not harbor ACS2-AS transcript and is therefore negative for its RNA. (C) Rate of ethylene evolution by fruits of wild type (WT), 2AS-AS antisense line (2AS-OH), $2 \mathrm{AS}-\mathrm{AS} \times 579 \mathrm{HO}(579 \times 2 \mathrm{AS}-\mathrm{HO})$ and $579-\mathrm{HO}$ at various stages of ripening. Each time point represents the means \pm SE from a minimum of three fruits except for the pink (PK) fruits from $579 \mathrm{HO}$ line where only two fruits were analyzed. transgenic tomato behaved similar to one of its parental, highpolyamine genetic line as regards the following metabolites: Asp, Asn, Glu, Gln, citrate, choline, and ethylene. However, the following metabolites seem to have become a target of the interaction of the two transgenes harbored in the double transgenic fruit: Trp, GABA, succinate, and malate.
Ripening fruits of Ohio 8245 showed increases in the levels of Glu, Asp, His, and Trp but decreased in the levels of GABA, Ile, Val, Gln, Asn, Tyr, and Phe (Figures 2A,B). Some of these patterns are similar while others are in contrast with the previously published ripening fruit metabolome of Moneymaker and Ailsa Craig cultivars (Carrari et al., 2006; Osorio et al., 2011). We observed 
increases in the levels of Glu and Asp but not of Phe, Ile, and Ala as reported in Moneymaker and Ailsa Craig cultivars. Phe Ile and Ala declined during the fruit ripening of Ohio 8245 cultivar (Figure 2A). Levels of GABA and Val declined in all three investigations (present study; Carrari et al., 2006; Osorio et al., 2011), but the levels of Thr, Gln, Asn, His, and Trp did not show significant changes during ripening of fruit from Ohio8245 line. Unlike the $N r$ mutant fruit, which exhibited decrease in Thr, fruit from 2AS-AS exhibited ripening-associated increase in this amino acid. Fruits from the 2AS-AS line showed decline in Val, Ala, Glu Gln Asp, Asn, Phe, and GABA during ripening, implying that even $50 \%$ reduction in ethylene production has a major effect on fruit metabolome. It is possible that different threshold levels of ethylene may regulate particular events/processes in a fruit. However, decline in $\beta$-Ala and GABA in rin, Nor, and 2AS-AS (ethylene biosynthesis attenuated) mutants indicates that ethylene regulates their accumulation during fruit ripening.

Among the organic acids, the levels of malate and fumarate declined noticeably in 2AS-AS, but increased several-fold in the double transgenic fruits, likely reflecting diminished respiration in 2AS-AS fruit in contrast to higher respiration in double transgenic fruit. We have previously shown that $579 \mathrm{HO}$ fruit exhibit several-fold higher respiration during ripening as compared to the WT and 556AZ fruit (Mattoo et al., 2006). These results are different from those reported in Moneymaker and Ailsa Craig where an increase in succinate and a decrease in malate was evident (Carrari et al., 2006; Osorio et al., 2011). Also, unlike ripening mutant(s) in which malate and succinate do not change during fruit ripening (Osorio et al., 2011), reduction in ethylene production significantly affected levels of citrate, succinate, and malate (Figure 2B), the data that are in line with ethylene regulation of respiration (Saltveit, 1999), largely involving the TCA cycle intermediates (Mattoo et al., 2006).

Levels of glucose and fructose remained similar throughout the fruit ripening of control fruit with a significant increase in sucrose levels only at the onset of ripening (breaker stage; Figure 2B). Contrasting results have been reported for changes in sugar levels in Moneymaker and Ailsa Craig fruits. Whereas a linear increase in glucose and fructose levels was reported in Moneymaker fruit, such changes were not seen in Ailsa Craig fruit (Carrari et al., 2006; Osorio et al., 2011). Such changes in 2AS-AS were not apparent in the present study, indicating a more complex role of ethylene/ripening associated with sugar metabolism. Reduction in glucose, fructose, citric, and malic acids has been reported in strawberry line with altered ethylene perception due to the overexpression of etr1-1 ethylene receptor mutant (Merchante et al., 2013).

The ingression of SAMdc overexpression in 2 AS-AS $\times 579 \mathrm{HO}$ cross resulted in partial recovery of several metabolites otherwise affected by reduction in ethylene in the 2AS-AS line (Figures 2A-C). The metabolic parameters that exhibit this partial to full recovery include Ile, Glu, Gln, Asn, succinate, fumarate, formate, compound B, Nucl1, and Nucl2. This recovery is associated with 2 to 3 -fold higher ethylene production in $556 \mathrm{HO}$ because of SAMdc overexpression (Mehta et al., 2002; see also Figure 4C). The enhanced metabolic activity due to higher polyamines, SPD, and SPM, is associated with increased levels of Ile, Val, Thr, Asn,
His, Trp, succinate, fumarate, formate, compound B, choline, adenosine, Nucl1, and $\mathrm{Nucl} 2$, as reported previously (Mattoo et al., 2006, 2007; Srivastava et al., 2007). Thus, polyamines SPD and SPM prominently alter the metabolic changes associated with the double transgenic tomato fruit. These findings are in line with the determined metabolome previously reported and discussed in relation to the genetic event of introducing ySAMdc transgene in tomato (Mattoo and Handa, 2008; Handa and Mattoo, 2010; Mattoo et al., 2010).

This study provides new dimension in light of other reports in the literature (and some mentioned above) showing that suppression of ethylene has measurable impact on the metabolome and aroma of a commodity. Thus, altering ethylene biosynthesis or signaling in plants, be that by a chemical treatment or using genetic means (mutants or transgenes), to prolong shelf life of fruits limits the metabolic potential (nutrition) of such fruit, which needs to be ascertained and then rectified/salvaged by additional treatments including applying exogenous ethylene or creating a double transgenic event as shown here.

\section{ACKNOWLEDGMENTS}

Mention of trade names or commercial products in this publication is solely for the purpose of providing specific information and does not imply recommendation or endorsement by the U.S. Department of Agriculture.

\section{REFERENCES}

Abeles, F. B., Morgan, P. W., and Saltveit, M. E. Jr. (1992). Ethylene in Plant Biology. San Diego: Academic Press.

Bauchot, A. D., Mottram, D. S., Dodson, A. T., and John, P. (1998). Effect of aminocyclopropane-1-carboxylic acid oxidase antisense gene on the formation of volatile esters in Cantaloupe Charentais melon (cv. Vedrantais). J. Agric. Food Chem. 46, 4787-4792. doi: 10.1021/jf980692z

Braun, S., Kalinowski, H. O., and Berger, S. (1998). 150 and More Basic NMR Experiments, 2nd Edn. Weinheim: Wiley-VCH.

Carrari, F., Baxter, C., Usadel, B., Urbanczyk-Wochniak, E., Zanor, M. I., NunesNesi, A., et al. (2006). Integrated analysis of metabolite and transcript levels reveals the metabolic shifts that underlie tomato fruit development and highlight regulatory aspects of metabolic network behavior. Plant Physiol. 142, 1380-1396. doi: 10.1104/pp.106.088534

Davies, C., and Böttcher, C. (2014). "Other hormonal signals during ripening," in Fruit Ripening, Physiology, Signalling and Genomics, eds P. Nath, M. Bouzayen, A. K. Mattoo, and J. C. Pech (Oxfordshire: CAB International), 202-216.

Defilippi, B. G., Dandekar, A. M., and Kader, A. A. (2004). Impact of suppression of ethylene action or biosynthesis on flavor metabolites in apple (Malus $\times$ domestica Borkh) fruits. J. Agric. Food Chem. 52, 5694-5701. doi: 10.1021/jf049504x

Defilippi, B. G., Dandekar, A. M., and Kader, A. A. (2005). Relationship of ethylene biosynthesis to volatile production, related enzymes, and precursor availability in apple peel and flesh tissues. J. Agric. Food Chem. 53, 3133-3141. doi: 10.1021/jf047892x

Fan, T. W. M. (1996). Metabolite profiling by one- and two-dimensional NMR analysis of complex mixtures. Prog. Nucl. Magn. Reson. Spectrosc. 28, 161-219. doi: 10.1016/0079-6565(95)01017-3

Flores, F., Yahyaoui, F. E., de Billerbeck, G., Romojaro, F., Latche, A., Bouzayen, M., et al. (2002). Role of ethylene in the biosynthetic pathway of aliphatic ester aroma volatiles in Charentais Cantaloupe melons. J. Exp. Bot. 53, 201-206. doi: 10.1093/jexbot/53.367.201

Fluhr, R., and Mattoo, A. K. (1996). Ethylene - biosynthesis and perception. CRC Crit. Rev. Plant Sci. 15, 479-523.

Gapper, N. E., Giovannoni, J. J., and Watkins, C. B. (2014). Understanding development and ripening of fruit crops in an 'omics' era. Hortic. Res. 1, 14034. doi: 10.1038/hortres.2014.34 
Grierson, D. (2014). "Ethylene biosynthesis," in Fruit Ripening, Physiology, Signalling and Genomics, eds P. Nath, M. Bouzayen, A. K. Mattoo, and J. C. Pech (Oxfordshire: CAB International), 178-192.

Hamilton, A., Lycett, G. W., and Grierson, D. (1990). Antisense gene that inhibits synthesis of the hormone ethylene in transgenic plants. Nature 346, 284-287. doi: $10.1038 / 346284 \mathrm{a} 0$

Handa, A. K., and Mattoo, A. K. (2010). Differential and functional interactions emphasize the multiple roles of polyamines in plants. Plant Physiol. Biochem. 48 540-546. doi: 10.1016/j.plaphy.2010.02.009

Harpaz-Saad, S., Yoon, G. M., Mattoo, A., and Kieber, J. J. (2012). The formation of ACC and competition between polyamines and ethylene for SAM. Ann. Plant Rev. 44, 53-81. doi: 10.1002/9781118223086.ch3

Heldt, H.-W. (2005). Plant Biochemisty. Burlington: Elsevier.

Hiwasa-Tanase, K., and Ezura, H. (2014). "Climacteric and non-climacteric ripening," in Fruit Ripening, Physiology, Signalling and Genomics, eds P. Nath, M. Bouzayen, A. K. Mattoo, and J. C. Pech (Oxfordshire: CAB International), 1-14.

Kausch, K. D., Sobolev, A. P., Goyal, R. K., Fatima, T., Laila-Beevi, R., Saftner, R. A., et al. (2012). Methyl jasmonate deficiency alters cellular metabolome, including the aminome of tomato (Solanum lycopersicum L.) fruit. Amino Acids 42, 843-856. doi: 10.1007/s00726-011-1000-5

Klee, H. (1993). Ripening physiology of fruit from transgenic tomato (Lycopersicon esculentum) plants with reduced ethylene synthesis. Plant Physiol. 102, 911-916.

Klie, S., Osorio, S., Tohge, T., Fabiana, M., Fait, A., Giovannoni, J. J., et al. (2013). Conserved changes in the dynamics of metabolic processes during fruit development and ripening across species. Plant Physiol. 164, 55-68. doi: 10.1104/pp.113.226142

Lasanajak, Y., Minocha, R., Minocha, S. C., Goyal, R., Fatima, T., Handa, A. K., et al. (2013). Enhanced flux of substrates into polyamine biosynthesis but not ethylene in tomato fruit engineered with yeast S-adenosylmethionine decarboxylase gene. Amino Acids 46, 729-742. doi: 10.1007/s00726-013-1624-8

Martens, H., and Martens, M. (2001). Multivariate Analysis of Quality. Chichester: JohnWiley and Sons.

Mattoo, A. K., Chung, S. H., Goyal, R. K., Fatima, T., Solomos, T., Srivastava, A., et al. (2007). Overaccumulation of higher polyamines in ripening transgenic tomato fruit revives metabolic memory, upregulates anabolism-related genes, and positively impacts nutritional quality. J. AOAC Int. 90, 1456-1464.

Mattoo, A. K., and Handa, A. K. (2008). Higher polyamines restore and enhance metabolic memory in ripening fruit. Plant Sci. 174, 386-393. doi: 10.1016/j.plantsci.2008.01.011

Mattoo, A. K., Minocha, S. C., Minocha, R., and Handa, A. K. (2010). Polyamines and cellular metabolism in plants: transgenic approaches reveal different responses to diamine putrescine versus higher polyamines spermidine and spermine. Amino Acids 38, 405-413. doi: 10.1007/s00726-009-0399-4

Mattoo, A. K., and Suttle, J. C. (1991). The Plant Hormone Ethylene. BocaRaton, FL: CRC Press.

Mattoo, A. K., Sobolev, A. P., Neelam, A., Goyal, R. K., Handa, A. K., and Segre, A L. (2006). Nuclear magnetic resonance spectroscopy-based metabolite profiling of transgenic tomato fruit engineered to accumulate spermidine and spermine reveals enhanced anabolic and nitrogen-carbon interactions. Plant Physiol. 142, 1759-1770. doi: 10.1104/pp.106.084400

Mehta, R. A., Cassol, T., Li, N., Ali, N., Handa, A. K., and Mattoo, A. K. (2002). Engineered polyamine accumulation in tomato enhances phytonutrient content, juice quality and vine life. Nat. Biotechnol. 20, 613-618. doi: 10.1038/nbt0602-613

Merchante, C., Vallarino, J. G., Osorio, S., Aragüez, I., Villarreal, N., Ariza, M. T. et al. (2013). Ethylene is involved in strawberry fruit ripening in an organ-specific manner. J. Exp. Bot. 64, 4421-4439. doi: 10.1093/jxb/ert257
Nath, P., Bouzayen, M., Mattoo, A. K., and Pech, J. C. (2014). Preface to Fruit Ripening, Physiology, Signalling and Genomics (Oxfordshire: CAB International). doi: 10.1079/9781845939625.0000

Oeller, P. W., Min-Wong, L., Taylor, L. P., Pike, D. A., and Theologis, A. (1991). Reversible inhibition of tomato fruit senescence by antisense RNA. Science 254, 437-439. doi: 10.1126/science.1925603

Osorio, S., Alba, R., Damasceno, C. M. B., Lopez-Casado, G., Lohse, M., Zanor, M. I., et al. (2011). Systems biology of tomato fruit development: combined transcript, protein, and metabolite analysis of tomato transcription factor (nor, rin) and ethylene receptor $(\mathrm{Nr})$ mutants reveals novel regulatory interactions. Plant Physiol. 157, 405-425. doi: 10.1104/pp.111.175463

Osorio, S., Scossa, F., and Fernie, A. R. (2013). Molecular regulation of fruit ripening. Front. Plant Sci. 4:198. doi: 10.3389/fpls.2013.00198

Paliyath, G., Murr, D. P., Handa, A. K., and Lurie, S. (2008). Postharvest Biology and Technology of Fruits, Vegetable, and Flowers. New Delhi: Wiley-Blackwell Publishing.

Perez-Fons, L., Wells, T., Corol, D. I., Ward, J. L., Gerrish, C., Beale, M. H., et al. (2014). A genome-wide metabolomic resource for tomato fruit from Solanum pennellii. Sci. Rep. 4, 3859. doi: 10.1038/srep03859

Pfitzner, A. J. P. (1998). "Transformation of tomato. Plant virology protocols: from virus isolation to transgenic resistance," in Methods in Molecular Biology, eds G. D. Foster and S. C. Taylor (Totowa, NJ: Humana Press Inc.), 359-363. doi: 10.1385/0-89603-385-6:359

Saltveit, M. E. (1999). Effect of ethylene on quality of fresh fruits and vegetables. Postharvest Biol. Technol. 15, 279-292. doi: 10.1016/S0925-5214(98) 00091-X

Sobolev, A. P., Segre, A. L., and Lamanna, R. (2003). Proton high-field NMR study of tomato juice. Magn. Reson. Chem. 41, 237-245. doi: 10.1002/mrc.1176

Srivastava, A., Chung, S. H., Fatima, T., Datsenka, T., Handa, A. K., and Mattoo, A. K. (2007). Polyamines as anabolic growth regulators revealed by transcriptome analysis and metabolite profiles of tomato fruits engineered to accumulate spermidine and spermine. Plant Biotechnol. 24, 57-70. doi: 10.5511/plantbiotechnology. 24.57

Wilkinson, J. Q., Lanahan, M. B., Clark, D. G., Bleecker, A. B., Chang, C., Meyerowitz, E. M., et al. (1997). A dominant mutant receptor from Arabidopsis confers ethylene insensitivity in heterologous plants. Nat. Biotechnol. 15, 444-447. doi: $10.1038 /$ nbt0597-444

Conflict of Interest Statement: The authors declare that the research was conducted in the absence of any commercial or financial relationships that could be construed as a potential conflict of interest.

Received: 27 August 2014; accepted: 24 October 2014; published online: 05 December 2014.

Citation: Sobolev AP, Neelam A, Fatima T, Shukla V, Handa AK and Mattoo AK (2014) Genetic introgression of ethylene-suppressed transgenic tomatoes with higherpolyamines trait overcomes many unintended effects due to reduced ethylene on the primary metabolome. Front. Plant Sci. 5:632. doi: 10.3389/fpls.2014.00632

This article was submitted to Plant Physiology, a section of the journal Frontiers in Plant Science.

Copyright (C) 2014 Sobolev, Neelam, Fatima, Shukla, Handa and Mattoo. This is an open-access article distributed under the terms of the Creative Commons Attribution License (CC BY). The use, distribution or reproduction in other forums is permitted, provided the original author(s) or licensor are credited and that the original publication in this journal is cited, in accordance with accepted academic practice. No use, distribution or reproduction is permitted which does not comply with these terms. 


\title{
Heterologous over-expression of ACC SYNTHASE8 (ACS8) in Populus tremula $x$ P. alba clone 717-1B4 results in elevated levels of ethylene and induces stem dwarfism and reduced leaf size through separate genetic pathways
}

\author{
Jonathan M. Plett ${ }^{1,2}$, Martin Williams ${ }^{3}$, Gaetan LeClair ${ }^{3}$, Sharon Regan ${ }^{1 \dagger}$ and Tannis Beardmore ${ }^{* t}$ \\ Department of Biology, Queen's University, Kingston, ON, Canada \\ ${ }^{2}$ Hawkesbury Institute for the Environment, University of Western Sydney, Richmond, NSW, Australia \\ ${ }^{3}$ Atlantic Forestry Centre, Canadian Forest Service, Natural Resources Canada, Fredericton, NB, Canada
}

Edited by:

Domenico De Martinis, Energy and Sustainable Economic

Development, Italy

Reviewed by:

Dierk Wanke, Tuebingen University, Germany

Sara Maldonado, University of

Buenos Aires, Argentina

*Correspondence:

Tannis Beardmore, Canadian Forest Service, Natural Resources Canada,

Fredericton, NB, E3B 5P7, Canada

e-mail: tannis.beardmore@

nrcan-rncan.gc.ca

tThese authors have contributed equally to this work.
Plant height is an important agronomic and horticultural trait that impacts plant productivity, durability and esthetic appeal. A number of the plant hormones such as gibberellic acid (GA), auxin and ethylene have been linked to control of plant architecture and size. Reduction in GA synthesis and auxin transport result in dwarfism while ethylene may have a permissive or repressive effect on tissue growth depending upon the age of plant tissues or the environmental conditions considered. We describe here an activation-tagged mutant of Populus tremula x P. alba clone 717-1B4 identified from 2000 independent transgenic lines due to its significantly reduced growth rate and smaller leaf size. Named dwarfy, the phenotype is due to increased expression of PtaACC SYNTHASE8, which codes for an enzyme in the first committed step in the biosynthesis of ethylene. Stems of $d$ warfy contain fiber and vessel elements that are reduced in length while leaves contain fewer cells. These morphological differences are linked to PtaACS8 inducing different transcriptomic programs in the stem and leaf, with genes related to auxin diffusion and sensing being repressed in the stem and genes related to cell division found to be repressed in the leaves. Altogether, our study gives mechanistic insight into the genetics underpinning ethylene-induced dwarfism in a perennial model organism.

Keywords: poplar, activation tagging, wood formation, plant stature, plant growth rate

\section{INTRODUCTION}

Reduced plant height, or dwarfism, is an important agronomic trait linked to higher yields (Huang et al., 1996; Yang and Hwa, 2008), easier harvesting (Adkins et al., 2010) and reduced nutrient demand on soils (Sieling and Kage, 2008). Leaf size, meanwhile, is linked to productivity, predation (Faeth, 1991) and the water status of the plant (Scoffoni et al., 2011). While both height and leaf size are complex traits, they appear to be genetically regulated by a similar panel of plant hormones (Valdovinos et al., 1967; Ephritikhine et al., 1999; Qi et al., 2011; Luo et al., 2013) and cytochrome P450s (Zhang et al., 2014), as well as abiotic factors such as temperature (Yang et al., 2014) and photoperiod (Li et al., 2014). Reductions in organ size are a result of two different physiological phenomena: smaller cells and impeded cellular division (Beemster et al., 2003). These two factors may work independently or synergistically to affect plant stature and organ size (Beemster et al., 2005; Skirycz et al., 2010). Newly produced plant tissues first exhibit growth due to rapid cellular division, a phase that is replaced in a distal-proximal manner by cellular expansion in progenitor cells (Donnelly et al., 1999). Due to the integrated control between these two processes, genetic mutations to single genes can have a drastic impact on plant stature as a whole or at the level of a specific tissue. Altered expression of genes such as ARABIDOPSIS VACUOLAR H+-PYROPHOSPHATASE1 (AVP1; Li et al., 2005), CYTOKININ RESISTANT1 (CNR1; Guo et al., 2010), and ISOPENTENYL TRANSFERASE3 (IPT3; Nobusawa et al., 2013) impact tissue size due to a difference in the total number of cells produced, while EXPANSIN10 (EXP10; Cho and Cosgrove, 2000), ARGOS-LIKE (Hu et al., 2006), and RETINOBLASTOMA-RELATED PROTEIN1 (RBR1; Sabelli et al., 2013) change the final size of plant tissues as a function of altered cell expansion.

The best studied genetic influences on dwarfism are genes and signaling pathways related to hormone production and sensitivity. Within these studies, ethylene, gibberellic acid (GA), auxin, and brassinosteroids (BR) have all been implicated with a role in cell division, cellular growth and overall plant architecture. Blocked BR synthesis (Nakaya et al., 2002) and reduced GA biosynthesis (Tong et al., 2007; Li et al., 2011) or increased GA catabolism (Busov et al., 2003; Schomburg et al., 2003; Curtis et al., 2005; Lee and Zeevaart, 2005; Dijkstra et al., 2008; Zawaski et al., 2011) induce dwarfism in a wide range of model plant systems. Auxin transport, meanwhile, is a critical component of proper plant stem elongation. In rice, auxin 
transport inhibition has been correlated to slower stem elongation (Yamamoto et al., 2007; Domingo et al., 2009) while reduced basipetal auxin transport in maize and Arabidopsis thaliana results in stunted plant development (Lantican and Muir, 1969; Geisler et al., 2003, 2005; Multani et al., 2003; Geisler and Murphy, 2006). Treatment of plant tissues with ethylene, a gaseous plant hormone, results in stunting (Vahala et al., 2013), a phenotype that has been linked to the induced expression of certain ETHYLENE RESPONSE FACTORs (ERFs; Dubois et al., 2013; Vahala et al., 2013). There also appears to be extensive cross-talk between the different hormone pathways with components of the ethylene pathway controlling GA biosynthesis (Qi et al., 2011) and the activity of DELLA proteins (Luo et al., 2013). Ethylene can also regulate auxin diffusion and biosynthesis (Valdovinos et al., 1967; Stepanova et al., 2005; Ruzicka et al., 2007; Swarup et al., 2007).

Here we characterize an activation tagged mutant of Populus tremula $x$ P. alba clone 717 , named “dwarfy," exhibiting severe dwarfism with both reduced stature and smaller leaves. We show that the gene responsible for this phenotype is annotated as the poplar 1-aminocyclopropane-1-carboxylate synthase (ACS) gene PtaACS8. Ethylene is synthesized in two enzymatic steps from the substrate S-adenosyl-methionine (SAM). The first step is the conversion of SAM into 1-aminocyclopropane-1-carboxylic acid (ACC) by the activity of ACSs followed by the conversion of ACC to ethylene catalyzed by ACC OXIDASEs (ACOs). Ethylene is then perceived by a family of membrane bound receptors that induce the transcription of ETHYLENE RESPONSE FACTORs (ERFs) which, in turn, controls transcription and, ultimately, plant development. We demonstrate that increased expression of PtaACS8 in the dwarfy line results in significantly higher levels of ethylene in all aerial tissues of the plant. Morphologically, the increased expression of PtaACS8 in the stem results in shorter vessels and fibers in secondary growth while endogenous over-expression of the PtaACS8 gene in the leaves results in the production of fewer cells. The reduced growth of stem cells is accompanied by a repression of auxin transport and signaling genes while reduction in cell number in leaves is concurrent with a large reduction in the transcript abundance of a number of cell-cycle genes. Therefore, we conclude that increased expression of PtaACS8 induces stem dwarfism and reduced leaf size through separate genetic pathways.

\section{MATERIALS AND METHODS PLANT MATERIAL}

All plants used in Figures 1, 4, 6 were grown under greenhouse conditions at the Canadian Forest Service (CFS), Fredericton, New Brunswick, Canada, while plants used for data analysis in Figures 2, 3, 5 were grown under greenhouse conditions at Queen's University, Kingston, Ontario, Canada. In the former situation, plants were grown under natural daylight and temperature, while in the latter situation, photoperiod was maintained at $16 \mathrm{~h}$ per day and temperature at $25^{\circ} \mathrm{C}$. The dwarfy mutant was generated as described by Harrison et al. (2007) in a P. tremula $x$ $P$. alba clone 717-1B4 background and all comparisons of $d w a r f y$ were made with this hybrid (wildtype). The dwarfy mutant was initially identified based on the dwarf characteristics such as plant height and leaf size among others in the mutant.

\section{T-DNA INSERTION ANALYSIS}

Southern analysis of the dwarfy poplar mutant line was done and confirmed the presence of one T-DNA insertion event (Harrison et al., 2007). Genomic DNA (gDNA) was extracted from CFS greenhouse dwarfy mutant leaves using the cetyltrimethylammonium bromide (CTAB) method and gDNA was quantified using an Nanodrop 1000 spectrophotometer and quality was checked on $0.8 \%(\mathrm{w} / \mathrm{v})$ agarose Tris-acetate EDTA ethidium bromide gel. To identify the site of T-DNA insertion, the Genome Walker ${ }^{\mathrm{TM}}$ universal kit (Clontech, http://www.clontech.com) was used according to the manufacturer's protocol to create 4 restriction digested gDNA libraries. Each library was analyzed by primary and nested PCR using T-DNA vector specific primers (VSP 1 and VSP 2) designed from the T-DNA sequence and adapter primers AP1 \& AP2 provided in the kit (Table S1). Primary PCRs were done on $1 \mu \mathrm{l}$ of each library except using High Fidelity Platinum Taq (Invitrogen) for the PCR reaction mix. Primary PCR reactions from each library were diluted 50 times in $\mathrm{H}_{2} \mathrm{O}$ and $1 \mu \mathrm{l}$ of the each dilution was used for nested PCR analysis using the same PCR reaction components except primers VSP2 \& AP2 were used. Cycling parameters for both primary \& nested PCRs were the same ones stated in the kit except that the elongation time was increased to $5 \mathrm{~min}$. Primary and nested PCRs were analyzed by gel electrophoresis and bands from the nested PCR reaction that were over $1 \mathrm{~kb}$ in size were subcloned in pCR4-topo vector using the TOPO TA cloning kit (Invitrogen, http://www. invitrogen.com) and fully sequenced at the McGill University and Genome Quebec Innovation Centre (http://gqinnovationcenter. com). Based on flanking sequence information, a flanking gDNA primer FSP1 was designed and PCR was done on dwarfy gDNA using FSP1 and VSP 2 primers to confirm the location of the T-DNA insertion site. This amplicon was TOPO TA cloned and fully sequenced. Localisation of insertion site was determined by BLASTn using flanking sequence as query against the $P$. trichocarpa genome (Tuskan et al., 2006) (Populus trichocarpa v3.0, DOE-JGI, http:://www.phytozome.net/poplar).

\section{AFFYMETRIX ARRAY ANALYSIS}

For gene expression analysis, total RNA was extracted from $0.5 \mathrm{~g}$ of different tissues of wildtype and dwarfy taken from CFS greenhouse grown plants using a modification of Chang et al., 1993 and the RNeasy kit (QIAGEN). Total RNA quality and quantity was determined by Nanodrop1000 and by electrophoresis. Triplicate samples of $d$ warfy and wild-type leaf and stem total RNA were sent to the Microarray Centre (University Health Network, Toronto (UHN)) for sample processing and analysis. Sample quality was verified with the Agilent 2100 Bioanalyser before analysis with the GeneChip ${ }^{\circledR}$ Poplar Genome Array. Data was also analyzed by the Microarray Center (UHN) using Gene Spring software.

\section{ISOLATION OF ACS8 CANDIDATE GENE CODING SEQUENCE}

The ACS 8 cDNA was isolated from wild-type leaf tissues using the Smart RACE kit (Clontech, http://www.clontech.com) according to manufacturer's protocol. One microgram of total RNA was used to produce the $5^{\prime} \& 3^{\prime}$ RACE ready products and ACS 8 gene specific primers ACS8RACE.fwd and ACS8RACE.rev 


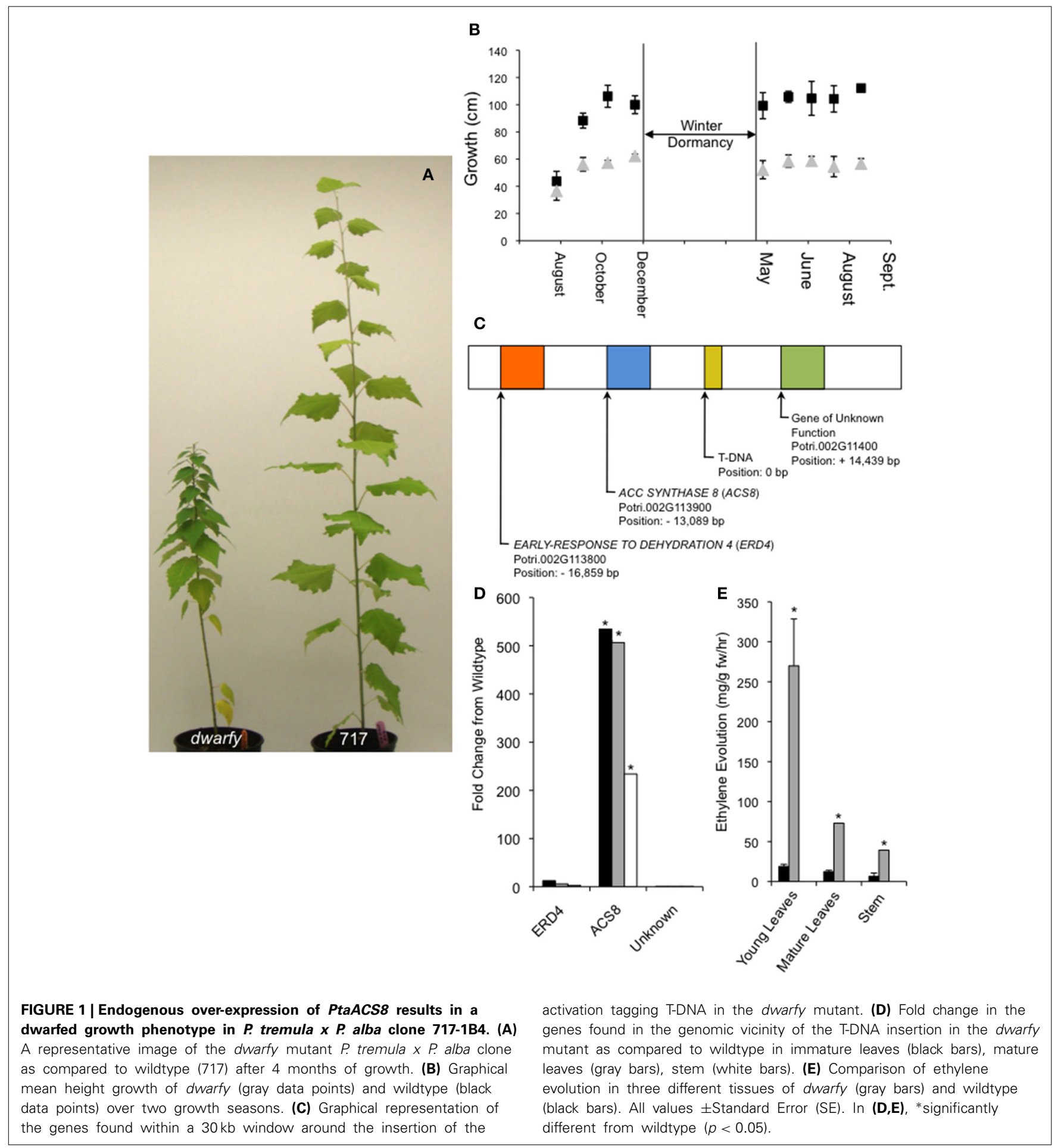

were used along with the Universal Primer (UP) provided in the kit (Table S1). $5^{\prime} \& 3^{\prime}$ RACE products were subcloned in pCR4-topo vector using the TOPO TA cloning kit and sequenced. Gene specific primers; ACS8-ATG.fwd and ACS8Stop.rev primers were designed and used to isolate the full ACS8 CDS using the $3^{\prime} \mathrm{RACE}$ ready product previously generated and the amplicon was cloned in pBluescript II $(+)$ (Fermentas, http://www.fermentas.com) using HindIII-XbaI restriction sites.
The resulting construct carrying the full ACS8 CDS was fully sequenced.

\section{AGROBACTERIUM TUMEFACIENS MEDIATED TRANFORMATION OF $\boldsymbol{P}$ TREMULA X P. ALBA CLONE 717-1B4}

In order to generate a binary plant transformation vector,ACS8/pBluescript II (+) construct was digested with EcoRI and subcloned in pART7 (Gleave, 1992) and verified by 


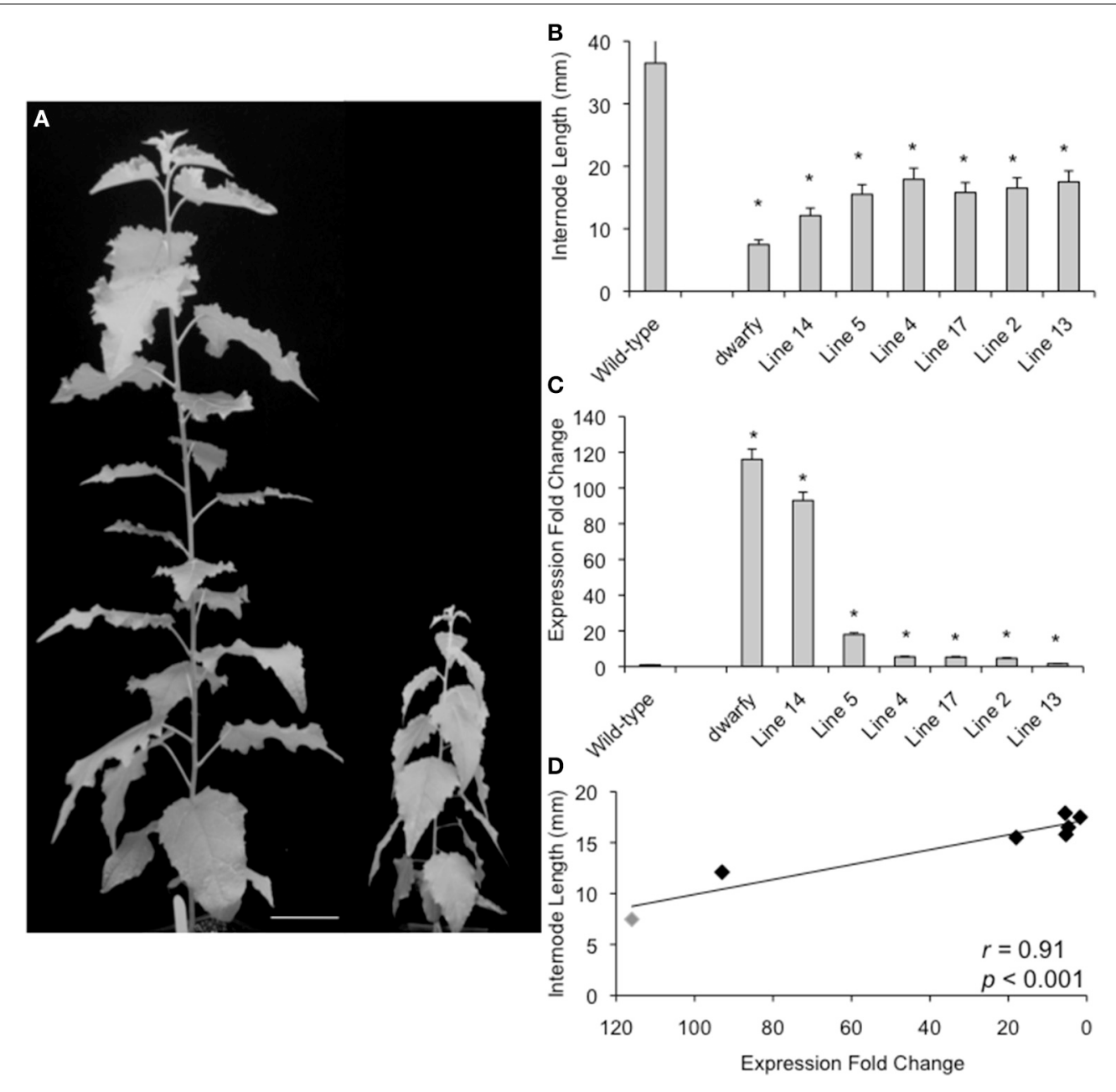

FIGURE 2 | Plant growth retardation in dwarfy mutant is significantly correlated to the expression level of PtaACS8. (A) A representative image of one independent line of the 35S::PtaACS8 mutant P. tremula $x$ $P$ alba clone as compared to wildtype (717) after 2 months of growth. Scale bar $=8 \mathrm{~cm}$. (B) Relative expression of PtaACS8 in wildtype, dwarfy and 6 independent transgenic lines containing the 35S::PtaACS8 construct. (C) Internode length of wildtype, dwarfy and 6 independent transgenic lines containing the 35S::PtaACS8 construct. (D) Correlation between PtaACS8 expression levels and internode length in wildtype, dwarfy and 6 independent transgenic lines containing the 35S::PtaACS8 construct. All values \pm Standard Error (SE). In (B,C), * significantly different from wildtype $(p<0.05)$. restriction digest for correct orientation between the CaMV promoter and ocs $3^{\prime}$ region. The ACS8/pART7 construct was subsequently digested with NotI and the whole cassette was ligated in the binary vector pART 27 (Gleave, 1992) prior to Agrobacterium tumefaciens transformation in line 717-1-B4 (Harrison et al., 2007). Out of 17 independent transgenic lines generated, 6 lines survived the transfer to greenhouse conditions and these lines were analyzed. Total RNA from leaf tissue was extracted from newly transformed lines and RT-qPCRs were done for gene expression analysis of lines generated using the procedures as stated below. Each line was analyzed in duplicate technical replicates. Internode lengths were measured after 3 months of growth.

\section{QUANTITATIVE GENE EXPRESSION ANALYSIS}

For in gene expression analysis in transgenic 35S::PtaACS8 lines, total RNA was extracted from $100 \mathrm{mg}$ of shoot apical tissue using the RNeasy kit (QIAGEN). Total RNA quality and quantity was determined by Nanodrop1000 and by electrophoresis. Two to four micrograms of total RNA was treated with Turbo DNaseI (Ambion, http://www.ambion.com) and RT-qPCR was done with $50 \mathrm{ng}$ of total RNA/reaction using the one step Quantitect SYBR Green RT-PCR kit (QIAGEN, http://www.qiagen.com). RT-qPCR cycling conditions were: $30 \mathrm{~min}$ at $50^{\circ} \mathrm{C}$ for reverse transcriptase reaction and $15 \mathrm{~min}$ at $95^{\circ} \mathrm{C}$ for enzyme inactivation followed by 40 cycles of $15 \mathrm{~s}$ at $94^{\circ} \mathrm{C}, 15 \mathrm{~s}$ denaturation at $55^{\circ} \mathrm{C}$ (annealing) and $30 \mathrm{~s}$ at $72^{\circ} \mathrm{C}$ elongation followed by fluorescence measurement. The relative expression of PtaACS8 was compared to the UBQ10 reference gene (Plett et al., 2010).

The amplification efficiencies of each gene primer set were determined by $E=10^{[-1 / \text { slope }]}$ and were calculated using the slopes of $\mathrm{n}$-fold serial dilution standard curves. Fold change ratios were determined using the comparative $\mathrm{Ct}$ method $(\Delta \Delta \mathrm{Ct}$ method) since amplification efficiencies were approximately equal in all target and reference genes measured in the study. 


\section{A \\ 717}

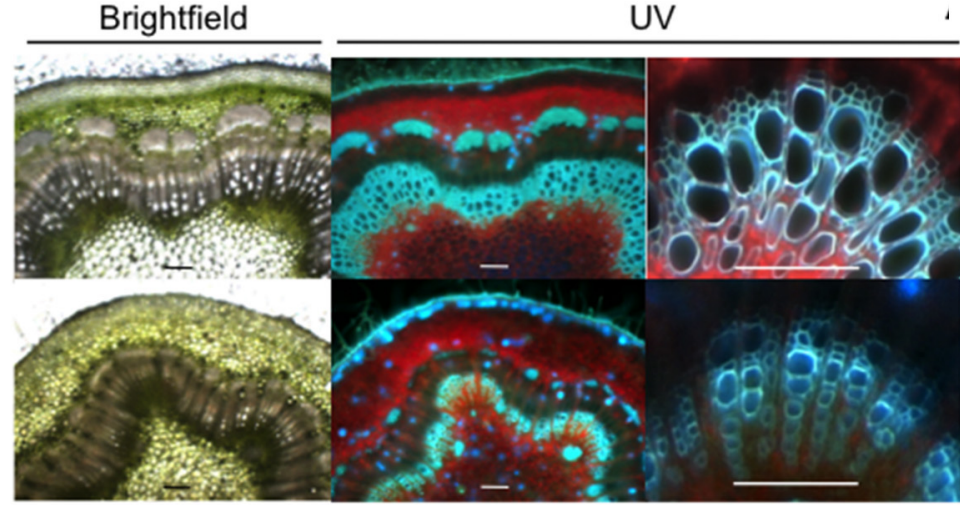

C

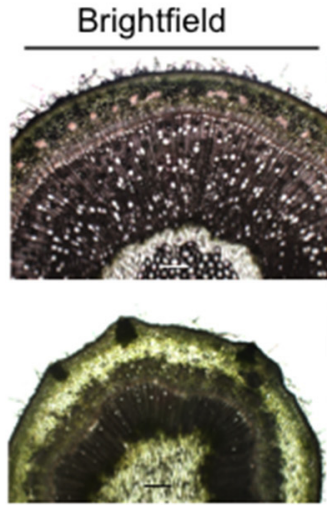

E

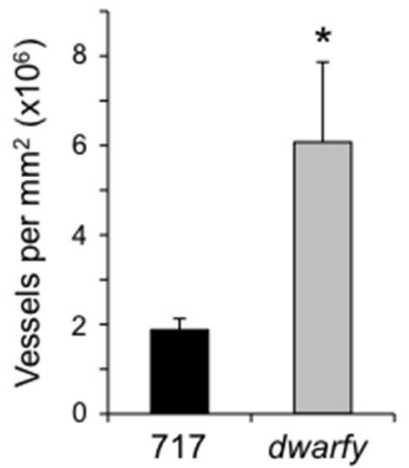

H

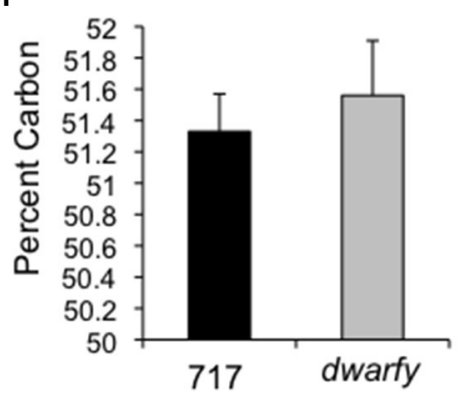

UV

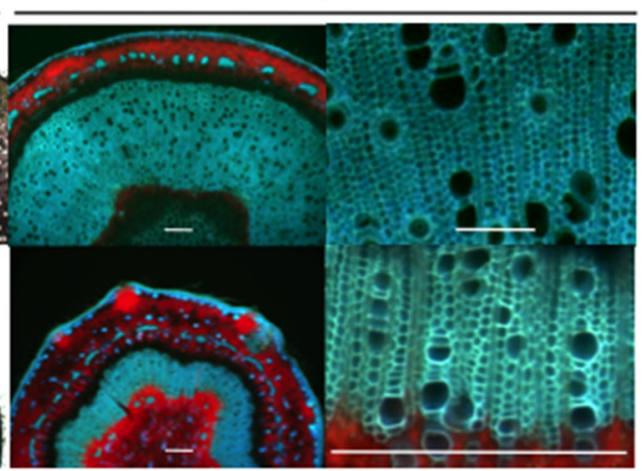

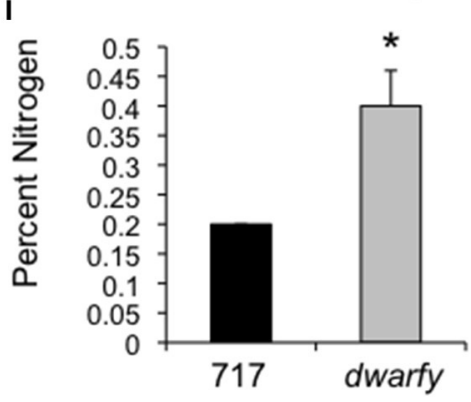

B

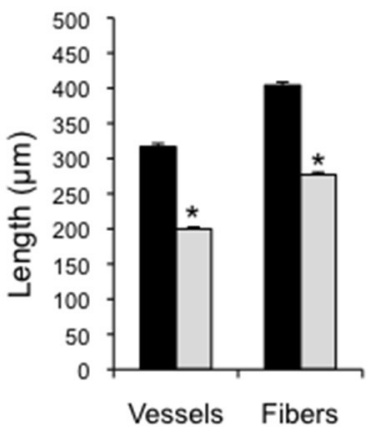

D

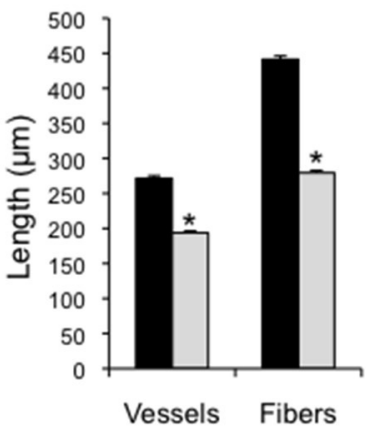

G

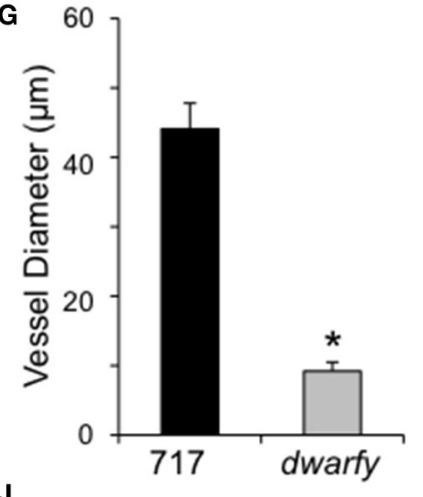

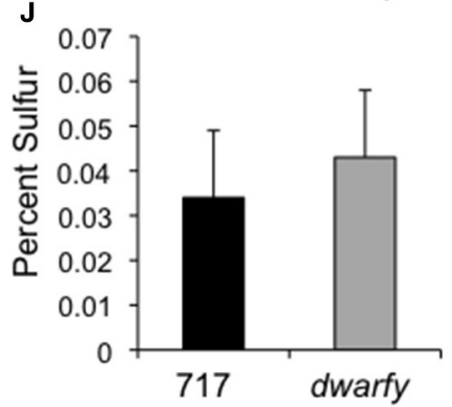

FIGURE 3 | Elevated expression levels of PtaACS8 result in significant changes in stem architecture and physical characteristics. (A) Transverse cross section of wild-type and dwarfy stems at the leaf 10-11 internode as observed under brightfield and UV autofluorescence. Scale bar $=1 \mathrm{~mm}$ for first two images and $100 \mu \mathrm{m}$ for the third panel (B) Vessel and fiber lengths of wild-type (black bars) and dwarfy (gray bars) stems between the leaf 10-11 internode. (C) Transverse cross section of wildtype and dwarfy stems at the leaf 20-21 internode as observed under brightfield and UV autofluorescence.

Scale bar $=0.5 \mathrm{~mm}$ for first two images and $100 \mu \mathrm{m}$ for the third panel. (D) Vessel and fiber lengths of wild-type (black bars) and dwarfy (gray bars) stems between the leaf 20-21 internode. Vessel (E) and fiber (F) density in wild-type and dwarfy stems between the leaf 20-21 internode. (G) Vessel diameter in wild-type and dwarfy stems between the leaf 20-21 internode. Relative percentage of carbon $\mathbf{( H )}$, nitrogen $\mathbf{( I )}$ and sulfur $\mathbf{( J )}$ in the stems of wildtype and $d$ warfy. All values \pm Standard Error. *Significantly different from wildtype $(p<0.05)$. 


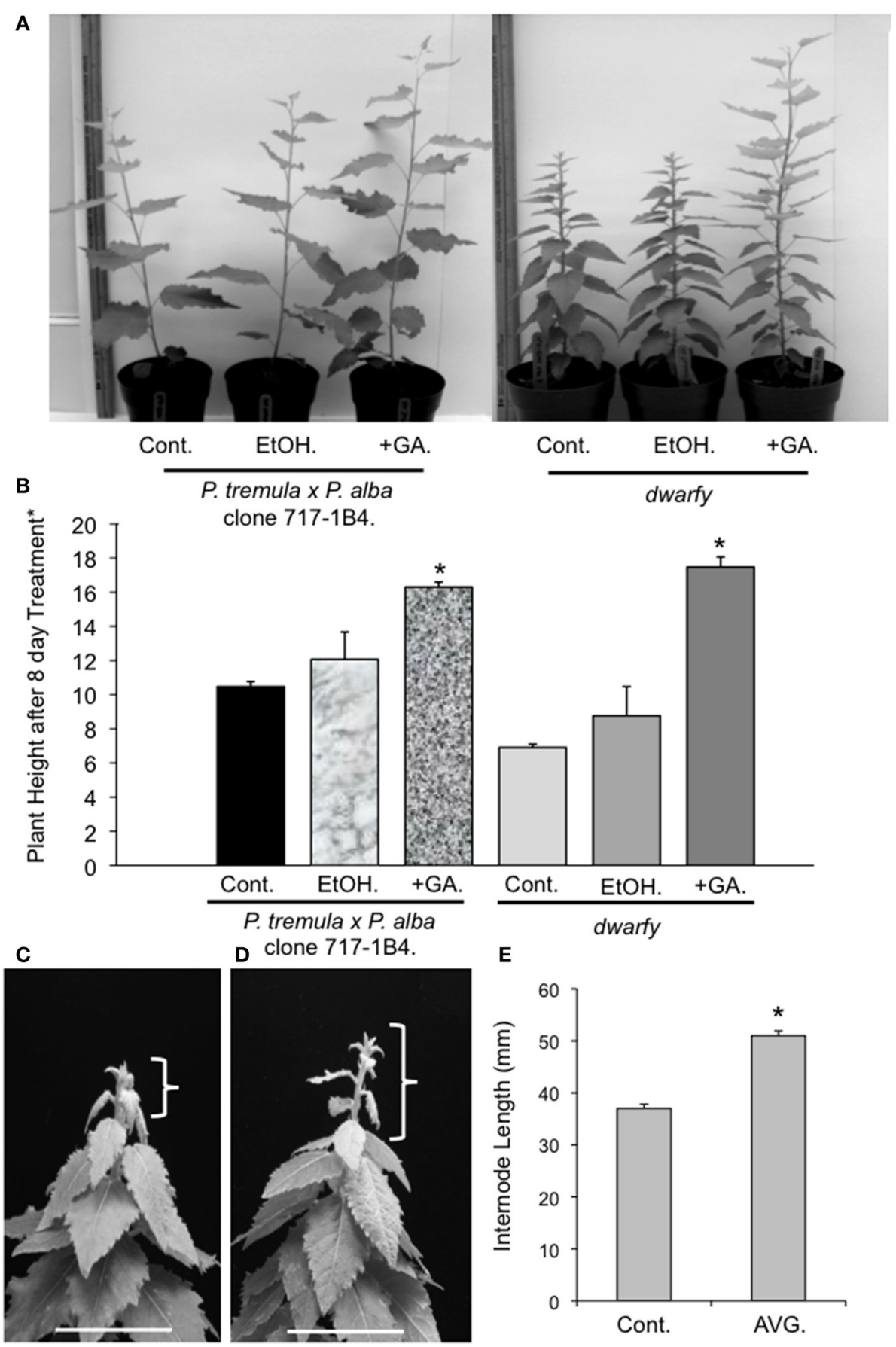

FIGURE 4 | Application of GA and AVG to dwarfy apexes induces faster growth rate. (A) Representative image of GA influence on the growth rates of dwarfy and wildtype (+GA) as compared to ethanol control $(+\mathrm{EtOH})$ and untreated control (Cont.). (B) Mean heights of wild-type and dwarfy saplings treated with $\mathrm{GA}(+\mathrm{GA})$ as compared to ethanol control (+EtOH) and untreated control (Cont.).
(C) Representative image of water and (D) AVG influence on the growth rates of dwarfy and wild-type. Parentheses indicate growth of main stem for the treatment period. Scale bar $=3 \mathrm{~cm}$. (E) Mean internode lengths wild-type and dwarfy saplings treated with AVG as compared to water control (Cont.). All values \pm SE. ${ }^{*}$ Significantly different from wildtype $(p<0.05)$.
Samples were analyzed in triplicates of each wildtype and the dwarfy mutant plants. Each total RNA sample was analyzed in duplicate. A No Reverse Transcriptase (NoRT) for each sample was included and a No Template Control (NTC) was included for each primer pair to make sure no contamination was present in the experiments. Amplicon specificity was confirmed by electrophoresis (single band at the right size), by melt curve analysis (single peak and $\mathrm{Tm}$ ) and by sequencing. 
A
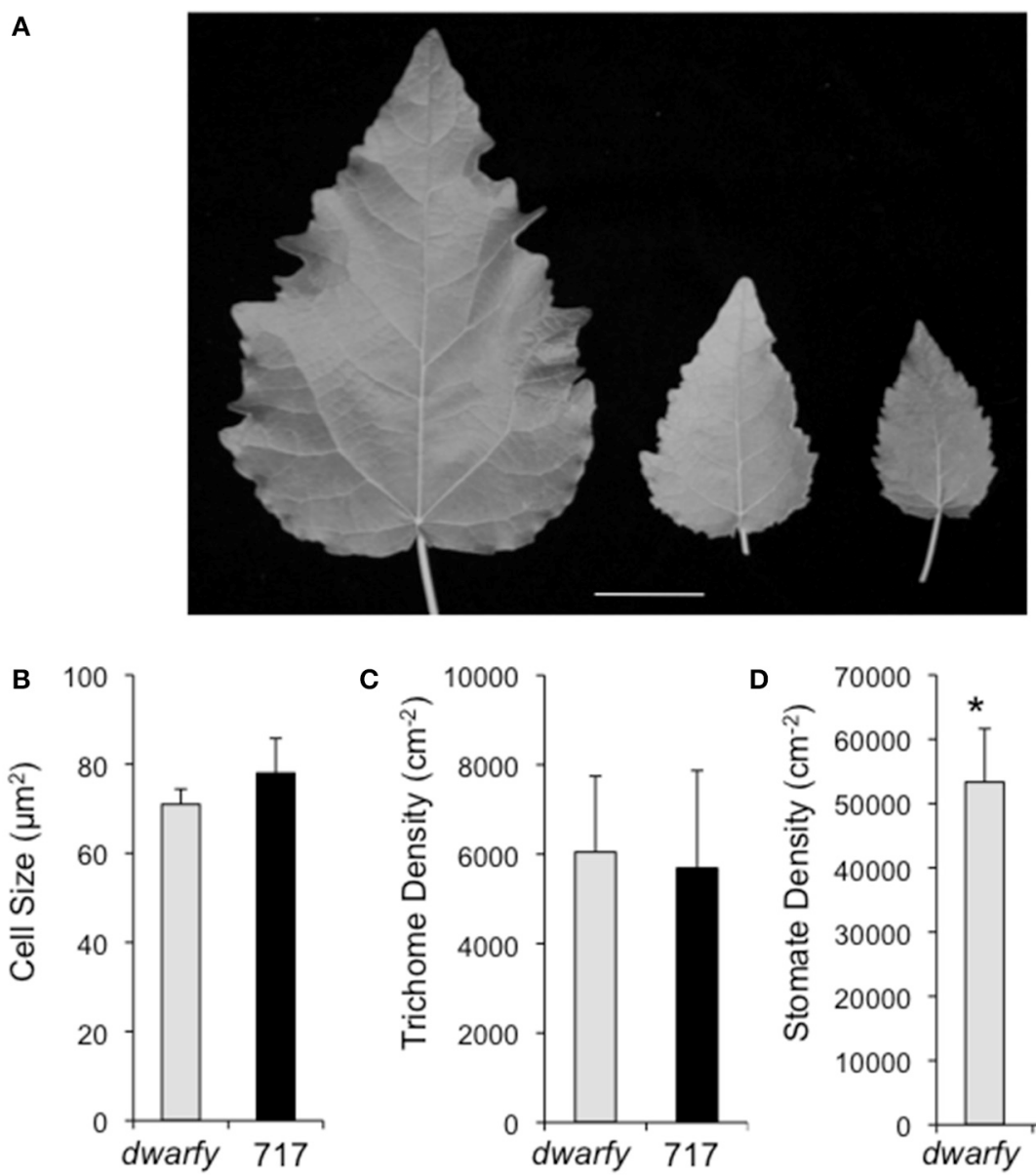

C

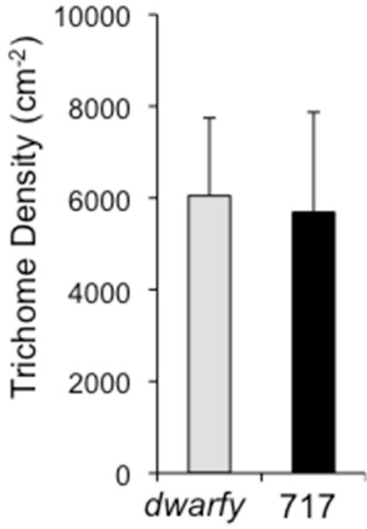

D
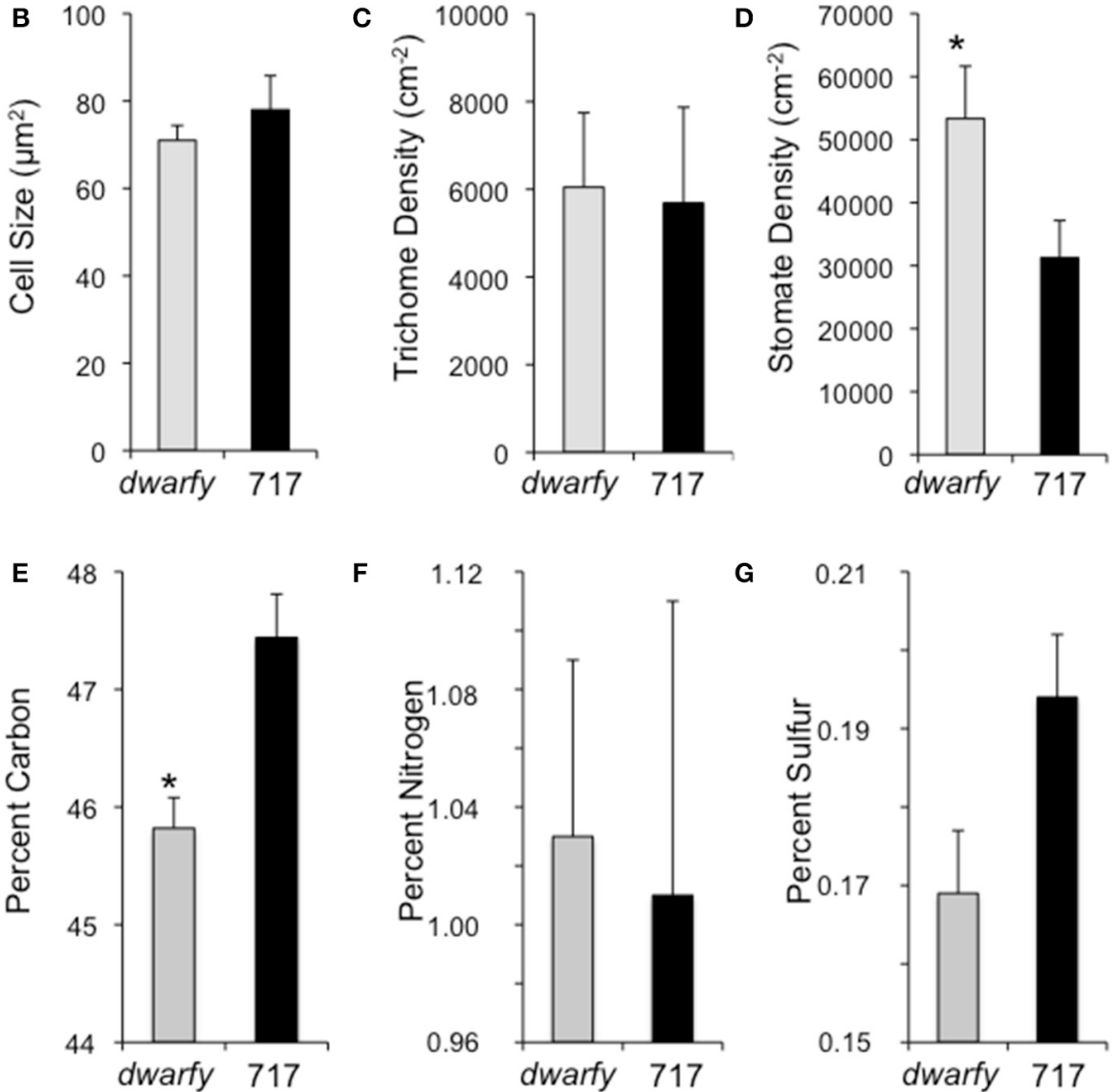

$\mathbf{F}$

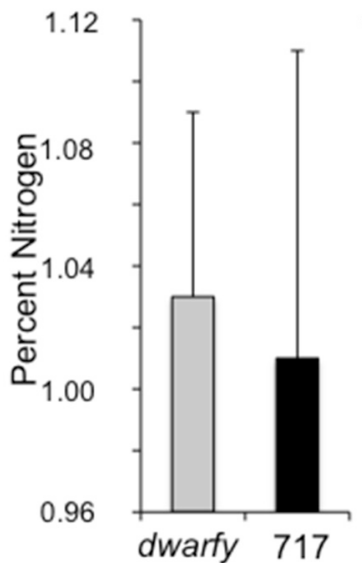

G

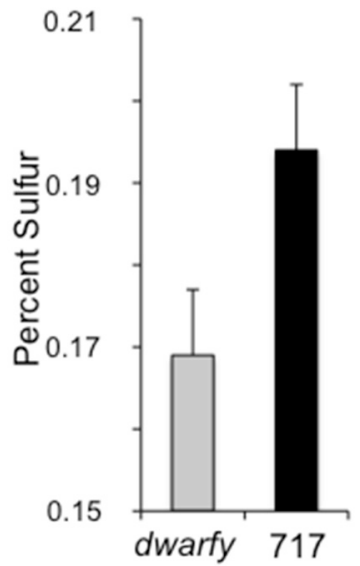

FIGURE 5 | Elevated expression levels of PtaACS8 result in significant changes in leaf architecture and physical characteristics. (A) A

representative image of fully expanded leaves of wildtype, one independent line of the 35S::PtaACS8 mutant $P$. tremula $\times$ P. alba clone 717-1B4 and $d$ warfy, respectively, after 2 months of growth. Scale bar $=2 \mathrm{~cm}$. Epidermal cell density (B), trichome density (C) and stomate density (D) in fully expanded leaves of $d$ warfy (gray bars) as compared to wild-type leaves (black bars). Relative percentage of carbon (E), nitrogen (F), and sulfur (G) in mature leaves of wildtype (black bars) and $d$ warfy (gray bars). All values $\pm \mathrm{SE}$. * Significantly different from wildtype $(p<0.05)$.

\section{ETHYLENE DETERMINATION}

Leaf and stem samples were removed from wild-type and dwarfy poplar plants between $10 \mathrm{AM}$ and $12 \mathrm{PM}$, and incubated in $20 \mathrm{~mL}$ headspace vials for $4 \mathrm{~h}$ at ambient temperature. Fresh weight was recorded and time between vial seal and sample injection were noted to have an exact incubation time. Ethylene content within this headspace was determined by gas chromatography coupled to a flame ionization detector (Gas Chromatography- Flame 

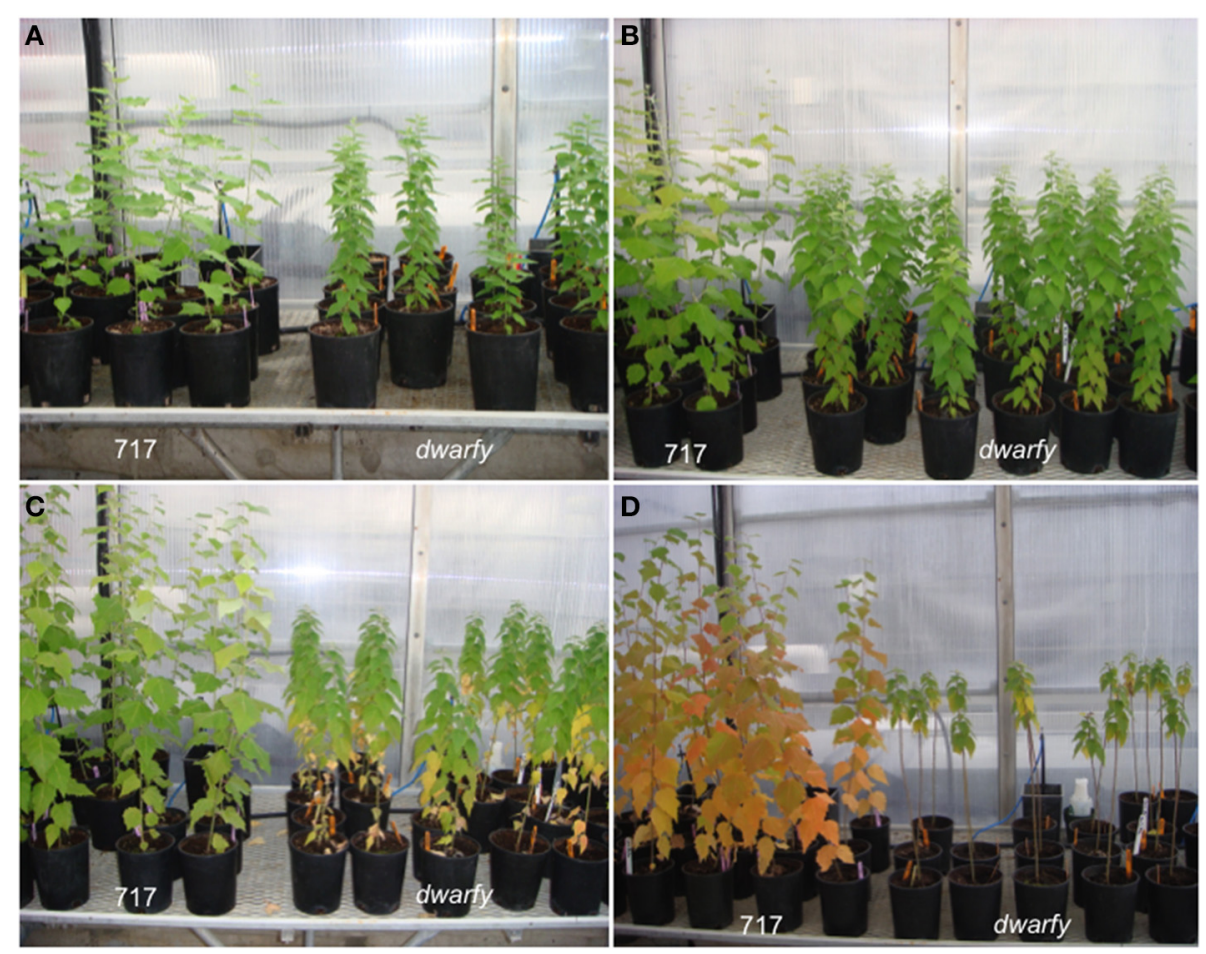

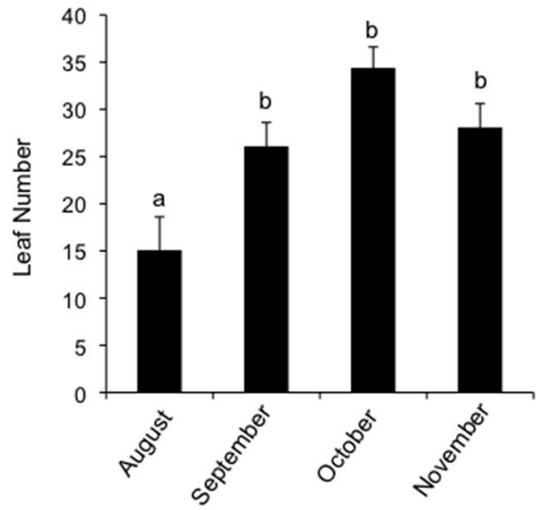

FIGURE 6 | dwarfy mutants exhibit pre-mature leaf senescence.

Comparison of leaf senescence rates in wildtype and dwarfy mutant clones within their first year of growth in August (A), September (B), October (C), and November (D). Leaf numbers on wild-type trees (E) and dwarfy trees (F)

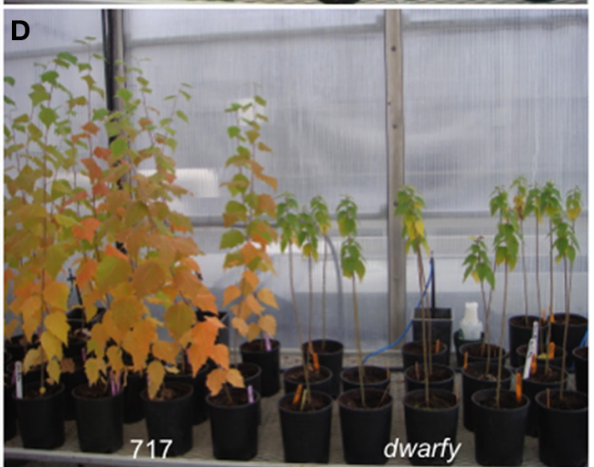

$\mathbf{F}$

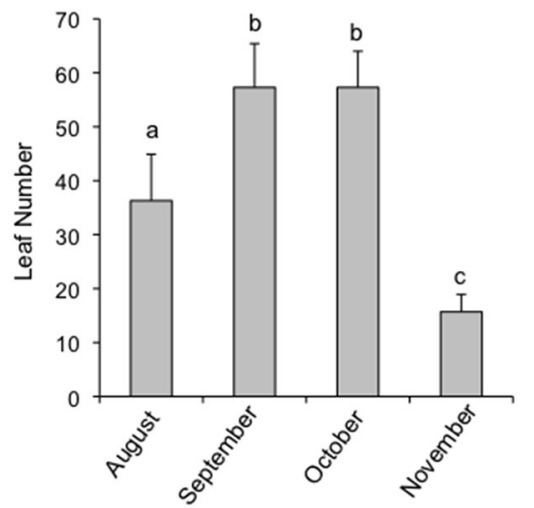

over the same time period are presented. \pm SE; superscript letters indicate significant differences between treatments as determined by One-Way analysis of variance (ANOVA) followed by a Tukey HSD (honestly significant difference) multiple comparison test $(p<0.05)$.
Ionization Detector GC-FID, Agilent 7890A) with an injector (splitless mode) temperature of $240^{\circ} \mathrm{C}$ and oven temperature at $60^{\circ} \mathrm{C}$ (isothermal) using helium as carrier gas $(3 \mathrm{~mL} / \mathrm{min})$. A30 $\mathrm{m} \times 0.53 \mathrm{~mm}$ ID (30 $\mu \mathrm{m}$ average thickness) Carboxen 1006 PLOT column (Supelco, Sigma Aldrich, Ontario Canada) was used to separate ethylene from the mixture. The FID (heated to $240^{\circ} \mathrm{C}$ ) hydrogen:air:makeup flows were $30: 400: 25(\mathrm{~mL} / \mathrm{min})$. Two measurements of $0.1 \mathrm{~mL}$ gas aliquot was taken from a headspace vial using a gas tight syringe (Hamilton 1700 series) and immediately injected for each sample. At these conditions, the observed retention time of ethylene was $3.14 \mathrm{~min}$. A calibration curve was generated to cover the range of $0.1-20 \mathrm{mg}$ ethylene. Ethylene standard gas mixture was made by drawing a volume of
99.5\% ethylene (Praxair) and injecting it in a previously vacuumpurged sealed headspace vial (volume determined by water capacity), then breaking the vacuum with a syringe needle and filling the vial with ambient air to atmospheric pressure. The diluted ethylene was allowed to stand for $1 \mathrm{~h}$ to reach dispersal equilibrium. Increasing volumes were injected to cover the desired ethylene range and each injection was repeated in triplicate.

\section{PHYSICAL CHARACTERISTICS ANALYSIS}

Cuttings of wildtype and dwarfy were established by cutting $4-5 \mathrm{~cm}$ shoot explants from stock plants. Cuttings were planted in Jiffy $42 \mathrm{~mm}$ peat plugs grown under greenhouse conditions June to August (natural lighting, watered twice daily) for 7 weeks. After 
7 weeks, cuttings were transferred to greenhouse pots $(15 \mathrm{~cm}$ diameter, $19 \mathrm{~cm}$ long). After a total of 9 weeks, 3 trees were randomly selected every month and height was measured and data analyzed using basic statistical tools in Excel (Microsoft Office). Leaf cell size, trichome density and cell density were performed as per Plett et al. (2010). Fiber and vessel isolation and measurements were performed as per Chaffey et al. (2002).

\section{GA AND ETHYLENE BIOSYNTHETIC INHIBITOR GROWTH EFFECT ANALYSIS}

Plants used for GA effect on growth were grown under normal greenhouse conditions as mentioned above. A triplicate (for GA) or a duplicate (for AVG) of wild-type and dwarfy plants of similar heights, grown for 5 weeks from cuttings, were used for each treatment for the experiment. Plant height was measured before the experiment and measured prior to each new application of GA or AVG. A total of 3 applications of $10 \mu \mathrm{l}$ of $3 \mathrm{mM} \mathrm{GA} /$ water or ETOH (for GA analysis) or of water or $100 \mu \mathrm{M}$ AVG were added every fourth day to the shoot apex of each plant and the total length of the experiment was 12 days. Data was analyzed using the height difference between the first measurement (before first application) and before 3rd application (3rd measurement), since 2 of the dwarfy/GA treated shoot apex samples had dried up and were dead before the final measurement.

\section{PERCENT CARBON, NITROGEN AND SULFUR ANALYSIS}

Dried leaf, stem, and roots samples from both wildtype and $d$ warfy were ground with a bead mill, and kept under vacuum in order to keep moisture out of the samples prior to carbon (C), nitrogen (N) and sulfur (S) analysis (CNS) by the CFS analytical laboratory according to the method of Kalra and Maynard (1991). A triplicate of each clone for each tissue types was measured and data was analyzed using basic statistical tools in Excel (Microsoft Office). Results presented are the measure of $\mathrm{C}, \mathrm{N}$ and $\mathrm{S}$ from healthy mature leaves and internode tissues harvested in June of the growing season.

\section{RESULTS \\ ENDOGENOUS OVER-EXPRESSION OF PtaACS8 INDUCES DWARFISM IN POPULUS}

From a large population of activation-tagged $P$. tremula $x P$. alba clone 717-1B4 (2000 independent transgenic lines; Harrison et al., 2007), we identified one line with a consistent reduction in growth rate over multiple growing seasons (Figures 1A,B). This mutant was named $d w a r f y$. Using Southern blotting only one T-DNA insert in dwarfy and located this insert on chromosome 2 using a modified TAIL PCR was identified. Within a window of $\pm 20 \mathrm{~Kb}$ around the T-DNA, 3 genes annotated in Phytozome (Figure 1C) were found as follows: a gene of unknown function (Potri.002G11400; +14.4 Kb up-stream), PtaACC SYNTHASE8 (PtaACS8; Potri.002G113900; $13.1 \mathrm{~Kb}$ down-stream) and PtaEARLY-RESPONSE TO DEHYDRATION 4 (ERD4; Potri.002G113800; $16.9 \mathrm{~Kb}$ down-stream). A quantification of the expression of these genes in the dwarfy mutant line relative to wild-type $P$. tremula $x P$. alba clone 717-1B4 demonstrated that only PtaACS 8 exhibited increased gene expression in all aerial tissues of the plant (Figure 1D). As ACC synthases are involved in the first step in the biosynthesis of the plant hormone ethylene, ethylene production was measured in the same three compartments as used for gene expression analysis in wildtype and mutant plants (i.e., young and mature leaves and stem tissues). Compared to wildtype, the mutant line produced $14 \times$ higher levels of ethylene in younger leaves and $6 \times$ higher levels of ethylene in mature leaves and the stems (Figure 1E).

To verify that increased transcript abundance of PtaACS 8 was indeed responsible for the dwarfism phenotype of the mutant, the Potri.002G113900 gene was cloned and expressed ectopically in the $P$. tremula $\times P$. alba clone 717-1B4 genetic background under the control of the $35 \mathrm{~S}$-cauliflower mosaic virus promoter. We were able to regenerate six independent transgenic lines from callus culture which, when grown alongside age-equivalent wildtype (i.e., propagated at the same time and treated in the same manner as the 35S::PtaACS8 lines), displayed a dwarf phenotype (Figure 2A). This reduction in growth and internode length was significant as compared to wildtype in all lines tested although the plants were consistently bigger than dwarfy (Figures 2A,B). The discrepancy in height difference is likely due to the fact that none of the 35S::PtaACS8 transgenic lines displayed the same level of PtaACS8 transcript accumulation as dwarfy (Figure 2C). As there was a significant correlation between the transcript abundance of PtaACS 8 and the dwarf phenotype in the transgenic lines (Figure 2D; $r=0.91 ; p<0.001$ ), we conclude that increased transcript abundance of PtaACS8 in the original $d w a r f y$ transgenic line is responsible for the reduction in plant stature.

\section{INCREASED TRANSCRIPT ABUNDANCE OF PtaACS8 LEADS TO ALTERED STEM CHARACTERISTICS}

The dwarfy mutant line exhibited alterations to the morphology of all aerial parts of the plant. While the internode length of the dwarfy line was significantly reduced (Figure 2B), there were also significant alterations to the microscopic anatomy of the stem (Figure 3). Due to the great difference in height of the two plants being compared, we used a plastochron index to identify and compare the same internode between the mutant line and wildtype. We used different microscopy techniques to observe different stem properties: brightfield to gain a general over-view of the stem architecture, UV excitation to differentiate chlorophyll autofluorescence (red signal) from secondary cell wall fluorescence (blue-green signal; Figures 3A,C). In young stems (internode between leaves 10 and 11), there was a reduction in the amount of secondary xylem formed in dwarfy as compared to wildtype (Figure $\mathbf{3 A}$ ) as well as a significant reduction in the length of xylem fibers and vessels (Figure 3B; $p<0.05$ ). In older stem tissues (internode between leaves 20 and 21), the reduction in secondary xylem formation (Figure 3C) and fiber/vessel lengths were still observed (Figure 3D). Detailed analysis of wood formation in these older tissues also revealed a difference in cell density: dwarfy had a higher density of xylem vessels per square millimeter with a significantly smaller outer diameter as compared to wild-type stems (Figures 3A,C,E-G) while there was no significant difference in the density of fibers. As alterations to the cell make-up of the stem and alteration in growth rate may influence nutrient deposition in the stem, we analyzed the percentage of carbon, nitrogen and sulfur in these mature internodes of both 
wildtype and $d$ warfy. No significant difference in percent accumulation of carbon and sulfur in the stems of $d$ warfy and wildtype were observed while the stems of the former accumulated a significantly higher concentration of nitrogen-containing compounds (Figures 3H-J; $p<0.05$ ).

\section{HORMONE- AND NUTRIENT-RELATED GENES DISPLAY ALTERED ABUNDANCE IN DWARFY STEMS}

In order to understand the transcriptomic profile of $d$ warfy stems, we performed a whole genome oligo-array transcriptomic analysis of whole stem tissues. We found 223 genes differentially expressed ( $\geq 2$-fold; $p<0.05$ ) as compared to wild-type P. tremula $\times$ P. alba clone 717-1B4 stems of the same age (Table S2). Within these genes we found that there were a number of ethylene and auxin related genes and genes coding for proteins involved in nutrient transport and biosynthesis (Table 1). Genes related to the ethylene pathway included PtaACS8 ( $>230$-fold increase) a number of ETHYLENE RESPONSE FACTOR (ERF) genes, two serine-threonine receptor kinases (PtaCTR3, PtaCTR4) and two ethylene receptor genes (PtaETR1, PtaETR5). The majority of genes associated with the auxin pathway, meanwhile, were repressed in the stems of dwarfy while a gene encoding an IAA-amido-synthetase glycosyl-hydrolase $(\mathrm{GH})$ family protein displayed increased abundance. Nutrient transport and synthesis was also affected with two sugar transporters and an amino acid transporter being repressed while the transcript accumulation of a glutamine synthase was increased (Table 1).

GA has been linked to enhanced growth phenotypes through the induction of auxin biosynthesis and polar transportation (Björklund et al., 2007). Therefore, as our transcriptional analysis of the dwarfy mutant indicated that auxin transport and signaling was affected, we tested whether GA application to the growing apex of $d$ warfy would be able to rescue the growth phenotype of the mutant. We found that the growth rate of $d$ warfy was significantly increased by treatment with GA (Figures 4A,B). Therefore GA is able to rescue the dwarfy phenotype. We also treated $d$ warfy with the ethylene biosynthetic inhibitor AVG. This treatment resulted in an increase in internode length (Figures 4C-E), demonstrating that blocking ethylene synthesis also rescues the dwarfy phenotype.

Table 1 | Genes found to have significantly different abundance in the stems of dwarfy as compared to wildtype.

\begin{tabular}{|c|c|c|c|c|}
\hline Probe & RefSeq protein ID & $E$-value & Fold change & Gene title \\
\hline \multicolumn{5}{|l|}{ ETHYLENE RELATED } \\
\hline PtpAffx.202003.1.S1_at & XP_002302380 & $0.00 \mathrm{E}+00$ & 233.9 & 1-Aminocyclopropane-1-carboxylate 8 (ACS8) \\
\hline Ptp.6619.1.S1_s_at & XP_002315490 & 8.00E-144 & 30.5 & AP2/ERF domain-containing transcription factor \\
\hline PtpAffx.75787.1.A1_s_at & XP_002297877 & $0.00 \mathrm{E}+00$ & 20.4 & AP2/ERF domain-containing transcription factor \\
\hline PtpAffx.75787.1.A1_at & XP_002304640 & $0.00 \mathrm{E}+00$ & 14.3 & AP2/ERF domain-containing transcription factor \\
\hline PtpAffx.129036.1.S1_at & XP_002316302 & $1.00 \mathrm{E}-16$ & 12.3 & Ethylene-responsive protein \\
\hline PtpAffx.219707.1.S1_at & XP_002326299 & $0.00 \mathrm{E}+00$ & 8.1 & AP2/ERF domain-containing transcription factor \\
\hline PtpAffx.4624.1.S1_at & XP_002328620 & $0.00 \mathrm{E}+00$ & 5.8 & AP2/ERF domain-containing transcription factor \\
\hline PtpAffx.572.3.S1_a_at & XP_002315958 & $0.00 \mathrm{E}+00$ & 5.3 & AP2/ERF domain-containing transcription factor \\
\hline Ptp.162.1.A1_at & XP_002302732 & $0.00 \mathrm{E}+00$ & 3.6 & Ethylene receptor 1 (PtETR1) \\
\hline PtpAffx.79014.1.S1_at & XP_002316514 & $0.00 \mathrm{E}+00$ & 3.5 & Serine/threonine protein kinase (PtCTR4) \\
\hline Ptp.866.1.S1_s_at & XP_002310408 & $4.00 \mathrm{E}-118$ & 3.3 & AP2/ERF domain-containing transcription factor \\
\hline PtpAffx.208193.1.S1_at & XP_002311669 & $0.00 \mathrm{E}+00$ & 2.8 & Ethylene receptor 5 (PtETR5) \\
\hline PtpAffx.122897.1.A1_at & XP_002308565 & $1.00 \mathrm{E}-96$ & 2.7 & REVERSION-TO-ETHYLENE SENSITIVITY1 (RTE1) \\
\hline PtpAffx.13062.4.S1_at & XP_002308982 & $0.00 \mathrm{E}+00$ & 2.5 & ein3-binding f-box protein 4 \\
\hline Ptp.2044.2.S1_a_at & XP_002311967 & $0.00 \mathrm{E}+00$ & 2.3 & Serine/threonine protein kinase (PtCTR3) \\
\hline \multicolumn{5}{|l|}{ AUXIN RELATED } \\
\hline PtpAffx.144034.1.S1_s_at & XP_002310372 & $1.00 \mathrm{E}-06$ & 3.2 & AUXIN-REGULATED GENE INVOLVED IN ORGAN SIZE (ARGOS) \\
\hline Ptp.6069.1.S1_at & XP_002320183 & $0.00 \mathrm{E}+00$ & 2.9 & GH3 family protein \\
\hline Ptp.8069.1.S1_at & XP_002306504 & $0.00 \mathrm{E}+00$ & -2.1 & NAKED PINS IN YUC MUTANTS 2 (NPY2) \\
\hline PtpAffx.155898.1.S1_at & XP_002320550 & $5.00 E-109$ & -2.3 & Dopamine beta-monooxygenase \\
\hline PtpAffx.97214.1.A1_at & XP_002302687 & $2.00 \mathrm{E}-132$ & -2.3 & Auxin-induced protein 5NG4 \\
\hline PtpAffx.117529.1.S1_at & XP_002323866 & $0.00 \mathrm{E}+00$ & -3.0 & MDR family $A B C$ transporter family \\
\hline PtpAffx.210100.1.S1_at & XP_002317029 & $2.00 \mathrm{E}-157$ & -3.1 & Auxin:hydrogen symporter \\
\hline PtpAffx.7696.4.S1_at & XP_002312567 & $1.00 \mathrm{E}-75$ & -4.3 & Auxin-responsive protein IAA4 \\
\hline \multicolumn{5}{|c|}{ NUTRIENT SYNTHESIS/TRANSPORT } \\
\hline PtpAffx.2311.1.S1_s_at & XP_002313246 & $0.00 \mathrm{E}+00$ & 6.2 & GLUTAMINE-DEPENDENT ASPARAGINE SYNTHASE 1 (ASN1) \\
\hline PtpAffx.217242.1.S1_at & XP_002331420 & $3.00 \mathrm{E}-169$ & -2.3 & Sugar transporter \\
\hline Ptp.5882.1.S1_at & XP_002301819 & $3.00 \mathrm{E}-43$ & -2.1 & RS21-C6 protein \\
\hline PtpAffx.111624.1.S1_at & XP_002302894 & $0.00 \mathrm{E}+00$ & -10.2 & Amino acid transporter \\
\hline
\end{tabular}

$(p<0.05 ;>2$-fold differential regulation). Note: In this table there are no column lines as there are in Table 2. 


\section{INCREASED TRANSCRIPT ABUNDANCE OF PtaACS8 LEADS TO ALTERED LEAF CHARACTERISTICS}

Mature leaves in the dwarfy mutant also showed altered size when compared to wild-type leaves (Figure 5A). The leaves of $d$ warfy were much smaller than those of wildtype (Figure 1A). Despite the alterations in leaf size, the epidermal cell size of $d$ warfy was not significantly altered compared to wildtype (Figure 5B). Trichome density was also not affected, but stomate density was significantly higher in the dwarfy mutant (Figures 5C,D). Only nitrogen content was significantly higher in dwarfy stems, as compared to wildtype (Figure 3I). Unlike stems, the total percentage of carbon in leaves was significantly reduced in dwarfy as compared to wildtype (Figure 5E), while nitrogen levels were not altered (Figure 5F). Sulfur levels in mutant leaves showed a tendency toward a lower accumulation compared to wildtype, but this difference was not found to be significant (Figure 5G; $p<0.05$ ). The date at which leaves became chlorotic and dropped off the stem in dwarfy as compared to wild-type plants was assessed as increased ethylene levels have been correlated to early leaf senescence (Breeze et al., 2011; Koyama et al., 2013). When grown under natural conditions, chlorosis of 1-year-old dwarfy leaves happens earlier as compared to wild-type plants (Figures 6A-D) and significant leaf drop occurred in dwarfy plants in the month of November while there was no significant leaf drop in the same period in wild-type trees (Figures 6E,F). It is interesting to speculate that the reduced $\mathrm{C}$ and $\mathrm{S}$ observed in the dwarfy leaves may be related to the shorter growing season for these leaves.

\section{SENESCENCE- AND CELL CYCLE-RELATED GENES EXHIBIT ALTERED ABUNDANCE IN DWARFY LEAVES}

We found that 183 genes were significantly regulated in fully expanded leaves of dwarfy as compared to wild-type P. tremula $x$ P. alba clone 717-1B4. A large number of hormone-related genes with altered transcription were observed in the stems of dwarfy, while only two of these genes (PtaACS 8 and a GH3 family protein) were significantly differentially regulated in mature dwarfy leaves ( $\geq 2$-fold; $p<0.05$; Table 2; Table S3). A number of nutrient transporters displayed altered transcript abundance, although they were different from those identified in dwarfy stems (Table 1). Three other classes of genes were differentially regulated in mature dwarfy leaves that were not observed in the stems: defense-, senescence- and cell cycle/expansion-related genes (Table 2). The majority of the defense-related genes were associated with pathogen attack, including a chitinase, a lipase and a glyoxal oxidase. As the leaf tissues were healthy at the time of harvest and displayed no infection structures, the activation of these genes is likely constitutive in the $d$ warfy background. Three genes associated with leaf senescence were also up-regulated. One group that only showed reduced levels of transcript abundance was that of genes associated with cell cycle and cellular growth (Table 2). Within this group of genes were a number of cyclins, calmodulin-like proteins and one expansin.

\section{DISCUSSION}

Due to ornamental value and to wide-ranging applications within agriculture, the genetic traits that control cell size and dwarfism in plants have been widely studied (Valdovinos et al., 1967;
Ephritikhine et al., 1999; Busov et al., 2003; Qi et al., 2011; Luo et al., 2013; Li et al., 2014; Yang et al., 2014; Zhang et al., 2014). Through breeding practices and applications of growth regulators, dozens of different dwarf plant varieties have been produced over the past few decades (Parker, 2012; Jiang et al., 2013; Wang et al., 2014a,b). Largely studied in annual models such as Arabidopsis, Zea and Oryza, control of plant stature has been linked most readily to plant hormones. For example, Arabidopsis mutants with increased ethylene production (e.g., eto; Woeste et al., 1999) exhibit thickening of the hypocotyl while increased ethylene signaling (e.g., $c t r 1-1$; $A C S 6^{D D D}$; Liu and Zhang, 2004) has been found to result in reduced stature and smaller leaf size. Due to advancements in insertional mutagenesis and other transgenic technologies, it is now becoming feasible to also screen perennial plants for the genes that control dwarfism (Busov et al., 2003; Harrison et al., 2007; Vahala et al., 2013). Here we characterize a mutant line of $P$. tremula $x P$. alba clone 717-1B4 that exhibits higher transcript accumulation of PtaACS 8 and that produces a significantly higher level of ethylene in all aerial tissues as compared to wild-type trees. Increased transcript abundance of PtaACS8 is correlated to reduced plant stature and smaller leaves while treatment of dwarfy shoots induces increases in internode length. The transcriptional cascade induced by altered levels of PtaACS8 are very different in stem and leaf tissue with a transcriptional reduction in genes associated with auxin transport and signaling evident in stems and repressed cell cycle genes in the leaves. These results place PtaACS8, and likely ethylene, as regulators that control two major morphological traits associated with dwarfism and reduced tissue size.

Using transformation technologies such as activation tagging is a very useful approach to identifying and characterizing the role of genes in a physiologically relevant manner. Rather than ectopic over-expression of a gene, the inserted enhancer used in activation tagging only enhances expression in its native expression pattern. This mutagenesis approach has been used in a number of model plant systems including Arabidopsis (Weigel et al., 2000), tomato (Mathews et al., 2003), rice (Jeong et al., 2006), and poplar (Harrison et al., 2007). Using this approach Busov et al. (2003) were able to identify a poplar GA2-OXIDASE that resulted in a plant with a very similar phenotype to that described here for $d w a r f y$; reduced stature and smaller leaves. Since their publication, dwarfism in a native dwarf plum tree cultivar has also been linked to a GA2-OXIDASE (El-Sharkawy et al., 2012) demonstrating that findings from activation tagging studies can be extended to natural plant populations.

As opposed to a strictly GA-dependent phenotype, our results support the hypothesis that growth retardation in dwarfy is driven largely by ethylene, the endpoint of the biochemical pathway in which PtaACS8 operates. This is based on the evidence that increased PtaACS 8 transcripts are correlated to significant increases in ethylene production in the stem (Figure 1E), whose signal is being relayed by the activation of several ERF genes (Table 1). Our results also demonstrate that blocking of ethylene biosynthesis resuces the dwarfy phenotype (Figures $4 \mathrm{C}-\mathbf{E}$ ). Further, the reduction in xylem fiber and vessel length described here-in has also previously been observed after ethylene treatment of poplar stems (Junghans et al., 2004; Love et al., 2009; 
Table 2 | Genes found to have significantly different abundance in the leaves of dwarfy as compared to wildtype.

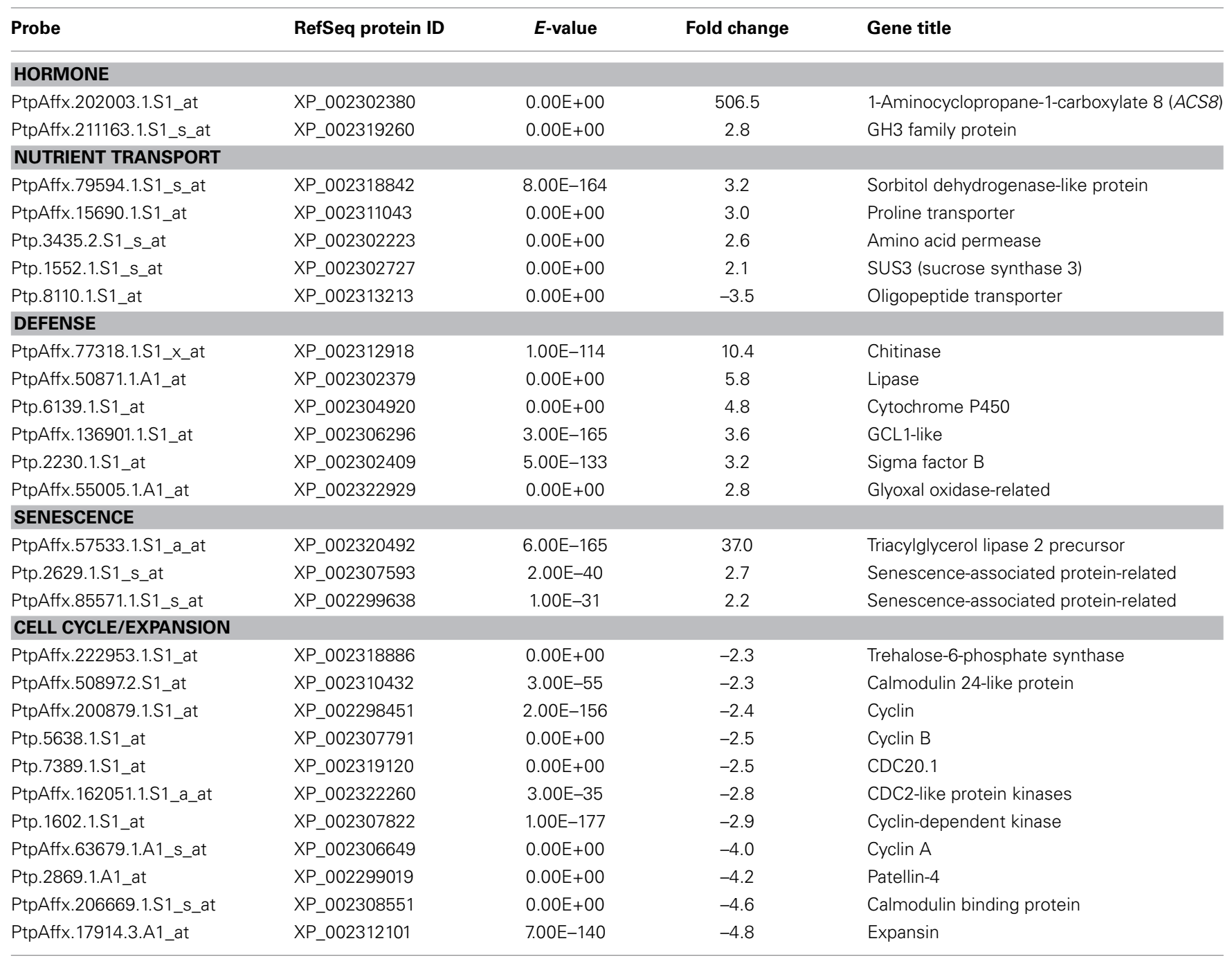

$p<0.05 ;>2$-fold differential regulation.

Vahala et al., 2013). While we cannot rule out the possibility that the reduced stature in dwarfy is a result of increased ACC accumulation, our results support the hypothesis that stunting of the dwarfy stem is controlled in an ethylene-dependent manner. Increased ethylene, however, is likely not the only causative factor in explaining the stature of dwarfy. Rather, ethylene appears to be influencing another pathway associated with plant stature: the auxin pathway. We found evidence for a repression of auxin-homeostasis and transport genes in the stem of $d$ warfy (Table 1). Ethylene has long been tied to a negative effect on auxin diffusion (von Guttenberg and Steinmetz, 1947; Morgan and Gausman, 1966; Valdovinos et al., 1967; Suttle, 1988; Andersson-Gunneras et al., 2003; Ruzicka et al., 2007; Stepanova et al., 2007; Swarup et al., 2007). As inhibition of auxin diffusion has been correlated to a reduction in stem cell elongation of poplar (Junghans et al., 2004), pea (Lantican and Muir, 1969), tomato (Higashide et al., 2014), tulip (Okubo and Uemoto, 1985), Arabidopsis (Franklin et al.,
2011; Chae et al., 2012), gourds (Wang et al., 2014a) amongst many other systems. Our results give a molecular framework by which ethylene affects $d$ warfy height where increased expression of PtaACS 8 results in greater production of ethylene which, upon perception in plant stem tissue, represses genes related to auxin diffusion and synthesis which would then curtail cell elongation in the stem. GA treatment of growth apexes can also rescue the dwarfy phenotype, although we cannot conclude from present data if GA generates this phenotype by acting downstream of the ethylene signal in the dwarfy mutant or in a separate pathway.

A different genetic pathway is likely responsible for the observed reduction in leaf size in dwarfy. While increases in PtaACS8 transcripts and ethylene evolution in the stem coincided with stunted fiber and vessel growth, no change in leaf epidermal cell size is observed despite higher levels of PtaACS 8 transcripts and higher ethylene evolution in the leaves. This would indicate that the leaf is smaller due to the absolute number of cells 
making up the tissue rather than the size of cell generated. It is interesting in the leaves of dwarfy that we see no evidence of compensation by leaf epidermal cells to maintain a larger leaf area. "Compensation" occurs when upstream inhibition of cell division initiates a secondary signaling pathway that increases cell size to maintain proper tissue growth (Hemerly et al., 1995; DeVeylder et al., 2001; Tsukaya, 2002; Horiguchi et al., 2006). Ethylene treatment has been associated with both stimulation of cell division (Love et al., 2009) and inhibition of cellular division (Edwards and Miller, 1972; Lee and LaRue, 1992; Heidstra et al., 1997; Dubois et al., 2013; Luo et al., 2013). In Arabidopsis, ethylene has been associated with reduced petal and leaf size in mutants with constitutive ethylene signaling (Kieber et al., 1993; Roman and Ecker, 1995; Luo et al., 2013) and under water limiting conditions due to the activity of ERF6 through its control of GA2-OXIDASE (Dubois et al., 2013). In the transcriptomic analysis of dwarfy leaves we do not see evidence of either $E R F$ or GA2-OXIDASE genes accumulating at altered abundances. Rather, within the group of genes regulated in dwarfy leaves, we observed the repression of a large class of cell cycle genes including CYCLIN-DEPENDENT KINASE1 (CDK1), CYCLIN A, and CYCLIN B1 (Table 2). In eukaryotic cells, CYCLIN A initiates the cellular transition from G2 to prophase after which CYCLIN B1 enters the nucleus and, together with CDK1, induces mitosis by phosphorylation and activation of enzymes regulating chromatin condensation, nuclear membrane breakdown and mitosis-specific microtubule and microfilament re-orientation (Nigg, 2001; Smits and Medema, 2001; Gavet and Pines, 2010; Suryadinata et al., 2010; Rattani et al., 2014). As this whole suite of proteins is necessary for cellular division, repression of their transcription in the leaves of $d w a r f y$, as compared to wild-type leaves, is likely the key pathway by which leaf size is being affected. These results are reminiscent of earlier observations that ethylene in Pisum sativum stopped cell division prior to entry into prophase (Apelbaum and Burg, 1972).

The leaf drop date of natural grown-year old dwarfy and wildtype plants was assessed as increased ethylene levels have been correlated to early leaf senescence (Breeze et al., 2011; Koyama et al., 2013). Leaf yellowing, considered to be the first visible senescent event (Quirino et al., 2000) was present in only the dwarfy basal leaves in October (Figure 6C), while in the wild-type, senescence related-color changes were prevalent in November basal leaves (Figure 6D) by which time dwarfy basal leaves had dehisced. Buchanan-Wollaston et al. (2003) noted that plants exposed to exogenous ethylene do exhibit premature senescence with the older leaves yellowing first; similar to the results here-in. November dwarfy and wild-type plants both had green apical leaves suggesting the onset of senescence and leaf dehiscence in all but the apical leaves in the dwarfy mutant were altered.

Our results support the hypothesis that there are two different developmental programs regulating tissue size in the dwarfy mutant. In the stem, we observe alterations to ethylene response factors and an inhibition of auxin homeostasis genes suggesting that ethylene inhibits stem elongation as previously observed in model organisms such as Arabidopsis (Guzman and Ecker, 1990), poplar (Junghans et al., 2004; Love et al., 2009; Vahala et al.,
2013), tobacco (Romano et al., 1993), and tomato (Huang and Lin, 2003) through its influence on the auxin pathway. In the leaves, we find that the leaves of dwarfy produce fewer cells and are, thereby, smaller. This phenotype is likely tied to the differential expression of the protein group responsible for the induction of mitosis. Altogether, our study of the dwarfy mutant poplar has given insight into the genetics underpinning ethylene-induced dwarfism.

\section{SUPPLEMENTARY MATERIAL}

The Supplementary Material for this article can be found online at: http://www.frontiersin.org/journal/10.3389/fpls.2014. 00514/abstract

\section{Table S1 | Primers used in this study.}

Table S2 | Genes differentially regulated in the stems of dwarfy as compared to wildtype stems.

Table S3 | Genes differentially regulated in the leaves of dwarfy as compared to wild-type leaves.

\section{REFERENCES}

Adkins, S., Foale, M., and Harries, H. (2010). "Growth and production of coconut. Soils, plant growth and crop production," in Encyclopedia of Life Support Systems (EOLSS), Developed Under the Auspices of UNESCO (Oxford, UK: Eolss Publishers). Available online at: http://greenplanet.eolss.net/EolssLogn/ mss/C10/E1-05A/E1-05A-40/E1-05A-40-TXT.aspx

Andersson-Gunneras, S., Hellgren, J. M., Bjorklund, S., Regan, S., Moritz, T., and Sundberg, B. (2003). Asymmetric expression of a poplar ACC oxidase controls ethylene production during gravitational induction of tension wood. Plant J. 34, 339-349. doi: 10.1046/j.1365-313X.2003.01727.x

Apelbaum, A., and Burg, S. P. (1972). Effect of ethylene on cell division and deoxyribonucleic acid synthesis in Pisum sativum. Plant Physiol. 50, 117-124. doi: 10.1104/pp.50.1.117

Beemster, G. T., de Veylder, L., Vercruysse, S., West, G., Rombault, D., van Hummelen, P., et al. (2005). Genome-wide analysis of gene expression profiles associated with cell cycle transitions in growing organs of Arabidopsis. Plant Physiol. 138, 734-743. doi: 10.1104/pp.104.053884

Beemster, G. T., Fiorani, F., and Inze, D. (2003). Cell cycle: the key to plant growth control? Trends Plant Sci. 8, 154-158. doi: 10.1016/S1360-1385(03)00046-3

Björklund, S., Antti, H., Uddestrand, I., Moritz, T., and Sundberg, B. (2007). Crosstalk between gibberellin and auxin in development of Populus wood: gibberellin stimulates polar auxin transport and has a common transcriptome with auxin. Plant J. 52, 499-511. doi: 10.1111/j.1365-313X.2007.03250.x

Breeze, E., Harrison, E., McHattie, S., Hughes, L., Hickman, R., Hill, C., et al. (2011). High-resolution temporal profiling of transcripts during Arabidopsis leaf senescence reveals a distinct chronology of processes and regulation. Plant Cell 23, 873-894. doi: 10.1105/tpc.111.083345

Buchanan-Wollaston, V., Earl, S., Harrison, E., Mathas, E., Navabpour, S., Page, T., et al. (2003). The molecular analysis of leaf senescence - a genomic approach. Plant Biotech. J. 1, 3-22. doi: 10.1046/j.1467-7652.2003.00004.x

Busov, V. B., Meilan, R., Pearce, D. W., Ma, C., Rood, S. B., and Strauss, S. H. (2003). Activation tagging of a dominant gibberellin catabolism gene (GA 2-OXIDASE) from poplar that regulates tree stature. Plant Physiol. 132, 1283-1291. doi: 10.1104/pp.103.020354

Chae, K., Isaacs, C. G., Reeves, P. H., Maloney, G. S., Muday, G. K., Nagpal, P., et al. (2012). Arabidopsis SMALL AUXIN UP RNA63 promotes hypocotyl and stamen filament elongation. Plant J. 71, 684-697. doi: 10.1111/j.1365313X.2012.05024.x

Chaffey, N., Cholewa, E., Regan, S., and Sundberg, B. (2002). Secondary xylem development in Arabidopsis: a model for wood formation. Physiol. Plant. 114, 594-600. doi: 10.1034/j.1399-3054.2002.1140413.x

Chang, S., Puryear, J., and Carney, J. (1993). A simple and efficient method for isolating RNA from pine trees. Plant Mol. Biol. Rep. 11, 113-116. doi: 10.1007/BF02670468 
Cho, H. T., and Cosgrove, D. J. (2000). Altered expression of EXPANSIN modulates leaf growth and pedicel abscission in Arabidopsis thaliana. Proc. Natl. Acad. Sci. U.S.A. 97, 9783-9788. doi: 10.1073/pnas.160276997

Curtis, I. S., Hanada, A., Yamaguchi, S., and Kamiya, Y. (2005). Modification of plant architecture through the expression of GA 2-OXIDASE under the control of an estrogen inducible promoter in Arabidopsis thaliana. Planta 222, 957-967. doi: 10.1007/s00425-005-0037-7

DeVeylder, L., Beeckman, T., Beemster, G. T. S., Krols, L., Terras, F., Landrieu, I., et al. (2001). Functional analysis of cyclin-dependent kinase inhibitors of Arabidopsis. Plant Cell 13, 1653-1667. doi: 10.1105/tpc.13.7.1653

Dijkstra, C., Adams, E., Bhattacharya, A., Page, A. F., Anthony, P., Kourmpetli, S., et al. (2008). Over-expression of a GIBBERELLIN 2-OXIDASE gene from Phaseolus coccineus L. enhances gibberellin inactivation and induces dwarfism in Solanum species. Plant Cell Rep. 27, 463-470. doi: 10.1007/s00299-0070471-z

Domingo, C., Andres, F., Tharreau, D., Iglesias, D. J., and Talon, M. (2009). Constitutive expression of OsGH3.1 reduces auxin content and enhances defense response and resistance to a fungal pathogen in rice. Mol. Plant Microbe Interact. 22, 201-210. doi: 10.1094/MPMI-22-2-0201

Donnelly, P. M., Bonetta, D., Tsukaya, H., Dengler, R. E., and Dengler, N. G. (1999) Cell cycling and cell enlargement in developing leaves of Arabidopsis. Dev. Biol. 215, 407-419. doi: 10.1006/dbio.1999.9443

Dubois, M., Skirycz, A., Claeys, H., Maleux, K., Dhondt, S., De Bodt, S., et al. (2013). ETHYLENE RESPONSE FACTOR6 acts as a central regulator of leaf growth under water-limiting conditions in Arabidopsis. Plant Physiol. 162, 1319-1332. doi: 10.1104/pp.113.216341

Edwards, M. E., and Miller, J. H. (1972). Growth regulation by ethylene in fern gametophytes. II. Inhibition of cell division. Am. J. Bot. 59, 450-457.

El-Sharkawy, I., El-Kayal, W., Prasath, D., Fernandex, H., Bouzayen, M., Svircev, A. M., et al. (2012). Identification and genetic characterization of GIBBERELLIN 2-OXIDASE gene that controls tree stature and reproductive growth in plum. J. Exp. Bot. 63, 1225-1239. doi: 10.1093/jxb/ err345

Ephritikhine, G., Gellner, M., Vannini, C., Lapous, D., and Barbier-Brygoo, H. (1999). The sax1 dwarf mutant of Arabidopsis thaliana shows altered sensitivity of growth responses to abscisic acid, auxin, gibberellins and ethylene and is partially rescued by exogenous brassinosteroid. Plant J. 18, 303-314. doi: 10.1046/j.1365-313X.1999.00454.x

Faeth, S. H. (1991). Effect of oak leaf size on abundance, dispersion and survival of the leafminer Cameraria sp. (Lepidoptera: Gracillariidae). Environ. Ent. 20, 196-204.

Franklin, K.A., Lee, S.H., Patel, D., Kumar, S.V., Spartz, A.K., Gu, C., et al. (2011). Phytochrome-interacting factor 4 (PIF4) regulates auxin biosynthesis at high temperature. Proc. Nat. Acad. Sci. U.S.A. 108, 20231-20235. doi: 10.1073/pnas. 1110682108

Gavet, O., and Pines, J. (2010). Progressive activation of CyclinB1-Cdk1 co-ordinates entry to mitosis. Develop. Cell 18, 533-543. doi: 10.1016/ j.devcel.2010.02.013

Geisler, M., Blakeslee, J. J., Bouchard, R., Lee, O. R., Vincenzetti, V., Brandyopadhyay, A., et al. (2005). Cellular efflux of auxin mediated by the Arabidopsis MDR/PGP transporter AtPGP1. Plant J. 44, 179-194. doi: 10.1111/j.1365-313X.2005.02519.x

Geisler, M., Kolukisaoglu, H. U., Bouchard, R., Billion, K., Berger, J., Saal, B., et al. (2003). TWISTED DWARF1, a unique plasma membrane-anchored immunophilin-like protein, interacts with Arabidopsis multidrug resistancelike transporters AtPGP1 and AtPGP19. Mol. Biol. Cell 14, 4238-4249. doi: 10.1091/mbc.E02-10-0698

Geisler, M., and Murphy, A. S. (2006). The ABC of auxin transport: the role of p-glycoproteins in plant development. FEBS Lett. 580, 1094-1102. doi: 10.1016/j.febslet.2005.11.054

Gleave, A. P. (1992). A versatile binary vector system with a T-DNA organizational structure conducive to efficient integreation of cloned DNA into the plant genome. Plant Mol. Biol. 20, 1203-1207.

Guo, M., Rupe, M. A., Dieter, J. A., Zou, J., Spielbauer, D., Duncan, K. E., et al. (2010). CELL NUMBER REGULATOR 2 affects plant and organ size in maize: implications for crop yield enhancement and heterosis. Plant Cell 22, 1057-1073. doi: 10.1105/tpc. 109.073676

Guzman, P., and Ecker, J. R. (1990). Exploiting the triple response of Arabidopsis to identify ethylene-related mutants. Plant Cell 2, 513-523.
Harrison, E. J., Bush, M., Plett, J. M., McPhee, D. P., Vitez, R., O’Malley, B., et al. (2007). Diverse developmental mutants revealed in an activation-tagged population of poplar. Can. J. Bot. 85, 1071-1081. doi: 10.1139/ B07-063

Heidstra, R., Yang, W. C., Yalcin, Y., Peck, S., Emons, A. M., van Kammen, A., et al. (1997). Ethylene provides positional information on cortical cell division but not involved in Nod factor-induced root hair tip growth in Rhizobium-legume interaction. Development 124, 1781-1787.

Hemerly, A., de Almeida Engler, J., Bergounioux, C., Van Montagu, M., Engler, G., Inze, D., et al. (1995). Dominant negative mutants of the CDC2 kinase uncouple cell division from iterative plant development. EMBO J. 14, 3925-3936.

Higashide, T., Narukawa, M., Shimada, Y., and Soeno, K. (2014). Suppression of elongation and growth of tomato seedlings by auxin biosynthesis inhibitors and modeling of the growth and environmental response. Sci. Rep. 4:4556. doi: 10.1038/srep04556

Horiguchi, G., Ferjani, A., Fujikura, U., and Tsukaya, H. (2006). Coordination of cell proliferation and cell expansion in the control of leaf size in Arabidopsis thaliana. J. Plant Res. 119, 37-42. doi: 10.1007/s10265-005-0232-4

Hu, Y., Poh, H. M., and Chua, N. H. (2006). The Arabidopsis ARGOS-LIKE gene regulates cell expansion during organ growth. Plant J. 47, 1-9. doi: 10.1111/j.1365-313X.2006.02750.x

Huang, J.-Y., and Lin, C.-H. (2003). Cold water treatment promotes ethylene production and dwarfing in tomato seedlings. Plant Physiol. Biochem. 41, 283-288. doi: 10.1016/S0981-9428(03)00020-2

Huang, N., Courtois, B., Khush, G. S., Lin, H., Wang, G., Wu, P., et al. (1996). Association of quantitative trait loci for plant height with major dwarfing genes in rice. Heredity 77, 130-137. doi: 10.1038/hdy.1996.117

Jeong, D.-H., An, S., Park, S., Kang, H.-G., Park, B.-B., Kim, S.-R., et al. (2006). Generation of a flanking sequence-tag database for activation-tagging lines in japonica rice. Plant J. 45, 123-132. doi: 10.1111/j.1365-313X.2005. 02610.x

Jiang, L., Liu, X., Xiong, G., Liu, H., Chen, F., Wang, L., et al. (2013). DWARF 53 act as a repressor of strigolactone signaling in rice. Nature 504, 401-405. doi: 10.1038 /nature 12870

Junghans, U., Langenfeld-Heyser, R., Polle, A., and Teichmann, T. (2004). Effects of auxin transport inhibitors and ethylene on the wood anatomy of poplar. Plant Biol. 6, 22-29. doi: 10.1055/s-2003-44712

Kalra, Y. P., and Maynard, D. G. (1991). Methods Manual for Forest Soil and Plant Analysis. Information Report NOR-X-319E. Edmonton, AB: Canadian Forest Service Publications.

Kieber, J. J., Rothenberg, M., Roman, G., Feldmann, K. A., and Ecker, J. R. (1993). CTR1, a negative regulator of the ethylene response pathway in Arabidopsis, encodes a member of the Raf family of protein kinases. Cell 72, 427-441.

Koyama, T., Nil, H., Mitsuda, N., Ohta, M., Kitajima, S., Ohme-Takagi, M., et al. (2013). A regulatory cascade involving class II ETHYLENE RESPONSE FACTOR transcriptional repressors operates in the progression of leaf senescence. Plant Physiol. 162, 991-1005. doi: 10.1104/pp.113.218115

Lantican, B. P., and Muir, R. M. (1969). Auxin physiology of dwarfism in Pisum sativum. Physiol. Plant. 22, 412-423. doi: 10.1111/j.1399-3054.1969.tb07393.x

Lee, D. J., and Zeevaart, J. A. (2005). Molecular cloning of GA 2-OXIDASE3 from spinach and its ectopic expression in Nicotiana sylvestris. Plant Physiol. 138, 243-254. doi: 10.1104/pp.104.056499

Lee, K. H., and LaRue, T. A. (1992). Exogenous ethylene inhibits nodulation of Pisum sativum L. cv Sparkle. Plant Physiol. 100, 1759-1763.

Li, J., Jiang, J., Qian, Q., Xu, Y., Zhang, C., Xiao, J., et al. (2011). Mutation of rice $B C 12 / G D D 1$, which encodes a kinesin-like protein that binds to a GA biosynthesis gene promoter, leads to dwarfism with impaired cell elongation. Plant Cell 23, 628-640. doi: 10.1105/tpc.110.081901

Li, J., Yang, H., Peer, W. A., Richter, G., Blakeslee, J., Bandyopadhyay, A., et al. (2005). Arabidopsis H+-PPase AVP1 regulates auxin-mediated organ development. Science 310, 121-125. doi: 10.1126/science.1115711

Li, R., Zia, J., Xu, Y., Zhao, X., Liu, Y.-G., and Chen, Y. (2014). Characterization and genetic mapping of a Photoperiod-sensitive dwarf 1 locus in rice (Oryze sativa L.). Theor. Appl. Genet. 127, 241-250. doi: 10.1007/s00122-013-2213-7

Liu, Y., and Zhang, S. (2004). Phosphorylation of 1-aminocyclopropane-1carboxylic acid synthase by MPK6, a stress-responsive mitogen-activated protein kinase, induces ethylene biosynthesis in Arabidopsis. Plant Cell 16, 3386-3399. doi: 10.1105/tpc.104.026609 
Love, J., Björklund, S., Vahala, J., Hertzberg, M., Kangasjärvi, J., and Sundberg, B. (2009). Ethylene is an endogenous stimulator of cell division in the cambial meristem of Populus. Proc. Natl. Acad. Sci. U.S.A. 106, 5984-5989. doi: 10.1073/pnas.0811660106

Luo, J., Ma, N., Pei, H., Chen, J., Li, J., and Gao, J. (2013). A DELLA gene, RhGAI1, is a direct target of EIN3 and mediates ethylene-regulated rose petal expansion via repressing the expression of RhCesA2. J. Exp. Bot. 64, 5075-5084. doi: $10.1093 /$ jxb/ert296

Mathews, H., Clendennen, S. K., Caldwell, C. G., Liu, X. L., Connors, K., Matheis, N., et al. (2003). Activation tagging in tomato identifies a transcriptional regulator of anthocyanin biosynthesis, modification and transport. Plant Cell 15, 1689-1703. doi: 10.1105/tpc.012963

Morgan, P. W., and Gausman, H. W. (1966). Effects of ethylene on auxin transport. Plant Physiol. 41, 45-52.

Multani, D. S., Briggs, S. P., Chamberlin, M. A., Blakeslee, J. J., Murphy, A. S., and Johal, G. S. (2003). Loss of an MDR transporter in compact stalks of maize br2 and sorgum dw3 mutants. Science 302, 81-84. doi: 10.1126/science.1086072

Nakaya, M., Tsukaya, H., Murakami, N., and Kato, M. (2002). Brassinosteroids control the proliferation in leaf cells of Arabidopsis thaliana. Plant Cell Physiol. 43, 239-244. doi: 10.1093/pcp/pcf024

Nigg, E. A. (2001). Mitotic kinases as regulators of cell division and its checkpoints. Nat. Rev. Mol. Cell Biol. 2, 21-32. doi: 10.1038/35048096

Nobusawa, T., Okushima, Y., Nagata, N., Kojima, M., Sakakibara, H., and Umeda, M. (2013). Synthesis of very-long-chain fatty acids in the epidermis controls plant organ growth by restricting cell proliferation. PLoS Biol. 11:e1001531. doi: 10.1371/journal.pbio.1001531

Okubo, H., and Uemoto, S. (1985). Changes in endogenous gibberellin and auxin activities during first internode elongation in tulip flower stalk. Plant Cell Physiol. 26, 709-719.

Parker, T. (2012). An apple a day!: new dwarf apple 'leprechaun' in the perfect planting partner for 'pinkbelle.' Medicus 52, 65. Available online at: http://search. informit.com.au/documentSummary; $\mathrm{dn}=214566906243183$;res=IELHEA

Plett, J. M., Wilkins, O., Campbell, M., Ralph, S., and Regan, S. (2010). Endogenous over-expression of Populus MYB186 increases trichome density, improves insect pest resistance, and impacts plant growth. Plant J. 64, 419-432. doi: 10.1111/j.1365-313X.2010.04343.X

Qi, W., Sun, F., Wang, Q., Chen, M., Huang, Y., Feng, Y.-Q., et al. (2011). Rice ethylene-response AP2/ERF factor OsEATB restricts internode elongation by down-regulating a gibberellin biosynthetic gene. Plant Physiol. 157, 216-228. doi: 10.1104/pp.111.179945

Quirino, B. F., Noh, Y. S., Himelblau, E., and Amasino, R. M. (2000). Molecular aspects of leaf senescence. Trends Plant Sci. 5, 278-282. doi: 10.1016/S13601385(00)01655-1

Rattani, A., Vinod, P. K., Godwin, J., Tachibana-Konwalski, K., Wolna, M., Malumbres, M., et al. (2014). Dependency of the spindle assembly checkpoint on Cdk1 renders the anaphase transition irreversible. Curr. Biol. 24, 630-637. doi: 10.1016/j.cub.2014.01.033

Roman, G., and Ecker, J. R. (1995). Genetic analysis of a seedling stress response to ethylene in Arabidopsis. Philos. Trans. R. Soc. Lond. B Biol. Sci. 350, 75-81.

Romano, C. P., Cooper, M. L., and Klee, H. J. (1993). Uncoupling auxin and ethylene effects in transgenic tobacco and Arabidopsis plants. Plant Cell 5, 181-189.

Ruzicka, K., Ljung, K., Vanneste, S., Podhorska, R., Beechman, T., Frimi, J., et al. (2007). Ethylene regulates root growth through effects on auxin biosynthesis and transport-dependent auxin distribution. Plant Cell 19, 2197-2212. doi: 10.1105/tpc.107.052126

Sabelli, P. A., Liu, Y., Dante, R. A., Lizarraga, L. E., Nguyen, H. N., Brown, S. W., et al. (2013). Control of cell proliferation, endoreduplicaiton, cell size, and cell death by the retinoblastoma-related pathway in maize endosperm. Proc. Natl. Acad. Sci. U.S.A. 110, E1827-E1836. doi: 10.1073/pnas.1304 903110

Schomburg, F. M., Bizzell, C. M., Lee, D. J., Zeevaart, J. A., and Amasino, R. M. (2003). Overexpression of a novel class of gibberellin 2-oxidases decreases gibberellin levels and creates dwarf plants. Plant Cell 15, 151-163. doi: 10.1105/tpc.005975

Scoffoni, C., Rawis, M., McKown, A., Cochard, H., and Sack, L. (2011). Decline of leaf hydraulic conductance with dehydration: relationship of leaf size and venation architecture. Plant Physiol. 156, 832-843. doi: 10.1104/pp.111. 173856
Sieling, K., and Kage, H. (2008). The potential of semi-dwarf oilseed rape genotypes to reduce the risk of $\mathrm{N}$ leaching. J. Agric. Sci. 146, 77-84. doi: $10.1017 /$ S0021859607007472

Skirycz, A., de Bodt, S., Obata, T., de Clercq, I., Claeys, H., de Rycke, R., et al. (2010). Developmental stage specificity and the role of mitochondrial metabolism in the response of Arabidopsis leaves to prolonged mild osmotic stress. Plant Physiol. 152, 226-244. doi: 10.1104/pp.109.148965

Smits, V. A., and Medema, R. H. (2001). Checking out the G2/M transition. Biochim. Biophys. Acta 1519, 1-12. doi: 10.1016/S0167-4781(01)00204-4

Stepanova, A. N., Hoyt, J. M., Hamilton, A. A., and Alonso, J. M. (2005). A link between ethylene and auxin uncovered by the characterization of two rootspecific ethylene-insensitive mutants in Arabidopsis. Plant Cell 17, 2230-2242. doi: 10.1105/tpc.105.033365

Stepanova, A. N., Yun, J., Likhacheva, A. V., and Alonso, J. M. (2007). Multilevel interactions between ethylene and auxin in Arabidopsis roots. Plant Cell 19, 2169-2185. doi: 10.1105/tpc.107.052068

Suryadinata, R., Sadowski, M., and Sarcevic, B. (2010). Control of cell cycle progression by phosphorylation of cyclin-dependent kinase (CDK) substrates. Biosci. Rep. 30:bsr0300243. doi: 10.1042/BSR20090171

Suttle, J. C. (1988). Effect of ethylene treatment on polar IAA transport, net IAA uptake and specific binding of N-1-naphthylphthalamic acid in tissues and microsomes isolated from etiolated pea epicotyls. Plant Physiol. 88, 795-799.

Swarup, R., Perry, P., Hagenbeek, D., van der Straeten, D., Beemster, G. T. S., Sandberg, G., et al. (2007). Ethylene upregulates auxin biosynthesis in Arabidopsis seedlings to enhance inhibition of root cell elongation. Plant Cell 19, 2186-2196. doi: 10.1105/tpc.107.052100

Tong, J.-P., Liu, X.-J., Zhang, S.-Y., Li, S.-Q., Peng, X.-J., Yang, J., et al. (2007). Identification, genetic characterization, GA response and molecular mapping of Sdt97: a dominant mutant gene conferring semi-dwarfism in rice (Oryza sativa L.). Genet. Res. 89, 221-230. doi: 10.1017/S00166723070 09020

Tsukaya, H. (2002). Interpretation of mutants in leaf morphology: genetic evidence for a compensatory system in leaf morphogenesis that provides a new link between cell and organismal theories. Int. Rev. Cytol. 217, 1-39. doi: 10.1016/S0074-7696(02)17011-2

Tuskan, G. A., DiFazio, S., Jansson, S., Bohlmann, J., Grigoriev, I., Hellsten, U., et al. (2006). The genome of black cottonwood, Populus trichocarpa (Torr.and Gray). Science 15, 1596-1604. doi: 10.1126/science.1128691

Vahala, J., Felten, J., Love, J., Gorzsás, A., Gerber, L., Lamminmäki, A., et al. (2013). A genome-wide screen for ethylene-induced Ethylene Response Factors (ERFs) in hybrid aspen stem identifies ERF genes that modify stem growth and wood properties. New Phytol. 200, 511-522. doi: 10.1111/nph. 12386

Valdovinos, J. G., Ernest, L. C., and Henry, E. W. (1967). Effect of ethylene and gibberellic acid on auxin synthesis in plant tissues. Plant Physiol. 42, 1803-1806.

von Guttenberg, H., and Steinmetz, E. (1947). The effects of ethylene on growth hormone and growth. Pharmazie 2, 17-21.

Wang, R., Huang, H., Lin, Y., Chen, Q., Liang, Z., and Wu, T. (2014a). Genetic and gene expression analysis of $d m 1$, a dwarf mutant from Cucurbita maxima Duch. ex Lam, based on the AFLP method. Can. J. Plant Sci. 94, 293-302. doi: 10.4141/cjps2013-016

Wang, W., Li, G., Zhao, J., Chu, H., Lin, W., Zhang, D., et al. (2014b). DWARF TILLER1, a WUSCHEL-related homeobox transcription factor, is required for tiller growth in rice. PLoS Genet. 10:e1004154. doi: 10.1371/journal.pgen.1004154

Weigel, D., Ahn, J. H., Blazquez, M. A., Borevitz, J. O., Christensen, S. K., Fankhause, C., et al. (2000). Activation tagging in Arabidopsis. Plant Physiol. 122, 1003-1014. doi: 10.1104/pp.122.4.1003

Woeste, K. E., Ye, C., and Kieber, J. J. (1999). Two Arabidopsis mutants that overproduce ethylene are affected in the posttranscriptional regulation of 1 aminocyclopropane-1-carboxylic acid synthase. Plant Physiol. 119, 521-530. doi: 10.1104/pp.119.2.521

Yamamoto, Y., Kamiya, N., Morinaka, Y., Matsuoka, M., and Sazuka, T. (2007). Auxin biosynthesis by the YUCCA genes in rice. Plant Physiol. 143, 1362-1371. doi: 10.1104/pp.106.091561

Yang, X. C., and Hwa, C. M. (2008). Genetic modification of plant architecture and variety improvement in rice. Heredity 101, 296-404. doi: 10.1038/hdy. 2008.90 
Yang, Y., Massa, G. D., and Mitchell, C. A. (2014). Temperature DIP at the beginning of the photoperiod reduces plant height but not seed yield of maize grown in controlled environments. Ind. Crops Prod. 53, 120-127. doi: 10.1016/j.indcrop.2013.12.002

Zawaski, C., Kadmiel, M., Pickens, J., Ma, C., Strauss, S., and Busov, V. (2011). Repression of gibberellin biosynthesis or signaling produces striking alterations in poplar growth, morphology and flowering. Planta 234, 1285-1298. doi: 10.1007/s00425-011-1485-x

Zhang, J., Liu, X., Li, S., Cheng, Z., and Li, C. (2014). The rice semi-dwarf mutant sd37, caused by a mutation in CYP96B4, plays an important role in the fine-tuning of plant growth. PLoS ONE 9:e88068. doi: 10.1371/journal.pone. 0088068

Conflict of Interest Statement: The authors declare that the research was conducted in the absence of any commercial or financial relationships that could be construed as a potential conflict of interest.
Received: 30 July 2014; accepted: 13 September 2014; published online: 04 November 2014.

Citation: Plett JM, Williams M, LeClair G, Regan S and Beardmore T (2014) Heterologous over-expression of ACC SYNTHASE8 (ACS8) in Populus tremula $x$ P. alba clone 717-1B4 results in elevated levels of ethylene and induces stem dwarfism and reduced leaf size through separate genetic pathways. Front. Plant Sci. 5:514. doi: 10.3389/fpls.2014.00514

This article was submitted to Plant Physiology, a section of the journal Frontiers in Plant Science.

Copyright (C) 2014 Plett, Williams, LeClair, Regan and Beardmore. This is an open-access article distributed under the terms of the Creative Commons Attribution License (CC BY). The use, distribution or reproduction in other forums is permitted, provided the original author(s) or licensor are credited and that the original publication in this journal is cited, in accordance with accepted academic practice. No use, distribution or reproduction is permitted which does not comply with these terms. 


\title{
The roles of ethylene and transcription factors in the regulation of onset of leaf senescence
}

\author{
Tomotsugu Koyama* \\ Bioorganic Research Institute - Suntory Foundation for Life Sciences, Osaka, Japan
}

\section{Edited by:}

Domenico De Martinis, Italian National Agency for New

Technologies, Energy and Sustainable

Economic Development, Italy

\section{Reviewed by:}

Victoria Fernandez, Technical University of Madrid, Spain Veronica Graciela Maurino, Heinrich-Heine-University Duesseldorf, Germany

\section{*Correspondence:}

Tomotsugu Koyama, Bioorganic Research Institute - Suntory Foundation for Life Sciences, Wakayamadai 1-1-1, Shimamoto, Osaka 618-8503, Japan

e-mail:koyama@sunbor.or.jp
Leaf senescence is the last stage of leaf development and is accompanied by cell death. In contrast to senescence in individual organisms that leads to death, leaf senescence is associated with dynamic processes that include the translocation of nutrients from old leaves to newly developing or storage tissues within the same plant. The onset of leaf senescence is largely regulated by age and internal and external stimuli, which include the plant hormone ethylene. Earlier studies have documented the important role of ethylene in the regulation of leaf senescence. The production of ethylene coincides with the onset of leaf senescence, whereas the application of ethylene to plants induces precocious leaf senescence. Recently, many studies have described the components of ethylene signaling and biosynthetic pathways that are involved in modulating the onset of leaf senescence. Particularly, transcription factors (TFs) integrate ethylene signals with those from environmental and developmental factors to accelerate or delay leaf senescence. This review aims to discuss the regulatory cascade involving ethylene and TFs in the regulation of onset of leaf senescence.

Keywords: AP2/ERF, ethylene, leaf development, leaf senescence, NAC, transcription factor, TCP, WRKY

\section{INTRODUCTION}

Leaf senescence occurs alongside color changes in leaves and is an easily visible phenomenon in the life cycle of a plant. Leaf senescence involves degradation of chlorophylls, carbohydrates, lipids, proteins, and nucleic acids and contributes to the mobilization of such nutrients from old leaves to growing or storage tissues. The importance of the efficient regulation of leaf senescence was reported by a study on the domestication of cultivated wheat. Loci tightly linked to the enrichment of several important nutrients in cereal grains encode transcription factors (TFs) that regulate the onset of leaf senescence in ancestral wheat plants (Uauy et al., 2006; Waters et al., 2009). The onset of leaf senescence is largely affected by the age of the plant, but is also influenced by changes in environmental conditions. Ethylene and other plant hormones accelerate or delay leaf senescence so that plants are better able to cope with severe environmental changes and achieve the maximum yield of seed and biomass production (Buchanan-Wollaston et al., 2003; Lim et al., 2007; Figure 1).

Upon leaf senescence, physiological events progress, which include chlorophyll breakdown, photosynthesis cessation, protein and nucleic acids degradation, catabolites and nutrients transport, and cell death responses, and the genes responsible for each event are dynamically up- or downregulated at the transcriptional level. Earlier studies have identified a group of senescence-associated genes (SAGs) that are induced upon senescence, and recent studies have shown specific roles for SAGs in leaf senescence (Gan and Amasino, 1997; Buchanan-Wollaston et al., 2005; Veyres et al., 2008). Indeed, treating plants with ethylene induces the expression of SAG genes (Jing et al., 2002). Dynamic changes in the expression profile of genes during leaf senescence can be visualized at the transcript and metabolite levels (Lin and Wu, 2004; BuchananWollaston et al., 2005; van der Graaff et al., 2006; Balazadeh et al., 2008; Breeze et al., 2011; Watanabe et al., 2013).

Extensive transcriptome analysis revealed differential expression patterns of various families of TFs during leaf senescence (Lin and Wu, 2004; Buchanan-Wollaston et al., 2005; Breeze et al., 2011). Analysis of the promoters of differentially expressed genes during leaf senescence has found enrichment of certain TF motifs such as, NO APICAL MERISTEM, Arabidopsis TRANSCRIPTION ACTIVATION FACTOR, CUP-SHAPED COTYLEDON (NAC), APETALA2/ETHYLENE RESPONSE FACTOR (AP2/ERF), and WRKY families (Breeze et al., 2011). Genetic and molecular studies also provide strong evidence that the activities of NAC, AP2/ERF, WRKY, and several other TF family members influence the onset of leaf senescence (Buchanan-Wollaston et al., 2003; Lim et al., 2007). Significantly, ethylene modulates the activity of these TFs. These findings illustrate that ethylene-mediated modulation of TF activities underlie the onset of leaf senescence.

This review aims to provide a detailed overview of the regulatory cascade involving ethylene and TFs in the regulation of the onset of leaf senescence. This review first provides a brief overview of the role of ethylene in this process and then focuses on the detailed actions of NAC, AP2/ERF, WRKY, and other developmental regulators (Table 1). Emphasis is also placed on how ethylene modulates TF activities and interacts with other hormones during the development of leaf senescence.

\section{ETHYLENE AS A REGULATOR OF THE ONSET OF LEAF SENESCENCE}

Earlier studies reported the involvement of ethylene in the regulation of leaf senescence. Ethylene production is associated with the 


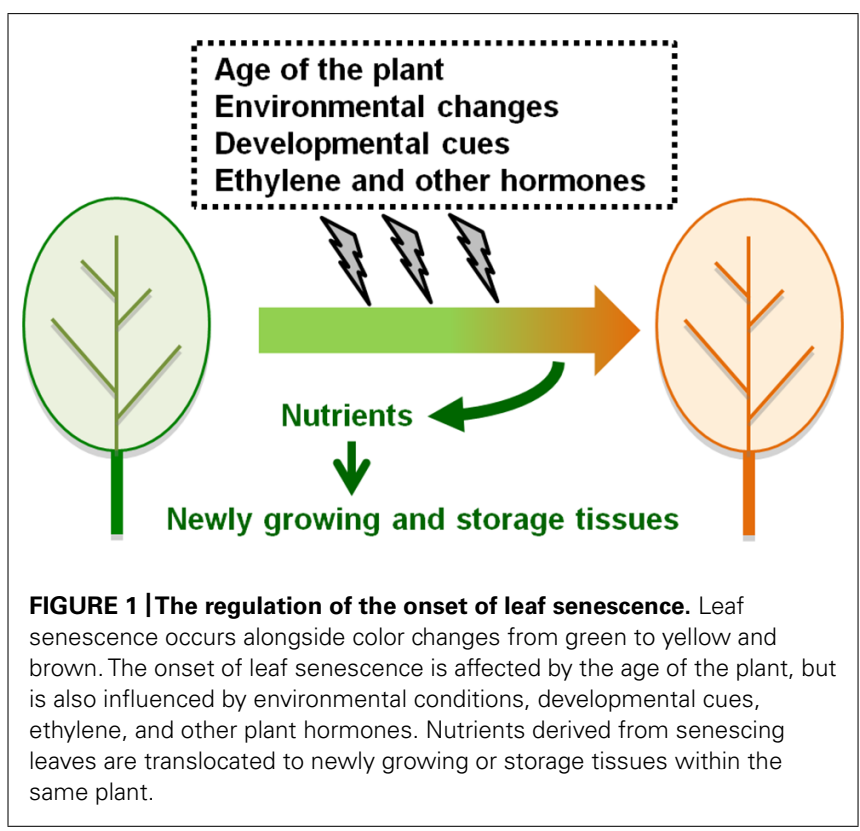

onset and progression of leaf senescence in various plant species (Abel et al., 1992). Application of ethylene to leaves stimulates senescence, but inhibitors of ethylene perception or biosynthesis delay leaf senescence (Aharoni and Lieberman, 1979; Kao and Yang, 1983). Furthermore, downregulation of an ethylene biosynthesis gene in tomato plants led to a decrease in ethylene production and substantially delayed leaf senescence, clearly suggesting that ethylene, produced as plants age, accelerates leaf senescence (John et al., 1995).

Knowledge of the ethylene signaling pathway will help to clarify the regulatory gene network involved in the onset of leaf senescence. As shown in Figure 2A, receptors localized on the endoplasmic reticulum (ER) membrane detect ethylene (Kendrick and Chang, 2008). Since these receptors repress the activity of downstream signaling components in the absence of ethylene (Figure 2B), ethylene reverses this repression and thus activates the signaling pathway. The signal generated following the detection of ethylene is subsequently transmitted to a complex composed of CONSTITUTIVE TRIPLE RESPONSE1 (CTR1), a Raf-like serine/threonine protien kinase, and ETHYLENE INSENSITIVE2 (EIN2), which is an integral ER membrane protein (Ju etal., 2012; Qiao etal., 2012). In the absence of the ethylene signal, CTR1 directly phosphorylates the cytosolic carboxyl-terminal domain of EIN2 (EIN2-C), whereas the ethylene signal prevents this phosphorylation and results in cleavage of EIN2-C, which then translocates to the nucleus and activates ETHYLENE-INSENSITIVE3 (EIN3) and EIN3-LIKE (EIL) TFs. The ethylene signal stabilizes EIN3 and EIL TFs, which are short-lived proteins in the absence of ethylene (Guo and Ecker, 2003; Potuschak et al., 2003), consequently inducing various physiological responses including the onset of leaf senescence.

Mutations in components of the ethylene signaling pathway exhibit differential timing of the onset of senescence, clearly suggesting that these components are involved in the regulation of such process. Consistent with the repressive role of ethylene receptors including ETHYLENE RESISTANT1 (ETR1) in the signaling pathway, a dominant-negative version of the receptors, such as the etr1 mutation, delays leaf senescence in Arabidopsis and petunia plants (Grbić and Bleecker, 1995; Wang et al., 2013). In contrast, an Arabidopsis null mutant that lacks two of five ethylene receptor genes has a phenotype consistent with constitutive ethylene response as well as accelerated leaf senescence (Qu et al., 2007). A pivotal role of EIN2 in the positive regulation of leaf senescence was documented by characterizing the genetic loci controlling the onset of leaf senescence in Arabidopsis (Oh et al., 1997; Kim et al., 2009). EIN3 positively regulates the onset of leaf senescence, since the ein 3 mutant delays leaf senescence whereas overexpression of EIN3 gene accelerates it (Li et al., 2013; Kim et al., 2014). In contrast, the $c$ trl mutant does not induce precocious leaf senescence and the involvement of CTR1 in the regulation of leaf senescence remains unclear. (Jing et al., 2005).

\section{ETHYLENE-REGULATED NAC AND OTHER TFs CONTROL THE ONSET OF LEAF SENESCENCE}

Several reports have attempted to elucidate the mechanism through which the ethylene signaling pathway modulates NAC activities during the onset of leaf senescence (Kim et al., 2009, 2014; Li et al., 2013; Figure 2A). The NAC TF family includes 105 members in Arabidopsis that are important during development and stress responses (Mitsuda and Ohme-Takagi, 2009). Among $N A C$ genes upregulated during leaf senescence, six NAC genes including ORESARA1 (ORE1)/ANAC092, ANAC019, NAClike activated by AP3 (AtNAP), ANAC047, ANAC055, and ORE1 SISTER1 (ORS1)/ANAC059 are activated through the EIN2dependent pathway (Kim et al., 2009, 2014; Figure 2A). ORE1 positively regulates the onset of leaf senescence and activates the expression of ORE1 itself, other NAC, nuclease, a sugar transporter, and various $S A G$ genes (Kim et al., 2009; Balazadeh et al., 2010; Breeze et al., 2011; Matallana-Ramirez et al., 2013; Rauf et al., 2013). ORE1 interacts with GOLDEN-LIKE2 (GLK2), the GARP family TF required for chloroplast development (Rauf et al., 2013). ORE1 attenuates GLK2 activity and may stop the maintenance of chloroplast development. ORE1 activity is modulated at both transcriptional and post-transcriptional levels (Kim et al., 2009; Figure 2A). ORE1 mRNA is targeted by the micro RNA miR164. The decrease in miR164 content with leaf aging is largely dependent on the EIN2 gene and thus leads to the accumulation of ORE1 mRNA in old leaves. Recent studies have further revealed that EIN3 directly activates expression of ORE1 (Li et al., 2013; Kim et al., 2014). Interestingly, EIN3 represses three miR164 precursor genes and is also involved in both positive and indirect regulation of the ORE1 gene (Li et al., 2013). Consistent with the molecular evidence, ORE1 expression is reduced in ein 3 mutant during leaf senescence. These observations suggest that EIN3, miR164, and ORE1 comprise a regulatory network that operates downstream of the ethylene signaling pathway (Figure 2A).

Among other NAC genes downstream of EIN2, the AtNAP gene is under the direct control of EIN3, whereas ORS1, ANAC019, ANAC047, and ANAC055 genes are activated in an EIN3-independent manner (Kim et al., 2014; Figure 2A). 
Table 1 | Transcription factors (TFs) regulating the onset of leaf senescence.

\begin{tabular}{|c|c|c|c|c|}
\hline $\mathrm{Name}^{\mathrm{a}}$ & Accession number ${ }^{b, c, d, e, f}$ & Family & Function $^{g}$ & Reference \\
\hline $\mathrm{ARF}^{\mathrm{a}}$ & AT5G62000 & ARF & Positive & Ellis et al. (2005), Lim etal. (2010) \\
\hline NtERF3 & D38124d & AP2/ERF & Positive & Koyama et al. (2013) \\
\hline AtERF4 & AT3G15210 & AP2/ERF & Positive & Koyama etal. (2013) \\
\hline AtERF8 & AT1G53170 & AP2/ERF & Positive & Koyama et al. (2013) \\
\hline SIERF36 & SGN-U564952 ${ }^{\mathrm{C}}$ & AP2/ERF & Positive & Upadhyay et al. (2013) \\
\hline EDF1 & AT1G25560 b & AP2/ERF & Negative & Chen etal. (2011) \\
\hline EDF2 & AT1G68840 & AP2/ERF & Negative & Chen etal. (2011) \\
\hline SUB1A & LOC_Os09g11480 & AP2/ERF & Negative & Fukao et al. (2012) \\
\hline CBF2 & AT4G25470 & AP2/ERF & Negative & Sharabi-Schwager et al. (2010) \\
\hline CBF3 & AT4G25480 b & AP2/ERF & Negative & Sharabi-Schwager et al. (2010) \\
\hline GLK2 & AT5G44190 b & GARP & Negative & Rauf et al. (2013) \\
\hline GBF1 & AT4G36730 b & GBF & Positive & Smykowski etal. (2010) \\
\hline $\mathrm{GAl}^{\mathrm{a}}$ & AT1G14920 & GRAS & Negative & Chen etal. (2014) \\
\hline GRF3 & AT2G36400 b & GRF & Negative & Debernardi etal. (2014) \\
\hline Knotted1 & AY312169d & homeodomain & Negative & Ori et al. (1999) \\
\hline KNAT2 & AT1G70510 & homeodomain & Negative & Hamant et al. (2002) \\
\hline FYF & AT5G62165 & MADS & Negative & Chen et al. (2011) \\
\hline MYBR1/MYB44 & AT5G67300 & MYB & Negative & Jaradat et al. (2013) \\
\hline NAM-B1 & DQ871219 & NAC & Positive & Uauy et al. (2006) \\
\hline AtNAP & AT1G69490 b & NAC & Positive & Guo and Gan (2006), Zhang and Gan (2012) \\
\hline JUB1 ${ }^{a}$ & AT2G43000 b & NAC & Negative & Wu et al. (2012) \\
\hline TCP2 & AT4G18390 & TCP & Positive & Schommer et al., 2008 \\
\hline TCP3 & AT1G53230 b & TCP & Positive & Schommer et al. (2008), Koyama et al. (2013) \\
\hline TCP4 & AT3G15030 & TCP & Positive & Schommer etal. (2008), Koyama et al. (2013) \\
\hline TCP5 & AT5G60970 b & TCP & Positive & Koyama et al. (2013) \\
\hline TCP10 & AT2G31070 & TCP & Positive & Schommer et al. (2008), Koyama etal. (2013) \\
\hline TCP13 & AT3G02150 b & TCP & Positive & Koyama et al. (2013) \\
\hline TCP19 & AT5G51910 b & TCP & Negative & Danisman et al. (2012) \\
\hline TCP20 & AT3G27010 & TCP & Negative & Danisman et al. (2012) \\
\hline TCP24 & AT1G30210 b & TCP & Positive & Schommer et al. (2008) \\
\hline WRKY6 & AT1G62300 b & WRKY & Positive & Robatzek and Somssich (2002) \\
\hline WRKY53 & AT4G23810 b & WRKY & Positive & Miao and Zentgraf (2007) \\
\hline WRKY54 & AT2G40750 b & WRKY & Negative & Besseau et al. (2012) \\
\hline WRKY57 & AT1G69310 b & WRKY & Negative & Jiang et al. (2014) \\
\hline WRKY70 & AT3G56400 b & WRKY & Negative & Besseau et al. (2012) \\
\hline SIZF2 & $A D Z 15317^{d}$ & Zn finger & Negative & Hichri et al. (2014) \\
\hline
\end{tabular}

a Abbreviations: GIBBERELLIC ACID INSENSITIVE (GAI) and JUNGBRUNNEN1 (JUB1). Other TF names are defined in the main text. ${ }^{\mathrm{b}, \mathrm{c}, \mathrm{d}, \mathrm{e}, \mathrm{f}}$ Accession numbers: The sequence data can be found in ${ }^{\mathrm{b}}$ Arabidopsis Genome Initiative, ${ }^{\mathrm{C}}$ Sol genomic network, ${ }^{\mathrm{d}}$ Genbank, ${ }^{\mathrm{e}}$ Michigan State University Rice Genome Annotation Project, and ${ }^{\mathrm{f}}$ Phytozome libraries. ${ }^{9}$ Function: Positive and negative indicate TFs that accelerate and delay leaf senescence, respectively. 

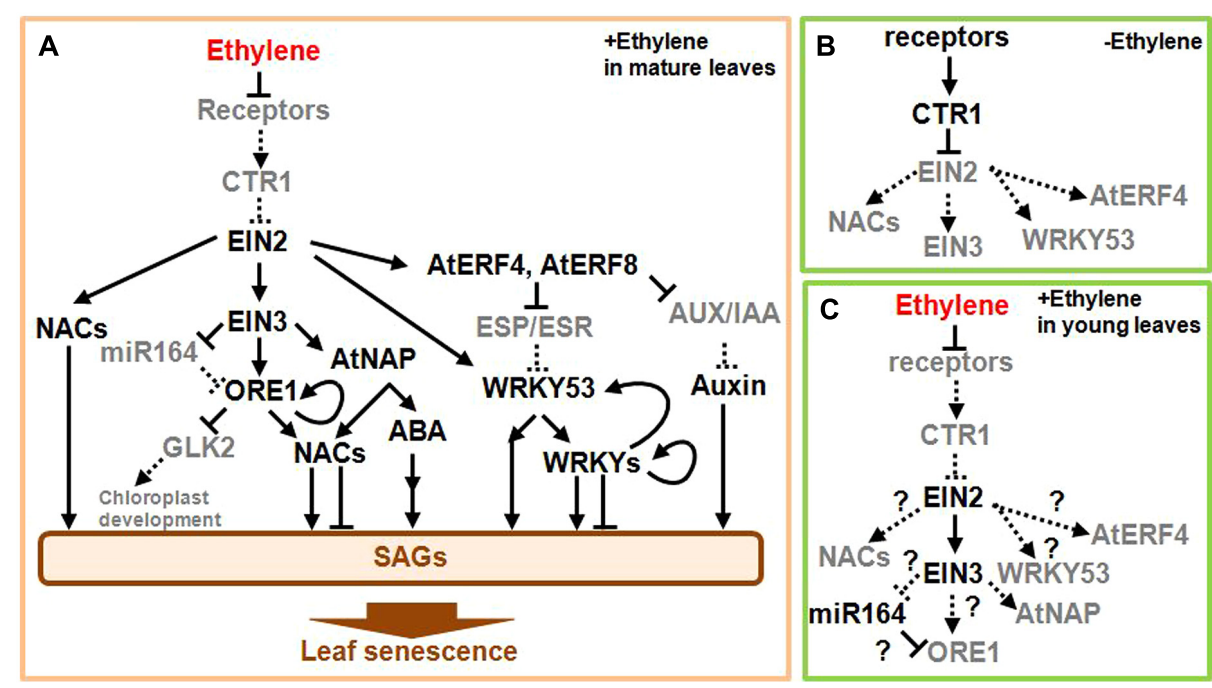

FIGURE 2 | Scheme of the ethylene signaling pathway leading to the onset of leaf senescence. (A) In mature leaves, the detection of ethylene activates the downstream signaling pathway leading to SAG induction and leaf senescence. (B) In young and mature leaves, the receptors constitutively repress the downstream signaling in the absence of ethylene. (C) In young leaves, the detection of ethylene activates the downstream signaling pathway, but does not itself induce leaf senescence. Note that EIN2 and EIN3 are active and induce some ethylene responses, but not leaf senescence by an uncharacterized mechanism, in which some regulators of leaf development are likely involved. Arrows and bars at the end of each line show positive and negative regulations, respectively. Solid lines and black gene names designate the active form, while dotted lines and gray gene names indicate the inactive form. Several transcription factors (TFs) and signals such as jasmonic acid are not drawn in this scheme owing to space limitations. A detailed description on the scheme is presented in the main text.
AtNAP positively regulates the onset of leaf senescence and activates a component of the abscisic acid (ABA) signaling pathway, which promotes both leaf senescence and stress responses (Guo and Gan, 2006; Zhang and Gan, 2012). A rice homolog gene, OsNAP1, acts as a positive regulator of leaf senescence and its product directly targets an ABA biosynthesis enzyme gene (Zhou etal., 2013; Liang etal., 2014). ORS1 positively regulates the onset of leaf senescence (Balazadeh et al., 2011). ANAC019 and ANAC055 seem to function under the control of C-REPEAT/DEHYDRATION RESPONSIVE ELEMENT BINDING FACTORS (CBFs) of AP2/ERF TFs and other TFs during stress response and leaf senescence (Hickman et al., 2013; See below). A role for ANAC047 in leaf senescence is yet to be determined.

Five additional NAC genes including VND-INTERACTING2 (VNI2) are thought to function downstream of ORE1 and AtNAP (Kim etal., 2014). VNI2 negatively regulates the onset of leaf senescence via the direct activation of COLD REGULATED (COR) and RESPONSIVE TO DEHYDRATION (RD) genes that are also responsive to environmental stimuli (Yang et al., 2011). By contrast, the functions of other NAC genes remain to be clarified. The regulation of EIN2, EIN3, NAC TFs, and the ABA response pathway are likely to be important in the integration of various inputs from diverse environmental factors as well as the age of the plant (Figure 2A).

\section{ETHYLENE-RESPONSIVE TFS IN THE REGULATION OF ONSET OF LEAF SENESCENCE}

Ethylene activates a substantial number of $A P 2 / E R F$ genes, and several of these regulate the onset of leaf senescence. The
AP2/ERF TFs comprise 146 members that include both activators and repressors of transcription (Mitsuda and OhmeTakagi, 2009). A subgroup of transcriptional repressors with the ERF-associated repression (EAR) motif, such as NtERF3, AtERF4, and AtERF8, positively regulate the onset of leaf senescence in Arabidopsis (Ohta etal., 2001; Koyama etal., 2013; Figure 2A). The finding of EIN2-dependent AtERF4 expression in leaves suggests that there is AtERF4 activity downstream of EIN2 (Fujimoto etal., 2000). AtERF4 and AtERF8 are degraded by a proteasomal-dependent pathway, but accumulate within the plant as a result of increasing age (Koyama et al., 2013). These ERF TFs directly repress expression of the EPITHIOSPECIFIER PROTEIN/EPITHIOSPECIFYING SENESCENCE REGULATOR (ESP/ESR) gene, a negative regulator of the onset of leaf senescence (Miao and Zentgraf, 2007; Koyama et al., 2013). The ESP/ESR transcript is highly expressed in young leaves, but decreased in old ones (Koyama et al., 2013). ESP/ESR inhibits the activity of WRKY53, a positive regulator of the onset of leaf senescence, at both transcriptional and posttranslational levels (Miao and Zentgraf, 2007; See below). These findings imply that AtERF4 and AtERF8 activate WRKY53 by removing the ESP/ESR-mediated inhibition. Therefore, AtERF4, AtERF8, ESP/ESR, and WRKY53 form another regulatory network for the onset of leaf senescence (Figure 2A). Moreover, AtERF4 and AtERF8 repress the expression of AUXIN/INDOLE3-ACETIC ACID (AUX/IAA) genes. AUX/IAA TFs generally suppress auxin responses that include positive effects on leaf senescence. Therefore, it is possible that the AtERF4- and AtERF8-mediated AUX/IAA repression enhances auxin response and then stimulates the onset of leaf senescence. In addition, 
a tomato homolog of AtERF4, SlERF36, accelerates leaf senescence when overexpressed in tomato plants (Upadhyay etal., 2013).

By contrast, RAV1 and GmRAV1, which possess another type of repression domain (Ikeda and Ohme-Takagi, 2009), negatively regulate the onset of leaf senescence, because overexpression of these $R A V 1$ genes delays leaf senescence in Arabidopsis (Woo et al., 2010). Other two Arabidopsis RAV genes, namely, ETHYLENE RESPONSE DNA BINDING FACTOR1 (EDF1) and EDF2, are proposed to regulate the onset of leaf senescence downstream of the MADS box TF, FOREVER YOUNG FLOWER (FYF; Chen et al., 2011). These $R A V$ genes are transcriptionally induced by ethylene (Alonso et al., 2003). Based on studies investigating EAR- and RAV-type AP2/ERF TFs, the ethylene signal appears to balance positive and negative regulations thus determining the rate of leaf senescence.

Transcriptional activators of ERF TFs are also involved in regulating the onset of leaf senescence. SUBMERGENCE1A (SUB1A) negatively regulates the onset of leaf senescence in rice (Fukao et al., 2012), while CYTOKININ RESPONSE FACTOR6 (CRF6) negatively regulates leaf senescence (Zwack etal., 2013). Overexpression of $C B F 2$ and $C B F 3$ genes delays leaf senescence in Arabidopsis (Sharabi-Schwager et al., 2010). Since CBFs target COR15 and RD29 genes and possibly control ANAC019 and ANAC055 (Yang etal., 2011; Hickman et al., 2013), CBFs seem to regulate the onset of leaf senescence via these downstream genes. However, there have been no reports on the involvement of ethylene in the regulation of these ERF activators.

\section{WRKY TFS INTEGRATE ETHYLENE AND JASMONIC ACID SIGNALS DURING LEAF SENESCENCE}

Jasmonic acid (JA) is another important factor regulating the onset of leaf senescence, because a mutant that lacks a JA-biosynthetic enzyme gene delays leaf senescence and application of JA to leaves accelerates senescence (He et al., 2002; Seltmann et al., 2010). JA often cooperatively interacts with ethylene to underpin many physiological responses (Lorenzo et al., 2004; Zhu et al., 2011). It has been well documented that JA, along with the age of the plant, induce transcription of many WRKY genes. (Lin and $\mathrm{Wu}$, 2004). Among WRKY TFs activated by JA, WRKY53 positively regulates the onset of leaf senescence and its activity is modulated by ESP/ESR at both transcriptional and post-translational levels (Miao and Zentgraf, 2007; Figure 2A). ESP/ESR physically interacts with WRKY53 and, presumably, prevents WRKY53 binding to DNA. ESP/ESR also inhibits the accumulation of WRKY53 transcripts in leaves. It has also been reported that WRKY6, WRKY54, WRKY57, and WRKY75 regulate leaf senescence (Robatzek and Somssich, 2002; Besseau et al., 2012; Li et al., 2012b). Since WRKY6 and WRKY53 have been shown to increase many WRKY transcripts in addition to SAGs (Robatzek and Somssich, 2002; Miao et al., 2004), some self-amplification of WRKY activity may contribute to the robust control of leaf senescence.

Several reports provide intriguing insights into the interactions between WRKY TFs and the ethylene signaling pathway. Nematode-induced WRKY53 expression in leaves requires a functional EIN2 gene, suggesting the involvement of EIN2 in the modulation of WRKY53 activity (Murray et al., 2007). WRKY75 is also involved in the ethylene-dependent defense-signaling pathway (Chen et al., 2013). WRKY33, whose transcript is markedly increased during leaf senescence, directly activates ethylene biosynthetic genes and is involved in ethylene production (Breeze et al., 2011; Li et al., 2012a). Thus, it is possible that WRKY TFs are activated by the cooperative action of the ethylene and JA signaling pathways in the regulation of the onset of leaf senescence.

In addition, the single stranded-DNA binding protein WHIRLY1A and a histone methyltransferase target the WRKY53 gene in Arabidopsis (Ay et al., 2009; Miao et al., 2013). A basic loop-helix-loop TF activates the WRKY53 gene and positively regulates the onset of leaf senescence in soybean (Meng et al., 2013). However, an association between ethylene and these WRKY53 regulators remains to be addressed.

\section{THE ONSET OF LEAF SENESCENCE IS AFFECTED BY TFs THAT ARE REOUIRED FOR LEAF DEVELOPMENT}

In contrast to the pivotal role of ethylene in the onset of leaf senescence, it is known that ethylene inhibits leaf expansion in young plants, but does not always induce leaf senescence (Kieber et al., 1993; Hua and Meyerowitz, 1998). Moreover, Arabidopsis ctr1 mutant has constitutive responses to ethylene resulting in the formation of a small rosette, but does not appear to induce precocious leaf senescence (Kieber et al., 1993; Jing et al., 2005). These apparent discrepancies suggest that the mechanism leading to leaf senescence might require some developmental regulators even when the ethylene signaling pathway is activated (Figure 2C). Whereas the functional interaction between ethylene and developmental regulators during leaf senescence is not fully understood, it is expected that such regulators have pivotal roles in the onset of leaf senescence.

KNOTTED1-like homeodomain (KNOX) TFs, which are required for shoot meristem and leaf development, negatively regulate the onset of leaf senescence (Ori et al., 1999; Hamant et al., 2002; Hay and Tsiantis, 2010). When KNOX genes are ectopically expressed in tobacco and Arabidopsis leaves, they markedly delay the onset of leaf senescence (Ori et al., 1999; Hamant et al., 2002). Ectopic KNOX expression confers an undifferentiated cell fate in leaves and inhibits their differentiation (Hay and Tsiantis, 2010). As a consequence, this disordered cellular regulation may indirectly delay the onset of leaf senescence. Otherwise, KNOX TFs regulate biosynthetic genes of cytokinin (Sakamoto et al., 2001; Hay et al., 2002), which acts as a negative regulator of the onset of leaf senescence, and therefore, possibly influences leaf senescence. In agreement with the antagonism between $K N O X$ genes and the gibberellin (GA) signaling pathway observed in shoot meristem and leaf development (Hay and Tsiantis, 2010), plants treated with GA and Arabidopsis mutants of the GRAS-type TF genes, which are negative regulators of the GA signaling pathway, accelerate leaf senescence (Chen et al., 2014).

Another class of regulators for both leaf senescence and development is the TEOSINTE BRANCHED1, CYCLOIDEA, PCNA BINDING FACTOR (TCP) TFs family. A combined analysis of high-resolution temporal clustering of genes differentially expressed during leaf senescence and TF-binding motif searching in the promoters of each cluster demonstrates that the 
TCP-binding motif is significantly enriched in certain downregulated gene clusters (Breeze et al., 2011). This indicates the co-regulation of these gene clusters and TCP activity. Consistent with bioinformatic surveillance, reverse genetic analysis revealed that inhibition of the CINCINNATA (CIN) subfamily of TCP (CIN-like TCP) delays leaf senescence whereas overexpression of a CIN-like TCP gene accelerates it (Schommer et al., 2008; Koyama et al., 2013). A possible scenario to explain the positive roles of CIN-like TCP TFs in the onset of leaf senescence is that CINTCP TFs activate JA biosynthetic enzyme genes (Schommer et al., 2008). Alternatively, CIN-like TCP TFs suppress an auxin signaling pathway, which is a negative regulator of leaf senescence, and also activates negative regulators of KNOX genes (Koyama et al., 2007, 2010). Moreover, CIN-like TCPs act as heterochronic regulators of leaf development and consequently influence the onset of leaf senescence (Efroni et al., 2008). By contrast, TCP19 and TCP20, which are grouped into a class I subgroup, negatively regulate the onset of leaf senescence and results in the opposite effects of CIN-like TCPs (Danisman et al., 2012).

In addition to KNOX and TCP TFs, Arabidopsis GROWTHREGULATING FACTOR (GRF) TFs and a tomato C2H2 typeEAR repressor regulate both leaf development and senescence (Debernardi et al., 2014; Hichri et al., 2014). Taking the roles of the developmental regulators into account, these regulators, thus, prevent precocious leaf senescence. Ethylene meditates various signals required for the induction of defense responses against biotic and abiotic stressors (Kendrick and Chang, 2008); however, these responses are not always followed by cell death. Therefore, such developmental regulators are likely to determine the fate of leaves upon ethylene exposure. In comparison to fully maturated leaves, young leaves accumulate low amounts of carbon and nitrogen sources that would be mobilized to growing and storage organs and therefore it is reasonable that young leaves are kept away from senescence even in the presence of ethylene.

\section{CONCLUSIONS AND PERSPECTIVES}

In addition to ethylene, JA and the developmental signals discussed in this review, additional factors such as cytokinin, auxin, $\mathrm{ABA}$, and hydrogen peroxide are involved in the regulation of leaf senescence (Ellis et al., 2005; Lim et al., 2010; Smykowski et al., 2010; Yang et al., 2011; Wu et al., 2012; Jaradat et al., 2013). Several TFs are reported to regulate the onset of leaf senescence under these additional signals and details of such TF are listed in Table $\mathbf{1}$. Ethylene and these signals are integrated for the regulation of the onset of leaf senescence; however, there have been no reports of a direct interaction between ethylene and such TFs acting downstream of these signals. It is interesting to investigate whether these TFs act in an ethylene-dependent manner during the onset of leaf senescence.

This review focuses on the roles of TFs and ethylene in the regulation of the onset of leaf senescence and emphasizes that regulation occurs at multiple levels downstream of the ethylene signaling pathway. Moreover, leaf development is tightly linked to the onset of senescence and further clarification of such mechanisms is in progress. Furthermore, the effect of ethylene on the stimulation of leaf senescence is dependent on the duration of ethylene exposure (Jing et al., 2005). Regulation of the appropriate duration of ethylene exposure could represent another candidate for modulating the ethylene signal and thus, the onset of leaf senescence. Further efforts to determine the mechanism that transforms the ethylene signal into the onset of leaf senescence will improve our current understanding of the roles of ethylene in leaf senescence.

\section{ACKNOWLEDGMENTS}

The author appreciates Drs. Makoto Suematsu and Honoo Satake for helpful comments on the manuscripts. This work is supported by JSPS KAKENHI Grant Number 26440158.

\section{REFERENCES}

Abel, F. B., Morgan, P. W., and Saltveit, M. S. Jr. (1992) "Regulation of ethylene production by internal, environmental, and stress factors," in Ethylene in Plant Biology, (San Diego, CA: Academic Press), 56-119.

Aharoni, N., and Lieberman, M. (1979). Ethylene as a regulator of senescence in tobacco leaf discs. Plant Physiol. 64, 801-804. doi: 10.1104/pp.64.5.801

Alonso, M., Stepanova, A. N., Leisse, T. J., Kim, C. J., Chen, H., Shinn, P., et al. (2003). Genome-wide insertional mutagenesis of Arabidopsis thaliana. Science 301, 653-657. doi: 10.1126/science.1086391

Ay, N., Irmler, K., Fischer, A., Uhlemann, R., Reuter, G., and Humbeck, K. (2009). Epigenetic programming via histone methylation at WRKY53 controls leaf senescence in Arabidopsis thaliana. Plant J. 58, 333-346. doi: 10.1111/j.13653139.2008.03782.x

Balazadeh, S., Kwasniewski, M., Caldana, C., Mehrnia, M., Zanor, M. I. Z., Xue, G.-P., et al. (2011). ORS1, an H2O2-Responsive NAC transcription factor, controls senescence in Arabidopsis thaliana. Mol. Plant 4, 346-360. doi: $10.1093 / \mathrm{mp} / \mathrm{ssq} 080$

Balazadeh, S., Riaño-Pachón, D. M., and Mueller-Roeber, B. (2008). Transcription factors regulating leaf senescence in Arabidopsis thaliana. Plant Biol. (Stuttg.) 10, 63-75. doi: 10.1111/j.1438-8677.2008.00088.x

Balazadeh, S., Siddiqui, H., Allu, A. D., Matallana-ramirez, L. P., and Caldana, C. (2010). A gene regulatory network controlled by the NAC transcription factor ANAC092/AtNAC2/ORE1 during salt-promoted senescence. Plant J. 62, 250-264. doi: 10.1111/j.1365-313X.2010.04151.x

Besseau, S., Li, J., and Palva, E. T. (2012). WRKY54 and WRKY70 co-operate as negative regulators of leaf senescence in Arabidopsis thaliana. J. Exp. Bot. 63, 2667-2679. doi: 10.1093/jxb/err450

Breeze, E., Harrison, E., McHattie, S., Hughes, L., Hickman, R., Hill, C., et al. (2011). High-resolution temporal profiling of transcripts during Arabidopsis leaf senescence reveals a distinct chronology of processes and regulation. Plant Cell 23, 873-894. doi: 10.1105/tpc.111.083345

Buchanan-Wollaston, V., Earl, S., Harrison, E., Mathas, E., Navabpour, S., Page, T., et al. (2003). The molecular analysis of leaf senescence - a genomics approach. Plant Biotechnol. J. 1, 3-22. doi: 10.1046/j.1467-7652.2003.00004.x

Buchanan-Wollaston, V., Page, T., Harrison, E., Breeze, E., Lim, P. O., Nam, H. G., et al. (2005). Comparative transcriptome analysis reveals significant differences in gene expression and signalling pathways between developmental and dark/starvation-induced senescence in Arabidopsis. Plant J. 42, 567-585. doi: 10.1111/j.1365-313X.2005.02399.x

Chen, M., Hsu, W., Lee, P., Thiruvengadam, M., Chen, H., and Yang, C. (2011). The MADS box gene, FOREVER YOUNG FLOWER, acts as a repressor controlling floral organ senescence and abscission in Arabidopsis. Plant J. 68, 168-185. doi: 10.1111/j.1365-313X.2011.04677.x

Chen, M., Maodzeka, A., Zhou, L., Ali, E., Wang, Z., and Jiang, L. (2014). Plant science removal of DELLA repression promotes leaf senescence in Arabidopsis. Plant Sci. 219-220, 26-34. doi: 10.1016/j.plantsci.2013.11.016

Chen, X., Liu, J., Lin, G., Wang, A., Wang, Z., and Lu, G. (2013). Overexpression of AtWRKY28 and AtWRKY75 in Arabidopsis enhances resistance to oxalic acid and Sclerotinia sclerotiorum. Plant Cell Rep. 32, 1589-1599. doi: 10.1007/s00299-0131469-3

Danisman, S., van der Wal, F., Dhondt, S., Waites, R., de Folter, S., Bimbo, A., et al. (2012). Arabidopsis class I and class II TCP transcription factors regulate jasmonic acid metabolism and leaf. Plant Physiol. 159, 1511-1523. doi: $10.1104 /$ pp.112.200303 
Debernardi, J. M., Mecchia, M. A., Vercruyssen, L., Smaczniak, C., Kaufmann, K., Inze, D., et al. (2014). Post-transcriptional control of GRF transcription factors by microRNA miR396 and GIF co-activator affects leaf size and longevity. Plant J. 79, 413-426. doi: 10.1111/tpj.12567

Efroni, I., Blum, E., Goldshmidt, A., and Eshed, Y. (2008). A protracted and dynamic maturation schedule underlies Arabidopsis leaf development. Plant Cell 20, 2293 2306. doi: $10.1105 /$ tpc. 107.057521

Ellis, C. M., Nagpal, P., Young, J. C., Hagen, G., Guilfoyle, T. J., and Reed, J. W. (2005). AUXIN RESPONSE FACTOR1 and AUXIN RESPONSE FACTOR2 regulate senescence and floral organ abscission in Arabidopsis thaliana. Development 132, 4563-4574. doi: 10.1242/dev.02012

Fujimoto, S. Y., Ohta, M., Usui, A., Shinshi, H., and Ohme-Takagi, M. (2000). Arabidopsis ethylene-responsive element binding factors act as transcriptional activators or repressors of GCC box-mediated gene expression. Plant Cell 12, 393-404. doi: 10.1105/tpc.12.3.393

Fukao, T., Yeung, E., and Bailey-serres, J. (2012). The submergence tolerance gene SUB1A delays leaf senescence under prolonged darkness through hormonal regulation in rice. Plant Physiol. 160, 1795-1807. doi: 10.1104/pp.112.207738

Gan, S., and Amasino, R. M. (1997). Making sense of senescence (molecular genetic regulation and manipulation of leaf senescence). Plant Physiol. 113, 313-319.

Grbić, V., and Bleecker, A. B. (1995). Ethylene regulates the timing of leaf senescence in Arabidopsis. Plant J. 8, 595-602. doi: 10.1046/j.1365-313X.1995.8040595.x

Guo, H., and Ecker, J. R. (2003). Plant responses to ethylene gas are mediated by SCF(EBF1/EBF2)-dependent proteolysis of EIN3 transcription factor. Cell 115, 667-677. doi: 10.1016/S0092-8674(03)00969-3

Guo, Y., and Gan, S. (2006). AtNAP, a NAC family transcription factor, has an important role in leaf senescence. Plant J. 46, 601-612. doi: 10.1111/j.1365313X.2006.02723.x

Hamant, O., Nogue, F., Belles-boix, E., Jublot, D., Grandjean, O., and Traas, J. (2002). The KNAT2 homeodomain protein interacts with ethylene and cytokinin signaling. Plant Physiol. 130, 657-665. doi: 10.1104/pp.004564.1996

Hay, A., Kaur, H., Phillips, A., Hedden, P., Hake, S., and Tsiantis, M. (2002). The gibberellin pathway mediates KNOTTED1-type homeobox function in plants with different body plans. Curr. Biol. 12, 1557-1565. doi: 10.1016/S0960-9822(02)01125-9

Hay, A., and Tsiantis, M. (2010). KNOX genes: versatile regulators of plant development and diversity. Development 137, 3153-3165. doi: 10.1242/dev.030049

He, Y., Fukushige, H., Hildebrand, D. F., and Gan, S. (2002). Evidence supporting a role of jasmonic acid in Arabidopsis leaf senescence. Plant Physiol. 128, 876-884. doi: 10.1104/pp.010843

Hichri, I., Muhovski, Y., Zi, E., Dobrev, P. I., Franco-zorrilla, J. M., Solano, R., et al. (2014). The Solanum lycopersicum zinc finger2 cysteine2/histidine2 repressor-like transcription factor regulates development and tolerance to salinity in Arabidopsis. Plant Physiol. 164, 1967-1990. doi: 10.1104/pp.113.225920

Hickman, R., Hill, C., Penfold, C. A., Breeze, E., Bowden, L., Moore, J. D., et al. (2013). A local regulatory network around three NAC transcription factors in stress responses and senescence in Arabidopsis leaves. Plant J. 75, 26-39. doi: $10.1111 /$ tpj.12194

Hua, J., and Meyerowitz, E. M. (1998). Ethylene responses are negatively regulated by a receptor gene family in Arabidopsis thaliana. Cell 94, 261-271. doi: 10.1016/S0092-8674(00)81425-7

Ikeda, M., and Ohme-Takagi, M. (2009). A novel group of transcriptional repressors in Arabidopsis. Plant Cell Physiol. 50, 970-975. doi: 10.1093/pcp/ pсp048

Jaradat, M. R., Feurtado, J. A., Huang, D., Lu, Y., and Cutler, A. J. (2013). Multiple roles of the transcription factor AtMYBR1/AtMYB44 in ABA signaling, stress responses, and leaf senescence. BMC Plant Biol. 13:192. doi: 10.1186/1471-222913-192

Jiang, Y., Liang, G., Yang, S., and Yu, D. (2014). Arabidopsis WRKY57 functions as a node of convergence for jasmonic acid - and auxin-mediated signaling in jasmonic acid - induced leaf senescence. Plant Cell 26, 230-245. doi: 10.1105/tpc.113.117838

Jing, H., Schippers, J. H. M., Hille, J., and Dijkwel, P. P. (2005). Ethylene-induced leaf senescence depends on age-related changes and OLD genes in Arabidopsis. J. Exp. Bot. 56, 2915-2923. doi: 10.1093/jxb/eri287

Jing, H., Sturre, M. J. G., Hille, J., and Dijkwel, P. P. (2002). Arabidopsis onset of leaf death mutants identify a regulatory pathway controlling leaf senescence. Plant $J$. 32, 51-63. doi: 10.1046/j.1365-313X.2002.01400.x
John, I., Drake, R., Farrell, A., Cooper, W., Lee, P., Horton, P., et al. (1995). Delayed leaf senescence in ethylene-deficient ACC-oxidase antisense tomato plants: molecular and physiological analysis. Plant J. 7, 483-490. doi: 10.1046/j.1365-313X.1995.7030483.x

Ju, C., Mee, G., Marie, J., Lin, D. Y., Ying, Z. I., Chang, J., et al. (2012). CTR1 phosphorylates the central regulator EIN2 to control ethylene hormone signaling from the ER membrane to the nucleus in Arabidopsis. Proc. Natl. Acad. Sci. U.S.A. 111, 10013-10018. doi: 10.1073/pnas.1214848109

Kao, C. H., and Yang, F. A. (1983). Role of ethylene in the senescence of detached rice leaves. Plant Physiol. 73, 881-885. doi: 10.1104/pp.73.4.881

Kendrick, M. D., and Chang, C. (2008). Ethylene signaling: new levels of complexity and regulation. Curr. Opin. Plant Biol. 11, 479-485. doi: 10.1016/j.pbi.2008.06.011

Kieber, J. J., Rothenberg, M., Roman, G., Feldmann, K. A., and Ecker, J. R. (1993). CTR1, a negative regulator of the ethylene response pathway in Arabidopsis, encodes a member of the raf family of protein kinases. Cell 72, 427-441. doi: 10.1016/0092-8674(93)90119-B

Kim, H. J., Hong, S. H., Kim, Y. W., Lee, I. H., Jun, J. H., Phee, B., et al. (2014). Gene regulatory cascade of senescence-associated NAC transcription factors activated by ETHYLENE-INSENSITIVE2-mediated leaf senescence signalling in Arabidopsis. J. Exp. Bot. 65, 4023-4036. doi: 10.1093/jxb/eru112

Kim, J. H., Woo, H. R., Kim, J., Lim, P. O., Lee, I. C., Choi, S. H., et al. (2009). Trifurcate feed-forward regulation of age-dependent cell death involving miR164 in Arabidopsis. Science 323, 1053-1057. doi: 10.1126/science.1166386

Koyama, T., Furutani, M., Tasaka, M., and Ohme-Takagi, M. (2007). TCP transcription factors control the morphology of shoot lateral organs via negative regulation of the expression of boundary-specific genes in Arabidopsis. Plant Cell 19, 473-484. doi: 10.1105/tpc. 106.044792

Koyama, T., Mitsuda, N., Seki, M., Shinozaki, K., and Ohme-Takagi, M. (2010). TCP transcription factors regulate the activities of ASYMMETRIC LEAVES1 and miR164, as well as the auxin response, during differentiation of leaves in Arabidopsis. Plant Cell 22, 3574-3588. doi: 10.1105/tpc.110. 075598

Koyama, T., Nii, H., Mitsuda, N., Ohta, M., Kitajima, S., Ohme-Takagi, M., et al. (2013). A regulatory cascade involving class II ETHYLENE RESPONSE FACTOR transcriptional repressors operates in the progression of leaf senescence. Plant Physiol. 162, 991-1005. doi: 10.1104/pp.113.218115

Li, G., Meng, X., Wang, R., Mao, G., Han, L., Liu, Y., et al. (2012a). Dual-level regulation of ACC synthase activity by MPK3/MPK6 cascade and its downstream WRKY transcription factor during ethylene induction in Arabidopsis. PLoS Genet. 8:e1002767. doi: 10.1371/journal.pgen.1002767

Li, Z., Peng, J., Wen, X., and Guo, H. (2012b). Gene network analysis and functional studies of senescence-associated genes reveal novel regulators of Arabidopsis leaf senescence. J. Integr. Plant Biol. 54, 526-539. doi: 10.1111/j.17447909.2012.01136.x

Li, Z., Peng, J., Wen, X., and Guo, H. (2013). Ethylene-insensitive3 is a senescence-associated gene that accelerates age-dependent leaf senescence by directly repressing miR164 transcription in Arabidopsis. Plant Cell 25, 3311-3328. doi: $10.1105 /$ tpc. 113.113340

Liang, C., Wang, Y., Zhu, Y., Tang, J., Hu, B., Liu, L., et al. (2014). OsNAP connects abscisic acid and leaf senescence by fine-tuning abscisic acid biosynthesis and directly targeting senescence-associated genes in rice. Proc. Natl. Acad. Sci. U.S.A. 111, 10013-10018. doi: 10.1073/pnas.1321568111

Lim, P. O., Kim, H. J., and Nam, H. G. (2007). Leaf senescence. Annu. Rev. Plant Biol. 58, 115-136. doi: 10.1146/annurev.arplant.57.032905.105316

Lim, P. O., Lee, I. C., Kim, J., Kim, H. J., Ryu, J. S., Woo, H. R., et al. (2010). Auxin response factor 2 (ARF2) plays a major role in regulating auxin-mediated leaf longevity. J. Exp. Bot. 61, 1419-1430. doi: 10.1093/jxb/erq010

Lin, J.-F., and Wu, S.-H. (2004). Molecular events in senescing Arabidopsis leaves. Plant J. 39, 612-628. doi: 10.1111/j.1365-313X.2004.02160.x

Lorenzo, O., Chico, J. M., and Sa, J. J. (2004). JASMONATE-INSENSITIVE1 encodes a MYC transcription factor essential to discriminate between different jasmonate-regulated defense responses in Arabidopsis. Plant Cell 16, 1938-1950. doi: $10.1105 /$ tpc.022319

Matallana-Ramirez, L. P., Rauf, M., Farage-barhom, S., Dortay, H., and Xue, G. (2013). NAC transcription factor orel and senescence- constitute a regulatory cascade in Arabidopsis. Mol. Plant 6, 1432-1452. doi: 10.1093/mp/ sst012 
Meng, Y., Li, H., Wang, Q., Liu, B., and Lin, C. (2013). Blue light-dependent interaction between cryptochrome2 and CIB1 regulates transcription and leaf senescence in soybean. Plant Cell 25, 4405-4420. doi: 10.1105/tpc.113.116590

Miao, Y., Jiang, J., Ren, Y., and Zhao, Z. (2013). The single-stranded DNA-binding protein WHIRLY1 represses WRKY53 expression and delays leaf senescence in a developmental stage-dependent manner in Arabidopsis. Plant Physiol. 163, 746756. doi: $10.1104 /$ pp.113.223412

Miao, Y., Laun, T., Zimmermann, P., and Zentgraf, U. (2004). Targets of the WRKY53 transcription factor and its role during leaf senescence in Arabidopsis. Plant Mol. Biol. 55, 853-867. doi: 10.1007/s11103-005-2142-1

Miao, Y., and Zentgraf, U. (2007). The antagonist function of Arabidopsis WRKY53 and ESR/ESP in leaf senescence is modulated by the jasmonic and salicylic acid equilibrium. Plant Cell 19, 819-830. doi: 10.1105/tpc.106.042705

Mitsuda, N., and Ohme-Takagi, M. (2009). Functional analysis of transcription factors in Arabidopsis. Plant Cell Physiol. 50, 1232-1248. doi: 10.1093/pcp/pcp075

Murray, S. L., Ingle, R. A., Petersen, L. N., and Denby, K. J. (2007). Basal resistance against Pseudomonas syringae in Arabidopsis involves WRKY53 and a protein with homology to a nematode resistance protein. Mol. Plant Microbe Interact. 20 1431-1438. doi: 10.1094/MPMI-20-11-1431

Oh, S. A., Park, J., Lee, G. I., Paek, K. H., Park, S. K., and Nam, H. G. (1997). Identification of three genetic loci controlling leaf senescence in Arabidopsis thaliana. Plant J. 12, 527-535. doi: 10.1046/j.1365-313X.1997.00527.x

Ohta, M., Matsui, K., Hiratsu, K., Shinshi, H., and Ohme-Takagi, M. (2001). Repression domains of class II ERF transcriptional repressors share an essential motif for active repression. Plant Cell 13, 1959-1968. doi: 10.1105/tpc.13.8.1959

Ori, N., Juarez, M. T., Jackson, D., Yamaguchi, J., Banowetz, G. M., and Hake, S. (1999). Leaf senescence is delayed in tobacco plants expressing the maize homeobox gene knotted 1 under the control of a senescence-activated promoter. Plant Cell 11, 1073-1080. doi: 10.1105/tpc.11.6.1073

Potuschak, T., Lechner, E., Parmentier, Y., Yanagisawa, S., Grava, S., Koncz, C., et al. (2003). EIN3-dependent regulation of plant ethylene hormone signaling by two Arabidopsis F box proteins: EBF1 and EBF2. Cell 115, 679-689. doi: 10.1016/S0092-8674(03)00968-1

Qiao, H., Shen, Z., Huang, S. C., Schmitz, R. J., Urich, M. A., Briggs, S. P., and Ecker J. R. (2012). Processing and subcellular trafficking of ER-tethered EIN2 control response to ethylene gas. Science 338, 390-393. doi: 10.1126/science.1225974

Qu, X., Hall, B. P., Gao, Z., and Schaller, G. E. (2007). A strong constitutive ethyleneresponse phenotype conferred on Arabidopsis plants containing null mutations in the ethylene receptors ETR1 and ERS1. BMC Plant Biol. 7:3. doi: 10.1186/14712229-7-3

Rauf, M., Arif, M., Dortay, H., Matallana-ram, L. P., Waters, M. T., Gil Nam, H., et al. (2013). ORE1 balances leaf senescence against maintenance by antagonizing G2-like-mediated transcription. EMBO Rep. 14, 382-388. doi: 10.1038/embor.2013.24

Robatzek, S., and Somssich, I. E. (2002). Targets of AtWRKY6 regulation during plant senescence and pathogen defense. Genes Dev. 16, 1139-1149. doi: 10.1101/gad.222702

Sakamoto, T., Kamiya, N., Ueguchi-Tanaka, M., Iwahori, S., and Matsuoka, M. (2001). KNOX homeodomain protein directly suppresses the expression of a gibberellin biosynthetic gene in the tobacco shoot apical meristem. Genes Dev. 15, 581-590. doi: 10.1101/gad.867901

Schommer, C., Palatnik, J. F., Aggarwal, P., Chételat, A., Cubas, P., Farmer, E. E., et al. (2008). Control of jasmonate biosynthesis and senescence by miR319 targets. PLoS Biol. 6:e230. doi: 10.1371/journal.pbio.0060230

Seltmann, M. A., Stingl, N. E., Lautenschlaeger, J. K., Krischke, M., Mueller, M. J., and Berger, S. (2010). Differential impact of lipoxygenase 2 and jasmonates on natural and stress-induced senescence in Arabidopsis. Plant Physiol. 152, 1940-1950. doi: $10.1104 /$ pp.110.153114

Sharabi-Schwager, M., Lers, A., Samach, A., Guy, C. L., and Porat, R. (2010). Overexpression of the CBF2 transcriptional activator in Arabidopsis delays leaf senescence and extends plant longevity. J. Exp. Bot. 61, 261-273. doi: $10.1093 / \mathrm{jxb} / \mathrm{erp} 300$

Smykowski, A., Zimmermann, P., and Zentgraf, U. (2010). G-Box Binding Factorl reduces CATALASE2 expression and regulates the onset of leaf senescence in Arabidopsis. Plant Physiol. 153, 1321-1331. doi: 10.1104/pp.110.157180

Uauy, C., Distelfeld, A., Fahima, T., Blechl, A., and Dubcovsky, J. (2006). A NAC gene regulating senescence improves grain protein, zinc, and iron content in Wheat. Science 314, 1298-1301. doi: 10.1126/science.1133649
Upadhyay, R. K., Soni, D. K., Singh, R., Dwivedi, U. N., Pathre, U. V., Nath, P., (2013). SlERF36, an EAR-motif-containing ERF gene from tomato, alters stomatal density and modulates photosynthesis and growth. J. Exp. Bot. 64, 3237-3247. doi: $10.1093 / \mathrm{jxb} / \mathrm{ert} 162$

van der Graaff, E., Schwacke, R., Schneider, A., Desimone, M., and Kunze, R. (2006). Transcription analysis of Arabidopsis membrane transporters and hormone pathways during developmental and induced leaf senescence. Plant Physiol. 141, 776-792. doi: $10.1104 /$ pp.106.079293

Veyres, N., Danon, A., Aono, M., Galliot, S., Karibasappa, Y. B., Diet, A., et al. (2008). The Arabidopsis sweetie mutant is affected in carbohydrate metabolism and defective in the control of growth, development and senescence. Plant J. 55, 665-686. doi: 10.1111/j.1365-313X.2008.03541.x

Wang, H., Liu, G., Li, C., Powell, A. L., Reid, M. S., Zhang, Z., et al. (2013). Defence responses regulated by jasmonate and delayed senescence caused by ethylene receptor mutation contribute to the tolerance of petunia to Botrytis cinerea. Mol. Plant Pathol. 14, 453-469. doi: 10.1111/mpp.12017

Watanabe, M., Balazadeh, S., Tohge, T., Erban, A., Giavalisco, P., Kopka, J., et al. (2013). Comprehensive dissection of spatiotemporal metabolic shifts in primary, secondary, and lipid metabolism during developmental senescence in Arabidopsis. Plant Physiol. 162, 1290-1310. doi: 10.1104/pp.113.217380

Waters, B. M., Uauy, C., Dubcovsky, J., and Grusak, M. A. (2009). Wheat (Triticum aestivum) NAM proteins regulate the translocation of iron, zinc, and nitrogen compounds from vegetative tissues to grain. J. Exp. Bot. 60, 4263-4274. doi: 10.1093/jxb/erp257

Woo, H. R., Kim, J. H., Kim, J., Kim, J., Lee, U., Song, I., et al. (2010). The RAV1 transcription factor positively regulates leaf senescence in Arabidopsis. J. Exp. Bot. 61, 3947-3957. doi: 10.1093/jxb/erq206

Wu, A., Allu, A. D., Garapati, P., Siddiqui, H., Dortay, H., Zanor, M. I., et al. (2012). JUNGBRUNNEN1, a reactive oxygen species-responsive NAC transcription factor, regulates longevity in Arabidopsis. Plant Cell 24, 482-506. doi: 10.1105/tpc.111.090894

Yang, S.-D., Seo, P. J., Yoon, H.-K., and Park, C.-M. (2011). The Arabidopsis NAC transcription factor VNI2 integrates abscisic acid signals into leaf senescence via the COR/RD genes. Plant Cell 23, 2155-2168. doi: 10.1105/tpc.111.084913

Zhang, K., and Gan, S.-S. (2012). An abscisic acid-AtNAP transcription factor-SAG113 protein phosphatase $2 \mathrm{C}$ regulatory chain for controlling dehydration in senescing Arabidopsis leaves. Plant Physiol. 158, 961-969. doi: 10.1104/pp.111.190876

Zhao, L., Luo, Q., Yang, C., Han, Y., and Li, W. (2008). A RAV-like transcription factor controls photosynthesis and senescence in soybean. Planta 227, 1389-1399. doi: 10.1007/s00425-008-0711-7

Zhou, Y., Huang, W., Liu, L., Chen, T., Zhou, F., and Lin, Y. (2013). Identification and functional characterization of a rice NAC gene involved in the regulation of leaf senescence. BMC Plant Biol. 13:1. doi: 10.1186/1471-2229-13-132

Zhu, Z., An, F., Feng, Y., Li, P., Xue, L., Mu, A., et al. (2011). Derepression of ethylenestabilized transcription factors (EIN3/EIL1) mediates jasmonate and ethylene signaling synergy in Arabidopsis. Proc. Natl. Acad. Sci. U.S.A. 108, 12539-12544. doi: 10.1073/pnas.1103959108

Zwack, P. J., Robinson, B. R., Risley, M. G., and Rashotte, A. M. (2013). Cytokinin response factor 6 negatively regulates leaf senescence and is induced in response to cytokinin and numerous abiotic stresses. Plant Cell Physiol. 54, 971-981. doi: $10.1093 /$ pcp/pct049

Conflict of Interest Statement: The author declares that the study was conducted in the absence of any financial, commercial or other relationships that might be perceived by the academic community as representing a potential conflict of interest.

Received: 26 August 2014; paper pending published: 13 October 2014; accepted: 03 November 2014; published online: 25 November 2014.

Citation: Koyama T (2014) The roles of ethylene and transcription factors in the regulation of onset of leaf senescence. Front. Plant Sci. 5:650. doi: 10.3389/fpls.2014.00650 This article was submitted to Plant Physiology, a section of the journal Frontiers in Plant Science.

Copyright $\odot 2014$ Koyama. This is an open-access article distributed under the terms of the Creative Commons Attribution License (CC BY). The use, distribution or reproduction in other forums is permitted, provided the original author(s) or licensor are credited and that the original publication in this journal is cited, in accordance with accepted academic practice. No use, distribution or reproduction is permitted which does not comply with these terms. 


\title{
To grow old: regulatory role of ethylene and jasmonic acid in senescence
}

\author{
Joonyup Kim ${ }^{1,2}$ *, Caren Chang ${ }^{2}$ and Mark L. Tucker ${ }^{1}$ * \\ 1 Soybean Genomics and Improvement Laboratory, United States Department of Agriculture-Agricultural Research Service, Beltsville, MD, USA \\ ${ }^{2}$ Department of Cell Biology and Molecular Genetics, University of Maryland, College Park, MD, USA
}

\author{
Edited by: \\ Zuhua He, Shanghai Institutes for \\ Biological Sciences, Chinese \\ Academy of Sciences, China \\ Reviewed by: \\ Saskia C. M. Van Wees, Utrecht \\ University, Netherlands \\ Chi-Kuang Wen, Shanghai Institutes \\ for Biological Sciences, Chinese \\ Academy of Sciences, China \\ *Correspondence: \\ Joonyup Kim and Mark L. Tucker, \\ Soybean Genomics and \\ Improvement Laboratory, United \\ States Department of \\ Agriculture-Agricultural Research \\ Service, 10300 Baltimore Avenue, \\ Building 006, Room 212, \\ BARC-WEST, Beltsville, \\ MD 20705, USA \\ e-mail: joonyup.kim@ars.usda.gov; \\ mark.tucker@ars.usda.gov
}

\begin{abstract}
Senescence, the final stage in the development of an organ or whole plant, is a genetically programmed process controlled by developmental and environmental signals. Age-related signals underlie the onset of senescence in specific organs (leaf, flower, and fruit) as well as the whole plant (monocarpic senescence). Rudimentary to most senescence processes is the plant hormone ethylene, a small gaseous molecule critical to diverse processes throughout the life of the plant. The role of ethylene in senescence was discovered almost 100 years ago, but the molecular mechanisms by which ethylene regulates senescence have been deciphered more recently primarily through genetic and molecular studies in Arabidopsis. Jasmonic acid (JA), another plant hormone, is emerging as a key player in the control of senescence. The regulatory network of ethylene and JA involves the integration of transcription factors, microRNAs, and other hormones. In this review, we summarize the current understanding of ethylene's role in senescence, and discuss the interplay of ethylene with JA in the regulation of senescence.
\end{abstract}

Keywords: ethylene, jasmonic acid, leaf senescence, cross-talk, miRNA, transcription factors

\section{INTRODUCTION}

Senescence, the cessation of growth in cells, organs or the whole plant, is a highly regulated developmental process affected by environmental factors (Lim et al., 2007; Guo and Gan, 2012; Li et al., 2012). It is a pivotal turning point in plant growth and development that most often terminates with a process termed programmed cell death (PCD). Senescence (and PCD) is an active degenerative process linked to physiological and biochemical changes at the cellular, organ, and whole plant level. For example, senescence of photosynthetic tissues (e.g., leaves, stems) is accompanied by a gradual de-greening process exhibited by the loss of chlorophyll and thylakoid membranes, and increase in lipid-containing plastoglobuli (Hensel et al., 1993; Grbić and Bleecker, 1995; Gepstein, 2004). In Arabidopsis, the transition of growth to senescence constitutes the conversion from maintaining somatic tissues to supporting reproductive development by rebalancing the allocation of plant resources (Gan and Amasino, 1997; Guo and Gan, 2005; Lim et al., 2007). Transcriptome analyses of several plant species reinforce earlier observations that many genes are differentially regulated during senescence (Garbarino and Belknap, 1994; Genschik et al., 1994; Nam, 1997; Guo et al., 2004; Breeze et al., 2011). Large-scale gene expression studies highlight changes in key regulatory components including receptor-like kinases (e.g., SARK and SIRK), transcription factors (e.g., WRKY, NAC), mitogen activated protein kinases (MAPKs), metabolic pathway, and signaling pathway components for plant hormones and stress responses (Guo et al., 2004; Breeze et al., 2011). Although senescence in polycarpic and monocarpic plants may share different outcomes, it is generally accepted that the major role for senescence is the recycling of nutrients and stored energy from somatic tissues to young developing organs and reproductive organs.

The plant hormone ethylene is critical to a diverse set of developmental programs and both abiotic and biotic stress responses. In addition, ethylene affects plant aging programs such as monocarpic senescence of the whole plant as well as individual leaves, flowers, and fruit (i.e., ripening; Abeles et al., 1992). Although the involvement of ethylene in senescence has been known for over 100 years, components in the ethylene-signaling pathway and the mechanisms of action were more recently identified mostly through work in Arabidopsis.

Jasmonic acid (JA) plays a major role in plant defense as well as growth and development, including leaf and reproductive organs. Identification of JA as a plant hormone signal occurred much more recently than that of ethylene (Sembdner and Parthier, 1993). One of the first reports of JA as a hormone was the identification of methyl-jasmonate (meJA) as the senescencepromoting substance in wormwood (Ueda and Kato, 1980). Studies in Arabidopsis indicate that JA is associated with the timing of senescence programs in both somatic tissues and reproductive organs (He et al., 2002; Castillo and Leon, 2008; Schommer et al., 2008; Danisman et al., 2012; Kim et al., 2013a). Notably, 
recent studies revealed that JA's role in senescence involves similar regulatory circuits as used by ethylene [e.g., microRNA (miRNA)transcription factors; Schommer et al., 2008]. In summary, the published results indicate that cross-talk between JA and ethylene fine-tunes the onset and timing of senescence in Arabidopsis.

Our objective here is to summarize current advances in understanding the mechanism by which ethylene regulates leaf senescence in Arabidopsis, and the role of JA in senescence in the context of its interplay with ethylene.

\section{ETHYLENE AND LEAF SENESCENCE}

All major hormones are found to be associated with leaf senescence either positively or negatively (Gan and Amasino, 1997; Khan et al., 2014). Although ethylene was one of the first plant hormones identified to accelerate senescence (Neljubow, 1901; Crocker, 1932), the mechanism by which ethylene regulates senescence remained inexplicit. Recent genetic and molecular studies in Arabidopsis have unraveled a fundamental signaling pathway for ethylene based on the ethylene regulated growth response (triple response) of etiolated seedlings. Briefly, ethylene is first perceived by its receptor [e.g., ETHYLENE RESPONSE 1 (ETR1)] residing at the endoplasmic reticulum (ER) membrane. Upon binding ethylene, the receptor stops activating the serine/threonine protein kinase CONSTITUTIVE RESPONSE 1 (CTR1), which causes CTR1 to stop phosphorylating the ER membrane-localized ETHYLENE INSENSITIVE 2 (EIN2) protein. When not phosphorylated, the cytosolic C-terminus of EIN2 is cleaved by an unidentified protease. After cleavage, the EIN2 Cterminus moves into the nucleus where it activates transcription (Ju et al., 2012; Qiao et al., 2012; Wen et al., 2012; Ji and Guo, 2013). ETHYLENE INSENSITIVE 3 (EIN3) and its close homolog, ETHYLENE INSENSITIVE3-LIKE 1 (EIL1), are key transcription factors activated by EIN2 (Alonso et al., 2003; Guo and Ecker, 2003; Potuschak et al., 2003; Binder et al., 2004, 2007; Gagne et al., 2004; An et al., 2010).

Much of what we currently know about the role of ethylene signaling in senescence comes from studies with genetic mutants that lack the ethylene dependent triple response in dark-grown seedlings. For example, the Arabidopsis ethyleneinsensitive mutants etr1-1, ein2-1, and ein1-1 (which is allelic to etr1-1) were shown to have a delayed onset of leaf senescence and expression of leaf senescence marker genes, e.g., SAG1, SAG2, and SAG12 (Grbić and Bleecker, 1995). The leaves of these mutants do, however, eventually senesce indicating that the role for ethylene in leaf senescence is not essential, but, in Arabidopsis, modulates the timing of senescence. In support of this role, when ethylene perception or biosynthesis was genetically inhibited in tobacco and tomato, the plants exhibited a delay in the onset of leaf senescence (Picton et al., 1993; John et al., 1995; Yang et al., 2008). In contrast, mutants with enhanced ethylene biosynthesis or a constitutive ethylene response (e.g., ctr1) did not always display an early onset of senescence (Guzman and Ecker, 1990; Kieber et al., 1993; Lanahan et al., 1994; Woeste et al., 1999). For instance, the ctrl mutant of Arabidopsis, which possesses constitutively activated ethylene signaling, did not display precocious leaf senescence, and the exogenous application of ethylene to the mutant did not affect the rate of senescence (Kieber et al., 1993). The lack of precocious leaf senescence in the ctrl mutant may be partly due to the fact that the $\operatorname{ctr} 1$ plants display a delayed flowering time compared to wild-type plants (Hua and Meyerowitz, 1998; Hall and Bleecker, 2003); however, when leaves were detached from the $\operatorname{ctr} 1$ mutant, the detached leaves clearly displayed accelerated senescence (Xu et al., 2014). Moreover, ectopic expression of 1aminocyclopropane-1-carboxylic acid (ACC) synthase in tomato, which overproduces ethylene, did not accelerate the rate of leaf senescence (Lanahan et al., 1994). These results suggest that, although ethylene plays a role in the timing of senescence, there are developmental conditions including flowering and/or other environmental cues that must precede ethylene if ethylene is to induce a senescence response.

Additional support for the role of ethylene in senescence comes from the identification of Arabidopsis mutants with a delayed leaf senescence phenotype (Oh et al., 1997). The oresara mutants (ore 1 , ore 2 , ore 3 , and ore 9 ) were identified as having a decrease in chlorophyll content, an indication of leaf senescence, and reduced photochemical efficiency of PSII (Fv/Fm), a parameter of functional leaf senescence (Oh et al., 1997). Genetic complementation of ore 2 and ore 3 confirmed that these two mutants are allelic to ein2, supporting earlier observations that the signaling cascade of ethylene is tightly associated with leaf senescence.

Between 2004 and 2005, several laboratories independently demonstrated that the miRNA, miR164, is involved in the control of mRNA levels for NAC-domain transcription factors, which were previously shown to be essential for organ differentiation and development (Laufs et al., 2004; Mallory et al., 2004; Baker et al., 2005; Guo et al., 2005). In 2009, Kim et al. (2009) reported that the ORE1, which was identified earlier in a screen for senescence associated genes (SAGs; Woo et al., 2004), is allelic to NAC2; moreover, they demonstrated that miR164ABC expression inversely correlated with the expression of NAC2 transcript. Through a combination of overexpression lines and genetically suppressed mutants of miR164, NAC2 and EIN2, they demonstrated a link between leaf senescence, ethylene signaling, and a decline in miR164 RNA, which leads to an increase in NAC2 expression (Kim et al., 2009; Li et al., 2013).

The role of ethylene signaling was further substantiated by the identification of EIN3 as another SAG in the ethylene-induced leaf senescence network and that elevated EIN3 expression accelerated the onset of senescence ( $\mathrm{Li}$ et al., 2013). In addition, Li et al. (2013) demonstrated by chromatin immunoprecipitation (ChIP) and electrophoretic mobility shift assays (EMSA) that EIN3 binds to the promoter for miR164. Subsequently, it was demonstrated by ChIP and a yeast one-hybrid (Y1H) assay that EIN3 not only binds to the miR164 promoter but also directly binds to the promoters of NAC2 and another NAC-domain gene, NAP, both of which were identified as SAGs (Kim et al., 2014). EIN3 and EIL1 are closely related homologues that appear to have redundant and distinct functions in ethylene responses (Chao et al., 1997; Binder et al., 2007). Both Li et al. (2013) and Kim et al. (2014) used ein3 eil1 double mutants to preclude complications in interpretation that might arise from their functional redundancy. They observed that ein 2 mutants displayed a more delayed senescence phenotype than the ein 3 eill double mutants. They concluded that EIN2, which precedes EIN3 and EIL1 in the ethylene-signaling pathway, 
controls senescence that is both dependent and independent of EIN3/EIL1. They proposed that EIN2 signaling somehow bypasses EIN3/EIL1. Although both Li et al. (2013) and Kim et al. (2014) suggested that EIN2-EIN3-miR164 regulate the timing of leaf senescence, further experiments may be necessary to firmly establish EIN3's role in the natural progression of leaf senescence. For instance, it may be useful to examine the expression of other EIL proteins (i.e., EIL2, EIL3, and EIL4) in relation to EIN3, particularly in leaf senescence.

To summarize, the proposed role for ethylene signaling in leaf senescence is to coordinate the timing of leaf senescence by integrating miR164-transcription factors in concert with environmental and age-related signals, which appears to ensure the timely and efficient transition from an active photosynthetic organ to a degenerating organ and to also salvage nutrients for development of reproductive organs and young leaves (Grbić and Bleecker, 1995).

\section{JASMONIC ACID AND LEAF SENESCENCE}

Jasmonic acid is another plant hormone that modulates defense responses, growth and development, and is also proposed to mediate leaf senescence (He et al., 2002; Schommer et al., 2008; Shan et al., 2011). A review of the literature on the involvement of JA in leaf senescence is, however, sometimes contradictory (Taylor and Whitelaw, 2001; Seltmann et al., 2010a,b) and not fully resolved. For example, many JA biosynthesis genes are differentially regulated, as some are up-regulated and others are downregulated in the progression of leaf senescence (He et al., 2002). In addition, several studies suggested that synthesis of JA during leaf senescence might be a secondary byproduct from the breakdown of macromolecules and membranes in the process of senescence. These studies suggested that the increase in oxylipins levels such as JA and 12-oxo-phytodienoic acid (OPDA) do not necessarily indicate a role for JA in natural leaf senescence but a byproduct of senescence (Seltmann et al., 2010a,b).

However, in Arabidopsis, recent studies suggest that JA does have a role in leaf senescence. Mutations in the JA receptor and JA biosynthesis genes, and physiological responses to JA or various JA derivatives, indicate that JA does mediate the timing of leaf senescence. Arabidopsis mutants allene oxide synthase (aos), oxophytodienoate-reductase 3 (opr3), which have reduced levels of JA, and coronatine insensitive 1 (coil), which is insensitive to JA, exhibit temporal shifts in the onset of natural and dark-induced senescence (He et al., 2002; Castillo and Leon, 2008; Schommer et al., 2008; Danisman et al., 2012). In addition, exogenous application of JA on wild-type Arabidopsis promotes leaf senescence and induces the expression of several $S A G$, including JA biosynthesis genes (He et al., 2002). Moreover, in naturally senescing leaves, a gradual increase in the transcript levels of JA biosynthesis genes (e.g., AOS, LIPOXYGENASE1 (LOX1), LOX3, LOX4, OPR1, and OPR3) was observed (He et al., 2002). Thus, results with Arabidopsis support earlier proposals that JA positively affects the timing of leaf senescence in conjunction with other age-related cues (He et al., 2002).

Recently, it was shown that the miRNA miR319 [JAGGED AND WAVY $(J A W)$ ] regulates the mRNA levels of the transcription factors called TEOSINTE BRANCHED/CYCLOIDEA/PCF
(TCP; Schommer et al., 2008). TCPs are plant-specific transcription factors identified as negative regulators of plant growth and development (Almeida et al., 1997; Doebley et al., 1997; Luo et al., 1999). In addition, TCPs were found to be critical for the expression of cell cycle regulators called proliferating cell nuclear antigen, PCNA (Kosugi and Ohashi, 1997). Schommer et al. (2008) showed that miR319, which affected the expression of class II TCP2/4/10, altered the expression of JA biosynthesis genes and the endogenous levels of JA. More specifically, they demonstrated that the miR319-TCP regulatory module regulated JA biosynthesis by repressing the expression of LOX2. They also showed that the reduced levels of JA resulted in a delay in leaf senescence, and that the delayed senescence phenotype was reversed by application of JA. They concluded that, whereas TCP2/4/10 negatively regulate leaf growth, they positively regulate leaf senescence.

In a separate study, it was revealed that a class I TCP (TCP20) also regulates LOX2 (Danisman et al., 2012). Mutations in TCP20 caused early onset of leaf senescence indicating that TCP20 acts as a negative regulator. They further demonstrated that TCP20 binds to the promoter of the LOX2 gene (Danisman et al., 2012). They proposed that class I and class II TCPs act antagonistically to regulate LOX gene expression. Interestingly, RNAi suppression of LOX2 expression, which was targeted specifically to mature leaves by using the $S A G 13$ promoter, greatly decreased the accumulation of JA as leaves aged naturally but had no effect on chlorophyll loss (Seltmann et al., 2010b). Conversely, when senescence was induced prematurely by treatment with sorbitol, chlorophyll loss and SEN1 and SAG13 expression were delayed. However, it is worth noting that the peak transcript levels of LOX2 preceded the onset of leaf senescence and declined as leaf senescence progressed (He et al., 2002), as reflected in a decline in the level of JA (Seltmann et al., 2010b). It is possible that the regulation of miR319-TCPLOX2 represents one of the early regulatory mechanisms in leaf development that alters leaf senescence and that expression of LOX2-RNAi with the SAG13 promoter occurred too late in the natural aging process to yield a delayed senescence response. In summary, although some results for JA signaling seem to contradict a role for JA in leaf senescence, it is worth emphasizing that the phenotype of the miR319 mutant, jaw-D, could be reversed by the exogenous application of meJA (Schommer et al., 2008).

\section{INTERPLAY BETWEEN ETHYLENE AND JA IN LEAF SENESCENCE}

Although the interaction between ethylene and JA has been well studied in diverse developmental, abiotic and biotic responses (Turner et al., 2002; Lorenzo et al., 2003; Memelink, 2009; Zhu et al., 2011; Kim et al., 2013a; Song et al., 2014; Zhang et al., 2014), a clear understanding of the interplay between ethylene and JA in mediation of leaf senescence is less well defined. Nevertheless, recent work on ethylene and JA signaling is beginning to shed light on the regulatory mechanisms by which these two senescenceaffecting hormones control the timing of leaf senescence.

Several studies have defined mechanisms by which ethylene and JA interact to regulate plant development and pathogen defense (Zhu et al., 2011; Song et al., 2014; Zhang et al., 2014). 
Zhu et al. (2011) showed that EIN3 and EIL1 positively regulate JA-mediated responses such as root hair development, resistance to necrotrophic pathogens, and related gene expression. They demonstrated that JAZ proteins (e.g., JAZ1, 3, and 9) physically interact with EIN3 and EIL1, and repress the transcriptional activities of EIN3/EIL1. They further showed that JAZ protein (JAZ1) recruits HISTONE DEACETYLASE 6 (HDA6), which deacetylates the chromatin of EIN3/EIL1 and represses EIL3/EIL1-dependent transcription and JA signaling. It would be interesting to know if a similar mechanism is utilized in senescence. In this regard, it has been demonstrated that HDA6 positively regulates JA-mediated leaf senescence as well as flowering time, which also influences the timing of senescence (Wu et al., 2008).

In addition to HDA6 control of EIN3/EIL1, Song et al. (2014) demonstrated that JA activated MYC2 repressed EIN3/EIL1 transcription. Conversely, EIN3/EIL1 repressed MYC2, which in turn inhibits JA responses. This reciprocating regulation may finetune ethylene/JA responses. Specifically, they showed that MYC2 represses transcriptional activities of EIN3/EIL1 to attenuate the mRNA levels of HOOKLESS 1 (HLS1, a positive regulator of apical hook development) and ETHYLENE RESPONSE FACTOR 1 (ERF1), which thereby inhibits ethylene-induced apical hook curvature. In addition, they showed that EIN3/EIL1 interact with MYC2/3/4 to attenuate JA-induced expression of woundherbivory-responsive genes, which reduces JA-regulated defense against herbivores.

In another independent study, Zhang et al. (2014) also demonstrated an antagonistic regulation between MYC2 and EIN3 in the ethylene-induced hook curvature formation. In this study, they showed that JA inhibits the formation of apical hook curvature by reducing HLS1 gene expression. They showed that JA-activated MYC2 transcriptionally represses EIN3 to reduce HLS1 activity. They proposed a dual mode of action for MYC2. Firstly, MYC2 promotes expression of the EIN3 BINDING F-BOX PROTEIN 1 (EBF1) that then promotes EIN3 degradation and, secondly, MYC2 repression of EIN3 inhibits the positive regulation by EIN3 of HSL1. However, it remains to be determined if these mutual antagonistic actions between ethylene and JA signaling in the apical hook formation also regulate the timing of leaf senescence.

More specific to senescence are two studies by Li et al. (2013) and Kim et al. (2013a). As discussed earlier, Li et al. (2013) demonstrated that JA regulation of senescence is dependent upon the key ethylene-signaling components EIN2 and EIN3/EIL1. In comparison to wild-type leaves, detached leaves of ein2 and ein3 eil1 mutants treated with $50 \mu \mathrm{M}$ of meJA largely stayed green. In addition, when wild-type leaves were co-treated with $100 \mu \mathrm{M}$ silver nitrate $(\mathrm{AgNO} 3)$, an inhibitor of ethylene action, and $50 \mu \mathrm{M}$ of meJA, the leaves remained largely green, suggesting that JA-induced leaf senescence is dependent upon ethylene signaling (Li et al., 2013).

In another project, Kim et al. (2013a) examined the interdependency of ethylene and JA in floral organ abscission. Although leaf senescence was not a major focus of that report, they did collect data for leaf senescence as well. In that project they observed that exogenous application of $200 \mu \mathrm{M}$ meJA accelerated the timing of floral organ abscission and leaf senescence in the ein2-1 mutant (Figure 1). Kim et al. (2013a) proposed that ethylene and
A

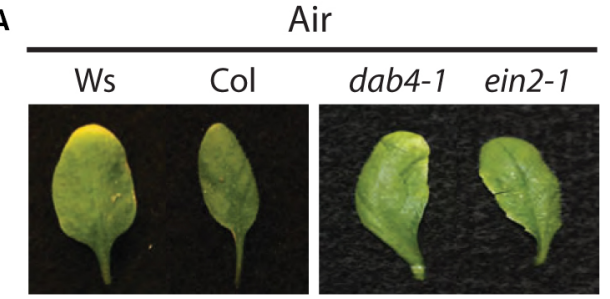

B

Ethylene (1ppm)

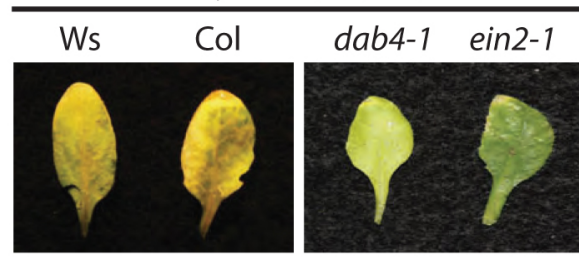

C

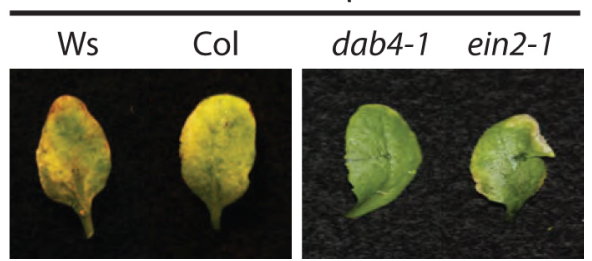

FIGURE 1 | Interdependency of ethylene and JA in leaf senescence. Leaf senescence phenotypes with air (A), ethylene (B), and meJA (C) treatments in wild-type plants (Ws and Col), a JA receptor mutant (dab4-1/coi1-37) and an ethylene insensitive mutant (ein2-1). This figure was originally published in Kim et al. (2013a) as supplemental Figure S4 Whole plant assays were carried out in a closed chamber 6 weeks after germination when the plants had bolted as previously described (Kim et al., 2013a,b).

JA act partly in parallel pathways to regulate the timing of both floral organ abscission and leaf senescence. Although the degree of accelerated leaf senescence in the Kim et al. (2013a) study appears to be minor, the discrepancy of JA dependency on EIN2 in the two separate senescence studies may be explained by differences in how the experiments were performed. In the Li et al. (2013) study, the leaves were detached from the wild type and ein2-5 plants at 3-4 weeks and then treated with a low concentration of $50 \mu \mathrm{M}$ meJA. In the Kim et al. (2013a) study, after 6 weeks when the plants had bolted and produced several inflorescences, the whole plant was sprayed twice a day for 3 days with $200 \mu \mathrm{M}$ meJA. In addition to the different meJA concentrations, which may have had a minor influence, the developmental stage of the leaves were different and, as previously mentioned, the development stage of the plant is also important to how JA influences the timing of senescence (Kim et al., 2013b; Li et al., 2013).

Of further interest in regard to ethylene and JA interdependency is that JA was found to alter ethylene signaling downstream of the ethylene receptors (Kim et al., 2013b). In this study, it was discovered that when JA synthesis was chemically or genetically inhibited with phenidone or dde2-2, respectively, ein2-1 mutants abscised earlier, and root growth was inhibited in responsive to $1 \mu \mathrm{L} \mathrm{L}^{-1}$ ethylene. Similar results were obtained with the coil 


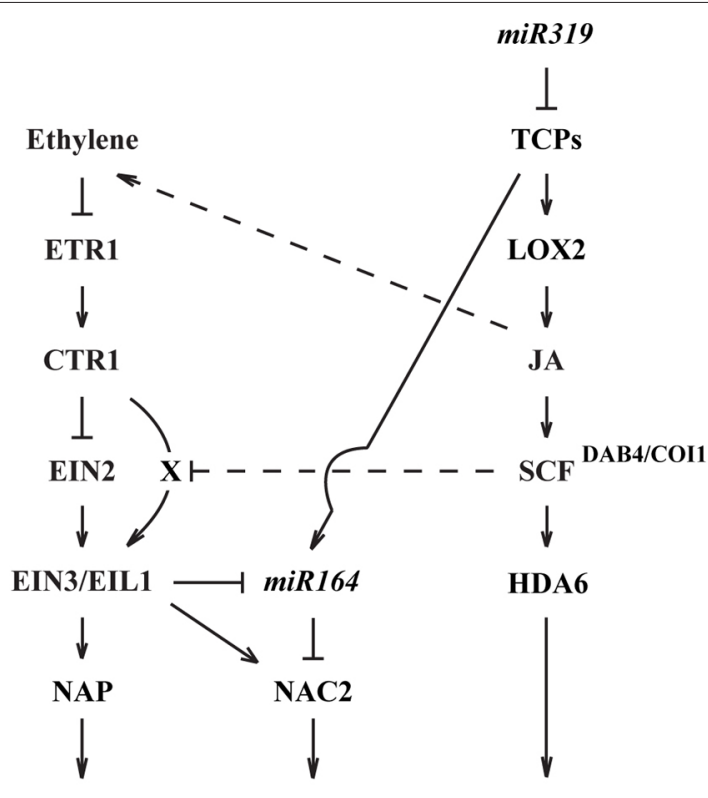

ONSET AND PROGRESSION OF LEAF SENESCENCE

FIGURE 2 | A schematic diagram for interactions between ethylene and JA in the regulation of leaf senescence. Known regulations are shown in solid lines and unidentified pathways are represented in dotted lines. $X$ denotes an unknown ethylene-signaling component that may be inhibited by JA or SCF ${ }^{\mathrm{DAB} 4 / \mathrm{COI} 1}$. See the text for details and supporting references.

ein2 double mutants. They concluded that there is an EIN2independent pathway for ethylene signaling that is inhibited by JA and, when JA is very low or JA signaling is blocked as in the coil mutant, the EIN2 independent ethylene signaling pathway is discernible in the ein 2 mutant. In this study, the authors did not examine leaf senescence. It would, however, be interesting to know if the EIN2 independent ethylene signaling pathway plays a role in leaf senescence and JA responsiveness (Figure 2).

\section{CONCLUDING REMARKS AND PERSPECTIVE}

Recent technological advances in transcriptome analyses, including mRNA and miRNA sequencing and ChIP, have greatly enhanced our understanding of signaling networks that control developmentally and environmentally affected process. Although these tools have helped to significantly advance our understanding of how ethylene and JA regulate senescence, there is still much more to learn. We have reviewed studies demonstrating the role of ethylene perception and its signaling pathway in the regulation of senescence. Of particular interest were recent findings demonstrating the interaction between miR164 and EIN3 to regulate the expression of NAC2, which in turn regulates the onset of senescence (Figure 2). We also reviewed independent studies of JA's control of senescence and how miR319 regulates the mRNA level of class II TCPs, which antagonistically work with class I TCPs to control the expression of LOX2, which is needed for synthesis of JA (Figure 2). In addition, we briefly reviewed the role of HDA6 and MYC2 in other develop- mental and defense responses where ethylene and JA signaling interact.

Senescence and the timing of the onset of senescence are complex. There is evidence to suggest that all of the known plant hormones have some influence over the timing of abscission, and many environmental factors and additional internal cues modulate this process (Gan and Amasino, 1997; Khan et al., 2014). Discovery of miRNAs and transcription factors involved in the regulation of the onset of leaf senescence identifies additional points in the network of senescence signals by which the complex process of senescence can be modulated by environmental and internal cues.

In addition to what we currently know about the regulation of leaf senescence, basic questions still need to be addressed. JA control of senescence appears to be dependent on ethylene perception and signaling except for a nuance, which suggests that at low JA levels ethylene signaling can bypass EIN2 (Kim et al., 2013b). Although ethylene signaling may bypass EIN2 under certain conditions, ethylene perception was shown to be necessary for JA responsiveness. It seems logical that JA might modulate senescence through regulation of ethylene biosynthesis (Figure 2). In fact, JA regulation of ethylene biosynthesis has been proposed for other processes (Wang et al., 2002), but the mechanism of regulation has not been well established. ACC is the immediate precursor to ethylene, and Staswick and Tiryaki (2004) discovered that JA can form a conjugate with ACC (JA-ACC). These authors suggested that conjugation of ACC by JA might play a role in regulating ethylene biosynthesis.

Also of interest are studies on miR319 and TCPs. It was demonstrated that miR319 and TCPs regulate CUC2 (NAC2/ ORE1) expression during meristem development (Koyama et al., 2007). This work on regulation of morphogenesis in the apical meristem suggests that NAC2 expression during senescence may also be regulated by miR319 and TCPs during leaf senescence. If true, this would tie together regulation of JA biosynthesis and ethylene signaling through regulation of EIN3 and NAC2 (Figure 2). The role of HDA6 and MYC2 in regulating ethylene and JA interactions in the control of apical hook formation and defense responses is interesting and should be examined further for a regulatory role in senescence. Much still needs to be done to understand the multitude of factors that mediate aging and the timing of senescence in both plant organs and whole plants.

\section{ACKNOWLEDGMENT}

This work was supported by a Binational Agricultural and Development Fund (BARD) US-4571-12C grant to Mark L. Tucker, and funding for Open Access was provided by the UMD Libraries Open Access Publishing Fund.

\section{REFERENCES}

Abeles, F., Morgan, P., and Saltveit, M. J. (1992). Ethylene in Plant Biology. San Diego, CA: Academic Press.

Almeida, J., Rocheta, M., and Galego, L. (1997). Genetic control of flower shape in Antirrhinum majus. Development 124, 1387-1392.

Alonso, J. M., Stepanova, A. N., Solano, R., Wisman, E., Ferrari, S., Ausubel, F. M., et al. (2003). Five components of the ethylene-response pathway identified in 
a screen for weak ethylene-insensitive mutants in Arabidopsis. Proc. Natl. Acad. Sci. U.S.A. 100, 2992-2997. doi: 10.1073/pnas.0438070100

An, F., Zhao, Q., Ji, Y., Li, W., Jiang, Z., Yu, X., et al. (2010). Ethylene-induced stabilization of ETHYLENE INSENSITIVE3 and EIN3-LIKE1 is mediated by proteasomal degradation of EIN3 binding F-Box 1 and 2 that requires EIN2 in Arabidopsis. Plant Cell 22, 2384-2401. doi: 10.1105/tpc.110.076588

Baker, C. C., Sieber, P., Wellmer, F., and Meyerowitz, E. M. (2005). The early extra petals1 mutant uncovers a role for microRNA miR164c in regulating petal number in Arabidopsis. Curr. Biol. 15, 303-315. doi: 10.1016/j.cub.2005.02.017

Binder, B. M., O’Malley, R. C., Wang, W., Moore, J. M., Parks, B. M., Spalding, E. P., et al. (2004). Arabidopsis seedling growth response and recovery to ethylene. A kinetic analysis. Plant Physiol. 136, 2913-2920. doi: 10.1104/pp.104.050369

Binder, B. M., Walker, J. M., Gagne, J. M., Emborg, T. J., Hemman, G., Bleecker, A. B., et al. (2007). The Arabidopsis EIN3-binding F-Box proteins, EBF1 and 2 have distinct but overlapping roles in regulating ethylene signaling. Plant Cell 19, 509-523. doi: 10.1105/tpc.106.048140

Breeze, E., Harrison, E., Mchattie, S., Hughes, L., Hickman, R., Hill, C., et al. (2011). High-resolution temporal profiling of transcripts during Arabidopsis leaf senescence reveals a distinct chronology of processes and regulation. Plant Cell 23, 873-894. doi: 10.1105/tpc.111.083345

Castillo, M. C., and Leon, J. (2008). Expression of the beta-oxidation gene 3-ketoacyl-CoA thiolase 2 (KAT2) is required for the timely onset of natural and dark-induced leaf senescence in Arabidopsis. J. Exp. Bot. 59, 2171-2179. doi: 10.1093/jxb/ern079

Chao, Q., Rothenberg, M., Solano, R., Roman, G., Terzaghi, W., and Ecker, J. R. (1997). Activation of the ethylene gas response pathway in Arabidopsis by the nuclear protein ETHYLENE-INSENSITIVE3 and related proteins. Cell 89, 1133-1144. doi: 10.1016/S0092-8674(00)80300-1

Crocker, W. (1932). The effect of ethylene upon living organisms. Proc. Am. Philos. Soc. 71, 295-298.

Danisman, S., Van Der Wal, F., Dhondt, S., Waites, R., De Folter, S., Bimbo, A., et al. (2012). Arabidopsis class I and class II TCP transcription factors regulate jasmonic acid metabolism and leaf development antagonistically. Plant Physiol. 159, 1511-1523. doi: 10.1104/pp.112.200303

Doebley, J., Stec, A., and Hubbard, L. (1997). The evolution of apical dominance in maize. Nature 386, 485-488. doi: 10.1038/386485a0

Gagne, J. M., Smalle, J., Gingerich, D. J., Walker, J. M., Yoo, S. D., Yanagisawa, S., et al. (2004). Arabidopsis EIN3-binding F-box 1 and 2 form ubiquitin-protein ligases that repress ethylene action and promote growth by directing EIN3 degradation. Proc. Natl. Acad. Sci. U.S.A. 101, 6803-6808. doi: 10.1073/pnas. 0401698101

Gan, S., and Amasino, R. (1997). Making sense of senescence. Plant Physiol. 113, 313-319.

Garbarino, J. E., and Belknap, W. R. (1994). Isolation of a ubiquitin-ribosomal protein gene (ubi3) from potato and expression of its promoter in transgenic plants. Plant Mol. Biol. 24, 119-127. doi: 10.1007/BF00040579

Genschik, P., Durr, A., and Fleck, J. (1994). Differential expression of several E2-type ubiquitin carrier protein genes at different developmental stages in Arabidopsis thaliana and Nicotiana sylvestris. Mol. Gen. Genet. 244, 548-556. doi: 10.1007/BF00583906

Gepstein, S. (2004). Leaf senescence-not just a 'wear and tear' phenomenon. Genome Biol. 5, 212. doi: 10.1186/gb-2004-5-3-212

Grbić, V., and Bleecker, A. (1995). Ethylene regulates the timing of leaf senescence in Arabidopsis. Plant J. 8, 595-602. doi: 10.1046/j.1365-313X.1995.8040595.x

Guo, H. S., Xie, Q., Fei, J. F., and Chua, N. H. (2005). MicroRNA directs mRNA cleavage of the transcription factor NACl to downregulate auxin signals for Arabidopsis lateral root development. Plant Cell 17, 1376-1386. doi: 10.1105/ tpc. 105.030841

Guo, H. W., and Ecker, J. R. (2003). Plant responses to ethylene gas are mediated by SCF (EBF1/EBF2)-dependent proteolysis of EIN3 transcription factor. Cell 115, 667-677. doi: 10.1016/S0092-8674(03)00969-3

Guo, Y., Cai, Z., and Gan, S. (2004). Transcriptome of Arabidopsis leaf senescence. Plant Cell Environ. 27, 521-549. doi: 10.1111/j.1365-3040.2003.01158.x

Guo, Y., and Gan, S. (2005). Leaf senescence: signals, execution, and regulation. Curr. Top. Dev. Biol. 71, 83-112. doi: 10.1016/S0070-2153(05)71003-6

Guo, Y., and Gan, S. S. (2012). Convergence and divergence in gene expression profiles induced by leaf senescence and 27 senescence-promoting hormonal, pathological and environmental stress treatments. Plant Cell Environ. 35, 644655. doi: 10.1111/j.1365-3040.2011.02442.x
Guzman, P., and Ecker, J. R. (1990). Exploiting the triple response of Arabidopsis to identify ethylene-related mutants. Plant Cell 2, 513-523. doi: 10.1105/tpc. 2.6.513

Hall, A. E., and Bleecker, A. B. (2003). Analysis of combinatorial loss-of-function mutants in the Arabidopsis ethylene receptors reveals that the ers 1 etr1 double mutant has severe developmental defects that are EIN2 dependent. Plant Cell 15, 2032-2041. doi: 10.1105/tpc.013060

He, Y., Fukushige, H., Hildebrand, D. F., and Gan, S. (2002). Evidence supporting a role of jasmonic acid in Arabidopsis leaf senescence. Plant Physiol. 128, 876-884. doi: 10.1104/pp.010843

Hensel, L. L., Grbic, V., Baumgarten, D. A., and Bleecker, A. B. (1993). Developmental and age-related processes that influence the longevity and senescence of photosynthetic tissues in Arabidopsis. Plant Cell 5, 553-564. doi: 10.1105/tpc. 5.5.553

Hua, J., and Meyerowitz, E. M. (1998). Ethylene responses are negatively regulated by a receptor gene family in Arabidopsis thaliana. Cell 94, 261-271. doi: $10.1016 / \mathrm{S} 0092-8674(00) 81425-7$

Ji, Y., and Guo, H. (2013). From endoplasmic reticulum (ER) to nucleus: EIN2 bridges the gap in ethylene signaling. Mol. Plant 6, 11-14. doi: 10.1093/mp/ sss 150

John, I., Drake, R., Farrell, A., Cooper, W., Lee, P., Horton, P., et al. (1995). Delayed leaf senescence in ethylene-deficient ACC-oxidase antisense tomato plants: molecular and physiological analysis. Plant J. 7, 483-490. doi: 10.1046/j.1365313X.1995.7030483.x

Ju, C., Yoon, G. M., Shemansky, J. M., Lin, D. Y., Ying, Z. I., Chang, J., et al. (2012). CTR1 phosphorylates the central regulator EIN2 to control ethylene hormone signaling from the ER membrane to the nucleus in Arabidopsis. Proc. Natl. Acad. Sci. U.S.A. 109, 19486-19491. doi: 10.1073/pnas.1214848109

Khan, M., Rozhon, W., and Poppenberger, B. (2014). The role of hormones in the aging of plants-a mini-review. Gerontology 60, 49-55. doi: 10.1159/000354334

Kieber, J. J., Rothenberg, M., Roman, G., Feldman, K. A., and Ecker, J. R. (1993). CTR1, a negative regulator of the ethylene response pathway in Arabidopsis, encodes a member of the raf family of protein kinases. Cell 72, 427-441. doi: 10.1016/0092-8674(93)90119-B

Kim, H. J., Hong, S. H., Kim, Y. W., Lee, I. H., Jun, J. H., Phee, B. K., et al. (2014). Gene regulatory cascade of senescence-associated NAC transcription factors activated by ETHYLENE-INSENSITIVE2-mediated leaf senescence signalling in Arabidopsis. J. Exp. Bot. 65, 4023-4036. doi: 10.1093/jxb/eru112

Kim, J., Dotson, B., Rey, C., Lindsey, J., Bleecker, A. B., Binder, B. M., et al. (2013a). New clothes for the jasmonic acid receptor COI1: delayed abscission, meristem arrest and apical dominance. PLoS ONE 8:e60505. doi: 10.1371/journal.pone. 0060505

Kim, J., Patterson, S. E., and Binder, B. M. (2013b). Reducing jasmonic acid levels causes ein2 mutants to become ethylene responsive. FEBS Lett. 587, 226-230. doi: 10.1016/j.febslet.2012.11.030

Kim, J. H., Woo, H. R., Kim, J., Lim, P. O., Lee, I. C., Choi, S. H., et al. (2009). Trifurcate feed-forward regulation of age-dependent cell death involving miR164 in Arabidopsis. Science 323, 1053-1057. doi: 10.1126/science.1166386

Kosugi, S., and Ohashi, Y. (1997). PCF1 and PCF2 specifically bind to cis elements in the rice proliferating cell nuclear antigen gene. Plant Cell 9, 1607-1619. doi: 10.1105/tpc.9.9.1607

Koyama, T., Furutani, M., Tasaka, M., and Ohme-Takagi, M. (2007). TCP transcription factors control the morphology of shoot lateral organs via negative regulation of the expression of boundary-specific genes in Arabidopsis. Plant Cell 19, 473-484. doi: 10.1105/tpc.106.044792

Lanahan, M. B., Yen, H. C., Giovannoni, J. J., and Klee, H. J. (1994). The never ripe mutation blocks ethylene perception in tomato. Plant Cell 6, 521-530. doi: $10.1105 /$ tpc.6.4.521

Laufs, P., Peaucelle, A., Morin, H., and Traas, J. (2004). MicroRNA regulation of the CUC genes is required for boundary size control in Arabidopsis meristems. Development 131, 4311-4322. doi: 10.1242/dev.01320

Li, Z., Peng, J., Wen, X., and Guo, H. (2012). Gene network analysis and functional studies of senescence-associated genes reveal novel regulators of Arabidopsis leaf senescence. J. Integr. Plant Biol. 54, 526-539. doi: 10.1111/j.1744-7909. 2012.01136.x

Li, Z., Peng, J., Wen, X., and Guo, H. (2013). Ethylene-insensitive3 is a senescenceassociated gene that accelerates age-dependent leaf senescence by directly repressing miR164 transcription in Arabidopsis. Plant Cell 25, 3311-3328. doi: $10.1105 /$ tpc. 113.113340 
Lim, P. O., Kim, H. J., and Nam, H. G. (2007). Leaf senescence. Annu. Rev. Plant Biol. 58, 115-136. doi: 10.1146/annurev.arplant.57.032905.105316

Lorenzo, O., Piqueras, R., Sanchez-Serrano, J. J., and Solano, R. (2003). ETHYLENE RESPONSE FACTOR1 integrates signals from ethylene and jasmonate pathways in plant defense. Plant Cell 15, 165-178. doi: 10.1105/tpc.007468

Luo, D., Carpenter, R., Copsey, L., Vincent, C., Clark, J., and Coen, E. (1999). Control of organ asymmetry in flowers of Antirrhinum. Cell 99, 367-376. doi: 10.1016/S0092-8674(00)81523-8

Mallory, A. C., Dugas, D. V., Bartel, D. P., and Bartel, B. (2004). MicroRNA regulation of NAC-domain targets is required for proper formation and separation of adjacent embryonic, vegetative, and floral organs. Curr. Biol. 14, 1035-1046. doi: 10.1016/j.cub.2004.06.022

Memelink, J. (2009). Regulation of gene expression by jasmonate hormones. Phytochemistry 70, 1560-1570. doi: 10.1016/j.phytochem.2009.09.004

Nam, H. G. (1997). The molecular genetic analysis of leaf senescence. Curr. Opin. Biotechnol. 8, 200-207. doi: 10.1016/S0958-1669(97)80103-6

Neljubow, D. (1901). Uber die horizontale Nutation der Stengel von Pisum sativum und einiger anderen Pflanzen. Beih. Bot. Zentralb. 10, 128-139.

Oh, S., Park, J.-H., Lee, G., Paek, K., Park, S., and Nam, H. (1997). Identification of three genetic loci controlling leaf senescence in Arabidopsis thaliana. Plant J. 12, 527-535. doi: 10.1046/j.1365-313X.1997.00489.x

Picton, S., Barton, S., Bouzayen, M., Hamilton, A., and Grierson, D. (1993). Altered fruit ripening and leaf senescence in tomatoes expressing an antisense ethylene-forming enzyme transgene. Plant J. 3, 469-481. doi: 10.1111/j.1365313X.1993.tb00167.x

Potuschak, T., Lechner, E., Parmentier, Y., Yanagisawa, S., Grava, S., Koncz, C., et al. (2003). EIN3-dependent regulation of plant ethylene hormone signaling by two Arabidopsis F box proteins: EBF1 and EBF2. Cell 115, 679-689. doi: 10.1016/S0092-8674(03)00968-1

Qiao, H., Shen, Z., Huang, S. S., Schmitz, R. J., Urich, M. A., Briggs, S. P., et al. (2012). Processing and subcellular trafficking of ER-tethered EIN2 control response to ethylene gas. Science 338, 390-393. doi: 10.1126/science.1225974

Schommer, C., Palatnik, J. F., Aggarwal, P., Chetelat, A., Cubas, P., Farmer, E. E., et al. (2008). Control of jasmonate biosynthesis and senescence by miR319 targets. PLoS Biol. 6:e230. doi: 10.1371/journal.pbio.0060230

Seltmann, M. A., Hussels, W., and Berger, S. (2010a). Jasmonates during senescence: signals or products of metabolism? Plant Signal. Behav. 5, 1493-1496. doi: $10.4161 /$ psb.5.11.13644

Seltmann, M. A., Stingl, N. E., Lautenschlaeger, J. K., Krischke, M., Mueller, M. J., and Berger, S. (2010b). Differential impact of lipoxygenase 2 and jasmonates on natural and stress-induced senescence in Arabidopsis. Plant Physiol. 152, 19401950. doi: 10.1104/pp.110.153114

Sembdner, G., and Parthier, B. (1993). The biochemistry and the physiological and molecular actions of jasmonates. Annu. Rev. Plant Physiol. Plant Mol. Biol. 44, 569-589. doi: 10.1146/annurev.pp.44.060193.003033

Shan, X., Wang, J., Chua, L., Jiang, D., Peng, W., and Xie, D. (2011). The role of Arabidopsis Rubisco activase in jasmonate-induced leaf senescence. Plant Physiol. 155, 751-764. doi: 10.1104/pp.110.166595

Song, S., Huang, H., Gao, H., Wang, J., Wu, D., Liu, X., et al. (2014). Interaction between MYC2 and ETHYLENE INSENSITIVE3 modulates antagonism between jasmonate and ethylene signaling in Arabidopsis. Plant Cell 26, 263 279. doi: 10.1105/tpc.113.120394

Staswick, P. E., and Tiryaki, I. (2004). The oxylipin signal jasmonic acid is activated by an enzyme that conjugates it to isoleucine in Arabidopsis. Plant Cell 16, 2117 2127. doi: $10.1105 /$ tpc. 104.023549
Taylor, J. E., and Whitelaw, C. A. (2001). Signals in abscission. New Phytol. 151, 323-340. doi: 10.1046/j.0028-646x.2001.00194.x

Turner, J. G., Ellis, C., and Devoto, A. (2002). The jasmonate signal pathway. Plant Cell 14(Suppl. 1), S153-S164. doi: 10.1105/tpc.000679

Ueda, J., and Kato, J. (1980). Isolation and identification of a senescence-promoting substance from wormwood (Artemisia absinthium L.). Plant Physiol. 66, 246249. doi: 10.1104/pp.66.2.246

Wang, K. L., Li, H., and Ecker, J. R. (2002). Ethylene biosynthesis and signaling networks. Plant Cell 14(Suppl. 1), S131-S151. doi: 10.1105/tpc.001768

Wen, X., Zhang, C., Ji, Y., Zhao, Q., He, W., An, F., et al. (2012). Activation of ethylene signaling is mediated by nuclear translocation of the cleaved EIN2 carboxyl terminus. Cell Res. 22, 1613-1616. doi: 10.1038/cr.2012.145

Woeste, K. E., Ye, C., and Kieber, J. J. (1999). Two Arabidopsis mutants that overproduce ethylene are affected in the posttranscriptional regulation of 1 aminocyclopropane-1-carboxylic acid synthase. Plant Physiol. 119, 521-530. doi: 10.1104/pp.119.2.521

Woo, H. R., Kim, J. H., Nam, H. G., and Lim, P. O. (2004). The delayed leaf senescence mutants of Arabidopsis, ore1, ore3, and ore9 are tolerant to oxidative stress. Plant Cell Physiol. 45, 923-932. doi: 10.1093/pcp/pch110

Wu, K., Zhang, L., Zhou, C., Yu, C. W., and Chaikam, V. (2008). HDA6 is required for jasmonate response, senescence and flowering in Arabidopsis. J. Exp. Bot. 59, 225-234. doi: 10.1093/jxb/erm300

Xu, A., Zhang, W., and Wen, C. K. (2014). ENHANCING ctr1-10 ETHYLENE RESPONSE2 is a novel allele involved in CONSTITUTIVE TRIPLERESPONSE1-mediated ethylene receptor signaling in Arabidopsis. BMC Plant Biol. 14:48. doi: 10.1186/1471-2229-14-48

Yang, T. F., Gonzalez-Carranza, Z. H., Maunders, M. J., and Roberts, J. A. (2008). Ethylene and the regulation of senescence processes in transgenic Nicotiana sylvestris plants. Ann. Bot. 101, 301-310. doi: 10.1093/aob/mcm229

Zhang, X., Zhu, Z., An, F., Hao, D., Li, P., Song, J., et al. (2014). Jasmonate-activated MYC2 represses ETHYLENE INSENSITIVE3 activity to antagonize ethylenepromoted apical hook formation in Arabidopsis. Plant Cell 26, 1105-1117. doi: 10.1105/tpc.113.122002

Zhu, Z., An, F., Feng, Y., Li, P., Xue, L., A, M., Jiang, Z., et al. (2011). Derepression of ethylene-stabilized transcription factors (EIN3/EIL1) mediates jasmonate and ethylene signaling synergy in Arabidopsis. Proc. Natl. Acad. Sci. U.S.A. 108, 12539-12544. doi: 10.1073/pnas.1103959108

Conflict of Interest Statement: The authors declare that the research was conducted in the absence of any commercial or financial relationships that could be construed as a potential conflict of interest.

Received: 10 October 2014; accepted: 10 January 2015; published online: 29 January 2015.

Citation: Kim J, Chang C and Tucker ML (2015) To grow old: regulatory role of ethylene and jasmonic acid in senescence. Front. Plant Sci. 6:20. doi: 10.3389/fpls. 2015.00020

This article was submitted to Plant Physiology, a section of the journal Frontiers in Plant Science.

Copyright (C) $2015 \mathrm{Kim}$, Chang and Tucker. This is an open-access article distributed under the terms of the Creative Commons Attribution License (CC BY). The use, distribution or reproduction in other forums is permitted, provided the original author(s) or licensor are credited and that the original publication in this journal is cited, in accordance with accepted academic practice. No use, distribution or reproduction is permitted which does not comply with these terms. 


\title{
1-aminocyclopropane-1-carboxylic acid (ACC) in plants: more than just the precursor of ethylene!
}

\author{
Bram Van de Poel ${ }^{1,2}$ and Dominique Van Der Straeten ${ }^{2 *}$ \\ ' Department of Cell Biology and Molecular Genetics, University of Maryland, College Park, MD, USA \\ ${ }^{2}$ Laboratory of Functional Plant Biology, Department of Physiology, Ghent University, Ghent, Belgium
}

\section{Edited by:}

Domenico De Martinis, ENEA Italian National Agency for New

Technologies, Energy and Sustainable Economic Development, Italy

\section{Reviewed by:}

Brad Binder, University of

Tennessee-Knoxville, USA

Chi-Kuang Wen, Chinese Academy of

Sciences, China

\section{${ }^{*}$ Correspondence:}

Dominique Van Der Straeten,

Laboratory of Functional Plant Biology, Department of Physiology, Ghent University, K.L. Ledeganckstraat 35, 9000 Ghent, Belgium

e-mail:dominique.vanderstraeten@ ugent.be
Ethylene is a simple two carbon atom molecule with profound effects on plants. There are quite a few review papers covering all aspects of ethylene biology in plants, including its biosynthesis, signaling and physiology. This is merely a logical consequence of the fascinating and pleiotropic nature of this gaseous plant hormone. Its biochemical precursor, 1-aminocyclopropane-1-carboxylic acid (ACC) is also a fairly simple molecule, but perhaps its role in plant biology is seriously underestimated. This triangularly shaped amino acid has many more features than just being the precursor of the lead-role player ethylene. For example, ACC can be conjugated to three different derivatives, but their biological role remains vague. ACC can also be metabolized by bacteria using ACC-deaminase, favoring plant growth and lowering stress susceptibility. ACC is also subjected to a sophisticated transport mechanism to ensure local and long-distance ethylene responses. Last but not least, there are now a few exciting studies where ACC has been reported to function as a signal itself, independently from ethylene. This review puts ACC in the spotlight, not to give it the lead-role, but to create a picture of the stunning co-production of the hormone and its precursor.

Keywords: 1-aminocyclopropane-1-carboxylic acid (ACC), ethylene, conjugation, deaminase, transport, signaling

\section{THE DISCOVERY OF ACC}

The discovery of ethylene as a plant growth regulator can be attributed to the work of the Russian scientist Neljubov (1901). He reported that dark-grown pea seedlings showed a reduced hypocotyl growth in combination with an exaggerated hypocotyl bending when exposed to illumination gas (Neljubov, 1901). Neljubov (1901) could pinpoint ethylene gas as the active component that caused dark-grown pea seedlings to bend, by flowing the illumination gas over several filters prior to exposing the seedlings. This typical ethylene response of dark-grown seedlings was later defined as the triple response: (1) shortening of the hypocotyl and roots, (2) radial swelling of the hypocotyl, and (3) the exaggeration of the apical hook (Knight et al., 1910). In 1934, conclusive evidence that ethylene is a natural product from plants, was presented by the English scientist (Gane, 1934). It took another 30 years before the primary steps of the ethylene biosynthesis pathway were elucidated (see Figure 1). Lieberman and Mapson (1964) first reported that ethylene could be produced from the amino acid methionine, taking advantage of the high rates of ethylene production from apples for their experimental work (Figure 1). 13 years later, Adams and Yang (1977) made tremendous progress in understanding the biosynthesis pathway of ethylene, when they discovered that S-adenosyl-L-methionine (SAM) was an intermediate between methionine and ethylene. Yang and co-workers also showed that $5^{\prime}$-methylthioadenosine (MTA) was formed as a by-product from SAM and that MTA could be recycled back to methionine (Murr and Yang, 1975). The elaboration of the different reaction steps of the methionine cycle in plants, now often referred to as the Yang-cycle, was mainly inspired by the biochemical similarities between the plant pathway and the methionine salvage cycle which was already known for prokaryotes, yeast, and mammalians. An-up-to-date overview of the methionine and SAM metabolism in plants is given by Sauter et al. (2013). The major discovery that made the methionine cycle in plants unique from all other organisms, was the characterization of 1-aminocyclopropane-1-carboxylic acid (ACC) as the intermediate between SAM and ethylene (Adams and Yang, 1979). Adams and Yang (1979) were able to identify ACC as the precursor for ethylene by feeding experiments on apple tissue, using radio-labeled methionine. Upon incubation of apple disks, they observed a shift from ethylene production in air, toward an unknown compound that was retained in the tissue when treated with nitrogen (lack of oxygen inhibits oxidation of ACC toward ethylene). By using a $\mathrm{pH}$-dependent ion mobility assay, they could characterize this unknown component as an amino acid. Subsequently, the component was identified as ACC, using co-migration of synthetic ACC for both paperchromatography and paper-electrophoresis (Adams and Yang, 1979). They further showed that the conversion of radioactively labeled methionine toward ethylene decreased when unlabeled ACC was supplemented, yet the conversion of labeled ACC to ethylene was almost not affected when unlabeled methionine was supplemented, suggesting that externally supplied ACC is in fact used to produce ethylene. Additional evidence for ACC being the intermediate precursor between SAM and ethylene was obtained by treating apple tissue with [S]-trans-2-amino4(2'-aminoethoxy)trans-3-butenoic acid, also known as AVG (2-amino-ethoxy-vinylglycine), a pyridoxal-5' -phosphate (PLP or 


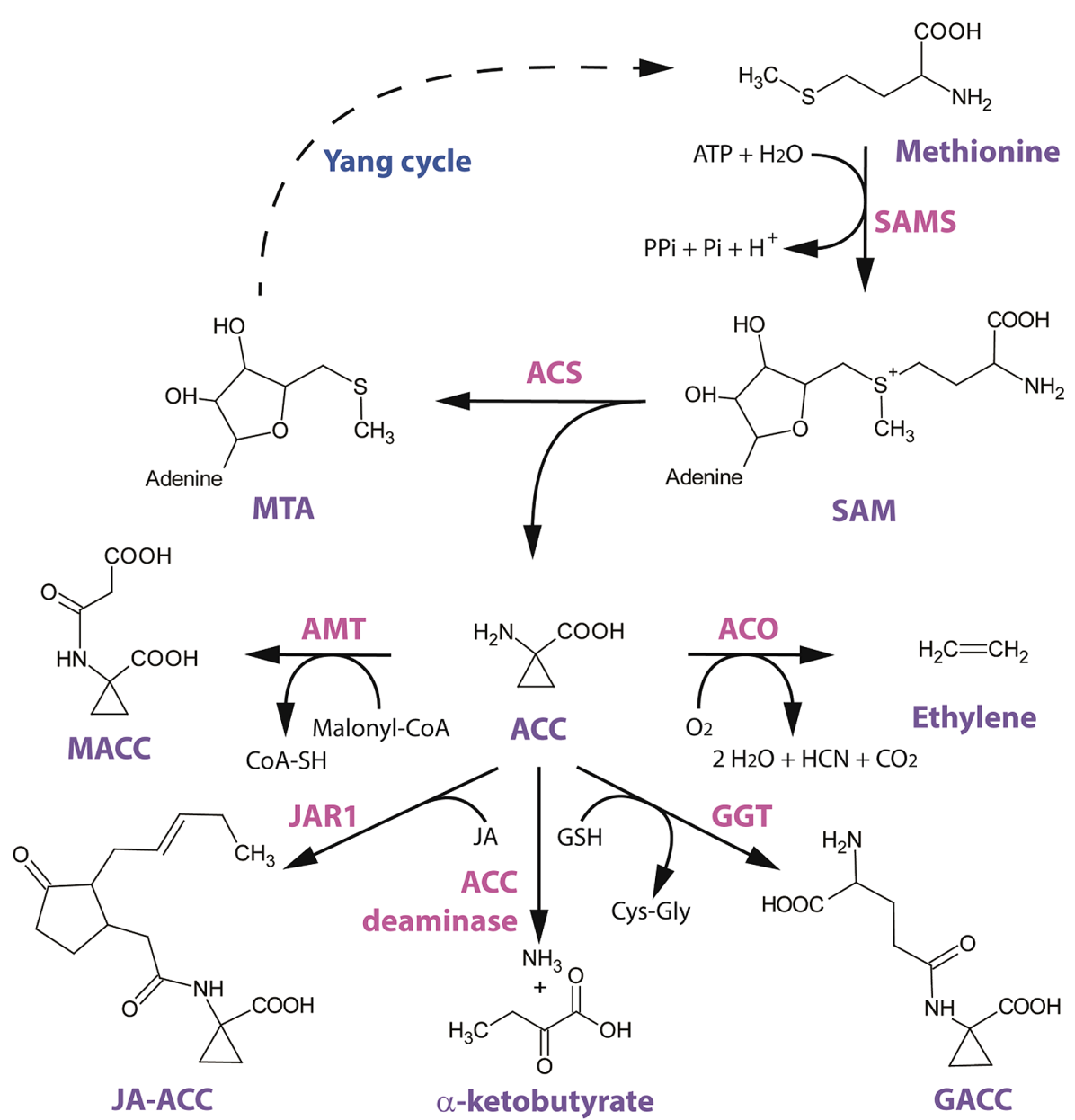

FIGURE 1 | Structural scheme of ethylene biosynthesis and 1-aminocyclopropane-1-carboxylic acid (ACC) conjugation/metabolism. The amino acid methionine is converted to S-adenosyl-L-methionine (SAM) by SAM-synthetase (SAMS) with the requirement of ATP. The general precursor SAM is then converted to ACC by ACC-synthase (ACS). This reaction also involves the cleavage of $5^{\prime}$-methylthioadenosine (MTA), which is recycled back to methionine by the Yang cycle (dotted line indicates multiple enzymatic steps). ACC can be converted to ethylene by ACC-oxidase (ACO) in the presence of oxygen. ACC can also be converted to its major conjugate 1-malonyl-ACC (MACC) by the yet uncharacterized ACC-N-malonyl transferase (AMT) with the requirement of malonyl-Coenzyme-A. A second derivate of ACC is $\gamma$-glutamyl-ACC (GACC) which is formed by $\gamma$-glutamyl-transpeptidase (GGT) with the requirement of glutathione (GSH). Another novel derivate of ACC is jasmonyl-ACC (JA-ACC), which is formed by jasmonic acid resistance 1 (JAR1). ACC can also be metabolized by the bacterial (and plant) ACC deaminase into ammonium and $\alpha$-ketobutyrate. vitamin $\mathrm{B}_{6}$ ) dependent enzyme inhibitor, which was later known to inhibit the enzymatic conversion of SAM toward ACC. The identification of ACC as the precursor of ethylene was a major breakthrough in the understanding of the ethylene biosynthesis pathway in plants, and was part of the foundation for many new discoveries in the field of ethylene biology.

\section{ACC AND ETHYLENE BIOSYNTHESIS}

As mentioned above, ACC is produced from SAM, releasing MTA. This reaction is catalyzed by the enzyme ACC-synthase (ACS; Boller etal., 1979). ACS is a member of the PLP-dependent enzymes, which use vitamin B6 as a co-factor for its enzymatic function. ACS was localized in the cytosol by activity assays on extracts retrieved after differential centrifugation (Boller et al., 1979). ACS genes were first characterized in zucchini by Sato and Theologis (1989) and in tomato by Van Der Straeten et al.
(1990). ACS is encoded by a multigene family of 12 members in Arabidopsis, eight of which encode functional ACC synthases [ACS2 (named ACS1 in Van Der Straeten et al., 1992), ACS4-9, ACS11]. In addition, there is one inactive isoform (AtACS1) and one pseudogene (AtACS3; Yamagami et al., 2003). ACS was found to form functional dimers of which the 3D structure was determined by Capitani et al. (1999). The formation of heterodimers increases the structural and functional complexity of the ACS protein family (Tsuchisaka et al., 2009). The large ACS gene family displays a tissue-specific and differential expression pattern in Arabidopsis (Tsuchisaka and Theologis, 2004). Using single and multiple acs knock-out mutants, it was demonstrated that there are specific developmental and physiological roles for individual members of the ACS gene-family, but also that there is a complex combinatorial interplay amongst them (Tsuchisaka et al., 2009). A diverse group of internal and external signals modulate the 
level of ethylene biosynthesis in numerous plant species, acting at the level of ACS gene expression. These inducers include auxin, cytokinin, brassinosteroids, ethylene, copper, mechanostimuli, ozone, pathogens and wounding (Van Der Straeten et al., 1992; Rodrigues-Pousada etal., 1993; Botella et al., 1995; Cary etal., 1995; Liang etal., 1996; Vahala etal., 1998; Woeste et al., 1999).

Three types of ACS proteins are recognized, based on their $C$-terminal structure. Type I ACS proteins contain in their $C$ terminal domain one putative calcium-dependent protein kinase (CDPK) phosphorylation target site and three mitogen-activated protein kinase (MAPK) phosphorylation sites (Yoon and Kieber, 2013). Type II ACS proteins only contain the MAPK phosphorylation sites, while type III ACS do not contain any phosphorylation sites (Yoon and Kieber, 2013). These post-translational phosphorylation sites play an important role in the stability of the ACS protein (Chae and Kieber, 2005). Both in Arabidopsis (Chae etal., 2003; Kim etal., 2003; Liu and Zhang, 2004; Wang etal., 2004; Yoshida etal., 2005, 2006; Joo et al., 2008; Christians et al., 2009; Lyzenga et al., 2012) and in tomato (Tatsuki and Mori, 2001; Kamiyoshihara et al., 2010) it was shown that differential phosphorylation of certain ACS members directed the protein for proteasomal degradation. Protein stability of certain ACS members is further regulated by the protein phosphatase 2A (PP2A; Skottke et al., 2011) and PP2C (Ludwikow et al., 2014), demonstrating a complex balance between phosphorylation and dephosphorylation to secure protein activity and stability.

The second ethylene biosynthesis protein is ACC-oxidase (ACO), which converts ACC to ethylene in the presence of oxygen. It took a long time before ACO activity could be demonstrated in vitro. The key aspect in isolating ACO was the addition of ascorbic acid (vitamin C) to the extraction media, as was first reported by Ververidis and John (1991) who isolated ACO from melon tissue and quantified in vitro ACO activity. Although the exact role of ascorbic acid for protein stability/activity remained uncertain for a long time (Rocklin et al., 2004), it was recently clarified that ascorbic acid participates in the ring opening of ACC, by providing a single-electron to the active site (Murphy et al., 2014). This catalytic reaction releases ethylene and a cyanoformate ion $\left[\mathrm{NCCO}_{2}\right]^{-}$, which is subsequently decomposed into $\mathrm{CO}_{2}$ and $\mathrm{CN}^{-}$(Murphy et al., 2014). The reactive cyanide $\left(\mathrm{CN}^{-}\right)$ is subsequently detoxified by $\beta$-cyanoalanine synthase to produce $\beta$-cyanoalanine (Miller and Conn, 1980). ACO belongs to the superfamily of dioxygenases that require iron $\left(\mathrm{Fe}^{2+}\right)$ as $\mathrm{CO}-$ factor and bicarbonate as activator (Dong et al., 1992; Zhang et al., 2004). The subcellular localization of ACO remains vague, as some studies localize ACO in the cytosol (Reinhardt et al., 1994; Chung etal., 2002; Hudgins et al., 2006), while other localize ACO at the plasma membrane (Rombaldi et al., 1994; Ramassamy etal., 1998). Although the ACO protein sequence does not contain any predicted transmembrane domains, it is still possible that the protein associates with the plasma membrane via (in)direct interactions. ACO is also encoded by a multigene family of five members in Arabidopsis [ACO1, 2, 4, At1g12010 (AOC3) and At1g77330 (ACO5)]. Expression of different members of the tomato ACO family in Escherichia coli showed that each isoform had a specific in vitro enzyme activity (Bidonde et al., 1998).

It is well accepted that ACS is the rate limiting step of ethylene biosynthesis in plants (Yang and Hoffman, 1984) although there are examples where ACO is the rate limiting step, e.g., during post-climacteric ripening of tomato fruit (Van de Poel et al., 2012). Three ACO genes are also auto-regulated by ethylene in Arabidopsis (De Paepe et al., 2004). This might suggest that the regulation of ACO expression and/or activity is more complex than anticipated. There are some hints for a putative post-transcriptional and/or post-translational regulatory mechanisms of ACO as suggested by Dilley et al. (2013) and as investigated through mathematical modeling by Van de Poel et al. (2014a).

Both ACS and ACO are two well-studied enzymes that exclusively participate in the ethylene biosynthesis pathway. Both proteins are well characterized, but many more questions remain unanswered. While the post-translational regulation of ACS has been revealed, the biochemical and mechanistic details of this protein modification are still unclear. Transcriptional and functional characterization of the different ACS gene-family members has shone light on the combinatorial interplay, nevertheless, much more work is needed to elucidate the exact role of each isoform and how they interact with each other. Much less is known about the post-translational regulation and combinatorial interplay of ACO. A lack of genetic studies focusing on ACO, raises the question whether or not ACO shares a similar structural, biochemical and post-translational complexity as ACS.

\section{ACC AS A PIVOTAL MOLECULE: ACC CONJUGATES AND THE CONTROL OF ETHYLENE BIOSYNTHESIS}

1-Aminocyclopropane-1-carboxylic acid is best known as the direct precursor of ethylene in the ethylene biosynthesis pathway. However, there also exist three different conjugates of ACC, suggesting that the biochemical regulation of the available ACC pool is more complex than anticipated, which in turn can possibly affect the eventual levels of the plant hormone ethylene.

Shortly after the identification of ACC as the intermediate between SAM and ethylene in the ethylene biosynthesis pathway, a first conjugated form of ACC, called malonyl-ACC (MACC), was discovered by Amrhein et al. (1981) in buckwheat seedlings and by Hoffman et al. (1982) in wheat leaves. MACC is formed by ACC$\mathrm{N}$-malonyl transferase (AMT) which was purified from tomato extracts (Martin and Saftner, 1995), although not structurally characterized. It was shown that the conjugation of ACC into MACC was stimulated by ethylene in preclimacteric tomatoes (Liu et al., 1985a), grapefruit flavedo (outer peel; Liu et al., 1985b) and tobacco leaves (Philosoph-Hadas et al., 1985), indicative for a feedback control of ethylene biosynthesis. Martin and Saftner (1995) also showed that the activity of AMT was ethylene inducible and that its activity correlated with the increase in ethylene production during climacteric ripening of tomato (Martin and Saftner, 1995). The exact amino acid and gene sequences of AMT are not yet known, and no putative AMT gene is annotated in the Arabidopsis genome, limiting more in-depth genetic and molecular studies. Because MACC does not participate in any other known biological conversions, MACC formation might be a mechanism to control the available ACC pool. This hypothesis was further strengthened 
by the observation that MACC could be translocated from the cytosol into the vacuole (and back) by ATP-dependent tonoplast carriers (Bouzayen et al., 1988, 1989; Tophof et al., 1989). Nonetheless, the reconversion of MACC toward ACC by an unknown MACC-hydrolase was reported twice in literature (Jiao et al., 1986; Hanley et al., 1989). The ability to hydrolyze MACC back into ACC and the ability to 'store' MACC in the vacuole is an interesting mechanism to regulate the cellular availability of MACC. Moreover, because MACC has no other biochemical role besides being an ACC conjugate, the regulation of MACC levels can also affect the available pool of ACC and possibly ethylene production levels. This hypothesis was investigated by in silico mathematical modeling, showing that the reconversion of MACC to ACC could have a potential stimulating effect on ethylene production during climacteric fruit ripening of tomato (Van de Poel et al., 2014a).

A second important derivative of ACC is $\gamma$-glutamyl-ACC (GACC), which was discovered in crude tomato extracts of ACC$\mathrm{N}$-malonyltransferase (Martin et al., 1995). These crude protein extracts were able to form a new ACC derivative, which could be identified as GACC (Martin et al., 1995). GACC is formed by the reaction of ACC with the tripeptide glutathione (GSH) by a $\gamma$-glutamyl-transpeptidase (also called $\gamma$-glutamyl-transferase, GGT; Martin etal., 1995; Martin and Slovin, 2000). While a first report, based on in vitro studies, stated that GACC was the most abundant ACC derivative in tomato (Martin and Saftner, 1995; Martin et al., 1995), another study found that MACC was the most abundant ACC derivative in vivo in tomato fruit during climacteric ripening (Peiser and Yang, 1998). The Arabidopsis genome contains four genes (GGT1-4), of which only GGT1 and GGT2 are catalytically active (Martin etal., 2007). Both GGT1 and GGT2 are co-expressed predominantly in rapidly growing tissue, and are localized extracellularly, which raised the question about the role of extracellular GACC (Martin et al., 2007). A knock-out mutant of GGT1 shows rapid senescence, while GGT3 knock-outs have a reduced rosette size and silique number (Martin et al., 2007). The effect of GACC formation by GGT on ACC availability and possibly ethylene biosynthesis remains to be investigated.

A third derivative of ACC is jasmonyl-ACC (JA-ACC). This molecule was discovered by screening for amino acid conjugates of JA, using GC-MS (Staswick and Tiryaki, 2004). Four amino acid conjugates of JA (JA-Ile, JA-Val, JA-Leu, and JA-Phe) were quantified in Arabidopsis tissue (Staswick and Tiryaki, 2004). Interestingly, the same authors also demonstrated that JA forms a conjugate with ACC (JA-ACC) in Arabidopsis leaves (Staswick and Tiryaki, 2004). Recombinant JAR1 enzyme was found to be able to form JA-ACC in vitro. Strangely, levels of JA-ACC were higher in the leaves of jar1 mutants compared to wild-type plants. It was also shown that JA-ACC inhibits root growth in Arabidopsis. Elegant genetic experiments with JA signaling mutants (coi1-35) showed that the JA-ACC-induced root inhibition was independent of JA signaling. Furthermore, the ethylene signaling mutant etr $1-1$ and the double mutant etr1-1 jar1-1 were insensitive to the JA-ACC treatment and displayed no inhibition of root growth, indicating that JA-ACC acts via the ethylene signaling pathway (Staswick and Tiryaki, 2004). Most likely the ACC moiety of JA-ACC is responsible for an increase in ethylene production which results in the root growth inhibition response. These experiments suggest that JA-ACC might serve as a pivotal molecule which can function as a modulator of the hormonal cross-talk between the ethylene and jasmonic acid pathway, although the exact molecular and biochemical mechanism of JA-ACC function remains unclear.

The three above-mentioned derivatives of ACC (MACC, GACC, and JA-ACC) are perhaps not the only ones. Future metabolic studies might reveal additional conjugates of ACC. Nonetheless, these three derivatives can potentially play an important role in the regulation of the pool of available ACC, which in turn can affect eventual ethylene production levels, with physiological and developmental consequences. Genetic perturbations of the formation of ACC derivatives could be a useful tool to unravel their exact roles. In addition, a more detailed structural and biochemical characterization of the enzymes involved in the formation of ACC derivatives is essential.

\section{ACC DEAMINASE AND PLANTS}

As mentioned above, plants possess several mechanisms to control their pool of ACC, for example by converting it to ethylene or to conjugates like MACC, GACC, or JA-ACC. Another unique way to metabolize ACC, is the deamination of ACC. ACC deaminase was first discovered in bacteria. Some plant growth-promoting bacteria are capable of processing the plant-borne ACC by converting it into ammonia and $\alpha$-ketobutyrate using the enzyme ACC deaminase (Honma and Shimomura, 1978). ACC deaminase was retrieved in for example, Pseudomonas sp. strain ACP (Honma and Shimomura, 1978), Pseudomonas chloroaphis 6G5 (Klee et al., 1991), Pseudomonas putida GR12-2 (Jacobson et al., 1994) and Pseudomonas putida UW4 (Hontzeas et al., 2004).

The bacterial ACC deaminase is a PLP-dependent enzyme with a rather low affinity for ACC (the reported $\mathrm{Km}$ value is $1.5-15 \mathrm{mM}$; Glick et al., 2007). Nonetheless, relatively low concentrations of ACC $(100 \mathrm{nM})$ can already induce the expression of the ACC deaminase gene ( $a c d S$ ), but so do other amino acids like L-alanine, DL-alanine, and DL-valine (Jacobson et al., 1994). acdS expression is also under the regulation of the nitrogen fixation ( $n$ if) promotor of some Rhizobia, linking ACC deaminase with nodule formation (Nukui et al., 2006; Nascimento et al., 2012).

Plant growth-promoting bacteria that harbor ACC deaminase must interact with the root environment in order to access plantproduced ACC. It was shown that root exudates contain certain amounts of ACC, which might attract ACC deaminase containing bacteria and establish the rhizosphere interaction (Penrose et al., 2001). It has been proposed that bacterial ACC deaminase can reduce the endogenous ethylene levels of plant roots by limiting the amount of available ACC, which will in turn prevent ethyleneinduced root growth inhibition, and thus promote plant growth (Glick et al., 1998, 2007; Glick, 2014). Another model proposes that plant growth-promoting bacteria produce IAA which can be taken up by the plant, and can induce the expression of $A C S$, resulting in an increase in ACC production, providing a nitrogen supply for the bacteria (Glick et al., 1998, 2007; Glick, 2014).

There are many beneficial effects of ACC deaminase containing bacteria on plant growth, particularly in relation to stress 
tolerance. For instance, ACC deaminase containing bacteria can reduce stress susceptibility of plants during flooding (Barnawal et al., 2012; Li et al., 2013), drought (Mayak et al., 2004a), salinity (Mayak et al., 2004b; Nadeem et al., 2007, 2010), flower senescence (Nayani et al., 1998; Ali et al., 2012), metal pollution (Glick, 2010), organic pollution (Gurska et al., 2009) and pathogens (Glick, 2014 and references therein). In addition, it has been reported that the presence of ACC deaminase can increase the symbiotic performance of Rhizobial strains (Ma et al., 2003).

Hence, bacterial ACC deaminase is also used as a biotechnological tool to control endogenous ACC levels and consequently lower ethylene production in plants. Transgenic plants overexpressing bacterial ACC deaminase were shown to be more resistant to growth inhibition when confronted with fungal pathogens (Lund et al., 1998; Robison et al., 2001), salt stress (Sergeeva et al., 2006), and metals (Grichko et al., 2000; Nie et al., 2002).

Plants themselves also contain a homolog of the bacterial ACC deaminase. In Arabidopsis, it was demonstrated that the previously known enzyme D-cysteine desulfydrase also possesses ACC deaminase activity (McDonnell et al., 2009). Antisense lines showed a decreased ACC deaminase activity, an increased sensitivity to ACC and produced more ethylene (McDonnell et al., 2009). These results indicate that the plant-specific ACC deaminase might be another metabolic shunt regulating ACC levels and ethylene production in plants.

\section{ACC TRANSPORT AND ITS ROLE DURING ROOT STRESS}

Besides the biosynthesis, conjugation, and catabolism of a hormone, short or long range transport is another important aspect to regulate proper dosage of a hormonal signal within an organism. Often, hormones are synthesized at one site and transported to another site for their action. Hormonal transport from one cell to another is an advanced process, that facilitates tissue specific or long-distance physiological processes or stress responses. Because ethylene is a gaseous molecule, it can freely diffuse from one cell to a neighboring cell, evoking mainly local responses. The presence of aerenchyma or large intercellular voids facilitates rapid long-distance transport of ethylene gas in plant organs. But longdistance ethylene responses can also be achieved by transport of its precursor ACC. Often, but not always, ACC is transported from the roots to the shoot, when the roots are exposed to stress (McManus, 2012). Yet, local ACC transport between cells of the same tissue type and intracellular transport is also possible, illustrating the molecular complexity of ACC transport.

One of the best characterized ACC transport systems is the translocation of ACC from the roots to the shoots of tomato plants suffering from flooding or root hypoxia. A lack of oxygen in the rhizosphere will induce the expression of ACS in the roots (Olson et al., 1995; Shiu et al., 1998) resulting in an increased ACS activity (Bradford et al., 1982; Wang and Arteca, 1992). The excess of ACC in the roots is not converted to ethylene due to a lack of oxygen and the absence of ACO in the roots. Rather, ACC is loaded into the xylem and transported to the shoots (Bradford and Yang, 1980). Once arrived at the shoots, ACC is converted into ethylene by ACO, which is already present in the leaves (English et al., 1995). In tomato, root hypoxia will result in an epinastic response of leaves due to an increased

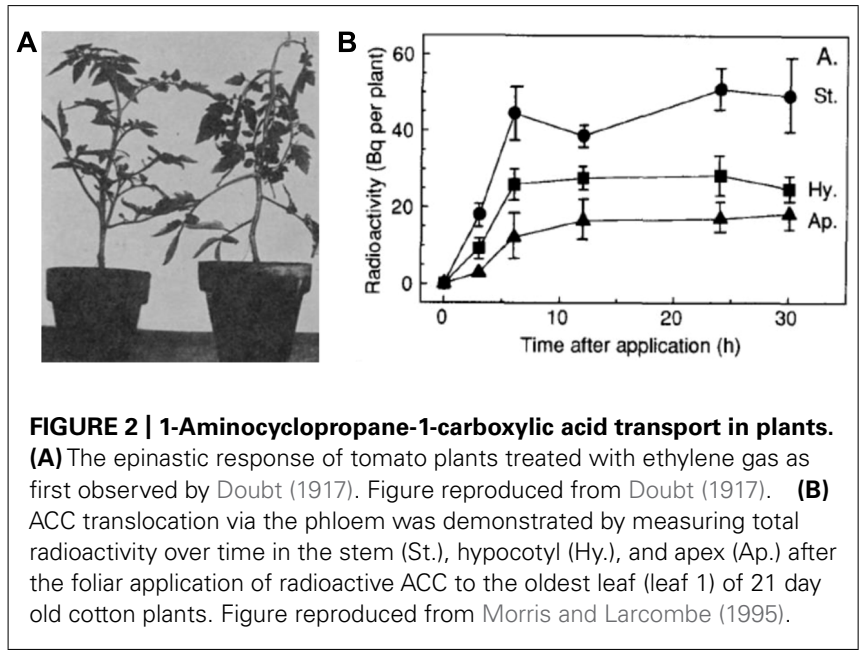

ethylene production (Figure 2A; Doubt, 1917; Jackson and Campbell, 1976). Differential expression of both ACS and ACO during hypoxia was also observed in Arabidopsis (Peng et al., 2005), sunflower seedlings (Finlayson et al., 1991), maize (Atwell et al., 2006; Geisler-Lee et al., 2010), and rice (Zarembinski and Theologis, 1993, 1997; Van Der Straeten et al., 1997, 2001; Zhou et al., 2001). Interestingly, long-distance transport of ACC has also been suggested to occur during other root stress conditions as for example drought (Davies etal., 2000; Sobeih etal., 2004; Skirycz et al., 2011), rehydration after drought (Tudela et al., 1992), nutrient stress (Lynch and Brown, 1997) and salinity (Feng and Barker, 1992; Ghanem et al., 2008).

Another long-distance ACC transport system is achieved via the phloem. Foliar applied radioactive ACC was found to be transported via the phloem to other aerial parts in tomato (Amrhein et al., 1982) and in cotton plants (Morris and Larcombe, 1995; Figure 2B). It should be noted that the foliar applied ACC was also rapidly converted into MACC, which was not found to be transported via the phloem (Morris and Larcombe, 1995). This immobility of MACC is in accordance with the earlier observations that MACC is actively transported from the cytosol into the vacuoles, where it could be subsequently stored (Bouzayen et al., 1988, 1989; Tophof et al., 1989). Interestingly, there could be a link between phloem transport of ACC and the Yang cycle. Pommerrenig et al. (2011) showed that Yang cycle genes were specifically expressed in phloem, indicating that recycling of MTA toward SAM is preferentially carried out in this tissue. Perhaps MTA recycling is stimulated by high rates of ACC synthesis in phloem cells, or the recycled SAM forms a pool for phloem-specific ACC production. In roots, spatiotemporal gene expression profiling demonstrated that different ACS isoforms are expressed (but not exclusively) in the vascular tissue (Brady et al., 2007; Dugardeyn et al., 2008). Moreover, the loading of ACC to this tissue could affect the homeostasis of SAM and consequently, polyamines (Pommerrenig et al., 2011).

The exact molecular mechanism by which ACC is loaded into the xylem and/or the phloem and subsequently transported throughout the plant is still unknown, but is an important element in our understanding of long-distance ethylene signaling 
via ACC and how plants deal with different root and leaf stress conditions.

Long- or medium-long-distance ACC transport was also observed (or speculated) during different developmental processes. Tissue specific gene expression profiling of maize root cells showed that there are differences between ACS and ACO expression patterns (Gallie et al., 2009). ACO was predominantly expressed in the protophloem sieve elements and the companion cells, while ACS was expressed only in the root cortex. This discrepancy led the authors to hypothesize that ACC could be transported from the site of synthesis to the site of consumption, in order to ensure the ethylene production levels observed (Gallie et al., 2009). Differences in ACS and ACO expression patterns predicted in silico in Arabidopsis roots support the same hypothesis (Dugardeyn et al., 2008). A similar reasoning was made by Jones and Woodson (1997, 1999), who observed differences in ACO and ACS transcripts in different cell-types of carnation flower. They also postulated that ACC transport from sites with a high ACS expression (in e.g., petals and styles) secured the ability of ACO to produce ethylene in cells with a high ACO expression (for example the ovaries; Jones and Woodson, 1997, 1999).

Of course one should take into account that gene expression levels not always reflect actual protein levels, and that posttranslational modifications can play an important role in protein stability and activity. A targeted metabolomics and proteomics study by Van de Poel et al. (2014b) investigated the tissue specificity of the ethylene biosynthesis pathway in tomato fruit. They observed that the pericarp tissue produced the highest amount of ethylene (and high ACO activity), while the pericarp had the lowest ACS activity and ACC content compared to other tissues. Perhaps ACC is transported from neighboring tissues with a high ACS activity or ACC content such as the locular gel, toward the pericarp in order to secure high rates of ethylene production during climacteric ripening of tomato (Van de Poel et al., 2014b).

Besides long-distance, short-distance intracellular transport of ACC was also observed in barley and wheat mesophyll cells (Tophof et al., 1989) and maize mesophyll cells (Saftner and Martin, 1993). ACC is transported across the tonoplast by carriers that rely on an electrochemical potential gradient of protons, and which are stimulated by the supplementation of ATP (Saftner and Martin, 1993). This intracellular compartmentalization of ACC allows the plant to precisely regulate the cellular pool of ACC, possibly also affecting ethylene biosynthesis.

Clearly, more research is needed to further unravel the exact molecular and biochemical mechanisms which assure intracellular, inter- and intra-tissue and long-distance ACC transport in plants, and their corresponding physiological effects.

\section{ACC AS A SIGNALING MOLECULE}

1-Aminocyclopropane-1-carboxylic acid holds a key position in many physiological processes as it is the direct precursor in the biosynthesis of ethylene. A balanced supply and consumption of ACC is essential to achieve the necessary production level of ethylene within a given spatial and temporal context. As shown above, the pool of ACC is regulated by a complex interaction of production, consumption, modification, and transport.
Interestingly, recent findings have suggested a perhaps even more important role for ACC, as a signaling molecule independent from ethylene (Yoon and Kieber, 2013).

A first report by Xu et al. (2008) investigated the role of ACC signaling in relation with FEI1 and FEI2, which are leucinerich repeat receptor-like kinases. The feil fei2 mutant displays a severe defect in anisotropic root growth due to a reduced cellulose microfiber content in the cell wall at the root tip (Figure 3). The feil fei2 phenotype can be reversed by the application of ethylene biosynthesis inhibitors, but not by ethylene signaling inhibitors. The application of both aminooxy-acetic acid (AOA) or $\alpha$-aminoisobutyric acid (AIB) specifically inhibits ethylene biosynthesis and can reverse the phenotype of the feil fei2 mutant (Figure 3). AOA is an inhibitor of PLP-dependent enzymes, and will affect the activity of ACS resulting in a reduced ethylene production. AIB on the other hand is a structural analog of ACC and acts as a competitive inhibitor of ACC preventing ethylene production at the level of ACO. Ethylene signaling inhibitors such as 1-methylcyclopropane (1-MCP) and silver ions, did not affect the feil fei2 phenotype (Figure 3). Similarly, genetics showed that the feil fei2 mutant crossed with etr1-3 (a mutation in the ethylene receptor causing severe ethylene insensitivity), nor ein2-50 (a central regulator of ethylene signaling causing ethylene insensitivity) could reverse the phenotype. All together this study showed that the typical feil fei2 phenotype was not affected by ethylene signaling, but could be reversed by ethylene biosynthesis inhibitors. This suggests that the signal reversing the feil fei2 phenotype originated independent from ethylene signaling, involved ACS and is possibly ACC itself (Xu etal., 2008).

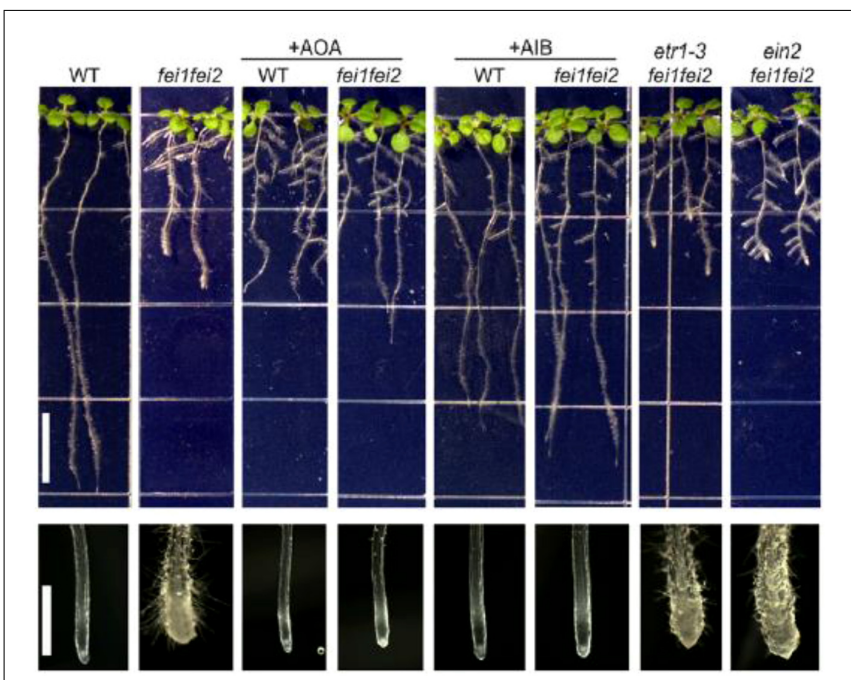

FIGURE 3 | Role of ACC/ethylene on the fei phenotype. Root phenotypes of seedlings grown on MS medium containing $0 \%$ sucrose for 4 days and then transferred to MS medium containing $4.5 \%$ sucrose, or additionally supplemented with AOA $(0.375 \mathrm{mM})$ or AIB $(1 \mathrm{mM})$ as indicated. Note that the distribution of lateral roots in the fei1 fei2 mutants in the presence of high sucrose is variable; the architecture of the fei1 fei2 ein2 triple mutant is not substantially different from that of the fei1 fei2 parent. The close-ups of the root tips clearly show the typical swelling of the fei1 fei2 mutant. (Bar $=1 \mathrm{~cm}$ ). Figure reproduced from Xu et al. (2008). 
A second report by Tsang et al. (2011) linked ACC signaling with cell elongation and cell wall composition of roots. Specific ethylene biosynthesis inhibitors [AVG, AOA, and 2-anilino7-(4-methoxyphenyl)-7,8-dihydro-5(6H)-quinazolinone (7303)] could reverse the inhibition of root cell expansion which was induced by an isoxaben treatment (a cellulose biosynthesis inhibitor causing cell wall stress). Similarly, as observed by $\mathrm{Xu}$ et al. (2008), an ethylene signaling inhibitor (silver ions) could not reverse the isoxaben-induced reduction in root cell elongation. Also, the ein3 eill ethylene insensitive mutant responded to ACC and isoxaben, providing genetic evidence of an ACC signaling mechanism independent of ethylene signaling. They also showed that the application of ACC without isoxaben, inhibited root cell elongation and was partially ethylene-dependent and partially ethylene-independent. Altogether, their results demonstrate that monitoring of cell wall integrity requires an ACC sensing/signaling mechanism, which can result in a reduction of root cell elongation, when disrupted. In addition, Tsang et al. (2011) showed that this inhibition of root cell elongation required auxin and reactive oxygen species (ROS) signaling, downstream of ACC signaling.

In a third report (Tsuchisaka et al., 2009) an octuple acs mutant was made to study the interplay between different ACS isoforms. The octuple line was created by introduction of two amiRNA lines (ACS8 and ACS11) into the hexuple mutant acs2,4,5,6,7,9 creating an octuple mutant line with complete or severe inhibition of ACS function. The lines that showed a very strong silencing of ACS8 and ACS11, displayed embryo lethality. This suggests that ethylene biosynthesis (or ACC biosynthesis) is essential for Arabidopsis viability, while this is not the case for the single (ctr1 and ein2) and double (ctr1 ein2) ethylene signaling mutants (Kieber et al., 1993; Roman et al., 1995; Alonso et al., 1999). This phenotypic discrepancy between ethylene biosynthesis and signaling once more suggests that ACC can acts as a signaling molecule itself, independent from ethylene, at least during embryo development and Arabidopsis viability. In addition, Tsuchisaka et al. (2009) characterized a wide variety of physiological and developmental processes of their single and multiple acs knock-out lines, and observed many phenotypes which were similar as for previously described ethylene signaling mutants. But interestingly they also observed several phenotypes like reduced branching, which were not observed in ethylene-insensitive mutants. These discrepancies could again be caused by ACC acting as a signaling molecule. However, it is also possible that individual ACS members have unique roles in developmental processes, and that knocking-out multiple ACS members might result in pleiotropic effects irrelevant to ACC or ethylene metabolism. Finally, it must be noted that such a severe genetic interference in ACC metabolism might also affect upstream SAM or MTA levels or the pool of downstream ACC conjugates, which in turn could signal themselves and affect cell physiology to contribute to the phenotypic differences observed.

All together these reports suggest a role for ACC as a signaling molecule to regulate plant development and growth, independent from ethylene. The exact molecular mechanism by which ACC signaling operates, and whether or not there is an ACC receptor and downstream signaling components, remains to be investigated. Future biochemical studies with specific ethylene biosynthesis and signaling inhibitors, in combination with genetics to create higher order ethylene biosynthesis/signaling mutants (like etr1ers1etr2ein4ers2ctr1ein2 or multiple aco knock-outs), could shed light on the role of ACC as a signaling molecule. It also still needs to be elucidated whether this unique title of "signaling molecule" is to be awarded to ACC, or rather to one if its (unknown) downstream derivatives.

\section{CONCLUSION}

Over the years, a lot of work has been done on ACC since its discovery in 1979, and it has become clear that ACC is more than just the precursor of ethylene. Its role in ethylene biosynthesis is well characterized, although there are still many questions concerning the two unique proteins that are associated with ACC in ethylene biosynthesis: ACS and ACO. Pioneering work on the characterization of post-translational modifications and the combinatorial interplay of ACS isoforms, has opened our eyes to the complex regulation of ethylene biosynthesis at the protein level. More mechanistic details are to be uncovered, probably including a complex post-translational control of ACO. Furthermore, ACC is conjugated into MACC, GACC, and JA-ACC. These derivatives are an elegant biochemical shunt to regulate the pool of ACC available for ethylene production, although it remains rather speculative what the exact biological roles are for these ACC conjugates. A better characterization of the participating enzymes is necessary to further elucidate the importunateness of ACC derivatives. Furthermore, ACC can also be used by bacterial (and plant) ACCdeaminase, adding another layer of metabolic complexity to the regulation of ACC levels. It is also well established that ACC can be easily transported over short (intracellular and intra-tissue) and long-distances (via the xylem and phloem), providing the plant with an elaborate system to control local and remote ethylene responses. Last but not least, ACC has been identified as a potential signaling molecule, independent of ethylene. This property of ACC is perhaps the most exciting, opening new avenues in ACC research, with potentially profound effects on plant physiology. The molecular mechanism by which ACC is signaling and the identity of other putative signaling components in such an 'ACC pathway' remain to be discovered.

\section{AUTHOR CONTRIBUTIONS}

Bram Van de Poel and Dominique Van Der Straeten conceived the topic and wrote the manuscript

\section{ACKNOWLEDGMENTS}

This work was supported by a Belgian American Educational Foundation Fellowship, a CBMG/CMNS Merit Postdoctoral Fellowship (University of Maryland) and a BOF Postdoctoral Fellowship (Ghent University) to Bram Van de Poel. Dominique Van Der Straeten gratefully acknowledges support from the Research Foundation Flanders (FWO) and Ghent University.

\section{REFERENCES}

Adams, D. O., and Yang, S. F. (1977). Methionine metabolism in apple tissue implication of S-adenosylmethionine as an intermediate in conversion of methionine to ethylene. Plant Physiol. 60, 892-896. doi: 10.1104/pp.60.6.892

Adams, D. O., and Yang, S. F. (1979). Ethylene biosynthesis - identification of 1-aminocyclopropane-1-carboxylic acid as an intermediate in the conversion 
of methionine to ethylene. Proc. Natl. Acad. Sci. U.S.A. 76, 170-174. doi: 10.1073/pnas.76.1.170

Ali, S., Charles, T. C., and Glick, B. R. (2012). Delay of carnation flower senescence by bacterial endo- phytes expressing ACC deaminase. J. Appl. Microbiol. 113, 1139-1144. doi: 10.1111/j.1365-2672.2012.05409.x

Alonso, J. M., Hirayama, T., Roman, G., Nourizadeh, S., and Ecker, J. R. (1999). EIN2, a bifunctional transducer of ethylene and stress responses in Arabidopsis. Science 284, 2148-2152. doi: 10.1126/science.284.5423.2148

Amrhein, A., Schneebeck, D., Skorupka, H., Stockigt, J., and Tophof, S. (1981). Identification of a major metabolite of the ethylene precursor 1aminocyclopropane-l-carboxylic acid in higher Plants. Naturwissenschaften 68, 619-620. doi: 10.1007/BF00398617

Amrhein, N., Breuing, F., Eberle, J., Skorupka, H., and Tophof, S. (1982). "The metabolism of 1-aminocyclopropane-1-carboxylic acid," in Plant Growth Substances, ed. P. H. Wareigng (London: Academic Press), 249-258

Atwell, B. J., Drew, M. C., and Jackson, M. B. (2006). The influence of oxygen deficiency on ethylene synthesis, 1-aminocyclopropane-1-carboxylic acid levels and aerenchyma formation in roots of Zea mays. Physiol. Plant. 71, 15-22.

Barnawal, D., Bharti, N., Maji, D., Chanotiya, C. S., and Kalra, A. (2012). 1-Aminocyclopropane-1-carboxylic acid (ACC) deaminase-containing rhizobacteria protect Ocimum sanctum plants during waterlogging stress via reduced ethylene generation. Plant Physiol. Biochem. 58, 227-235. doi: 10.1016/j.plaphy.2012.07.008

Bidonde, S., Ferrer, M. A., Zegzouti, H., Ramassamy, S., Latche, A., Pech, J. C., et al. (1998). Expression and characterization of three tomato 1-aminocyclopropane1-carboxylate oxidase cDNAs in yeast. Eur. J. Biochem. 253, 20-26. doi: 10.1046/j.1432-1327.1998.2530020.x

Boller, T., Herner, R. C., and Kende, H. (1979). Assay for and enzymatic formation of an ethylene precursor, 1-aminocyclopropane-l-carboxylic acid. Planta 145, 293-303. doi: 10.1007/BF00454455

Botella, J. R., Arteca, R. N., and Frangos, J. (1995). A mechanical strain-induced 1aminocyclopropane-1-carboxylic acid synthase gene. Proc. Natl. Acad. Sci. U.S.A 92, 1595-1598. doi: 10.1073/pnas.92.5.1595

Bouzayen, M., Latche, A., Alibert, G., and Pech, J. C. (1988). Intracellular sites of synthesis and storage of 1-(malonylamino)cyclopropane-1-carboxylic acid in acer-pseudoplatanus cells. Plant Physiol. 88, 613-617. doi: 10.1104/pp.88.3.613

Bouzayen, M., Latche, A., Pech, J. C., and Marigo, G. (1989). Carrier-mediated uptake of 1-(malonylamino)cyclopropane-1-carboxylic acid in vacuoles isolated from catharanthus-roseus cells. Plant Physiol. 91, 1317-1322. doi: 10.1104/pp.91.4.1317

Bradford, K. J., Hsiao, T. C., and Yang, S. F. (1982). Inhibition of ethylene synthesis in tomato plants subjected to anaerobic root stress. Plant Physiol. 70, 1503-1507. doi: 10.1104/pp.70.5.1503

Bradford, K. J., and Yang, S. F. (1980). Xylem transport of 1-aminocyclopropane1 -carboxylic acid, an ethylene precursor, in waterlogged tomato plants. Plant Physiol. 65, 322-326. doi: 10.1104/pp.65.2.322

Brady, S. M., Orlando, D. A., Lee, J.-Y., Koch, J., Dinney, J. R., Mace, D., et al. (2007). A high-resolution root spatiotemporal map reveals dominant expression patterns. Science 318, 801-806. doi: 10.1126/science.1146265

Capitani, G., Hohenester, E., Feng, L., Storici, P., Kirsch, J. F., and Jansonius, J. N. (1999). Structure of 1-aminocyclopropane-1-carboxylate synthase, a key enzyme in the biosynthesis of the plant hormone ethylene. J. Mol. Biol. 294, 745-756. doi: 10.1006/jmbi.1999.3255

Cary, A. J., Liu, W., and Howell, S. H. (1995). Cytokinin action is coupled to ethylene in its effects on the inhibition of root and hypocotyl elonagion. Plant Physiol. 107, 1075-1082. doi: 10.1104/pp.107.4.1075

Chae, H. S., Faure, F., and Kieber, J. J. (2003). The eto1, eto2, and eto3 mutations and cytokinin treatment increase ethylene biosynthesis in Arabidopsis by increasing the stability of ACS protein. Plant Cell 15, 545-559. doi: 10.1105/tpc.0 06882

Chae, H. S., and Kieber, J. J. (2005). Eto Brute? Role of ACS turnover in regulating ethylene biosynthesis. Trends Plant Sci. 10, 291-296. doi: 10.1016/j.tplants.2005.04.006

Christians, M. J., Gingerich, D. J., Hansen, M., Binder, B. M., Kieber, J. J., and Vierstra, R. D. (2009). The BTB ubiquitin ligases ETO1, EOL1, and EOL2 act collectively to regulate ethylene biosynthesis in Arabidopsis by controlling type2 ACC synthase levels. Plant J. 57, 332-345. doi: 10.1111/j.1365-313X.2008.0 3693.x
Chung, M. C., Chou, S. J., Kuang, L. Y., Charng, Y. Y., and Yang, S. F. (2002). Subcellular localization of 1-aminocyclopropane-1-carboxylic acid oxidase in apple fruit. Plant Cell Physiol. 43, 549-554. doi: 10.1093/pcp/pcf067

Davies, W. J., Bacon, M. A., Thompson, D. S., Sobeih, W., and Rodriguez, L. G. (2000). Regulation of leaf and fruit growth in plants growing in drying soil: exploitation of the plants' chemical signalling system and hydraulic architecture to increase the efficiency of water use in agriculture. J. Exp. Bot. 51, 1617-1626. doi: 10.1093/jexbot/51.350.1617

De Paepe, A., Vuylsteke, M., Van Hummelen, P., Zabeau, M., and Van Der Staeten, D. (2004). Transcriptional profiling by cDNA-AFLP and microarray analysis reveals novel insights into the early response to ethylene in Arabidopsis. Plant J. 39, 537-559. doi: 10.1111/j.1365-313X.2004.02156.x

Dilley, D. R., Wang, W., Kadirjan-Kalbach, D., Ververidis, F., Beaudry, R., and Padmanabhan, K. (2013). 1-Aminocyclopropane-1-carboxylic acid oxidase reaction mechanism and putative post-translational activities of the ACCO protein. AoB Plants 5:plt031. doi: 10.1093/aobpla/plt031

Dong, J. G., Fernandezmaculet, J. C., and Yang, S. F. (1992). Purification and characterization of 1-aminocyclopropane-1-carboxylate oxidase from apple fruit. Proc. Natl. Acad. Sci. U.S.A. 89, 9789-9793. doi: 10.1073/pnas.89.20.9789

Doubt, S. L. (1917). The response of plants to illuminating gas. Bot. Gaz. 63, 209-224. doi: 10.1086/332006

Dugardeyn, J., Vandenbussche, F., and Van Der Straeten, D. (2008). To grow or not to grow: what can we learn on ethylene-gibberellin cross-talk by in silico gene expression analysis. J. Exp. Bot. 59, 1-16. doi: 10.1093/jxb/erm349

English, P. J., Lycett, G. W., Roberts, J. A., and Jackson, M. B. (1995). Increased 1-aminocyclopropane-1-carboxylic acid oxidase activity in shoots of flooded tomato plants raises ethylene production to physiologically active levels. Plant Physiol. 109, 1435-1440.

Feng, J. N., and Barker, A. V. (1992). Ethylene evolution and ammonium accumulation by tomato plants under water and salinity stresses. J. Plant Nutr. 15, 2471-2490. doi: 10.1080/01904169209364488

Finlayson, S. A., Foster, K. R., and Reid, D. M. (1991). Transport and metabolism of 1-aminocyclopropane- 1-carboxylic acid in sunflower (Helianthus annuus L.) Seedlings. Plant Physiol. 96, 1360-1367. doi: 10.1104/pp.96.4.1360

Gallie, D. R., Geisler-Lee, J., Chen, J., and Jolley, B. (2009). Tissue-specific expression of the ethylene biosynthetic machinery regulates root growth in maize. Plant Mol. Biol. 69, 195-211. doi: 10.1007/s11103-008-9418-1

Gane, R. (1934). Production of ethylene by some ripening fruits. Nature 134, 10081008. doi: 10.1038/1341008a0

Geisler-Lee, J., Caldwell, C., and Gallie, D. R. (2010). Expression of the ethylene biosynthetic machinery in maize roots is regulated in response to hypoxia. J. Exp. Bot. 61, 857-871. doi: 10.1093/jxb/erp362

Ghanem, M. E., Albacete, A., Martinez-Andujar, C., Acosta, M., Romero-Aranda, R., Dodd, I. C., et al. (2008). Hormonal changes during salinity-induced leaf senescence in tomato (Solanum lycopersicum L.). J. Exp. Bot. 59, 3039-3050. doi: 10.1093/jxb/ern153

Glick, B. R. (2010). Using soil bacteria to facilitate phytoremediation. Biotechnol. Adv. 28, 367-374. doi: 10.1016/j.biotechadv.2010.02.001

Glick, B. R. (2014). Bacteria with ACC deaminase can promote plant growth and help to feed the world. Microbiol. Res. 169, 30-39. doi: 10.1016/j.micres.2013.09.009

Glick, B. R., Cheng, Z., Czarny, J., and Duan, J. (2007). Promotion of plant growth by ACC deaminase-producing soil bacteria. Eur. J. Plant Pathol. 119, 329-339. doi: 10.1007/s10658-007-9162-4

Glick, B. R., Penrose, D. M., and Li, J. (1998). A model for the lowering of plant ethylene concentrations by growth-promoting rhizobacteria. J. Theor. Biol. 190, 63-68. doi: 10.1006/jtbi.1997.0532

Grichko, V. P., Filby, B., and Glick, B. R. (2000). Increased ability of transgenic plants expressing the bacterial enzyme ACC deaminase to accumulate $\mathrm{Cd}, \mathrm{Co}, \mathrm{Cu}, \mathrm{Ni}$, $\mathrm{Pb}$, and Zn. J. Biotechnol. 81, 45-53. doi: 10.1016/S0168-1656(00)00270-4

Gurska, J., Wang, W., Gerhardt, K. E., Khalid, A. M., Isherwood, D. M., Huang, X.-D., et al. (2009). Field test of a multi-process phytoremediation system at a petroleum sludge contam-inated land farm. Environ. Sci. Technol. 43, 4472-4479. doi: $10.1021 /$ es $801540 \mathrm{~h}$

Hanley, K. M., Meir, S., and Bramlage, W. J. (1989). Activity of aging carnation flower parts and the effects of 1-(malonylamino)cyclopropane-1-carboxylic acidinduced ethylene. Plant Physiol. 91, 1126-1130. doi: 10.1104/pp.91.3.1126

Hoffman, N. E., Yang, S. F., and Mckeon, T. (1982). Identification of 1-(malonylamino)cyclopropane-1-carboxylic acid as a major conjugate of 
1-aminocyclopropane-1-carboxylic acid, an ethylene precursor in higherplants. Biochem. Biophys. Res. Commun. 104, 765-770. doi: 10.1016/0006291X(82)90703-3

Honma, M., and Shimomura, T. (1978). Metabolism of 1-aminocyclopropane-1carboxylic acid. Agric. Biol. Chem. 43, 1825-1831. doi: 10.1271/bbb1961.42.1825

Hontzeas, N., Zoidakis, J., Glick, B. R., and Abu-Omar, M. M. (2004). Expression and characterization of 1-aminocyclopropane-1-carboxylate deaminase from the rhizobacterium Pseudomonas putida UW4: a key enzyme in bacterial plant growth promotion. Biochim. Biophys. Acta 1703, 11-19. doi: 10.1016/j.bbapap.2004.09.015

Hudgins, J. W., Ralph, S. G., Franceschi, V. R., and Bohlmann, J. (2006). Ethylene in induced conifer defense: cDNA cloning, protein expression, and cellular and subcellular localization of 1-aminocyclopropane-1-carboxylate oxidase in resin duct and phenolic parenchyma cells. Planta 224, 865-877. doi: 10.1007/s00425006-0274-4

Jackson, M. B., and Campbell, D. J. (1976). Waterlogging and petiole epinasty in tomato - role of ethylene and low oxygen. New Phytol. 76, 21-29. doi 10.1111/j.1469-8137.1976.tb01434.x

Jacobson, C. B., Pasternak, J. J., and Glick, B. R. (1994). Partial purification and characterization of ACC deaminase from the plant growth-promoting rhizobacterium Pseudomonas putida GR12-2. Can. J. Microbiol. 40, 1019-1025. doi: 10.1139/m94-162

Jiao, X. Z., Philosoph-Hadas, S., Su, L. Y., and Yang, S. F. (1986). The conversion of 1-(malonylamino)cyclopropane-1-carboxylic acid to 1-aminocyclo propane- 1-carboxylic acid in plant tissues. Plant Physiol. 81, 637-641. doi: 10.1104/pp.81.2.637

Jones, M., and Woodson, W. (1999). Differential expression of three members of the 1-aminocyclopropane-1-carboxylate synthase gene family in carnation. Plant Physiol. 119, 755-764. doi: 10.1104/pp.119.2.755

Jones, M. L., and Woodson, W. R. (1997). Pollination-induced ethylene incarnation. Role of stylar ethylene in corolla senescence. Plant Physiol. 115, 205-212.

Joo, S., Liu, Y., Lueth, A., and Zhang, S. (2008). MAPK phosphorylation-induced stabilization of ACS6 protein is mediated by the non-catalytic C-terminal domain, which also contains the cis-determinant for rapid degradation by the $26 \mathrm{~S}$ proteasome pathway. Plant J. 54, 129-140. doi: 10.1111/j.1365-313X.2008.03404.x

Kamiyoshihara, Y., Iwata, M., Fukaya, T., Tatsuki, M., and Mori, H. (2010). Turnover of LeACS2, a wound-inducible 1-aminocyclopropane-1-carboxylic acid synthase in tomato, is regulated by phosphorylation/dephosphorylation. Plant J. 64, 140150. doi: 10.1111/j.1365-313X.2010.04316.x

Kieber, J. J., Rothenberg, M., Roman, G., Feldmann, K. A., and Ecker, J. R. (1993). CTR1, a negative regulator of the ethylene response pathway in Arabidopsis, encodes a member of the raf family of protein kinases. Cell 72, 427-441. doi: 10.1016/0092-8674(93)90119-B

Kim, C. Y., Liu, Y., Thorne, E. T., Yang, H., Fukushige, H., Gassmann, W., et al. (2003). Activation of a stress-responsive mitogen-activated protein kinase cascade induces the biosynthesis of ethylene in plants. Plant Cell 15, 2707-2718. doi: 10.1105/tpc.011411

Klee, H. J., Hayford, M. B., Kretzmer, K. A., Barry, G. F., and Kishmore, G. M. (1991). Control of ethylene synthesis by expression of a bacterial enzyme in transgenic tomato plants. Plant Cell 3, 1187-1193. doi: 10.1105/tpc.3.11.1187

Knight, L. I., Rose, R. C., and Crocker, W. (1910). Effects of various gases and vapors upon etiolated seedlings of the sweet pea. Science 31, 635-636.

Li, J., McConkey, B. J., Cheng, Z., Guo, S., and Glick, B. R. (2013). Identification of plant growth-promoting bacteria-responsive proteins in cucumber roots under hypoxic stress using a proteomic approach. J. Proteomics 84, 119-131. doi: 10.1016/j.jprot.2013.03.011

Liang, X., Shen, N. F., and Theologis, A. (1996). $\mathrm{Li}^{+}$-regulated 1aminocyclopropane-1-carboxylate synthase gene expression in Arabidopsis thaliana. Plant J. 10, 1027-1036. doi: 10.1046/j.1365-313X.1996.10061027.x

Lieberman, M., and Mapson, L. W. (1964). Genesis and biogenesis of ethylene. Nature 204, 343-345. doi: 10.1038/204343a0

Liu, Y., Su, L. Y., and Yang, S. F. (1985a). Ethylene promotes the capability to malonylate 1-aminocyclopropane-1-carboxylic acid and D-amino acids in preclimacteric tomato fruits. Plant Physiol. 77, 891-895. doi: 10.1104/pp.77.4.891

Liu, Y., Hoffman, N. E., and Yang, S. F. (1985b). Ethylene-promoted malonylation of 1-aminocyclopropane-1-carboxylic acid participated in autoinhibition of ethylene synthesis in grapefruit flavedo disks. Planta 164, 565-568. doi: 10.1007/BF00395976
Liu, Y., and Zhang, S. (2004). Phosphorylation of 1-aminocyclopropane-1carboxylic acid synthase by MPK6, a stress-responsive mitogen-activated protein kinase, induces ethylene biosynthesis in Arabidopsis. Plant Cell 16, 3386-3399. doi: 10.1105/tpc.104.026609

Ludwikow, A., Ciesla, A., Kasprowicz-Maluski, A., Mitula, F., Tajdel, M., Galganski, L., et al. (2014). Arabidopsis protein phosphatase 2C ABIl interacts with type I ACC synthases and is involved in the regulation of ozone-induced ethylene biosynthesis. Mol. Plant 7, 960-967. doi: 10.1093/mp/ssu025

Lund, S. T., Stall, R. E., and Klee, H. J. (1998). Ethylene regulates the susceptible response to pathogen infection in tomato. Plant Cell 10, 371-382. doi: 10.1105/tpc.10.3.371

Lynch, J., and Brown, K. M. (1997). Ethylene and plant responses to nutritional stress. Physiol. Plant. 100, 613-619. doi: 10.1111/j.1399-3054.1997.tb03067.x

Lyzenga, W. J., Booth, J. K., and Stone, S. L. (2012). The Arabidopsis RING-type E3 ligase XBAT32 mediates the proteasomal degradation of the ethylene biosynthetic enzyme, 1-aminocyclopropane-1-carboxylate synthase 7. Plant J. 71, 23-34. doi: 10.1111/j.1365-313X.2012.04965.x

Ma, W., Guinel, F. C., and Glick, B. R. (2003). Rhizobium leguminosarum biovar viciae 1-aminocyclopropane-1-carboxylate deaminase promotes nodulation of pea plants. Appl. Environ. Microbiol. 69, 4396-4402. doi: 10.1128/AEM.69.8.43964402.2003

Martin, M. N., Cohen, J. D., and Saftner, R. A. (1995). A new 1-aminocyclopropane1-carboxylic acid-conjugating activity in tomato fruit. Plant Physiol. 109, 917926. doi: 10.1104/pp.109.3.917

Martin, M. N., and Saftner, R. A. (1995). Purification and characterization of 1aminocyclopropane-1-carboxylic acid N-malonyltransferase from tomato fruit. Plant Physiol. 108, 1241-1249.

Martin, M. N., Saladores, P. H., Lambert, E., Hudson, A. O., and Leustek, T. (2007). Localization of members of the gamma-glutamyl transpeptidase family identifies sites of glutathione and glutathione S-conjugate hydrolysis. Plant Physiol. 144, 1715-1732. doi: 10.1104/pp.106.094409

Martin, M. N., and Slovin, J. P. (2000). Purified $\gamma$-glutamyl transpeptidases from tomato exhibit high affinity for glutathione and glutathione S-conjugates. Plant Physiol. 122, 1417-1426. doi: 10.1104/pp.122.4.1417

Mayak, S., Tirosh, T., and Glick, B. R. (2004a). Plant growth-promoting bacteria that confer resistance to water stress in tomatoes and peppers. Plant Sci. 166, 525-530. doi: 10.1016/j.plantsci.2003.10.025

Mayak, S., Tirosh, T., and Glick, B. R. (2004b). Plant growth-promoting bacteria that confer resistance in tomato to salt stress. Plant Physiol. Biochem. 42, 565-572. doi: 10.1016/j.plaphy.2004.05.009

McDonnell, L., Plett, J. M., Andersson-Gunneras, S., Kozela, C., Dugardeyn, J., Van Der Straeten, D., et al. (2009). Ethylene levels are regulated by a plant encoded 1-aminocyclopropane-1-carboxylic acid deaminase. Physiol. Plant. 136, 94-109. doi: 10.1111/j.1399-3054.2009.01208.x

McManus, M. T. (2012). The Plant Hormone Ethylene - Annual Plant Reviews. Hoboken, NJ: Wiley-Blackwell. doi: 10.1002/9781118223086

Miller, J. M., and Conn, E. E. (1980). Metabolism of hydrogen cyanide by higher plants. Plant Physiol. 65, 1199-1202. doi: 10.1104/pp.65.6.1199

Morris, D., and Larcombe, N. J. (1995). Phloem transport and conjugation of foliarapplied 1-aminoc clopropane-1-carboxylic acid in cotton (Gossypium hirsutum L.). J. Plant Physiol. 146, 429-436. doi: 10.1016/S0176-1617(11)82004-3

Murphy, L. J., Robertson, K. N., Harroun, S. G., Brosseau, C. L., WernerZwanziger, U., Moilanen, J., et al. (2014). A simple complex on the verge of breakdown: isolation of the elusive cyanoformate ion. Science 344, 75-78. doi: $10.1126 /$ science. 1250808

Murr, D. P., and Yang, S. F. (1975). Conversion of $5^{\prime}$-methylthioadenosine to methionine by apple tissue. Phytochemistry 14, 1291-1292. doi: 10.1016/S00319422(00)98613-8

Nadeem, S. M., Zahair, Z. A., Naveed, M., and Arshad, M. (2007). Preliminary investigations on inducing salt tolerance in maize through inoculation with rhizobacteria containing ACC deaminase activity. Can. J. Microbiol. 53, 1141-1149. doi: 10.1139/W07-081

Nadeem, S. M., Zahair, Z. A., Naveed, M., Asghar, H. N., and Asghar, M. (2010). Rhizobacteria capable of producing ACC-deaminase may mitigate salt stress in wheat. Soil Sci. Soc. Am. J. 74, 533-542. doi: 10.2136/sssaj2008.0240

Nascimento, F. X., Brígido, C., Glick, B. R., Oliveira, S., and Alho, L. (2012). Mesorhizobium ciceri LMS-1 expressing an exogenous 1-aminocyclopropane1-carboxylate (ACC) deaminase increases its nodulation abilities and chickpea 
plant resistance to soil constraints. Lett. Appl. Microbiol. 55, 15-21. doi: 10.1111/j.1472-765X.2012.03251.x

Nayani, S., Mayak, S., and Glick, B. R. (1998). The effect of plant growth promoting rhizobacteria on the senescence of flower petals. Ind. J. Exp. Biol. 36, 836-839.

Neljubov, D. (1901). Uber die horizontale nutation der Stengel von Pisum sativum und einiger anderer Pflanzen. Beih. Bot. Centralb. 10, 128-139.

Nie, L., Shah, S., Burd, G. I., Dixon, D. G., and Glick, B. R. (2002). Phytoremediation of arsenate contam-inated soil by transgenic canola and the plant growth-promoting bacterium Enterobacter cloacae CAL2. Plant Physiol. Biochem. 40, 355-361. doi: 10.1016/S0981-9428(02)01375-X

Nukui, N., Minamisawa, K., Ayabe, S.-I., and Aoki, T. (2006). Expression of the 1-aminocyclopropane-1-carboxylic acid deaminase gene requires symbiotic nitrogen-fixing regulator gene nifA2 in Mesorhizobium loti MAFF303099. Appl. Environ. Microbiol. 72, 4964-4969. doi: 10.1128/AEM.02745-05

Olson, D. C., Oetiker, J. H., and Yang, S. F. (1995). Analysis of LE-ACS3, a 1-aminocyclopropane-1-carboxylic acid synthase gene expressed during flooding in the roots of tomato plants. J. Biol. Chem. 270, 14056-14061. doi $10.1074 /$ jbc. 270.23 .14056

Peiser, G., and Yang, S. F. (1998). Evidence for 1-(malonylamino)cyclopropane-1carboxylic acid being the major conjugate of aminocyclopropane-1-carboxylic acid in tomato fruit. Plant Physiol. 116, 1527-1532. doi: 10.1104/pp.116.4.1527

Peng, H.-P., Lin, T.-Y., Wang, N.-N., and Shih, M. C. (2005). Differential expression of genes encoding 1-aminocyclopropane-1-carboxylate synthase in Arabidopsis during hypoxia. Plant Mol. Biol. 58, 15-25. doi: 10.1007/s11103-005-3573-4

Penrose, D. M., Moffatt, B. A., and Glick, B. R. (2001). Determination of 1-aminocyclopropane-1-carboxylic acid (ACC) to assess the effects of ACC deaminase-containing bacteria on roots of canola seedlings. Can. J. Microbiol. 47, 77-80. doi: 10.1139/w00-128

Philosoph-Hadas, S., Meir, S., and Aharoni, N. (1985). Autoinhibition of ethylene production in tobacco leaf-disks - enhancement of 1-aminocyclopropane-1carboxylic acid conjugation. Physiol. Plant. 63, 431-437. doi: 10.1111/j.13993054.1985.tb02322.x

Pommerrenig, B., Feussner, K., Zierer, W., Rabinovych, V., Klebl, F., Feussner, I., et al. (2011). Phloem-specific expression of Yang cycle genes and identification of novel Yang cycle enzymes in Plantago and Arabidopsis. Plant Cell 23, 1904-1919. doi: $10.1105 /$ tpc.110.079657

Ramassamy, S., Olmos, E., Bouzayen, M., Pech, J. C., and Latche, A. (1998). 1 aminocyclopropane-1-carboxylate oxidase of apple fruit is periplasmic. J. Exp. Bot. 49, 1909-1915. doi: 10.1093/jexbot/49.329.1909

Reinhardt, D., Kende, H., and Boller, T. (1994). Subcellular-localization of 1aminocyclopropane-1-carboxylate oxidase in tomato cells. Planta 195, 142-146. doi: 10.1007/BF00206302

Robison, M. M., Shah, S., Tamot, B., Pauls, K. P., Moffatt, B. A., and Glick, B. R. (2001). Reduced symptoms of Verticillium wilt in transgenic tomato expressing a bacterial ACC deaminase. Mol. Plant Pathol. 2, 135-145. doi: 10.1046/j.13643703.2001.00060.x

Rocklin, A. M., Kato, K., Liu, H. W., Que, L., and Lipscomb, J. D. (2004). Mechanistic studies of 1-aminocyclopropane-1-carboxylic acid oxidase: single turnover reaction. J. Biol. Inorg. Chem. 9, 171-182. doi: 10.1007/s00775-003-0510-3

Rodrigues-Pousada, R. A., De Rycke, R., Dedonder, A., Van Caeneghem, W., Engler, G., Van Montagu, M., et al. (1993). The Arabidopsis 1-amioncyclopropane-1carboxylate synthase gene 1 is expressed during early development. Plant Cell 5, 897-891. doi: 10.1105/tpc.5.8.897

Roman, G., Lubarsky, B., Kieber, J. J., Rothenberg, M., and Ecker, J. R. (1995). Genetic analysis of ethylene signal transduction in Arabidopsis thaliana: five novel mutant loci integrated into a stress response pathway. Genetics 139, 1393-1409. doi: 10.1186/1471-2164-9-44

Rombaldi, C., Lelievre, J. M., Latche, A., Petitprez, M., Bouzayen, M., and Pech, J. C. (1994). Immunocytolocalization of 1-aminocyclopropane-1-carboxylic acid oxidase in tomato and apple fruit. Planta 192, 453-460. doi: 10.1007/BF00 203582

Saftner, R. A., and Martin, M. N. (1993). Transport of 1-aminocyclopropane-1carboxylic acid into isolated maize mesophyll vacuoles. Physiol. Plant. 87, 535543. doi: 10.1111/j.1399-3054.1993.tb02504.x

Sato, T., and Theologis, A. (1989). Cloning the mRNA encoding 1aminocycloproane-1-carboxylate synthase, the key enzyme for ethylene biosynthesis in plants. Proc. Natl. Acad. Sci. U.S.A. 86, 6621-6625. doi: 10.1073/pnas.86.17.6621
Sauter, M., Moffatt, B. M., Saechao, M. C., Hell, R., and Wirtz, M. (2013). Methionine salvage and S-adenosylmethionine: essential links between sulfur, ethylene and polyamine biosynthesis. Biochem. J. 451, 145-154. doi: 10.1042/ BJ20121744

Sergeeva, E., Shah, S., and Glick, B. R. (2006). Tolerance of transgenic canola expressing a bacterial ACC deaminase gene to high concentrations of salt. World J. Microbiol. Biotechnol. 22, 277-282. doi: 10.1007/s11274-005-9032-1

Shiu, O. Y., Oetiker, J. H., Yip, W. K., and Yang, S. F. (1998). The promoter of LE-ACS 7, an early flooding-induced 1-aminocyclopropane-1-carboxylate synthase gene of the tomato, is tagged by a Sol 3 transposon. Proc. Natl. Acad. Sci. U.S.A. 95, 10334-10339. doi: 10.1073/pnas.95.17.10334

Skirycz, A., Claes, H., De Bodt, S., Oikawa, A., Shinoda, S., Andriankaja, M., et al. (2011). Pause-and-stop: the effects of osmotic stress on cell proliferation during early leaf development in Arabidopsis and a role for ethylene signaling in cell cycle arrest. Plant Cell 23, 1876-1888. doi: 10.1105/tpc.111.084160

Skottke, K. R., Yoon, G. M., Kieber, J. J., and DeLong, A. (2011). Protein phosphatase $2 \mathrm{~A}$ controls ethylene biosynthesis by differentially regulating the turnover of ACC synthase isoforms. PLoS Genet. 7:e1001370. doi: 10.1371/journal.pgen.10 01370.

Sobeih, W. Y., Dodd, I. C., Bacon, M. A., Grierson, D., and Davies, W. J. (2004). Long-distance signals regulating stomatal conductance and leaf growth in tomato (Lycopersicon esculentum) plants subjected to partial root-zone drying. J. Exp. Bot. 55, 2353-2363. doi: 10.1093/jxb/erh204

Staswick, P. E., and Tiryaki, I. (2004). The oxylipin signal jasmonic acid is activated by an enzyme that conjugates it to isoleucine in Arabidopsis. Plant Cell 16, 21172127. doi: 10.1105/tpc.104.023549

Tatsuki, M., and Mori, H. (2001). Phosphorylation of tomato 1aminocyclopropane-1-carboxylic acid synthase, LE-ACS2, at the C-terminal region. J. Biol. Chem. 276, 28051-28057. doi: 10.1074/jbc.M101543200

Tophof, S., Martinoia, E., Kaiser, G., Hartung, W., and Amrhein, N. (1989). Compartmentation and transport of 1-aminocyclopropane-1-carboxylic acid and N-malonyl-1-aminocyclopropane-1-carboxylic acid in barley and wheat mesophyll-cells and protoplasts. Physiol. Plant. 75, 333-339. doi: 10.1111/j.13993054.1989.tb04635.x

Tsang, D. L., Edmond, C., Harrington, J. L., and Nühse, T. S. (2011). Cell wall integrity controls root elongation via a general 1-aminocyclopropane-1carboxylic acid-dependent, ethylene-independent pathway. Plant Physiol. 156, 596-604. doi: 10.1104/pp.111.175372

Tsuchisaka, A., and Theologis, A. (2004). Unique and overlapping expression patterns among the Arabidopsis 1-amino-cyclopropane-1-carboxylate synthase gene family members. Plant Physiol. 136, 2982-3000. doi: 10.1104/pp.104. 049999

Tsuchisaka, A., Yu, G., Jin, H., Alonso, J. M., Ecker, J. R., Zhang, X., et al. (2009). A combinatorial interplay among the 1-aminocyclopropane-1-carboxylate isoforms regulates ethylene biosynthesis in Arabidopsis thaliana. Genetics 183, 979-1003. doi: 10.1534/genetics.109.107102

Tudela, D., Primo-millo, E., Citricultura, D., De Valencia, I., and Horta, M. D. (1992). 1-aminocyclopropane-1-carboxylic acid transported from roots to shoots promotes leaf abscission in Cleopatra Mandarin (Citrus reshni Hort. ex Tan.) seedlings rehydrated after water stress. Plant Physiol. 100, 131-137. doi: 10.1104/pp.100.1.131

Vahala, J., Schlagnhaufer, C. D., and Pell, E. J. (1998). Induciton of an ACC synthase cDNA by ozone in light-grown Arabidopsis thaliana leaves. Physiol. Plant. 103, 45-50. doi: 10.1034/j.1399-3054.1998.1030106.x

Van de Poel, B., Bulens, I., Hertog, M. L. A. T. M., Nicolai, B., and Geeraerd, A. (2014a). A transcriptomics-based kinetic model for ethylene biosynthesis in tomato (Solanum lycopersicum) fruit: development, validation and exploration of novel regulatory mechanisms. New Phytol. 202, 952-963. doi: 10.1111/ nph. 12685

Van de Poel, B., Bulens, I., Markoula, A., Hertog, M. L. A. T., Dreesen, R., Wirtz, M., et al. (2012). Targeted systems biology profiling of tomato fruit reveals coordination of the Yang cycle and a distinct regulation of ethylene biosynthesis during postclimacteric ripening. Plant Physiol. 160, 1498-1514. doi: 10.1104/pp.112.206086

Van de Poel, B., Vandenzavel, N., Smet, C., Nicolay, T., Bulens, I., Mellidou, I., et al. (2014b). Tissue specific analysis reveals a differential organization and regulation of both ethylene biosynthesis and E8 during climacteric ripening of tomato. BMC Plant Biol. 14:11. doi: 10.1186/1471-2229-14-11 
Van Der Straeten, D., Anuntalabhochai, S., Van Caeneghem, W., Zhou, Z., Gielen, J., and Van Montagu, M. (1997). Expression of three members of the ACC synthase gene family in deepwater rice by submergence, wounding and hormonal treatments. Plant Sci. 124, 79-87. doi: 10.1016/S0168-9452(97)04609-8

Van Der Straeten, D., Rodrigues-Pousada, R. A., Villarroel, R., Hnaley, S., Goodman, H. M., and Van Montagu, M. (1992). Cloning, genetic mapping, and expression analysis of an Arabidopsis thaliana gene that encodes 1-aminocycloropane1-carboxlylate synthase. Proc. Natl. Acad. Sci. U.S.A. 89, 9969-9973. doi: 10.1073/pnas.89.20.9969

Van Der Straeten, D., Van Wiemeersch, L., Goodman, H. M., and Van Montagu, M. (1990). Cloning and sequence of two different cDNA's encoding 1aminocyclopropane-1-carboxylate synthase in tomato. Proc. Natl. Acad. Sci. U.S.A. 87, 4859-4863. doi: 10.1073/pnas.87.12.4859

Van Der Straeten, D., Zhou, Z., Prinsen, E., Van Onckelen, H. A., and Van Montagu, M. C. (2001). A comparative molecular-physiological study of submergence response in lowland and deepwater rice. Plant Physiol. 125, 955-968. doi: $10.1104 /$ pp.125.2.955

Ververidis, P., and John, P. (1991). Complete recovery in vitro of ethylene-forming enzyme-activity. Phytochemistry 30, 725-727. doi: 10.1016/0031-9422(91)8 5241-Q

Wang, K. L.-C., Yoshida, H., Lurin, C., and Ecker, J. R. (2004). Regulation of ethylene gas biosynthesis by the Arabidopsis ETO1 protein. Nature 428, 945-950. doi: $10.1038 /$ nature 02516

Wang, T., and Arteca, R. N. (1992). Effects of low root stress on ethylene biosynthesis in tomato plants (Lycopersicon esculentum Mill cv Heinz 1350). Plant Physiol. 98, 97-100. doi: 10.1104/pp.98.1.97

Woeste, K. E., Vogel, J. P., and Kieber, J. J. (1999). Factors regulating ethylene biosynthesis in etiolated Arabidopsis thaliana seedlings. Physiol. Plant. 105, 478484. doi: 10.1034/j.1399-3054.1999.105312.x

Xu, S.-L., Rahman, A., Baskin, T. I., and Kieber, J. J. (2008). Two leucine-rich repeat receptor kinases mediate signaling, linking cell wall biosynthesis and ACC synthase in Arabidopsis. Plant Cell 20, 3065-3079. doi: 10.1105/tpc.108.063354

Yamagami, T., Tsuchisaka, A., Yamada, K., Haddon, W. F., Harden, L. E., and Theologis, A. (2003). Biochemical diversity among the 1-amino-cyclopropane1-carboxylate synthase isozymes encoded by the Arabidopsis gene family. J. Biol. Chem. 278, 49102-49112. doi: 10.1074/jbc.M308297200

Yang, S. F., and Hoffman, N. E. (1984). Ethylene biosynthesis and its regulation in higher-plants. Annu. Rev. Plant Physiol. Mol. Biol. 35, 155-189. doi: 10.1146/annurev.pp.35.060184.001103

Yoon, G. M., and Kieber, J. J. (2013). 1-Aminocyclopropane-1-carboxylic acid as a signalling molecule in plants. AoB Plants 5:plt017. doi: 10.1093/aobpla/plt017
Yoshida, H., Nagata, M., Saito, K., Wang, K. L. C., and Ecker, J. R. (2005) Arabidopsis ETO1 specifically interacts with and negatively regulates type 2 1-aminocyclopropane-1-carboxylate synthases. BMC Plant Biol. 5:14. doi: 10.1186/1471-2229-5-14

Yoshida, H., Wang, K. L., Chang, C. M., Mori, K., Uchida, E., and Ecker, J. R. (2006). The ACC synthase TOE sequence is required for interaction with ETO1 family proteins and destabilization of target proteins. Plant Mol. Biol. 62, 427-437. doi: 10.1007/s11103-006-9029-7

Zarembinski, T. I., and Theologis, A. (1993). Anaerobiosis and plant growth hormones induce two genes encoding 1-aminocyclopropane-1-carboxylate synthase in rice (Oryza sativa L.). Mol. Biol. Cell 4, 363-373. doi: 10.1091/mbc.4.4.363

Zarembinski, T. I., and Theologis, A. (1997). Expression characteristics of OS-ACS1 and OS-ACS2, two members of the 1-aminocyclopropane-1-carboxylate synthase gene family in rice (Oryza sativa L. cv. Habiganj Aman II) during partial submergence. Plant Mol. Biol. 33, 71-77. doi: 10.1023/B:PLAN.0000009693.26740.c3

Zhang, Z. H., Ren, J. S., Clifton, I. J., and Schofield, C. J. (2004). Crystal structure and mechanistic implications of 1-aminocyclopropane-1-carboxylic acid oxidase - The ethylene-forming enzyme. Chem. Biol. 11, 1383-1394. doi: 10.1016/j.chembiol.2004.08.012

Zhou, Z., Vriezen, W., Caeneghem, W., Van Montagu, M., and Van Der Straeten, D. (2001). Rapid induction of a novel ACC synthase gene in deepwater rice seedlings upon complete submergence. Euphytica 121, 137-143. doi: 10.1023/A:1012059425624

Conflict of Interest Statement: The authors declare that the research was conducted in the absence of any commercial or financial relationships that could be construed as a potential conflict of interest.

Received: 10 October 2014; accepted: 28 October 2014; published online: 11 November 2014.

Citation: Van de Poel B and Van Der Straeten D (2014) 1-aminocyclopropane-1carboxylic acid (ACC) in plants: more than just the precursor of ethylene!. Front. Plant Sci. 5:640. doi: 10.3389/fpls.2014.00640

This article was submitted to Plant Physiology, a section of the journal Frontiers in Plant Science.

Copyright (C) 2014 Van de Poel and Van Der Straeten. This is an open-access article distributed under the terms of the Creative Commons Attribution License (CC BY). The use, distribution or reproduction in other forums is permitted, provided the original author(s) or licensor are credited and that the original publication in this journal is cited, in accordance with accepted academic practice. No use, distribution or reproduction is permitted which does not comply with these terms. 


\title{
The role of ethylene and ROS in salinity, heavy metal, and flooding responses in rice
}

\author{
Bianka Steffens * \\ Department of Plant Physiology, Faculty of Biology, Philipps University, Marburg, Germany
}

\section{Edited by:}

Domenico De Martinis, National Agency for New Technologies,

Energy and Sustainable Economic

Development, Italy

\section{Reviewed by:}

Nicolas L. Taylor, The University of Western Australia, Australia

Francesca Cardinale, University of

Turin, Italy

${ }^{*}$ Correspondence:

Bianka Steffens, Department of

Plant Physiology, Faculty of Biology,

Philipps University,

Karl-von-Frisch-Strasse 8, 35043

Marburg, Germany

e-mail:

bianka.steffens@biologie.uni-

marburg.de

\begin{abstract}
Plant growth and developmental processes as well as abiotic and biotic stress adaptations are regulated by small endogenous signaling molecules. Among these, phytohormones such as the gaseous alkene ethylene and reactive oxygen species (ROS) play an important role in mediating numerous specific growth or cell death responses. While apoplastic ROS are generated by plasma membrane-located respiratory burst oxidase homolog proteins, intracellular ROS are produced mainly in electron transfer chains of mitochondria and chloroplasts. Ethylene accumulates in plants due to physical entrapment or by enhanced ethylene biosynthesis. A major crop that must endure high salt and heavy metal concentrations upon flooding in regions of Asia is rice. Ethylene and ROS have been identified as the major signals that mediate salinity, chromium, and flooding stress in rice. This mini review focuses on (i) what is known about ethylene and ROS level control during these abiotic stresses in rice, (ii) how the two signals mediate growth or death processes, and (iii) feedback mechanisms that in turn regulate ethylene and ROS signaling.
\end{abstract}

Keywords: abiotic stress response, chromium, ethylene, flooding, reactive oxygen species, rice, salt

\section{INTRODUCTION}

Flooding is a major abiotic stress which results in crop yield losses in a wide range of different landscapes. Some crop species can endure soil waterlogging for some hours while other flood tolerant crops can cope with partial or complete flooding for some days or months (Bailey-Serres and Voesenek, 2008). In addition to flooding, salt concentrations may rise when salts delivered by water from the ocean or from flushing out salts from wet soil. In addition, heavy metal availability may change due to changing $\mathrm{pH}$ values upon flooding. High salinity and heavy metal concentrations can similarly reduce crop growth and may even cause plant death (e.g., Li et al., 2014; Trinh et al., 2014).

Flood tolerant crops possess anatomical, metabolic, or morphological adaptations. One metabolic response to flooding, heavy metals and high salinity, is the induction of ethylene production (Kende, 1993; Li et al., 2014; Trinh et al., 2014). Ethylene production is enhanced in crops such as barley and rice (Kende, 1993; Vassilev et al., 2004; Li et al., 2014). Only tolerant rice plants, however, endure longer phases of soil waterlogging or submergence (Bailey-Serres and Voesenek, 2008). Ethylene is produced in a two-step reaction. The first specific step is the formation of 1-aminocyclopropane-1-carboxylate (ACC) by ACC synthase (ACS), the second step implies ethylene formation from ACC by ACC oxidase (ACO; Kende, 1993). Ethylene is not only produced during these abiotic stresses, but has also been identified as regulator of stress-related morphological responses such as primary and secondary root growth or aerenchyma formation in internodes, roots, and leaves (e.g., Parlanti et al., 2011; Steffens et al., 2011). In Arabidopsis, ethylene is perceived by five ER- localized two-component receptors such as ETR1 (ETHYLENE RESISTANT1; Chen et al., 2002). Downstream of ethylene receptors the signaling cascade consists, of a member of the Nramp family of ion transporters, EIN2 (ETHYLENE INSENSITIVE2), of transcription factors such as EIN3 and of members of the APETALA2/ETHYLENE RESPONSE FACTOR (AP2/ERF) multi gene family.

Reactive oxygen species (ROS) have been identified as a second class of small molecules that mediate responses to flooding, heavy metals, and high salinity. ROS are generated from molecular oxygen and their origin is diverse. Apoplastic ROS are generated through plasma membrane-located Rboh (respiratory burst oxidase homolog) proteins. Rboh proteins in plants are homologs of mammalian NADPH oxidase subunit gp91 phox (Torres et al., 1998) that produce superoxide anions $\left(\mathrm{O}_{2}^{\bullet-}\right)$. Short-lived $\mathrm{O}_{2}^{--}$dismutate to the non-radical hydrogen peroxide $\left(\mathrm{H}_{2} \mathrm{O}_{2}\right)$ either spontaneously, or catalyzed by superoxide dismutase (SOD) or ascorbate peroxidases (POD). In addition, $\mathrm{O}_{2}^{\bullet-}$, hydroxyl radicals, hydroperoxyl radicals, ozone, and singlet oxygen are produced through the reductive power provided by electron transport chains of mitochondria and chloroplasts and through peroxisomal activity (Blokhina and Fagerstedt, 2010; Chang et al., 2012). Non-enzymatic ROS scavenging proteins such as cysteine-rich metallothioneins (MTs) as well as antioxidant enzymes, e.g., SOD, catalase, and glutathione reductase (GR) are essential for ROS homeostasis. During biotic and abiotic stress, the cellular ROS balance is disturbed by either enhancing ROS generation or reducing ROS scavenging abilities (Steffens et al., 2013). 
Plants react to different incoming signals when flooding, high salinity, and heavy metal stress occur in combination. The plants' ability to coordinate these signals and start the adaptive survival responses requires mainly two internal signals: ethylene and different ROS. This review focuses on ethylene and ROS as signaling intermediates in salinity, chromium, and flooding stress responses in the crop species rice.

\section{MAPK-MEDIATED PHOSPHORYLATION AFFECTS ETHYLENE AND ROS HOMEOSTASIS DURING SALT STRESS SIGNALING}

In all other plant species than halophytes, high sodium chloride concentrations cause growth retardation and may result in plant death because of drastic changes in ion and ROS homeostasis and in altered gene expression (Li et al., 2014). In addition, salt stress induces ethylene generation, ethylene can function as a downstream signal and alter gene expression as well (Wang et al., 2002). Interestingly, enhanced synthesis of ACC, the natural precursor of ethylene, seems to reduce salt tolerance (Dong et al., 2011). Ethylene signaling in turn is required for salinity tolerance of plants (Dong et al., 2011), showing the importance of ethylene homeostasis during salt stress.

Lectin receptor-like kinases (RLKs) are a family with 173 members in rice. The lectin RLK protein consist of an N-terminal lectin, a transmembrane domain, and a C-terminal kinase domain (Vaid et al., 2012). They are involved in developmental processes, in biotic stress signaling or in self-incompatibility (Vaid et al., 2012). Like other RLKs, lectin RLKs mediate the incoming signals through phosphorylation of mitogen-activated protein kinases (MAPKs). Activation of MAPK cascade signaling and enhanced ROS generation are further salt stress responses (Kiegerl et al., 2000; Teige et al., 2004).

The plasma membrane-located lectin RLK SIT1 (SALT INTOLERANCE1) is mainly located at the surface of rice root cells (Li et al., 2014). SIT1 is activated under high salinity conditions and mediates salt stress signaling through direct phosphorylation of OsMPK3 and OsMPK6 (Figure 1). Activated OsMPK3 and OsMPK6 in turn phosphorylate ACS proteins. Phosphorylation of ACS proteins results in increased protein stability and activity, hence in enhanced ethylene production. SIT1 upregulates ACS2 during salt stress, pointing to an additional transcriptional regulation of genes relevant for ethylene biosynthesis. Ethylene signaling through ETR1, EIN2, and EIN3 is also part of the SIT1 signaling pathway during salt stress. During salt stress, OsMPK3 and OsMPK6 activities act upstream of ethylene signaling (Li et al., 2014). Ethylene was shown to be required for ROS accumulation upon salt stress (Li et al., 2014). ROS accumulation is dependent on reduced POD and GR activities ( $\mathrm{Li}$ et al., 2014).

Ethylene responsive transcription factors of other species have been described to be involved in the control of ROS generation and signaling. Transcription factors of the AP2/ERF multi gene family link the signaling pathways of ROS and ethylene during abiotic stress. In Tamarix hispida, ERF1 inhibits the expression of SOD and POD genes during drought or high salinity, thus leading to enhanced ROS levels due to reduced scavenging ability (Wang et al., 2014). In rice, this link has not yet been analyzed.

\section{STRESS \\ High salinity

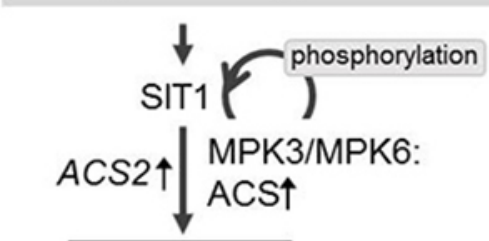 \\ Ethylene $\uparrow$

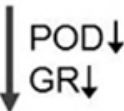

$$
\text { ROS } \uparrow
$$

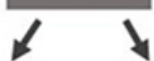 \\ High $\mathrm{Cr}(\mathrm{VI})$

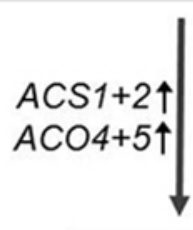 \\ Ethylene $\uparrow$
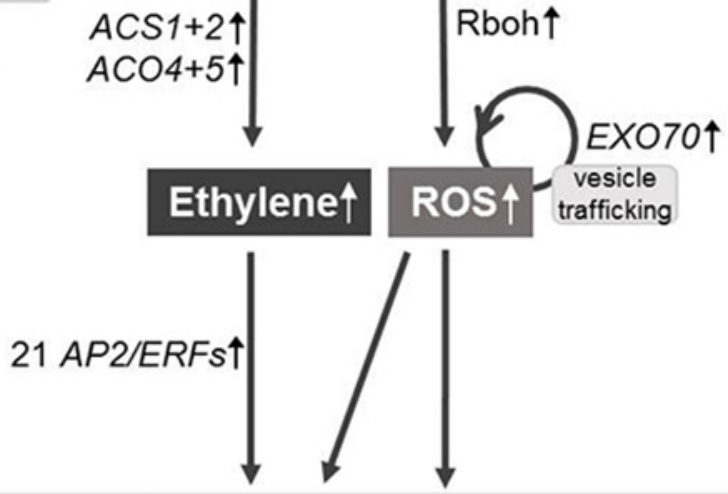 \\ Flooding

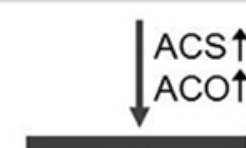 \\ Ethylene $\uparrow$

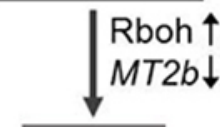 \\ ROS $\uparrow$

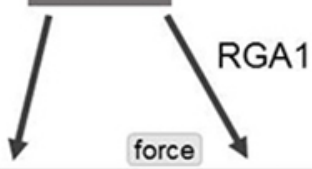
Root growth
Plant
inhibition

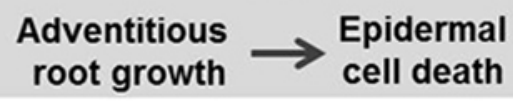 \\ RESPONSE \\ FIGURE 1 | Simplified model of salinity-, chromium-, and flooding- induced signaling pathways in rice. Ethylene and ROS are the major internal signals. Plant responses comprise growth regulation and death. \\ Other hormones and signals are not shown. Abbreviations and details are described in the text (based on Steffens et al., 2012; Li et al., 2014; Trinh et al., 2014).}




\section{ETHYLENE, ROS, AND VESICLE TRAFFICKING-TEAM PLAYERS DURING CHROMIUM STRESS IN RICE}

A side effect of waterlogging or flooding may be the $\mathrm{pH}$ dependent increased availability of the heavy metal chromium from soils. Principal component analysis of different data sets from soil studies in Asia suggested that the concentration of chromium correlated with the soil $\mathrm{pH}$ values (Zarcinas et al., 2004). Toxicity of heavy metals is based on (i) exchange of required ions from protein binding sites, (ii) cellular redox perturbation through altering ROS production and scavenging, and (iii) membrane damage; toxicity often depends (iv) on the valence state of the heavy metal ion (Sharma and Dietz, 2009). Copper and chromium are merely two redox-active heavy metals that at high concentrations in soil induce oxidative stress and hence growth retardation in plants such as pea (Palma et al., 1987) or red cabbage (Posmyk et al., 2008). Upon heavy metal stress ROS are produced mainly through electron transport chains of mitochondria and chloroplasts and through peroxisomal activity (Dixit et al., 2002; Pandey et al., 2009). In addition, Rboh contribute to apoplastic ROS generation induced by heavy metals (Trinh et al., 2014).

Induction of oxidative stress by the most toxic form of chromium $\mathrm{Cr}(\mathrm{VI})$ was identified as the major problem for seedling growth in rice (Panda, 2007; Zeng et al., 2012). Cr stress resulted in ROS generation through plasma membranelocated Rboh (Trinh et al., 2014) and elevated lipid peroxidation (Panda, 2007). Based on transcriptome profiles of rice seedling roots that were obtained after 1 and $3 \mathrm{~h}$ of application of $\mathrm{Cr}(\mathrm{VI})$, ethylene biosynthesis and signaling, vesicle trafficking and ROS level modulation were identified as being part of the $\mathrm{Cr}$ signaling pathway (Huang et al., 2014; Trinh et al., 2014; Figure 1).

Various plant hormone increase upon different heavy metal stresses. During high cadmium concentrations in pea and barley ethylene levels are elevated (Vassilev et al., 2004; RodríguezSerrano et al., 2006). Upregulation of two of the six ACS gene family members, ACS1 and ACS2, and of two of the seven ACO gene family members in rice, $\mathrm{ACO} 4$ and $\mathrm{ACO} 5$ (Rzewuski and Sauter, 2008), indicates that ethylene synthesis is part of $\mathrm{Cr}$ signaling (Trinh et al., 2014). In rice, ACS1 is also induced by hypoxia, anoxia, ethylene, and $\mathrm{H}_{2} \mathrm{O}_{2}$ (Zarembinski and Theologis, 1993; Steffens and Sauter, 2009a), and ACS2 and ACO5 are both induced by infection with the fungus Magnaporthe grisea (Iwai et al., 2006), linking these genes to ethylene biosynthesis upon abiotic and biotic stress. Ethylene, together with other plant hormones may contribute to growth inhibition during $\mathrm{Cr}$ stress.

Upregulation of more than twofold of AP2/ERF genes by $\mathrm{Cr}$ treatment was observed in 21 members of different gene family subgroups and downregulation of a member of subgroup Ib, ERF120 (Nakano et al., 2006; Trinh et al., 2014). Three genes, namely ERF67 (subgroup VIIa), ERF68 (subgroup VIIa), and ERF77 (subgroup VIIIa), were upregulated by $\mathrm{Cr}$ and drought in rice (Nakano et al., 2006; Wang et al., 2011; Trinh et al., 2014). In addition, subgroup VII AP2/ERFs such as SNORKEL1 (SK1), SNORKEL2 (SK2), and SUBMERGENCE1A-1 (SUB1A-1) were previously identified as being crucial for ethylene-mediated growth regulation of rice upon submergence (Fukao et al., 2006;
Hattori et al., 2009). Hence, ERF67, ERF68, and ERF77 are subject to regulation by different abiotic stresses, indicating that they may be general abiotic stress response genes.

Vesicle trafficking contributes to exocytosis, hence cell growth and other adaptive reactions of plants to stresses are affected. Exocyst subunit Exo70 is part of a protein complex with eight subunits that mediates vesicle trafficking from the post-Golgi to the plasma membrane. Upregulation upon $\mathrm{Cr}$ treatment of five Exo70 genes of different groups of the 41 Exo70 gene family members in rice indicates that exocytosis contributes to early Cr signaling (Trinh et al., 2014). Vesicle trafficking-associated gene expression was also found in roots of Salix during $\mathrm{Cr}(\mathrm{VI})$ stress (Quaggiotti et al., 2007). Inhibition of vesicle trafficking by pharmacological approaches using brefeldin $\mathrm{A}$ resulted in reduced ROS generation during $\mathrm{Cr}(\mathrm{VI})$ and $\mathrm{Cu}$ stress, indicating that vesicle trafficking mediated by the exocyst complex increases oxidative stress during heavy metal stress (Lin et al., 2013; Trinh et al., 2014). The involvement of vesicle trafficking itself or vesicle trafficking-associated gene expression have been, to my knowledge, not analyzed yet for the signaling pathway or biosynthesis.

\section{ETHYLENE, ROS, AND MECHANICAL SIGNALING MEDIATE EPIDERMAL CELL DEATH UPON FLOODING IN RICE}

Morphological adaptive responses of submerged rice plants, such as internodal growth or spatially controlled death of parenchymal cells during aerenchyma formation in various organs, are regulated through ethylene, abscisic acid, and/or gibberellin signaling (e.g., Raskin and Kende, 1984a,b; Justin and Armstrong, 1991; Hoffmann-Benning and Kende, 1992; Steffens et al., 2011). The gaseous hormone ethylene accumulates upon submergence due to physical entrapment and enhanced biosynthesis. Ethylene is accepted to be the major regulator. In deepwater rice, ethylene helps the foliage to escape submergence by fast stem growth mediated by SK1 and SK2 (Hattori et al., 2009). In floodingresistant cultivars ethylene inhibits stem growth during the quiescence response regulated by another subgroup VII AP2/ERF transcription factor, SUB1A-1 (Xu et al., 2006). The involvement of subgroup VII AP2/ERFs in aerenchyma formation in internodes, primary and secondary roots is largely unknown. Interestingly, analysis of aerenchyma formation in leaf sheaths of a variety where SublA is absent and a variety containing the Sub1A gene revealed that either ethylene accumulation and signaling or ROS signaling are important (Parlanti et al., 2011).

Furthermore, ethylene mediates submergence-induced growth of adventitious roots (Lorbiecke and Sauter, 1999; Steffens et al., 2012) and death of the epidermal cells overlying adventitious root primordia (Mergemann and Sauter, 2000; Steffens and Sauter, 2009a; Steffens et al., 2012). These ethylene-regulated responses are mediated by both ROS accumulation and/or ROS signaling. Transcriptome analysis revealed that $A C O 1$ is upregulated in epidermal cells overlying adventitious root primordia, hence locally increased ACO1 activity could result in spatial control of ethylene biosynthesis (Mekhedov and Kende, 1996; Steffens and Sauter, 2009a). Death of epidermal cells overlying adventitious root primordia depends on Rboh activity, ethylene and in addition on 
a mechanical signal. Force generated by the growing adventitious root primordium combined with chemical signaling results in locally induced epidermal cell death (Steffens et al., 2012; Figure 1). Epidermal cell death is furthermore mediated by RGA1 (rice heterotrimeric G protein alpha subunit; D1) that encodes the single $G \alpha$ subunit of heterotrimeric $G$ protein in rice (Steffens and Sauter, 2009b). G protein signaling is an essential part of epidermal cell death. $d 1$ mutant plants that have a repressed expression of RGA1 showed a reduction of epidermal cell death in response to ethylene and $\mathrm{H}_{2} \mathrm{O}_{2}$. The role of heterotrimeric $\mathrm{G}$ protein in salinity and heavy metal stress has not been analyzed yet in rice.

\section{ETHYLENE AND ROS-SMALL MOLECULES AND THEIR COMPLEXITY}

Various abiotic stresses occur during a plants' life due to its sessile way of life. Crops like rice have to cope with high levels of salt and soil contaminations such as chromium that occur with rising water levels. Responses to these tremendous stresses are mediated by ethylene and ROS which act as internal signals. Ethylene and ROS are intermediators of gene expression as far as growth regulation or specific cell death are affected. Sometimes the plant dies due to these stresses, but processes triggered by ethylene and ROS often prevent plants' death. Besides the chemical signals, phosphorylation cascades, vesicle trafficking, or mechanosignaling were identified as being part of salinity, chromium, or submergence signaling, respectively. These and other results not mentioned here pinpoint to the complexity of ethylene and ROS signaling upon abiotic stress responses. A closer look into ethylene signaling and ROS homeostasis in the future will help to itemize the regulatory network that leads to plant survival responses upon abiotic stresses.

\section{REFERENCES}

Bailey-Serres, J., and Voesenek, L. A. (2008). Flooding stress: acclimations and genetic diversity. Annu. Rev. Plant Biol. 59, 313-339. doi: 10.1146/annurev. arplant.59.032607.092752

Blokhina, O., and Fagerstedt, K. V. (2010). Oxidative metabolism, ROS and NO under oxygen deprivation. Plant Physiol. Biochem. 48, 359-373. doi: 10.1016/j.plaphy.2010.01.007

Chang, R., Jang, C. J., Branco-Price, C., Nghiem, P., and Bailey-Serres, J. (2012). Transient MPK6 activation in response to oxygen deprivation and reoxygenation is mediated by mitochondria and aids seedling survival in Arabidopsis. Plant Mol. Biol. 78, 109-122. doi: 10.1007/s11103-011-9850-5

Chen, Y. F., Randlett, M. D., Findell, J. L., and Schaller, E. G. (2002). Localization of the ethylene receptor ETR1 to the endoplasmic reticulum in Arabidopsis. J. Biol. Chem. 277, 19861-19866. doi: 10.1074/jbc.M201286200

Dixit, V., Pandey, V., and Shyam, R. (2002). Chromium ions inactivate electron transport and enhance superoxide generation in vivo in pea (Pisum sativum L. cv. Azad) root mitochondria. Plant Cell Environ. 25, 687-690. doi: 10.1046/j.1365-3040.2002.00843.x

Dong, H., Zhen, Z., Peng, J., Chang, L., Gong, Q., and Wang, N. N. (2011). Loss of ACS7 confers abiotic stress tolerance by modulating ABA sensitivity and accumulation in Arabidopsis. J. Exp. Bot. 62, 4875-4887. doi: 10.1093/jxb/err143

Fukao, T., Xu, K., Ronald, P. C., and Bailey-Serres, J. (2006). A variable cluster of ethylene response factor-like genes regulates metabolic and developmental acclimation responses to submergence in rice. Plant Cell 18, 2021-2034. doi: 10.1105/tpc. 106.043000

Hattori, Y., Nagai, K., Furukawa, S., Song, X. J., Kawona, R., Sakakibara, H., et al. (2009). The ethylene response factors SNORKEL1 and SNORKEL2 allow rice to adapt to deep water. Nature 460, 1026-1030. doi: 10.1038/nature08258
Hoffmann-Benning, S., and Kende, H. (1992). On the role of abscisic acid and gibberellin in the regulation of growth in rice. Plant Physiol. 99, 1156-1161. doi: 10.1104/pp.99.3.1156

Huang, T. L., Huang, L. Y., Fu, S. F., Trinh, N. N., and Huang, H. J. (2014). Genomic profiling of rice roots with short- and long-term chromium stress. Plant Mol. Biol. 86, 157-170. doi: 10.1007/s11103-014-0219-4

Iwai, T., Miyasaka, A., Seo, S., and Ohashi, Y. (2006). Contribution of ethylene biosynthesis for resistance to blast fungus infection in young rice plants. Plant Physiol. 142, 1202-1215. doi: 10.1104/pp.106.085258

Justin, S. H. F. W., and Armstrong, W. (1991). Evidence for the involvement of ethylene in aerenchyma formation in adventitious roots of rice (Oryza sativa L.). New Phytol. 118, 49-62. doi: 10.1111/j.1469-8137.1991.tb00564.x

Kende, H. (1993). Ethylene biosynthesis. Annu. Rev. Plant Physiol. Plant Mol. Biol. 44, 283-307. doi: 10.1146/annurev.pp.44.060193.001435

Kiegerl, S., Cardinale, F., Siligan, C., Gross, A., Baudouin, E., Liwosz, A., et al. (2000). SIMKK, a mitogen-activated protein kinase (MAPK) kinase, is a specific activator of the salt stress-induced MAPK, SIMK. Plant Cell 12, 2247-2258. doi: 10.1105/tpc.12.11.2247

Li, C. H., Wang, G., Zhao, J. L., Zhang, L. Q., Ai, L. F., Han, Y. F., et al. (2014). The receptor-like kinase SIT1 mediates salt sensitivity by activating MAPK3/6 and regulating ethylene homeostasis in rice. Plant Cell 26, 2538-2553. doi: 10.1105/tpc.114.125187

Lin, C. Y., Trinh, N., Fu, S. F., Hsiung, Y. C., Chia, L. C., Lin, C. W., et al. (2013). Comparison of early transcriptome responses to copper and cadmium in rice roots. Plant Mol. Biol. 81, 507-522. doi: 10.1007/s11103-013-0020-9

Lorbiecke, R., and Sauter, M. (1999). Adventitious root growth and cell-cycle induction in deepwater rice. Plant Physiol. 119, 21-29. doi: 10.1104/pp.119. 1.21

Mekhedov, S. I., and Kende, H. (1996). Submergence enhances expression of a gene encoding 1-aminocyclopropane-1-carboxylate oxidase in deepwater rice. Plant Cell Physiol. 37, 531-537. doi: 10.1093/oxfordjournals.pcp.a028976

Mergemann, H., and Sauter, M. (2000). Ethylene induces epidermal cell death at the site of adventitious root emergence in rice. Plant Physiol. 124, 609-614. doi: 10.1104/pp.124.2.609

Nakano, T., Suzuki, K., Fujimura, T., and Shinshi, H. (2006). Genome-wide analysis of the ERF gene family in Arabidopsis and rice. Plant Physiol. 140, 411-432. doi: $10.1104 /$ pp.105.073783

Palma, J. M., Gómez, M., Yáñez, J., and Del Río, L. A. (1987). Increased levels of peroxisomal active oxygen-related enzymes in copper-tolerant pea plants. Plant Physiol. 85, 570-574. doi: 10.1104/pp.85.2.570

Panda, S. K. (2007). Chromium-mediated oxidative stress and ultrastructural changes in root cells of developing rice seedlings. J. Plant Physiol. 164, 14191428. doi: 10.1016/j.jplph.2007.01.012

Pandey, V., Dixit, V., and Shyam, R. (2009). Chromium effect on ROS generation and detoxification in pea (Pisum sativum) leaf chloroplasts. Protoplasma 236, 85-95. doi: 10.1007/s00709-009-0061-8

Parlanti, S., Kudahettige, N. P., Lombardi, L., Mensuali-Sodi, A., Alpi, A., Perata, P., et al. (2011). Distinct mechanisms for aerenchyma formation in leaf sheaths of rice genotypes displaying a quiescence or escape strategy for flooding tolerance. Ann. Bot. 107, 1335-1343. doi: 10.1093/aob/mcr086

Posmyk, M. M., Kontek, R., and Janas, K. M. (2008). Antioxidant enzymes activity and phenolic compounds content in red cabbage seedlings exposed to copper stress. Ecotoxicol. Environ. Saf. 72, 596-602. doi: 10.1016/j.ecoenv.2008. 04.024

Quaggiotti, S., Barcaccia, G., Schiavon, M., Nicole, S., Galla, G., Rossignolo, V., et al. (2007). Phytoremediation of chromium using Salix species: cloning ESTs and candidate genes involved in the Cr response. Gene 402, 68-80. doi: 10.1016/j.gene.2007.07.021

Raskin, I., and Kende, H. (1984a). Regulation of growth in stem sections of deep water rice. Planta 160, 66-72. doi: 10.1007/BF00392467

Raskin, I., and Kende, H. (1984b). Role of gibberellin in the growth response of deep water rice. Plant Physiol. 76, 947-950. doi: 10.1104/pp.76.4.947

Rodríguez-Serrano, M., Romero-Puertas, M. C., Zabalza, A., Corpas, F. J., Gómez, M., Del Río, L. A., et al. (2006). Cadmium effect on oxidative metabolism of pea (Pisum sativum L.) roots. Imaging of reactive oxygen species and nitric oxide accumulation in vivo. Plant Cell Environ. 29, 1532-1544. doi: 10.1111/j.13653040.2006.01531.x

Rzewuski, G., and Sauter, M. (2008). Ethylene biosynthesis and signaling in rice. Plant Sci. 175, 32-42. doi: 10.1016/j.plantsci.2008.01.012 
Sharma, S. S., and Dietz, K. J. (2009). The relationship between metal toxicity and cellular redox imbalance. Trends Plant Sci. 14, 43-50. doi: 10.1016/j.tplants.2008.10.007

Steffens, B., Geske, T., and Sauter, M. (2011). Aerenchyma formation in the rice stem and its promotion by $\mathrm{H}_{2} \mathrm{O}_{2}$. New Phytol. 190, 369-378. doi: 10.1111/j.1469-8137.2010.03496.x

Steffens, B., Kovalev, A., Gorb, S. N., and Sauter, M. (2012). Emerging roots alter epidermal cell fate through mechanical and reactive oxygen species signaling. Plant Cell 24, 3296-3306. doi: 10.1105/tpc.112.101790

Steffens, B., and Sauter, M. (2009a). Epidermal cell death in rice is confined to cells with a distinct molecular identity and is mediated by ethylene and $\mathrm{H}_{2} \mathrm{O}_{2}$ through an autoamplified signal pathway. Plant Cell 21, 184-196. doi: 10.1105/tpc.108.061887

Steffens, B., and Sauter, M. (2009b). Heterotrimeric G protein signaling is required for epidermal cell death in rice. Plant Physiol. 151, 732-740. doi: 10.1104/pp.109.142133

Steffens, B., Steffen-Heins, A., and Sauter, M. (2013). Reactive oxygen species mediate growth and death in submerged plants. Front. Plant Sci. 4:179. doi: 10.3389/fpls.2013.00179

Teige, M., Scheikl, E., Eulgem, T., Dóczi, R., Ichimura, K., Shinozaki, K., et al. (2004). The MKK2 pathway mediates cold and salt stress signaling in Arabidopsis. Mol. Cell 15, 141-152. doi: 10.1016/j.molcel.2004.06.023

Torres, M. A., Onouchi, H., Hamada, S., Machida, C., Hammond-Kosack, K. E., and Jones, J. D. (1998). Six Arabidopsis thaliana homologues of the human respiratory burst oxidase (gp91phox). Plant J. 14, 365-370. doi: 10.1046/j.1365313X.1998.00136.x

Trinh, N. N., Huang, T. L., Chi, W. C., Fu, S. F., Chen, C. C., and Huang, H. J. (2014). Chromium stress response effect on signal transduction and expression of signaling genes in rice. Physiol. Plant. 150, 205-224. doi: 10.1111/ppl.12088

Vaid, N., Pandey, P. K., and Tuteja, N. (2012). Genome-wide analysis of lectin receptor-like kinase family from Arabidopsis and rice. Plant Mol. Biol. 80, 365388. doi: 10.1007/s11103-012-9952-8

Vassilev, A., Lidon, F., Scotti, P., da Graca, M., and Yordanov, I. (2004). Cadmiuminduced changes in chloroplast lipids and photosystem activities in barley plants. Biol. Plant. 48, 153-156. doi: 10.1023/B:BIOP.0000024295.27419.89

Wang, D., Pan, Y., Zhao, X., Zhu, L., Fu, B., and Li, Z. (2011). Genome-wide temporal-spatial gene expression profiling of drought responsiveness in rice. BMC Genomics 12:149. doi: 10.1186/1471-2164-12-149
Wang, K. L., Li, H., and Ecker, J. R. (2002). Ethylene biosynthesis and signaling networks. Plant Cell 14(Suppl.), S131-S151. doi: 10.1105/tpc.001768

Wang, L., Qin, L., Liu, W., Zhang, D., and Wang, Y. (2014). A novel ethyleneresponsive factor from Tamarix hispida, ThERF1, is a GCC-box- and DRE-motif binding protein that negatively modulates abiotic stress tolerance in Arabidopsis. Physiol. Plant. 152, 84-97. doi: 10.1111/ppl.12159

Xu, K., Xu, X., Fukao, T., Canlas, P., Maghirang-Rodriguez, R., Heuer, S., et al. (2006). SublA is an ethylene-response-factor-like gene that confers submergence tolerance to rice. Nature 442, 705-708. doi: 10.1038/nature 04920

Zarcinas, B. A., Isahk, C. F., McLaughlin, M. J., and Cozens, G. (2004). Heavy metals in soils and crops in Southeast Asia. 1. Peninsular Malaysia. Environ. Geochem. Health 26, 343-357. doi: 10.1007/s10653-005-4669-0

Zarembinski, T. I., and Theologis, A. (1993). Anaerobiosis and plant growth hormones induce two genes encoding 1-aminocyclopropane-1-carboxylate synthase in rice (Oryza sativa L.). Mol. Biol. Cell 4, 363-373. doi: 10.1091/mbc.4. 4.363

Zeng, F., Qiu, B., Wu, X., Niu, S., Wu, F., and Zhang, G. (2012). Glutathionemediated alleviation of chromium toxicity in rice plants. Biol. Trace Elem. Res. 148, 255-263. doi: 10.1007/s12011-012-9362-4

Conflict of Interest Statement: The author declares that the research was conducted in the absence of any commercial or financial relationships that could be construed as a potential conflict of interest.

Received: 28 August 2014; accepted: 18 November 2014; published online: 04 December 2014.

Citation: Steffens B (2014) The role of ethylene and ROS in salinity, heavy metal, and flooding responses in rice. Front. Plant Sci. 5:685. doi: 10.3389/fpls.2014.00685

This article was submitted to Plant Physiology, a section of the journal Frontiers in Plant Science.

Copyright (C) 2014 Steffens. This is an open-access article distributed under the terms of the Creative Commons Attribution License (CC BY). The use, distribution or reproduction in other forums is permitted, provided the original author(s) or licensor are credited and that the original publication in this journal is cited, in accordance with accepted academic practice. No use, distribution or reproduction is permitted which does not comply with these terms. 


\title{
Loss of the ETR1 ethylene receptor reduces the inhibitory effect of far-red light and darkness on seed germination of Arabidopsis thaliana
}

\author{
Rebecca L. Wilson, Arkadipta Bakshi and Brad M. Binder* \\ Department of Biochemistry, Cellular, and Molecular Biology, University of Tennessee, Knoxville, TN, USA
}

\section{Edited by: \\ Caren Chang, University of Maryland, USA \\ Reviewed by: \\ Ute Hoecker, University of Cologne, Germany \\ Rongcheng Lin, Chinese Academy of Sciences, China}

\section{${ }^{*}$ Correspondence:}

Brad M. Binder, Department of Biochemistry, Cellular, and Molecular Biology, University of Tennessee, M407 Walters Life Sciences Building, Knoxville, TN 37996-7994, USA e-mail: bbinder@utk.edu
When exposed to far-red light followed by darkness, wild-type Arabidopsis thaliana seeds fail to germinate or germinate very poorly. We have previously shown that the ethylene receptor ETR1 (ETHYLENE RESPONSE1) inhibits and ETR2 stimulates seed germination of Arabidopsis during salt stress. This function of ETR1 requires the full-length receptor. These roles are independent of ethylene levels and sensitivity and are mainly mediated by a change in abscisic acid (ABA) sensitivity. In the current study we find that etr1-6 and etr 1-7 loss-offunction mutant seeds germinate better than wild-type seeds after illumination with far-red light or when germinated in the dark indicating an inhibitory role for ETR1. Surprisingly, this function of ETR1 does not require the receiver domain. No differences between these mutants and wild-type are seen when germination proceeds after treatment with white, blue, green, or red light. Loss of any of the other four ethylene receptor isoforms has no measurable effect on germination after far-red light treatment. An analysis of the transcript abundance for genes encoding $A B A$ and gibberellic acid (GA) metabolic enzymes indicates that etr1-6 mutants may produce more GA and less ABA than wild-type seeds after illumination with far-red light which correlates with the better germination of the mutants. Epistasis analysis suggests that ETR1 may genetically interact with the phytochromes (phy), PHYA and PHYB to control germination and growth. This study shows that of the five ethylene receptor isoforms in Arabidopsis, ETR1 has a unique role in modulating the effects of red and far-red light on plant growth and development.

Keywords: ethylene receptors, ethylene, phytochrome, light signaling, cross-talk, seed germination

\section{INTRODUCTION}

Ethylene is a gaseous plant hormone that is involved in many developmental and physiological processes in higher plants (Mattoo and Suttle, 1991; Abeles et al., 1992). In Arabidopsis thaliana, there are five receptor isoforms that mediate responses to ethylene called ETHYLENE RESPONSE1 (ETR1), ETR2, ETHYLENE INSENSITIVE4 (EIN4), ETHYLENE RESPONSE SENSOR1 (ERS1), and ERS2 (Chang etal., 1993; Hua and Meyerowitz, 1998; Hua et al., 1998; Sakai et al., 1998). Genetic studies have shown that in the absence of ethylene, the receptors positively regulate CONSTITUTIVE TRIPLE RESPONSE1 (CTR1) which acts as a negative regulator of the pathway. The role of CTR1 is to inhibit downstream components of the pathway and prevent ethylene responses (Kieber et al., 1993). According to current models, ethylene binding to the receptors reduces the activity of the receptors. This leads to reduced activity of the CTR1 kinase resulting in reduced phosphorylation of EIN2 protein (Chen et al., 2011; Ju et al., 2012; Qiao et al., 2012). This reduction in EIN2 phosphorylation leads to a decrease in ubiquitination of EIN2 causing a rise in EIN2 protein levels and proteolytic separation of the C-terminal portion of the protein from the N-terminal portion (Qiao et al., 2009, 2012; Ju etal., 2012; Wen et al., 2012). The C-terminal region of EIN2 causes a rise in the levels of the EIN3 and EIL1 transcription factors (Guo and Ecker, 2003;
Yanagisawa et al., 2003; Gagne et al., 2004) which leads to most ethylene responses.

The plant ethylene receptors fall into two subfamilies with subfamily 1 comprising ETR1 and ERS1 and subfamily 2 comprising ETR2, EIN4, and ERS2. These receptors have homology to bacterial two-component receptors that signal via histidine autophosphorylation followed by phosphotransfer to an aspartate on a receiver domain. However, only subfamily 1 receptors have histidine kinase activity in vitro with the subfamily 2 receptors having serine/threonine kinase activity in vitro (Gamble et al., 1998; Moussatche and Klee, 2004). ETR1 is unique in that it the only ethylene receptor in Arabidopsis with both histidine kinase activity and a receiver domain and it may be the only receptor with histidine kinase activity in vivo (Moussatche and Klee, 2004). ETR1 histidine kinase activity is not required for ethylene signaling in plants (Wang et al., 2003; Xie et al., 2006; Qu et al., 2007) but does modulate signal output via an unknown mechanism (Binder et al., 2004; Kim etal., 2011; Hall etal., 2012). All of the Arabidopsis ethylene receptor isoforms are involved in ethylene signaling (Hua et al., 1995, 1998; Hall and Bleecker, 2003). However, even though the receptors have overlapping roles for certain traits, it is also clear that they have non-redundant roles (Hall and Bleecker, 2003; Binder et al., 2004, 2006; Seifert et al., 2004; O’Malley et al., 2005; Xie et al., 2006; Qu et al., 2007; Plett 
et al., 2009a,b; Liu et al., 2010; Kim et al., 2011). In some cases, individual isoforms have roles that are opposite from the other isoforms in the control of certain phenotypes (Binder et al., 2006; Liu et al., 2010; Wilson et al., 2014). This is likely to be a common attribute of ethylene signaling in land plants since certain receptors have a prominent role in controlling specific phenotypes in other species including both eudicots and monocots (Tieman et al., 2000; Kevany etal., 2007; Chen et al., 2009; Wuriyanghan et al., 2009). The mechanistic basis for these unique roles is unknown.

Seed germination is a critical developmental stage of the plant that is controlled by both hormones such as abscisic acid (ABA), gibberellic acid (GA), and ethylene and environmental factors such as light quality and quantity (reviewed by Bentsink and Koorneef, 2008; Linkies and Leubner-Metzger, 2012). Ethylene stimulates germination of Arabidopsis seeds (Bleecker et al., 1988; Wilson et al., 2014) and ethylene insensitive etr1-1 mutants have diminished germination (Chiwocha et al., 2005). Ethylene appears to function in this process by antagonizing the inhibitory effects of ABA on germination (Chiwocha et al., 2005; Linkies et al., 2009). However, we recently showed that loss of ETR1 enhances and loss of ETR2 diminishes seed germination during salt stress (Wilson et al., 2014). These contrasting roles for ETR1 and ETR2 are not predicted by current models of ethylene signaling and appear to largely be independent of ethylene. Rather, ETR1 and ETR2 are affecting seed germination during salt stress predominantly via regulation of ABA synthesis or signaling (Wilson et al., 2014). These prior observations suggested that there might be other traits regulated by the ethylene receptors that are unpredicted for by current models of ethylene signaling.

Light intensity and quality affect Arabidopsis seed germination and the phytochrome (phy) family of photoreceptors has a critical role in this regulation (Shinomura et al., 1994, 1996). The phy are evolutionarily related to the ethylene receptors with both receptors having homology to bacterial two-component receptors and are thought to have been acquired from cyanobacteria (Bleecker, 1999; Mount and Chang, 2002). It is noteworthy that even though the phy are evolutionarily related to the ethylene receptors and also contain putative histidine kinase domains, plant phy have serine/threonine protein kinase activity (Yeh and Lagarias, 1998; Fankhauser et al., 1999) much like the subfamily two ethylene receptors. The phy are red/far-red photoreversible receptors that affect many developmental and growth processes in plants including germination (Neff et al., 2000). Illumination with red light drives phy to the active form of the receptor that is far-red absorbing $\left(\mathrm{P}_{\mathrm{FR}}\right)$ and illumination with far-red light drives it toward the red absorbing form $\left(P_{R}\right)$ that is inactive. There are complex interactions between light and phytohormones in the control of germination (reviewed by Bae and Choi, 2008; Finkelstein et al., 2008; Jaillais and Chory, 2010; Lau and Deng, 2010; Leivar and Quail, 2010). Here we report that loss of ETR1 reduces the effect of far-red light on germination and is affecting the transcript abundance of genes encoding metabolic enzymes for both GA and ABA. Epistasis analysis shows that ETR1 genetically interacts with PHYA and $P H Y B$. However, the effects of ETR1 on seed germination may be independent of light suggesting a model where ETR1 acts in parallel with the photoreceptors to control seed germination.

\section{MATERIALS AND METHODS PLANT MATERIALS AND CHEMICALS}

The etr1-6, etr1-7, etr2-3, and ein4-4 mutants are lab stocks that have been previously characterized (Hua and Meyerowitz, 1998). The phya- $t$ (SALK 014575C) and phyb-9 (CS6217) mutants were obtained from the Arabidopsis Biological Resource Center and have previously been described (Rösler et al., 2007; Sung et al., 2007). The receptor combinatorial mutants and transformants used in this study have previously been described (Hua and Meyerowitz, 1998; Kim et al., 2011; Wilson et al., 2014). All mutants are in the Columbia background. GA was from ACROS Organics (Belgium) and the ABA biosynthesis inhibitor norflurazon was from Fluka (Switzerland). L- $\alpha$-(2-amino ethoxyvinyl)-glycine (AVG) was a gift from Rohm Haas (Philadelphia).

The etr1-6;phya-t and etr1-6;phyb-9 mutants were obtained by crossing phya-t to etr1-6 and phyb-9 to etr1-6. The double mutants were selected for in the F2 generation by first growing seedlings in constant far-red light to identify homozygous phya-t mutants or constant red light to identify homozygous phyb-9 mutants. Tall seedlings were transferred to soil and subsequently genotyped with dCAPs primers to identify homozygous etr1-6 plants. The dCAPs primers used for genotyping etr16 were $5^{\prime}$-ACTCGTTGAAGTCGTCCCTGATC-3' (forward) and 5'-ATGTGAGAGAGCTACAGCCAC-3' (reverse). These primers yield an expected polymerase chain reaction (PCR) product of 121 bp. Digestion of this PCR product with BslI results in a 23 and $98 \mathrm{bp}$ fragment for wild-type ETR1 and does not digest product from etr1-6, while digestion with Hpy188I results in a 24 and 97 bp product from etr1-6 and does not digest PCR product from wild-type ETR1. Homozygous F3 or F4 seeds were used for germination assays and analysis of hypocotyl lengths.

\section{SEED GERMINATION EXPERIMENTS}

To minimize biological variation, seeds were harvested on the same day from plants grown together under similar conditions (Hensel et al., 1993), stored in a desiccator at room temperature, and allowed to after-ripen for at least 3 weeks. These were then mechanically sorted as previously described (Ellwell et al., 2011). Seeds between 250 and $300 \mu \mathrm{m}$ in size were surface sterilized in $70 \%$ ethanol for $30 \mathrm{~s}$, dried on filter paper and placed on agar plates containing half-strength Murashige and Skoog (MS) basal medium with Gamborg's vitamins (Sigma, St. Louis, MO, USA), $\mathrm{pH}$ 5.7, 0.8\% (w/v) agar, with no added sugar. Plates were sealed with micropore surgical tape (3M, St. Paul, MN, USA) so that ethylene did not accumulate (Buer et al., 2003). Twenty seeds of one genotype were plated per plate with a spacing of $5 \mathrm{~mm}$ between seeds and three plates per genotype per condition were used in each experiment.

Where used, GA and the ABA synthesis inhibitor, norflurazon were prepared as $10,000 \times$ stocks in ethanol, filter sterilized, and added to the media at the indicated concentration after autoclaving the media. Solvent control plates contained $0.01 \%$ ethanol. In some cases, $5 \mu \mathrm{M}$ AVG was added to block ethylene biosynthesis. Ethylene treatments were conducted by placing the plates of seeds in sealed containers and injecting ethylene to yield the indicated concentrations. 
Unless otherwise specified, seeds were treated with 45$55 \mu \mathrm{mol} \mathrm{m} \mathrm{m}^{-2} \mathrm{~s}^{-1}$ white light for $4 \mathrm{~h}$. This was followed by a $3 \mathrm{~h}$ treatment with $12 \mu \mathrm{mol} \mathrm{m}{ }^{-2} \mathrm{~s}^{-1}$ blue $\left(\lambda_{\max }=470 \mathrm{~nm}\right)$, green $\left(\lambda_{\max }=525 \mathrm{~nm}\right)$, red $\left(\lambda_{\max }=672 \mathrm{~nm}\right)$, or far-red $\left(\lambda_{\max }=732 \mathrm{~nm}\right)$ light. Monochromatic light was provided by LED arrays (Quantum Devices, Inc., Barneveld, WI, USA). For positive controls, seeds were treated for $3 \mathrm{~h}$ with $45-55 \mu \mathrm{mol} \mathrm{m}{ }^{-2} \mathrm{~s}^{-1}$ white light. After light treatment, seeds were allowed to germinate for 7 days in darkness at which time germination was evaluated. For dark controls, seeds were treated for 5 min with far-red light immediately after sowing without a pre-treatment with white light (Oh et al., 2007) and then allowed to germinate in darkness for 7 days. In some experiments, seeds were germinated on $150 \mathrm{mM}$ $\mathrm{NaCl}$ under continuous white light.

Germination was scored as the visible rupture of the testa (seed coat). After each experiment, plates containing seeds that did not germinate were transferred to white light conditions $(16 \mathrm{~h}$ of light/8 h of dark photoperiod) and germination evaluated after 7 days. In all cases, seeds reached at least 95\% germination showing that the seeds were viable.

\section{ETHYLENE MEASUREMENTS}

To measure ethylene production, $22 \mathrm{mg}$ of seeds were placed on $1 \mathrm{~mL}$ of half-strength MS media in sealed $6 \mathrm{~mL}$ glass vials, treated with white or far-red light, and ethylene levels in the headspace measured every $6 \mathrm{~h}$ over several days using an ETD-300 photoacoustic ethylene detector (Sensor Sense, The Netherlands) as previously described (Wilson et al., 2014).

\section{HYPOCOTYL GROWTH EXPERIMENTS}

To examine the growth of hypocotyls, seeds were placed on agar plates and illuminated with $45-55 \mu \mathrm{mol} \mathrm{m}^{-2} \mathrm{~s}^{-1}$ white light for $24 \mathrm{~h}$. The plates of seeds were then grown vertically for 6 days under continuous red or far-red light. At that time, the plates were scanned with a flat-bed scanner and seedling length measured using ImageJ (ver. 1.43u).

\section{RNA ISOLATION AND QUANTITATIVE REAL-TIME REVERSE TRANSCRIPTASE (qRT)-PCR}

The transcript abundance of several Arabidopsis genes that encode GA and ABA metabolic enzymes was examined using quantitative real-time reverse transcriptase (qRT)-PCR. This included gene transcripts for GA3 oxidase 1 and 2 (GA3ox1, GA3ox2) encoding for two GA biosynthesis enzymes, GA2ox2 encoding for a GA degradation enzyme, zeaxanthin epoxidase (ZEP), 9-cisepoxycarotenoid dioxygenase 6 and 9 (NCED6, NCED9) encoding for three ABA biosynthesis enzymes, and ABA 8'-hydroxylase (CYP707A2) encoding for an ABA degradation enzyme. For this, total RNA was isolated from either $25 \mathrm{mg}$ dry seeds or $25 \mathrm{mg}$ (dry weight) of seeds that were imbibed in half strength MS and light treated as specified for the indicated times. RNA was isolated according to methods modified from Meng and Feldman (2010). Instead of resuspending the pellet in Trizol, the RNA was further purified using the Spectrum Plant Total RNA Kit (Sigma, St. Louis, MO, USA). Total RNA was treated with DNase I (Invitrogen, Carlsbad, CA, USA) and 800 ng of the RNA was used for cDNA synthesis with the ImProm-II Reverse Transcription System
(Promega, Madison, WI, USA) according to the manufacturer's instructions. Each qPCR reaction consisted of $5 \mu \mathrm{L}$ of SsoFast EvaGreen Supermix (Bio-Rad, Hercules, CA, USA), $0.5 \mu \mathrm{L}$ each of the forward and Reverse primers $(10 \mu \mathrm{M})$ and $4 \mu \mathrm{L}$ of cDNA diluted 1:8. The qPCR reactions were run on a Bio-Rad iQ5 RealTime PCR Detection System (Bio-Rad, Hercules, CA, USA) with the following conditions: an initial denaturation step of $95^{\circ} \mathrm{C}$ for $1 \mathrm{~min}$ followed by 45 cycles of $15 \mathrm{~s}$ at $95^{\circ} \mathrm{C}, 30 \mathrm{~s}$ at $58^{\circ} \mathrm{C}$, and $10 \mathrm{~s}$ at $72^{\circ} \mathrm{C}$.

Transcript data were normalized to At3g12210 (Dekkers et al., 2011) using the method of Livak and Schmittgen (2001) for each seed line for each condition to obtain the relative amounts of target gene transcripts between plant backgrounds for each treatment. The primers used for GA3ox2 were $5^{\prime}$-GTTCTTTAATAAGAAGATGTGGTCCG-3' (forward) and $5^{\prime}$ CATCAACTTGGCTGCCAACTTT- $3^{\prime}$ (reverse). Other primers used have been previously described (Seo et al., 2004; Kim et al., 2012; Shu et al., 2013).

\section{STATISTICS}

Data were analyzed with Student's $t$-tests and considered statistically different with a $P$ value $<0.05$.

\section{RESULTS ETR1 INHIBITS SEED GERMINATION AFTER EXPOSURE TO FAR-RED LIGHT}

We recently reported that loss of ETR1 led to better seed germination during salt stress when seeds were germinated in white light (Wilson et al., 2014). During the course of those experiments we noted that loss of ETR1 also caused better seed germination during salt treatment when the seeds were germinated in darkness (data not shown). This led us to determine the role of individual ethylene receptor isoforms on seed germination under different light conditions. To do this, we measured the percent of seed germination for receptor loss-of-function mutants 7 days following a $3 \mathrm{~h}$ treatment with white, blue, green, red, or far-red light as described in Section "Materials and Methods." Following white light treatment, all seed lines reached at least 95\% germination (Figure 1). The extent of wild-type seed germination was not altered by blue, green, or red light. However, germination of wild-type seeds was drastically reduced by treatment with farred light. This is consistent with prior research (Shinomura et al., 1994). By contrast, the effect of far-red light on the germination of etr1-6 and etr1-7 loss-of-function mutants was significantly reduced even though responses to blue, green, and red light were not altered by the loss of ETR1. This effect of etr1 mutants was observed with multiple seed batches. Loss of ETR2 or EIN4 did not affect responses to far-red light, but did have a small and statistically significant effect on germination in blue light (Figure 1). Loss of ERS1 or ERS2 had no measurable effect on germination in any of the light conditions tested (data not shown). Interestingly, when germination occurred in darkness without a white light pretreatment, wild-type, etr2-3, and ein4-4 seeds failed to germinate or germinated very poorly, whereas etr1 loss-of-function mutants germinated to an extent that was similar to germination after farred illumination (Figure 1). These data suggest that ETR2 and EIN4 promote seed germination after exposure to blue light and 


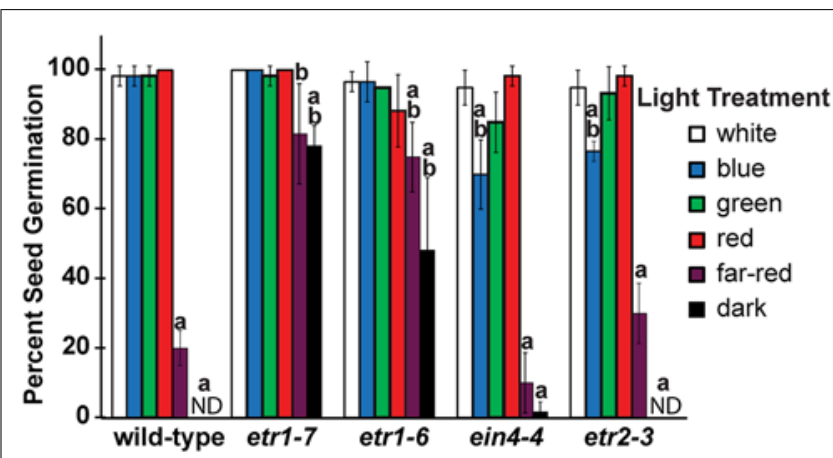

FIGURE 1 | Ethylene receptors affect germination in response to different wavelengths of light. Seed germination of wild-type seeds was compared to ethylene receptor loss-of-function mutants. Seeds were treated with $4 \mathrm{~h}$ of white light, followed by $3 \mathrm{~h}$ of different colors of monochromatic light or white light as indicated. For dark controls, seeds were treated for 5 min with far-red light immediately after sowing without a pre-treatment with white light. The seeds were then placed in darkness for 7 days. At that time seed germination was measured and the percent of seed germination calculated. Plots show the average \pm SD from at least three biological replicates. ND denotes no germination detected. Data were analyzed by $t$-tests and differences considered statistically significant with $P<0.05$. ${ }^{\text {a }}$ Significant difference from white light for that ecotype; b Significant difference of mutant from wild-type after the same light treatment.

ETR1 inhibits germination after exposure to far-red light and in darkness.

\section{ETR1 FUNCTIONS OPPOSITELY TO ETR2 TO INFLUENCE SEED GERMINATION AFTER EXPOSURE TO FAR-RED LIGHT}

In seed germination under salt stress, etr2-3 loss-of-function mutants were shown to have reduced germination compared to wild-type seeds and ETR1 and ETR2 were found to act additively (Wilson et al., 2014). In contrast, under the far-red light conditions used, the germination of etr2-3 mutants was indistinguishable from wild-type seeds (Figure 1). However, an effect of ETR2 on seed germination in these conditions might have been masked by the fact that the wild-type seeds germinated poorly and a further reduction might have been obscured. To determine whether or not ETR1 and ETR2 act additively to control seed germination after far-red light, we compared seed germination of etr1-6 and etr2-3 to germination of etr1-6;etr2-3 double mutants. Following a $3 \mathrm{~h}$ white light treatment, the seed germination of the single and double mutants were not significantly different and reached at least 95\% germination (Figure 2). A different pattern emerged after a $3 \mathrm{~h}$ far-red light treatment. Here, the etr $1-6$ seeds germinated significantly better and the etr2-3 seeds germinated significantly worse than the etr1-6;etr2-3 double mutants. These results show that ETR1 and ETR2 have opposite effects on seed germination after far-red light treatment.

\section{THE ETR1 RECEIVER DOMAIN IS NOT REQUIRED FOR ETR1 FUNCTION IN SEED GERMINATION AFTER EXPOSURE TO FAR-RED LIGHT}

We previously showed that etr1-6;etr2-3;ein4-4 triple mutants germinate better than wild-type seeds during salt stress under white light (Wilson et al., 2014). Interestingly, transformation of this triple mutant with a cDNA construct for full-length ETR1

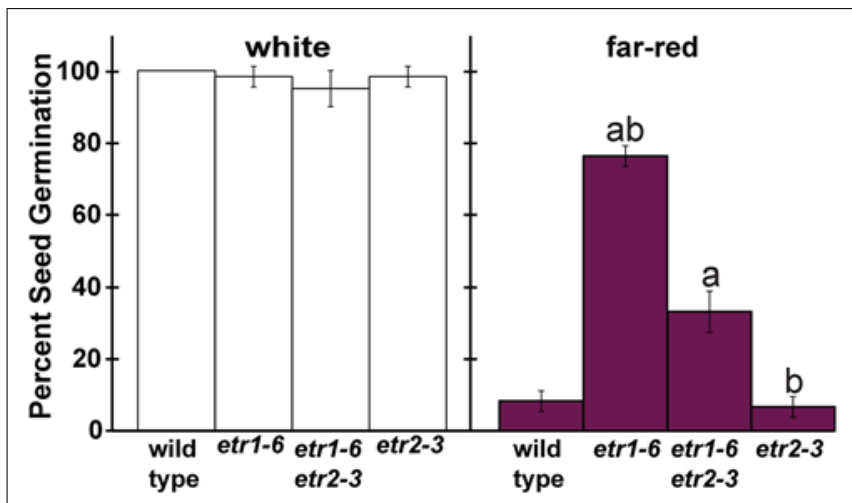

FIGURE 2 | ETR1 functions oppositely to ETR2 to control seed germination after treatment with far-red light. The percent of wild-type, etr1-6, etr2-3, and etr1-6;etr2-3 seed germination was determined 7 days after a far-red light treatment. For comparison, germination after a white light treatment is included. Plots show the average \pm SD from at least three biological replicates. Data were analyzed by $t$-tests and differences considered statistically significant with $P<0.05$. ${ }^{\text {S }}$ Statistical difference from wild-type in that light condition; ${ }^{b}$ Significant difference of the single mutant from the double.

(cETR1) resulted in a reduction in germination, whereas transformation with a truncated ETR1 transgene lacking the receiver domain $(\operatorname{cetr} 1-\Delta R)$ failed to alter germination (Wilson et al., 2014). Both constructs are under the control of the ETR1 promoter and have been shown to be expressed and functional (Kim et al., 2011) supporting the idea that the full-length ETR1 receptor is required to affect seed germination during salt stress under white light. To determine whether or not the ETR1 receiver domain is also required to modulate seed germination after treatment with far-red light, we examined the seed germination of these transformants. Comparable to our results with germination on salt, the etr1-6;etr2-3;ein4-4 triple mutants germinated better than wild-type seeds after illumination with far-red light (Figure 3). However, in contrast to germination on salt, both the full-length and truncated ETR1 transgenes reduced seed germination to wild-type levels after far-red illumination indicating that the receiver domain of ETR1 is not required to function in this trait. When transferred to white light conditions for 7 days, all seed lines reached at least $98 \%$ germination (data not shown). This pattern of rescue is different from what we obtained when studying the role of ETR1 in the control of germination during salt stress in white light (Wilson et al., 2014). To confirm this difference, we germinated seeds in white light under salt stress. Under salt stress, wild-type seeds reached approximately $72 \%$ germination while the etr1-6;etr2-3;ein4-4 triple mutants reached 100\% germination (Figure 3). The full-length $c E T R 1$, but not the truncated cetr $1-\Delta R$ transgene, reduced germination to wild-type levels as previously reported (Wilson et al., 2014). These results reveal that the function of ETR1 varies depending upon environmental conditions.

\section{ETHYLENE MAY HAVE A ROLE IN MEDIATING THE EFFECTS OF ETR1 ON GERMINATION AFTER EXPOSURE TO FAR-RED ILLUMINATION}

In order to determine whether differences in ethylene production or sensitivity are responsible for the difference between the 


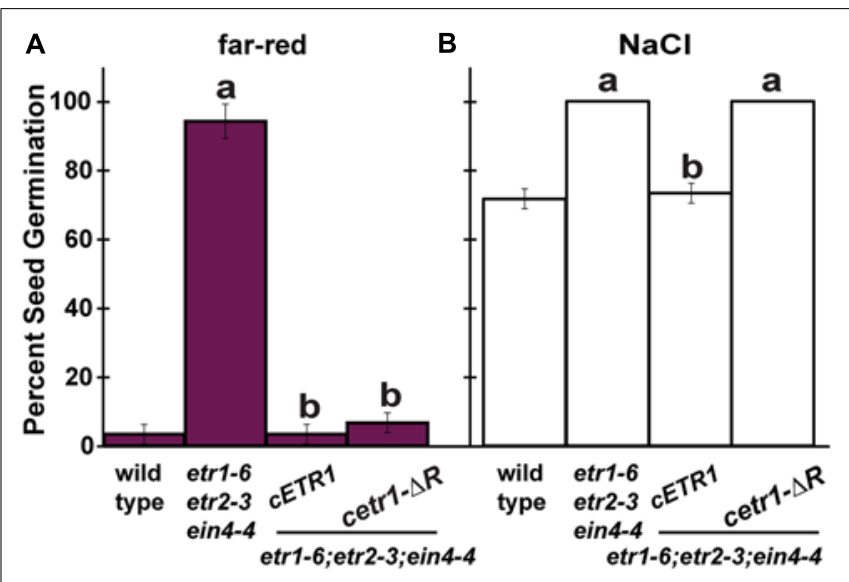

FIGURE 3 | After far-red illumination, the better germination of etr1;etr2; ein4 triple mutants is reversed by both a full-length and truncated ETR1 transgene lacking the receiver domain. (A) The percent of germination 7 days after treatment with far-red light for wild-type, etr1-6;etr2-3; ein4-4 triple mutants and these triple mutants transformed with cDNA for full-length ETR1 (cETR1) or a truncated ETR1 lacking the receiver domain (cetr $1-\Delta R$ ) was determined. (B) For comparison, the percent germination of these seed lines 7 days after sowing on $150 \mathrm{mM}$ $\mathrm{NaCl}$ in the presence of continuous white light was determined. Data were analyzed by $t$-tests and differences considered statistically significant with

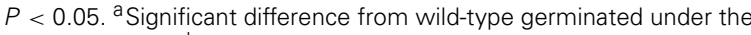
same conditions. ${ }^{\text {b }}$ Significant rescue of germination by the transgene.

etr1 loss-of-function mutants and wild-type in germination following far-red treatment, we examined the effect of ethylene and the ethylene biosynthesis inhibitor, AVG, on germination. Following white light treatment, AVG did not have a statistically significant effect on the germination of wild-type, etr1-6 or etr2-3 seeds (Figure 4A). AVG also did not affect wild-type or etr2-3 germination following far-red treatment. However, germination of etr $1-6$ was statistically reduced by $20 \%$ by AVG after far-red light (Figure 4A). Although AVG reduced germination of etr1-6 seeds in far-red, this mutant still had better seed germination than wild-type. These observations suggest that part of the difference in germination between wild-type and the etr1-6 mutants may be due to alterations in ethylene levels. To more directly determine this, we measured ethylene levels of germinating seeds after white or far-red light treatment. We found that ethylene levels were indistinguishable between etr1-6 and wild-type seeds during germination in both conditions (data not shown) suggesting that higher ethylene levels are not responsible for the enhanced germination of etr1-6 seeds after far-red illumination.

Ethylene can stimulate seed germination of lettuce and cocklebur after far-red illumination (Abeles and Lonski, 1969; Burdett and Vidaver, 1971; Esashi et al., 1987). We wished to determine if this was the case for Arabidopsis too and to see if the etr1-6 and etr2-3 mutants had altered ethylene sensitivity when assayed in these conditions. One explanation for etr1 loss-of-function mutants germinating better than wild-type is that they are more sensitive to ethylene. This has been observed when assaying growth of dark-grown Arabidopsis seedlings (Hua and Meyerowitz, 1998; Cancel and Larsen, 2002), but is not true for seeds germinating
A

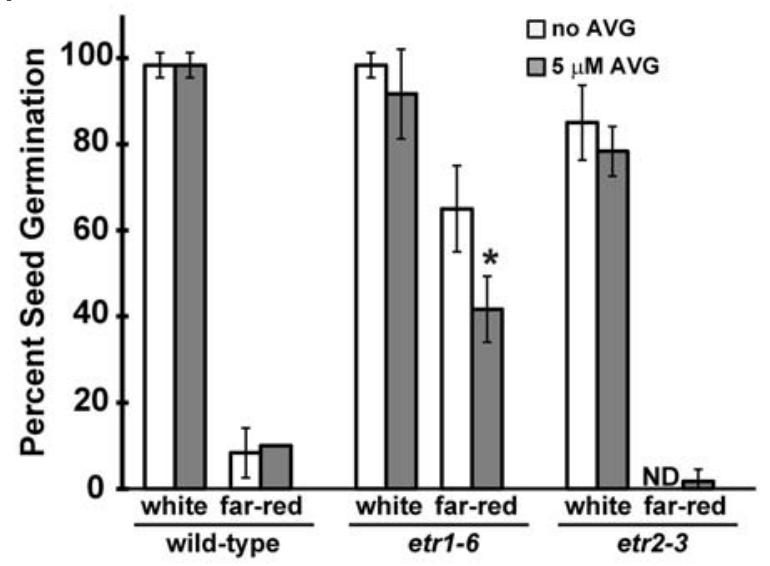

B

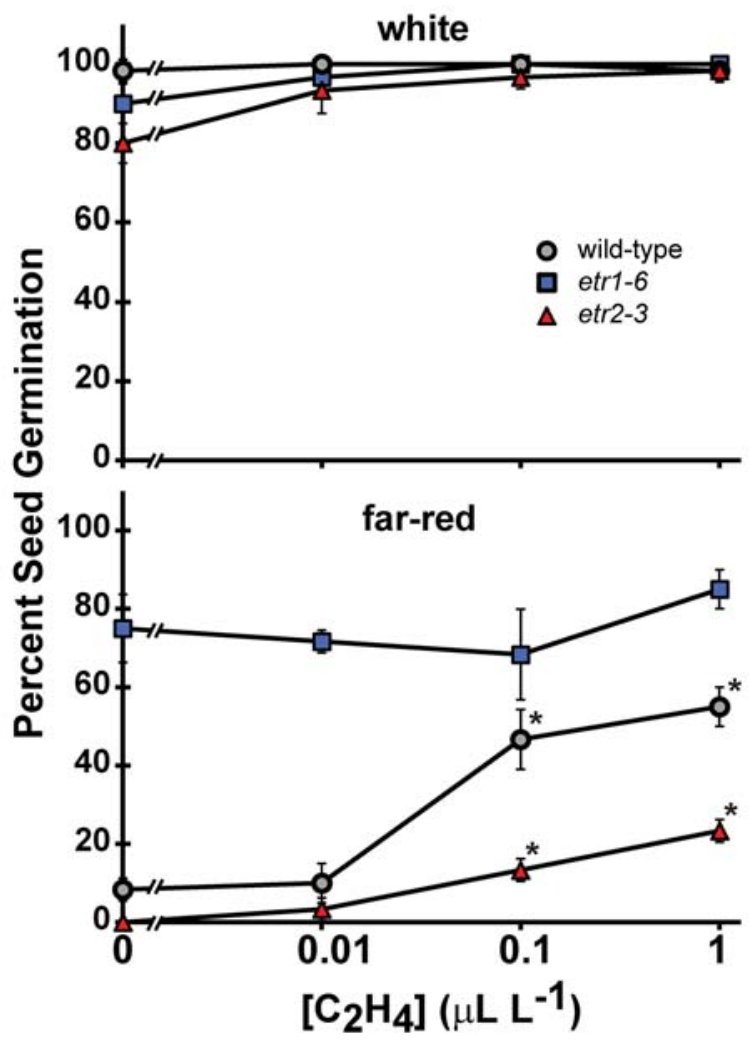

FIGURE 4 |The effects of L- $\alpha$-(2-amino ethoxyvinyl)-glycine (AVG) and ethylene on seed germination after treatment with far-red light. The number of seeds that germinated after far-red or white illumination was counted and the percent of seeds that germinated was calculated.

(A) Seeds on plates containing the ethylene biosynthesis inhibitor, AVG, were treated with far-red light and then germinated for 7 days in the dark. The average $\pm S D$ from at least three biological replicates is plotted. ND denotes no germination detected. Data were analyzed by $t$-tests and differences considered statistically significant with $P<0.05$. ${ }^{*}$ Denotes that AVG had a significant effect compared to the non-treated control in that light condition. (B) The effects of increasing concentrations of ethylene as indicated were measured after treatment with white light or far-red light. The average \pm SD from at least three biological replicates is plotted. Data were analyzed by $t$-tests and differences considered statistically significant with $P<0.05$. *Denotes that ethylene significantly increased the percent of germination after-far red light over the ethylene untreated control. 
on salt (Wilson et al., 2014). After white light, 0.01 ppm ethylene increased the germination of etr $2-3$ seeds slightly, but significantly $(P<0.05)$, and higher concentrations of ethylene caused no measurable additional increase in germination. By contrast, ethylene had no measurable effect on the germination of wild-type or etr16 seeds after white light illumination (Figure 4B). After treatment with far-red light, ethylene increased seed germination of wildtype seeds (Figure 4B). By contrast, ethylene had no measurable effect on germination of the etr1-6 mutants indicating that these mutants are largely un-responsive to ethylene for this trait. We also examined etr2-3 mutants and found that ethylene enhanced germination of these seeds after far-red illumination, but the effect was smaller than seen with wild-type seeds. The highest concentration of ethylene used ( $1 \mathrm{ppm}$ ) failed to completely eliminate the differences in germination between etr1-6, etr2-3, and wild-type seeds. This is at least 1000-fold higher than the concentration of ethylene released by the germinating seeds under these conditions. Thus, ethylene may have a role in mediating the differences in germination between wild-type and etr 1 mutants, but other factors are likely to be more important.

\section{ABA AND GA HAVE A ROLE IN MEDIATING THE EFFECTS OF ETR1 ON GERMINATION AFTER EXPOSURE TO FAR-RED ILLUMINATION}

There are complex interactions between hormones and light to control germination (reviewed by Finkelstein et al., 2008; Lau and Deng, 2010; Leivar and Quail, 2010). We previously found that ETR1 predominantly affects ABA sensitivity to alter seed germination during salt stress (Wilson et al., 2014). We therefore were curious to know if ETR1 is also predominantly affecting seed germination after treatment with far-red light in the same way.

Abscisic acid is well known for inhibiting germination and GA for stimulating Arabidopsis seed germination (Hilhorst and Karssen, 1992; Garciarrubio et al., 1997). In order to test whether differences in ABA or GA or both were responsible for the differences in germination following far-red light treatment, we treated the seeds with an ABA biosynthesis inhibitor, norflurazon, or with GA. Initially, these chemicals were dissolved in ethanol to yield a final concentration of $0.1 \%(\mathrm{v} / \mathrm{v})$ ethanol. However, this concentration of ethanol significantly $(P<0.05)$ reduced germination of etr1-6 and etr2-3 seeds after white light treatment to $55 \pm 22$ and $45 \pm 9 \%$, respectively. As previously observed, this concentration of ethanol had no measureable effect on the germination of Columbia seeds (Hirayama et al., 2004). Even though norflurazon and GA affected germination as expected, this added effect of the ethanol made interpretation of the data difficult. Because of this, we used stocks that yielded a final ethanol concentration of $0.01 \%(\mathrm{v} / \mathrm{v})$. At this concentration of ethanol, there was no statistical difference $(P<0.05)$ between wild-type and the mutants following white light treatment (Figure 5). However, following far-red treatment, this concentration of ethanol significantly $(P<0.05)$ reduced the germination of etr1-6 (Figure 1 vs. Figure 5). In Figure 1, that has no added ethanol, etr1-6 reached $75 \pm 10 \%$ germination, but when ethanol was added to the plates (Figure 5) etr1-6 only reached $33 \pm 6 \%$ germination following far-red treatment. These experiments were conducted at the same time and with the same seed batches.

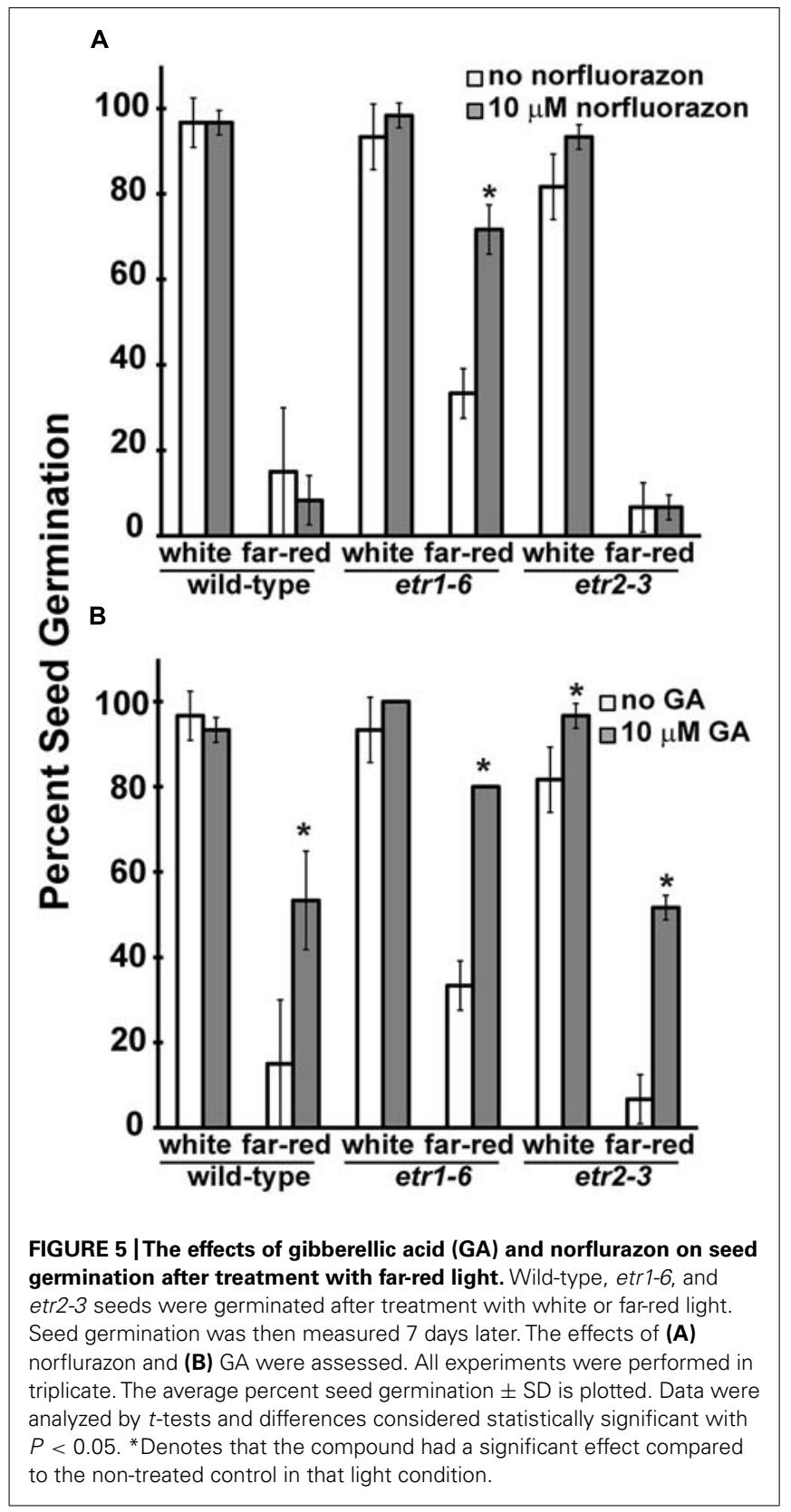

We found that addition of $10 \mu \mathrm{M}$ norflurazon, resulted in a significant improvement of etr1-6 germination following far-red treatment, but surprisingly had no effect on wild-type or etr2-3 seed germination (Figure 5A). Treatment with $10 \mu \mathrm{M}$ GA significantly increased the germination following far-red treatment of all three seed lines tested $(P<0.05)$, but was not able to improve the germination of wild-type and etr2-3 to that seen by etr1-6 (Figure 5B). These data suggest that ETR1 is having a complex effect on $\mathrm{ABA}$ and GA to control seed germination after far-red illumination. This is somewhat different from the role of ETR1 in controlling seed germination during salt stress (Wilson et al., 2014).

To explore this complex interaction more, we used qRTPCR to examine the time-course of changes in transcript 
levels of selected genes during germination under the conditions used above. We chose genes encoding for enzymes for GA biosynthesis (GA3ox1, GA3ox2), GA degradation (GA2ox2), ABA biosynthesis (ZEP, NCED6, NCED9), and ABA degradation (CYP707A2). These genes are important for seed development and germination (Yamauchi et al., 2004, 2007; Lefebvre etal., 2006; Okamoto etal., 2006). Generally consistent with prior studies (Yamauchi etal., 2004; Seo et al., 2006; Finch-Savage etal., 2007), imbibing wild-type seeds in white light led to large increases in the transcript abundance of GA3ox1, GA3ox2, and CYP707A2 and significant $(P<0.05)$ decreases in GA2ox2, ZEP, and NCED6 (Figure 6). The transcript abundance of NCED9 increased slightly, but overall these results are predicted to lead to increased GA levels and decreased ABA levels. Immediately following treatment with farred light, the transcript abundance of GA3ox1, GA3ox2, ZEP, NCED9, and CYP707A2 decreased and the levels of GA2ox2 and NCED6 increased in wild-type seeds. Overall, these transcriptional changes are predicted to cause $\mathrm{ABA}$ levels to rise and GA levels to decrease compared to white light and lead to inhibited germination. In the wild-type seeds, the transcript levels of each gene showed different patterns of change over the $24 \mathrm{~h}$ period following far-red illumination. GA3ox 1 and GA3ox2 showed a small decrease $6 \mathrm{~h}$ following far-red light but then plateaued at a very low level and CYP707A2 showed very little change in transcript abundance after farred illumination. By contrast, the transcript levels of $Z E P$, NCED6, and NCED9 increased with time after far-red light treatment.

The effect of the etr1-6 mutation on the transcript levels of these genes was evident at various times during germination. After white-light treatment, the etr1-6 mutants had higher levels of GA3ox1, GA3ox2, and ZEP and slightly lower levels of $G A 20 \times 2(P<0.05)$ compared to the wild-type seeds (Figure 6). Immediately following treatment with far-red light, the levels of GA3ox1, GA2ox2, NCED6, and CYP707A2 were slightly but significantly $(P<0.05)$ lower in etr1-6 mutants compared to wild-type and GA3ox2 and ZEP were slightly, but significantly higher in the mutants compared to wild-type. More differences between the etr1-6 seeds and wild-type became apparent with increasing time after treatment with far-red light with a trend toward higher levels of GA3ox1 and GA3ox2 and lower levels of ZEP, NCED6, and NCED9 in the etr1-6 mutants compared to wild-type. The differences in the levels of ZEP, NCED6, and NCED9 appear to arise because the levels of these gene transcripts increased in the wild-type seeds, but remained fairly constant in the etr1-6 mutants. Since the transcript levels of GA and ABA metabolic genes generally correlate with the levels of GA and $A B A$, respectively (Qin and Zeevaart, 1999; Iuchi etal., 2001; Reid etal., 2002; Seo et al., 2004; Lefebvre etal., 2006; Reinecke et al., 2013), our results suggest that the etr1-6 seeds are likely to have higher levels of GA and lower levels of ABA than wild-type seeds following treatment with far-red light. These alterations in GA and ABA levels are predicted to cause better germination of the mutant seeds compared to the wild-type seeds after far-red illumination.

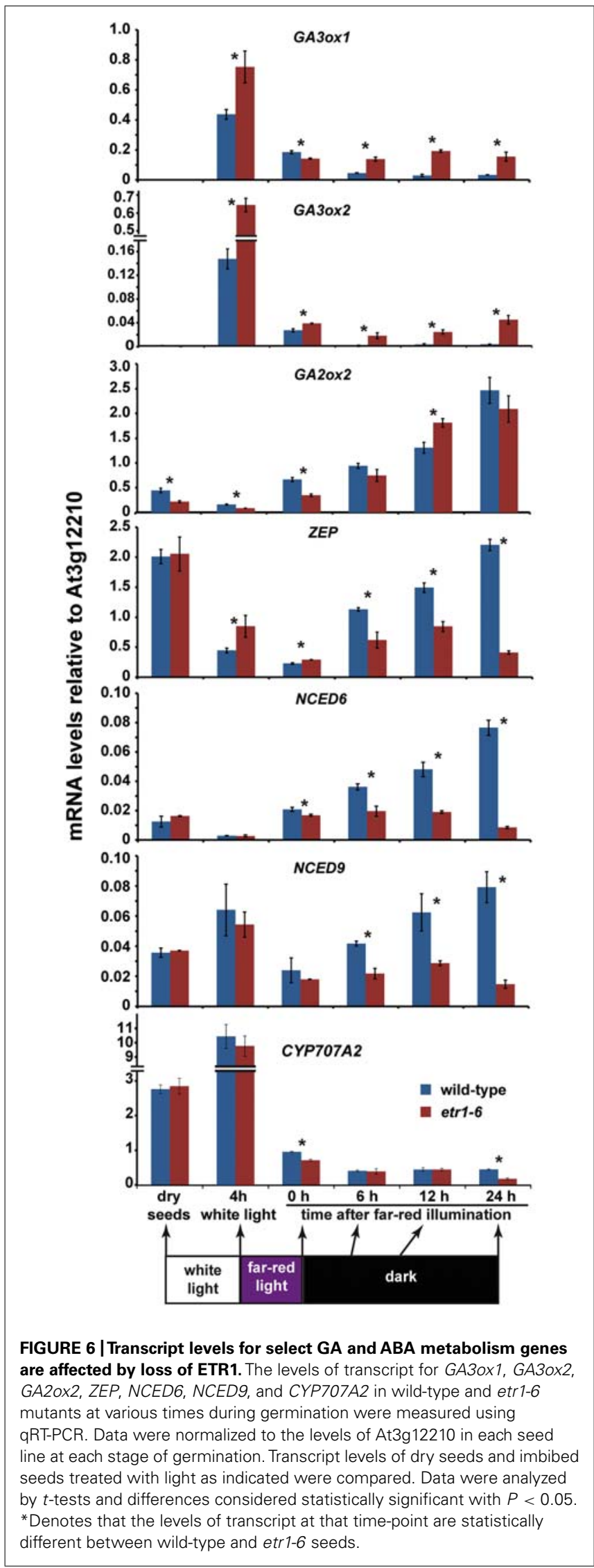




\section{ETR1 GENETICALLY INTERACTS WITH PHYA AND PHYB TO CONTROL GERMINATION}

The phy have an important role in seed germination and responses to far-red light are mediated by the phy (Shinomura et al., 1994, 1996; Poppe and Schäfer, 1997; Hennig etal., 2002; Rösler et al., 2007) suggesting that ETR1 is affecting events influenced by the phy. The phy act as photoreversible switches driven by red and far-red light to control germination of seeds including germination of Arabidopsis (Shinomura et al., 1994; Casal and Sánchez, 1998). To confirm that loss of ETR1 is affecting phy-influenced germination, we treated seedlings with a series of $5 \mathrm{~min}$ pulses of red and far-red light followed by darkness (Figure 7). From this we found that there was no measurable difference in wild-type and etr1-6 germination rates in seeds treated with a pulse series that ended with red light. By contrast, if the pulse series ended with far-red light, wild-type seedlings failed to germinate, but approximately $20 \%$ of the etr1- 6 seeds germinated.

Of the phy, phyA and phyB have the predominant roles in controlling Arabidopsis seed germination (Shinomura et al., 1994, 1996; Rösler etal., 2007). To further explore the interaction between ETR1 and the phy, we generated etr1-6;phya- $t$ and etr1-6;phyb-9 double mutants. After treatment with white light, the germination rates of etr1-6, etr1-6;phya-t, and phya$t$ were indistinguishable from wild-type seeds (Figure 8A). By contrast, the $p h y b-9$ seeds had severely diminished germination after white light treatment, consistent with previous reports (Reed et al., 1994; Shinomura et al., 1994). The etr1-6;phyb-9 double mutants had a percent germination worse than the etr1-6 single mutants and slightly better than the phyb-9 mutants following white light treatment. After treatment with far-red light, the phya$t$ and phyb-9 mutants had significantly worse germination than wild-type seeds and the etr1-6 mutants significantly better germination (Figure 8A). Both the etr1-6;phya-t and etr1-6;phyb-9

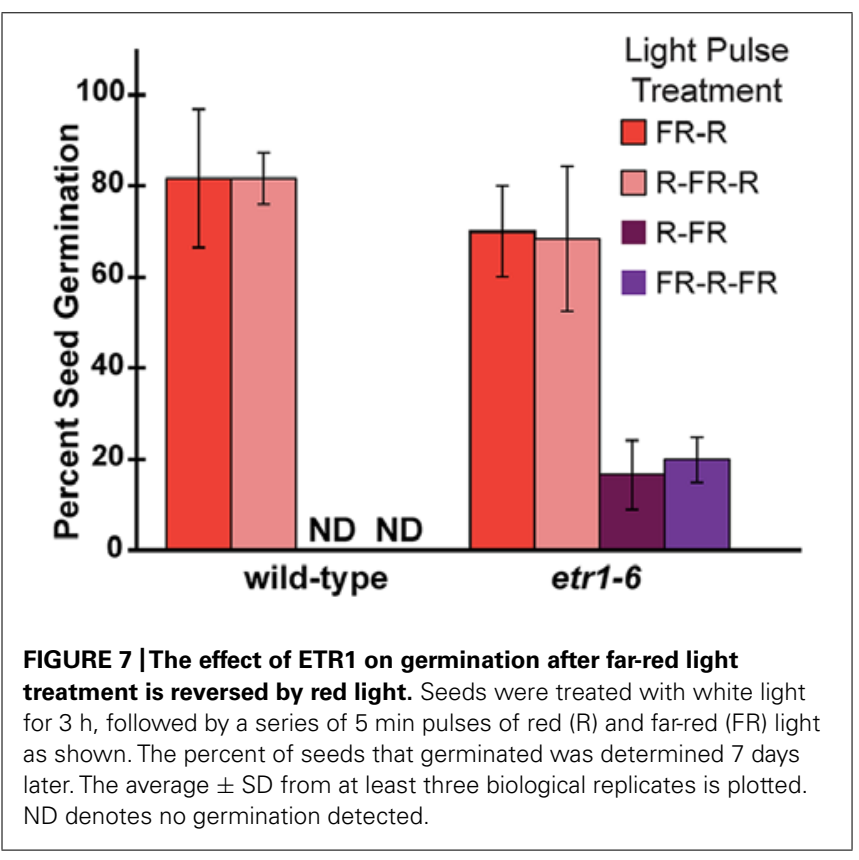

double mutants had a percent germination intermediate between the single mutants. However, these were not statistically different.

We also examined hypocotyl growth in red and far-red light (Figure 8B). Consistent with previous studies, the $p h y B$ mutants grew taller than wild-type in red light and the phyA mutants grew taller than wild-type in far-red light (Reed et al., 1994; Quail et al., 1995; Smith et al., 1997). The growth of the etr1-6 seedlings was similar to wild-type in both light conditions. In red light, the etr16;phyb-9 double mutants were shorter than the phyb-9 mutants and taller than the etr1-6 mutants. Similarly, in far-red light the etr1-6;phya- $t$ double mutants were shorter than the phya- $t$ seedlings and taller than the etr1-6 seedlings. The etr1-6;phya- $t$ double mutants were also slightly, but significantly, shorter than either single mutant in red light. Together, these results on seed germination and hypocotyl growth show that ETR1 genetically interacts with $P H Y A$ and $P H Y B$.

\section{ETR1 AFFECTS GERMINATION INDEPENDENTLY OF LIGHT}

From the above experiments, it is unclear whether or not ETR1 is affecting responses to light by affecting signaling from phyA or phyB. We have previously shown that etr1-6 loss-of-function mutants are more sensitive to ABA when measuring seed germination under white light (Wilson et al., 2014) indicating that the effects of ETR1 could be independent of these photoreceptors. Since phyB has the major role in controlling germination in the dark (Bentsink and Koorneef, 2008), we examined the effect of ETR1 on the germination of phyb-9 mutants in the dark. In dark conditions, wild-type and phyb-9 seeds failed to germinate, whereas etr1-6 mutants did germinate (Figure 9). The etr1-6;phyb-9 also germinated under these conditions but had a percent germination lower than the etr1-6 single mutant seeds. This indicates that ETR1 can affect germination independently of light.

\section{DISCUSSION}

It has been suggested that there is a network of two-component receptors in plants that fine-tune signaling (Hwang et al., 2002; Hass et al., 2004; Wilson et al., 2014). In this study, we found that ETR1, a two-component-like receptor for ethylene, inhibits germination after far-red light treatment and in the dark. This is similar to results we obtained when examining germination during salt stress (Wilson et al., 2014). However, in the prior study, the effect of ETR1 was to mainly affect ABA signaling or synthesis. In the current study, ETR1 was found to affect ABA, GA, and perhaps ethylene. It has previously been shown that in Arabidopsis phyA and B affect the transcript abundance of ETR2 and ERS1 (Tepperman et al., 2004), and phy interacting factor1 (PIF1), which is important for phy signaling, affects the transcript abundance of ETR2 and EIN4 (Oh et al., 2009). Additionally, phyB is involved in the circadian control of ethylene synthesis in sorghum (Finlayson et al., 1998) showing that there is likely to be signaling cross-talk from the phy to ethylene signaling and synthesis. Results presented in the current study show that there is also signaling from ETR1, but not the other four ethylene receptor isoforms, that affects responses to red and far-red light.

Previous research has shown that the receiver domain of ETR1 affects receptor function in the control of growth of dark-grown 

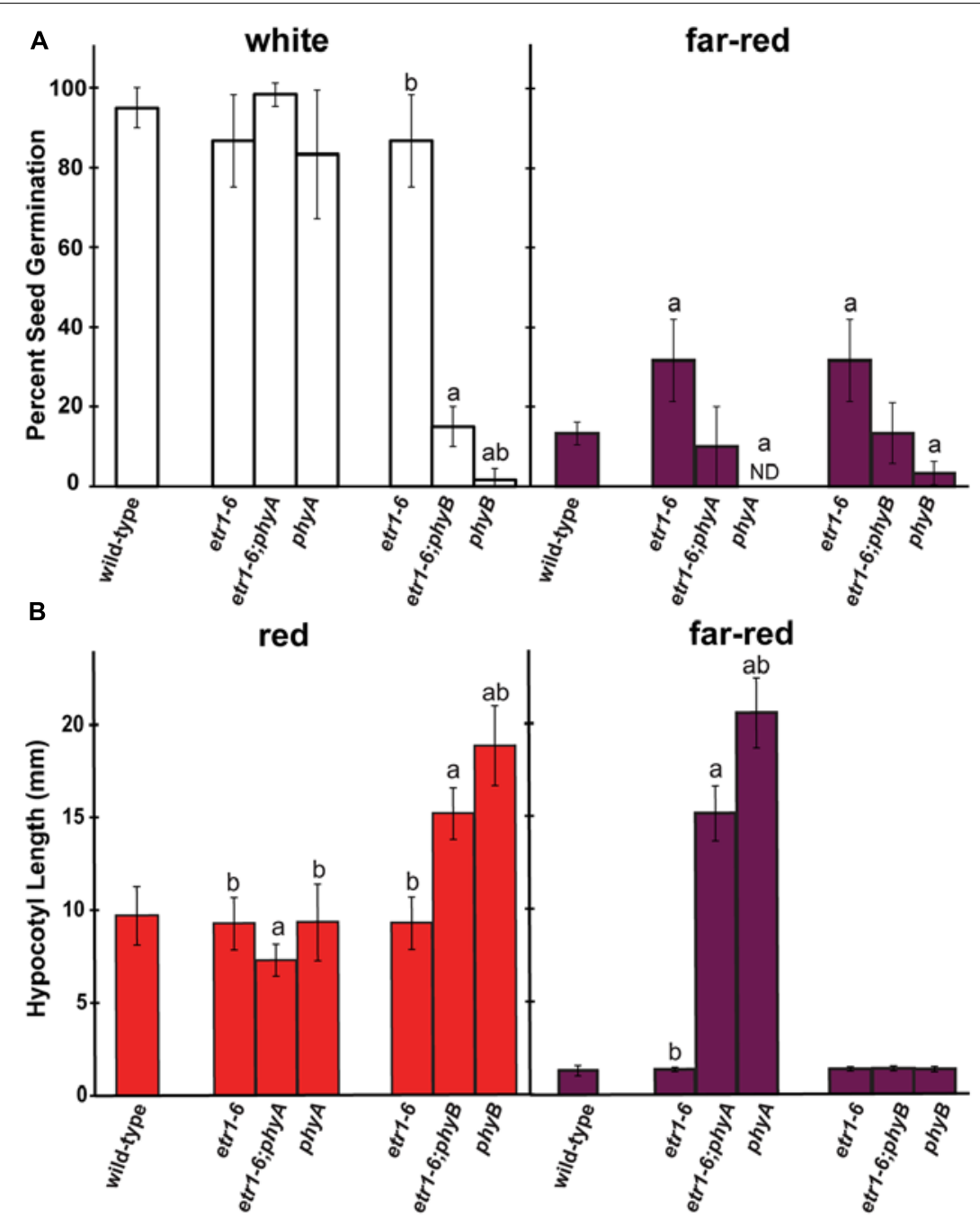

FIGURE 8 | Epistasis analysis of ETR1 and the phytochromes (phy), PHYA and $\boldsymbol{P H Y B}$ after light treatment. (A) Seeds were treated with white light for $3 \mathrm{~h}$, followed by either $4 \mathrm{~h}$ of white or far-red light as shown. The percent of seed germination was then determined 7 days later. The average \pm SD from at least three biological replicates is plotted. ND denotes no germination detected. (B) Seeds were treated with white light for $24 \mathrm{~h}$ followed by continuous illumination with red or far-red light for 6 days as shown. The lengths of the hypocotyls were then measured. The average \pm SD is plotted. In both panels, the data were analyzed by $t$-tests and differences considered

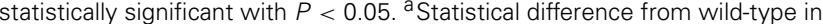
that light condition; b Significant difference of the single mutant from the double. seedlings (Binder et al., 2004, 2006; Qu and Schaller, 2004; Kim et al., 2011) and germination during salt stress under white light (Wilson et al., 2014). In the current study, we found that transformation of etr1-6;etr2-3;ein4-4 triple mutants with either cETR1 or cetr $1-\Delta R$ rescued germination after far-red light treatment to wild-type levels showing that the receiver domain of ETR1 is not required in the control of this trait. This is intriguing because the receiver domain is required for ETR1 function in the control of germination during salt stress under white light (Wilson et al., 2014; this study). These results indicate that environmental conditions affect the function of the ETR1 ethylene receptor to regulate seed germination. The mechanism for this is not known but one possibility is that a downstream target unique to ETR1 is altered upon illumination. There is precedence for this since light regulates the levels of one downstream target of EIN3, PIF3, to alter the effects of ethylene on plant growth in the dark versus light (Zhong et al., 2012).

A major question is whether or not the effect of ETR1 on seed germination is ethylene-dependent or not. We found that the ethylene biosynthesis inhibitor, AVG, partially reversed the effect of etr1-6 on germination after treatment far-red light, but addition of ethylene up to $1 \mathrm{ppm}$ had no effect on germination of the mutant after far-red illumination. It is possible that the etr1 loss-of-function mutants are producing high levels of ethylene and are saturated for ethylene responses. However, this seems unlikely since etr1-6 produced very low levels of ethylene that were indistinguishable from the germinating wild-type seeds after illumination with far-red light. Transfer of these seeds to white light conditions resulted in an increase in germination showing that the seeds were still capable of better germination. Additionally, AVG 


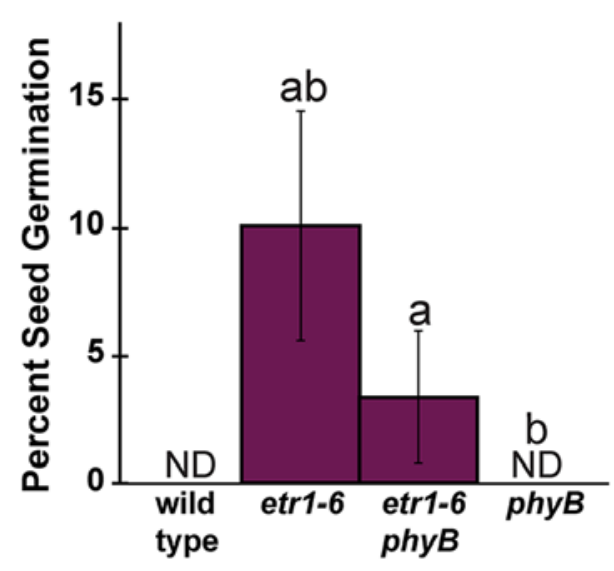

FIGURE 9 | Epistasis analysis of ETR1 and PHYB in the dark. Seeds were germinated in the dark for 7 days and the percent of seed germination was then determined. The average \pm SD from at least three biological replicates is plotted. ND denotes no germination detected. The data were analyzed by $t$-tests and differences considered statistically significant with $P<0.05$. ${ }^{a}$ Statistical difference from wild-type in that light condition; bSignificant difference of the single mutant from the double mutant.

has effects that are independent of its effect on ethylene biosynthesis (Larsen and Chang, 2001; Soeno et al., 2010) confounding the results with this compound. Thus, it is seems unlikely that ethylene is the major factor contributing to the differences in germination between wild-type and etr1 loss-of-function mutants observed in this study.

Results presented here indicate that ETR1 is affecting germination by affecting the levels of GA and ABA. We observed that the transcript abundance of genes encoding certain GA and ABA metabolic genes are altered in etr1-6 mutants consistent with higher GA levels and lower ABA levels after far-red light treatment in the etr1 loss-of-function mutants compared to wild-type. Additionally, we have previously shown that etr1-6 mutants are less sensitive to ABA than wild-type (Wilson et al., 2014). Together, these results would explain the better germination of the etr1 loss-of-function mutants. Consistent with these results, application of GA improved the germination of all seed lines and norflurazon improved seed germination of etr1-6 mutants.

Another major question is whether ETR1 is affecting germination via the phy signaling pathway, via a phy-independent pathway, or both. Previous studies provide a tentative link between ETR1 and phyB (Urao et al., 2000; Sweere et al., 2001; To et al., 2004; Salomé et al., 2006; Scharein et al., 2008). However, our data suggest that ETR1 is affecting responses to light that are independent of the phy. Support for this is our observation that etr1 loss-offunction mutants are more sensitive to ABA in white light (Wilson et al., 2014) and germinate better than wild-type seeds in the dark (this study). Additionally, etr1;phyb double mutants germinated in the dark, whereas phyb single mutants did not. Similarly, after treatment with far-red light the phyA mutants did not germinate but the etrl;phyA double mutants did germinate. Thus, the effects of ETR1 on germination are occurring in the absence of actively signaling phy. These observations coupled with our observation that ETR1 affects the transcript levels of genes encoding for ABA and GA metabolic enzymes support a model where ETR1 is affecting germination by altering ABA and GA levels and $\mathrm{ABA}$ sensitivity in parallel with the effects of phy signaling on these parameters.

\section{ACKNOWLEDGMENTS}

The authors acknowledge the technical assistance of Abigail Hall, Joonyup Kim, and Daniel Rose and thank Kenneth McFarland and Albrecht von Arnim for the use of equipment. We also thank Albrecht von Arnim for feedback on this manuscript. This research was supported by a NSF fellowship to Rebecca L. Wilson and a NSF grant (MCB-0918430) to Brad M. Binder.

\section{REFERENCES}

Abeles, F., Morgan, P., and Saltveit, M. J. (1992). Ethylene in Plant Biology. San Diego, CA: Academic Press.

Abeles, F. B., and Lonski, J. (1969). Stimulation of lettuce seed germination by ethylene. Plant Physiol. 44, 277-280. doi: 10.1104/pp.44.2.277

Bae, G., and Choi, G. (2008). Decoding of light signals by plant phytochromes and their interacting proteins. Annu. Rev. Plant Biol. 59, 281-311. doi: 10.1146/annurev.arplant.59.032607.092859

Bentsink, L., and Koorneef, M. (2008). Seed dormancy and germination. Arabidopsis Book 6:e0119. doi: 10.1199/tab.0119

Binder, B. M., O’Malley, R. C., Wang, W., Moore, J. M., Parks, B. M., Spalding, E. P., et al. (2004). Arabidopsis seedling growth response and recovery to ethylene. A kinetic analysis. Plant Physiol. 136, 2913-2920. doi: 10.1104/pp.104.050369

Binder, B. M., O’Malley, R. C., Wang, W., Zutz, T. C., and Bleecker, A. B. (2006). Ethylene stimulates nutations that are dependent on the ETR1 receptor. Plant Physiol. 142, 1690-1700. doi: 10.1104/pp.106.087858

Bleecker, A. B. (1999). Ethylene perception and signaling: an evolutionary perspective. Trends Plant Sci. 4, 269-274. doi: 10.1016/S1360-1385(99)01427-2

Bleecker, A. B., Estelle, M. A., Somerville, C., and Kende, H. (1988). Insensitivity to ethylene conferred by a dominant mutation in Arabidopsis thaliana. Science 241, 1086-1089. doi: 10.1126/science.241.4869.1086

Buer, C. S., Wasteneys, G. O., and Masle, J. (2003). Ethylene modulates root-wave responses in Arabidopsis. Plant Physiol. 132, 1085-1096. doi: 10.1104/pp.102.019182

Burdett, A. N., and Vidaver, W. E. (1971). Synergistic action of ethylene with gibberellin or red light in germinating lettuce seeds. Plant Physiol. 48, 656-657. doi: $10.1104 /$ pp.48.5.656

Cancel, J. D., and Larsen, P. B. (2002). Loss-of-function mutations in the ethylene receptor ETR1 cause enhanced sensitivity and exaggerated response to ethylene in Arabidopsis. Plant Physiol. 129, 1557-1567. doi: 10.1104/pp.003780

Casal, J. J., and Sánchez, R. A. (1998). Phytochromes and seed germination. Seed Sci. Res. 8, 317-329. doi: 10.1017/S0960258500004256

Chang, C., Kwok, S. F., Bleecker, A. B., and Meyerowitz, E. M. (1993). Arabidopsis ethylene-response gene ETR1: similarity of product to two-component regulators. Science 262, 539-544. doi: 10.1126/science.8211181

Chen, R., Binder, B. M., Garrett, W. M., Tucker, M. L., Cooper, B., and Chang, C. (2011). Proteomic responses in Arabidopsis thaliana seedlings treated with ethylene. Mol. Biosyst. 7, 2637-2650. doi: 10.1039/c1mb05159h

Chen, T., Liu, J., Lei, G., Liu, Y.-F., Li, Z.-G., Tao, J.-J., et al. (2009). Effects of tobacco ethylene receptor mutations on receptor kinase activity, plant growth and stress responses. Plant Cell Physiol. 50, 1636-1650. doi: 10.1093/pcp/pcp107

Chiwocha, S., Cutler, A., Abrams, S. R., Ambrose, S., Yang, J., Ross, A. R., et al. (2005). The etr1-2 mutation in Arabidopsis thaliana affects the abscisice acid, auxin, cytokinin and gibberellin metabolic pathways during maintenance of seed dormancy, moist-chilling and germination. Plant J. 42, 35-48. doi: 10.1111/j.1365-313X.2005.02359.x

Dekkers, B. J. W., Willems, L., Bassel, G. W., Van Bolderen-Veldkamp, R. P., Ligterink, W., Hilhorst, H. W. M., et al. (2011). Identification of reference genes for RT-qPCR expression analysis in Arabidopsis and tomato seeds. Plant Cell Physiol. 53, 28-37. doi: $10.1093 / \mathrm{pcp} / \mathrm{pcr} 113$

Ellwell, A. L., Gronwald, D. S., Miller, N. D., Spalding, E. P., and Brooks, T. L. D. (2011). Separating parental environment from seed size effects on next generation 
growth and development in Arabidopsis. Plant Cell Environ. 34, 291-301. doi: 10.1111/j.1365-3040.2010.02243.x

Esashi, Y., Hase, S., and Kojima, K. (1987). Light actions in the germination of cocklebur seeds. J. Exp. Bot. 189, 702-710. doi: 10.1093/jxb/ 38.4.702

Fankhauser, C., Yeh, K.-C., Lagarias, C. J., Zhang, H., Elich, T. D., and Chory, J. (1999). PKS1, a substrate phosphorylated by phytochrome that modulates light signaling in Arabidopsis. Science 284, 1539-1541. doi: 10.1126/science.284.5419.1539

Finch-Savage, W. E., Cadman, C. S. C., Toorop, P. E., Lynn, J. R., and Hilhorst, H. W. M. (2007). Seed dormancy release in Arabidopsis Cvi by dry after-ripening, low temperature, nitrate and light shows common quantitative patterns of gene expression directed by environmentally specific sensing. Plant J. 51, 60-78. doi: 10.1111/j.1365-313X.2007.03118.x

Finkelstein, R., Reeves, W., Ariizumi, T., and Steber, C. (2008). Molecular aspects of seed dormancy. Annu. Rev. Plant Biol. 59, 387-415. doi 10.1146/annurev.arplant.59.032607.092740

Finlayson, S. A., Lee, I.-J., and Morgan, P. W. (1998). Phytochrome B and the regulation of circadian ethylene production in Sorghum. Plant Physiol. 116, 17-25. doi: $10.1104 / \mathrm{pp} .116 .1 .17$

Gagne, J. M., Smalle, J., Gingerich, D. J., Walker, J. M., Yoo, S. D., Yanagisawa, S., et al (2004). Arabidopsis EIN3-binding F-box 1 and 2 form ubiquitin-protein ligases that repress ethylene action and promote growth by directing EIN3 degradation. Proc. Natl. Acad. Sci. U.S.A. 101, 6803-6808. doi: 10.1073/pnas.0401698101

Gamble, R. L., Coonfield, M. L., and Schaller, G. E. (1998). Histidine kinase activity of the ETR1 ethylene receptor from Arabidopsis. Proc. Natl. Acad. Sci. U.S.A. 95 7825-7829. doi: 10.1073/pnas.95.13.7825

Garciarrubio, A., Legaria, J. P., and Covarrubias, A. A. (1997). Abscisic acid inhibits germination of mature Arabidopsis seeds by limiting the availability of energy and nutrients. Planta 203, 182-187. doi: 10.1007/s004250050180

Guo, H. W., and Ecker, J. R. (2003). Plant responses to ethylene gas are mediated by SCF (EBF1/EBF2)-dependent proteolysis of EIN3 transcription factor. Cell 115 667-677. doi: 10.1016/S0092-8674(03)00969-3

Hall, A. E., and Bleecker, A. B. (2003). Analysis of combinatorial loss-of-function mutants in the Arabidopsis ethylene receptors reveals that the ers1 etr1 double mutant has severe developmental defects that are EIN2 dependent. Plant Cell 15 2032-2041. doi: 10.1105/tpc.013060

Hall, B. P., Shakeel, S. N., Amir, M., Haq, N. U., Qu, X., and Schaller, G. E. (2012). Histidine kinase activity of the ethylene receptor ETR1 facilitates the ethylene response in Arabidopsis. Plant Physiol. 159, 682-695. doi: 10.1104/pp.112.196790

Hass, C., Lohrmann, J., Albrecht, V., Sweere, U., Hummel, F., Yoo, S., et al. (2004). The response regulator 2 mediates ethylene signalling and hormone integration in Arabidopsis. EMBO J. 23, 3290-3302. doi: 10.1038/si.emboj. 7600337

Hennig, L., Stoddart, W. M., Dieterle, M., Whitelam, G. C., and Schäfer, E. (2002) Phytochrome E controls light-induced germination of Arabidopsis. Plant Physiol. 128, 194-200. doi: 10.1104/pp.010559

Hensel, L. L., Grbic, V., Baumgarten, D. A., and Bleecker, A. B. (1993) Developmental and age-related processes that influence the longevity and senescence of photosynthetic tissues in Arabidopsis. Plant Cell 5, 553-564. doi: 10.1105/tpc.5.5.553

Hilhorst, H. W. M., and Karssen, C. M. (1992). Seed dormancy and germination: the role of abscisic acid and gibberellins and the importance of hormone mutants. Plant Growth Regul. 11, 225-238. doi: 10.1007/BF00024561

Hirayama, T., Fujishige, N., Kunii, T., Nishimura, N., Iuchi, S., and Shinozaki, K. (2004). A novel ethanol-hypersensitive mutant of Arabidopsis. Plant Cell Physiol. 45, 703-711. doi: 10.1093/pcp/pch078

Hua, J., Chang, C., Sun, Q., and Meyerowitz, E. M. (1995). Ethylene insensitivity conferred by Arabidopsis ERS gene. Science 269, 1712-1714. doi: 10.1126/science.7569898

Hua, J., and Meyerowitz, E. M. (1998). Ethylene responses are negatively regulated by a receptor gene family in Arabidopsis thaliana. Cell 94, 261-271. doi: 10.1016/S0092-8674(00)81425-7

Hua, J., Sakai, H., Nourizadeh, S., Chen, Q. H. G., Bleecker, A. B., Ecker, J. R., et al. (1998). EIN4 and ERS2 are members of the putative ethylene receptor gene family in Arabidopsis. Plant Cell 10, 1321-1332. doi: 10.1105/tpc.10.8.1321

Hwang, D., Chen, H. C., and Sheen, J. (2002). Two-component signal transduction pathways in Arabidopsis. Plant Physiol. 129, 500-515. doi: 10.1104/pp.005504
Iuchi, S., Kobayashi, M., Taji, T., Naramoto, M., Seki, M., Kato, T., et al. (2001) Regulation of drought tolerance by gene manipulation of 9-cis-epoxycarotenoid dioxygenase, a key enzyme in abscisic acid biosynthesis in Arabidopsis. Plant J. 27, 325-333. doi: 10.1046/j.1365-313x.2001.01096.x

Jaillais, Y., and Chory, J. (2010). Unraveling the paradoxes of plant hormone signaling integration. Nat. Struct. Mol. Biol. 17, 642-645. doi: 10.1038/ nsmb0610-642

Ju, C., Yoon, G. M., Shemansky, J. M., Lin, D., Yin, I., Chang, J., et al. (2012). CTR1 phosphorylates the central regulator EIN2 to control ethylene hormone signaling from the ER membrane to the nucleus in Arabidopsis. Proc. Natl. Acad. Sci. U.S.A. 109, 19486-19491. doi: 10.1073/pnas.1214848109

Kevany, B. M., Tieman, D. M., Taylor, M. G., Cin, V. D., and Klee, H. J. (2007) Ethylene receptor degradation controls the timing of ripening in tomato fruit. Plant J. 51, 458-467. doi: 10.1111/j.1365-313X.2007.03170.x

Kieber, J. J., Rothenberg, M., Roman, G., Feldman, K. A., and Ecker, J. R. (1993) CTR1, a negative regulator of the ethylene response pathway in Arabidopsis, encodes a member of the Raf family of protein kinases. Cell 72, 427-441. doi: 10.1016/0092-8674(93)90119-B

Kim, H., Helmbrecht, E. E., Stalans, M. B., Schmitt, C., Patel, N., Wen, C.-K., et al. (2011). Ethylene receptor ETR1 domain requirements for ethylene responses in Arabidopsis seedlings. Plant Physiol. 156, 417-429. doi: 10.1104/pp.110. 170621

Kim, J., Wilson, R. L., Case, J. B., and Binder, B. (2012). A comparative study of ethylene growth response kinetics in eudicots and monocots reveals a role for gibberellin in growth inhibition and recovery. Plant Physiol. 160, 1567-1580. doi: 10.1104/pp.112.205799

Larsen, P. B., and Chang, C. (2001). The Arabidopsis eer1 mutant has enhanced ethylene responses in the hypocotyl and stem. Plant Physiol. 125, 1061-1073. doi: 10.1104/pp.125.2.1061

Lau, O. S., and Deng, X. W. (2010). Plant hormone signaling lightens up: integrators of light and hormones. Curr. Opin. Plant Biol. 13, 571-577. doi: 10.1016/j.pbi.2010.07.001

Lefebvre, V., North, H., Frey, A., Sotta, B., Seo, M., Okamoto, M., et al. (2006). Functional analysis of Arabidopsis NCED6 and NCED9 genes indicates that ABA synthesized in the endosperm is involved in the induction of seed dormancy. Plant J. 45, 309-319. doi: 10.1111/j.1365-313X.2005.02622.x

Leivar, P., and Quail, P. H. (2010). PIFs: pivotal components in a cellular signaling hub. Trends Plant Sci. 16, 19-28. doi: 10.1016/j.tplants.2010. 08.003

Linkies, A., and Leubner-Metzger, G. (2012). Beyond gibberellins and abscisic acid: how ethylene and jasmonates control seed germination. Plant Cell Rep. 31, 253270. doi: 10.1007/s00299-011-1180-1

Linkies, A., Müller, K., Morris, K., Turečková, V., Wenk, M., Cadman, C. S. C., et al. (2009). Ethylene interacts with abscisic acid to regulate endosperm rupture during germination: a comparative approach using Lepidium sativum and Arabidopsis thaliana. Plant Cell 21, 3803-3822. doi: 10.1105/tpc.109.070201

Liu, Q., Xu, C., and Wen, C.-K. (2010). Genetic and transformation studies reveal negative regulation of ERS1 ethylene receptor signaling in Arabidopsis. BMC Plant Biol. 10:60. doi: 10.1186/1471-2229-10-60

Livak, K. J., and Schmittgen, T. D. (2001). Analysis of relative gene expression data using real-time quantitative PCR and the $2^{-\Delta \Delta C T}$ method. Methods 25, 402-408. doi: 10.1006/meth.2001.1262

Mattoo, A. K., and Suttle, J. C. (eds). (1991). The Plant Hormone Ethylene. Boca Raton: CRC Press, Inc.

Meng, L., and Feldman, L. (2010). A rapid TRIzol-based two-step method for DNAfree RNA extraction from Arabidopsis siliques and dry seeds. Biotechnol. J. 5, 183-186. doi: 10.1002/biot.200900211

Mount, S. M., and Chang, C. (2002). Evidence for a plastid origin of plant ethylene receptor genes. Plant Physiol. 130, 10-14. doi: 10.1104/pp.005397

Moussatche, P., and Klee, H. J. (2004). Autophosphorylation activity of the Arabidopsis ethylene receptor multigene family. J. Biol. Chem. 279, 48734-48741. doi: 10.1074/jbc.M403100200

Neff, M. M., Fankhauser, C., and Chory, J. (2000). Light: an indicator of time and place. Genes Dev. 14, 257-271.

O’Malley, R. C., Rodriguez, F. I., Esch, J. J., Binder, B. M., O’Donnell, P., Klee, H. J., et al. (2005). Ethylene-binding activity, gene expression levels, and receptor system output for ethylene receptor family members from Arabidopsis and tomato Plant J. 41, 651-659. doi: 10.1111/j.1365-313X.2004.02331.x 
Oh, E., Kang, H., Yamaguchi, S., Park, J., Lee, D., Kamiya, Y., et al. (2009). Genomewide analysis of genes targeted by PHYTOCHROME INTERACTING FACTOR 3-LIKE5 during seed germination in Arabidopsis. Plant Cell 21, 403-419. doi: 10.1105/tpc.108.064691

Oh, E., Yamaguchi, S., Hu, J., Yusuke, J., Jung, B., Paik, I., et al. (2007). PIL5, a phytochrome-interacting bHLH protein, regulates gibberellin responsiveness by binding directly to the GAI and RGA promoters in Arabidopsis seeds. Plant Cell 19, 1192-1208. doi: 10.1105/tpc.107.050153

Okamoto, M., Kuwahara, A., Seo, M., Kushiro, T., Asami, T., Hirai, N., et al. (2006) CYP707A1 and CYP707A2, which encode abscisic acid 8-hydroxylases, are indispensable for proper control of seed dormancy and germination in Arabidopsis. Plant Physiol. 141, 97-107. doi: 10.1104/pp.106.079475

Plett, J. M., Cvetkovska, M., Makenson, P., Xing, T., and Regan, S. (2009a). Arabidopsis ethylene receptors have different roles in Fumonisin B1-induced cell death. Physiol. Mol. Plant Pathol. 74, 18-26. doi: 10.1016/j.pmpp.2009.08.004

Plett, J. M., Mathur, J., and Regan, S. (2009b). Ethylene receptor ETR2 controls trichome branching by regulating microtubule assembly in Arabidopsis thaliana. J. Exp. Bot. 60, 3923-3933. doi: 10.1093/jxb/erp228

Poppe, C., and Schäfer, E. (1997). Seed germination of Arabidopsis thaliana phyA/phyB double mutants is under phytochrome control. Plant Physiol. 114, 1487-1492. doi: 10.1104/pp.114.4.1487

Qiao, H., Chang, K. N., Yazaki, J., and Ecker, J. R. (2009). Interplay between ethylene, ETP1/ETP2 F-box proteins, and degradation of EIN2 triggers ethylene responses in Arabidopsis. Genes Dev. 23, 512-521. doi: 10.1101/gad.1765709

Qiao, H., Shen, Z., Huang, S.-S. C., Schmitz, R. J., Urich, M. A., Briggs, S. P., et al. (2012). Processing and subcellular trafficking of ER-tethered EIN2 control response to ethylene gas. Science 338, 390-393. doi: 10.1126/science.1225974

Qin, X., and Zeevaart, J. A. D. (1999). The 9-cis-epoxycarotenoid cleavage reaction is the key regulatory step of abscisic acid biosynthesis in water-stressed bean. Proc. Natl. Acad. Sci. U.S.A. 96, 15354-15361. doi: 10.1073/pnas.96. 26.15354

Qu, X., Hall, B., Gao, Z., and Schaller, G. E. (2007). A strong constitutive ethyleneresponse phenotype conferred on Arabidopsis plants containing null mutations in the ethylene receptors ETR1 and ERS1. BMC Plant Biol. 7:3. doi: 10.1186/14712229-7-3

Qu, X., and Schaller, G. E. (2004). Requirement of the histidine kinase domain for signal transduction by the ethylene receptor ETR1. Plant Physiol. 136, 2961-2970. doi: 10.1104/pp.104.047126

Quail, P. H., Boylan, M. T., Parks, B. M., Short, T. W., Xu, Y., and Wagner, D. (1995). Phytochromes: photosensory perception and signal transduction. Science 268, 675-680. doi: 10.1126/science.7732376

Reed, J. W., Nagatani, A., Elich, T. D., Fagan, M., and Chory, J. (1994). Phytochrome A and Phytochrome B have overlapping but distinct functions in Arabidopsis development. Plant Physiol. 104, 1139-1149.

Reid, J. B., Botwright, N. A., Smith, J. J., O’Neill, D. P., and Kerckhoffs, L. H. J. (2002). Control of gibberellin levels and gene expression during de-etiolation in pea. Plant Physiol. 128, 734-741. doi: 10.1104/pp.010607

Reinecke, D. M., Wickramarathna, A. D., Ozga, J. A., Kurepin, L. V., Jin, A. L., Good, A. G., et al. (2013). Gibberellin 3-oxidase gene expression patterns influence gibberellin biosynthesis, growth, and development in pea. Plant Physiol. 163, 929-945. doi: 10.1104/pp.113.225987

Rösler, J., Klein, I., and Zeidler, M. (2007). Arabidopsis fhl/fhyl double mutant reveals a distinct cytoplasmic action of phytochrome A. Proc. Natl. Acad. Sci U.S.A. 104, 10737-10742. doi: 10.1073/pnas.0703855104

Sakai, H., Hua, J., Chen, Q. H. G., Chang, C., Medrano, L. J., Bleecker, A. B., et al. (1998). ETR2 is an ETR1-like gene involved in ethylene signaling in Arabidopsis. Proc. Natl. Acad. Sci. U.S.A. 95, 5812-5817. doi: 10.1073/pnas.95. 10.5812

Salomé, P. A., To, J. P. C., Kieber, J. J., and Mcclung, C. R. (2006). Arabidopsis response regulators ARR3 and ARR4 play cytokinin-independent roles in the control of circadian period. Plant Cell 18, 55-69. doi: 10.1105/tpc.105. 037994

Scharein, B., Voet-Van-Vormizeele, J., Harter, K., and Groth, G. (2008). Ethylene signaling: identification of a putative ETR1-AHP1 phosphorelay complex by fluorescence spectroscopy. Anal. Biochem. 377, 72-76. doi: 10.1016/j.ab.2008. 03.015

Seifert, G. J., Barber, C., Wells, B., and Roberts, K. (2004). Growth regulators and the control of nucleotide sugar flux. Plant Cell 16, 723-730 doi: 10.1105/tpc.019661
Seo, M., Aoki, H., Koiwai, H., Kamiya, Y., Nambara, E., and Koshiba, T. (2004) Comparative studies on the Arabidopsis Aldehyde Oxidase (AAO) gene family revealed a major role of AAO3 in ABA biosynthesis in seeds. Plant Cell Physiol. 45, 1694-1703. doi: 10.1093/pcp/pch198

Seo, M., Hanada, A., Kuwahara, A., Endo, A., Okamoto, M., Yamauchi, Y., et al. (2006). Regulation of hormone metabolism in Arabidopsis seeds: phytochrome regulation of abscisic acid metabolism and abscisic acid regulation of gibberellin metabolism. Plant J. 48, 354-366. doi: 10.1111/j.1365-313X.2006. 02881.x

Shinomura, T., Nagatani, A., Chory, J., and Furuya, M. (1994). The induction of seed germination in Arabidopsis thaliana is regulated principally by phytochrome B and secondarily by phytochrome A. Plant Physiol. 104, 363-371.

Shinomura, T., Nagatani, A., Hanzawa, H., Kubota, M., Watanabe, M., and Furuya, M. (1996). Action spectra for phytochrome A- and B-specific photoinduction of seed germination in Arabidopsis thaliana. Proc. Natl. Acad. Sci. U.S.A. 93, 8129-8133. doi: 10.1073/pnas.93.15.8129

Shu, K., Zhang, H., Wang, S., Chen, M., Wu, Y., Tang, S., et al. (2013). ABI4 Regulates primary seed dormancy by regulating the biogenesis of abscisic acid and gibberellins in Arabidopsis. PLoS Genet. 9:e1003577. doi: 10.1371/journal.pgen. 1003577

Smith, H., Xu, Y., and Quail, P. H. (1997). Antagonistic but complementary actions of phytochromes A and B allow optimum seedling de-etiolation. Plant Physiol. 114, 637-641. doi: 10.1104/pp.114.2.637

Soeno, K., Goda, H., Ishii, T., Ogura, T., Tachikawa, T., Sasaki, E., et al. (2010). Auxin biosynthesis inhibitors, identified by a genomics-based approach, provide insights into auxin biosynthesis. Plant Cell Physiol. 51, 524-536. doi: 10.1093/pcp/ pcq032

Sung, D.-Y., Lee, D., Harris, H., Raab, A., Feldmann, J., Meharg, A., et al. (2007). Identification of an arsenic tolerant double mutant with a thiol-mediated component and increased arsenic tolerance in phyA mutants. Plant J. 49, 1064-1075. doi: 10.1111/j.1365-313X.2006.03018.x

Sweere, U., Eichenberg, K., Lohrmann, J., Mira-Rodado, V., Bäurle, I., Kudla, J., et al. (2001). Interaction of the response regulator ARR4 with Phytochrome B in modulating red light signaling. Science 294, 1108-1111. doi: 10.1126/science. 1065022

Tepperman, J. M., Hudson, M. E., Khanna, R., Zhu, T., Chang, S. H., Wang, X., etal. (2004). Expression profiling of phyB mutant demonstrates substantial contribution of other phytochromes to red-light-regulated gene expression during seedling de-etiolation. Plant J. 38, 725-739. doi: 10.1111/j.1365313X.2004.02084.x

Tieman, D. V., Taylor, M. G., Ciardi, J. A., and Klee, H. J. (2000). The tomato ethylene receptors NR and LeETR4 are negative regulators of ethylene response and exhibit functional compensation within a multigene family. Proc. Natl. Acad. Sci. U.S.A. 97, 5663-5668. doi: 10.1073/pnas.090550597

To, J. P. C., Haberer, G., Ferreira, F. J., Deruère, J., Mason, M. G., Schaller, G. E., et al. (2004). Type-A Arabidopsis response regulators are partially redundant negative regulators of cytokinin signaling. Plant Cell 16, 658-671. doi: 10.1105/tpc. 018978

Urao, T., Miyata, S., Yamaguchi-Shinozaki, K., and Shinozaki, K. (2000). Possible his to Asp phosphorelay signaling in an Arabidopsis twocomponent system. FEBS Lett. 478, 227-232. doi: 10.1016/S0014-5793(00) 01860-3

Wang, W., Hall, A. E., O’Malley, R., and Bleecker, A. B. (2003). Canonical histidine kinase activity of the transmitter domain of the ETR1 ethylene receptor from Arabidopsis is not required for signal transmission. Proc. Natl. Acad. Sci. U.S.A. 100, 352-357. doi: 10.1073/pnas.0237085100

Wen, X., Zhang, C., Ji, Y., Zhao, Q., He, W., An, F., et al. (2012). Activation of ethylene signaling is mediated by nuclear translocation of the cleaved EIN2 carboxyl terminus. Cell Res. 22, 1613-1616. doi: 10.1038/cr. 2012.145

Wilson, R. L., Kim, H., Bakshi, A., and Binder, B. M. (2014). The ethylene receptors ETHYLENE RESPONSE1 and ETHYLENE RESPONSE2 have contrasting roles in seed germination of Arabidopsis during salt stress. Plant Physiol. 165, 1353-1366. doi: 10.1104/pp.114.241695

Wuriyanghan, H., Zhang, B., Cao, W.-H., Ma, B., Lei, G., Liu, Y.-F., et al. (2009). The ethylene receptor ETR2 delays floral transition and affects starch accumulation in rice. Plant Cell 21, 1473-1494. doi: 10.1105/tpc.108.065391 
Xie, F., Liu, Q., and Wen, C.-K. (2006). Receptor signal output mediated by the ETR1 N-terminus is primarily subfamily I receptor dependent. Plant Physiol. 142, 492-508. doi: 10.1104/pp.106. 082628

Yamauchi, Y., Ogawa, M., Kuwahara, A., Hanada, A., Kamiya, Y., and Yamaguchi, S (2004). Activation of gibberellin biosynthesis and response pathways by low temperature during imbibition of Arabidopsis thaliana seeds. Plant Cell 16, 367-378. doi: 10.1105/tpc.018143

Yamauchi, Y., Takeda-Kamiya, N., Hanada, A., Ogawa, M., Kuwahara, A., Seo, M., et al. (2007). Contribution of gibberellin deactivation by AtGA2ox2 to the suppression of germination of dark-imbibed Arabidopsis thaliana seeds. Plant Cell Physiol. 48, 555-561. doi: 10.1093/pcp/ pcm023

Yanagisawa, S., Yoo, S. D., and Sheen, J. (2003). Differential regulation of EIN3 stability by glucose and ethylene signalling in plants. Nature 425, 521-525. doi: 10.1038 /nature01984

Yeh, K.-C., and Lagarias, J. C. (1998). Eukaryotic phytochromes: lightregulated serine/threonine protein kinases with histidine kinase ancestry. Proc. Natl. Acad. Sci. U.S.A. 95, 13976-13981. doi: 10.1073/pnas.95.23. 13976
Zhong, S., Shi, H., Xue, C., Wang, L., Xi, Y., Li, J., et al. (2012). A molecular framework of light-controlled phytochrome action in Arabidopsis. Curr. Biol. 22, 1530-1535. doi: 10.1016/j.cub.2012.06.039

Conflict of Interest Statement: The authors declare that the research was conducted in the absence of any commercial or financial relationships that could be construed as a potential conflict of interest.

Received: 28 May 2014; accepted: 13 August 2014; published online: 28 August 2014. Citation: Wilson RL, Bakshi A and Binder BM (2014) Loss of the ETR1 ethylene receptor reduces the inhibitory effect of far-red light and darkness on seed germination of Arabidopsis thaliana. Front. Plant Sci. 5:433. doi: 10.3389/fpls.2014.00433

This article was submitted to Plant Physiology, a section of the journal Frontiers in Plant Science.

Copyright $(2) 2014$ Wilson, Bakshi and Binder. This is an open-access article distributed under the terms of the Creative Commons Attribution License (CC BY). The use, distribution or reproduction in other forums is permitted, provided the original author(s) or licensor are credited and that the original publication in this journal is cited, in accordance with accepted academic practice. No use, distribution or reproduction is permitted which does not comply with these terms. 


\title{
An alternate route of ethylene receptor signaling
}

\author{
Jingyi Zhang, Jing $\mathrm{Yu}^{\dagger}$ and Chi-Kuang Wen* \\ National Key Laboratory of Plant Molecular Genetics and National Center for Plant Gene Research (Shanghai), Institute of Plant Physiology and Ecology, \\ Shanghai Institutes for Biological Sciences, Chinese Academy of Sciences, Shanghai, China
}

\section{Edited by:}

Caren Chang, University of Maryland, USA

\section{Reviewed by:}

Brad Binder, University of Tennessee-Knoxville, USA Uener Kolukisaoglu, University of Tuebingen, Germany

\section{*Correspondence.}

Chi-Kuang Wen, National Key Laboratory of Plant Molecular Genetics and National Center for Plant Gene Research (Shanghai), Institute of Plant Physiology and Ecology, Shanghai Institutes for Biological Sciences, Chinese Academy of Sciences, 300 Fenglin Road, Shanghai 200032, China e-mail:qgwen@sibs.ac.cn

\section{${ }^{\dagger}$ Present address}

Jing Yu, State Key Laboratory of Hybrid Rice, School of Life Sciences and Biotechnology, Shanghai Jiao Tong University, Shanghai 200240, China
The gaseous plant hormone ethylene is perceived by a family of ethylene receptors and mediates an array of ethylene responses. In the absence of ethylene, receptor signaling is conveyed via the C-terminal histidine kinase domain to the N-terminus of the CONSTITUTIVETRIPLE RESPONSE1 (CTR1) protein kinase, which represses ethylene signaling mediated by ETHYLENE INSENSITIVE2 (EIN2) followed by EIN3. In the presence of ethylene, the receptors are inactivated when ethylene binds to their $\mathrm{N}$-terminal domain, and consequently CTR1 is inactive, allowing EIN2 and EIN3 to activate ethylene signaling. Recent findings have shown that the ethylene receptor $\mathrm{N}$-terminal portion can conditionally mediate the receptor signal output in mutants lacking CTR1, thus providing evidence of an alternative pathway from the ethylene receptors not involving CTR1. Here we highlight the evidence for receptor signaling to an alternative pathway and suggest that receptor signaling is coordinated via the $\mathrm{N}$ - and $\mathrm{C}$-termini, as we address the biological significance of the negative regulation of ethylene signaling by the two pathways.

Keywords: ethylene signaling, ETR1, CTR1, EIN2, Arabidopsis

\section{INTRODUCTION}

Signal transduction of the gaseous plant hormone ethylene has been studied for more than 2 decades, mainly with the dicotyledonous model plant Arabidopsis, and a linear signal transduction pathway has been proposed (Figure 1A; Ju and Chang, 2012; Ju et al., 2012; Shakeel et al., 2013). In the absence of ethylene, the ethylene receptors at the endoplasmic reticulum (ER) are active, and the docking of CONSTITUTIVE TRIPLE RESPONSE1 (CTR1) at the receptor histidine kinase domain facilitates CTR1 activation by unknown mechanisms. Serine/threonine kinase activity of CTR1 (Huang etal., 2003) results in phosphorylation of the C-terminal domain of ETHYLENE INSENSITIVE2 (EIN2; Ju etal., 2012). This phosphorylation prevents the EIN2 C-terminal domain from moving into the nucleus, and thusly prevents ethylene signaling. With ethylene binding to the receptors or in the absence of the receptors, CTR1 cannot be activated to phosphorylate EIN2. Underphosphorylated EIN2 undergoes proteolytic cleavage by an unknown mechanism to release a nuclear localization signal (NLS)-containing C-terminus, which enters the nucleus to mediate signaling to the EIN3 and EIN3-LIKE1 (EIL1) transcription factors. Such factors directly activate an array of primary ethylene response genes, including the ETHYLENE RESPONSE FACTOR1 (ERF1) transcription factor gene (Chao etal., 1997; Solano etal., 1998; Qiao etal., 2012; Chang etal., 2013).

This model illustrates a framework of ethylene signaling; however, the dynamic fine-tuning of ethylene signaling remains to be fully addressed. One question we have been focusing on is a pathway that does not involve CTR1, as proposed 16 years ago (Hua and Meyerowitz, 1998). The finding that expression of the ethylene receptor ETHYLENE RESPONSE1 (ETR1) $\mathrm{N}$-terminus represses ethylene signaling without involving CTR1 reveals an alternative pathway that greatly represses ethylene signaling (Gallie, 2012; Qiu et al., 2012). In this perspective article, we highlight studies that have revealed this alternative pathway for receptor signaling and discuss the dynamic coordination of the two pathways in negatively regulating ethylene signaling. We also discuss other alternative pathways that were have been previously suggested.

\section{EVIDENCE FOR ETHYLENE RECEPTOR SIGNALING NOT INVOLVING CTR1}

Results from various studies imply that ethylene receptor signaling could be in part independent of CTR1. The ctr1-1 mutation, encoding a D694E substitution, reduces CTR1 kinase activity to approximately $<0.1 \%$ that of the wild-type activity and $\operatorname{ctr} 1-3$, encoding an R435STOP early termination, has a stronger phenotype than $\operatorname{ctr} 1-1$ and the putative ctr1-3 protein does not have the kinase domain (Huang et al., 2003). The weak in vitro kinase activity of ctr1-1 that was detected could be due to the residual activity with the D694E substitution (Huang etal., 2003), nonspecific phosphorylation, or a possible trace amount of kinase contamination during protein purification. Both ctr1-1 and ctr13 are responsive to ethylene treatment, indicating that ethylene 


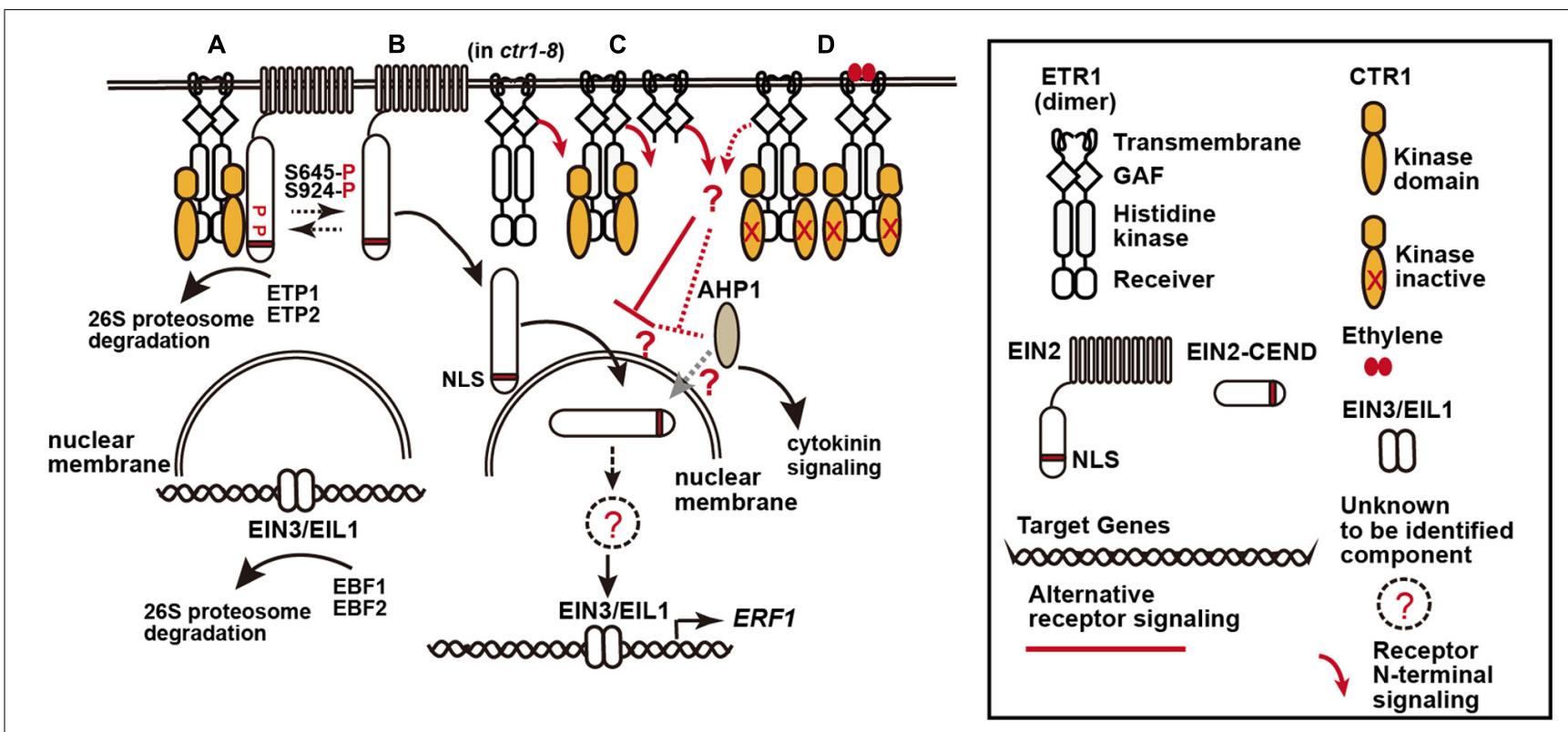

FIGURE 1 | A model of the ethylene receptor signaling via the $\mathrm{C}$ - and $\mathbf{N}$-termini of ethylene receptor. (A) Ethylene receptor C-terminal signaling, here represented by ETHYLENE RESPONSE1 (ETR1), is mediated via the histidine kinase domain to CTR1 to phosphorylate EIN2 residues S645 and S924, and EIN2 is retained at the endoplasmic reticulum for degradation mediated by the F-box proteins EIN2 TARGETING PROTEIN1 (ETP1) and ETP2. Ethylene signaling mediated by EIN2 to the nuclear EIN3/EIL1 is inhibited, and EIN3 and EIL1 undergo degradation mediated by the F-box proteins EIN3 BINDING F-BOX PROTEIN1 (EBF1) and EBF2. (B) "Default" ethylene signaling by EIN2. Without the receptors (in the receptor quintuple mutant), EIN2-mediated ethylene signaling fully occurs and the mutants produce an extremely strong constitutive ethylene response phenotype. With the receptors, a portion of EIN2 could be underphosphorylated, presumably because of interconversion between the phosphorylated and underphosphorylated state or incomplete phosphorylation by CTR1, and cleaved to potentially activate ethylene signaling. (C,D) Ethylene signaling suppression by the receptor $\mathrm{N}$-terminus: (C) With the receptors, the "default" ethylene signaling mediated by EIN2 can be repressed in part by the receptor $\mathrm{N}$-terminal signaling (red line), regardless of CTR1 docking. (D) Ethylene binding or docking of a kinase-inactive ctr1 at the receptors inhibits the receptor C-terminal signaling, and the cleaved EIN2 C-terminus is translocated to the nucleus to induce EIN3/EIL1-directed expression of ethylene response genes. The $\mathrm{N}$-terminal signaling is also inhibited (red dotted line), and the inhibition of EIN2-mediated signaling is largely alleviated. Components involved in the receptor $\mathrm{N}$-terminal signaling remain to be identified. AHP1 and EIN2 are proposed to be involved in the CTR1-independent pathway because of their interaction with ETR1, and AHP1 also mediates cytokinin signaling. receptor signaling can be mediated bypassing CTR1 (Larsen and Chang, 2001). A reciprocal evidence is the stronger constitutive ethylene response phenotype of the Arabidopsis quadruple and quintuple mutants (respectively, lacking 4 and 5 of the five homologous ethylene receptors) compared to the ctr1-1 mutant (Hua and Meyerowitz, 1998; Liu et al., 2010).

It was considered that the loss of multiple ethylene receptors could impact seedling growth, and the severe mutant phenotype might not be solely due to strong constitutive ethylene responses (Hua and Meyerowitz, 1998). Nevertheless, the possibility of an alternative pathway was supported by experimental evidence showing that expression of ETR1p:etr $1^{1-349}$, which encodes the ETR1 N-terminus (residues 1-349) lacking the CTR1 docking site, largely rescued the $c t r 1-1$ and $c t r 1-2$ mutant phenotypes and reduced the ERF1 transcript level. The ctr1-2 allele has a 17-bp deletion and probably encodes a truncated protein with 462 residues (Huang et al., 2003; Qiu et al., 2012), which was not immunologically detectable at the molecular-weight position of wild-type CTR1 (Gao et al., 2003). Ethylene insensitivity conferred by the dominant etr1-1 mutation was prevented by $\operatorname{ctr} 1-1$, whereas expression of ETR1p:etr1-1 $1^{1-349}$ in ctr1-1 conferred ethylene insensitivity. Moreover, expression of an ETR1p:etr1 $1^{1-349}$ transgene in the etr1-1 ctr1-1 double mutant largely restored ethylene insensitivity conferred by the etr1-1 allele, revealing the role of the ETR1 N-terminus in receptor cooperation and signal output without involving CTR1. Consistently, the ctr1-1 allele prevents ethylene insensitivity by other ethylene-insensitive receptor genes, and ethylene insensitivity was restored to various degrees by the wild-type ETR1 N-terminus (Gallie, 2012; Qiu et al., 2012).

The CTR1 N-terminus is a regulatory domain that physically interacts with the ETR1 C-terminal histidine kinase domain (Clark et al., 1998). Excess CTR1 N-terminus (residues 7-560) generated by expression of a $35 S: C T R 1^{7-560}$ transgene, lacking the kinase domain, most likely occupies the ethylene receptors and prevents the normal receptor signal output, and the overexpressor (CTR1-Nox) consequently shows the typical constitutive ethylene response phenotype (Huang et al., 2003; Qiu et al., 2012). These studies define a role of the CTR1 N-terminus in receptor docking to mediate the receptor signaling to the CTR1 C-terminal kinase domain. However, there is more to this story. The constitutive ethylene response of CTR1-Nox was largely rescued by expression of the ETR1 N-terminus. etr $1^{1-349}$ lacks the CTR1 docking site; without the docking of CTR1 N-terminus, the receptor N-terminal signaling may be mediated by an alternative pathway (Qiu et al., 2012). 
Another line of evidence for the alternative pathway may come from studies of $c t r 1-8$. In contrast to CTR1, which associates with ethylene receptors at the ER membrane, the ctr1-8 protein (with a G354E substitution) does not associate with ethylene receptors and is detected in the cellular soluble fraction (Gao et al., 2003; Huang et al., 2003). With these features, theoretically ctr18 cannot be activated by the receptors to phosphorylate EIN2, thus resulting in constitutive ethylene responses. However, the ctr1-8 mutant has a relatively mild constitutive ethylene response phenotype as compared with $c t r 1-1$ and $c t r 1-2$ (Xie et al., 2012). Given that ctr1-8 has a wild-type kinase domain, the weak phenotype could be due to some ctr1-8 protein (below the limit of detection) still associating with the receptors to repress ethylene signaling. This scenario, however, is not supported by the result that overexpressing the N-terminus of wild-type CTR1 but not ctr1-8 increases ethylene sensitivity (Huang et al., 2003); whether the mutant ctr1-8 protein can still mediate receptor signaling is yet to be determined. Thus, the weak $\operatorname{ctr} 1-8$ mutant phenotype could indicate suppression of ethylene signaling by the receptors without involving CTR1.

There are two models for an alternative ethylene signaling pathway, based on evidence from biochemical studies and protein-protein interactions. (1) Ethylene binding to the receptors results in the dynamic dissociation of CTR1 from, and association of EIN2 with, the receptors; in this case, the ethylene receptors might directly mediate ethylene signaling to EIN2 (Bisson and Groth, 2010, 2011). (2) Ethylene binding also facilitates the dissociation of the ETR1-interacting phosphotransfer protein ARABIDOPSIS HISTIDINE-CONTAINING PHOSPHOTRANSMITTER1 (AHP1) that is phosphorylated by ETR1 prior to ethylene binding (Scharein et al., 2008; Scharein and Groth, 2011). A conjectured two-component signaling model was proposed for ethylene signaling mediated by the receptors via AHPs to type $\mathrm{B}$ response regulator proteins known as ARABIDOPSIS RESPONSE REGULATORs (ARRs; Shakeel et al.,
2013). S-nitrosylation of AHP1 Cys115 inhibits protein phosphorylation and subsequent phosphotransfer to ARR1, thereby suppressing cytokinin signaling (Feng et al., 2013). A complex formed by the two-component histidine kinase ARABIDOPSIS HISTIDINE KINASE5 (AHK5) and AHP1 is involved in a variety of biological processes (Bauer et al., 2013). Thus, AHP1 might differentially mediate signaling from various upstream histidine kinase proteins.

\section{ALTERNATIVE PATHWAY SUGGESTED BY ctr1-1 EIN2/ein2}

In a genetic screen for suppressors of $\operatorname{ctr1-1}$, we isolated $\operatorname{ctr1-1}$ EIN2/ein2, which showed a weaker constitutive ethylene response phenotype than ctr1-1 (Figure 2). That is, with ethylene treatment, seedling hypocotyl elongation of this mutant (with ein2 heterozygous) was inhibited to a lesser extent than that of ethylene-treated wild-type (Col-0) seedlings, with the ctr1-1 ein2 seedling hypocotyl elongation unresponsive to ethylene (Figure 2A). Light-grown ctr1-1 EIN2/ein2 seedlings produced a weaker growth-inhibition phenotype than $c t r 1-1$ with or without ethylene treatment, while ctr1-1 ein2 seedling growth was unresponsive to ethylene (Figure 2B). The transcript level of the ethylene-dependent ERF1 gene in the ctr1-1 EIN2/ein2 mutant was slightly higher than in the ctr1-1 ein2 double homozygote but much lower than in the ctr1-1 mutant alone, and ethylene induction of ERF1 expression was higher in ctr1-1 EIN2/ein2 than ctr1-1 ein2 but much lower than in the wild type (Figure 2C).

Sequence analysis revealed that a G1440A transition mutation caused a W308stop early termination of EIN2 in the newly isolated allele. The allele could conceivably produce a truncated EIN2 protein lacking the C-terminus for ethylene signaling and is likely a loss-of-function mutation. With a single-copy of wild-type EIN2, the EIN2 protein level in ctr1-1 EIN2/ein2 may not be sufficient to fully induce the ethylene response to the same degree as $\operatorname{ctr} 1-1$. The mutation could be recessive or partially dominant because
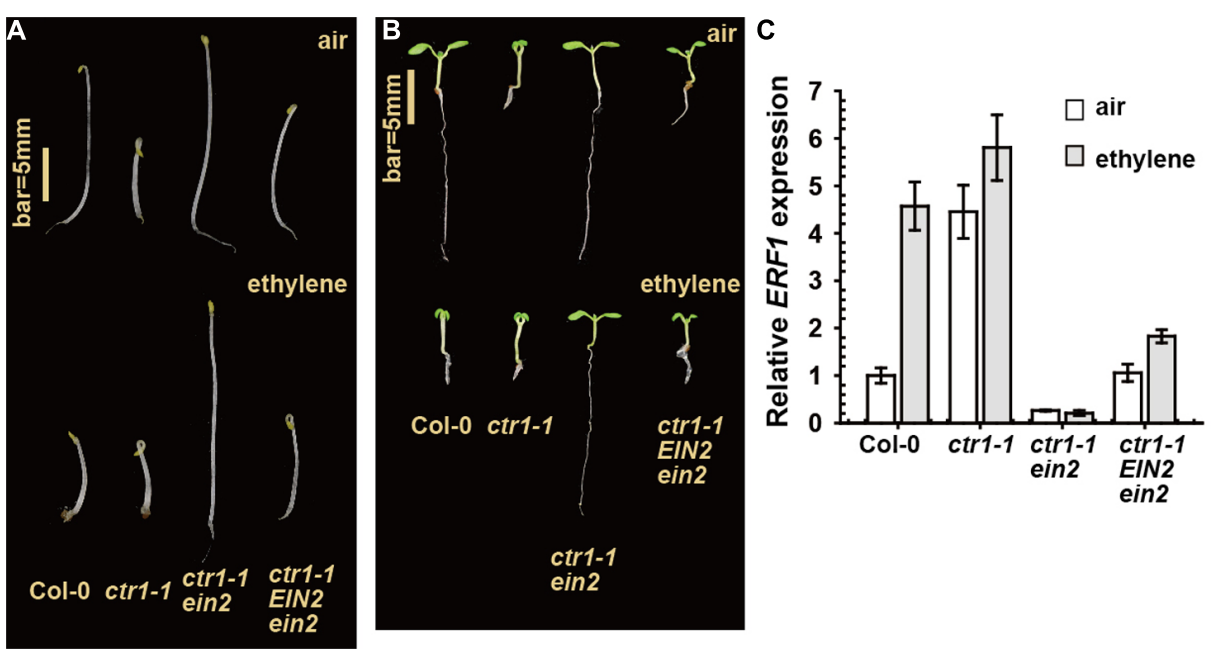

FIGURE 2 | The ethylene response phenotype of ctr1-1 EIN2/ein2. The ethylene response phenotype for etiolated (A) and light-grown (B) seedlings and ERF1 levels in rosettes (C). Data are mean \pm SE. 
the heterozygote but not homozygote was responsive to ethylene. An important finding is that the ctr1-1 EIN2/ein2 mutant appears to be slightly responsive to ethylene (Figure 2). This slight responsiveness to ethylene could reflect a pathway that does not involve CTR1. Without an alternative pathway to activate the wild-type copy of EIN2, the ethylene response would not be expected.

We therefore propose that ethylene binding could prevent the receptor $\mathrm{N}$-terminal signaling so that ethylene signaling is relieved from suppression. Alternatively, ethylene signaling could be mediated by the receptors via AHPs to ARRs or directly to EIN2 (Bisson and Groth, 2011; Scharein and Groth, 2011; Shakeel et al., 2013).

\section{SIGNIFICANCE OF THE ALTERNATIVE PATHWAY IN ETHYLENE SIGNALING}

Gene function can be partially inferred from the phenotype of loss-of-function mutants, and the effects conferred by different lesions of a gene may differ. The role of the alternative pathway in ethylene signaling could be minor given that ctr1-1 and ctr13 are slightly responsive to ethylene (Larsen and Chang, 2001) or the mutant ctr1-1 protein retains a trace of kinase activity (Huang etal., 2003). In contrast, the constitutive ethylene response phenotype is stronger in the ethylene-receptor quintuple mutant than in the ctr1-1 mutant (Liu et al., 2010). The findings that ctr1-8, ETR1p:etr1 $1^{1-349}$ ctr1-2, and ctr1-1 EIN2/ein2 have a much weaker constitutive ethylene response phenotype than $\operatorname{ctr} 1-1 /$ ctr1-2 suggest that the role of the alternative receptorsignaling pathway seems to be more pronounced than previously thought.

We explain these two conflicting interpretations for significance of the alternative pathway as follows. The inhibition of receptor signaling by the full-length receptors but not the truncated ETR1 N-terminus with expression of excess CTR1 N-terminus (Huang et al., 2003; Qiu et al., 2012) implies that the docking of a kinase-defective CTR1 protein at the receptors could inhibit the receptor $\mathrm{N}$-terminal signaling to the alternative pathway (Xie et al., 2012). Conceivably, ctr1-1 and ctr1-2 could dock at the receptor C-terminus to inhibit the $\mathrm{N}$-terminal signaling. In $\operatorname{ctr} 1-8$, the receptor $\mathrm{N}$-terminal signaling to the alternative pathway is not inhibited, and the mutant produces a weak phenotype while being fully responsive to ethylene treatment that inhibits the N-terminal signaling. ETR1 and CTR1 may dissociate, with the Kd for ETR1CTR1 interaction to be determined; the N-terminal signaling may not be fully inhibited by kinase-defective CTR1 proteins so that the constitutive ethylene response phenotype is weaker in ctr11 and ctr1-2 than the quintuple mutant, with ctr1-1 EIN2/ein2 responsive to ethylene.

Whether the truncated ctr1-2 protein could dock at the receptors and ctr1-8 could mediate receptor signaling are to be demonstrated. More lines of experimental evidence are required to reveal the significance of the alternative ethylene signaling via the receptors to EIN2 or AHP1.

\section{DYNAMIC ETHYLENE RECEPTOR SIGNALING VIA THE C- AND N-TERMINI}

A proposed model for the dynamic receptor ethylene signaling via the two termini is described in Figure 1. The model explains the following scenarios. (1) In the receptor quintuple mutants, the default "ethylene signaling" mediated by EIN2 is not affected because of the absence of the receptor $\mathrm{C}$ - and $\mathrm{N}$ terminal signaling, and the mutant shows an extremely strong constitutive ethylene response phenotype (Figure 1B). (2) ctr18 does not dock at the receptors, and the N-terminal signaling is not inhibited; although the EIN2 C-terminus is theoretically released, ethylene signaling is nevertheless inhibited by the receptor N-terminal signaling, so that $\operatorname{ctr} 1-8$ shows a relatively weak constitutive ethylene response phenotype (Figure 1C). (3) In mutants defective in CTR1 kinase activity (i.e., ctr1-1 and ctr1-2), the mutant CTR1 proteins dock at the receptors to inhibit but not fully prevent receptor N-terminal signaling, whereas the EIN2 C-terminus is released for ethylene signaling (Figure 1D). Thus, the ctrl mutants produce a typical ethylene response phenotype that is weaker than the receptor quintuple mutant phenotype. (4) In ctr1-1 EIN2/ein2, receptor N-terminal signaling is inhibited; however, it is still sufficient to repress in part the ethylene signaling that is mediated by the EIN2 C-terminus, which exists at a reduced amount. Thus, the heterozygote shows a weaker phenotype than ctr1-1. Ethylene treatment inhibits the receptor $\mathrm{N}$-terminal signaling, and the mutant is ethylene responsive.

\section{CONCLUSION AND REMARKS}

Little is known about the components involved in the possible alternative pathway of ethylene perceived by ethylene receptors and mediation of receptor $\mathrm{N}$-terminal signaling; mutants isolated from an ongoing suppressor screen in our laboratory for ETR1p:etr1-1 $1^{1-349}$ ctr1-1 could potentially isolate the involved components. The isolation of several ENHANCED ETHYLENE RESPONSE (EER) genes suggests a resetting mechanism damping ethylene signaling by components involved in various biological processes (Larsen and Chang, 2001; Robles et al., 2007; Christians et al., 2008; Deslauriers and Larsen, 2010; Lu et al., 2010). REVERSION-TO-ETHYLENE SENSITIVITY1 (RTE1) is an ER- and Golgi-associated protein facilitating ETR1 receptor Nterminal signaling (Resnick et al., 2006; Zhou et al., 2007; Gallie, 2012; Qiu et al., 2012), with its functions involving cytochrome b (Chang et al., 2014). The RTE1-cytrochrome b interaction may have a role in the alternative pathway. Unlike the ETR1 N-terminal signaling, the conjectured two-component signaling via AHPs and ARRs (Scharein and Groth, 2011; Shakeel et al., 2013) is presumably mediated via the receptor C-terminal histidine kinase domain. With a long half-life for ethylene binding to the receptors, a desensitizing mechanism is expectedly required for sustained AHP1 phosphorylation and dissociation from ETR1 and for EIN2 recruitment for ethylene signaling. The possibility for regulating ethylene signaling by multiple pathways or components is therefore likely.

Ethylene receptor signaling via the $\mathrm{C}$ - and $\mathrm{N}$-termini could act independently, with distinct signaling components and targets; alternatively, the signaling could be mediated to different components, converged at the same signaling component, and sharing a common downstream pathway. EIN2 might mediate ethylene signaling via the receptor N-terminus because ETR1 and EIN2 physically interact and a single copy of the ein $2^{G 1440 A}$ allele 
partially suppressed ctr1-1; evidence for an interaction of ETR1 Nterminus with EIN2 is required to strengthen this scenario. EIN3 and EIL1 are the prime transcription factors responsible for inducing an array of ethylene response genes (Chao et al., 1997; An et al., 2010; Chang et al., 2013). The two pathways could converge and share a common signaling pathway that involves EIN3 and EIL1.

The signaling components may be dynamically present in a pathway for an immediate response. EIN2 could be present in a steady-state interconversion between the phosphorylated and underphosphorylated state in the absence of ethylene treatment; alternatively, a small portion, but not most, of EIN2 could escape the phosphorylation by CTR1 to ensure immediate ethylene signaling. Conceivably, a level of constitutive ethylene signaling could occur, if not repressed, to trigger stronger ethylene signaling. Alternative receptor signaling could have a role in minimizing degrees of the basal-level ethylene signaling. The two-level control may facilitate a dynamic fine-tuning of ethylene signaling in response to a wide range of ethylene concentrations.

\section{AUTHOR CONTRIBUTIONS}

Jing $\mathrm{Yu}$ isolated the heterozygous EIN2/ein2 ctr1-1 mutant, Jingyi Zhang characterized the ethylene response, and Chi-Kuang Wen wrote the paper.

\section{ACKNOWLEDGMENTS}

This work was supported by the Chinese Ministry of Science and Technology (2011CB100700 and 2012AA10A302-2) to Chi-Kuang Wen.

\section{REFERENCES}

An, F., Zhao, Q., Ji, Y., Li, W., Jiang, Z., Yu, X., et al. (2010). Ethyleneinduced stabilization of ETHYLENE INSENSITIVE3 and EIN3-LIKE1 Is mediated by proteasomal degradation of EIN3 binding F-Box 1 and 2 that requires EIN2 in Arabidopsis. Plant Cell 22, 2384-2401. doi: 10.1105/tpc.110. 076588

Bauer, J., Reiss, K., Veerabagu, M., Heunemann, M., Harter, K., and Stehle, T. (2013). Structure-function analysis of Arabidopsis thaliana histidine kinase AHK5 bound to its cognate phosphotransfer protein AHP1. Mol. Plant 6, 959-970. doi: $10.1093 / \mathrm{mp} / \mathrm{sss} 126$

Bisson, M. M. A., and Groth, G. (2010). New insight in ethylene signaling: autokinase activity of ETR1 modulates the interaction of receptors and EIN2. Mol. Plant 3, 882-889. doi: $10.1093 / \mathrm{mp} / \mathrm{ssq} 036$

Bisson, M. M. A., and Groth, G. (2011). New paradigm in ethylene signaling: EIN2, the central regulator of the signaling pathway, interacts directly with the upstream receptors. Plant Signal. Behav. 6, 164-166. doi: 10.4161/psb.6. 1.14034

Chang, J., Clay, J. M., and Chang, C. (2014). Association of cytochrome b5 with ETR1 ethylene receptor signaling through RTE1 in Arabidopsis. Plant J. 77, 558-567. doi: $10.1111 /$ tpj. 12401

Chang, K. N., Zhong, S., Weirauch, M. T., Hon, G., Pelizzola, M., Li, H., et al. (2013). Temporal transcriptional response to ethylene gas drives growth hormone cross-regulation in Arabidopsis. Elife 2, e00675. doi: 10.7554/eLife. 00675

Chao, Q., Rothenberg, M., Solano, R., Roman, G., Terzaghi, W., and Ecker, J. (1997). Activation of the ethylene gas response pathway in Arabidopsis by the nuclear protein ETHYLENE-INSENSITIVE3 and related proteins. Cell 89, 1133-1144. doi: 10.1016/S0092-8674(00)80300-1

Christians, M. J., Robles, L. M., Zeller, S. M., and Larsen, P. B. (2008). The eer5 mutation, which affects a novel proteasome-related subunit, indicates a prominent role for the COP9 signalosome in resetting the ethylene-signaling pathway in Arabidopsis. Plant J. 55, 467-477. doi: 10.1111/j.1365-313X.2008. 03521.x
Clark, K. L., Larsen, P. B., Wang, X., and Chang, C. (1998). Association of the Arabidopsis CTR1 Raf-like kinase with the ETR1 and ERS ethylene receptors. Proc. Natl. Acad. Sci. U.S.A. 95, 5401-5406. doi: 10.1073/pnas.95. 9.5401

Deslauriers, S. D., and Larsen, P. B. (2010). FERONIA is a key modulator of brassinosteroid and ethylene responsiveness in Arabidopsis hypocotyls. Mol. Plant 3, 626-640. doi: 10.1093/mp/ssq015

Feng, J., Wang, C., Chen, Q., Chen, H., Ren, B., Li, X., et al. (2013). S-nitrosylation of phosphotransfer proteins represses cytokinin signaling. Nat. Commun. 4, 1529. doi: $10.1038 /$ ncomms 2541

Gallie, D. (2012). F1000 prime recommendation of [Qiu L etal., Plant Physiol. 2012, 159(3):1263-76]. Faculty of 1000. doi: 10.3410/f.717953741.793459406. f1000.com/prime/717953741\#eval793459406

Gao, Z., Chen, Y. F., Randlett, M. D., Zhao, X. C., Findell, J. L., Kieber, J. J., et al. (2003). Localization of the raf-like kinase CTR1 to the endoplasmic reticulum of Arabidopsis through participation in ethylene receptor signaling complexes. J. Biol. Chem. 278, 34725-34732. doi: 10.1074/jbc.M305548200

Hua, J., and Meyerowitz, E. M. (1998). Ethylene responses are negatively regulated by a receptor gene family in Arabidopsis thaliana. Cell 94, 261-271. doi: 10.1016/S0092-8674(00)81425-7

Huang, Y., Li, H., Hutchison, C. E., Laskey, J., and Kieber, J. J. (2003). Biochemical and functional analysis of CTR1, a protein kinase that negatively regulates ethylene signaling in Arabidopsis. Plant J. 33, 221-233. doi: 10.1046/j.1365-313X.2003.01620.x

Ju, C., and Chang, C. (2012). Advances in ethylene signaling: protein complexes at the endoplasmic reticulum membrane. AoB Plants 2012, pls031. doi: 10.1093/aobpla/pls031

Ju, C., Yoon, G. M., Shemansky, J. M., Lin, D. Y., Ying, Z. I., Chang, J., et al. (2012). CTR1 phosphorylates the central regulator EIN2 to control ethylene hormone signaling from the ER membrane to the nucleus in Arabidopsis. Proc. Natl. Acad. Sci. U.S.A. 109, 19486-19491. doi: 10.1073/pnas.1214848109

Larsen, P. B., and Chang, C. (2001). The Arabidopsis eer1 mutant has enhanced ethylene responses in the hypocotyl and stem. Plant Physiol. 125, 1061-1073. doi: 10.1104/pp.125.2.1061

Liu, Q., Xu, C., and Wen, C.-K. (2010). Genetic and transformation studies reveal negative regulation of ERS1 ethylene receptor signaling in Arabidopsis. BMC Plant Biol. 10:60. doi: 10.1186/1471-2229-10-60

Lu, Q., Tang, X., Tian, G., Wang, F., Liu, K., Nguyen, V., et al. (2010). Arabidopsis homolog of the yeast TREX-2 mRNA export complex: components and anchoring nucleoporin. Plant J. 61, 259-270. doi: 10.1111/j.1365-313X.2009. 04048.x

Qiao, H., Shen, Z., Huang, S.-S. C., Schmitz, R. J., Urich, M. A., Briggs, S. P., et al. (2012). Processing and subcellular trafficking of ER-tethered EIN2 control response to ethylene Gas. Science 338, 390-393. doi: 10.1126/science. 1225974

Qiu, L., Xie, F., Yu, J., and Wen, C.-K. (2012). Arabidopsis RTE1 Is essential to ethylene receptor ETR1 amino-terminal signaling independent of CTR1. Plant Physiol. 159, 1263-1276. doi: 10.1104/pp.112.193979

Resnick, J. S., Wen, C.-K., Shockey, J. A., and Chang, C. (2006). From the cover: REVERSION-TO-ETHYLENE SENSITIVITY1, a conserved gene that regulates ethylene receptor function in Arabidopsis. Proc. Natl. Acad. Sci. U.S.A. 103, 79177922. doi: $10.1073 /$ pnas. 0602239103

Robles, L. M., Wampole, J. S., Christians, M. J., and Larsen, P. B. (2007). Arabidopsis enhanced ethylene response 4 encodes an EIN3-interacting TFIID transcription factor required for proper ethylene response, including ERF1 induction. J. Exp. Bot. 58, 2627-2639. doi: 10.1093/jxb/erm080

Scharein, B., and Groth, G. (2011). Phosphorylation alters the interaction of the Arabidopsis phosphotransfer protein AHP1 with Its sensor kinase ETR1. PLoS ONE 6:e24173. doi: 10.1371/journal.pone.0024173

Scharein, B., Voet-Van-Vormizeele, J., Harter, K., and Groth, G. (2008). Ethylene signaling: identification of a putative ETR1-AHP1 phosphorelay complex by fluorescence spectroscopy. Anal. Biochem. 377, 72-76. doi: 10.1016/j.ab.2008. 03.015

Shakeel, S. N., Wang, X., Binder, B. M., and Schaller, G. E. (2013). Mechanisms of signal transduction by ethylene: overlapping and non-overlapping signaling roles in a receptor family. AoB Plants 5, plt010. doi: 10.1093/aobpla/plt010

Solano, R., Stepanova, A., Chao, Q., and Ecker, J. (1998). Nuclear events in ethylene signaling: a transcriptional cascade mediated by ETHYLENE-INSENSITIVE3 
and ETHYLENE-RESPONSE-FACTOR1. Genes Dev. 12, 3703-3714. doi: 10.1101/gad.12.23.3703

Xie, F., Qiu, L., and Wen, C.-K. (2012). Possible modulation of Arabidopsis ETR1 N-terminal signaling by CTR1. Plant Signal. Behav. 7, 1243-1245. doi: $10.4161 /$ psb. 21545

Zhou, X., Liu, Q., Xie, F., and Wen, C.-K. (2007). RTE1 Is a golgi-associated and ETR1-dependent negative regulator of ethylene responses. Plant Physiol. 145, 75-86. doi: 10.1104/pp.107.104299

Conflict of Interest Statement: The authors declare that the research was conducted in the absence of any commercial or financial relationships that could be construed as a potential conflict of interest.
Received: 18 August 2014; accepted: 03 November 2014; published online: 20 November 2014.

Citation: Zhang J, Yu J and Wen C-K (2014) An alternate route of ethylene receptor signaling. Front. Plant Sci. 5:648. doi: 10.3389/fpls.2014.00648

This article was submitted to Plant Physiology, a section of the journal Frontiers in Plant Science.

Copyright (C) 2014 Zhang, Yu and Wen. This is an open-access article distributed under the terms of the Creative Commons Attribution License (CC BY). The use, distribution or reproduction in other forums is permitted, provided the original author(s) or licensor are credited and that the original publication in this journal is cited, in accordance with accepted academic practice. No use, distribution or reproduction is permitted which does not comply with these terms. 


\title{
Novel connections and gaps in ethylene signaling from the ER membrane to the nucleus
}

\author{
Young-Hee Cho and Sang-Dong Yoo* \\ Division of Life Sciences, College of Life Sciences and Biotechnology, Korea University, Seoul, South Korea
}

Edited by:

Tomotsugu Koyama, Suntory

Foundation for Life Sciences, Japan

Reviewed by:

Caren Chang, University of

Maryland, USA

Hongwei Guo, Peking University,

China

*Correspondence:

Sang-Dong Yoo, Division of Life

Sciences, College of Life Sciences

and Biotechnology, Korea

University, 145 Anamro,

Sungbuk-gu, Seoul 136-713, South

Korea

e-mail: sangdong@korea.ac.kr
The signaling of the plant hormone ethylene has been studied genetically, resulting in the identification of signaling components from membrane receptors to nuclear effectors. Among constituents of the hormone signaling pathway, functional links involving a putative mitogen-activated protein kinase kinase CONSTITUTIVE TRIPLE RESPONSE1 (CTR1) and a membrane transporter-like protein ETHYLENE INSENSITIVE2 (EIN2) have been missing for a long time. We now learn that EIN2 is cleaved and its $\mathrm{C}$-terminal end moves to the nucleus upon ethylene perception at the membrane receptors, and then the C-terminal end of EIN2 in the nucleus supports EIN3-dependent ethylene-response gene expression. CTR1 kinase activity negatively controls the EIN2 cleavage process through direct phosphorylation. Despite the novel connection of CTR1 with EIN2 that explains a large portion of the missing links in ethylene signaling, our understanding still remains far from its completion. This focused review will summarize recent advances in the EIN3-dependent ethylene signaling mechanisms including CTR1-EIN2 functions with respect to EIN3 regulation and ethylene responses. This will also present several emerging issues that need to be addressed for the comprehensive understanding of signaling pathways of the invaluable plant hormone ethylene.

Keywords: ethylene, signaling, MAPkinasekinaseKinase, CTR1, EIN2, EIN3

\section{INTRODUCTION}

Ethylene is a small volatile hydrocarbon gas and mediates diverse physiological responses in plant cells. The plant hormone is synthesized by a simple two-step biochemical pathway involving conversion of S-adenosyl-L-methionine (SAM) to 1aminocyclopropane-1-carboxylic acid (ACC) and then to ethylene, which occurs in all higher plants (Dorling and McManus, 2012; Harpaz-Saad et al., 2012). Ethylene regulates a wide variety of physiological responses throughout the life of various plants. This covers physiological regulations from seed dormancy release and germination, seedling growth, vegetative organ growth and shaping, reproductive organ growth and sex determination, fruit ripening, organ senescence, and abscission to plant-microbe interactions (McManus, 2012). This diverse ethylene physiology results from the fine-tuning of ethylene production and signaling that are under the control of complex interactions among ethylene and other signaling pathways. Therefore, ethylene signaling functions and mechanisms need to be understood at the higher order of complexity integrating other signaling pathways.

Ethylene is the first plant hormone, for which signaling pathway has been elucidated with mainly Arabidopsis genetics (Bleecker et al., 1988; Guzman and Ecker, 1990). ETHYLENE INSENSITIVE3 (EIN3) and EIN3-LIKE1 (EIL1) are the key transcription factors for ethylene immediate early gene expression (Figure 1). Protein stability regulation plays the major controlling step in the modulation of the transcription factors. EIN2, an NRAMP-like integral membrane protein located at the endoplas- mic reticulum (ER), is another necessary genetic component for EIN3-dependent ethylene signaling. Recently three independent research groups (Ju et al., 2012; Qiao et al., 2012; Wen et al., 2012) have reported novel observations that EIN2 is cleaved and its processed C-terminal product (EIN2C) is translocated to the nucleus in response to ethylene. Despite the EIN2 translocation that correlates well with ethylene responses, the protease involved in EIN2 cleavage and a mechanistic function of EIN2C in EIN3dependent gene expression have not been elucidated in these studies. Furthermore, it has yet to be examined whether or not any additional biochemical modification is required for EIN2C to be processed and translocated to the nucleus for ethylene signaling.

This focused review summarizes the new discoveries of EIN2EIN3 process in the ethylene signaling and raises specific questions that need to be investigated for comprehensive understanding of ethylene signaling. Readers are advised to consider many excellent reviews to cover other exciting discoveries including functional modes of ethylene receptors (Hall et al., 2007; Cho and Yoo, 2009; Yoo et al., 2009; Lacey and Binder, 2014). Any newly identified EIN2-EIN3 regulatory processes will extend our understanding of integrated physiological responses of ethylene and other hormones that are involved in model and crop plant growth and development.

\section{ETHYLENE SIGNALING}

In Arabidopsis, ethylene is binding to membrane proteins that are composed of five partially redundant receptors; ETHYLENE 


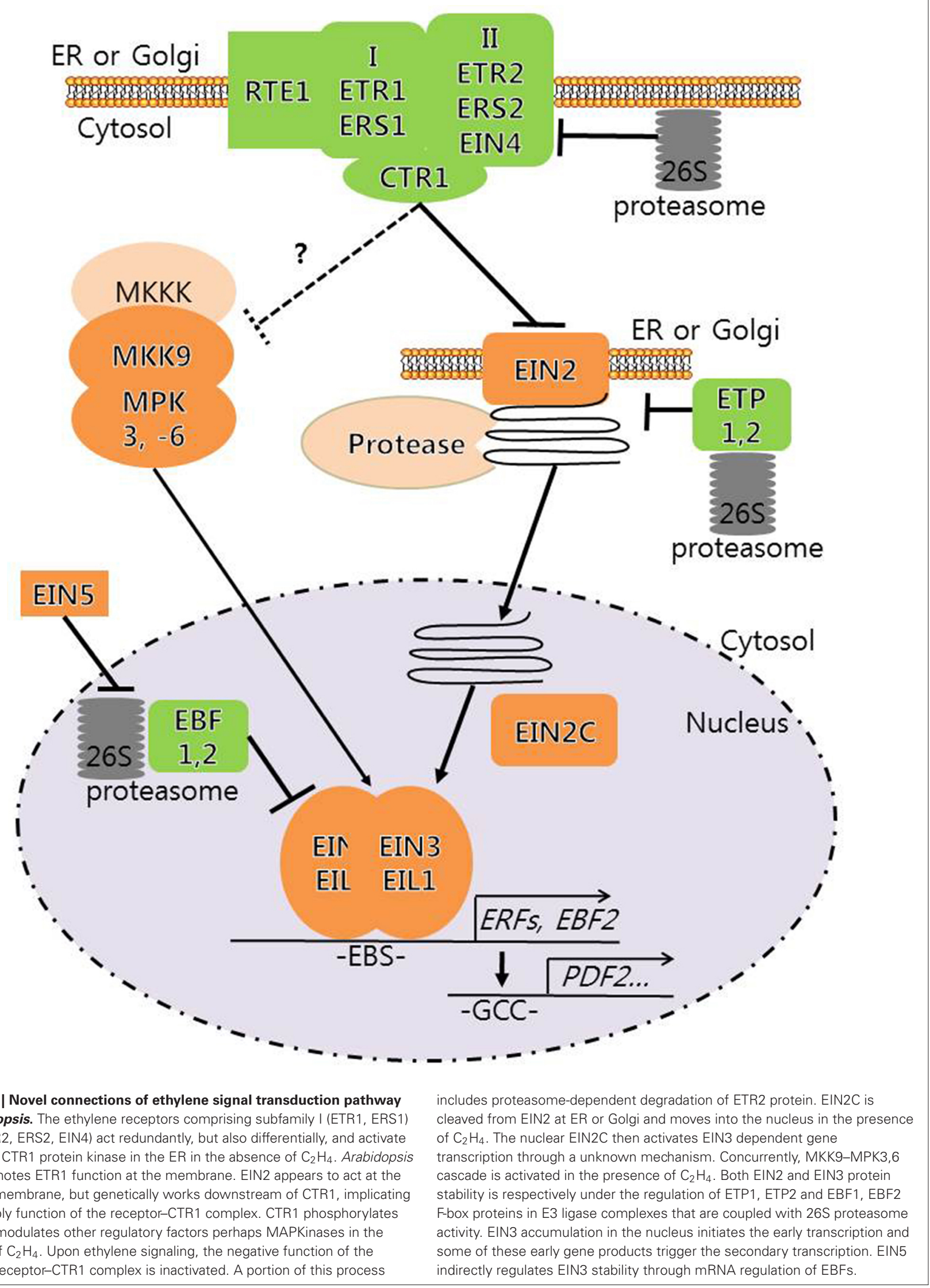


RESPONSE1 (ETR1), ETR2, ETHYLENE RESPONSE SENSOR1 (ERS1), ERS2, and EIN4. The receptors differentially express and act in different tissues and at distinct developmental stages (Bleecker et al., 1988; Chang et al., 1993; Hua et al., 1995, 1998; Hua and Meyerowitz, 1998; Sakai et al., 1998). In the absence of ethylene, ETR1 and other receptors have inverse agonistic roles in the hormone signaling and suppress ethylene responses (Hua and Meyerowitz, 1998; Wang et al., 2006). This negative action of the ethylene-free receptors is linked to another genetically identified regulator, CONSTITUTIVE TRIPLE RESPONSE1 (CTR1), which encodes a putative Raf-like mitogen-activated protein kinase kinase kinase (MAPKKK; Kieber et al., 1993; Clark et al., 1998; Huang et al., 2003). REVERSION-TO-ETHYLENE SENSITIVITY1 (RTE1) directly interacts with ETR1 in the ER membrane and Golgi-apparatus (Resnick et al., 2006; Zhou et al., 2007; Dong et al., 2008, 2010), perhaps for $\mathrm{Cu}^{+}$-dependent ethylene perception of the membrane receptors.

A membrane-integrated metal transporter-like protein EIN2 has been identified as a necessary component in the downstream of ethylene receptor signaling (Alonso et al., 1999). In the absence of ethylene, EIN2 is degraded by 26S proteasome activity under the action of two F-box proteins in E3 ubiquitin ligase complexes, EIN2 TARGETING F-BOX PROTEIN1 (ETP1) and ETP2 (Qiao et al., 2009). In the presence of ethylene, a part of EIN2 accumulates in plant nuclei (Ju et al., 2012; Qiao et al., 2012; Wen et al., 2012), which correlates well with the accumulation of EIN3 and EIL1 in the nucleus. Likewise, EIN3 is constantly degraded in the absence of ethylene, a process which is under the control of two F-box proteins in E3 ubiquitin ligase complexes, EIN3 BINDING F-BOX PROTEIN1 (EBF1) and EBF2 (Guo and Ecker, 2003; Potuschak et al., 2003; Yanagisawa et al., 2003; Gagne et al., 2004). The accumulation of EIN3 and EIL1 in the nucleus triggers primary transcription through EIN3-binding sites in the promoters of target genes such as ETHYLENE RESPONSE FACTOR1 (ERF1) and EBF2 (Solano et al., 1998; Alonso et al., 2003; Konishi and Yanagisawa, 2008). ERF1 then itself serves as a transcriptional activator by specifically recognizing and binding to a GCC element in the promoters of ethylene secondary responsive genes (Ohme-Takagi and Shinshi, 1995; Solano et al., 1998). All the gene products of these transcription cascades lead to cellular and biochemical changes that execute the physiological and developmental adaptation of plants in response to ethylene.

Ethylene signaling feeds back to dampen ethylene responses once executed. ERS1, ETR2, and EBF2 transcript abundance increases in response to ethylene (Hua et al., 1995; Sakai et al., 1998). These newly synthesized negative regulators in ethylene signaling may diminish and/or reset ethylene responses in the tissues that initially transduce the hormone signal.

Ethylene signaling induces gene expression and brings up the overall physiological changes of plants eventually to adapt to various biotic and abiotic stresses that trigger ethylene synthesis and signaling. For the rapid intracellular signaling, cellular protein kinases and nuclear transcription factors need to be connected instantly and dynamically. A newly identified molecular mechanism of EIN2 is the key signaling process of the ethylene immediate response and is the main point of discussion in the following section.

\section{EIN2 REGULATION IN ETHYLENE SIGNALING}

EIN2 encodes a membrane protein with 1294 amino acids that has a hydrophobic domain at the N-terminal end (480 amino acids), containing $21 \%$ sequence identity to NRAMP metal ion transporter proteins (Alonso et al., 1999). Even so, no transporter activity has been seen with EIN2 in ethylene signaling. The EIN2 C-terminal half (840 amino acids) partially complemented the light-dependent hypocotyl response to ethylene (Smalle et al., 1997), but failed to restore the triple response that was originally used for identifying ein2 (Alonso et al., 1999). The ein2 mutant was also insensitive to paraquat and jasmonate (Alonso et al., 1999), but hypersensitive to ABA (Beaudoin et al., 2000; Ghassemian et al., 2000), indicating either that responses to these other signals may require EIN2-dependent ethylene signaling pathway and/or that EIN2 has multiple functions in the stress signaling responses.

The strong ethylene insensitivity of ein 2 correlates well with the diminished EIN3 protein levels in the mutant (Guo and Ecker, 2003; Wen et al., 2012). Thus, an ER membrane protein EIN2 appears to convey ethylene signaling by stabilizing the nuclear protein EIN3 through an unidentified mechanism. It has been proposed that EIN2C enters the nucleus and binds to EBF1 and EBF2 to inactivate and then stabilizes EIN3.

EIN2 has a short half-life of $30 \mathrm{~min}$ or less because of its degradation by the $26 \mathrm{~S}$ proteasome activity coupled with ETP1 and ETP2 functions in E3 ligase complexes (Qiao et al., 2009). These F-box proteins interact with the C-terminal EIN2 ${ }^{1047-1294}$ and cause degradation of EIN2 in the absence of ethylene. On the other hand, ethylene can stabilize EIN2 by diminishing ETP1 and ETP2 activity with an unknown mechanism. Null EIN2-targeting E3 ligase activity in the etp1 etp2 double mutant causes EIN2 accumulation and leads to constitutive ethylene responses. This finding implies that EIN2 protein accumulation is necessary and sufficient for EIN3 protein accumulation and downstream ethylene responses in plant cells. However, this view has to be examined carefully with respect to the new discovery of EIN2 cleavage that is necessary for EIN2 function in ethylene signaling (Ju et al., 2012; Qiao et al., 2012; Wen et al., 2012).

Qiao et al. (2012) has reported that EIN2C, with its intrinsic nuclear localization signal (NLS), is cleaved from EIN2 and moves into the nucleus within $10 \mathrm{~min}$ after ethylene application. Since NLS-less EIN2 is unable to complement the loss of function ein2 mutant phenotype in the light, EIN2C needs to be present in the nucleus to mediate ethylene signaling. EIN2 movement to the nucleus is faster than EIN2 stabilization, which mostly happens 1 to $4 \mathrm{~h}$ after ethylene application (Qiao et al., 2009). Protein phospho-modification analysis has revealed that $\mathrm{Ser}^{645}$ of EIN2 $\left(\mathrm{EIN} 2^{\mathrm{S} 645}\right)$ that is the experimentally determined amino acid residue as a cleavage site of EIN2 is the main phosphorylation and dephosphorylation site in the absence and presence of ethylene, respectively (Qiao et al., 2012). Complementation of ein2-5 with EIN2 ${ }^{\text {S645A }}$ (Ser to Ala) preventing phosphorylation at this residue results in induction of the EIN2C cleavage and translocation to the nucleus, and constitutive ethylene responses in the absence of ethylene. This implicates that EIN2 ${ }^{\mathrm{S} 645}$ is phosphorylated and its phosphorylation prevents the protein cleavage and translocation 
to the nucleus to suppress the hormone signaling in the absence of ethylene.

Upon ethylene treatment, two different patterns of EIN2 protein accumulation were reported (Qiao et al., 2009; Ju et al., 2012). EIN2 protein levels were increased in total proteins (Qiao et al., 2009), but decreased in microsomal fractions (Ju et al., 2012). Since EIN2C movement to the nucleus plays a key role in ethylene signaling responses, observation of ethylene-dependent EIN2 protein reduction at microsomal fraction supports the signaling process. However, EIN2 accumulation in response to ethylene is a little difficult to connect to the functional mode of EIN2.

CTR1 is identified as the protein kinase responsible for phosphorylating EIN2 on several conserved residues (Ju et al., 2012). Null mutation of CTR1 displays a constitutive ethylene response and thus CTR1 acts as a negative regulator in ethylene signaling. Although CTR1 has protein domains similar to MAPKKKs, its downstream targets MAPKK and MAPK have never been identified in any plant species. Ju et al. (2012) demonstrated that CTR1 phosphorylates EIN2 at six amino acids, including S645, in vitro. In another report, CTR1 phosphorylates four amino acids in EIN2 (Chen et al., 2011) and the phosphorylation of two amino acid sites appears important in the ethylene signaling context. More specifically, ein2 plants expressing either EIN2 $2^{S 645 A}$ or EIN2 $2^{S 924 A}$ conferred ethylene responses in the absence of ethylene. The lack of EIN2 phosphorylation at S645 or S645S924 was accompanied with EIN2C translocation to the nucleus and constitutive ethylene responses. It still remains to be examined whether the lack of EIN2 phosphorylation at S924 also brings about EIN2C translocation in a manner similar to the other variants. The functional complementation assays indicate that S645 and S924 phosphorylations are relevant to ethylene signaling suppression.

Recently, the discrepancy between the cleavage site of EIN2C reported by Qiao et al. (2012) and by Ju et al. (2012) was questioned (Cooper, 2013; Qiao et al., 2013). Furthermore, genetic complementation of ein 2 with $E I N 2^{S 645 A}$ results in a relatively weak ethylene response compared to $E I N 2^{S 924 A}$, which implicates that EIN2S645 phosphorylation perhaps plays minor role in the hormone signaling. The clear protein accumulation pattern, precise cleavage site and major phosphorylation sites of EIN2 that are responsible for ethylene intracellular signaling are still in debates and need to be resolved.

Is EIN2C in the nucleus enough for ethylene signaling responses? Wen et al. (2012) have constructed a chimerical gene of a glucocorticoid receptor-fused to C-terminal half of EIN2 and demonstrated transgenic plants expressing the Dex-inducible EIN2C displayed ethylene-induced Arabidopsis rosette growth inhibition and also its hypocotyl growth promotion as Dexinducible EIN2 caused EIN3 protein accumulation. Although no triple response assay was reported, EIN2C expression was shown to be enough to confer ethylene response in the light.

These recent studies have provided mechanistic evidence supporting the necessity of EIN2 in ethylene signaling, but how EIN2C in the nucleus modulates EIN3 function to drive the downstream physiological responses of ethylene still remains unknown. Since EIN3 fails to accumulate in ein2 (Guo and Ecker, 2003), EIN2C most likely controls EIN3 protein stability directly or indirectly. Another important question is whether nuclear localized EIN2C is entirely sufficient for driving EIN3-dependent ethylene-response gene expression. EIN2C-complemented transgenic ein2 lines have hitherto never been able to complement the triple response. Thus, ethylene signaling appears to require additional processes for full execution of the hormone signaling such as other CTR1-dependent and/or -independent pathways.

EIN2 interacts directly with ETR1 in the ER membrane (Bisson et al., 2009; Bisson and Groth, 2010). Fluorescence resonance energy transfer (FRET) and intrinsic tryptophan fluorescence quenching for protein interaction assays showed that ETR1 autophosphorylation is required for ETR1 and EIN2 interaction. Upon ethylene perception, ETR1 appears to be dephosphorylated to bind more efficiently to the C-terminal end of EIN2. However, only little changes of $\mathrm{K}_{d}$ of ETR1 and EIN2 with a very high affinity at a nanomolar scale make it difficult to substantiate its involvement in ethylene signaling. Then, the obvious question would be how to fit these interaction dynamics of ETR1 and EIN2 with ethylene-inducible EIN2C cleavage and translocation processes. Since nuclear localization of EIN2C is pivotal in ethylene signaling response, ETR1-EIN2 interaction needs to be further examined to secure its significance in ethylene signaling.

\section{EIN3 REGULATION IN ETHYLENE SIGNALING}

The ethylene insensitivity of ein 3 is less severe than that of etr 1 and ein2 (Chao et al., 1997). This is apparently due to its functional redundancy with EIN3-LIKE1, and thus ein3 eil1 double mutants can block most ethylene responses as like ein2 (Alonso et al., 2003; Binder et al., 2004).

EIN3 transcription factor protein accumulates in the nucleus in the presence of ethylene (Yanagisawa et al., 2003). In the absence of ethylene, EIN3 is negatively regulated and constantly degraded in plant cells (Guo and Ecker, 2003, 2004; Potuschak et al., 2003; Gagne et al., 2004). Such EIN3 protein degradation is controlled by $26 \mathrm{~S}$ proteasomal activity under the regulation of two redundant F-box proteins $\mathrm{EBF} 1$ and $\mathrm{EBF} 2$. In the process, both F-box proteins bind EIN3 directly in yeast and in vitro systems (Solano et al., 1998; Guo and Ecker, 2003; Potuschak et al., 2003). In the ebf1 ebf2 double mutant, EIN3 and EIL1 accumulate in the absence of ethylene and cause a seedling-arrestment phenotype (Gagne et al., 2004; Binder et al., 2007). Normal seedling growth is, however, restored in the quadruple ein 3 eill ebf1 ebf2 mutant indicating that EBF1 and EBF2 act more or less specifically to control EIN3 and EIL1 protein stability (Binder et al., 2007).

Apart from common functions of EBF1 and EBF2 on EIN3 and EIL1 degradation, each F-box protein has also a unique role in ethylene signaling. Individual ebf1 and ebf2 mutants show differential growth responses to ethylene (Binder et al., 2007) and ctr1 ebf1 and ctr1 ebf2 display obviously distinct phenotypes.

Although many studies have indicated that the control of EIN3 protein stability is a key regulatory process in ethylene signaling, how ethylene signaling modulates EIN3 stability in the nucleus has not been clearly elucidated. Shi et al. (2012) found that an EIN3 dependent seedling response to cold/freezing is led by EIN2 that destabilizes EBF1, causing EIN3 accumulation in the nucleus. Ethylene signaling somehow modulates protein stability 
of EBF1 to influence EIN3 stability. This mechanism has not been demonstrated experimentally.

Once ethylene signaling is initiated, EBF2 appears to be activated transcriptionally. Unlike $E B F 1, E B F 2$ expression is induced by EIN3-dependent transcription and also by a regulatory step of mRNA stability depending on the $3^{\prime}$-untranslated region of EBF2 in the presence of ethylene (Olmedo et al., 2006; Potuschak et al., 2006; Gregory et al., 2008). The mRNA stability of EBF2 is under the indirect/direct control of a ribonuclease EIN5/EXORIBONUCLEASE4 (XNR4)/ACC INSENSITIVE1 (AIN1) activity.

EIN5 activity also controls the stability of many other RNAs including small RNAs (Olmedo et al., 2006). Therefore, it would be important to conduct further experiments to test whether or not the EIN5-dependent EBF2 regulation serves as a part of the ethylene signaling pathway.

Several studies now report that EIN3 protein levels are changed under different conditions (Lee et al., 2006; Laluk et al., 2011; Shi et al., 2012; Kim et al., 2013). In the absence of ethylene, EIN3 and EIL1 are stabilized in the light, but these proteins are degraded in the dark (Lee et al., 2006). Cold/freezing also causes EIN3 to accumulate but suppresses ethylene production, and thus the EIN3 accumulation by cold/freezing seems to be independent of ethylene signaling response (Shi et al., 2012). Furthermore, EIN3 stability regulation and plant immunity responses now point out that triple response which is one of the typical seedling responses to ethylene in the dark can be uncoupled from EIN3 accumulation in the nucleus that is a typical biochemical process in ethylene signaling. EIN3 accumulates in a null mutant botrytis induced kinase1 (bik1), but this protein accumulation does not result in a triple response (Laluk et al., 2011). Instead, bik 1 is rather insensitive to ethylene. In another case, the protein level of EIN3 is down-regulated in the ectopic expression of GDSL lipase1 (GLIP1; Kim et al., 2013). However, the seedlings display hypersensitivity to ethylene instead of hyposensitivity. In both cases BIK1 and GLIP1 act downstream of EIN3 but upstream of the triple response so that these mutants are interfered in EIN3 dependent responses (Liu et al., 2013; Kim et al., 2014). All these studies simply demonstrate the complexity of ethylene signaling with respect to a seemingly simple phenotypic response.

\section{EIN3-DEPENDENT ETHYLENE RESPONSIVE GENE EXPRESSION}

Nuclear EIN3 activates primary ethylene-dependent transcription through binding to the cis-element AYGWAYCT within promoters of ethylene early response genes (Yamasaki et al., 2005). The DNA binding activity of EIN3, and its close homolog EIL1, often results in transcriptional activation of target genes such as ERF1, AtERFs, $E R S 1$, and EBF2 as a primary response of ethylene signaling. EIN3 also binds to the promoter regions of PHYTOCHROME INTERACTION FACTOR1 (PIF1) and PIF3 directly to activate their transcription and causes seedling greening and hypocotyl growth under lights, respectively (Zhong et al., 2012a,b).

In contrast to the well characterized transcription activator role of EIN3/EIL1, recent studies on EIN3 function have proposed transcriptional repression functions through direct binding to target gene promoters as well. EIN3 binds to the $5^{\prime}$-flanking region of SA INDUCTION DEFICIENT2 (SID2) involved in salicylic acid (SA) biosynthesis (Chen et al., 2009) to negatively modulate target gene expression. Consequently, EIN3 activation compromises SAdependent defense and causes systemic vulnerability to bacterial pathogens. Similarly, EIN3 is reported to bind to the promoter of CRT/DRE BINDING FACTOR3 (CBF3), which is a key transcription factor in cold/freezing resistance response, and suppresses its transcription (Shi et al., 2012). Thus, ethylene sensitivity seems to compromise $\mathrm{CBF} 3$ gene expression and cold/freezing tolerance. Such direct repressor function of EIN3 on gene expression is still rare when compared to its activator function, and needs to be further characterized more thoroughly.

Zhu et al. (2011) have investigated the interaction of jasmonate and ethylene signaling in plant defense and revealed a link between these two hormones and further investigated a molecular basis of repressor function of EIN3 in gene expression. JASMONATE ZIM DOMAIN (JAZ) transcription factors interact directly with EIN3 and suppress EIN3 transcription activity. The JAZ proteins do so in part by recruiting a histone deacetylase (HDAC6) repressor component. HDAC6 obstructs EIN3 from binding to its targeted promoters by removing acetyl groups from the histones at target chromatins. In summary, EIN3 recruits a transcriptional repressor complex to a target gene and suppresses gene expression. In the presence of both jasmonate and ethylene, JAZ destabilizes and EIN3 accumulates in the nucleus, and eventually activates target genes cooperatively. Even so, this mechanism is not enough to explain how EIN3 can act as an activator for some gene transcriptions, but as a repressor for others.

\section{GAPS IN OUR KNOWLEDGE OF ETHYLENE INTRACELLULAR SIGNALING}

Ethylene is a key signaling molecule mediating physiological events underlying plant growth and development. Multifaceted functions of ethylene ensure developmental plasticity of plants in response to diverse environmental stress conditions. Our understanding of ethylene signaling is advanced by recent functional characterization of CTR1 phosphorylation of EIN2 and its phosphorylation status-dependent movement into the nucleus that controls EIN3 stability mediating ethylene signaling (Figure 1). Discovery of CTR1 and EIN2 connection does provide an epic moment in the field of ethylene signaling research. However, several issues and questions still remain unresolved for comprehensive understanding of ethylene signaling, including (1) which phosphorylation sites and what sizes of EIN2C are truly involved in ethylene signaling since discrepancy has been documented in the literature, (2) which protease functions in EIN2 cleavage, (3) how EIN2C manipulates EIN3 protein stability, (4) whether CTR1 is the only protein kinase that is involved in EIN2 phosphorylation, and (5) whether CTR1-dependent phosphorylation of EIN2 is sufficient for ethylene signaling. For example, a search for MAPKKs and MPKs downstream of the MAPKKK CTR1 could identify additional CTR1 substrates. MAPK cascades of MKK9-MPK3 and MPK6 also involve in EIN3 phosphorylation and its stability regulation (Yoo et al., 2008). As EIN2C cannot fully complement EIN2 functions, such a parallel pathway may exist in ethylene signaling. Taken together, our understanding of 
the pathways and processes of ethylene signaling is far from its completion at this stage.

Since ethylene mediates so many physiological traits important for plant-based biomass productivity and its genetic constituents in signaling pathways are largely conserved in diverse plant genomes, detailed understanding of ethylene signaling in the model plant Arabidopsis will provide invaluable information to screen and characterize regulatory chemicals to specifically manipulate ethylene signaling in crops and certainly be able to manipulate ethylene-dependent physiology for practical purposes.

\section{ACKNOWLEDGMENTS}

We appreciate kind suggestions on the manuscript from Dr. Donald A. Hunter (New Zealand Institute of Plant \& Food Research Limited). This work was supported by grants from the Korean National Research Foundation (NRF-2012R1A2A2A01005933) to Sang-Dong Yoo, and by grants from the Korean National Research Foundation (NRF-2013R1A1A2A 10006458) and Korea University to Young-Hee Cho.

\section{REFERENCES}

Alonso, J. M., Hirayama, T., Roman, G., Nourizadeh, S., and Ecker, J. R. (1999). EIN2, a bifunctional transducer of ethylene and stress responses in Arabidopsis. Science 284, 2148-2152. doi: 10.1126/science.284.5423.2148

Alonso, J. M., Stepanova, A. N., Solano, R., Wisman, E., Ferrari, S., Ausubel, F. M., et al. (2003). Five components of the ethylene-response pathway identified in a screen for weak ethylene-insensitive mutants in Arabidopsis. Proc. Natl. Acad. Sci. U.S.A. 100, 2992-2997. doi: 10.1073/pnas.0438070100

Beaudoin, N., Serizet, C., Gosti, F., and Giraudat, J. (2000). Interactions between abscisic acid and ethylene signaling cascades. Plant Cell 12, 1103-1115. doi: 10.1105/tpc.12.7.1103

Binder, B. M., Mortimore, L. A., Stepanova, A. N., Ecker, J. R., and Bleecker, A. B. (2004). Short term growth responses to ethylene in Arabidopsis seedlings are EIN3/EIL1 independent. Plant Physiol. 136, 2921-2927. doi: 10.1104/pp.104.050393

Binder, B. M., Walker, J. M., Gagne, J. M., Emborg, T. J., Hemmann, G., Bleecker, A. B., et al. (2007). The Arabidopsis EIN3 binding F-Box proteins EBF1 and EBF2 have distinct but overlapping roles in ethylene signaling. Plant Cell 19, 509-523. doi: 10.1105/tpc.106.048140

Bisson, M. A., and Groth, G. (2010). New insight in ethylene signaling: autokinase activity of ETR1 modulates the interaction of receptors and EIN2. Mol. Plant 3, 882-889. doi: $10.1093 / \mathrm{mp} / \mathrm{ssq} 036$

Bisson, M. M., Bleckmann, A., Allekotte, S., and Groth, G. (2009). EIN2, the central regulator of ethylene signaling, is localized at the ER membrane where it interacts with the ethylene receptor ETR1. Biochem. J. 424, 1-6. doi: 10.1042/BJ20091102

Bleecker, A. B., Estelle, M. A., and Somerville, C. (1988). Insensitive to ethylene conferred by a dominant mutation in Arabidopsis thaliana. Science 241, 10861089. doi: 10.1126/science.241.4869.1086

Chang, C., Kwok, S. F., Bleecker, A. B., and Meyerowitz, E. M. (1993). Arabidopsis ethylene response gene ETR1: similarity of product to two component regulators. Science 262, 539-544. doi: 10.1126/science.8211181

Chao, Q., Rothenberg, M., Solano, R., Roman, G., Terzaghi, W., and Ecker, J. R. (1997). Activation of the ethylene gas response pathway in Arabidopsis by the nuclear protein Ethylene-Insensitive3 and related proteins. Cell 89, 1133-1144. doi: 10.1016/S0092-8674(00)80300-1

Chen, H., Xue, L., Chintamanani, S., Germain, H., Lin, H., Cui, H., et al. (2009). ETHYLENE INSENSITIVE3 and ETHYLENE INSENSITIVE3-LIKE1 repress SALICYLIC ACID INDUCTION DEFICIENT2 expression to negatively regulate plant innate immunity in Arabidopsis. Plant Cell 21, 2527-2540. doi: 10.1105/tpc.108.065193

Chen, R., Binder, B., Garrett, W. M., Tucker, M. L., Chang, C., and Cooper, B. (2011). Proteomic responses in Arabidopsis thaliana seedlings treated with ethylene. Mol. Biosyst. 7, 2637-2650. doi: 10.1039/c1mb05159h
Cho, Y. H., and Yoo, S. D. (2009). Emerging complexity of ethylene signal transduction. J. Plant Biol. 52, 283-288. doi: 10.1007/s12374-009-9038-6

Clark, K. L., Larsen, P. B., Wang, X., and Chang, C. (1998). Association of the Arabidopsis CTR1 raf-like kinase with the ETR1 and ERS ethylene receptors. Proc. Natl. Acad. Sci. U.S.A. 95, 5401-5406. doi: 10.1073/pnas.95.9.5401

Cooper, B. (2013). Separation anxiety: an analysis of ethylene-induced cleavage of EIN2. Plant Signal. Behav. 8:e24721. doi: 10.4161/psb.24721

Dong, C. H., Rivarola, M., Resnick, J. S., Maggin, B. D., and Chang, C. (2008). Subcellular co-localization of Arabidopsis RTE1 and ETR1 supports a regulatory role for RTE1 in ETR1 ethylene signaling. Plant J. 53, 275-286. doi: 10.1111/j.1365313X.2007.03339.x

Dong, C. H., Jang, M., Scharein, B., Malach, A., Rivarola, M., Liesch, J., et al. (2010). Molecular association of the Arabidopsis ETR1 ethylene receptor and a regulator of ethylene signaling, RTE1. J. Biol. Chem. 285, 40706-40713. doi: 10.1074/jbc.M110.146605

Dorling, S., and McManus, M. T. (2012). "The fate of ACC in higher plants," in Annual Plant Reviews, Vol. 44, The Plant Hormone Ethylene, ed. M. T. McManus (Oxford: Blackwell Publishing), 83-115.

Gagne, J. M., Smalle, J., Gingerich, D. J., Walker, J. M., Yoo, S. D., Yanagisawa, S., et al. (2004). Arabidopsis EIN3-binding F-box 1 and 2 form ubiquitinprotein ligases that repress ethylene action and promote growth by directing EIN3 degradation. Proc. Natl. Acad. Sci. U.S.A. 101, 6803-6808. doi: 10.1073/pnas.0401698101

Ghassemian, M., Nambara, E., Cutler, S., Kawaide, H., Kamiya, Y., and McCourt, P. (2000). Regulation of abscisic acid signaling by the ethylene response pathway in Arabidopsis. Plant Cell 12, 1117-1126. doi: 10.1105/tpc.12.7.1117

Gregory, B. D., O’Malley, R. C., Lister, R., Urich, M. A., Tonti-Filippini, J., Chen, H., et al. (2008). A link between RNA metabolism and silencing affecting Arabidopsis development. Dev. Cell 14, 854-866. doi: 10.1016/j.devcel.2008. 04.005

Guo, H., and Ecker, J. R. (2003). Plant responses to ethylene gas are mediated by $\mathrm{SCF}^{\mathrm{EBF} 1 / \mathrm{EBF} 2}$-dependent proteolysis of EIN3 transcription factor. Cell 115, 667677. doi: 10.1016/S0092-8674(03)00969-3

Guo, H., and Ecker, J. R. (2004). The ethylene signaling pathway: new insights. Curr. Opin. Plant Biol. 7, 40-49. doi: 10.1016/j.pbi.2003.11.011

Guzman, P., and Ecker, J. R. (1990). Exploiting the triple response of Arabidopsis to identify ethylene-related mutants. Plant Cell 2, 513-523. doi: 10.1105/tpc.2.6.513

Hall, B. P., Shakeel, S. N., and Schaller, G. E. (2007). Ethylene receptors: ethylene perception and signal transduction. J. Plant Growth Regul. 26, 118-130. doi: 10.1007/s00344-007-9000-0

Harpaz-Saad, S., Yoon, G. M., Mattoo, A. K., and Kieber, J. J. (2012). “The formation of ACC and competition between polyamines and ethylene form SAM," in Annual Plant Review, Vol. 44, The Plant Hormone Ethylene, ed. M. T. McManus (Oxford: Blackwell Publishing), 53-81.

Hua, J., Chang, C., Sun, Q., and Meyerowitz, E. M. (1995). Ethylene sensitivity conferred by Arabidopsis ERS gene. Science 269, 1712-1714. doi: 10.1126/science. 7569898

Hua, J., and Meyerowitz, E. M. (1998). Ethylene responses are negatively regulated by a receptor gene family in Arabidopsis thaliana. Cell 94, 261-271. doi: 10.1016/S0092-8674(00)81425-7

Hua, J., Sakai, H., Nourizadeh, S., Chen, Q. G., Bleecker, A. B., Ecker, J. R., et al. (1998). EIN4 and ERS2 are members of the putative ethylene receptor family in Arabidopsis. Plant Cell 10, 1321-1332. doi: 10.1105/tpc.10.8.1321

Huang, Y., Li, H., Hutchison, C. E., Laskey, J., and Kieber, J. J. (2003). Biochemical and functional analysis of CTR1, a protein kinase that negatively regulates ethylene signaling in Arabidopsis. Plant J. 33. 221-233. doi: 10.1046/j.1365313X.2003.01620.x

Ju, C.,Yoon, G., Shemansky, J., Lin, D., Ying, Z. I., Chang, J., et al. (2012). CTR1 phosphorylates the central regulator EIN2 to control ethylene hormone signaling from the ER membrane to the nucleus in Arabidopsis. Proc. Natl. Acad. Sci. U.S.A. 109, 19486-19491. doi: 10.1073/pnas.1214848109

Kieber, J. J., Rothenberg, M., Roman, G., Feldmann, K. A., and Ecker, J. R. (1993). CTR1, a negative regulator of the ethylene response pathway in Arabidopsis, encodes a member of the raf family of protein kinases. Cell 72, 427-441. doi: 10.1016/0092-8674(93)90119-B

Kim, H. G., Kwon, S. J., Jang, Y. J., Nam, M. H., Chung, J. H., Na, Y.-C., et al. (2013). GDSL LIPASE1 modulates plant immunity through feedback regulation of ethylene signaling. Plant Physiol. 163, 1776-1791. doi: 10.1104/pp.113.225649 
Kim, H. G., Kwon, S. J., Jang, Y. J., Chung, J. H., Nam, M. H., and Park, O. K. (2014). GDSL lipase 1 regulates ethylene signaling and ethyleneassociated systemic immunity in Arabidopsis. FEBS Lett. 588, 1652-1658. doi: 10.1016/j.febslet.2014.02.062

Konishi, M., and Yanagisawa, S. (2008). Arabidopsis ethylene signaling involves feedback regulation via the elaborate control of EBF2 expression by EIN3. Plant J. 55, 821-831. doi: 10.1111/j.1365-313X.2008.03551.x

Lacey, R. F., and Binder, B. M. (2014). How plants sense ethylene gas-the ethylene receptors. J. Inorg. Biochem. 133, 58-62. doi: 10.1016/j.jinorgbio.2014.01.006

Laluk, K., Luo, H., Chai, M., Dhawan, R., Lai, Z., and Mengiste, T. (2011). Biochemical and genetic requirements for function of the immune response regulator BOTRYTIS-INDUCED KINASE1 in plant growth, ethylene signaling and PAMP-triggered immunity in Arabidopsis. Plant Cell 23, 2831-2849. doi: 10.1105/tpc.111.087122

Lee, J. H., Deng, X. W., and Kim, W. T. (2006). Possible role of light in the maintenance of EIN3?EIL1 stability in Arabidopsis seedlings. Biochem. Biophys. Res. Commun. 350, 484-491. doi: 10.1016/j.bbrc.2006.09.074

Liu, Z., Wu, Y., Yang, F., Zhang, Y., Chen, S., Xie, Q., et al. (2013). BIK1 interacts with PEPRs to mediate ethylene-induced immunity. Proc. Natl. Acad. Sci. U.S.A. 110, 6205-6210. doi: 10.1073/pnas.1215543110

McManus, M. T. (2012). The Plant Hormone Ethylene. Annual Plant Review, Vol. 44. Oxford: Wiley-Blackwell.

Ohme-Takagi, M., and Shinshi, H. (1995). Ethylene-inducible DNA binding proteins that interact with an ethylene-responsive element. Plant Cell 7, 173-182. doi: 10.1105/tpc.7.2.173

Olmedo, G., Guo, H., Gregory, B. D., Nourizadeh, S. D., Aguilar-Henonin, L., Li, H., et al. (2006). ETHYLENE-INSENSITIVE5 encodes a $5^{\prime} \longrightarrow 3^{\prime}$ exoribonuclease required for regulation of the EIN3-targeting F-box proteins EBF1/2. Proc. Natl. Acad. Sci. U.S.A. 103, 13286-13293. doi: 10.1073/pnas.0605528103

Potuschak, T., Lechner, E., Parmentier, Y., Yanagisawa, S., Grava, S., Koncz, C., et al. (2003). EIN3-dependent regulation of plant ethylene hormone signaling by two Arabidopsis F box proteins: EBF1 and EBF2. Cell 115, 679-689. doi: 10.1016/S0092-8674(03)00968-1

Potuschak, T., Vansiri, A., Binder, B. M., Lechner, E., Vierstra, R. D., and Genschik, P. (2006). The exoribonuclease XRN4 is a component of the ethylene response pathway in Arabidopsis. Plant Cell 18, 3047-3057. doi: 10.1105/tpc.106. 046508

Qiao, H., Chang, K. N., Yazaki, J., and Ecker, J. R. (2009). Interplay between ethylene, ETP1/ETP2 F-box proteins, and degradation of EIN2 triggers ethylene responses in Arabidopsis. Genes Dev. 23, 512-521. doi: 10.1101/gad. 1765709

Qiao, H., Shen, Z., Huang, S. S. C., Schmitz, R. J., Urich, M. A., Briggs, S. P., et al. (2012). Processing and subcellular trafficking of ER-tethered EIN2 control response to ethylene gas. Science 338, 390-393. doi: 10.1126/science. 1225974

Qiao, H., Shen, Z., Huang, S. S. C., Schmitz, R. J., Urich, M. A., Briggs, S. P., et al. (2013). Response to perspective: "separation anxiety: an analysis of ethyleneinduced cleavage of EIN2”. Plant Signal. Behav. 8:e25037. doi: 10.4161/psb.25037

Resnick, J. S., Wen, C. K., Shockey, J. A., and Chang, C. (2006). REVERSION-TOETHYLENE SENSITIVITY1, a conserved gene that regulates ethylene receptor function in Arabidopsis. Proc. Natl. Acad. Sci. U.S.A. 103, 7917-7922. doi: 10.1073/pnas.0602239103

Sakai, H. M., Hua, J., Chen, Q. G., Chang, C., Medrano, L. J., Bleecker, A. B., et al. (1998). ETR2 is an ETR1-like gene involved in ethylene signaling in Arabidopsis. Proc. Natl. Acad. Sci. U.S.A. 95, 5812-5817. doi: 10.1073/pnas.95.10.5812

Shi, Y., Tian, S., Hou, L., Huang, X., Zhang, X., Guo, H., et al. (2012). Ethylene signaling negatively regulates freezing tolerance by repressing expression of CBF and Type-A ARR genes in Arabidopsis. Plant Cell 24, 2578-2595. doi: 10.1105/tpc.112.098640
Smalle, J., Haegman, M., Kurepa, J., Van Montagu, M., and Straeten, D. V. (1997). Ethylene can stimulate Arabidopsis hypocotyl elongation in the light. Proc. Natl. Acad. Sci. U.S.A. 94, 2756-2761. doi: 10.1073/pnas.94.6.2756

Solano, R., Stepanova, A., Chao, Q., and Ecker, J. R. (1998). Nuclear events in ethylene signaling: a transcriptional cascade mediated by ETHYLENEINSENSITVE3 and ETHYLENE-RESPONSE-FACTOR1. Genes Dev. 12, 37033714. doi: 10.1101/gad.12.23.3703

Wang, W., Esch, J. J., Shiu, S., Agula, H., Binder, B. M., Chang, C., et al. (2006). Identification of important regions for ethylene binding and signaling in the transmembrane domain of the ETR1 ethylene receptor of Arabidopsis. Plant Cell 18, 3429-3442. doi: 10.1105/tpc.106.044537

Wen, X., Zhang, C., Ji, Y., Zhao, Q., He, W., An, F., et al. (2012). Activation of ethylene signaling is mediated by nuclear translocation of the cleaved EIN2 carboxyl terminus. Cell Res. 22, 1613-1616. doi: 10.1038/cr.2012.145

Yamasaki, K., Kigawa, T., Inoue, M., Yamasaki, T., Yabuki, T., Aoki, M., et al. (2005). Solution structure of the major DNA-binding domain of Arabidopsis thaliana ethylene-insensitive3-like3. J. Mol. Biol. 348, 253-264. doi: 10.1016/j.jmb.2005.02.065

Yanagisawa, S., Yoo, S. D., and Sheen, J. (2003). Differential regulation of EIN3 stability by glucose and ethylene signalling in plants. Nature 425, 521-525. doi: 10.1038/nature01984

Yoo, S. D., Cho, Y. H., Tena, G., Xiong, Y., and Sheen, J. (2008). Dual control of nuclear EIN3 by bifurcated MAPK cascades in $\mathrm{C}_{2} \mathrm{H}_{4}$ signalling. Nature 451, 789-795. doi: 10.1038/nature06543

Yoo, S. D., Cho, Y. H., and Sheen, J. (2009). Emerging connections in the ethylene signaling network. Trends Plant Sci. 14, 270-279. doi: 10.1016/j.tplants.2009.02.007

Zhong, S., Shi, H., Xue, C., Wang, L., Xi, Y., Li, J., et al. (2012a). A molecular framework of light-controlled phytochrome action in Arabidopsis. Curr. Biol. 22, 1530-1535. doi: 10.1016/j.cub.2012.06.039

Zhong, S., Zhao, M., Shi, T., Shi, H., An, F., Zhao, Q., et al. (2012b). EIN3/EIL1 cooperates with PIF1 to prevent photo-oxidation and to promote greening of Arabidopsis seedlings. Proc. Natl. Acad. Sci. U.S.A. 106, 21431-21436. doi: 10.1073/pnas.0907670106

Zhou, X., Liu, Q., Xie, F., and Wen, C. K. (2007). RTE1 is a Golgi-associated and ETR1-dependent negative regulator of ethylene responses. Plant Physiol. 145, 75-86. doi: 10.1104/pp.107.104299

Zhu, Z., An, F., Feng, Y., Li, P., Xue, L., Mu, A., et al. (2011). Derepression of ethylene-stabilized transcription factors (EIN3/EIL1) mediates jasmonate and ethylene signaling synergy in Arabidopsis. Proc. Natl. Acad. Sci. U.S.A. 108, 12539-12544. doi: 10.1073/pnas.1103959108

Conflict of Interest Statement: The authors declare that the research was conducted in the absence of any commercial or financial relationships that could be construed as a potential conflict of interest.

Received: 27 August 2014; accepted: 02 December 2014; published online: 05 January 2015.

Citation: Cho Y-H and Yoo S-D (2015) Novel connections and gaps in ethylene signaling from the ER membrane to the nucleus. Front. Plant Sci. 5:733. doi: $10.3389 / f p l s .2014 .00733$

This article was submitted to Plant Physiology, a section of the journal Frontiers in Plant Science.

Copyright (C) 2015 Cho and Yoo. This is an open-access article distributed under the terms of the Creative Commons Attribution License (CC BY). The use, distribution or reproduction in other forums is permitted, provided the original author(s) or licensor are credited and that the original publication in this journal is cited, in accordance with accepted academic practice. No use, distribution or reproduction is permitted which does not comply with these terms. 


\section{Ethylene, a key factor in the regulation of seed dormancy}

\section{Françoise Corbineau*, Qiong Xia, Christophe Bailly and Hayat El-Maarouf-Bouteau}

Biologie des Semences (Seed Biology), UMR7622 CNRS-UPMC, Sorbonne Universités - Université Pierre et Marie Curie-Paris, Paris, France

\section{Edited by:}

Domenico De Martinis, Italian

National Agency for New

Technologies, Energy and Sustainable

Economic Development, Italy

\section{Reviewed by:}

Wendy A. Peer, University of

Maryland, USA

Luciano Freschi, University of São

Paulo, Brazil

\section{*Correspondence:}

Françoise Corbineau, Biologie des Semences (Seed Biology), Laboratoire de Biologie du Développement, Sorbonne Universités - Université Pierre et Marie Curie-Paris, UMR 7622, Bat C2, Boîte Courrier 24, 4 Place Jussieu, 75005 Paris, France e-mail: francoise.corbineau@upmc.fr
Ethylene is an important component of the gaseous environment, and regulates numerous plant developmental processes including seed germination and seedling establishment. Dormancy, the inability to germinate in apparently favorable conditions, has been demonstrated to be regulated by the hormonal balance between abscisic acid (ABA) and gibberellins (GAs). Ethylene plays a key role in dormancy release in numerous species, the effective concentrations allowing the germination of dormant seeds ranging between 0.1 and $200 \mu \mathrm{L} \mathrm{L}^{-1}$. Studies using inhibitors of ethylene biosynthesis or of ethylene action and analysis of mutant lines altered in genes involved in the ethylene signaling pathway (etr1, ein2, ain 1, etr1, and erf1) demonstrate the involvement of ethylene in the regulation of germination and dormancy. Ethylene counteracts $A B A$ effects through a regulation of $A B A$ metabolism and signaling pathways. Moreover, ethylene insensitive mutants in Arabidopsis are more sensitive to ABA and the seeds are more dormant. Numerous data also show an interaction between ABA, GAs and ethylene metabolism and signaling pathways. It has been increasingly demonstrated that reactive oxygen species (ROS) may play a significant role in the regulation of seed germination interacting with hormonal signaling pathways. In the present review the responsiveness of seeds to ethylene will be described, and the key role of ethylene in the regulation of seed dormancy via a crosstalk between hormones and other signals will be discussed.

Keywords: abscisic acid, dormancy, ethylene, gibberellins, reactive oxygen species, seed germination

\section{INTRODUCTION}

In soil, seeds are exposed to various environmental factors including temperature, moisture, oxygen and light, which regulate seed germination, and subsequent seedling growth. Phase I of the germination process is initiated by imbibition, which is required to activate the respiratory metabolism, and transcriptional and translational activities. In phase II called germination sensu stricto, water uptake ceases and reserve mobilization starts. Phase III is characterized by radicle protrusion (Bewley and Black, 1994; Bewley, 1997; Nonogaki et al., 2010; Weitbrecht et al., 2011). Germination requires specific temperatures, oxygen levels and light, the exact proportions being species specific. However, the seeds of species (or even within species) do not germinate, or do so with difficulty, even when incubated under apparently favorable conditions; these are considered as dormant and cannot germinate in the same conditions (i.e., water, air, temperature) under which non-dormant seeds do (Bewley and Black, 1994; Corbineau and Côme, 1995; Bewley, 1997). Dormancy is a heritable trait, but its intensity at harvest and its maintenance after harvest is highly modulated by the environmental conditions throughout seed development and ripening on the plant, and during seed storage (Bewley, 1997). Factors inhibiting germination can reside within the embryo itself (embryo dormancy) or can result from an inhibitory action of the covering structures (seed coat imposed dormancy; Bewley and Black, 1994; Hilhorst, 2007). Primary dormancy sets during seed development, but a secondary dormancy can develop in mature seeds with some degree of primary dormancy or in non-dormant seeds in response to unfavorable conditions for germination (Hilhorst, 2007; Hilhorst et al., 2010).

The involvement of the hormonal balance between abscisic acid (ABA) and gibberellins (GAs) in the regulation of seed germination and dormancy in response to environmental signals is well documented and discussed in recent reviews (Finkelstein et al., 2008; Nambara et al., 2010; Nonogaki et al., 2010; Weitbrecht etal., 2011; Graeber et al., 2012; Rajjou et al., 2012; Arc et al., 2013; Miransari and Smith, 2014). ABA is well known to play a crucial role in induction of dormancy in the developing seeds and in maintenance of dormancy during seed imbibition, while GAs are involved in dormancy release and/or germination (Finkelstein et al., 2008; Cutler et al., 2010; Nambara et al., 2010; Miransari and Smith, 2014). In addition to GAs and ABA, other hormones (ethylene, jasmonates, auxins) also play a role in the control of seed germination (Linkies and Leubner-Metzger, 2012; Arc etal., 2013; Miransari and Smith, 2014). Ethylene $\left(\mathrm{C}_{2} \mathrm{H}_{4}\right)$ in particular regulates germination and dormancy of numerous species via a complex hormonal signaling network (Matilla, 2000; Brady and McCourt, 2003; Feurtado and Kermode, 2007; Matilla and Matilla-Vazquez, 2008; Arc et al., 2013).

The role of reactive oxygen species (ROS) in seed biology has progressively emerged and evolved this last decade. Originally considered as harmful compounds, causing deleterious reactions toward a wide range of biomolecules and thus to seeds, ROS are now widely acknowledged as signaling compounds regulating the germination process through an 
hormonal network (Bailly et al., 2008; Diaz-Vivancos et al., 2013; El-Maarouf-Bouteau et al., 2014).

In this review, we describe how ethylene interacts with other plant hormones in regulation of germination and dormancy, concentrating on its interactions with ABA, GAs, and ROS.

\section{ETHYLENE BIOSYNTHESIS DURING GERMINATION}

Ethylene production by seeds begins immediately after the onset of imbibition and increases with time of germination. There is, however, a peak in ethylene emission concomitant with the radicle protrusion through the seed coat (Ketring and Morgan, 1969; Fu and Yang, 1983; Satoh and Esashi, 1983; Gallardo et al., 1991; Gorecki etal., 1991; Siriwitayawan etal., 2003; El-Maarouf-Bouteau et al., 2014). Seed ethylene production is species dependent (Kepczynski and Kepczynska, 1997; Matilla, 2000), but is generally below levels detectable by gas chromatography during imbibition. Using a high sensitivity laser photo acoustic spectroscopy (Cristescu et al., 2008), El-Maarouf-Bouteau et al. (2014) have confirmed the occurrence of an ethylene peak at the end of the germination process in sunflower (Helianthus annuus) seeds. Interestingly, a close relationship between the ability to produce ethylene and seed vigor has been reported in various species including rape (Brassica napus; Takayanagi and Harrington, 1971), cotton (Gossypium spp.; Ketring et al., 1974), peanut (Arachis hypogaea; Ketring et al., 1974), cocklebur (Xanthium pennsylvanicum; Gorecki et al., 1991), snap bean (Phaseolus vulgaris; Samimy and Taylor, 1983), sunflower (Chonowski etal., 1997) and pea (Pisum sativum; Gorecki et al., 1991), and 1-aminocyclopropane 1-carboxylic acid (ACC)-dependent $\mathrm{C}_{2} \mathrm{H}_{4}$ production was proposed as a marker of seed quality (Khan, 1994; Corbineau, 2012).

The pathway of ethylene biosynthesis in seeds is the same as that described for other plant organs, in which $S$-adenosylmethionine ( $S$-AdoMet) and ACC are the main intermediates (Yang and Hoffman, 1984; Wang et al., 2002; Rzewuski and Sauter, 2008; Figure 1). S-AdoMet synthesized from methionine by the $S$-AdoMet synthetase (or SAM synthetase), is converted to ACC, the direct precursor of ethylene, by ACC synthase ( $S$-adenosyl-L-methionine methylthioadenosine-lyase, ACS). The by-product $5^{\prime}$-methylthioadenosine (MTA) is recycled back to methionine through the Yang Cycle (Yang and Hoffman, 1984; Kende, 1993). S-AdoMet is also the precursor of the biosynthesis of polyamines, which can also play a role in seed germination (Matilla, 1996). Ethylene production results from the oxidation of ACC by ACC oxidase (ACO), which also generates $\mathrm{CO}_{2}$ and hydrogen cyanide (HCN; Figure 1). Autocatalytic synthesis of ethylene via induction of $A C C$ and $A C O$ transcription is well known in fruit ripening (Lin et al., 2009), ethylene also regulates ACO expression in pea (Petruzzelli et al., 2000, 2003), beechnut (Fagus sylvatica; Calvo et al., 2004b), and turnip (Brassica rapa; Puga-Hermida et al., 2003). In contrast, ethylene or ACC does not affect the abundance of ACO transcript in sugar beet (Beta vulgaris; Hermann etal., 2007) and, expression of SoACS7 in Sisymbrium officinale and PsAC1 in pea (Petruzzelli et al., 2000, 2003; Iglesias-Fernandez and Matilla, 2010).

Increased ethylene production during germination is associated with an increase in ACO activity, as well as a progressive accumulation of ACS and ACO transcripts (Gomez-Jimenez et al., 1998; Matilla and Matilla-Vazquez, 2008; Linkies et al., 2009; Iglesias-Fernandez and Matilla, 2010; Linkies and Leubner-Metzger, 2012). Although ACS is considered to be a key enzyme in the regulation of ethylene production in most plant responses to abiotic and biotic stresses (Wang et al., 2002), it was demonstrated in seeds that ACO activity plays a fundamental role during germination (Matilla and Matilla-Vazquez, 2008; Linkies and Leubner-Metzger, 2012). Both ACS and ACO are encoded by a multigene family, and the regulation of particular ACS and ACO genes differ among each other (Wang et al., 2002, 2005; Yamagami et al., 2003). In both Arabidopsis and cress (Lepidium sativum), ACO1 and ACO2 have been demonstrated to be the major ACOs involved in ethylene synthesis (Linkies et al., 2009; Linkies and Leubner-Metzger, 2012), and the correlation between the abundance of ACO transcripts and the ACO activity suggests its regulation at a transcriptional level during germination.

Ethylene is involved in various developmental processes and responses to biotic and abiotic stresses in plants (Bleecker and Kende, 2000; Wang etal., 2002). The key components in its signaling pathway have been identified using a molecular dissection of ethylene responsiveness in Arabidopsis (Wang etal., 2002; Stepanova and Alonso, 2009; Yoo et al., 2009). Five membrane-localized ethylene receptors, ethylene resistant 1 (ETR1), ETR2, ethylene response sensor 1 (ERS1), ERS2, and ethylene insensitive 4 (EIN4) exist in Arabidopsis (Wang et al., 2002). Among them, ETR1 and ERS1 contain three transmembrane domains in the $\mathrm{N}$-terminus and a histidine kinase domain in the C-terminus, when ETR2, EIN4, and ERS2 present four transmembrane regions and a serine-threonine kinase domain in the C-terminus (Wang etal., 2006; Kendrick and Chang, 2008). Binding of ethylene to its receptors results in inactivation of CTR1 (constitutive triple response 1) protein kinase, which in turn activates the kinase cascade controlling EIN2 and its transcription factors in the nucleus. These, such as EIN3, EILs, ethylene response element binding proteins (EREBPs)/ethylene responsive factors (ERFs) activate the transcription of ethylene response genes (Wang etal., 2002; Liu etal., 2004; Hall etal., 2007; Rzewuski and Sauter, 2008; Yoo etal., 2008, 2009; Stepanova and Alonso, 2009; Figure 1). Recently, it was demonstrated that EIN2 is phosphorylated by CTR1 kinase in the absence of ethylene, and that EIN2 protein level is regulated through its degradation by the proteasome (Qiao et al., 2009, 2012; Ju et al., 2012).

\section{SEED RESPONSIVENESS TO EXOGENOUS ETHYLENE}

Exogenous ethylene or ethephon, an ethylene releasing compound, improves germination in numerous species (Esashi, 1991; Corbineau and Côme, 1995; Kepczynski and Kepczynska, 1997; Matilla, 2000; Matilla and Matilla-Vazquez, 2008; Arc et al., 2013). It stimulates germination of non-dormant seeds incubated in non-optimal environmental conditions such as too high temperatures (Rao et al., 1975; Abeles, 1986; Gallardo et al., 1991), osmotic stress (Negm and Smith, 1978; Kepczynski, 1986; Khan et al., 2009), hypoxia (Esashi et al., 1989; Corbineau and Côme, 1992), and salinity (Zapata et al., 2003; Wang et al., 2011; Lin etal., 2013; Silva etal., 2014). It can also break primary and 


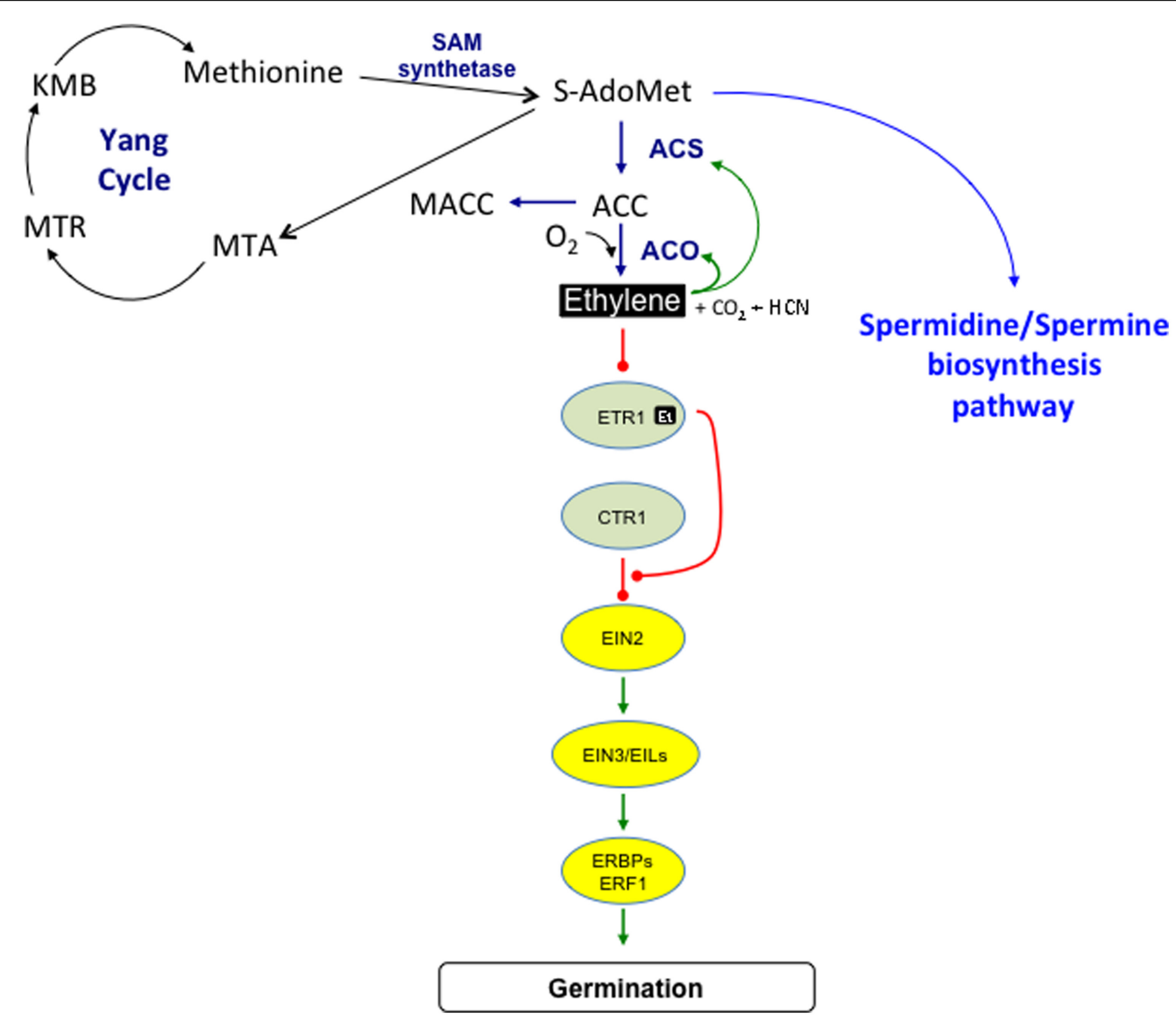

FIGURE 1 | Ethylene biosynthesis and signaling pathways. S-adenosyl methionine ( $S$-AdoMet) is synthesized from methionine by the SAM synthetase, it is then converted to 1-aminocyclopropane-1-carboxylic acid (ACC) by the ACC synthase (ACS), 5-methylthioadenosine (MTA) being a by-product. MTA is recycled to methionine through the Yang Cycle by successive enzymatic reactions involving different intermediates among which 5-methylthioribose (MTR) and 2-keto-4-methylthiobutyrate (KMB). $S$-AdoMet is also the precursor of the spermidine/spermine biosynthesis pathway. Ethylene production results from the ACC oxidation catalyzed by the ACC oxidase (ACO) that also generates carbon dioxide and cyanide. Malonylation of ACC to malonyl-ACC (MACC) reduces $\mathrm{ACC}$ content and consequently ethylene production.
Ethylene can stimulate its own biosynthesis, by improving ACC synthesis catalyzed by ACS, and conversion to ethylene by ACO. Ethylene binds to receptors (among which ethylene receptor 1, ETR1) located in the endoplasmic reticulum, which leads to the deactivation of the receptors that become able to recruit CTR1 (constitutive triple response). Release of CTR1 inhibition allows EIN2 to act as a positive regulator of ethylene signaling pathway. EIN2 acts upstream of nuclear transcription factors, such as EIN3 (ethylene insensitive), EILs (EIN3-like), ERBPs (ethylene responsive element binding protein), and ERFs (ethylene response factor). $\longrightarrow$ and $-\bullet$ arrows indicate positive and negative interactions between the different elements of the signaling cascade, respectively. secondary dormancy (Table 1). It breaks the embryo dormancy in apple (Malus domestica; Kepczynski et al., 1977; Sinska and Gladon, 1984; Sinska and Lewandowska, 1991) and beechnut (Calvo etal., 2004a), the dormancy of which is usually broken by chilling, and in sunflower (Corbineau et al., 1990), the dormancy of which is progressively alleviated during dry storage (after-ripening). It also promotes the germination of seeds exhibiting a seed coat imposed dormancy in various species such as cocklebur (Katoh and Esashi, 1975; Esashi et al., 1978), subterranean clover (Trifolium subterraneum; Esashi and Leopold, 1969), Rumex crispus (Taylorson, 1979), and Arabidopsis (Siriwitayawan et al., 2003). In particular, it can also overcome the secondary dormancy induced by high temperatures in lettuce (Lactuca sativa; Speer et al., 1974; Abeles, 1986), sunflower (Corbineau et al., 1988), Amaranthus caudatus (Kepczynski et al., 1996a) and Amaranthus paniculatus (Kepczynski and Kepczynska, 1993). In Rhus coriaria, a post-fire pioneer, low ethylene concentrations $\left(0.03-0.10 \mu \mathrm{L} \mathrm{L}^{-1}\right)$ released by wet ash stimulates germination (Ne'eman et al., 1999), although it does not improve that of many other species in which germination is smoke-induced (Brown and van Staden, 1997). Ethylene also improves germination of seeds from parasitic plants such as Striga asiatica, Striga lutea and Striga hermonthica (Egley and Dale, 1970; Bebawi and Eplee, 1986).

The stimulatory effect of ethylene is dose dependent, the hormone being efficient when applied at concentration ranging from 0.1 to $200 \mu \mathrm{L} \mathrm{L}^{-1}$ depending on the species, the depth of dormancy and the environmental conditions. Breaking of dormancy either during chilling in apple (Sinska, 1989) or dry storage in sunflower (Corbineau and Côme, 2003), Amaranthus retroflexus (Kepczynski and Sznigir, 2014) and Stylosanthes 
Table 1 | Plant species whose seed dormancy is broken by ethylene, ethephon, an ethylene releasing compound, or 1-aminocyclopropane1-carboxylic acid, the direct precursor of ethylene.

\begin{tabular}{|c|c|c|}
\hline Type of dormancy & Species & Reference \\
\hline \multirow[t]{9}{*}{ Primary dormancy } & Amaranthus caudatus & Kepczynski and Karssen (1985) \\
\hline & Arabidopsis thaliana & Siriwitayawan et al. (2003) \\
\hline & Arachis hypogaea & Ketring and Morgan (1969) \\
\hline & Chenopodium album & Machabée and Saini (1991) \\
\hline & Helianthus annuus & Corbineau et al. (1990) \\
\hline & Pyrus malus & Kepczynski etal. (1977) \\
\hline & & Sinska and Gladon (1984) \\
\hline & Rhus coriaria & Ne'eman et al. (1999) \\
\hline & Rumex crispus & Taylorson (1979) \\
\hline Thermo-dormancy & Lactuca sativa & Speer etal. (1974) \\
\hline \multirow[t]{6}{*}{ Secondary dormancy } & Amaranthus caudatus & Kepczynski and Karssen (1985) \\
\hline & Amaranthus paniculatus & Kepczynski and Kepczynska (1993) \\
\hline & Helianthus annuus & Corbineau et al. (1988) \\
\hline & Lactuca sativa & Abeles (1986) \\
\hline & Rumex crispus & Samimy and Khan (1983) \\
\hline & Xanthium pennsy/vanicum & Esashi et al. (1978) \\
\hline
\end{tabular}

humilis (Ribeiro and Barros, 2006) is associated with an increasing sensitivity to ethylene. At harvest, dormant sunflower seeds require $50 \mu \mathrm{L} \mathrm{L}^{-1}$ ethylene in order to germinate at $15^{\circ} \mathrm{C}$, but only 10 and $3 \mu \mathrm{L} \mathrm{L}^{-1}$ after 8 and 15 weeks of dry-storage at $5^{\circ} \mathrm{C}$, respectively (Corbineau and Côme, 2003). In contrast, the responsiveness to the hormone decreases progressively during seed incubation under environmental conditions that induce a secondary dormancy (Negm et al., 1973; Speer et al., 1974; Esashi et al., 1978; Jones and Hall, 1984; Corbineau and Côme, 2003).

Although ethylene stimulates the germination of numerous light sensitive seeds, it does not overcome the light requirement in Amaranthus retroflexus (Schönbeck and Egley, 1981), celery (Apium graveolens; Thomas etal., 1975), lettuce (Burdett and Vidaver, 1971), and Spergula arvensis (Olatoye and Hall, 1973). Recently, Wilson et al. (2014b) demonstrate that loss of ETR1 reduces the inhibitory effect of far-red on the germination of Arabidopsis seeds through expression of genes involved in ABA and GAs metabolism. An epistasis analysis performed by the same authors also suggests that ETR1 may interact with phytochromes to control seed germination.

An additive or synergistic effect of $\mathrm{CO}_{2}$ and ethylene has also been demonstrated in seeds of peanut (Ketring and Morgan, 1972), Spergula arvensis (Jones and Hall, 1984), cocklebur (Katoh and
Esashi, 1975), and lettuce (Negm and Smith, 1978; Saini et al., 1986). In the case of cocklebur (Esashi et al., 1986) and sunflower (Corbineau et al., 1990) seeds, it was suggested that the improving effect of $\mathrm{CO}_{2}$ results from an enhancement of $\mathrm{C}_{2} \mathrm{H}_{4}$ biosynthesis, since it is suppressed in the presence of inhibitors of ethylene synthesis.

\section{INVOLVEMENT OF ETHYLENE IN SEED GERMINATION AND DORMANCY}

Numerous studies demonstrate that the ability to germinate correlates with ethylene production, suggesting that ethylene is involved in the regulation of seed germination and dormancy (reviewed in Kepczynski and Kepczynska, 1997; Matilla and Matilla-Vazquez, 2008; Arc et al., 2013). For example, induction of thermodormancy at high temperatures is associated with a reduced ethylene production in chickpea (Cicer arietinum; Gallardo et al., 1991), sunflower (Corbineau et al., 1988), and lettuce (Prusinski and Khan, 1990). This decrease in $\mathrm{C}_{2} \mathrm{H}_{4}$ production may result from an increase in ACC-malonyltransferase activity, thus from a decrease in ACC content as demonstrated in chickpea (Martinez-Reina et al., 1996), an inhibition of ACO activity (Corbineau et al., 1988; Gallardo et al., 1991), or a reduced expression of ACS and ACO (Argyris etal., 2008). In contrast, breaking of dormancy by various treatments (e.g., chilling, GAs, NO, HCN) leads to an increase in ethylene production 
(Kepczynski and Kepczynska, 1997; Arc et al., 2013). In Arabidopsis, the inductive effect of chilling is associated with a reduced expression of $A C O$, but in a transient induction of ACS (Narsai etal., 2011; Linkies and Leubner-Metzger, 2012). However, after-ripening of Sisymbrium officinale seeds inhibits expression of SoACS7 and SoACO2 which are involved in ethylene biosynthesis, during early seed incubation, but stimulates that of SoGA20ox2, SoGA3ox2, and SoGA2ox6 involved in GA metabolism (Iglesias-Fernandez and Matilla, 2009).

Data obtained using inhibitors of ethylene biosynthesis pathway or mutants altered in ethylene biosynthesis and signaling pathways demonstrated that endogenous ethylene plays a key role in the regulation of germination and dormancy. Incubation of seeds in the presence of aminoethoxyvinylglycine (AVG) and aminooxyacetic acid (AOA), inhibitors of ACS activity, $\mathrm{CoCl}_{2}$ and $\alpha$-aminoisobutyric acid $(\alpha$-AIB), inhibitors of ACO activity, or 2,5-norbornadiene (2,5 NBD) and silver thiosulfate (STS), inhibitors of ethylene action, allowed demonstration of the involvement of endogenous ethylene in germination and dormancy breakage (Kepczynski etal., 1977, 2003; Sinska and Gladon, 1989; Corbineau etal., 1990; Longan and Stewart, 1992; Gallardo et al., 1994; Hermann et al., 2007). In contrast, seed incubation in the presence of ACC, the direct precursor of ethylene, improves seed germination in numerous species such as lettuce (Fu and Yang, 1983), sunflower (Corbineau et al., 1990), cocklebur (Satoh et al., 1984), Amaranthus sp. (Kepczynski, 1986; Kepczynski et al., 1996b), chickpea (Gallardo et al., 1994), and sugar beet (Hermann et al., 2007). This effect of ACC suggests that ACO is potentially active, and that dormancy might result from insufficient ACC level due to low ACS activity.

It is important to notice that HCN, a co-product of ACC oxidation, can also break seed dormancy in apple (Perino et al., 1984; Lewak, 2011; Krasuska and Gniazdowska, 2012; Krasuska et al., 2014), sunflower (Oracz et al., 2008) and Amaranthus retroflexus (Kepczynski and Sznigir, 2014).

Using Arabidopsis lines altered in ethylene biosynthesis and signaling allowed to characterize the regulation of dormancy by ethylene (Table 2). Seeds of ethylene insensitive etr1 (ethylene resistant) as well as ein2 (ethylene insensitive 2) mutants display enhanced primary dormancy relative to wild type, probably due to high ABA sensitivity, whereas ctr1 (constitutive triple responses) mutant have a slightly enhanced rate of germination (Bleecker et al., 1988; Leubner-Metzger etal., 1998; Beaudoin et al., 2000; Ghassemian et al., 2000; Hall et al., 2001; Chiwocha et al., 2005; Subbiah and Reddy, 2010). EIN2 plays a key role in the ethylene signaling pathway, and loss of its function results in a hypersensitivy to salt and osmotic stress during germination and early seedling development in Arabidopsis (Wang et al., 2007). ERFs genes might also play a pivotal role in ethylene responsiveness and regulation of germination (Leubner-Metzger et al., 1998; Pirrello et al., 2006). FsERF1 expression is minimal in dormant beechnut embryo, but increases during moist chilling which breaks dormancy (Jimenez et al., 2005). In sunflower, ERF1 expression is fivefold higher in non-dormant than in dormant embryos, and expression is markedly stimulated by HCN, which breaks dormancy (Oracz et al., 2008). In tomato (Solanum lycopersicon), SlERF2 transcript abundance is higher in germinating seeds than in non-germinating ones, and its overexpression

Table 2 | Dormancy and ABA sensitivity of various mutants of Arabidopsis thaliana affected in ethylene biosynthesis or signaling pathway.

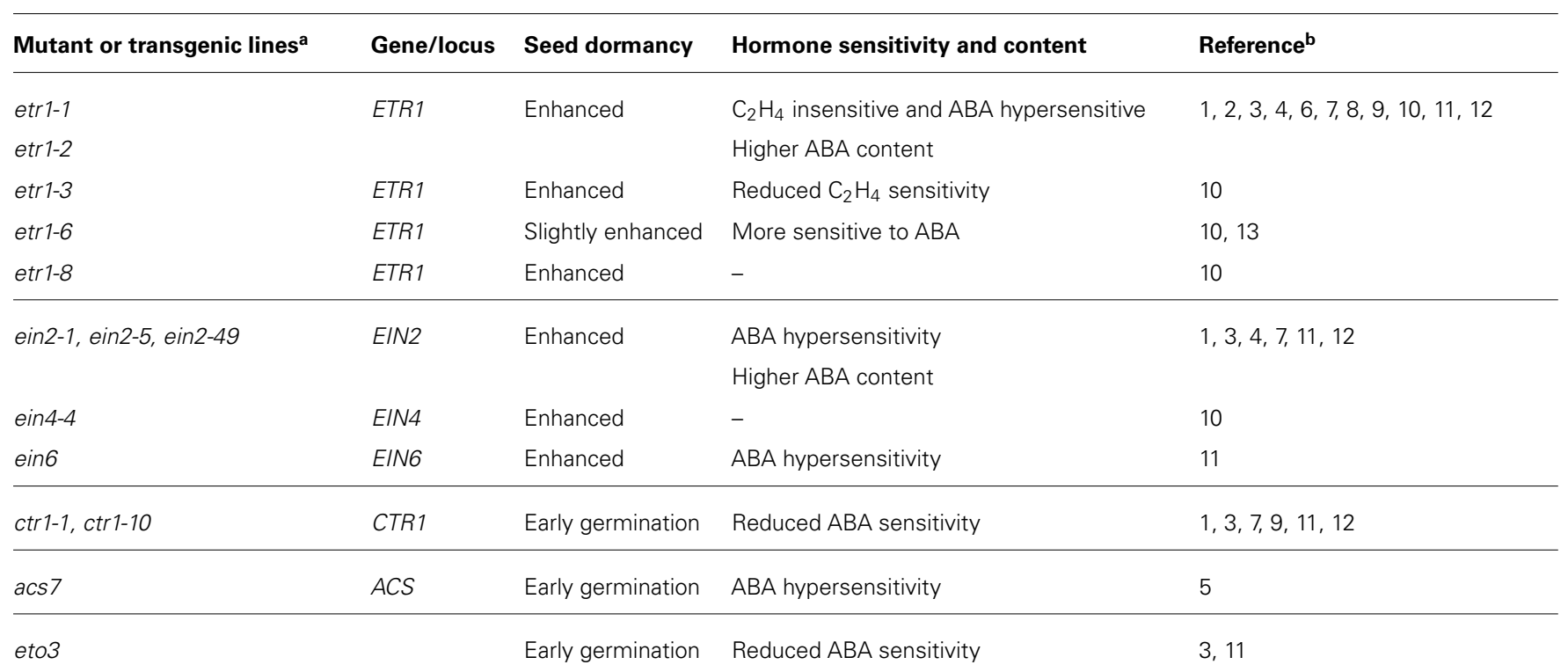

${ }^{a}$ mutant abbreviations: acs7 = ACC synthetase 7; ctr1 = constitutive triple response1; ein2, ein4, ein $6=$ ethylene insensitive 1, 4, 6; etr1 = ethylene resistant1; eto3 $=$ ethylene overproducer3.

breferences: (1) Beaudoin etal. (2000); (2) Bleecker etal. (1988); (3) Cheng etal. (2009); (4) Chiwocha etal. (2005); (5) Dong etal. (2011); (6) Ghassemian etal. (2000): (7) Hall etal. (2001); (8) Leubner-Metzger et al. (1998); (9) Ouaked etal. (2003); (10) Siriwitayawan et al. (2003); (11) Subbiah and Reddy (2010); (12) Wang etal. (2007); (13) Wilson etal. (2014a). 
in transgenic lines results in premature seed germination (Pirrello et al., 2006).

Transcriptional arrays have been used to draw a global view of gene expression in germinating and non-germinating seeds and core sets of genes were analyzed with respect to hormone responsive elements. The analysis of transcriptome data of dormant and after-ripened states in Arabidopsis performed by Cadman et al. (2006) showed that ACS2 gene expression was up-regulated in the dormant state and AtERF5 was up-regulated in germinating state. In lettuce, Argyris et al. (2008) have shown that ethylene responsive genes are regulated by thermo-inhibition; $A C O$ and ACS gene expression is reduced while CTR1, EIN2, ETR1 expression is increased at high temperature. These results point out the gap that exists between hormone metabolism and signaling regulation at the level of gene expression. In wheat seeds, 78 probesets annotated as ethylene metabolism and signaling genes have been differentially expressed between dormant and after-ripened seeds (Chitnis et al., 2014). ACO is represented by four probesets that are up-regulated in after-ripened wheat seeds but no ACS corresponding probeset has been found. Ethylene signaling element as reversion to ethylene sensitivity 1, ERS1, EBF1 (EIN3 binding box protein1), prohibitin 3 or ERF have been shown to be up-regulated in after-ripened seeds at 12 or $24 \mathrm{~h}$ of imbibition. The number of genes related to ethylene involved in germination is underestimated since only direct known ethylene signaling components are targeted for analysis in omic studies. It has been shown that treatment with ACC of 7 days germinated seedlings triggers change in expression of 544 genes, among them 244 were common to seeds given an ABA treatment (Nemhauser et al., 2006). These results have been used to compare genes regulated in Lepidium seed tissues during germination (Linkies et al., 2009). Pectate Lyase 1, Argos-like, Expansin A2, B-1,3-glucanase and chitinase $B$ which play an important role in endosperm weakening and/or radicle growth in germination of Lepidium seeds, are proposed as putative ethylene response down-stream genes. Cell wall loosening enzymes expressed in endosperm are also controlled by both ABA and GA (Groot et al., 1988; Toorop et al., 2000).

\section{ETHYLENE CROSSTALK WITH ABA/GAs AND SEED GERMINATION INTERRELATIONSHIP BETWEEN ETHYLENE AND ABA}

The antagonistic effects of $\mathrm{ABA}$ and ethylene in the regulation of seed germination and dormancy have been extensively studied (Leubner-Metzger et al., 1998; Beaudoin et al., 2000; Kucera et al., 2005; Matilla and Matilla-Vazquez, 2008; Linkies et al., 2009; Arc et al., 2013). Ethylene overcomes the inhibitory action of ABA on germination of numerous species among which are Amaranthus caudatus (Kepczynski, 1986), Chenopodium album (Karssen, 1976), cotton (Halloin, 1976), tobacco (Nicotiana tabacum), and Arabidopsis (Leubner-Metzger et al., 1998). In Arabidopsis and Lepidium sativum, ethylene also counteracts the inhibition by ABA of endosperm cap weakening and rupture (Linkies et al., 2009). On the contrary, ABA increases the ethylene requirement in order to release dormancy in sunflower (Corbineau and Côme, 1995, 2003) and Amaranthus caudatus (Kepczynski et al., 2003). The negative interaction between ABA and ethylene is also supported by data obtained with various mutants affected in the signaling pathway of both hormones. Ethylene insensitive mutants (etr1, ein2, and ein6) are hypersensitive to ABA, whereas seeds of ein3, ein4, ein5, and ein7 germinate normally. Conversely, eto1, eto3, and ctr 1 mutants (characterized by an increase in $\mathrm{C}_{2} \mathrm{H}_{4}$ production) exhibit a reduced sensitivity to ABA (Table 2; Beaudoin et al., 2000; Ghassemian et al., 2000; Subbiah and Reddy, 2010). Loss of function of CRT1 enhances the tolerance to ABA of abil-1 seeds (Beaudoin et al., 2000). Genetic approaches, using double mutants obtained by crossing ethylene insensitive mutants (ctr1, ein1, ein3, and ein6) with aba2 mutant, demonstrate that $\mathrm{ABA}$ and $\mathrm{C}_{2} \mathrm{H}_{4}$ may also act in parallel, since they exibit phenotypes resulting from both ABA deficiency and altered ethylene sensitivity (Cheng et al., 2009).

Although ACC and exogenous ethylene do not affect ABA content in Lepidium sativum (Linkies et al., 2009) and sugar beet (Hermann et al., 2007), Arabidopsis seeds from ethylene insensitive mutants, etr1 and ein2, have a higher ABA content than that of wild type seeds (Kende et al., 1998; Beaudoin et al., 2000; Ghassemian et al., 2000; Chiwocha et al., 2005; Wang et al., 2007). For example, mutation in ETR1 results in an 8-fold higher ABA content in mature seeds than in wild type, probably due to a decrease in ABA conjugation (Chiwocha et al., 2005). Loss of function of ACS7, one of type 3 ACS with a very short C-terminus and no phosphorylation site, results in reduced $\mathrm{C}_{2} \mathrm{H}_{4}$ emission and hypersensitivity to ABA, consequently conferring abiotic stress tolerance to Arabidopsis seeds (Dong et al., 2011). ABA accumulation is also associated with stimulation of ABA biosynthesis through an upregulation of NCED and a down-regulation of CYP707A2 in seeds of the etr1 mutant (Cheng et al., 2009), or an up-regulation of NCED3 associated with an up-regulation of $A B A 1$ in ein2 seeds (Wang et al., 2007).

Inhibition of germination by $\mathrm{ABA}$ is associated with an inhibition of in vivo ACO activity and is correlated with a reduction in ACO transcript accumulation (Bailly et al., 1992; Petruzzelli etal., 2000, 2003; Linkies et al., 2009), leading to a reduction of ethylene production (Kepczynski and Kepczynska, 1997; Matilla, 2000). In Arabidopsis, accumulation of $A C O 1$ and $A C O 2$ transcripts during germination is inhibited by $\mathrm{ABA}$, and the high level of $A C O 1$ transcript in ABA-insensitive mutants suggest a tight regulation of ACO expression by ABA (Penfield et al., 2006; Carrera et al., 2008; Linkies et al., 2009). In Lepidium sativum, ABA inhibits expression of both ACO1 and ACO2 in the endosperm cap (Linkies et al., 2009). Upregulation of $A C O$ transcript has also been detected by microarray analysis in the aba2 mutant in Arabidopsis (Cheng etal., 2009). In contrast, there is an ABA-mediated up-regulation of ACC accumulation and ACO expression in sugar beet seeds (Hermann et al., 2007).

\section{INTERRELATIONSHIP BETWEEN ETHYLENE AND GAs}

Gibberellins improve the germination of dormant seeds in numerous species whose dormancy is broken by ethylene, ethephon, or ACC (c.f. Table 1). Both hormones promote the germination of primary dormant seeds of Arabidopsis (Ogawa et al., 2003; Siriwitayawan et al., 2003), Amaranthus retroflexus (Kepczynski et al., 1996b; Kepczynski and Sznigir, 2014), beechnut (Calvo 
et al., 2004a,b), apple (Kepczynski et al., 1977; Sinska and Gladon, 1984; Sinska, 1989; Lewak, 2011), and Sisymbrium officinale (Iglesias-Fernandez and Matilla, 2010). They also break secondary dormancy in Rumex crispus (Samimy and Khan, 1983) and cocklebur seeds (Esashi et al., 1975), and thermodormancy in lettuce achenes (Keys et al., 1975). In Arabidopsis, $\mathrm{C}_{2} \mathrm{H}_{4}$ restores the germination of the GA-deficient mutant ga1-3 (Karssen et al., 1989), and $\mathrm{GA}_{3}$ stimulates that of the etr1 mutant (Bleecker et al., 1988), while no stimulatory effect is noted on the germination of the GAdeficient gib-1 mutant in tomato (Groot and Karssen, 1987). All these data suggest that GAs and ethylene pathways interact (Brady and McCourt, 2003; Feurtado and Kermode, 2007; Matilla and Matilla-Vazquez, 2008; Miransari and Smith, 2014).

In beechnut, incubation of embryos in the presence of $\mathrm{GA}_{3}$ results in an accumulation of ACC and an increase in ACC oxidase activity and $\mathrm{C}_{2} \mathrm{H}_{4}$ production, concomitant with an increased expression of FsACO1 (Calvo et al., 2004a). Similarly, the improving effect of $\mathrm{GA}_{4}$ on the germination of Arabidopsis ga1-3 mutant seeds is associated with an increase in AtACO (Ogawa etal., 2003). Decrease of the expression of FsACO1 in the presence of paclobutrazol, a GAs biosynthesis inhibitor, confirms that GAs activates the ethylene biosynthesis pathway (Calvo et al., 2004a,b). However, in Sisymbrium officinale, IglesiasFernandez and Matilla (2010) demonstrate that expression of SoACS7 and SoACO2 during germination is inhibited by paclobutrazol, but is not affected by application of either ethrel or $\mathrm{GA}_{4+7}$. In addition, the up-regulation of AtERS1 (ETHYLENE RESPONSE SENSOR encoding a member of ethylene receptor family) in Arabidopsis in the presence of $\mathrm{GA}_{4}$ (Ogawa etal., 2003) and of an EIN-3 like in beechnut in the presence of $\mathrm{GA}_{3}$ (Lorenzo et al., 2000) suggest an effect of GAs on ethylene response.

Numerous data also suggest that ethylene stimulates seed germination by affecting the GAs biosynthesis or signaling pathway. $\mathrm{GA}_{1}, \mathrm{GA}_{4}$, and $\mathrm{GA}_{7}$ strongly accumulate in dry mature seeds of the etr1-2 Arabidopsis mutant relative to wild type, and both $\mathrm{GA}_{4}$ and $\mathrm{GA}_{7}$ contents remain higher than in wild type during the two first days of imbibition (Chiwocha et al., 2005). The changes in GA content during germination suggest that lack of ETR1, i.e., of ethylene signaling pathway, results (i) in alteration of GAs biosynthesis pathway, and (ii) in a requirement for higher levels of GAs than wild type, to promote germination (Chiwocha et al., 2005). In beechnut, expression of FsGA20ox1, which is involved in the synthesis of active GAs, remains low in stratified seeds (i.e., non-dormant seeds) and seeds treated with $\mathrm{GA}_{3}$ or ethephon, but inhibition of ethylene biosynthesis by AOA (2-aminoxyacetic acid) results in an increase in this transcript indicating the involvement of ethylene in the regulation of GA biosynthesis (Calvo et al., 2004b). Studies of expression of genes involved in GA synthesis (SoGA3ox2 and SoGA20ox2) and degradation (SoGA2ox6) during imbibition of Sisymbrium officinale seeds in the presence of $\mathrm{GA}_{4+7}$, ethylene, and inhibitors of GA synthesis or ethylene synthesis and signaling, indicate that GA biosynthesis is strongly regulated by GA and ethylene (Iglesias-Fernandez and Matilla, 2010).

Gibberellin signaling pathways depend on DELLA proteins including GAI (GA INSENSITIVE), RGA (REPRESSOR OF ga1-3), RGL1 (RGA LIKE1), RGL2 and RGL3 (Sun and Gubler, 2004; Davière and Achard, 2013). GAs destabilizes the DELLA proteins, which act as growth repressors by targeting GAs for ubiquitination and degradation (Dill et al., 2004). In Arabidopsis, Achard et al. $(2003,2007)$ reported that a part of ethylene action on hypocotyl growth and floral transition was mediated via its effects on the DELLA proteins. This may be true too in the control of germination since DELLA proteins seem to play a key role in the regulation of seed germination (Lee et al., 2002; Tyler et al., 2004; Cao et al., 2006; Steber, 2007; Piskurewicz et al., 2008; Schwechheimer, 2008). Thus, the seed GA content and responsiveness may result from a regulation of DELLA accumulation by ethylene.

\section{ROS AND ETHYLENE INTERACT TO REGULATE SEED GERMINATION}

It has been shown in various seed species (Oracz et al., 2007; Ishibashi et al., 2008, 2013; Müller et al., 2009; Bahin et al., 2011), including Arabidopsis (Liu et al., 2010; Leymarie et al., 2012), that radicle protrusion is associated with and/or required a controlled accumulation of ROS. Regarding the role of plant hormones in seed germination and dormancy, several studies have investigated the possible relationship between metabolic and signaling pathways of these hormones, mainly ABA and GAs, and ROS homeostasis (Bailly et al., 2008). Up-to-date, however, the relationship between ROS and ethylene has been scarcely studied within the context of seed germination, although this is well documented in other contexts such as plant pathogen interactions (Mersmann et al., 2010) or cell death regulation (Overmyer et al., 2003).

In sunflower embryos, whose dormancy is released by exogenous ethylene (Corbineau etal., 1990), it has been recently demonstrated that ethylene markedly enhanced ROS accumulation within dormant embryonic axes, probably through the activation of NADPH oxidase (El-Maarouf-Bouteau et al., 2014). Whether ethylene produced in response to ROS has a direct effect on cell wall properties and cell elongation or if it stimulates cell signaling pathways related to germination, is however not known. Contrasting results obtained by Lin et al. (2012, 2013) demonstrates that ethylene decreases ROS content in Arabidopsis seeds germinating under salinity stress. In the case of the former species, ethylene has an antagonistic effect to ROS that are detrimental for germination, probably because their production increases to excessive levels in response to stress. This highlights the plasticity of seed responses to ROS but also the complexity of their interaction with ethylene (Bailly et al., 2008).

Several authors have also studied the effect of ROS on ethylene production during seed germination. Dormant sunflower embryos treated by methylviologen, a ROS generating compound, germinate rapidly at temperatures that would otherwise prevent their germination (Oracz et al., 2007). However, this improving effect is not associated with an increase in ethylene production which peaks at the time radicle elongates, and which therefore must be considered as a post-germinative event (El-MaaroufBouteau et al., 2014). These authors propose that ethylene might participate in association with ROS to facilitate the initiation of cell elongation, the first visible symptom of germination. In dormant apple embryos, Gniazdowska et al. (2010) suggest 
that the improving effect of $\mathrm{NO}$ and $\mathrm{HCN}$ results from a transient ROS production leading to an ethylene production required in termination of the sensu stricto germination process before radicle elongation and propose that this might result from a nonenzymatic oxidation of ACC. Ishibashi et al. (2013) have proposed that ROS produced in soybean (Glycine max) embryonic axes during imbibition induces ethylene production, which promotes cell elongation in the radicle. However, in that case, ethylene was measured after the onset of radicle protrusion, and this production was probably more related to the kinetics of seedling elongation than on a direct effect of $\mathrm{H}_{2} \mathrm{O}_{2}$. In contrast, in pea, which germination is not strongly regulated by ethylene, Barba-Espin et al. (2011) demonstrate that $\mathrm{H}_{2} \mathrm{O}_{2}$ treatment results in a reduction in PsACS2 transcript abundance consistent with a decrease in ACC content. These results suggest that ROS and ethylene probably do not interact directly, but rather through a complex hormonal network (Diaz-Vivancos et al., 2013).

All together these data suggest that the interaction between ROS and ethylene in seeds can operate in both directions, depending on the physiological context of germination, i.e., on the environmental conditions prevailing during imbibition, and is highly species related. One can predict that the use of seeds of the plant model Arabidopsis will help decipher the molecular bases of this interaction. In particular it will be interesting to determine whether ROS can trigger expression of the ethylene signaling pathway components and vice versa. For example, Oracz et al. (2009) has demonstrated the occurrence of such a cross talk, since the treatment of dormant sunflower embryos by methylviologen induced the expression of ETR2 and of the transcription factor ERF1. The involvement of ethylene transcription factors in response to ROS appears to be worth investigating since studies with other plant systems have also implicated such a relationship (Sewelam et al., 2013).

\section{CONCLUSION: NETWORK BETWEEN ETHYLENE, PLANT HORMONES, AND ROS}

Seed germination is regulated by ethylene in a complex signaling network, which is also operational in numerous developmental processes, including vegetative growth, flowering timing, fruit ripening and organ senescence and abscission (Yoo et al., 2009; Muday et al., 2012; Arc et al., 2013). As mentioned above, ethylene interacts with ABA and GAs, both hormones being essential regulators of germination and dormancy (Feurtado and Kermode, 2007; Nambara et al., 2010; Nonogaki et al., 2010; Miransari and Smith, 2014). Thus, the improving effect of ethylene may occur via the involvement of $\mathrm{C}_{2} \mathrm{H}_{4}$-GAs-ABA crosstalk but whether its action is direct or indirect needs clarification. Research on the effect of $\mathrm{ABA}$ and $\mathrm{GAs}$ on $\mathrm{C}_{2} \mathrm{H}_{4}$ biosynthesis and signaling pathways, especially in seeds, would then require further investigation, specifically in relation with ROS. Figure 2 summarizes the current data concerning ABA-GAs- $\mathrm{C}_{2} \mathrm{H}_{4}$ networks based on genetic analyses, microarray data, and physiological studies. ABA inhibits the $\mathrm{C}_{2} \mathrm{H}_{4}$ biosynthesis pathway via an inhibitory action on ACO activity and on the ACO transcript accumulation. On the contrary, $\mathrm{C}_{2} \mathrm{H}_{4}$ counteracts both ABA synthesis and signaling, ETR1 having a key role. In addition, $\mathrm{C}_{2} \mathrm{H}_{4}$ affects the synthesis of GAs via modification of

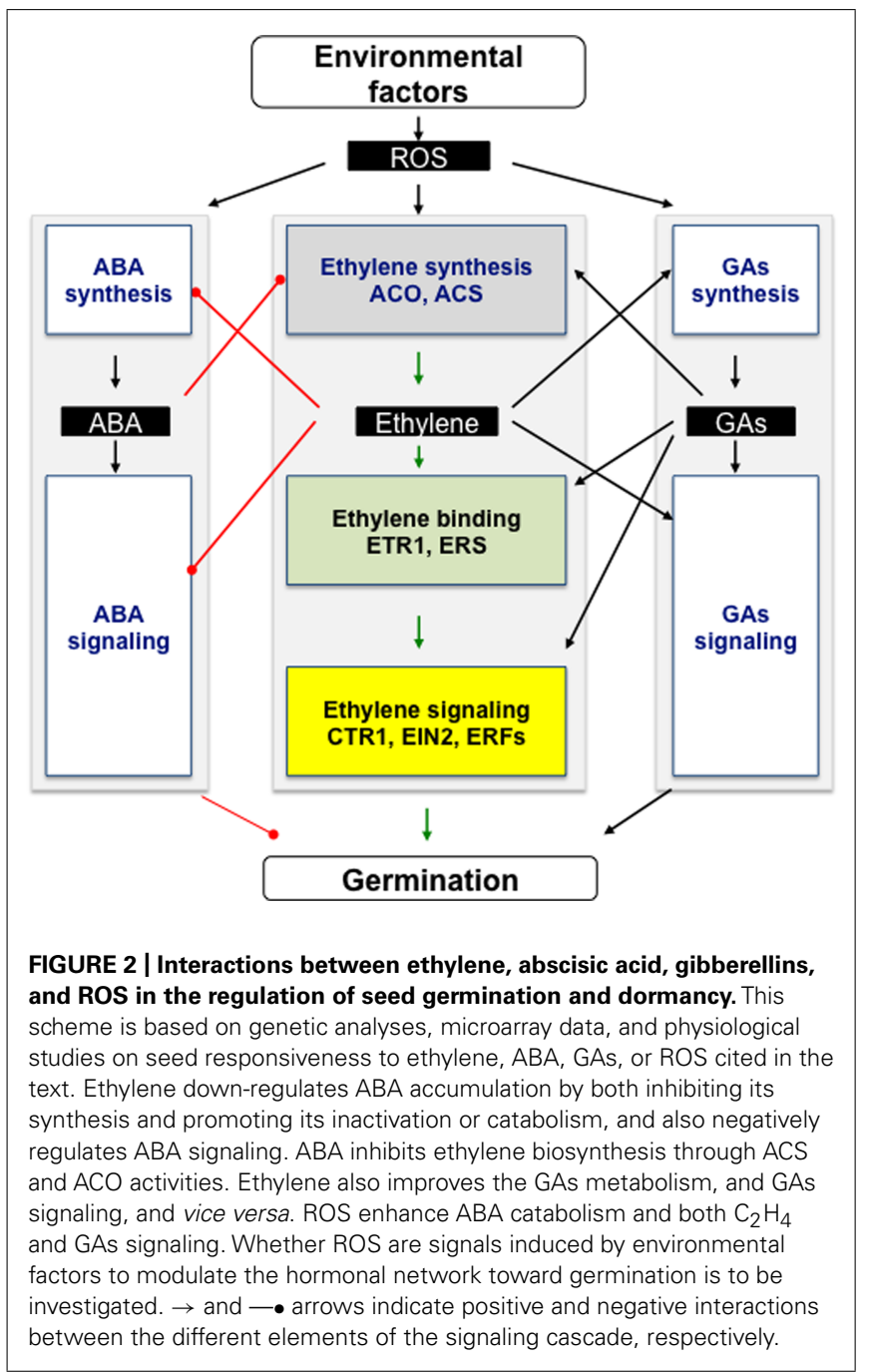

expression of genes (GA3ox and GA20ox) involved in GAs synthesis. Ethylene also probably modifies the GAs signaling pathway via a regulation of DELLA proteins, as demonstrated in growth processes (Achard et al., 2003, 2007). To add to the complexity of the ABA-GAs- $\mathrm{C}_{2} \mathrm{H}_{4}$ network, there are antagonistic interactions between $\mathrm{ABA}$ and $\mathrm{GAs}, \mathrm{C}_{2} \mathrm{H}_{4}$ and brassinosteroids, jasmonates and auxins (Wang et al., 2002; Brady and McCourt, 2003; Weiss and Ori, 2007; Finkelstein et al., 2008; Matilla and Matilla-Vazquez, 2008; Cheng et al., 2009; Divi et al., 2010; Linkies and LeubnerMetzger, 2012). ROS also regulate seed germination through hormonal networks, in particular with ABA and GAs (Bethke et al., 2007; Liu et al., 2010; Diaz-Vivancos et al., 2013). It would be then important to discriminate the hierarchy of the different signaling pathways, and their role as sensor of environmental signals.

Omics studies are now available in the field of seed germination but efforts to develop transcriptomic analysis of ethylene action are required to understand ethylene involvement in seed germination. Analysis of the effects of ethylene on specific cellular processes highlighted by dormancy and germination studies such as transcription regulation, cell cycle activity and endosperm weakening 
should help to understand the regulatory network of germination process in seeds. Moreover, although hormonal signaling network share common components, they may work in specific territories in seeds.

\section{REFERENCES}

Abeles, F. B. (1986). Role of ethylene in Lactuca sativa cv Grand Rapids seed germination. Plant Physiol. 81, 780-787. doi: 10.1104/pp.81.3.780

Achard, P., Baghour, M., Chapple, A., Hedden, P., Van Der Straeten, D., Genschik, P., et al. (2007). The plant stress hormone ethylene controls floral transition via DELLA-dependent regulation of floral meristem-identity genes. Proc. Natl. Acad. Sci. U.S.A. 104, 6484-6489. doi: 10.1073/pnas.061 0717104

Achard, P., Vriezen, W. H., Van Der Straeten, D., and Harberd, N. P. (2003). Ethylene regulates Arabidopsis development via the modulation of DELLA protein growth repressor function. Plant Cell 15, 2816-2825. doi: 10.1105/tpc.015685

Arc, E., Sechet, J., Corbineau, F., Rajjou, L., and Marion-Poll, A. (2013). ABA crosstalk with ethylene and nitric oxide in seed dormancy and germination. Front. Plant Sci. 4:63. doi: 10.3389/fpls.2013.00063

Argyris, J., Dahal, P., Hayashi, E., Still, D. W., and Bradford, K. J. (2008). Genetic variation for lettuce seed thermoinhibition is associated with temperature-sensitive expression of abscisic acid, gibberellin, and ethylene biosynthesis, metabolism, and response genes. Plant Physiol. 148, 926-947. doi: 10.1104/pp.108. 125807

Bahin, E., Bailly, C., Sotta, B., Kranner, I., Corbineau, F., and Leymarie, J. (2011). Crosstalk between reactive oxygen species and hormonal signaling pathways regulates grain dormancy in barley. Plant Cell Environ. 34, 980-993. doi: 10.1111/j.1365-3040.2011.02298.x

Bailly, C., Corbineau, F., and Côme, D. (1992). The effects of abscisic acid and methyl jasmonate on 1-aminocyclopropane 1-carboxylic acid conversion to ethylene in hypocotyl segments of sunflower seedlings, and their control by calcium and calmodulin. Plant Growth Regul. 11, 349-355. doi: 10.1007/BF00130641

Bailly, C., El-Maarouf-Bouteau, H., and Corbineau, F. (2008). From intracellular signaling networks to cell death: the dual role of reactive oxygen species in seed physiology. C. R. Biol. 331, 806-814. doi: 10.1016/j.crvi.2008.07.022

Barba-Espin, G., Diaz-Vivancos, P., Job, D., Belghazi, M., Job, C., and Hernandez, J. A. (2011). Understanding the role of $\mathrm{H}_{2} \mathrm{O}_{2}$ during pea seed germination: a combined proteomic and hormone profiling approach. Plant Cell Environ. 34, 1907-1919. doi: 10.1111/j.1365-3040.2011.02386.x

Beaudoin, N., Serizet, C., Gosti, F., and Giraudat, J. (2000). Interactions between abscisic acid and ethylene signaling cascades. Plant Cell 12, 1103-1115. doi: 10.1105/tpc.12.7.1103

Bebawi, F. F., and Eplee, R. E. (1986). Efficacy of ethylene as a germination stimulant of Striga hermonthica. Weed Sci. 34, 694-698.

Bethke, P. C., Libourel, I. G. L., and Jones, R. L. (2007). "Nitric oxide in seed dormancy and germination," in Annual Plant Reviews, Vol. 27, Seed Development, Dormancy and Germination, eds K. Bradford and H. Nonogaki (Oxford: Blackwell Publishing Ltd), 153-175.

Bewley, J. D. (1997). Seed germination and dormancy. Plant Cell 9, 1055-1066. doi: 10.1105/tpc.9.7.1055

Bewley, J. D., and Black, M. (1994). Seeds: Physiology of Development and Germination. NewYork: Plenum Press. doi: 10.1007/978-1-4899-1002-8

Bleecker, A. B., Estelle, M. A., Somerville, C., and Kende, H. (1988). Insensitivity to ethylene conferred by a dominant mutation in Arabidopsis thaliana. Science 241, 1086-1089. doi: 10.1126/science. 241.4869 .1086

Bleecker, A. B., and Kende, H. (2000). Ethylene: a gaseous signal molecule in plants. Annu. Rev. Cell Dev. Biol. 16, 1-18. doi: 10.1146/annurev.cellbio.16.1.1

Brady, S. M., and McCourt, P. (2003). Hormone cross-talk in seed dormancy. J. Plant Growth 22, 25-31. doi: 10.1007/s00344-003-0018-7

Brown, N. A. C., and van Staden, J. (1997). Smoke as a germination cue: a review. Plant Growth Regul. 22, 115-124. doi: 10.1023/A:1005852018644

Burdett, A. N., and Vidaver, W. (1971). Synergistic action of ethylene with gibberellin or red light in germinating lettuce seeds. Plant Physiol. 48, 656-657. doi: 10.1104/pp.48.5.656

Cadman, C. S. C., Toorop, P. E., Hilhorst, H. W. M., and Finch-Savage, W. E. (2006). Gene expression profiles of Arabidopsis Cvi seeds during dormancy cycling indicate a common underlying dormancy control mechanism. Plant J. 46, 805822. doi: 10.1111/j.1365-313X.2006.02738.x
Calvo, A. P., Nicolas, C., Lorenzo, O., Nicolas, G., and Rodriguez, D. (2004a). Evidence for positive regulation by gibberellins and ethylene of ACC oxidase expression and activity during transition from dormancy to germination in Fagus sylvatica L. seeds. J. Plant Growth Regul. 23, 44-53. doi: 10.1007/s00344-0040074-7

Calvo, A. P., Nicolas, C., Nicolas, G., and Rodriguez, D. (2004b). Evidence of a crosstalk regulation of a GA 20-oxidase (FsGA20oxl) by gibberellins and ethylene during the breaking of dormancy in Fagus sylvatica seeds. Physiol. Plant. 120, 623-630. doi: 10.1111/j.0031-9317.2004.0270.x

Cao, D., Cheng, H., Wu, W., Soo, H. M., and Peng, J. (2006). Gibberellin mobilizes distinct DELLA-dependent transcriptomes to regulate seed germination and floral development in Arabidopsis. Plant Physiol. 142, 509-525. doi: 10.1104/pp.106.082289

Carrera, E., Holman, T., Medhurst, A., Dietrich, D., Footitt, S., Theodoulou, F. L., etal. (2008). Seed after-ripening is a discrete developmental pathway associated with specific gene networks in Arabidopsis. Plant J. 142, 1493-1510.

Cheng, W. H., Chiang, M. H., Hwang, S. G., and Lin, P. C. (2009). Antagonism between abscisic acid and ethylene in Arabidopsis acts in parallel with the reciprocal regulation of their metabolism and signaling pathways. Plant Mol. Biol. 71, 61-80. doi: 10.1007/s11103-009-9509-7

Chitnis, V. R., Gao, F., Yao, Z., Jordan, M. C., Park, S., and Ayele, B. T. (2014). After-ripening induced transcriptional changes of hormonal genes in wheat seeds: the cases of brassinosteroids, ethylene, cytokinin and salicylic acid. PLoS ONE 9:e87543. doi: 10.1371/journal.pone.0087543

Chiwocha, S. D. S., Cutler, A. J., Abrams, S. R., Ambrose, S. J., Yang, J., and Kermode, A. R. (2005). The ert1-2 mutation in Arabidopsis thaliana affects the abscisic acid, auxin, cytokinin and gibberellin metabolic pathways during maintenance of seed dormancy, moist-chilling and germination. Plant J. 42, 35-48. doi: $10.1111 /$ j.1365-313X.2005.02359.x

Chonowski, M., Corbineau, F., and Côme, D. (1997). Physiological and biochemical changes induced in sunflower seeds by osmopriming and subsequent drying, storage and aging. Seed Sci. Res. 7, 323-332. doi: 10.1017/S0960258500 $00372 \mathrm{X}$

Corbineau, F. (2012). Markers of seed quality: from present to future. Seed Sci. Res. 22, S61-S68. doi: 10.1017/S0960258511000419

Corbineau, F., Bagniol, S., and Côme, D. (1990). Sunflower (Helianthus annuus L.) seed dormancy and its regulation by ethylene. Isr. J. Bot. 39, 313-325.

Corbineau, F., and Côme, D. (1992). "Germination of sunflower seeds and its regulation by ethylene," in Advances in the Science and Technology of Seeds, eds F. Jiarui and A. A. Khan (Beijing, NY: Science Press), 277-287.

Corbineau, F., and Côme, D. (1995). "Control of seed germination and dormancy by gaseous environment," in Seed Development and Germination, eds J. Kigel and G. Galili (New York: Marcel Dekker), 397-424.

Corbineau, F., and Côme, D. (2003). "Germination of sunflower seeds as related to ethylene synthesis and sensitivity - an overview," in Biology and Biotechnology of the Plant Hormone Ethylene III, eds M. Vendrell, H. Klee, J. C. Pech, and F. Romojaro (Amsterdam: IOS Press), 216-221.

Corbineau, F., Rudnicki, R. M., and Côme, D. (1988). Induction of secondary dormancy in sunflower seeds by high temperature. Possible involvement of ethylene biosynthesis. Physiol. Plant. 73, 368-373. doi: 10.1111/j.13993054.1988.tb00612.x

Cristescu, S. M., Persijn, S. T., Lintel Hekkert, S., and Harren, F. J. M. (2008). Laserbased systems for trace gas detection in life sciences. Appl. Phys. B 92, 343-349. doi: $10.1007 / \mathrm{s} 00340-008-3127-y$

Cutler, S. R., Rodriguez, P. L., Finkelstein, R. R., and Abrams, S. R. (2010). Abscisic acid: emergence of a core signaling network. Annu. Rev. Plant Biol. 61, 651-679. doi: 10.1146/annurev-arplant-042809-112122

Davière, J.-M., and Achard, P. (2013). Gibberellin signalling in plants. Development 140, 1147-1151. doi: 10.1242/dev.087650

Diaz-Vivancos, P., Barba-Espin, G., and Hernandez, J. A. (2013). Elucidating hormonal/ROS networks during germination: insights and perspectives. Plant Cell Rep. 32, 1491-1502. doi: 10.1007/s00299-013-1473-7

Dill, A., Thomas, S. G., Steber, C. M., and Sun, T.-p. (2004). The Arabidopsis F-box protein SLEEPY1 targets gibberellin signaling repressors for gibberellin-induced degradation. Plant Cell 16, 1392-1405. doi: 10.1105/tpc.020958

Divi, U. K., Rahman, T., and Krishna, P. (2010). Brassinosteroid-mediated stress tolerance in Arabidopsis shows interactions with abscisic acid, ethylene and salicylic acid pathways. BMC Plant Biol. 10:151. doi: 10.1186/1471-2229-10-151 
Dong, H., Zhen, Z., Peng, J., Chang, L., Gong, Q., and Wang, N. N. (2011). Loss of ACS7 confers abiotic stress tolerance by modulating ABA sensitivity and accumulation in Arabidopsis. J. Exp. Bot. 62, 4875-4887. doi: 10.1093/jxb/ err 143

Egley, G. H., and Dale, J. E. (1970). Ethylene, 2-chloroethylphosphonic acid and witchweed germination. Weed Sci. 18, 586-589.

El-Maarouf-Bouteau, H., Sajjad, Y., Bazin, J., Langlade, N., Cristescu, S. M., Balzergue, S., et al. (2014). Reactive oxygen species, abscisic acid and ethylene interact to regulate sunflower seed germination. Plant Cell Environ. doi: 10.1111/pce.12371 [Epub ahead of print].

Esashi, Y. (1991). "Ethylene and seed germination," in The Plant Hormone Ethylene, eds A. K. Mattoo and J. C. Suttle (Boca Raton, FL: CRC Press), 133-157.

Esashi, Y., Abe, Y., Ashino, H., Ishizawa, K., and Saitoh, K. (1989). Germination of cocklebur seeds and growth of their axial and cotyledonary tissues in response to $\mathrm{C}_{2} \mathrm{H}_{4}, \mathrm{CO}_{2}$ and/or $\mathrm{O}_{2}$ under water stress. Plant Cell Environ. 12, 183-190. doi 10.1111/j.1365-3040.1989.tb01931.x

Esashi, Y., Hata, Y., and Katoh, H. (1975). Germination of cocklebur seeds: interactions between gibberellic acid, benzyladenine, thiourea, KNO3 and gaseous factors. Aust. J. Plant Physiol. 2, 569-579. doi: 10.1071/PP9750569

Esashi, Y., and Leopold, A. C. (1969). Dormancy regulation in subterranean clover seeds by ethylene. Plant Physiol. 44, 1470-1472. doi: 10.1104/pp.44.10.1470

Esashi, Y., Okazaki, M., Yanai, N., and Hishinuma, K. (1978). Control of the germination of secondary dormant cocklebur seeds by various germination stimulants. Plant Cell Physiol. 19, 1497-1506.

Esashi, Y., Ooshima, Y., Abe, M., Kurota, A., and Satoh, S. (1986). CO2-enhanced $\mathrm{C}_{2} \mathrm{H}_{4}$ production in tissues of imbibed cocklebur seeds. Aust. J. Plant Physiol. 13, 417-429. doi: 10.1071/PP9860417

Feurtado, J. A., and Kermode, A. R. (2007). "A merging of paths: abscisic acid and hormonal cross-talk in the control of seed dormancy maintenance and alleviation," in Annual Plant Reviews, Vol. 27, Seed Development, Dormancy and Germination, eds K. Bradford and H. Nonogaki (Oxford: Blackwell Publishing Ltd), 176-223. doi: 10.1002/9780470988848.ch8

Finkelstein, R. R., Reeves, W., Ariizumi, T., and Steber, C. (2008). Molecular aspects of seed dormancy. Annu. Rev. Plant Biol. 59, 387-415. doi: 10.1146/annurev.arplant.59.032607.092740

Fu, J. R., and Yang, S. F. (1983). Release of heat pretreatment-induced dormancy in lettuce seeds by ethylene or cytokinin in relation to the production of ethylene and the synthesis of 1-aminocyclopropane-1-carboxylic acid during germination. J. Plant Growth Regul. 2, 185-192. doi: 10.1007/BF02042247

Gallardo, M., Del Mar Delgado, M., Sanchez-Calle, I. M., and Matilla, A. J. (1991) Ethylene production and 1-aminocyclopropane-1-carboxylic acid conjugation in thermoinhibited Cicer arietinum L. seeds. Plant Physiol. 97, 122-127. doi: 10.1104/pp.97.1.122

Gallardo, M., Gallardi, M. E., Matilla, A., Munoz de Ruedo, P., and Sanchez-Calle, I. M. (1994). Inhibition of polyamine synthesis by cyclohexylamine stimulates the ethylene pathway and accelerates the germination of Cicer arietinum seeds. Physiol. Plant. 91, 9-16. doi: 10.1111/j.1399-3054.1994.tb00652.x

Ghassemian, M., Nambara, E., Cutler, S., Kawaide, H., Kamiya, Y., and McCourt, P. (2000). Regulation of abscisic acid signaling by the ethylene response pathway in Arabidopsis. Plant Cell 12, 1117-1126. doi: 10.1105/tpc.12.7.1117

Gniazdowska, A., Krasuska, U., and Bogatek, R. (2010). Dormancy removal in apple embryos by nitric oxide or cyanide involves modifications in ethylene biosynthetic pathway. Planta 232, 1397-1407. doi: 10.1007/s00425-010-1262-2

Gomez-Jimenez, M. C., Matilla, A. J., and Garrido, D. (1998). Isolation and characterization of a cDNA encoding an ACC oxidase from Cicer arietinum and its expression during embryogenesis and seed germination. Aust. J. Plant Physiol. 25, 765-773. doi: 10.1071/PP97166

Gorecki, R. J., Ashino, H., Satoh, S., and Esashi, Y. (1991). Ethylene production in pea and cocklebur seeds of differing vigour. J. Exp. Bot. 42, 407-414. doi: 10.1093/jxb/42.3.407

Graeber, K., Nakabayashi, K., Miatton, E., Leubner-Metzger, G., and Soppe, W. J. (2012). Molecular mechanisms of seed dormancy. Plant Cell Environ. 35, 1769 1786. doi: 10.1111/j.1365-3040.2012.02542.x

Groot, S. P., and Karssen, C. M. (1987). Gibberellins regulate seed germination in tomato by endosperm weakening: a study with gibberellin-deficient mutants. Planta 171, 525-531. doi: 10.1007/BF00392302

Groot, S. P., Kieliszewska-Rokicka, B., Vermeer, E., and Karssen, C. M. (1988). Gibberellin-induced hydrolysis of endosperm cell walls in gibberellin- deficient tomato seeds prior to radicle protrusion. Planta 174, 500-504. doi: 10.1007/BF00634479

Hall, B., Shakeel, S., and Schaller, G. (2007). Ethylene receptors: ethylene perception and signal transduction. J. Plant Growth Regul. 26, 118-130. doi: 10.1007/s00344007-9000-0

Hall, M. A., Moshkov, I. E., Novikova, G. V., Mur, L. A. J., and Smith, A. R. (2001). Ethylene signal perception and transduction: multiple paradigms? Biol. Rev. 76, 103-128. doi: 10.1017/S1464793100005649

Halloin, J. M. (1976). Inhibition of cotton seed germination with abscisic acid and its reversal. Plant Physiol. 57, 454-455. doi: 10.1104/pp.57.3.454

Hermann, K., Meinhard, J., Dobrev, P., Linkies, A., Pesek, B., Hess, B., et al. (2007). 1-amynocyclopropane-1-carboxylic acid and abscisic acid during the germination of sugar beet (Beta vulgaris L.): a comparative study of fruits and seeds. J. Exp. Bot. 58, 3047-3060. doi: 10.1093/jxb/erm162

Hilhorst, H. W. M. (2007). "Definitions and hypotheses of seed dormancy," in Annual Plant Reviews, Vol. 27, Seed Development, Dormancy and Germination, eds K. Bradford and H. Nonogaki (Oxford: Blackwell Publishing Ltd), 50-71. doi: 10.1002/9780470988848.ch3

Hilhorst, H. W. M., Finch-Savage, W. E., Buitink, J., Bolingue, W., and LeubnerMetzger, G. (2010). "Dormancy in plant seeds," in Dormancy and Resistance in Harsh Environment, eds E. Lubzens, J. Cerdà, and M. Clarck (Berlin: Springer), 43-67. doi: 10.1007/978-3-642-12422-8_4

Iglesias-Fernandez, R., and Matilla, A. (2009). After-ripening alters the gene expression pattern of oxidases involved in the ethylene and gibberellin pathways during early imbibition of Sisymbrium officinale L. seeds. J. Exp. Bot. 60, 1645-1661. doi: 10.1093/jxb/erp029

Iglesias-Fernandez, R., and Matilla, A. (2010). Genes involved in ethylene and gibberellins metabolism are required for endosperm-limited germination of Sisymbrium officinale L. seeds. Planta 231, 653-664. doi: 10.1007/s00425-0091073-5

Ishibashi, Y., Koda, Y., Zheng, S.-H., Yuasa, T., and Iwaya-Inoue, M. (2013). Regulation of soybean seed germination through ethylene production in response to reactive oxygen species. Ann. Bot. 111, 95-102. doi: 10.1093/aob/ $\operatorname{mcs} 240$

Ishibashi, Y., Yamamoto, K., Tawaratsumida, T., Yuasa, T., and Iwaya-Inoue, M. (2008). Hydrogen peroxide scavenging regulates germination ability during wheat (Triticum aestivum L.) seed maturation. Plant Signal. Behav. 3, 183-188. doi: $10.4161 /$ psb.3.3.5540

Jimenez, J. A., Rodriguez, D., Calvo, A. P., Mortensen, L. C., Nicolas, G., and Nicolas, C. (2005). Expression of a transcription factor (FsERF1) involved in ethylene signaling during the breaking of dormancy in Fagus sylvatica seeds. Physiol. Plant. 125, 373-380. doi: 10.1111/j.1399-3054.2005.00571.x

Jones, J. F., and Hall, M. A. (1984). Studies on the requirement for carbon dioxide and ethylene for germination of Spergula arvensis L. seeds. Plant Sci. Lett. 16, 87-93. doi: 10.1016/0304-4211(79)90012-9

Ju, C., Yoon, G. M., Shemansky, J. M., Lin, D. Y., Ying, Z. I., Chang, J., et al. (2012). CTR1 phosphorylates the central regulator EIN2 to control ethylene hormone signaling from the ER membrane to the nucleus in Arabidopsis. Proc. Natl. Acad. Sci. U.S.A. 109, 19486-19491. doi: 10.1073/pnas.1214848109

Karssen, C. M. (1976). Two sites of hormonal action during germination of Chenopodium album seeds. Physiol. Plant. 36, 264-270. doi: 10.1111/j.13993054.1976.tb04426.x

Karssen, C. M., Zagórsky, S., Kepczynski, J., and Groot, S. P. C. (1989). Key role for endogenous gibberellins in the control of seed germination. Ann. Bot. 63, 71-80.

Katoh, H., and Esashi, Y. (1975). Dormancy and impotency of cocklebur seeds. I. $\mathrm{CO}_{2}, \mathrm{C}_{2} \mathrm{H}_{4}, \mathrm{O}_{2}$ and high temperature. Plant Cell Physiol. 16, 687-696. doi: $10.1093 / \mathrm{jxb} / 35.10 .1515$

Kende, H. (1993). Ethylene biosynthesis. Annu. Rev. Plant Physiol. Plant Mol. Biol. 44, 283-307. doi: 10.1146/annurev.pp.44.060193.001435

Kende, H., van der Knaap, E., and Cho, H. T. (1998). Deepwater rice: a model plant to study stem elongation. Plant Physiol. 118, 1105-1110. doi: 10.1104/pp.118.4.1105 Kendrick, M. D., and Chang, C. (2008). Ethylene signaling: new levels of complexity and regulation. Curr. Opin. Plant Biol. 11, 479-485. doi: 10.1016/j.pbi.2008.06.011

Kepczynski, J. (1986). Inhibition of Amaranthus caudatus seed germination by polyethylene glycol-6000 and abscisic acid and its reversal by ethephon or aminocyclopropane-1-carboxylic acid. Physiol. Plant. 67, 588-591. doi: 10.1111/j.1399-3054.1986.tb05060.x 
Kepczynski, J., Bihun, M., and Kepczynska, E. (1996a). Induction and release of secondary dormancy in Amaranthus caudatus L. seeds. Plant Physiol. Biochem. Special Issue S03-S50, 42.

Kepczynski, J., Corbineau, F., and Côme, D. (1996b). Responsiveness of Amaranthus retroflexus seeds to ethephon, 1-aminocyclopropane-1-carboxylic acid and gibberellic acid in relation to temperature and dormancy. Plant Growth Regul. 20, 259-265. doi: 10.1007/BF00043316

Kepczynski, J., Bihun, M., and Kepczynska, E. (2003). The release of secondary dormancy by ethylene in Amaranthus caudatus L. seeds. Seed Sci. Res. 13, 69-74. doi: $10.1079 /$ SSR2002125

Kepczynski, J., and Karssen, C. M. (1985). Requirement for the action of endogenous ethylene during germination of non-dormant seeds of Amaranthus caudatus. Physiol. Plant. 63, 49-52. doi: 10.1111/j.1399-3054.1985.tb02816.x

Kepczynski, J., and Kepczynska, E. (1993). "The effect of putrescine, ethephon and ACC on germination of thermodormant Amaranthus paniculatus L. seed," in Basic and Applied Aspects of Seed Biology, Fourth International Workshop on Seeds, ed D. Côme and F. Corbineau (Paris: ASFIS), 537-554.

Kepczynski, J., and Kepczynska, E. (1997). Ethylene in seed dormancy and germination. Physiol. Plant. 101, 720-726. doi: 10.1034/j.1399-3054.1997.1010407.x

Kepczynski, J., Rudnicki, R. M., and Khan, A. A. (1977). Ethylene requirement for germination of partly after-ripened apple embryo. Physiol. Plant. 40, 292-295. doi: 10.1111/j.1399-3054.1977.tb04075.x

Kepczynski, J., and Sznigir, P. (2014). Participation of GA3, ethylene, NO and HCN in germination of Amaranthus retroflexus L. seeds with various dormancy levels. Acta Physiol. Plant. 36, 1463-1472. doi: 10.1007/s11738-014-1524-x

Ketring, D. L., and Morgan, P. W. (1969). Ethylene as a component of the emanations from germinating peanut seeds and its effect on dormant Virginia-type seeds. Plant Physiol. 44, 326-330. doi: 10.1104/pp.44.3.326

Ketring, D. L., and Morgan, P. W. (1972). Physiology of oil seeds. IV. Role of endogenous ethylene and inhibitory regulators during natural and induced afterripening of dormant Virginia-type peanut seeds. Plant Physiol. 50, 382-387. doi: 10.1104/pp.50.3.382

Ketring, D. L., Morgan, P. W., and Powell, R. D. (1974). "Relations of ethylene production to germinability and growth of two oil seeds, cotton and peanuts," in Plant growth substances 1973, ed. Y. Sumiki (Tokyo: Hirokawa), 891-899.

Keys, R. D., Smith, O. E., Kumamoto, J., and Lyon, J. J. (1975). Effect of gibberellic acid, kinetin, and ethylene plus carbon dioxide on the thermodormancy of lettuce seed (Lactuca sativa L. cv Mesa 659). Plant Physiol. 56, 826-829. doi: 10.1104/pp.56.6.826

Khan, A. A. (1994). ACC-derived ethylene production, a sensitive test for seed vigor. J. Am. Soc. Hort. Sci. 119, 1083-1090.

Khan, M. A., Ansari, R., Gul, B., and Li, W. Q. (2009). Dormancy and germination responses of halophyte seeds to the application of ethylene. C. R. Biol. 332, 806815. doi: 10.1016/j.crvi.2009.05.002

Kucera, B., Cohn, M. A., and Leubner-Metzger, G. (2005). Plant hormone interactions during seed dormancy release and germination. Seed Sci. Res. 15, 281-307. doi: $10.1079 /$ SSR2005218

Krasuska, U., Ciacka, K., Debska, K., Bogatek, R., and Gniazdowska, A. (2014). Dormancy alleviation by NO or HCN leading to decline of protein carbonylation levels in apple (Malus domestica Borkh.) embryos. J. Plant Physiol. 171, 11321141. doi: 10.1016/j.jplph.2014.04.015

Krasuska, U., and Gniazdowska, A. (2012). Nitric oxide and hydrogen cyanide as regulating factors of enzymatic antioxidant system in germinating apple embryos. Acta Physiol. Plant. 34, 683-692. doi: 10.1007/s11738-011-0868-8

Lee, S., Cheng, H., King, K. E., King, K. E., Wang, W., He, Y., et al. (2002). Gibberellin regulates Arabidopsis seed germination via RGL2, a GAI/RGA-like gene whose expression is up-regulated following imbibition. Genes Dev. 16, 646-658. doi: 10.1101/gad.969002

Leubner-Metzger, G., Petruzzelli, L., Waldvogel, R., Vogeli-Lange, R., and Meins, F. (1998). Ethylene-responsive element binding protein (EREBP) expression and the transcriptional regulation of class I beta-1,3-glucanase during tobacco seed germination. Plant Mol. Biol. 38, 785-795. doi: 10.1023/A:1006040425383

Lewak, S. (2011). Metabolic control of embryonic dormancy in apple seed: seven decades of research. Acta Physiol. Plant. 33, 1-24. doi: 10.1007/s11738-0100524-8

Leymarie, J., Vitkauskaite, G., Hoang, H. H., Gendreau, E., Chazoule, V., Meimoun P., et al. (2012). Role of reactive oxygen species in the regulation of Arabidopsis seed dormancy. Plant Cell Physiol. 53, 96-106. doi: 10.1093/pcp/pcr129
Lin, Y., Yang, L., Paul, M., Zu, Y., and Tang, Z. (2013). Ethylene promotes germination of Arabidopsis seed under salinity by decreasing reactive oxygen species: evidence for the involvement of nitric oxide simulated by sodium niroprusside. Plant Physiol. Biochem. 73, 211-218. doi: 10.1016/j.plaphy.2013.10.003

Lin, Y. C., Wang, J. J., Zu, Y. G., and Tang, Z. H. (2012). Ethylene antagonizes the inhibition of germination in Arabidopsis induced by salinity by modulating the concentration of hydrogen peroxide. Acta Physiol. Plant. 34, 1895-1904. doi: 10.1007/s11738-012-0989-8

Lin, Z., Zhong, S., and Grierson, D. (2009). Recent advances in ethylene research. J. Exp. Bot. 60, 3311-3336. doi: 10.1093/jxb/erp204

Linkies, A., and Leubner-Metzger, G. (2012). Beyond gibberellins and abscisic acid: how ethylene and jasmonates control seed germination. Plant Cell Rep. 31, 253270. doi: 10.1007/s00299-011-1180-1

Linkies, A., Muller, K., Morris, K., Tureckova, V., Wenk, M., Cadman, C. S., et al. (2009). Ethylene interacts with abscisic acid to regulate endosperm rupture during germination: a comparative approach using Lepidium sativum and Arabidopsis thaliana. Plant Cell 21, 3803-3822. doi: 10.1105/tpc.109.070201

Liu, Q., Zhou, G. Y., and Wen, C. K. (2004). Ethylene signal transduction in Arabidopsis. J. Plant Physiol. Mol. Biol. 30, 241-250.

Liu, Y., Ye, N., Liu, R., Chen, M., and Zhang, J. (2010). $\mathrm{H}_{2} \mathrm{O}_{2}$ mediates the regulation of ABA catabolism and GA biosynthesis in Arabidopsis seed dormancy and germination. J. Exp. Bot. 61, 2979-2990. doi: 10.1093/jxb/erq125

Longan, D. C., and Stewart, G. R. (1992). Germination of the seeds of parasitic angiosperms. Seed Sci. Res. 2, 179-190.

Lorenzo,O., Rodriguez, D., Nicolas, C., and Nicolas, G. (2000). "Characterization and expression of two protein kinase and an EIN3-like genes, which are regulated by ABA and GA3 in dormant Fagus sylvatica seeds," in Seed Biology: Advances and Applications, eds M. Black, K. J. Bradford, and J. Vazquez-Ramos (Wallingford: CAB International), 329-340.

Machabée, S., and Saini, H. S. (1991). Differences in requirement for endogenous ethylene during germination of dormant and non-dormant seeds of Chenopodium album L. J. Plant Physiol. 138, 97-101. doi: 10.1016/S01761617(11)80737-6

Martinez-Reina, G., Matilla, A. J., Martin-Remesal, C., Gallardo, M., and Munoz De Rueda, P. (1996). Biochemical properties of 1-aminocyclopropane-1-carboxylate $N$-malonyl transferase activity from early growing embryonic axes of chickpea (Cicer arietinum L.) seeds. J. Exp. Bot. 47, 1771-1778. doi: 10.1093/jxb/47. 11.1771

Matilla, A. J. (1996). Polyamines and seed germination. Seed Sci. Res. 6, 81-93. doi: $10.1017 /$ S096025850000310X

Matilla, A. J. (2000). Ethylene in seed formation and germination. Seed Sci. Res. 10, 111-126. doi: 10.1017/S096025850000012X

Matilla, A. J., and Matilla-Vazquez, M. A. (2008). Involvement of ethylene in seed physiology. Plant Sci. 175, 87-97. doi: 10.1016/j.plantsci.2008.01.014

Mersmann, S., Bourdais, G., Rietz, S., and Robatzek, S. (2010). Ethylene signaling regulates accumulation of the FLS2 receptor and is required for the oxidative burst contributing to plant immunity. Plant Physiol. 154, 391-400. doi: 10.1104/pp.110.154567

Miransari, M., and Smith, D. L. (2014). Plant hormones and seed germination. Environ. Exp. Bot. 99, 110-121. doi: 10.1016/j.envexpbot.2013.11.005

Muday, G. K., Rahman, A., and Binder, B. M. (2012). Auxin and ethylene: collaborators or competitors? Trends Plant Sci. 17, 181-195. doi: 10.1016/j.tplants.2012.02.001

Müller, K., Linkies, A., Vreeburg, R. A. M., Fry, S. C., Krieger-Liszkay, A., and Leubner-Metzger, G. (2009). In vivo cell wall loosening by hydroxyl radicals during cress (Lepidium sativum L.) seed germination and elongation growth. Plant Physiol. 150, 1855-1865. doi: 10.1104/pp.109. 139204

Nambara, E., Okamoto, M., Tatematsu, K., Yano, R., Seo, M., and Kamiya, Y. (2010). Abscisic acid and the control of seed dormancy and germination. Seed Sci. Res. 20, 55-67. doi: 10.1017/S0960258510000012

Narsai, R., Law, S. R., Carrie, C., Xu, L., and Whelan, J. (2011). In depth temporal transcriptome profiling reveals a crucial developmental switch with roles for RNA processing and organelle metabolism that are essential for germination in Arabidopsis thaliana. Plant Physiol. 157, 1342-1362. doi: 10.1104/pp.111.183129

Ne'eman, G., Henig-Sever, N., and Eshel, A. (1999). Regulation of the germination of Rhus coriaria, a post-fire pioneer, by heat, ash, $\mathrm{pH}$, waterpotential and ethylene. Physiol. Plant. 106, 47-52. doi: 10.1034/j.1399-3054.1999.106107.x 
Negm, F. B., and Smith, O. E. (1978). Effects of ethylene and carbon dioxide on the germination of osmotically inhibited lettuce seeds. Plant Physiol. 49, 869-872. doi: 10.1104/pp.49.6.869

Negm, F. B., Smith, O. E., and Kumamoto, J. (1973). The role of phytochrome in an interaction with ethylene and carbon dioxide in overcoming lettuce seed thermodormancy. Plant Physiol. 51, 1089-1094. doi: 10.1104/pp.51.6.1089

Nemhauser, J. L., Hong, F., and Chory, J. (2006). Different plant hormones regulate similar processes through largely nonoverlapping transcriptional responses. Cell 126, 467-475. doi: 10.1016/j.cell.2006.05.050

Nonogaki, H., Bassel, G. W., and Bewley, J. D. (2010). Germination - Still a mystery. Plant Sci. 179, 574-581. doi: 10.1016/j.plantsci.2010.02.010

Ogawa, M., Hanada, A., Yamauchi, Y., Kuwahara, A., Kamiya, Y., and Yamaguchi, S. (2003). Gibberellin biosynthesis and response during Arabidopsis seed germination. Plant Cell 15, 1591-1604. doi: 10.1105/tpc.011650

Olatoye, S. T., and Hall, M. A. (1973). "Interaction of ethylene and light on dormant weed seeds," in Seed Ecology, ed. W. Heydecker (London: Butterworths), 233-240.

Oracz, K., El-Maarouf-Bouteau, H., Bogatek, R., Corbineau, F., and Bailly, C. (2008). Release of sunflower seed dormancy by cyanide: cross-talk with ethylene signaling pathway. J. Exp. Bot. 59, 2241-2251. doi: 10.1093/jxb/ern089

Oracz, K., El-Maarouf-Bouteau, H., Farrant, J. M., Cooper, K., Belghazi, M., Job, C., et al. (2007). ROS production and protein oxidation as a novel mechanism for seed dormancy alleviation. Plant J. 50, 452-465. doi: 10.1111/j.1365313X.2007.03063.x

Oracz, K., El-Maarouf-Bouteau, H., Kranner, I., Bogatek, R., Corbineau, F., and Bailly, C. (2009). The mechanisms involved in seed dormancy alleviation by hydrogen cyanide unravel the role of reactive oxygen species as key factors of cellular signaling during germination. Plant Physiol. 150, 494-505. doi: 10.1104/pp.109.138107

Ouaked, F., Rozhon, W., Lecourieux, D., and Hirt, H. (2003). A MAPK pathway mediates ethylene signaling in plants. EMBO J. 22, 1282-1288. doi: 10.1093/emboj/cdg131

Overmyer, K., Brosche, M., and Kangasjarvi, J. (2003). Reactive oxygen species and hormonal control of cell death. Trends Plant Sci. 8, 335-342. doi: 10.1016/S1360 1385(03)00135-3

Penfield, S., Li, Y., Gilday, A. D., Graham, S., and Graham, I. A. (2006). Arabidopsis ABA INSENSITIVE4 regulates lipid mobilization in the embryo and reveals repression of seed germination by the endosperm. Plant Cell 18, 1887-1899. doi: 10.1105/tpc.106.041277

Perino, C., Simond-Côte, E., and Côme, D. (1984). Effets du cyanure de potassium et de l'acide salicylhydroxamique sur la levée de dormance et l'activité respiratoire des embryons de Pommier (Pirus malus L.). C. R. Acad. Sci. Paris III 299, 249-251.

Petruzzelli, L., Coraggio, I., and Leubner-Metzger, G. (2000). Ethylene promotes ethylene biosynthesis during pea seed germination by positive feedback regulation of 1-amonocyclopropane-1-carboxylic acid oxidase. Planta 211, 144-149. doi $10.1007 /$ s004250000274

Petruzzelli, L., Sturaro, M., Mainieri, D., and Leubner-Metzger, G. (2003). Calcium requirement for ethylene-dependent responses involving 1-aminocyclopropane1 -carboxylic acid oxidase in radicle tissues of germinated pea seeds. Plant Cell Environ. 26, 661-671. doi: 10.1046/j.1365-3040.2003.01001.x

Pirrello, J., Jaimes-Miranda, F., Sanchez-Ballesta, M. T., Tournier, B., Khalil-Ahmad, Q., Regad, F., et al. (2006). Sl-ERF2, a tomato ethylene response factor involved in ethylene response and seed germination. Plant Cell Physiol. 47, 1195-1205. doi: $10.1093 / \mathrm{pcp} / \mathrm{pcj} 084$

Piskurewicz, U., Jikumaru, Y., Kinoshita, N., Nambara, E., Kamiya, Y., and Lopez-Molina, L. (2008). The gibberellic acid signaling repressor RGL2 inhibits Arabidopsis seed germination by stimulating abscisic acid synthesis and ABI5 activity. Plant Cell 20, 2729-2745. doi: 10.1105/tpc.108.061515

Prusinski, J., and Khan, A. A. (1990). Relationship of ethylene production to stress alleviation in seeds of lettuce cultivars. J. Am. Soc. Hort. Sci. 115, 294-298.

Puga-Hermida, M. I., Gallardo, M., Rodroguez-Gacio, M. D., and Matilla, A. J. (2003). The heterogeneity of turnip-tops (Brassica rapa) seeds inside the silique affects germination, the activity of the final step of the ethylene pathway, and abscisic acid and polyamine content. Funct. Plant Biol. 30, 767-775. doi 10.1071/FP03053

Qiao, H., Chang, K. N., Yazaki, J., and Ecker, J. R. (2009). Interplay between ethylene, ETP1/ETP2 F-box proteins, and degradation of EIN2 triggers ethylene responses in Arabidopsis. Genes Dev. 23, 512-521. doi: 10.1101/gad.1765709
Qiao, H, Shen, Z., Huand, S.-S. C., Schmitz, R. J., Urich, M. A., Briggs, S. P., et al. (2012). Processing and subcellular trafficking of ER-tethered EIN2 control response to ethylene gas. Science 338, 390-393. doi: 10.1126/science.1225974

Rajjou, L., Duval, M., Gallardo, K., Catusse, J., Bally, J., Job, C., et al. (2012). Seed germination and vigor. Annu. Rev. Plant Biol. 63, 507-533. doi: 10.1146/annurevarplant-042811-105550

Rao, V. S., Sankhla, N., and Khan, A. A. (1975). Additive and synergistic effects of kinetin and ethrel on germination, thermodormancy, and polyribosome formation in lettuce seeds. Plant Physiol. 56, 263-266. doi: 10.1104/pp.56.2.263

Ribeiro, D. M., and Barros, R. S. (2006). Sensitivity to ethylene as a major component in the germination of seeds of Stylosanthes humilis. Seed Sci. Res. 16, 37-45. doi: 10.1079 /SSR2005233

Rzewuski, G., and Sauter, M. (2008). Ethylene biosynthesis and signaling in rice. Plant Sci. 175, 32-42. doi: 10.1016/j.plantsci.2008.01.012

Saini, H. S., Consolacion, E. D., Bassi, P. K., and Spencer, M. S. (1986). Requirement for ethylene synthesis and action during relief of thermoinhibition of lettuce seed germination by combinations of gibberellic acid, kinetin, and carbon dioxide. Plant Physiol. 81, 950-953. doi: 10.1104/pp.81.4.950

Samimy, C., and Khan, A. A. (1983). Secondary dormancy, growth-regulator effects, and embryo growth potential in curly dock (Rumex crispus) seeds. Weed Sci. 31, $153-158$.

Samimy, C., and Taylor, A. G. (1983). Influence of seed quality on ethylene production of germinating snap bean seeds. J. Am. Soc. Hort. Sci. 108, 767-769.

Satoh, S., and Esashi, Y. (1983). Ethylene production, 1-aminocyclopropane-1carboxylic acid content and its conversion to ethylene in axial segments of dormant and nondormant cocklebur seeds. Plant Cell Physiol. 24, 883-887.

Satoh, S., Takeda, Y., and Esashi, Y. (1984). Dormancy and impotency of cocklebur seeds. IX. Changes in ACC-ethylene conversion activity and ACC content of dormant and non-dormant seeds during soaking. J. Exp. Bot. 35, 1515-1524. doi: 10.1093/jxb/35.10.1515

Schönbeck, M. W., and Egley, G. H. (1981). Phase-sequence of redroot pigweed seed germination responses to ethylene and other stimuli. Plant Physiol. 68, 175-179. doi: $10.1104 /$ pp.68.1.175

Schwechheimer, C. (2008). Understanding gibberellic acid signaling-are we there yet? Curr. Opin. Plant Biol. 11, 9-15. doi: 10.1016/j.pbi.2007.10.011

Sewelam, N., Kazan, K., Thomas-Hall, S. R., Kidd, B. N., Manners, J. M., and Schenk, P. M. (2013). Ethylene response factor 6 is a regulator of reactive oxygen species signaling in Arabidopsis. PLoS ONE 8:e70289. doi: 10.1371/journal.pone.0070289

Silva, P. O., Medina, E. F., Barros, R. S., and Ribeiro, D. M. (2014). Germination of salt-stressed seeds as related to ethylene biosynthesis ability in three Stylosanthes species. J. Plant Physiol. 171, 14-22. doi: 10.1016/j.jplph.2013. 09.004

Sinska, I. (1989). Interaction of ethephon with cytokinin and gibberellin during the removal of apple seed dormancy and germination of embryos. Plant Sci. 64, 39-44. doi: 10.1016/0168-9452(89) 90149-0

Sinska, I., and Gladon, R. (1984). Ethylene and the removal of embryonal apple seed dormancy. HortScience 19, 73-75.

Sinska, I., and Gladon, R. (1989). Effects of inhibitors of synthesis and action of ethylene on apple seed stratification and embryo germination. Acta Physiol. Plant. 11, 307-316

Sinska, I., and Lewandowska, U. (1991). Polyamines and ethylene in the removal of embryonal dormancy in apple seeds. Physiol. Plant. 81, 59-64. doi: 10.1111/j.1399-3054.1991.tb01713.x

Siriwitayawan, G., Geneve, R. L., and Downie, A. B. (2003). Seed germination of ethylene perception mutants of tomato and Arabidopsis. Seed Sci. Res. 13, 303-314. doi: 10.1079/SSR2003147

Speer, H. L., Hsiao, A. I., and Vidaver, W. (1974). Effects of germination promoting substances given in conjunction with red light on the phytochrome-mediated germination of dormant lettuce seeds (Lactuca sativa L.). Plant Physiol. 54, 852854. doi: $10.1104 /$ pp.54.6.852

Steber, C. M. (2007). "De-repression of seed germination by GA signaling," in Annual Plant Reviews, Vol. 27, Seed Development, Dormancy and Germination, eds K. Bradford and H. Nonogaki (Oxford: Blackwell Publishing Ltd), 248-263. doi: 10.1002/9780470988848.ch10

Stepanova, A. N., and Alonso, J. M. (2009). Ethylene signaling and response: where different regulatory modules meet. Curr. Opin. Plant Biol. 12, 548-555. doi: 10.1016/j.pbi.2009.07.009 
Subbiah, V., and Reddy, K. J. (2010). Interactions between ethylene, abscisisc acid and cytokinin during germination and seedling establishment in Arabidopsis. J. Biosci. 35, 451-458. doi: 10.1007/s12038-010-0050-2

Sun, T. P., and Gubler, F. (2004). Molecular mechanism of gibberellin in plants. Annu. Rev. Plant Biol. 7, 847-859. doi: 10.1146/annurev.arplant.55.031903.141753

Takayanagi, K., and Harrington, J. F. (1971). Enhancement of germination rate of aged seeds by ethylene. Plant Physiol. 47, 521-524. doi: 10.1104/pp.47.4.521

Taylorson, R. B. (1979). Response of weed seeds to ethylene and related hydrocarbons. Weed Sci. 27, 7-10.

Thomas, T. H., Palevitch, D., Biddington, N. L., and Austin, R. B. (1975). Growth regulators and the phytochrome-mediated dormancy of celery seeds. Physiol. Plant. 35, 101-106. doi: 10.1111/j.1399-3054.1975. tb03876.x

Toorop, P. E., van Aelst, A. C., and Hilhorst, H. W. (2000). The second step of the biphasic endosperm cap weakening that mediates tomato (Lycopersicon esculentum) seed germination is under control of ABA. J. Exp. Bot. 51, 371-379. doi 10.1093/jexbot/51.349.1371

Tyler, L., Thomas, S. G., Hu, J., Dill, A., Alonso, J. M., Ecker, J. R., et al. (2004). DELLA proteins and gibberellin-regulated seed germination and floral development in Arabidopsis. Plant Physiol. 135, 1008-1019. doi: 10.1104/pp.104. 039578

Wang, B., Zhang, J., Xia, X., and Zhang, W. H. (2011). Ameliorative effect of brassinosteroid and ethylene on germination of cucumber seeds in the presence of sodium chloride. Plant Growth Regul. 65, 407-413. doi: 10.1007/s10725-0119595-9

Wang, K. L. C., Li, H., and Ecker, J. R. (2002). Ethylene biosynthesis and signaling networks. Plant Cell 14(Suppl. ), S131-S151.

Wang, N. N., Shih, M. C., and Li, N. (2005). The GUS reporter-aided analysis of the promoter activities of Arabidopsis ACC synthase genes AtACS4, AtACS5, and AtACS7 induced by hormones and stresses. J. Exp. Bot. 56, 909-920. doi: 10.1093/jxb/eri083

Wang, W., Esch, J. J., Shiu, S.-H., Agula, H., Binder, B. M., Chang, C., et al. (2006). Identification of important regions for ethylene binding and signaling in the transmembrane domain of the ETR1 ethylene receptor of Arabidopsis. Plant Cell 18, 3429-3442. doi: 10.1105/tpc.106.044537

Wang, Y., Liu, C., Li, K., Sun, F., Hu, H., Li, X., et al. (2007). Arabidopsis EIN2 modulates stress response through abscisic acid response pathway. Plant Mol. Biol. 64, 633-644. doi: 10.1007/s11103-007-9182-7

Weiss, D., and Ori, N. (2007). Mechanisms of cross talk between gibberellin and other hormones. Plant Physiol. 144, 1240-1246. doi: 10.1104/pp.107. 100370

Weitbrecht, K., Müller, K., and Leubner-Metzger, G. (2011). First of the mark: early seed germination. J. Exp. Bot. 62, 3289-3309. doi: 10.1093/jxb/err030
Wilson, R. L., Kim, H., Bakshi, A., and Binder, B. M. (2014a). The ethylene receptors ETHYLENE RESPONSE1 and ETHYLENE RESPONES2 have contrasting roles in seed germination of Arabidopsis during salt stress. Plant Physiol. 165, 1353-1366. doi: 10.1104/pp.114.241695

Wilson, R. L., Bakshi, A., and Binder, B. M. (2014b). Loss of the ETR1 ethylene receptor reduces the inhibitory effect of far-red light and darkness on seed germination of Arabidopsis thaliana. Front. Plant Sci. 5:433. doi: 10.3389/fpls. 2014.00433

Yamagami, T., Tsuchisaka, A., Yamada, K., Haddon, W. F., Harden, L. A., and Theologis, A. (2003). Biochemical diversity among the 1aminocyclopropane-1-carboxylate synthase isozymes encoded by the Arabidopsis gene family. J. Biol. Chem. 278, 49102-49112. doi: 10.1074/jbc.M308 297200

Yang, S. F., and Hoffman, N. E. (1984). Ethylene biosynthesis and its regulation in higher plants. Annu. Rev. Plant Physiol. Plant Mol. Biol. 35, 155-189. doi: 10.1146/annurev.arplant.35.1.155

Yoo, S. D., Cho, Y. H., and Sheen, J. (2009). Emerging connections in the ethylene signaling network. Trends Plant Sci. 14, 270-279. doi: 10.1016/j.tplants.2009.02.007

Yoo, S. D., Cho, Y. H., Tena, G., Xiong, Y., and Sheen, J. (2008). Dual control of nuclear EIN3 by bifurcate MAPK cascades in $\mathrm{C}_{2} \mathrm{H}_{4}$ signalling. Nature 451, 789-795. doi: 10.1038/nature06543

Zapata, H., P. J., Serrano, M., Pretel, M. T., Amoros, A., and Botella, M. A. (2003). Changes in ethylene evolution and polyamine profiles of seedlings of nine cultivars of Lactuca sativa L. in response to salt stress during germination. Plant Sci. 164, 557-563. doi: 10.1016/S0168-9452(03)00005-0

Conflict of Interest Statement: The authors declare that the research was conducted in the absence of any commercial or financial relationships that could be construed as a potential conflict of interest.

Received: 14 August 2014; accepted: 22 September 2014; published online: 10 October 2014.

Citation: Corbineau F, Xia Q, Bailly C and El-Maarouf-Bouteau H (2014) Ethylene, a key factor in the regulation of seed dormancy. Front. Plant Sci. 5:539. doi: 10.3389/fpls.2014.00539

This article was submitted to Plant Physiology, a section of the journal Frontiers in Plant Science.

Copyright (c) 2014 Corbineau, Xia, Bailly and El-Maarouf-Bouteau. This is an openaccess article distributed under the terms of the Creative Commons Attribution License (CC BY). The use, distribution or reproduction in other forums is permitted, provided the original author(s) or licensor are credited and that the original publication in this journal is cited, in accordance with accepted academic practice. No use, distribution or reproduction is permitted which does not comply with these terms. 


\title{
Plant-Agrobacterium interaction mediated by ethylene and super-Agrobacterium conferring efficient gene transfer
}

\author{
Satoko Nonaka and Hiroshi Ezura* \\ Gene Research Center, Faculty of Life and Environmental Sciences, University of Tsukuba, Tsukuba, Japan
}

\section{Edited by:}

Tomotsugu Koyama, Suntory

Foundation for Life Sciences, Japan

Reviewed by:

Sakiko Okumoto, Virginia

Polytechnic Institute and State

University, USA

Nabil I. Elsheery, Tanta University,

Egypt

*Correspondence:

Hiroshi Ezura, Gene Research

Center, Faculty of Life and

Environmental Sciences, University

of Tsukuba, Tennodai 1-1-1,

Tsukuba, Ibaraki 305-8572, Japan

e-mail:

ezura.hiroshi.fa@u.tsukuba.ac.jp
Agrobacterium tumefaciens has a unique ability to transfer genes into plant genomes. This ability has been utilized for plant genetic engineering. However, the efficiency is not sufficient for all plant species. Several studies have shown that ethylene decreased the Agrobacterium-mediated transformation frequency. Thus, A. tumefaciens with an ability to suppress ethylene evolution would increase the efficiency of Agrobacteriummediated transformation. Some studies showed that plant growth-promoting rhizobacteria (PGPR) can reduce ethylene levels in plants through 1-aminocyclopropane-1-carboxylic acid (ACC) deaminase, which cleaves the ethylene precursor ACC into $\alpha$-ketobutyrate and ammonia, resulting in reduced ethylene production. The whole genome sequence data showed that $A$. tumefaciens does not possess an ACC deaminase gene in its genome. Therefore, providing ACC deaminase activity to the bacteria would improve gene transfer. As expected, A. tumefaciens with ACC deaminase activity, designated as super-Agrobacterium, could suppress ethylene evolution and increase the gene transfer efficiency in several plant species. In this review, we summarize plantAgrobacterium interactions and their applications for improving Agrobacterium-mediated genetic engineering techniques via super-Agrobacterium.

Keywords: Agrobacterium-mediated gene transfer, Agrobacterium tumefaciens, ACC deaminase, ethylene, Agrobacterium-plant interaction

\section{INTRODUCTION}

Agrobacterium tumefaciens is a soil-borne bacterium known to cause crown gall disease in plants. A. tumefaciens strains that induce crown gall have a large plasmid (tumor-inducing plasmid: Ti plasmid), which is essential for the establishment of crown gall disease (van Larebeke et al., 1974, 1975; Zaenen et al., 1974; Watson et al., 1975; Currier and Nester, 1976). Chilton et al. (1977) detected part of the Ti plasmid in the crown gall genome, showing that plant cell conversion resulted in genetic transformation. This work clearly showed that A. tumefaciens has the ability to transfer T-DNA into plant genomes (Chilton et al., 1977).

Since this discovery in 1977, many investigators have attempted to generate A. tumefaciens suitable for plant genetic engineering. In early techniques, the oncogenic T-DNA region of the Ti plasmid was replaced with genes of interest by single or double homologous recombination (Zambryski et al., 1983). Although the recombination steps are easy, they are limited by the potential recombination of repetitive sequences during or after recombination in the Ti plasmid replicon in A. tumefaciens. Hoekema et al. (1983) showed that A. tumefaciens is able to deliver the T-DNA even if the Ti plasmid is divided into two plasmids (the T-DNA region and the vir region). This finding made it possible to extract only the T-DNA region from A. tumefaciens, and the modification was easier without the sequence limitation. After this finding, a binary plant vector strategy became the standard method in plant genetic engineering (Hoekema et al., 1983; Bevan, 1984). Increasing the virulence of A. tumefaciens is a useful strategy for higher transformation efficiency. The identification and application of vir gene inducers, such as plant phenolic compounds (Stachel et al., 1985, 1986) and sugars (Cangelosi et al., 1990; He et al., 2009; Hu et al., 2013), enabled this technique to be adapted for a wide range of plant species, especially monocot crops. The selection of hyper-virulent strains also spread the adaptation throughout the host range. Superbinary vectors, which have shown higher vir gene expression, have provided critical improvements in transformation efficiency (Komari et al., 2006). Agrobacterium-mediated gene transfer has been well established through many modifications, and it is a basic technique in plant science, but the gene transfer efficiencies are not sufficient for many plant species, especially crops and biomass plants. Therefore, improvements in these techniques are required.

Some plant metabolites and phytohormones inhibit Agrobacterium-mediated transformation. In particular, ethylene showed a negative effect on Agrobacterium-mediated T-DNA transformation in many plant species. In this review, we focus on the effect of ethylene on Agrobacterium-mediated gene transfer into plant cells and introduce an engineering strategy by which to increase the transformation efficiency of $A$. tumefaciens via ethylene removal. The established A. tumefaciens strain is designated as superAgrobacterium. 


\section{ETHYLENE SUPPRESSES Agrobacterium-MEDIATED T-DNA TRANSFER}

The gaseous phytohormone ethylene regulates multiple physiological and developmental processes in plants, such as seedling emergence, leaf and flower senescence, ripening, organ abscission, growth-phase transitions, rhizobium-legume interaction, and plant-pathogen interaction (Beyer, 1981; Yang and Hoffman, 1984; Yang, 1985; Abeles, 1992; Ogawara et al., 2003). Ethylene synthesis is stimulated by biotic or abiotic stress. Ethylene also modulates A. tumefaciens-plant interactions. Ethylene is a crucial determinant of crown gall development. Plants treated with inhibitors of ethylene synthesis or perception, such as aminoethoxyvinylglycine (AVG), and ethyleneinsensitive Never-ripe mutants (tomato) suppress crown gall growth (Aloni et al., 1998). Because vascularization is essential for efficient assimilate import from the host plant into the tumor cells, if the vascularization is suppressed, nutrient supply stops (Malsy et al., 1992; Pradel et al., 1996, 1999), resulting in the inhibition of crown gall development. Ethylene stimulates crown gall development by inducing vascular development (Wächter et al., 2003), and it induces crown gall development but inhibits Agrobacterium-mediated genetic transformation of plant cells. The enhancement of ethylene production by supplying its immediate precursor, 1aminocyclopropane-1-carboxylic acid (ACC), suppresses gene transfer in tomatoes and melons (Davis et al., 1992; Ezura et al., 2000). Ethylene is increased by wounding and A. tumefaciens infection stress during co-cultivation (Ezura et al., 2000). Reducing ethylene production during co-cultivation using ethylene biosynthesis inhibitors such as AVG or suppressing plant ethylene perception by adding silver ions to the tissue culture medium has improved the transformation efficiency in melons (Ezura et al., 2000), cauliflowers (Chakrabarty et al., 2002), apricots (Burgos and Alburquerque, 2003), apple trees (Petri et al., 2005; Seong et al., 2005), and bottle gourds (Han et al., 2005). The stable transformation frequency was also increased in the Arabidopsis thaliana ethylene-insensitive mutants etr1-1 and etr1-2 (Nonaka et al., 2008a).

These results indicate that ethylene inhibits gene transfer in plants. One possible explanation for this phenomenon might involve the plant defense response via ethylene signaling. Previous studies have found that ethylene regulates several genes that are involved in the defense response, including those that encode the PR proteins chitinase, $\beta$-1, 3-glucanase, and PR1 (Deikman, 1997) in addition to phytoalexin synthetic enzymes (Ecker and Davis, 1987), defensins (Penninckx et al., 1996), and hydroxy-Pro-rich glycoproteins (Toppan et al., 1982). These compounds suppress bacterial growth because of their antibacterial activity. Indeed, in tomatoes, the reduced expression of LeETR4, which encodes a tomato ethylene receptor, resulted in increased sensitivity to ethylene, an enhanced hypersensitive response, and the reduced growth of Xanthomonas campestris pv. vesicatoria compared to wild-type (WT) plants (Ciardi et al., 2000). Ethylene insensitivity results in reduced resistance to the potato soft rot agent Erwinia carotovora subsp. carotovora in A. thaliana etr1-1 and ein2-1 mutants (Norman-Setterblad et al., 2000).

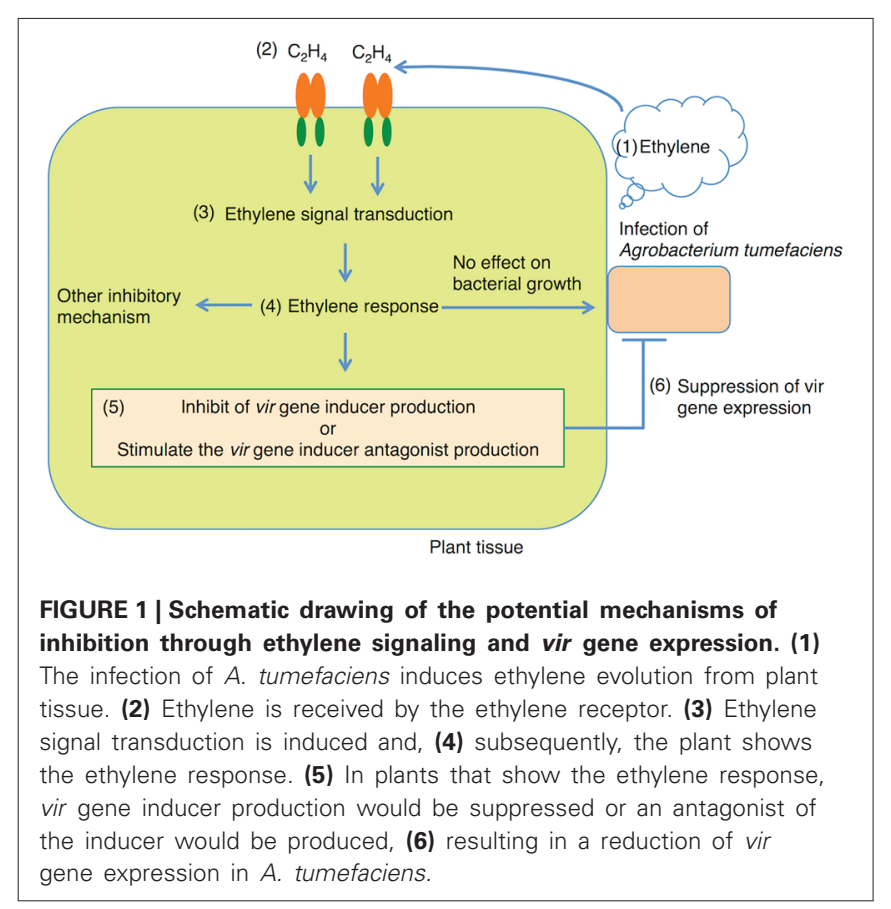

In general, ethylene levels increase in plant defense responses; however, the inhibitory effect of ethylene on Agrobacteriummediated gene transfer does not occur through plant defense. The model scheme is described in Figure 1. Microarray and differential display analysis showed that $A$. tumefaciens infection induces the plant genes necessary for transformation while simultaneously repressing host defense response genes (Veena et al., 2003). Ethylene evolution was induced by A. tumefaciens inoculation in the early stage of the infection (Nonaka et al., 2008b; Lee et al., 2009). Although A. tumefaciens infection increased ethylene levels, the plant genes that encode ethylene receptors and their downstream signaling components, including defense response genes, are not induced (Lee et al., 2009). These results support the hypothesis that the inhibitory effect of ethylene on Agrobacterium-mediated gene transfer is independent of the plant defense response. Indeed, the plant ethylene response did not affect $A$. tumefaciens growth during co-cultivation (Nonaka et al., 2008a). The plant ethylene response inhibits T-DNA transfer through the suppression of vir gene expression (Nonaka et al., 2008a). The application of exudate from plants showing an ethylene response to A. tumefaciens reduced vir gene expression, which decreased T-DNA transfer. Such an inhibitory effect was partially overcome by the application of acetosyringone, a vir gene inducer, and in an A. tumefaciens strain constitutively expressing the vir gene. From this result, two possibilities were considered: in plants showing an ethylene response, the amount of the vir gene inducer would be reduced or the antagonist of the vir gene inducer would be produced. This deficient recovery indicates that vir gene suppression is one of the reasons for a reduction in T-DNA transfer via the ethylene response. The partial restoration upon the application of the vir gene inducer suggests that there is another inhibitory mechanism of ethylene. 
Therefore, the suppression of the vir gene is not sufficient to explain the negative effect of ethylene on Agrobacterium-mediated gene transfer. Because the inhibitory mechanism of ethylene on Agrobacterium-mediated gene transfer still needs to be clarified, to improve the transformation frequency, the introduction of the ability to reduce ethylene in A. tumefaciens would be effective.

\section{STRATEGY TO REDUCE ETHYLENE PRODUCTION IN PLANT CELLS INOCULATED WITH Agrobacterium}

Ethylene is generated through the ethylene biosynthetic pathway, which was elucidated largely by the pioneering work of Yang and co-workers in the 1970s and 1980s (Wang et al., 2002). Ethylene is synthesized from the amino acid methionine, which is converted to $S$-adenosyl- $L$-methionine (SAM) by SAM synthase (ADS). SAM is the major methyl donor in plants and is involved in the methylation of lipids, proteins, and nucleic acids. SAM is converted by the enzyme ACC synthase (ACS) to $5^{\prime}$-methylthioadenosine (MTA), which is converted back to methionine via the Yang Cycle and to ACC, the precursor of ethylene. ACC is finally oxidized by ACC oxidase (ACO) to form ethylene, cyanide, and carbon dioxide. The conversion of SAM to ACC is considered to be the rate-limiting step in ethylene biosynthesis and consequently has been studied intensively. Therefore, the degradation of ACC, the immediate precursor of ethylene, effectively reduces ethylene production in plants. The reduction of ethylene via the degradation of ACC is found in some soil bacteria.

The pyridoxal 5-phosphate-dependent enzyme ACC deaminase catalyzes the decomposition of ACC to $\alpha$-ketobutyrate and ammonia (Honma and Shimomura, 1978; Minami et al., 1998; Hontzeas et al., 2004; McDonnell et al., 2009). Although ACC deaminase genes have been isolated in a wide range of organisms, the ACC deaminase gene was isolated from many plant growthpromoting rhizobacteria (PGPR; Table 1). As shown in. PGPR with ACC deaminase activity can lower the ethylene level in plant cells through the degradation of ACC.

In response to tryptophan and other small molecules present in root exudates, IAA is synthesized and secreted by a PGPR that is bound to the surface of the root (Whipps, 1990; Hong et al., 1991; Fallik et al., 1994; Dimkpa et al., 2012). Some of the newly synthesized IAA is taken up by the plant and can stimulate ACS to convert SAM to ACC (Kende, 1993). The uptake and subsequent cleavage of ACC by the PGPR decrease the amount of ACC outside the plant (Penrose and Glick, 2001).

The $K_{\mathrm{m}}$ of ACC deaminase is lower than ACC oxidase. The various plant ACC oxidase for ACC ranges from approximately $8 \mu \mathrm{M}$ (for ripening apples) to $120 \mu \mathrm{M}$ (for etiolated beans; Smith et al., 1992). The $K_{\mathrm{m}}$ of ACC deaminase for ACC ranges from approximately 1.5 to $3.4 \mathrm{mM}$ (Honma and Shimomura, 1978; Hontzeas et al., 2004). This means that ACC oxidase has about a 100-fold greater affinity for ACC than does ACC deaminase. Despite the fact that ACC oxidase has a much higher affinity for ACC, the kinetic calculations indicate that ACC deaminase can be more effective in lowering ACC than ACC oxidase when the amount of ACC deaminase is much greater than the amount of
Table 1 | Organisms with ACC deaminase activity.

\begin{tabular}{ll}
\hline Source organisms & Reference \\
\hline Bacteria (Gram-negative) & \\
\hline Enterobacter cloacae & Holguin and Glick (2001) \\
Achromobacter xylosoxidans & Belimov et al. (2001) \\
Rhizobium leguminosarum & Ma et al. (2003) \\
Pseudomonas putida & Hontzeas et al. (2004) \\
Burkholderia phytofirmans & Sessitsch et al. (2005) \\
Variovorax paradoxus & Madhaiyan et al. (2006) \\
Methylobacterium fujisawaense & Belimov et al. (2009) \\
Cronobacter sakazakii & Jha et al. (2012) \\
Mesorhizobium sp. & Gontia-Mishra et al. (2014) \\
Haererehalobacter sp. & Gontia-Mishra et al. (2014) \\
Halomonas sp. & Gontia-Mishra et al. (2014)
\end{tabular}

Bacteria (Gram-positive)
Rhodococcus sp.

Brevibacterium iodinum

Bacillus licheniformis

Zhihengliuela alba

Micrococcus sp.

Brachybacterium saurashtrense

Brevibacterium casei

Archaebacteria

Pyrococcus horikoshii

Yeasts

Hansenula saturnus

Issatchenkia occidentalis

Fungi

Penicillium citrinum

Trichoderma asperellum

Phytophthora sojae

Plants

Arabidopsis thaliana

Populus tremula

Solanum lycopersicum
Belimov et al. (2001)

Dastager et al. (2010)

Siddikee et al. (2011)

Gontia et al. (2011)

Jha et al. (2012)

Gontia-Mishra et al. (2014)

Gontia-Mishra et al. (2014)

Fujino et al. (2004)

Minami et al. (1998)

Palmer et al. (2007)

Jia et al. (1999)

Viterbo et al. (2010)

Singh and Kashyap (2012)

McDonnell et al. (2009)

Plett et al. (2009)

Gontia-Mishra et al. (2014)
ACC oxidase (Glick et al., 1998). These results indicate that the ACC metabolite reaction in PGPR was more effective than that in plant cells and that the ACC level are lower in inner plant cells than in external cells. Therefore, to maintain equilibrium between internal and external ACC levels, the plant increases the level of ACC exudate. PGPRs have the ability to utilize ACC as a sole source of nitrogen in plant roots (Glick et al., 1994; Jacobson et al., 1994) and to proliferate under conditions in which other soil bacteria cannot grow. The reduction of the inner ACC level caused by the utilization of ACC in bacteria causes the plant to synthesize more ACC and to effectively exude ACC from the plant.

A significant correlation was found between in vitro bacterial ACC deaminase activity and the growth-promoting activity of these bacteria under pot and field trial conditions (Shaharoona et al., 2006a,b). In fact, many types of PGPR containing the ACC deaminase gene reduced ethylene production, resulting in physiological changes in many types of plants. The rhizobacterium 
Variovorax paradoxus 5C-2, which possesses ACC deaminase, promotes the growth (leaf area and shoot biomass) and development of WT A. thaliana and the ethylene-overproducing mutant eto11 but does not have these effects in ethylene-insensitive mutants (etr1-1 and ein2-1; Chen et al., 2013). Furthermore, V. paradoxus $5 \mathrm{C}-2$ decreased the ACC concentrations in the rosette leaves of WT plants by $59 \%$ and foliar ethylene emission in both WT plants and eto1-1 mutants by 42 and 37\%, respectively, (Chen et al., 2013). Rhizobacteria possessing ACC deaminase induced maximum waterlogging tolerance in Ocimum sanctum, as treated waterlogged plants exhibited the maximum growth and biomass yield with minimal ethylene levels (Barnawal et al., 2012). Bacterial endophytes expressing ACC deaminase delay flower senescence (Ali et al., 2012). Mesorhizobium ciceri LMS-1 expressing an exogenous ACC deaminase increases nodulation abilities and chickpea plant resistance to soil constraints (Nascimento et al., 2012).

\section{SUPER-Agrobacterium WITH ACC DEAMINASE ACTIVITY INCREASES T-DNA TRANSFER EFFICIENCY VIA $A$. \\ tumefaciens}

The introduction of ACC deaminase activity into A. tumefaciens may reduce ethylene levels during co-cultivation and increase $A$. tumefaciens-mediated transformation efficiency. Whole-genome sequence analysis revealed that the A. tumefaciens strain C58 does not have an ACC deaminase gene (Wood et al., 2001); therefore, this strategy seems to be effective in this species. The ACC deaminase gene was amplified from Pseudomonas sp. via PCR. This amplified fragment was inserted into pBBR1MCS5 (pBBRacdS). The pBBR1MCS-5 plasmid is compatible with IncP, IncQ, and IncW plasmids, and it has a different origin of the RK2 vector, which is used as a binary vector (Kovach et al., 1994). Because their origins are different, pBBRacdS and the binary vector are able to coexist in A. tumefaciens. The expression of ACC deaminase was controlled by the lac $Z$ promoter, which constitutively and strongly expresses genes in A. tumefaciens. The A. tumefaciens strain harboring ACC deaminase genes showed ACC deaminase activity and effectively inhibited ethylene production in melons (Figure 2A), resulting in increased transient T-DNA transformation in melons (Figure 2B; Nonaka et al., 2008b).

For the further improvement of Agrobacterium-mediated transformation, we attempted to increase the expression level of ACC deaminase. Klüsener et al. (2010) showed that virB1, virD1, and virE1 of A. tumefaciens were among the most highly expressed genes in acidic medium ( $\mathrm{AB}$ medium at $\mathrm{pH} 5.5$ ) containing $100 \mathrm{~mol} / \mathrm{L}$ acetosyringone (Klüsener et al., 2010). We compared the activities of these three promoters to select the highest one, and the virD1 promoter showed the strongest transcription activity. The virD1 promoter conferred fourfold increased transcriptional activity compared with the lac $Z$ promoter. The time course analysis (0-72 h) showed that ACC deaminase expression was induced at $6 \mathrm{~h}$ by adding acetosyringone to MS medium, and the high expression level was maintained until $72 \mathrm{~h}$. By contrast, the expression of the ACC deaminase gene driven by the lac $Z$ promoter started to decrease at $6 \mathrm{~h}$.

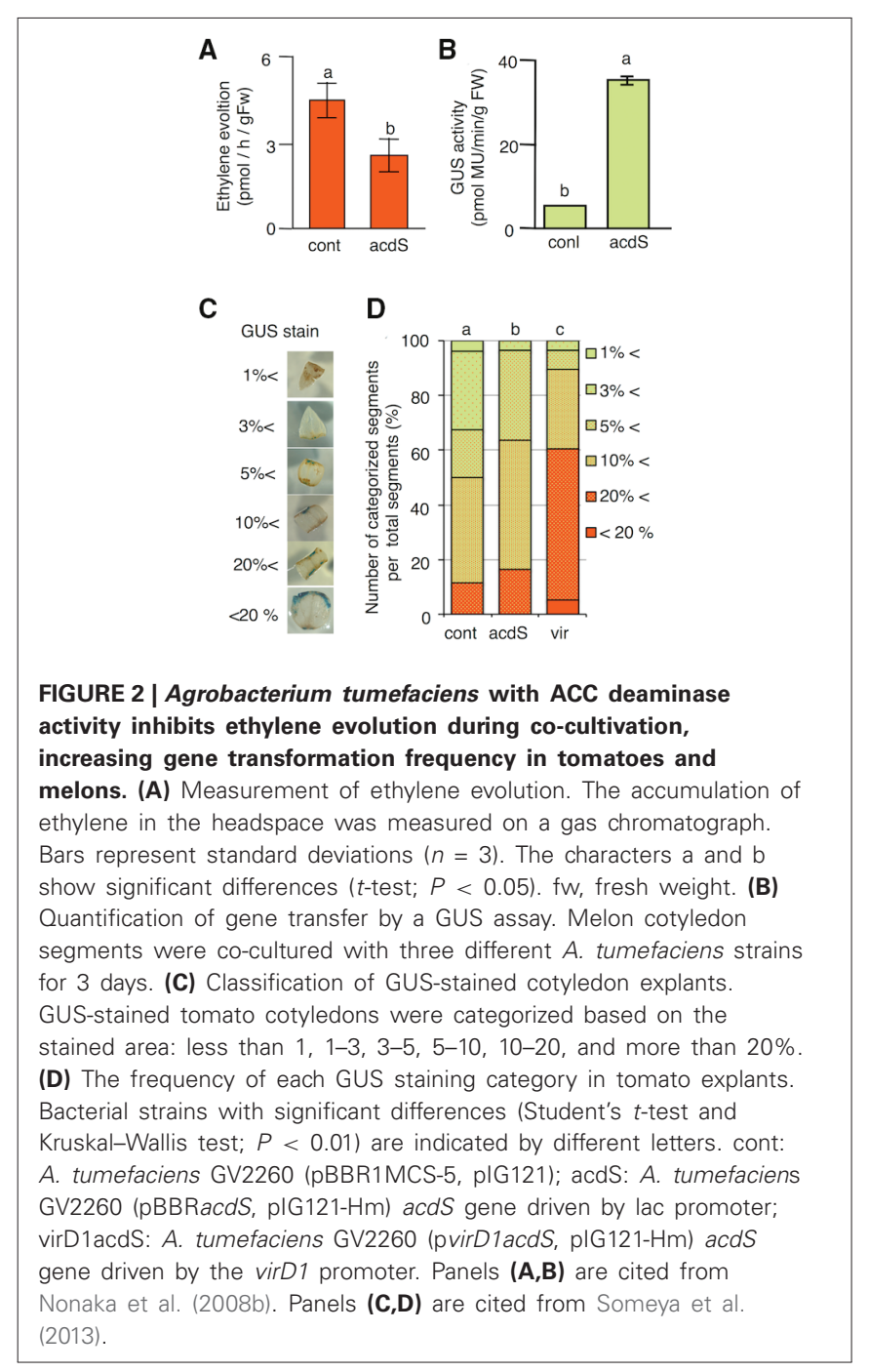

Compared with the lac $Z$ promoter, the virD1 promoter maintained ACC deaminase gene expression at a higher level for a long time, increasing the ACC deaminase activity in A. tumefaciens. Generally, the co-cultivation period is 72 to $96 \mathrm{~h}$, so the virD1 promoter seems to be suitable as the ACC deaminase gene activator. This newly developed A. tumefaciens strain showed higher TDNA transformation efficiency in tomatoes (Figures 2C,D) and higher biomass production than Erianthus ravennae, which shows very low transformation frequency. Therefore, A. tumefaciens with higher ACC deaminase activity is a powerful tool for the Agrobacterium-mediated genetic engineering of plants (Someya et al., 2013). Introducing ACC deaminase into A. tumefaciens is effective at increasing the stable transformation frequency. $A$. tumefaciens with ACC deaminase succeeded in increasing the stable transformation frequency in Egusi melons (Ntui et al., 2009) and three canola cultivars (Brassica napus cv. Westar, B. napus cv. Hyola 401 and B. napus cv. 4414RR; Hao et al., 2010). We herein designate $A$. tumefaciens with ACC deaminase activity as super-Agrobacterium, which has the potential to improve the transformation efficiency of recalcitrant plant species. 


\section{CONCLUSION}

Agrobacterium-mediated transformation is an important tool for plant genetic engineering. Although a sophisticated protocol has been established for model plants, such as A. thaliana, tobacco, and rice, the transformation efficiency was not sufficiently high for commercially important crops such as maize, sorghum, soybean, barley, and E. ravennae. There has therefore been a need for the improvement of this methodology. Among the various negative factors contributing to low Agrobacteriummediated transformation rates, ethylene has been well known as an inhibitor of transformation efficiency for a long time. There are chemicals to reduce ethylene production or ethylene perception. However, they are hard to use because they are expensive, in a gaseous form or toxic to bacteria; they are thus not suitable for improving Agrobacterium-mediated transformation. Therefore, we attempted to reduce ethylene in A. tumefaciens. Some PGPRs have an enzyme that degrades the ethylene precursor ACC. This strategy is not toxic for bacteria, and it is very easy to perform. We introduced the enzyme ACC deaminase into A. tumefaciens. The strain has been designated as superAgrobacterium. It showed increased transient gene delivery into melon cotyledons (Nonaka et al., 2008b), tomatoes, and E. ravennae (Someya et al., 2013). In addition, the super-Agrobacterium also increased stable transformation in Egusi melon and three canola species (Ntui et al., 2009; Hao et al., 2010). To improve the "super-Agrobacterium," a thorough analysis will be required in the future. One of the negative effects of ethylene is the suppression of vir gene expression in A. tumefaciens. However, the constitutive vir gene expression strain or the addition of the vir gene expression inducer could only partially overcome the inhibitory effect of ethylene on vir gene expression. This result showed the possibility of a different inhibitory mechanism that is caused by ethylene; therefore, a thorough analysis will be required in the future.

Our study showed that there are two points that should be considered when improving the efficiency of Agrobacterium-mediated gene transfer: first, the transient transformation frequency is important for increasing the stable transformation efficiency. Second, the regulation of plant-derived signals is an effective method for increasing the A. tumefaciens-mediated gene transfer frequency. For a more sophisticated strategy by which to increase transformation, it is effective to analyze the plant-derived signals in Agrobacterium-plant interactions. The removal of these signals would further increase the A. tumefaciens-mediated transformation frequency in recalcitrant plants.

\section{ACKNOWLEDGMENTS}

We thank Prof. M. Mii (Chiba University, Japan) and Dr. I. Nakamura (Chiba University, Japan) for kindly providing the $E$. ravennae calli and binary plasmid, respectively. This research was supported in part by the New Energy and Industrial Technology Development Organization (NEDO) to Hiroshi Ezura This work was also supported by JSPS KAKENHI, a Grant-in-Aid for Young Scientists (B); (Grant Number 24780001) to Satoko Nonaka, and the Cooperative Research Grant of Gene Research Center, the University of Tsukuba to Hiroshi Ezura and Satoko Nonaka.

\section{REFERENCES}

Abeles, F. B. (1992). "Regulation of ethylene production by internal, environmental, and stress factors," in Ethylene in Plant Biology, 2nd Edn, eds F. B. Abeles, P. W. Morgan II, and M. E. Saltveit (San Diego: Academic Press), 56-119.

Ali, S., Charles, T. C., and Glick, B. R. (2012). Delay of flower senescence by bacterial endophytes expressing 1-aminocyclopropane-1-carboxylate deaminase. J. Appl. Microbiol. 113, 1139-1144. doi: 10.1111/j.1365-2672.2012.05409.x

Aloni, R., Wolf, A., Feigenbaum, P., Avni, A., and Klee, H. J. (1998). The never ripe mutant provides evidence that tumor-induced ethylene controls the morphogenesis of Agrobacterium tumefaciens-induced crown galls on tomato stems. Plant Physiol. 117, 841-849. doi: 10.1104/pp.117.3.841

Barnawal, D., Bharti, N., Maji, D., Chanotiya, C. S., and Kalra, A. (2012). 1-Aminocyclopropane-1-carboxylic acid (ACC) deaminase-containing rhizobacteria protect Ocimum sanctum plants during waterlogging stress via reduced ethylene generation. Plant Physiol. Biochem. 58, 227-235. doi: 10.1016/j.plaphy.2012.07.008

Belimov, A., Dodd, I. C., Safronova, V. I., and Davies, W. J. (2009). ACC deaminase-containing rhizobacteria improve vegetative development and yield of potato plants grown under water-limited conditions. Asp. Appl. Biol. 98, 163-169.

Belimov, A. A., Safronova, V. I., Sergeyeva, T. A., Egorova, T. N., Matveyeva, V. A., Tsyganov, V. E., et al. (2001). Characterization of plant growth promoting rhizobacteria isolated from polluted soils and containing 1-aminocyclopropane1-carboxylate deaminase. Can. J. Microbiol. 47, 642-652. doi: 10.1139/cjm-477-642

Bevan, M. (1984). Binary Agrobacterium vectors for plant transformation. Nucleic Acids Res. 12, 8711-8721. doi: 10.1093/nar/12.22.8711

Beyer, E. M. (1981). "Ethylene action and metabolism," in Recent Advances in the Biochemistry of Fruits and Vegetables, eds J. Friend and M. J. C. Rhodes (New York: Academic Press), 107-121.

Burgos, L., and Alburquerque, N. (2003). Ethylene inhibitors and low kanamycin concentrations improve adventitious regeneration from apricot leaves. Plant Cell Rep. 21, 1167-1174. doi: 10.1007/s00299-003-0625-6

Cangelosi, G. A., Ankenbauer, R. G., and Nester, E. W. (1990). Sugars induce the Agrobacterium virulence genes through a periplasmic binding protein and a transmembrane signal protein. Proc. Natil. Acad. Sci. U.S.A. 87, 6708-6712. doi: 10.1073/pnas.87.17.6708

Chakrabarty, R., Viswakarma, N., Bhat, S. R., Kirti, P. B., Singh, B. D., and Chopra, V. L. (2002). Agrobacterium-mediated transformation of cauliflower: optimization of protocol and development of Bt-transgenic cauliflower. J. Biosci. 27, 495-502. doi: 10.1007/BF02705046

Chen, L., Dodd, I. C., Theobald, J. C., Belimov, A. A., and Davies, W. J. (2013). The rhizobacterium Variovorax paradoxus 5C-2, containing ACC deaminase, promotes growth and development of Arabidopsis thaliana via an ethylenedependent pathway. J. Exp. Bot. 64, 1565-1573. doi: 10.1093/jxb/ert031

Chilton, M. D., Drummond, M. H., Merio, D. J., Sciaky, D., Montoya, A. L., Gordon, M. P., et al. (1977). Stable incorporation of plasmid DNA into higher plant cells: the molecular basis of crown gall tumorigenesis. Cell 11, 263-271. doi: 10.1016/0092-8674(77)90043-5

Ciardi, J. A., Tieman, D. M., Lund, S. T., Jones, J. B., Stall, R. E., and Klee, H. J. (2000). Response to Xanthomonas campestris pv. vesicatoria in tomato involves regulation of ethylene receptor gene expression. Plant Physiol. 123, 81-92. doi: 10.1104/pp.123.1.81

Currier, T. C., and Nester, E. W. (1976). Evidence for diverse types of large plasmids in tumor-inducing strains of Agrobacterium. J. Bacteriol. 126, 157-165.

Dastager, S. G., Deepa, C. K., and Pandey, A. (2010). Isolation and characterization of novel plant growth promoting Micrococcus sp NII-0909 and its interaction with cowpea. Plant Physiol. Biochem. 48, 987-992. doi: 10.1016/j.plaphy.2010. 09.006

Davis, M. E., Miller, A. R., and Lineberger, R. D. (1992). Studies on the effects of ethylene on transformation of tomato cotyledons (Lycopersicon esculentum Mill.) by Agrobacterium tumefaciens. J. Plant Physiol. 139, 309-312. doi: 10.1016/S0176-1617(11)80343-3

Deikman, J. (1997). Molecular mechanisms of ethylene regulation of gene transcription. Physiol. Plant. 100, 561-566. doi: 10.1111/j.1399-3054.1997. tb03061.x

Dimkpa, C. O., Zeng, J., McLean, J. E., Britt, D. W., Zhan, J., and Anderson, A. J. (2012). Production of indole-3-acetic acid via the indole-3acetamide pathway in the plant-beneficial bacterium Pseudomonas 
chlororaphis $\mathrm{O} 6$ is inhibited by $\mathrm{ZnO}$ nanoparticles but enhanced by $\mathrm{CuO}$ nanoparticles. Appl. Environ. Microbiol. 78, 1404-1410. doi: 10.1128/AEM. 07424-11

Ecker, J. R., and Davis, R. W. (1987). Plant defense genes are regulated by ethylene. Proc. Natl. Acad. Sci. U.S.A. 84, 5202-5206. doi: 10.1073/pnas.84.15.5202

Ezura, H., Yuhashi, K., Yasuta, T., and Minamisawa, K. (2000). Effect of ethylene on Agrobacterium tumefaciens-mediated gene transfer to melon. Plant Breed. 119, 75-79. doi: 10.1046/j.1439-0523.2000.00438.x

Fallik, E., Sarig, S., and Okon, Y. (1994). "Morphology and physiology of plant roots associated with Azospirillum," in Azospirillum-Plant Associations, ed. Y. Okon (Boca Raton: CRC Press), 77-85.

Fujino, A., Ose, T., Yao, M., Tokiwano, T., Honma, M., Watanabe, N., et al. (2004). Structural and enzymatic properties of 1-aminocyclopropane-1-carboxylate deaminase homologue from Pyrococcus horikoshii. J. Mol. Biol. 341, 999-1013. doi: 10.1016/j.jmb.2004.06.062

Gontia, I., Kavita, K., Schmid, M., Hartmann, A., and Jha, B. (2011). Brachybacterium saurashtrense sp. nov., a halotolerant root associated bacterium with plant growth-promoting potential. Int. J. Syst. Evol. Microbiol. 61, 2799-2804. doi: 10.1099/ijs.0.023176-0

Gontia-Mishra, I., Sasidharan, S., and Tiwari, S. (2014). Recent developments in use of 1-aminocyclopropane-1-carboxylate (ACC) deaminase for conferring tolerance to biotic and abiotic stress. Biotechnol. Lett. 36, 889-898. doi: 10.1007/s10529-014-1458-9

Glick, B. R., Jacobson, C. B., Schwarze, M. M. K., and Pasternak, J. J. (1994). 1-Aminocyclopropane-1-carboxylic acid deaminase mutants of the plant growth promoting rhizobacterium Pseudomonas putida GR12-2 do not stimulate canola root elongation. Can. J. Microbiol. 40, 911-915. doi: 10.1139/ m94-146

Glick, B. R., Penrose, D. M., and Li, J. (1998). A model for the lowering of plant ethylene concentrations by plant growth promoting bacteria. J. Theor. Biol. 190, 63-68. doi: 10.1111/j.1574-6968.2010.01977.x

Han, J. S., Kim, C. K., Park, S. H., Hirschi, K. D., and Mok, I. G. (2005). Agrobacterium-mediated transformation of bottle gourd (Lagenaria siceraria Standl.). Plant Cell Rep. 23, 692-698. doi: 10.1007/s00299-004-0874-z

Hao, Y., Charles, T. C., and Glick, B. R. (2010). ACC deaminase increases the Agrobacterium tumefaciens-mediated transformation frequency of commercial canola cultivars. FEMS Microbiol. Lett. 7, 185-190. doi: 10.1111/j.15746968.2010.01977.x

He, F., Nair, G. R., Soto, C. S., Chang, Y., Hsu, L., Ronzone, E., et al. (2009). Molecular basis of ChvE function in sugar binding, sugar utilization, and virulence in Agrobacterium tumefaciens. J. Bacteriol. 191, 5802-5813. doi: 10.1128/jb. 00451-09

Hoekema, A., Hirsch, P. R., Hooykaas, P. J. J., and Schilperoort, R. A. (1983). A binary plant vector strategy based on separation of the Vir- and T-region on the Agrobacterium tumefaciens Ti plasmid. Nature 303, 179-180. doi: 10.1038/303179a0

Holguin, G., and Glick, B. R. (2001). Expression of the ACC deaminase gene from Enterobacter cloacae UW4 in Azospirillum brasilense. Microb. Ecol. 41, 281-288. doi: $10.1007 / \mathrm{s} 002480000040$

Hong, Y., Glick, B. R., and Pasternak, J. J. (1991). Plant-microbial interactions under gnotobiotic conditions: a scanning electron microscope study. Curr. Microbiol. 23, 111-114. doi: 10.1007/BF02092259

Honma, S., and Shimomura, T. (1978). Metabolism of 1-aminocyclopropane-1carboxylic acid. Agric. Biol. Chem. 42, 1825-1831. doi: 10.1271/bbb1961.42.1825

Hontzeas, N., Zoidakis, J., Glick, B. R., and Abu-Omar, M. M. (2004). Expression and characterization of 1-aminocyclopropane-1-carboxylate deaminase from the rhizobacterium Pseudomonas putida UW4: a key enzyme in bacterial plant growth promotion. Biochim. Biophys. Acta 1703, 11-19. doi: 10.1016/j.bbapap.2004.09.015

Hu, X., Zhao, J., Degrado, W. F., and Vinns, A. N. (2013). Agrobacterium tumefaciens recognizes its host environment using ChvE to bind divers plant sugars as virulence signals. Proc. Natil. Acad. Sci. U.S.A. 110, 678-683. doi: $10.1073 /$ pnas. 1215033110

Jacobson, C. B., Pasternak, J. J., and Glick, B. R. (1994). Partial purification and characterization of 1-aminocyclopropane-1-carboxylate deaminase from the plant growth promoting rhizobacterium Pseudomonas putida GR12-2. Can. J. Microbiol. 40, 1019-1025. doi: 10.1139/m94-162

Jha, B., Gontia, I., and Hartmann, A. (2012). The roots of the halophyte Salicornia brachiata are a source of new halotolerant diazotrophic bacteria with plant growth-promoting potential. Plant Soil 356, 265-277. doi: 10.1007/s11104-0110877-9

Jia, Y. J., Kakuta, Y., Sugawara, M., Igarashi, T., Oki, N., Kisaki, M., et al. (1999). Synthesis and degradation of 1-aminocyclopropane-1-carboxylic acid by Penicillium citrinum. Biosci. Biotechnol. Biochem. 63, 542-549. doi: 10.1271/bbb.63.542

Kende, H. (1993). Ethylene biosynthesis. Annu. Rev. Plant Physiol. Plant Mol. Biol. 44, 283-307. doi: 10.1146/annurev.pp.44.060193.001435

Komari, T., Takakura, Y., Ueki, J., Kato, N., Ishida, Y., and Hiei, Y. (2006). Binary vectors and super-binary vectors. Methods Mol. Biol. 343, 15-41. doi: 10.1385/159745-130-4:15

Kovach, M. E., Phillips, R. W., Elzer, P. H., Roop, R. M. II, and Peterson, K. M. (1994). pBBR1MCS: a broad-host-range cloning vector. Biotechniques 16, 800-802.

Klüsener, S., Hacker, S., Tsai, Y. L., Bandow, J. E., Gust, R., Lai, E. M., et al. (2010). Proteomic and transcriptomic characterization of a virulence-deficient phosphatidylcholine-negative Agrobacterium tumefaciens mutant. Mol. Genet. Genomics 283, 575-589. doi: 10.1007/s00438-010-0542-7

Lee, C. W., Efetova, M., Engelmann, J. C., Kramell, R., Wasternack, C., LudwigMuller, J., et al. (2009). Agrobacterium tumefaciens promotes tumor induction by modulating pathogen defense in Arabidopsis thaliana. Plant Cell 21, 29482962. doi: 10.1105/tpc.108.064576

Ma, W., Guinel, F. C., and Glick, B. R. (2003). Rhizobium leguminosarum biovar viciae 1-aminocyclopropane-1-carboxylate deaminase promotes nodulation of pea plants. Appl. Environ. Microbiol. 69, 4396-4402. doi: 10.1128/AEM.69.8.4396-4402.2003

Madhaiyan, M., Poonguzhali, S., Ryu, J., and Sa, T. (2006). Regulation of ethylene levels in canola (Brassica campestris) by 1-aminocyclopropane-1-carboxylate deaminase-containing Methylobacterium fujisawaense. Planta 224, 268-278. doi: 10.1007/s00425-005-0211-y

Malsy, S., van Bel, A. J. E., Kluge, M., Hartung, W., and Ullrich, C. I. (1992). Induction of crown galls by Agrobacterium tumefaciens (strain C-58) reverses assimilate translocation and accumulation in Kalanchoë daigremontiana. Plant Cell Environ. 15, 519-529. doi: 10.1111/j.1365-3040.1992.tb01485.x

McDonnell, L., Plett, J. M., Andersson-Gunnerås, S., Kozela, C., Dugardeyn, J., Van Der Straeten, D., et al. (2009). Ethylene levels are regulated by a plant encoded 1-aminocyclopropane-1-carboxylic acid deaminase. Physiol. Plant. 136, 94-109. doi: 10.1111/j.1399-3054.2009.01208.x

Minami, R., Uchiyama, K., Murakami, T., Kawai, J., Mikami, K., Yamada, T., et al. (1998). Properties, sequence, and synthesis in Escherichia coli of 1- aminocyclopropane-1-carboxylate deaminase from Hansenula saturnus. J. Biochem. 123, 1112-1118. doi: 10.1093/oxfordjournals.jbchem.a022050

Nascimento, F. X., Brigido, C., Glick, B. R., Oliveira, S., and Alho, L. (2012). Mesorhizobium ciceri LMS-1 expressing an exogenous 1-aminocyclopropane1-carboxylate (ACC) deaminase increases its nodulation abilities and chickpea plant resistance to soil constraints. Lett. Appl. Microbiol. 55, 15-21. doi: 10.1111/j.1472-765X.2012.03251.x

Nonaka, S., Yuhashi, K., Takada, K., Sugaware, M., Minamisawa, K., and Ezura, H. (2008a). Ethylene production in plants during transformation suppresses vir gene expression in Agrobacterium tumefaciens. New Phytol. 178, 647-656. doi: 10.1111/j.1469-8137.2008.02400.x

Nonaka, S., Sugawara, M., Minamisawa, K., Yuhashi, K., and Ezura, H. (2008b). 1Aminocyclopropane-1-carboxylate deaminase enhances Agrobacterium tumefaciens-mediated gene transfer into plant cells. Appl. Environ. Microbiol. 74, 25262528. doi: 10.1128/AEM.02253-07

Norman-Setterblad, C., Vidal, S., and Palva, E. T. (2000). Interacting signal pathways control defense gene expression in Arabidopsis in response to cell wall degrading enzymes from Erwinia carotovora. Mol. Plant Microbe Interact. 13, 430-438. doi: 10.1094/MPMI.2000.13.4.430

Ntui, V. O., Thirukkumaran, G., Iioka, S., and Mii, M. (2009). Efficient plant regeneration via organogenesis in "Egusi" melon (Colocynthis citrullus L.). Sci. Hortic. 119, 397-402. doi: 10.1016/j.scienta.2008.08.031

Ogawara, T., Higashi, K., Kamada, H., and Ezura, H. (2003). Ethylene advances the transition from vegetative growth to flowering in Arabidopsis thaliana. J. Plant Physiol. 160, 1335-1340. doi: 10.1078/0176-1617-01129

Palmer, C., Golden, K., Danniels, L., and Ahmad, H. (2007). ACC deaminase from Issatchenkia occidentalis. J. Biol. Sci. 7, 188-193. doi: 10.3923/jbs.2007.188.193

Pradel, K. S., Ullrich, C. I., Santa Cruz, S., and Oparka, K. J. (1999). Symplastic continuity in Agrobacterium tumefaciens-induced tumours. J. Exp. Bot. 50, 183192. doi: $10.1093 / \mathrm{jxb} / 50.331 .183$ 
Pradel, S. K., Rezmer, C., Krausgrill, S., Rausch, T., and Ullrichm, C. I. (1996). Evidence for symplastic phloem unloading with concomitant high activity of acid cell wall invertase in Agrobacterium tumefaciens-induced plant tumors. Bot. Acta 109, 397-404. doi: 10.1111/j.1438-8677.1996.tb00589.x

Penninckx, I., Eggermont, K., Terras, F. R. G., Thomma, B. P. H. J., Samblanx, G. W. D., Buchala, A., et al. (1996). Pathogen-induced systemic activation of a plant defensin gene in Arabidopsis follows a salicylic acid-independent pathway. Plant Cell 8, 2309-2323. doi: 10.1105/tpc.8.12.2309

Penrose, D. M., and Glick, B. R. (2001). Levels of ACC and related compounds in exudate and extracts of canola seeds treated with ACC deaminasecontaining plant growth-promoting bacteria. Can. J. Microbiol. 47, 368-372. doi: 10.1139/w01-014

Petri, C., Alburquerque, N., Perez-Tornero, O., and Burgos, L. (2005). Auxin pulses and a synergistic interaction between polyamines and ethylene inhibitors improve adventitious regeneration from apricot leaves and Agrobacteriummediated transformation of leaf tissues. Plant Cell Tissue Organ Cult. 82, 105111. doi: 10.1007/s11240-004-7013-y

Plett, J. M., McDonnell, L., and Regan, S. (2009). Plant encoded 1aminocyclopropane-1-carboxylic acid deaminase activity implicated in different aspects of plant development. Plant Signal. Behav. 4, 1186-1189. doi: 10.1111/j.1399-3054.2009.01208.x

Seong, E. S., Song, K. J., Jegal, S., Yu, C. Y., and Chung, I. M. (2005). Silver nitrate and aminoethoxyvinylglycine affect Agrobacterium-mediated apple transformation. Plant Growth Regul. 45, 75-82. doi: 10.1007/s10725-004-6126-y

Sessitsch, A., Coenye, T., Sturz, A. V., Vandamme, P., Barka, E., Wang-Pruski, G., et al. (2005). Burkholderia phytofirmans sp. nov., a novel plant-associated bacterium with plant-beneficial properties. Int. J. Syst. Evol. Microbiol. 55, 11871192. doi: 10.1099/ijs.0.63149-0

Shaharoona, B., Arshad, M., and Zahir, Z. A. (2006a). Effect of plant growth promoting rhizobacteria containing ACC-deaminase on maize (Zea mays L.) growth under axenic conditions and on nodulation in mung bean (Vigna radiata L.). Lett Appl. Microbiol. 42, 155-159. doi: 10.1111/j.1472-765X.2005. 01827.x

Shaharoona, B., Arshad, M., Zahir, Z. A., and Khalid, A. (2006b). Performance of Pseudomonas spp. containing ACC-deaminase for improving growth and yield of maize (Zea mays L.) in the presence of nitrogenous fertilizer. Soil Biol. Biochem. 38, 2971-2975. doi: 10.1016/j.soilbio.2006.03.024

Stachel, S. E., Messens, E., van Montagu, M., and Zambryski, P. (1985). Identification of the signal molecules produced by wounded plant cells that activate T-DNA transfer in Agrobacterium tumefaciens. Nature 318, 624-629. doi: $10.1038 / 318624 \mathrm{a} 0$

Stachel, S. E., Nester, E. W., and Zambryski, P. C. (1986). A plant cell factor induces Agrobacterium tumefaciens vir gene expression. Proc. Natil. Acad. Sci. U.S.A. 83, 379-383. doi: 10.1073/pnas.83.2.379

Siddikee, M. A., Glick, B. R., Chauhan, P. S., Yim, W. J., and Sa, T. (2011). Enhancement of growth and salt tolerance of red pepper seedlings (Capsicum annuum L.) by regulating stress ethylene synthesis with halotolerant bacteria containing 1-aminocyclopropane-1-carboxylic acid deaminase activity. Plant Physiol. Biochem. 49, 427-434. doi: 10.1016/j.plaphy.2011. 01.015

Singh, N., and Kashyap, S. (2012). In silico identification and characterization of 1aminocyclopropane-1-carboxylate deaminase from Phytophthora sojae. J. Mol. Model. 18, 4101-4111. doi: 10.1007/s00894-012-1389-0

Smith, J. J., Ververidis, P., and John, P. (1992). Characterization of the ethyleneforming enzyme partially purified from melon. Phytochemistry 31, 1485-1494. doi: 10.1016/0031-9422(92)83092-D

Someya, T., Nonaka, S., Nakamura, K., and Ezura, H. (2013). Increased 1aminocyclopropane-1-carboxylate deaminase activity enhances Agrobacterium tumefaciens-mediated gene delivery into plant cells. Microbiologyopen 2, 873880. doi: $10.1002 / \mathrm{mbo} 3.123$

Toppan, A., Roby, D., and Esquerre-Tugaye, M. T. (1982). Cell surfaces in plant-microorganism interactions: III. In vivo effect of ethylene on hydroxyproline-rich glycoprotein accumulation in the cell wall of diseased plants. Plant Physiol. 70, 82-86. doi: 10.1104/pp.70.1.82

van Larebeke, N., Engler, G., Holsters, M., van den Elsacker, S., Schelperoort, R. A., and Schell, J. (1974). Large plasmid in Agrobacterium tumefaciens essential for crown gall-inducing ability. Nature 252, 169-170. doi: 10.1038/ 252169a0

van Larebeke, N., Genetello, C., Schell, J., Schilperoort, R. A., Hermans, A. K., van Montagu, M., et al. (1975). Acquisition of tumour-inducing ability by nononcogenic agrobacteria as a result of plasmid transfer. Nature $255,742-743$. doi: $10.1038 / 255742 \mathrm{a} 0$

Veena, Jiang, H., Doerge, R. W., and Gelvin, S. B. (2003). Transfer of T-DNA and Vir proteins to plant cells by Agrobacterium tumefaciens induces expression of host genes involved in mediating transformation and suppresses host defense gene expression. Plant J. 35, 219-236. doi: 10.1046/j.1365-313X.2003. 01796.x

Viterbo, A., Landau, U., Kim, S., Chernin, L., and Chet, I. (2010). Characterization of ACC deaminase from the biocontrol and plant growth-promoting agent Trichoderma asperellum T203. FEMS Microbiol. Lett. 305, 42-48. doi: 10.1111/j.1574-6968.2010.01910.x

Wang, K. L., Li, H., and Ecker, J. R. (2002). Ethylene biosynthesis and signaling networks. Plant Cell 14(Suppl.), S131-S151. doi: 10.1105/tpc.001768

Wächter, R., Langhans, M., Aloni, R., Götz, S., Weilmünster, A., Koops, A., et al. (2003). Vascularization, high-volume solution flow, and localized roles for enzymes of sucrose metabolism during tumorigenesis by Agrobacterium tumefaciens. Plant Physiol. 133, 1024-1037. doi: 10.1104/pp.103.028142

Watson, B., Currier, T. C., Gordon, M. P., Chilton, M. D., and Nester, E. W. (1975). Plasmid required for virulence of Agrobacterium tumefaciens. J. Bacteriol. 123, 255-264.

Whipps, J. M. (1990). “Carbon utilization," in The Rhizosphere, ed. J. M. Lynch (Chichester: Wiley), 59-97.

Wood, D. W., Setubal, J. C., Kaul, R., Monks, D. E., Kitajima, J. P., Okura, V. K., et al. (2001). The genome of the natural genetic engineer Agrobacterium tumefaciens C58. Science 294, 2317-2323. doi: 10.1126/science.1066804

Yang, S. F. (1985). Biosynthesis and action of ethylene. HortScience 20, 41-45.

Yang, S. F., and Hoffman, N. E. (1984). Ethylene biosynthesis and its regulation in higher plants. Annu. Rev. Plant Physiol. 35, 155-189. doi: 10.1146/annurev.pp.35.060184.001103

Zaenen, I., van Larebeke, N., Van Montagu, M., and Schell, J. (1974). Supercoiled circular DNA in crown-gall inducing Agrobacterium strains. J. Mol. Biol. 86, 109-127. doi: 10.1016/S0022-2836(74)80011-2

Zambryski, P., Joos, H., Genetello, C., Leemans, J., Montagu, M. V., and Schell, J. (1983). Ti plasmid vector for the introduction of DNA into plant cells without alteration of their normal regeneration capacity. EMBO J. 2, 21432150 .

Conflict of Interest Statement: The authors declare that the research was conducted in the absence of any commercial or financial relationships that could be construed as a potential conflict of interest.

Received: 03 September 2014; paper pending published: 04 October 2014; accepted: 15 November 2014; published online: 03 December 2014.

Citation: Nonaka S and Ezura H (2014) Plant-Agrobacterium interaction mediated by ethylene and super-Agrobacterium conferring efficient gene transfer. Front. Plant Sci. 5:681. doi: 10.3389/fpls.2014.00681

This article was submitted to Plant Physiology, a section of the journal Frontiers in Plant Science.

Copyright (C) 2014 Nonaka and Ezura. This is an open-access article distributed under the terms of the Creative Commons Attribution License (CC BY). The use, distribution or reproduction in other forums is permitted, provided the original author(s) or licensor are credited and that the original publication in this journal is cited, in accordance with accepted academic practice. No use, distribution or reproduction is permitted which does not comply with these terms. 


\section{ADVANTAGES OF PUBLISHING IN FRONTIERS}
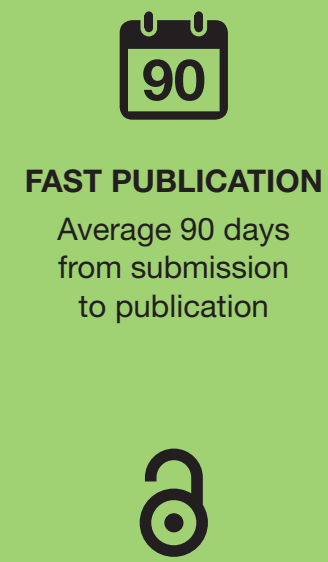

OPEN ACCESS

Articles are free to read, for greatest visibility

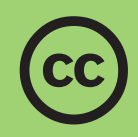

\section{COPYRIGHT TO AUTHORS}

No limit to article distribution and re-use

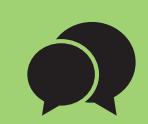

COLLABORATIVE

PEER-REVIEW

Designed to be rigorous yet also collaborative, fair and constructive

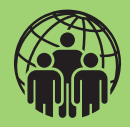

\section{TRANSPARENT}

Editors and reviewers acknowledged by name on published articles

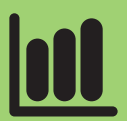

IMPACT METRICS

Advanced metrics track your article's impact

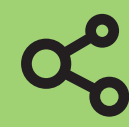

\section{RESEARCH NETWORK}

Our network increases readership

for your article

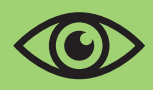

\section{GLOBAL SPREAD}

Six million monthly page views worldwide

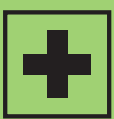

SUPPORT

By our Swiss-based editorial team 